\title{
ARRAKIS: atlas of resonance rings as known in the $S^{4} G^{\star}$
}

\author{
S. Comerón ${ }^{1,2,3}$, H. Salo ${ }^{1}$, E. Laurikainen ${ }^{1,2}$, J. H. Knapen ${ }^{4,5}$, R. J. Buta ${ }^{6}$, M. Herrera-Endoqui ${ }^{1}$, J. Laine ${ }^{1}$, \\ B. W. Holwerda ${ }^{7}$, K. Sheth ${ }^{8}$, M. W. Regan ${ }^{9}$, J. L. Hinz ${ }^{10}$, J. C. Muñoz-Mateos ${ }^{11}$, A. Gil de Paz ${ }^{12}$, \\ K. Menéndez-Delmestre ${ }^{13}$, M. Seibert ${ }^{14}$, T. Mizusawa ${ }^{8,15}$, T. $_{\text {Kim }}^{8,11,14,16}$, S. Erroz-Ferrer ${ }^{4,5}$, D. A. Gadotti ${ }^{10}$, \\ E. Athanassoula ${ }^{17}, \mathrm{~A}$. Bosma ${ }^{17}$, and L. C. $\mathrm{Ho}^{14,18}$ \\ 1 University of Oulu, Astronomy Division, Department of Physics, PO Box 3000, 90014 Oulu, Finland \\ e-mail: seb.comeron@gmail.com \\ ${ }^{2}$ Finnish Centre of Astronomy with ESO (FINCA), University of Turku, Väisäläntie 20, 21500 Piikkiö, Finland \\ 3 Korea Astronomy and Space Science Institute, 776, Daedeokdae-ro, Yuseong-gu, 305-348 Daejeon, Republic of Korea \\ ${ }^{4}$ Instituto de Astrofísica de Canarias, 38205 La Laguna, Tenerife, Spain \\ 5 Departamento de Astrofísica, Universidad de La Laguna, 38200 La Laguna, Tenerife, Spain \\ 6 Department of Physics and Astronomy, University of Alabama, Box 870324, Tuscaloosa, AL 35487, USA \\ 7 European Space Agency, ESTEC, Keplerlaan 1, 2200 AG Noorwijk, The Netherlands \\ 8 National Radio Astronomy Observatory/NAASC, 520 Edgemont Road, Charlottesville, VA 22903, USA \\ 9 Space Telescope Science Institute, 3700 San Antonio Drive, Baltimore, MD 21218, USA \\ 10 European Southern Observatory, 19001 Casilla, Santiago 19, Chile \\ 11 MMTO, University of Arizona, 933 North Cherry Avenue, Tucson, AZ 85721, USA \\ 12 Departamento de Astrofísica, Universidad Complutense de Madrid, 28040 Madrid, Spain \\ 13 Universidade Federal do Rio de Janeiro, Observatório do Valongo, Ladeira Pedro Antônio, 43, CEP 20080-090 Rio de Janeiro, \\ Brazil \\ 14 The Observatories of the Carnegie Institution for Science, 813 Santa Barbara Street, Pasadena, CA 91101, USA \\ 15 Florida Institute of Technology, Melbourne, FL 32901, USA \\ 16 Astronomy Program, Department of Physics and Astronomy, Seoul National University, 151-742 Seoul, Republic of Korea \\ 17 Aix Marseille Université, CNRS, LAM (Laboratoire d'Astrophysique de Marseille) UMR 7326, 13388 Marseille, France \\ 18 Kavli Institute for Astronomy and Astrophysics, Peking University, 100871 Beijing, PR China
}

Received 3 April 2013 / Accepted 2 December 2013

\begin{abstract}
Context. Resonance rings and pseudorings (here collectively called rings) are thought to be related to the gathering of material near dynamical resonances caused by non-axisymmetries in galaxy discs. This means that they are the result of secular evolution processes that redistribute material and angular momentum in discs. Studying them may give clues on the formation and growth of bars and other disc non-axisymmetries.

Aims. Our aims are to produce a catalogue and an atlas of the rings detected in the Spitzer Survey of Stellar Structure in Galaxies $\left(\mathrm{S}^{4} \mathrm{G}\right)$ and to conduct a statistical study of the data in the catalogue.

Methods. We traced the contours of rings previously identified and fitted them with ellipses. We found the orientation of bars by studying the galaxy ellipse fits from the $S^{4} G$ pipeline 4 . We used the galaxy orientation data obtained by the $S^{4} G$ pipeline 4 to obtain intrinsic ellipticities and orientations of rings and the bars.

Results. ARRAKIS contains data on 724 ringed galaxies in the $\mathrm{S}^{4} \mathrm{G}$. The frequency of resonance rings in the $\mathrm{S}^{4} \mathrm{G}$ is of $16 \pm 1 \%$ and $35 \pm 1 \%$ for outer and inner features, respectively. Outer rings are mostly found in Hubble stages $-1 \leq T \leq 4$. Inner rings are found in a broad distribution that covers the range $-1 \leq T \leq 7$. We confirm that outer rings have two preferred orientations, namely parallel and perpendicular to the bar. We confirm a tendency for inner rings to be oriented parallel to the bar, but we report the existence of a significant fraction (maybe as large as 50\%) of inner features that have random orientations with respect to the bar. These misaligned inner rings are mostly found in late-type galaxies $(T \geq 4)$. We find that the fraction of barred galaxies hosting outer (inner) rings is $\sim 1.7$ times ( 1.3 times) that in unbarred galaxies.

Conclusions. We confirm several results from previous surveys as well as predictions from simulations of resonant rings and/or from manifold flux tube theory. We report that a significant fraction of inner rings in late-type galaxies have a random orientation with respect to the bar. This may be caused by spiral modes that are decoupled from the bar and dominate the Fourier amplitude spectrum at the radius of the inner ring. The fact that rings are only mildly favoured by bars suggests that those in unbarred galaxies either formed because of weak departures from the axisymmetry of the galactic potential or that they are born because of bars that were destroyed after the ring formation.
\end{abstract}

Key words. atlases - catalogs - galaxies: statistics - galaxies: structure

$\star$ Tables A.1 and A.2 (catalogue) are only available at the CDS via anonymous ftp to cdsarc.u-strasbg.fr (130.79.128.5) or via http://cdsarc.u-strasbg.fr/viz-bin/qcat?]/A+A/562/A121 


\section{Introduction}

Galaxies are constantly evolving. Their properties change because of fast interactions and mergers (Toomre 1977) and also because of slow secular evolution (e.g., Kormendy \& Kennicutt 2004; Athanassoula 2012a). Part of the secular evolution in disc galaxies is driven by non-axisymmetries such as bars and oval distortions. Long-lived non-axisymmetries efficiently redistribute material and angular momentum across the discs in a Hubble-Lemaître time, which makes understanding them crucial to describe present-day galaxies. This slow process is, among many other consequences, responsible for the creation of pseudobulges (Kormendy \& Kennicutt 2004), for the radial spread of outer parts of discs (Schwarz 1984; Athanassoula 2012b), and also for building spectacular rings and pseudorings. In this paper we study rings and pseudorings in a representative sample of nearby galaxies.

Rings are beautiful closed structures made of stars and/or gas. Pseudorings are their open counterparts, sometimes incomplete versions of rings and sometimes formed by spiral arms that almost connect. Here we use the word rings to refer to the set that includes both rings and pseudorings. The set of closed features is referred to as closed rings.

Rings often host intense star formation and/or are made of young blue stars (see, e.g., Buta \& Crocker 1993; Knapen et al. 1995; Crocker et al. 1996; Buta 2002; Knapen 2005; Buta et al. 2007; Comerón et al. 2010; Grouchy et al. 2010). However, "dead" purely stellar rings exist as well (Buta 1991; Erwin \& Sparke 1999, 2002; Comerón 2013).

The majority of rings seen in normal disc galaxies are likely caused by the influence of dynamical orbital resonances on the motions of gas clouds in the plane of the disc. The presence of a rotating bar or oval sets up a pattern speed and probably drives spiral patterns that can, via action of gravity torques, secularly evolve into more closed, ring-like features (Schwarz 1981, 1984). The main evidence in support of this idea has come from observations of ring morphologies, intrinsic ring shapes, and intrinsic bar and ring major axis orientations as well as test-particle and $n$-body simulations (see review by Buta \& Combes 1996 and Rautiainen \& Salo 2000).

There are four major and two secondary dynamical resonances that are believed to be important for ring formation (for detailed reviews of barred galaxy dynamics, see Sellwood \& Wilkinson 1993; Athanassoula 2012a). These are defined (in the epicyclic approximation) by the relation between the bar pattern speed, $\Omega_{\mathrm{p}}$, the circular angular speed $\Omega$, and the radial epicyclic frequency $\kappa$. The major resonances are the outer Lindblad resonance (OLR, where $\Omega_{\mathrm{p}}=\Omega+\kappa / 2$ ), the corotation resonance (CR, where $\Omega_{\mathrm{p}}=\Omega$ ), and the two inner Lindblad resonances (ILRs, where $\Omega_{p}=\Omega-\kappa / 2$ ). The secondary resonances are the inner 4:1 resonance (often called the UHR for ultraharmonic resonance, but which is here referred to as I4R, where $\Omega_{p}=\Omega-\kappa / 4$ ) and the outer $4: 1$ resonance $\left(\mathrm{O} 4 \mathrm{R}\right.$, where $\left.\Omega_{\mathrm{p}}=\Omega+\kappa / 4\right)$. Because $\Omega$ and $\kappa$ depend on the rotation curve of a galaxy, resonance locations will be tied to the gravitational potential of the system. For example, depending on the bar pattern speed and the central mass concentration, one or both ILRs may be absent. Typically, the OLR is located at a radius of roughly twice the bar length, the CR is located slightly outside the end of the bar, and the ILRs are well inside the bar.

Between the outermost ILR and the CR, the main family of orbits, called $x_{1}$ orbits, is parallel to the bar. In the framework of the epicyclic approximation, each time a main resonance is crossed, the orbits change their orientation by $90^{\circ}$. Because of this, orbits that are slightly inside a resonance will intersect with those slightly outside it. Graphic depictions of this can be found, for instance, in Fig. 11 in Knapen et al. (1995) and Fig. 2 in Englmaier \& Gerhard (1997). Growing bars redistribute the angular momentum in a galaxy. Gas outside CR tends to be moved to the OLR radius (Lynden-Bell \& Kalnajs 1972; Sellwood 1981; Schwarz 1981). Gas inside CR tends to move inwards and is collected either close to the I4R and/or close to the ILRs (Schwarz 1984). Thus, orbits near resonances are fed with gas by the bar angular momentum redistribution. Because of the high gas densities reached there and because of the collisions of gas clumps moving in intersecting orbits, star formation starts and resonance rings are created. Although strictly speaking the epicyclic approximation is no longer valid for strongly perturbed potentials such as those affected by a strong bar (for a good example of that see Salo et al. 1999), the picture described here remains qualitatively valid.

Rings in barred galaxies are classified according to their size compared to that of the bar. The precise location of a resonance cannot be determined unless the rotation curve of the galaxy and the pattern speed of the bar are known. Thus the resonance interpretations come from a confrontation between theory and observation:

- Outer rings are found at a radius roughly twice as large as that of the bar and are thought to be typically related to the OLR. Occasionally, some outer rings may be linked to the O4R as in the case of the dimpled ones in Byrd et al. (1998), those in simulations by Rautiainen \& Salo (2000), and in the modelling of NGC 1433 by Treuthardt et al. (2008). The first outer feature that has been described is that in NGC 1291 (Perrine 1922).

- Inner rings are found slightly outside the bar and are thought to be related to the I4R. They have been observed since R. J. Mitchell's observations of NGC 4725 in 1858 (Parsons 1880). They were first described by Curtis (1918).

- Nuclear rings are found well inside the bar and are generally thought to be related to the ILRs (but see Kim et al. 2012, where nuclear rings are suggested to be caused by the centrifugal barrier encountered by gas migrating to the inner regions of the galaxy). They were first seen in NGC 4321 by Keeler (1908) and described in NGC 3351 by Curtis (1918). Nuclear rings are especially bright and correlate with the presence of sigma-drops (sigma-drops are nuclear regions in the centre of galaxies where the stellar velocity dispersion is measured to be lower than in the surroundings; Comerón et al. 2008b).

We caution about the naming conventions and note that inner rings in barred galaxies are not thought to be related to the inner Lindblad resonances, but to the I4R.

Based on data from the Third Reference Catalogue of Bright Galaxies (RC3; de Vaucouleurs et al. 1991), Buta \& Combes (1996) found that roughly $10 \%$ of disc galaxies host outer rings and that $45 \%$ of disc galaxies host inner rings. Comerón et al. (2010) found that the fraction of nuclear rings is roughly $20 \%$ for galaxies with Hubble stages between $T=-3$ and $T=7$.

Rings have been associated to resonances since the pioneering works by Marochnik et al. (1972) and Schommer \& Sullivan (1976). Subsequently, this connection has been made in simulations by, among others, Schwarz (1981, 1984), Byrd et al. (1994), and Knapen et al. (1995). Because of that, outer, inner, and nuclear rings have historically been called resonance rings.

A complementary theory explains the formation of at least some rings. This theory, called manifold theory, shows in a 
single framework the creation of outer spirals and outer and inner rings. It proposes that they are made of particles trapped in invariant manifolds (tubes of orbits) that start and end in one of the two unstable Lagrangian points at the end of the bar, $L_{1}$ and $L_{2}$ (Romero-Gómez et al. 2006, 2007; Athanassoula et al. 2009b,a, 2010; Athanassoula 2012b). This theory predicts the existence of inner rings and that they are mainly oriented along the bar. It also predicts the existence of the outer $R_{1}, R_{2}$, and $\mathrm{R}_{1} \mathrm{R}_{2}$ rings and their orientation with respect to the bar (for a description of outer ring morphologies see Sect. 3.1). It also predicts the locations and axial ratios of rings. In fact, there is no prediction of the resonance ring simulations that the flux tube manifold theory does not predict as well, while the manifold shapes reproduce well that of rings in the response simulations mentioned above. The latter is possible because the values of the radii of the $L_{1}, L_{2}, L_{3}$, and $L_{4}$ Lagrangian points in barred galaxies are so close to that of the corotation radius that for observed galaxies they can be considered as equal. In this way it is possible to overcome the approximation of a resonant radius that is only valid in the linear and epicyclic approximations, that is, not in realistic barred galaxies. In the following we write manifold theory instead of flux tube manifold theory, and we use the generic name resonant rings for resonant and flux tube manifold rings.

Rings are also observed in galaxies that do not host a bar (see, e.g., Figs. 3e and 3f in Buta 1995). Comerón et al. (2010) claimed that the frequency of rings with sizes comparable with those of nuclear rings in unbarred galaxies $(19 \pm 4 \%)$ is similar to the frequency of unbarred disc galaxies. One possibility to explain the existence of such rings is that they are remnants left after the bar dissolved (for possible bar dissolution mechanisms, see e.g., Raha et al. 1991; Friedli \& Benz 1993; Bournaud \& Combes 2002; Bournaud et al. 2005) or was destroyed in interactions with other galaxies (Athanassoula 1996; Berentzen et al. 2003). However, the possibility of bar dissolution has to be taken with caution because many simulations showed that bars cannot be easily erased without interactions (see, e.g., Martinez-Valpuesta \& Shlosman 2004; Debattista et al. 2004, 2006; Berentzen et al. 2007; Athanassoula et al. 2013). Alternatively, these rings that apparently do not fit in the resonance theory might be explained by resonances caused by weak oval distortions, strong spiral patterns (Jungwiert \& Palous 1996; Rautiainen \& Salo 2000), or by the gravitational potential distortions induced during minor mergers and/or interactions. This last possibility has been suggested by Knapen et al. (2004) for the pseudoring in NGC 278 and by some numerical experiments (Tutukov \& Fedorova 2006). Moreover, rings can form out of satellite material during minor mergers (Eliche-Moral et al. 2011).

Another kind of interesting morphological feature in disc galaxies are the lenses. Lenses are typically found in early-type disc galaxies, they have flat luminosity profiles and fairly sharp edges. Lenses, like rings, can be classified as outer, inner, or nuclear, based on their sizes. They have been studied in detail in the Near-Infrared atlas of S0-Sa galaxies (NIRSOS; Laurikainen et al. 2011). Some features, called ring-lenses and first reported by Kormendy (1979), have properties between those of rings and lenses; they are lens-like features with a significant enhancement of luminosity close to their edges. In this paper we included ringlenses together with classical rings.

Rings can be responsible for a significant fraction of the light emitted by a galaxy. For example, the exceptionally bright nuclear ring in NGC 1097 emits $\sim 10 \%$ of the light of the galaxy at $3.6 \mu \mathrm{m}$ (Sheth et al. 2010). This can be considered as an estimate of the upper limit of the fraction of light coming from rings in present-day galaxies. However, since rings are often regions of intense star formation with a low mass-to-light ratio even in the mid-infrared, the fraction of the galaxy baryonic mass found in them is smaller than that.

Non-resonant mechanisms also produce rings, but they often have an appearance fairly different from that of resonance rings. A ring can be formed at the largest non-looping orbit whose major axis is parallel to the major axis of the bar. Such a ring is called an $x_{1}$-ring (Regan \& Teuben 2004). Collisional rings occur when a disc galaxy collides head-on with a satellite (Lynds $\&$ Toomre 1976; Theys \& Spiegel 1977). Polar rings occur when part of the mass of a satellite is accreted in an orbit perpendicular to the disc plane of the main galaxy (Schweizer et al. 1983). These three types of rings are rarer than resonance rings.

Moreover, some of the smallest nuclear rings (also called ultra-compact nuclear rings; Comerón et al. 2008a,c) might be related to the interaction of an AGN with the surrounding interstellar medium (Comerón et al. 2010).

Some rings that are coplanar with the galaxy disc may be related to polar rings. In a few galaxies, in-plane rings are found to be made of counterrotating material postulated to come from a recent minor merger. This could be the case, for instance, for the outer ring in IC 2006 (Schweizer et al. 1989; Bettoni et al. 2001), the inner ring in NGC 3593 (Corsini et al. 1998), the inner ring in NGC 4138 (Jore et al. 1996; Thakar et al. 1997), and the innermost ring in NGC 7742 (Sil'chenko \& Moiseev 2006; Mazzuca et al. 2006).

In this paper we present a catalogue (Appendix A) and an atlas (Appendix B) of the rings identified in the Spitzer Survey of Stellar Structure in Galaxies ( $S^{4} G$; Sheth et al. 2010). The focus is on resonance rings, hence the title ARRAKIS: atlas of resonance rings as known in the $\mathrm{S}^{4} \mathrm{G}$. In the catalogue section, we present data on the projected and intrinsic ring major and minor axes and orientations. We also present the projected and intrinsic highest bar ellipticities, which can be used as a rough estimate of the bar strength, and the bar orientations. The atlas presents images of all the galaxies with rings. We overlay the measured contour of the rings on these images.

The paper is structured as follows: in Sect. 2 we briefly describe the $S^{4} \mathrm{G}$ and the ring identification process, in Sect. 3 we describe the types of rings detected in the $S^{4} G$, and in Sect. 4 we describe the production process of the catalogue. We compare our measured ring properties with those in previous studies in Sect. 5, we present some statistical results on the ring fraction, their intrinsic axis ratio, their position angle (PA) offset with bars, and sizes in Sect. 6, and we discuss some of these points in Sect. 7. We summarise our results and conclusions in Sect. 8. The catalogue is presented in Appendix A and the atlas of rings is presented in Appendix B. Appendix C contains a list of galaxies in the catalogue with a duplicated NGC/IC identification.

\section{Identification of rings and pseudorings in the $S^{4} G$}

\subsection{The $S^{4} G$}

The rings in this paper are identified and described using $\mathrm{S}^{4} \mathrm{G}$ images. The $S^{4} \mathrm{G}$ is a mid-infrared survey that has observed a sample of galaxies representative of the local Universe using the InfraRed Array Camera (IRAC; Fazio et al. 2004) of the Spitzer Space Telescope (Werner et al. 2004). The goal of the $S^{4} \mathrm{G}$ is to describe the stellar mass distribution in the local Universe. The mid-infrared is the ideal band to do so because it 
has little dust absorption. The selection criteria of the sample are the following:

- Radio radial heliocentric velocity $v_{\text {radio }}<3000 \mathrm{~km} \mathrm{~s}^{-1}$, which is equivalent to a distance of $D<41 \mathrm{Mpc}$ when using a Hubble-Lemaître constant of $H_{0}=73 \mathrm{~km} \mathrm{~s}^{-1} \mathrm{Mpc}^{-1}$.

- Integrated blue magnitude $m_{B \text {,corr }}<15.5$ mag. The magnitude considered here is that corrected for Galactic extinction, inclination, and $K$-correction.

- Angular diameter $D_{25}>1^{\prime}$.

- Galactic latitude $|b|>30^{\circ}$ to avoid the observations to be overly affected by foreground stars and other Galactic emission.

All these parameters were taken from HyperLeda (Paturel et al. 2003). One of the reasons why the sample is representative and not volume-limited is that the galaxies in the sample are limited in diameter and in brightness. Another source of incompleteness is that one of the main inputs of HyperLeda is the RC3, which is only reasonably complete at $m_{B}<15.5 \mathrm{mag}$ (this time the blue magnitude is uncorrected). An additional reason is that some galaxies, especially those of earlier types, did not have a radial velocity in radio in HyperLeda at the time when the sample was defined. The final $\mathrm{S}^{4} \mathrm{G}$ sample size is 2352 galaxies.

The images were taken to map the galaxies at least up to $1.5 \times D_{25}$ and mosaics were produced when needed. The galaxies were observed in the 3.6 and $4.5 \mu \mathrm{m}$ filters of IRAC with a total integration time of four minutes, obtaining images with a surface brightness sensitivity of $\mu(\mathrm{AB})_{3.6 \mu \mathrm{m}} \sim 27 \mathrm{mag} \operatorname{arcsec}^{-2}$. Such deep images are unprecedented for a large survey of galaxies in the mid-infrared.

Because the distances used here are redshift-independent or Hubble-Lemaître flow-corrected, some of the galaxy distances listed in the table in Appendix A exceed $41 \mathrm{Mpc}$, as explained in Sect. 6.5.

The $\mathrm{S}^{4} \mathrm{G}$ data is now public and can be downloaded from the NASA/IPAC Infrared Science Archive (IRSA) website ${ }^{1}$.

Some galaxies in the $S^{4} G$ have two identifications in the NGC and IC catalogues (Dreyer 1888, 1895). To facilitate the comparison of the data presented here with other samples, Appendix C presents a list of the galaxies with a double identification that have been included in ARRAKIS. This list has been made by using mostly data from The Historically Corrected New General Catalogue ${ }^{2}$, but also from NED and HyperLeda.

\subsection{Morphological classification of $S^{4} G$ galaxies}

Buta et al. (in prep.) classified most of the galaxies in the $S^{4} G$ using the criteria described in Buta et al. (2010) and Laurikainen et al. (2011). In brief, the galaxies were classified using the de Vaucouleurs revised Hubble-Sandage system (de Vaucouleurs 1959) and its revision (de Vaucouleurs 1963), which has three dimensions, namely the stage $\left(\mathrm{E}, \mathrm{E}^{+}, \mathrm{S}^{-}, \mathrm{S}^{\circ}, \mathrm{S}^{+}, \mathrm{S} 0 / \mathrm{a}, \mathrm{Sa}\right.$, $\mathrm{Sab}, \mathrm{Sb}, \mathrm{Sbc}, \mathrm{Sc}, \mathrm{Scd}, \mathrm{Sd}, \mathrm{Sdm}, \mathrm{Sm}, \mathrm{Im}$ ), the family (SA, SAB, $\mathrm{SAB}, \mathrm{SA} \underline{\mathrm{B}}, \mathrm{SB})$, and the variety (r, rs, rs, rs, s). Additional dimensions were added to the classification by indicating which galaxies are highly inclined (spindle or $\mathrm{sp}$ ), which galaxies are double-staged, which galaxies have shells, lenses, nuclear bars, nuclear discs, triaxial bulges, ansæ, X-shaped bars, tidal debris, pseudobulges, and other peculiarities. Most important for this work, they also classified which galaxies host outer and nuclear rings. For an extensive review of this matter see Buta (2013).

\footnotetext{
1 http://irsa.ipac.caltech.edu/

2 http://www.ngcicproject.org
}

The galaxies were classified using the $S^{4} \mathrm{G} 3.6 \mu \mathrm{m}$ band and interpreting the images as if they were blue light. The classifications in both bands are in general very similar and only differ in galaxies with stages between S0/a and Sc, for which the classification in $3.6 \mu \mathrm{m}$ is on average one stage earlier than in the $B$ band (Buta et al. 2010). This one-stage shift should be taken into account when comparing ARRAKIS results with those in previous studies based on $B$-band imaging.

A problem may appear when, assuming that rings are related to resonances, those in unbarred galaxies are classified as outer, inner, and nuclear rings. In that case, they were classified comparing the ring size with the galaxy radius and/or the location where they appear in relation to spiral arms. This approach may cause some rings to be misclassified, as discussed in Buta (1995) and Comerón et al. (2010).

A few of the classified galaxies have two or even three features that fit in the outer, inner, or nuclear categories. For example, NGC 5055 is an SA(rs,rl)bc galaxy and has two inner features. In this case, the feature that appears first in the classification (rs) is the outermost feature. The only exceptions for this rule are $R_{1} R_{2}^{\prime}$ and $R_{1}^{\prime} R_{2}^{\prime}$ combinations, for which the order is reversed because of historical reasons.

Because of the limited amount of dust obscuration it suffers, the $3.6 \mu \mathrm{m}$ band presents an advantage over optical wavelengths at detecting and describing rings that otherwise could remain hidden. This, combined with the angular resolution of the images in the $S^{4} \mathrm{G}(0.75$ pixel size and a full width at half maximum of $\sim 2^{\prime \prime}$ ), allowed us to identify rings with diameters down to $\sim 10^{\prime \prime}$ in low-inclination galaxies. We here considered low-inclination galaxies to be those with $i \leq 60^{\circ}$. This value is based on our ability to measure bar properties as explained in Sects. 4.5 and 4.6. The ring identification becomes increasingly difficult for higher disc inclinations due to higher optical depth and ring foreshortening (see, e.g., Tables IV and V in Buta \& Combes 1996). A diameter of $10^{\prime \prime}$ corresponds to $1.0 \mathrm{kpc}$ at a distance $20 \mathrm{Mpc}$, and to $2.4 \mathrm{kpc}$ at a distance of $50 \mathrm{Mpc}$, below which $98 \%$ of ARRAKIS galaxies are found.

The completeness regarding outer rings is hard to assess; some outer rings are known to be exceedingly faint and some may have surface brightnesses below the sensitivity level of the $\mathrm{S}^{4} \mathrm{G}$. Additionally, in some cases, outer features are better seen in blue light: for example, the outer ring in NGC 7743, which is distinguishable in the $B$-band image in Buta et al. (2007), is not seen in the mid-infrared. Moreover, in at least one case (NGC 4151) an outer ring has not been detected here because it is larger than the region covered by the $S^{4} \mathrm{G}$ frame.

The completeness in the detection of inner rings is very high. When looking at galaxies with $D<20 \mathrm{Mpc}$, we find that only $5 \%$ of inner rings have a diameter smaller than $2.4 \mathrm{kpc}$ and therefore could not be detected for galaxies at $D=50 \mathrm{Mpc}$. We therefore expect to be missing less than $5 \%$ of the inner rings due to spacial resolution problems.

Regarding nuclear features, we cannot expect to be as complete as we are at finding inner rings: at a distance $D=20 \mathrm{Mpc}$, $5^{\prime \prime}$ correspond to a ring radius of $r=500 \mathrm{pc}$, which is similar to the average size of the nuclear rings detected in Comerón et al. (2010). Therefore, any statistics based on the nuclear rings in ARRAKIS must be considered uncertain and only representative of the behaviour of the largest nuclear rings.

A total of $2347 \mathrm{~S}^{4} \mathrm{G}$ galaxies have been classified in Buta et al. (in prep.). One of these galaxies, NGC 7204 (also named PGC 68061), is a pair composed of NGC 7204A and NGC 7204B, leading the count of $S^{4} G$ classified galaxies to 2348. Four $S^{4} \mathrm{G}$ galaxies could not be classified because they 


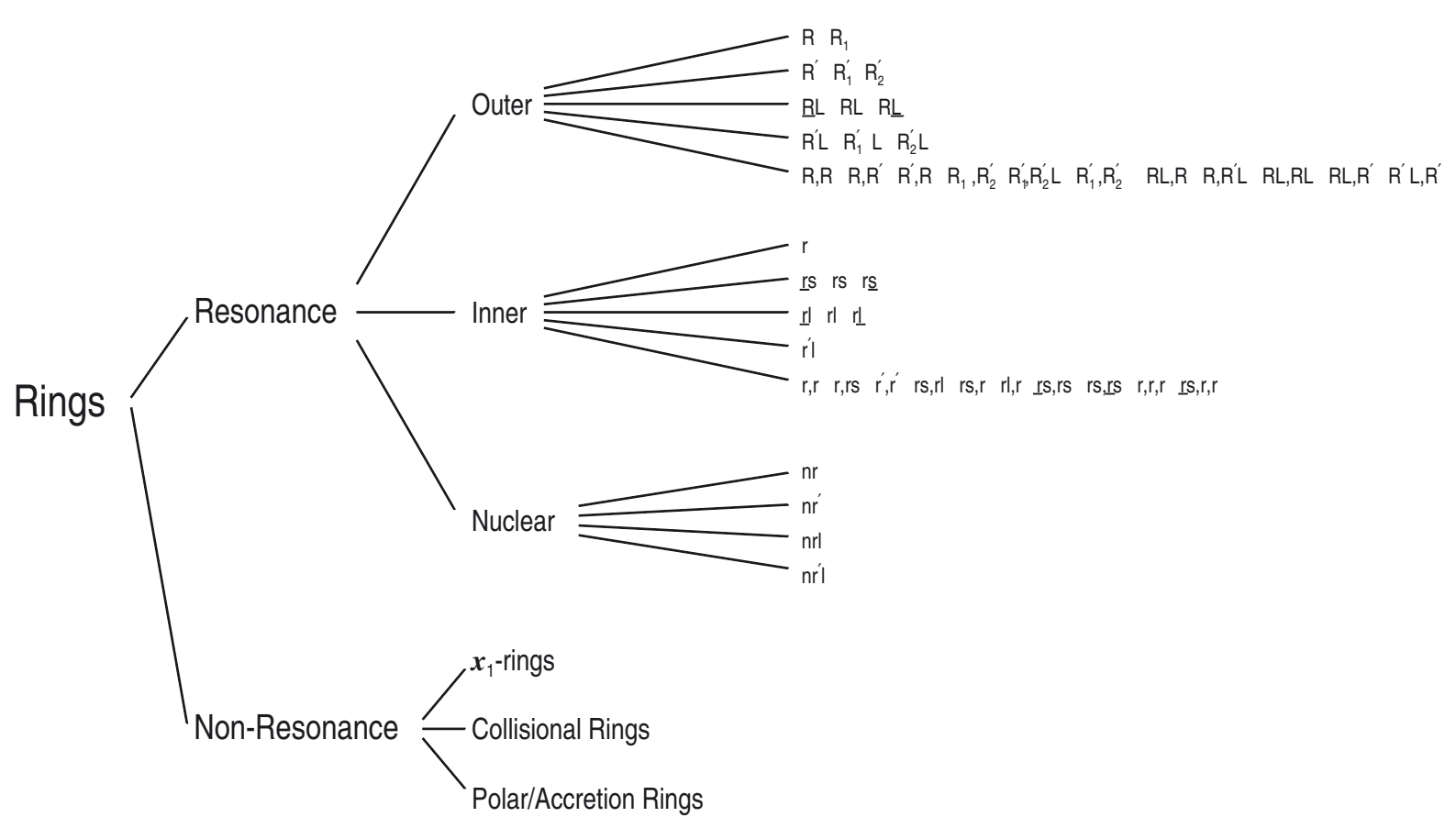

Fig. 1. Schematic classification of the ring types found in ARRAKIS. Multiple ring combinations are also included.

appear close to very bright saturated stars which makes the galaxy morphology hard to describe. In addition, 70 galaxies were classified that not belong to the $S^{4} G$ sample, but appear in the $\mathrm{S}^{4} \mathrm{G}$ frames. Most of these galaxies are satellites of galaxies in the $S^{4} \mathrm{G}$ sample or background galaxies. Thus, we have data of a total of 2418 galaxies for which there is a classification that considers the presence of rings.

The total number of galaxies that appear in $\mathrm{S}^{4} \mathrm{G}$ frames and have been classified to host rings is 735 . Of these, 724 galaxies are included in the original $\mathrm{S}^{4} \mathrm{G}$ sample. These 724 galaxies have 295 outer rings, 609 inner rings, 47 nuclear rings, 4 x -rings, , 4 collisional rings, and 7 polar rings.

To ensure the reliability of the ring statistics we considered only the galaxies in the $\mathrm{S}^{4} \mathrm{G}$ sample, which, as said before, is representative of the local Universe. However, for the sake of completeness, in the appendices we also included the data and the images corresponding to rings in galaxies not included in the original $\mathrm{S}^{4} \mathrm{G}$ sample.

\section{Ring morphologies}

The types of rings studied here are described below. A schematic description of the different ring categories can be found in Fig. 1. A glossary of some common morphological features is presented in Table 1. For more information on ring classification and morphology see, for example, Buta et al. (2007) and Buta (2013).

\subsection{Outer rings}

In barred galaxies, outer rings are those with roughly twice the size of the bar. They are thought to be related to the OLR or, occasionally, to the O4R. Since the work from de Vaucouleurs (1959), outer features have been divided into closed rings (R) and pseudorings $\left(\mathrm{R}^{\prime}\right)$, depending on whether the feature is closed/complete or not.

Additional subdivisions have been made later based on the morphology of the outer features in the simulations of Schwarz (1981, 1984) (see Fig. 2 in Buta 1986a). $R_{1}$ closed rings and $R_{1}^{\prime}$
Table 1. Glossary with some notation used in galaxy classification.

\begin{tabular}{|c|c|}
\hline Notation & Feature \\
\hline $\mathrm{R}$ & Outer closed ring \\
\hline $\mathrm{R}_{1}$ & Outer closed ring with Type 1 morphology \\
\hline $\mathrm{R}_{2}$ & Outer closed ring with Type 2 morphology \\
\hline $\mathrm{RL}, \mathrm{RL}, \mathrm{RL}$ & Outer closed ring-lenses \\
\hline$\overline{\mathrm{L}}$ & Outer lens \\
\hline $\mathrm{R}^{\prime}$ & Outer pseudoring \\
\hline $\mathrm{R}_{1}^{\prime}$ & Outer pseudoring with Type 1 morphology \\
\hline $\mathrm{R}_{2}^{\prime}$ & Outer pseudoring with Type 2 morphology \\
\hline$\underline{\mathrm{R}}^{\prime} \mathrm{L}, \mathrm{R}^{\prime} \mathrm{L}, \mathrm{R}^{\prime} \underline{\mathrm{L}}$ & Outer pseudoring-lenses \\
\hline $\mathrm{r}$ & Inner closed ring \\
\hline$\underline{\mathrm{r}}, \mathrm{rl}, \mathrm{r} \underline{\mathrm{l}}$ & Inner closed ring-lenses \\
\hline & Inner lens \\
\hline $\mathrm{r}^{\prime}, \underline{\mathrm{r} s}, \mathrm{rs}, \mathrm{rs}$ & Inner pseudorings \\
\hline $\mathrm{r}^{\prime} 1, \mathrm{r}^{\prime} 1, \mathrm{r}^{\prime}{ }^{-}$ & Inner pseudoring-lenses \\
\hline$\overline{\mathrm{nr}} \quad-$ & Nuclear closed ring \\
\hline $\mathrm{nl}$ & Nuclear lens \\
\hline $\mathrm{nrl}$ & Nuclear closed ring-lens \\
\hline $\mathrm{nr}^{\prime}$ & Nuclear pseudoring \\
\hline $\mathrm{nr}^{\prime} 1$ & Nuclear pseudoring-lens \\
\hline$x_{1} r$ & $\mathrm{x}_{1}$-ring \\
\hline RG & Ring galaxy (collisional ring) \\
\hline PRG & Polar ring galaxy \\
\hline
\end{tabular}

pseudorings consist of arms that start at one end of the bar and end after a $180^{\circ}$ bend in the other end of the bar. These rings typically have a dimpling in the region of the end of the bar and thus are 8-shaped. $\mathrm{R}_{2}$ closed rings and $\mathrm{R}_{2}^{\prime}$ pseudorings consist of two spiral arms, each starting at one of the ends of the bar and intersecting with each other at a position roughly perpendicular to the main axis of the bar after a $270^{\circ}$ bend. ARRAKIS includes some $\mathrm{R}_{2}^{\prime}$ pseudorings, but no $\mathrm{R}_{2}$ rings. According to the simulations from Schwarz $(1981,1984), R_{1}$ features are expected to be elongated and perpendicular to the major axis of the bar, and $\mathrm{R}_{2}$ features are expected to be elongated and parallel to the major axis of the bar. Sometimes galaxies host an $R_{1}^{\prime} R_{2}^{\prime}$ or an $R_{1} R_{2}^{\prime}$ combination. 

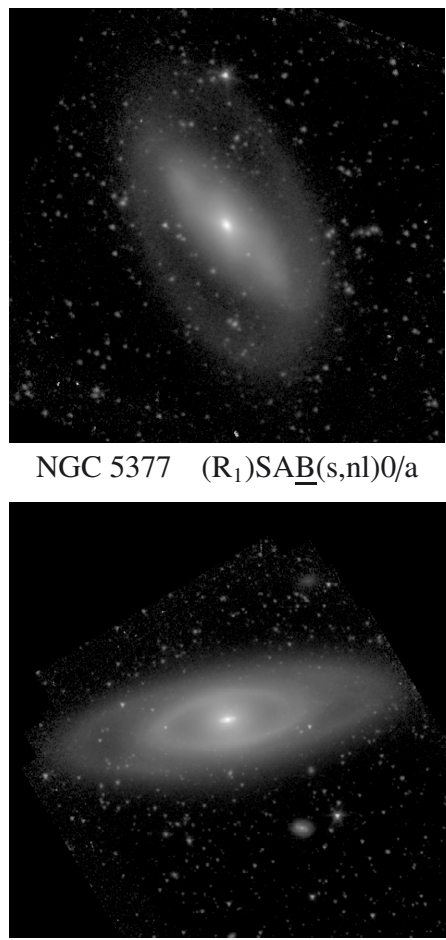

NGC 4274 (R)SB(r,nr)0/a

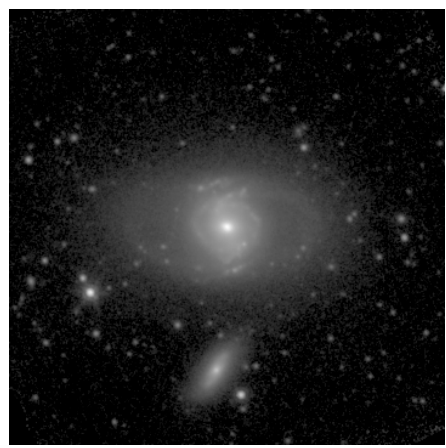

NGC $4045 \quad\left(\mathrm{R}_{1}^{\prime} \mathrm{L}\right) \mathrm{SAB}(\underline{\mathrm{rs}}, \mathrm{nl}) \mathrm{ab}$

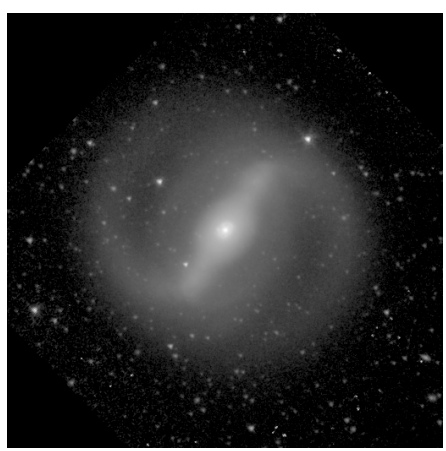

NGC $4314 \quad\left(\mathrm{R}_{1}^{\prime}\right) \mathrm{SB}(\mathrm{rl}, \mathrm{nr}) \mathrm{a}$
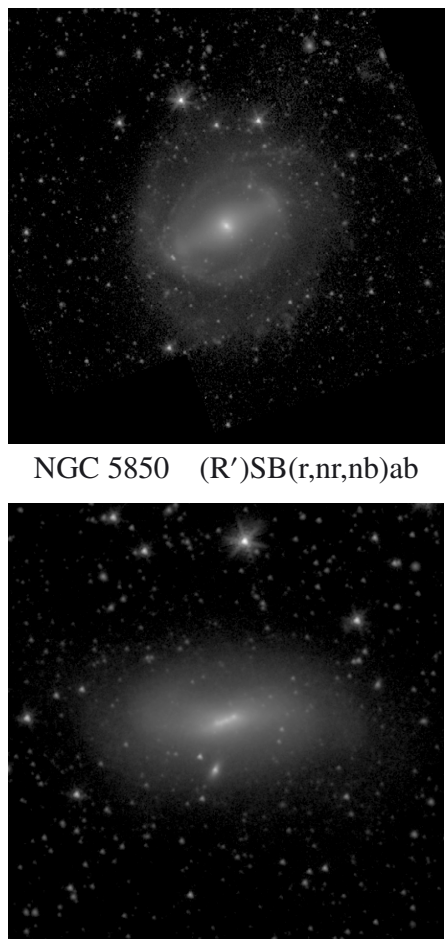

NGC $4424 \quad\left(\mathrm{R}_{2}^{\prime} \mathrm{L}\right) \mathrm{SB}(\mathrm{s}) 0 / \mathrm{a}$ pec

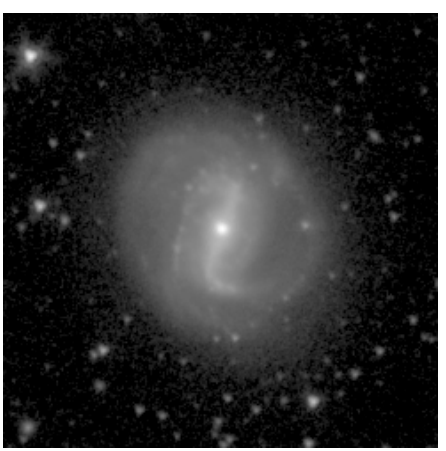

NGC $5757 \quad\left(\mathrm{R}_{2}^{\prime}\right) \mathrm{SB}(\mathrm{rs}) \underline{\mathrm{ab}}$

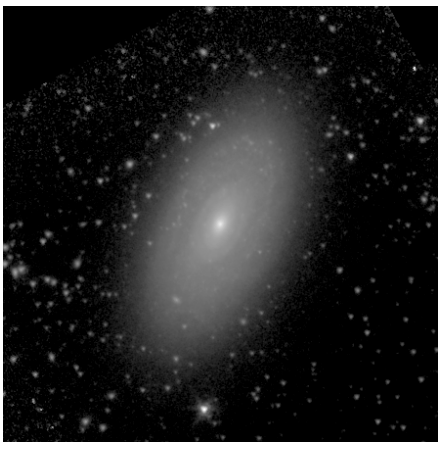

NGC 4380 (R)SA(r,l)ab

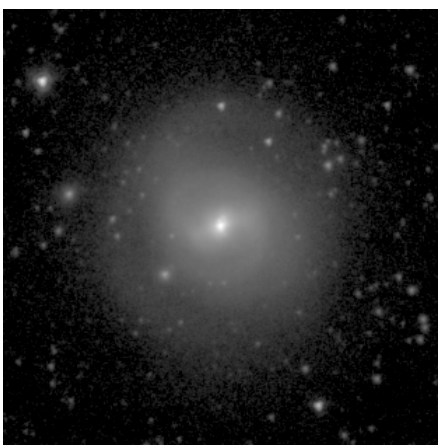

NGC 1022 (RL)SAB(rs)0/a
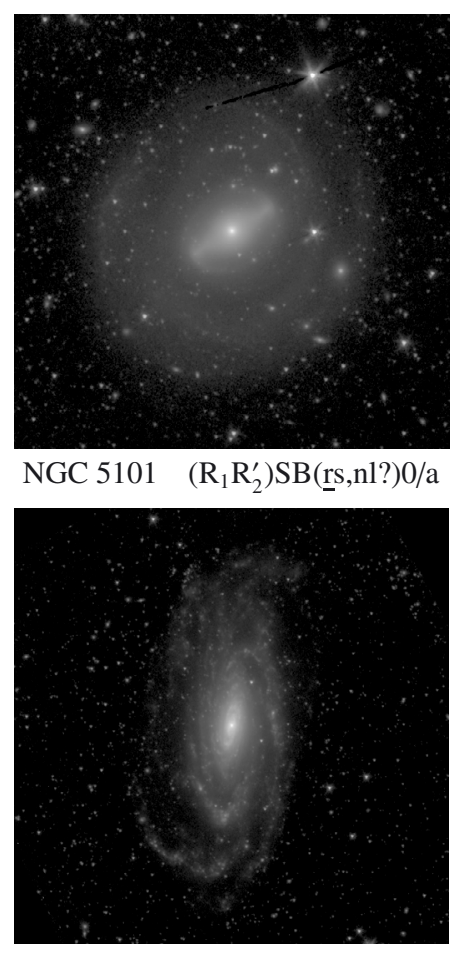

NGC $5033\left(\mathrm{R}^{\prime}\right) \mathrm{SA}(\mathrm{rs}) \mathrm{c}$

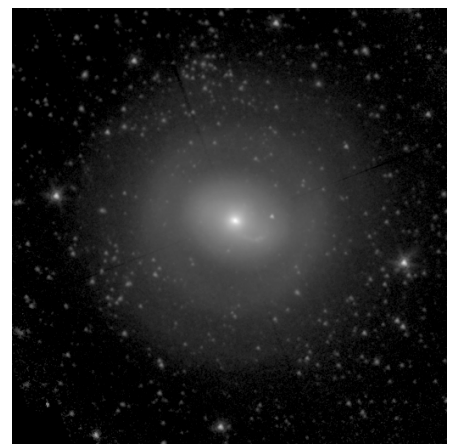

NGC 4457 (RR)SAB(1)0 $0^{+}$

Fig. 2. Selection of $S^{4} \mathrm{G}$ galaxies hosting a variety of outer rings. The images are in the $3.6 \mu \mathrm{m}$ band, have a size twice that of the $\mu_{3.6} \mu \mathrm{m}=$ $25.5 \mathrm{mag} \mathrm{arcsec}^{-2}$ diameter isophote and are oriented with north up and east left. The top row shows galaxies with outer features compatible with the $\mathrm{R}_{1}$ and $\mathrm{R}_{2}$ ring morphologies seen in simulations by Schwarz $(1981,1984)$ and defined by Buta (1986a). The second row shows outer rings that cannot be easily recognised to belong to these categories in either barred or unbarred galaxies. The third row has three example of ring-lenses and a rare example of a galaxy with two outer rings. The names of the galaxies and their morphological classification (from Buta et al., in prep.) can be found below each image.

When an outer feature could not be unambiguously classified in the $\mathrm{R}_{1}$ and $\mathrm{R}_{2}$ categories (for example in unbarred galaxies), it was classified as $\mathrm{R}$ in the case of closed rings and as $\mathrm{R}^{\prime}$ in the case of pseudorings.

Some outer features have properties intermediate between rings and lenses and were classified as outer ring-lenses. They are indicated by adding an $\mathrm{L}$ to the morphological classifications, for instance, $\mathrm{RL}$ and $\mathrm{R}_{1}^{\prime} \mathrm{L}$. Subtler degrees of ringness of outer ring-lenses are sometimes described by underlines, forming the sequence R, RL, RL, RL, L.

A selection of galaxies showing a variety of outer rings observed in the $S^{4} \mathrm{G}$ is presented in Fig. 2.

\subsection{Inner rings}

In barred galaxies, inner rings are those that are roughly the size of the bar or slightly larger than the bar. They are thought to be related to the I4R. They are classified in a sequence of openness of the spiral arms that curve to form the ring. This sequence ranges from completely closed inner rings to spirals in the following order, r, rs, rs, rs, s (de Vaucouleurs 1963). Underlines indicate transitions between the $r$, rs, and $s$ varieties. Intermediate varieties between $r$ and $s$ may also indicate rings that are not complete. Features classified as inner pseudorings ( $\underline{\mathrm{r}} \mathrm{s}, \mathrm{rs}, \mathrm{rs}$ ) are sometime denoted as $\mathrm{r}^{\prime}$ in the literature.

Inner features can also be ring-lenses. Closed inner ringlenses are indicated as $\mathrm{rl}$ and inner pseudoring-lenses are indicated as $\mathrm{r}^{\prime}$ l. Subtler degrees of ringness of inner ring-lenses are sometimes described by underlines, forming the sequence r, $\underline{\mathrm{rl}}$, $\mathrm{rl}, \mathrm{rl}, 1$.

A selection of galaxies showing a variety of inner rings observed in the $S^{4} G$ is presented in Fig. 3. 


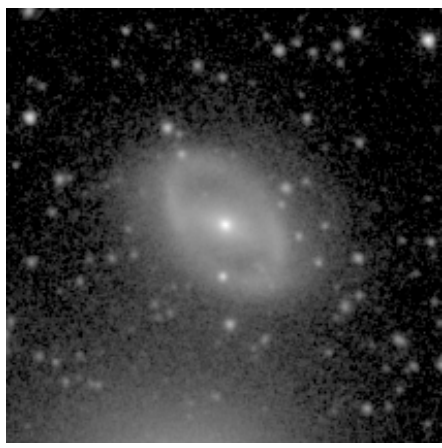

NGC 5636 SAB(r)0/a

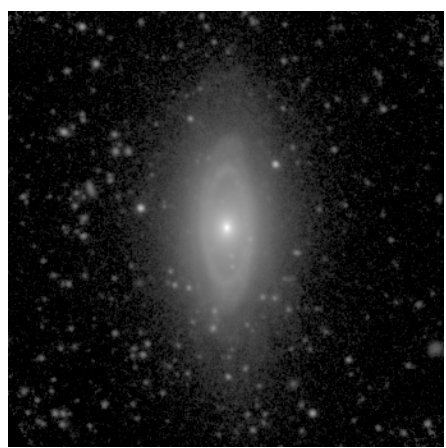

NGC 3900 SA(r)0/a

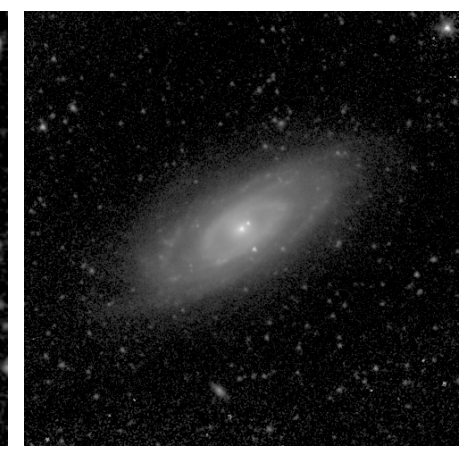

NGC 3705 (R')SAB(rs)b

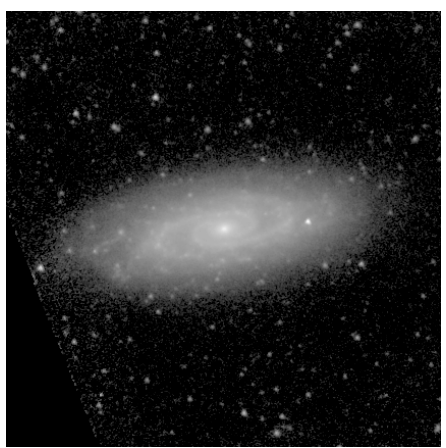

NGC 4062 SA(rs)b

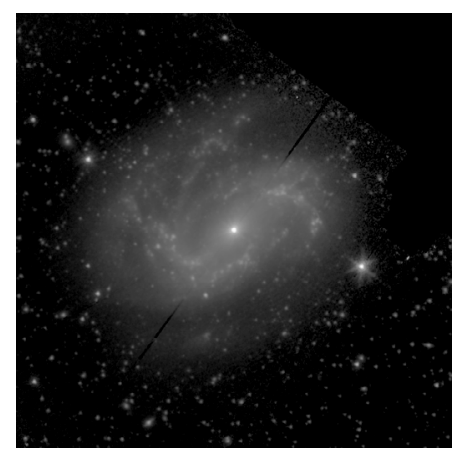

NGC $4051 \quad$ SAB(rs)b

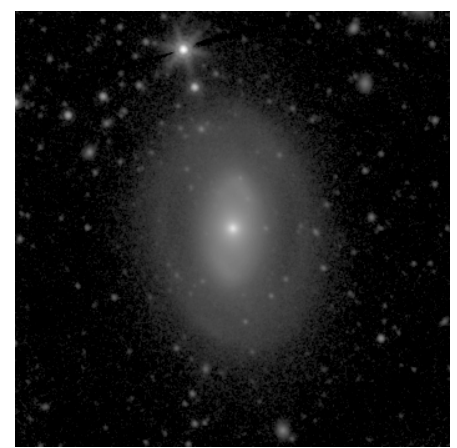

NGC 2962 (R)SAB $(\mathrm{rl}) 0^{+}$

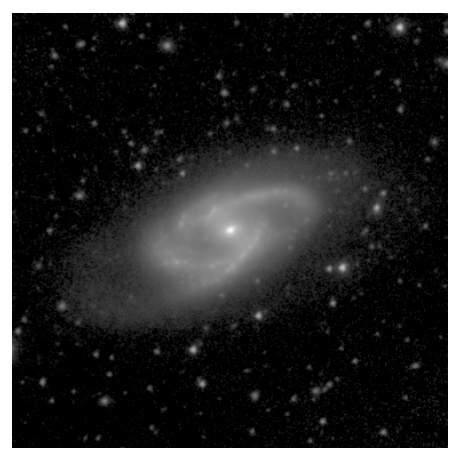

NGC 150 SAB(rs)ab

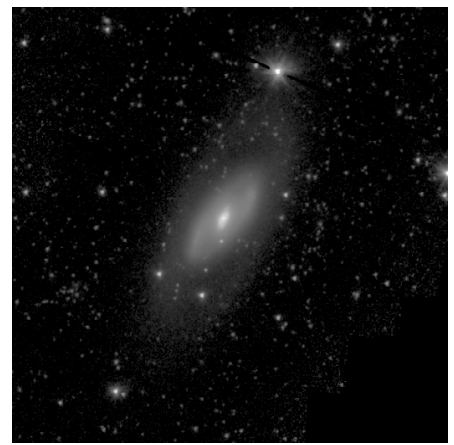

NGC $1415 \quad(\mathrm{RL}) \mathrm{SAB}_{\mathrm{a}}\left(\mathrm{r}^{\prime} 1, \mathrm{nr}\right) 0^{+}$

Fig. 3. Selection of $\mathrm{S}^{4} \mathrm{G}$ galaxies hosting a variety of inner rings presented in the same way as those in Fig. 2. The top row presents a succession of increasingly more spiral-like inner features in a series of SAB or moderately barred galaxies. The second line shows example of inner rings in unbarred galaxies and two examples of ring-lenses in early-type disc galaxies.

\subsection{Nuclear rings}

Although generally small, several nuclear features are visible in the $S^{4} \mathrm{G}$ images. In barred galaxies, nuclear rings are found inside bars. They are thought to be related to the ILRs (but see Kim et al. 2012). They are expected to be located between the outer and the inner ILR when both are present and inside the ILR when the galaxy has only one of them (see, e.g., Shlosman 1999; Sheth et al. 2000). Nuclear rings have been studied in detail in the atlas of images of nuclear rings (AINUR; Comerón et al. 2010).

Nuclear features are classified into the closed nuclear ring (nr) and nuclear pseudoring ( $\left.\mathrm{nr}^{\prime}\right)$ subtypes depending on whether the feature appears completely closed or not. Closed nuclear ring-lenses and pseudoring-lenses are denoted as $\mathrm{nrl}$ and $\mathrm{nr}^{\prime} \mathrm{l}$, respectively.

A selection of galaxies showing a variety of nuclear rings observed in the $S^{4} \mathrm{G}$ is presented in Fig. 4 .

\section{4. $x_{1}$, collisional, polar, and accretion rings}

Although the rings discussed in this subsection have a nonresonant origin, they have been included in ARRAKIS for the sake of completeness.

$\mathrm{x}_{1}$-rings, indicated as $\mathrm{x}_{1} \mathrm{r}$ in the morphological classification, are very elongated and parallel to bars. Collisional rings, indicated as RG, are in general as large as outer rings, but their centres are typically significantly offset from the galaxy nucleus. They often appear to be intrinsically elliptical (see, e.g., Appleton \& Struck-Marcell 1996). Finally, polar rings, indicated as PRG, are typically seen as needle-like features roughly perpendicular to the major axis of the galaxy, although they can be at other angles, like in the case of NGC 660, which is shown in Fig. 5 (Whitmore et al. 1990; van Driel et al. 1995).

Accretion rings are thought to be similar to polar rings, but in their case the material has been accreted at a small angle compared to the galaxy main plane. Since accretion rings are indistinguishable from resonance rings unless kinematic data are available, we included them in the statistics together with resonance rings. To our knowledge, the only rings in this paper that belong to this category are the inner ring in NGC 3593 (Corsini et al. 1998), the inner ring in NGC 4138 (Jore et al. 1996; Thakar et al. 1997), and the innermost ring in NGC 7742 (Sil'chenko \& Moiseev 2006; Mazzuca et al. 2006).

A selection of images showing galaxies with $\mathrm{x}_{1}$, collisional, and polar rings observed in the $\mathrm{S}^{4} \mathrm{G}$ is presented in Fig. 5.

\section{Preparation of the catalogue}

\subsection{Description of the projected shapes of rings}

To describe the shapes of rings, we assumed that they are intrinsically circular or elliptical, as was done in other statistical ring studies (de Vaucouleurs \& Buta 1980; Buta 1986a, 1995; Buta \& Crocker 1993; Knapen et al. 2002; Comerón et al. 2010; Grouchy et al. 2010; Laurikainen et al. 2011). Because an ellipse in projection is also an ellipse, the geometrical description of a projected ring in these studies was done by giving the sizes of its major and minor axes $\left(D_{\mathrm{r}}\right.$ and $\left.d_{\mathrm{r}}\right)$ or by giving its major axis and its axial ratio $\left(q_{\mathrm{r}}=d_{\mathrm{r}} / D_{\mathrm{r}}\right)$. To fully characterize the ring geometrical properties, its projected major axis position angle, $\mathrm{PA}_{\mathrm{r}}$, is sometimes given as well.

Although the original $S^{4} \mathrm{G}$ frames have been used for the identification of the rings in Buta et al. (in prep.), working with these images is not always optimal when describing their shape 

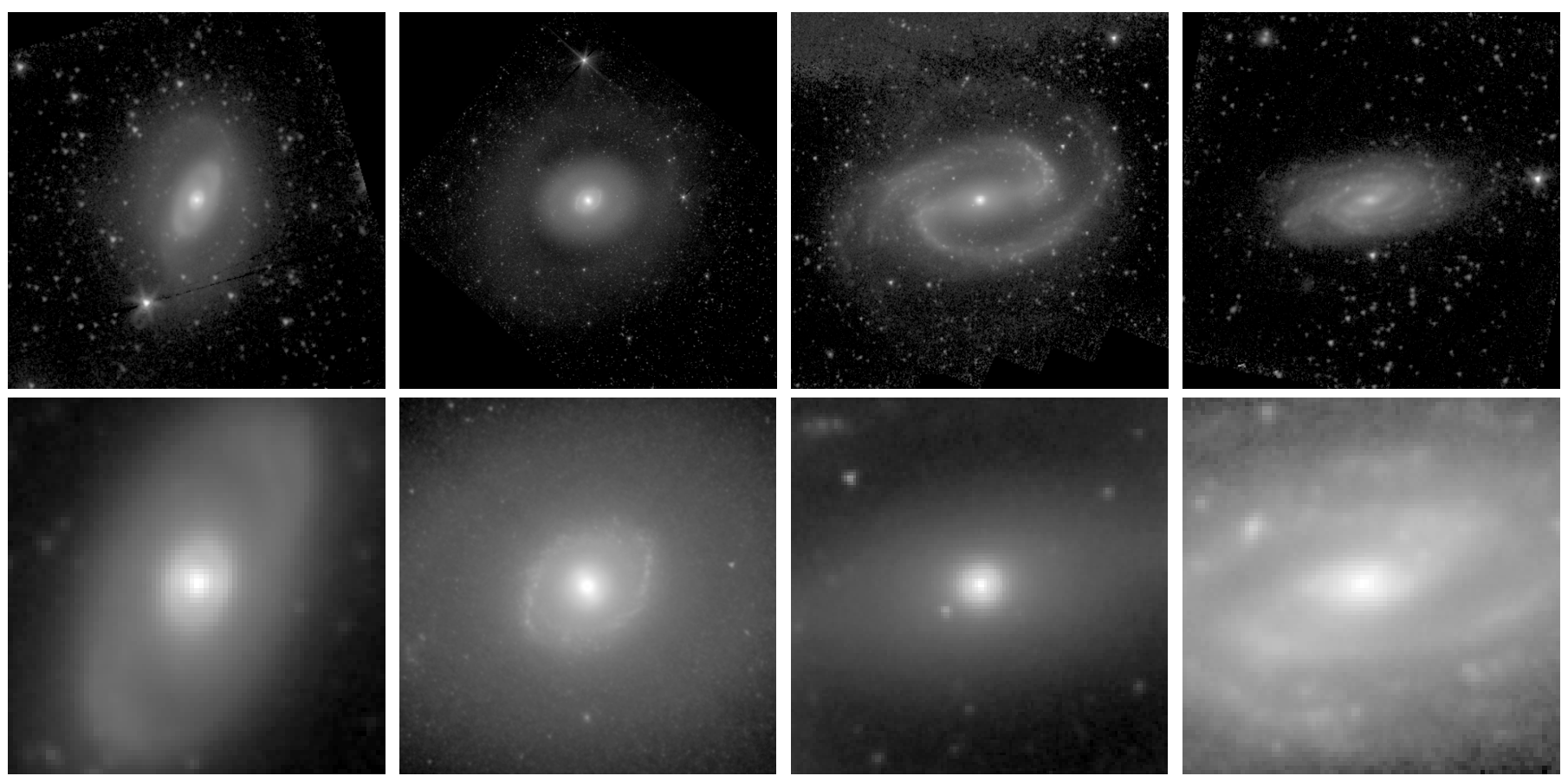

IC $4214 \quad\left(\mathrm{R}_{1}\right) \underline{S A B}_{\mathrm{a}}\left(\mathrm{r}^{\prime} 1, \mathrm{nr}, \mathrm{nb}\right) 0 / \mathrm{a} \quad \mathrm{NGC} 4736 \quad(\mathrm{R}) \mathrm{SAB}\left(\mathrm{rl}, \mathrm{nr}^{\prime}, \mathrm{nl}, \mathrm{nb}\right) \mathrm{a}$

NGC $1300 \quad\left(\mathrm{R}^{\prime}\right) \mathrm{SB}(\mathrm{s}, \mathrm{nrl}) \mathrm{b}$

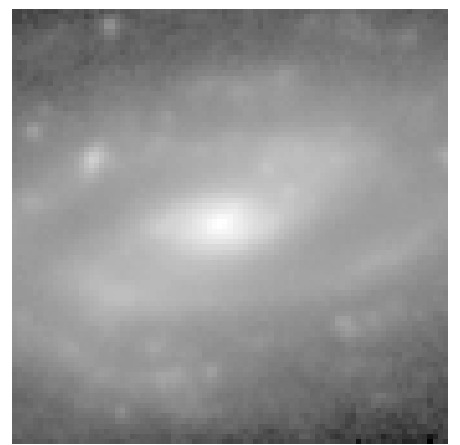

NGC $1090 \quad \mathrm{SAB}\left(\mathrm{s}, \mathrm{nr}^{\prime} 1\right) \mathrm{bc}$

Fig. 4. Selection of $\mathrm{S}^{4} \mathrm{G}$ galaxies hosting a variety of nuclear features. The images in the top row are presented in the same way as those in Fig. 2 . The bottom panels are zoomed by a factor of five compared with those in the top row. The frames show galaxies hosting a ring, a pseudoring, a ring-lens, and a pseudoring-lens, respectively.

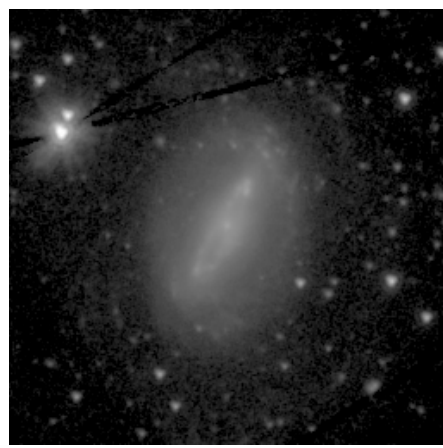

NGC $6012 \quad\left(\mathrm{R}^{\prime}\right) \mathrm{SB}\left(\mathrm{r}, \mathrm{x}_{1} \mathrm{r}\right) \mathrm{ab}$

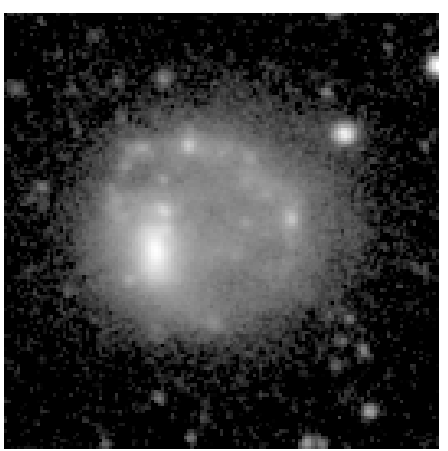

NGC 2793 RG pec

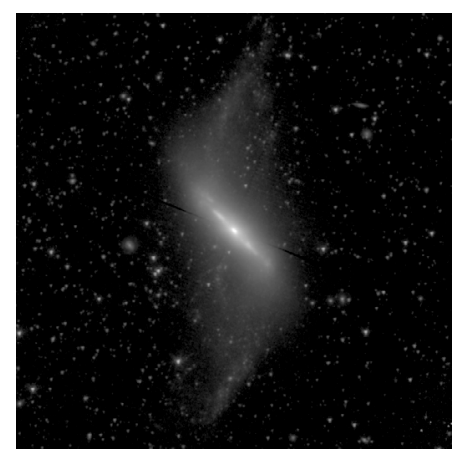

NGC 660 PRG

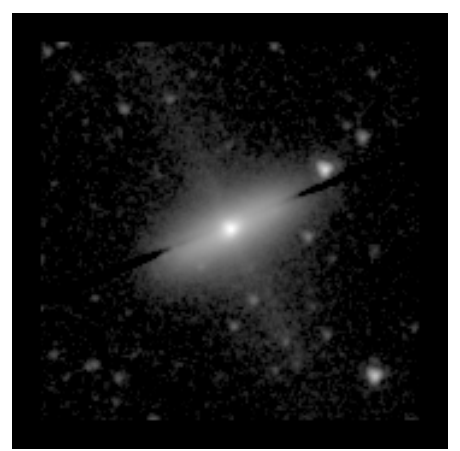

NGC $5122 \mathrm{~S}^{-} \mathrm{sp}+\mathrm{PRG} \mathrm{sp}$

Fig. 5. Selection of $\mathrm{S}^{4} \mathrm{G}$ galaxies hosting a variety of non-resonance rings presented in the same way as those in Fig. 2. The first frame shows a galaxy with an $\mathrm{x}_{1}$-ring, the second one a galaxy with a collisional one, and the last two show galaxies with polar rings.

because the contrast between rings and the rest of the galaxy is sometimes very low. A way to overcome this problem is to model the emission of the main components of the galaxy (bulge, bar, disc, etc.) and subtract this model from the original image. This results in the so-called residual image where the disc substructure, such as rings and spiral arms, appears contrastenhanced. This procedure was preferred to other methods to enhance rings such as unsharp masking because the galaxies were modelled by the $\mathrm{S}^{4} \mathrm{G}$ pipeline 4 (P4; Salo et al., in prep.). In short, $\mathrm{P} 4$ models were made by fitting the $\mathrm{S}^{4} \mathrm{G}$ images with up to four components (nucleus, bulges, discs, and/or bars) using version 3.0 of GALFIT (Peng et al. 2002, 2010). When fitting the model, the disc ellipticity $\left(\epsilon_{\mathrm{d}}=1-q_{\mathrm{d}}\right)$ and position angle $\left(\mathrm{PA}_{\mathrm{d}}\right)$ were fixed to the values corresponding to deep outerdisc isophotes obtained with an ellipse-fitting routine. A model galaxy image and a residual image were obtained as an output of P4. For the few ARRAKIS galaxies not included in the $S^{4} G$ sample, additional ellipse fits and decompositions were made using the P4 software.
We examined the original and residual images of the galaxies and decided for each ring in which image, original or residual, it was better defined, and thus in which image its shape was easier to measure. Although a priori this should always be the residual image (see, e.g., the example in Fig. 6), because of the galaxies with complicated morphologies and/or because P4 models were intentionally kept simple, some residual images have artefacts that hinder defining the outline of a ring. This occurs more often when looking at the inner features in galaxies with strong bars.

After selecting the image in which the rings were better defined, we used IRAF's ${ }^{3}$ (Tody 1986, 1993) TVMARK task to describe them. When used in interactive mode, TVMARK allows marking points in an image displayed in DS9 (Joye \& Mandel 2003). This was used to characterise the shape of rings

IRAF is distributed by the National Optical Astronomy Observatories, which are operated by the Association of Universities for Research in Astronomy, Inc., under cooperative agreement with the National Science Foundation. 

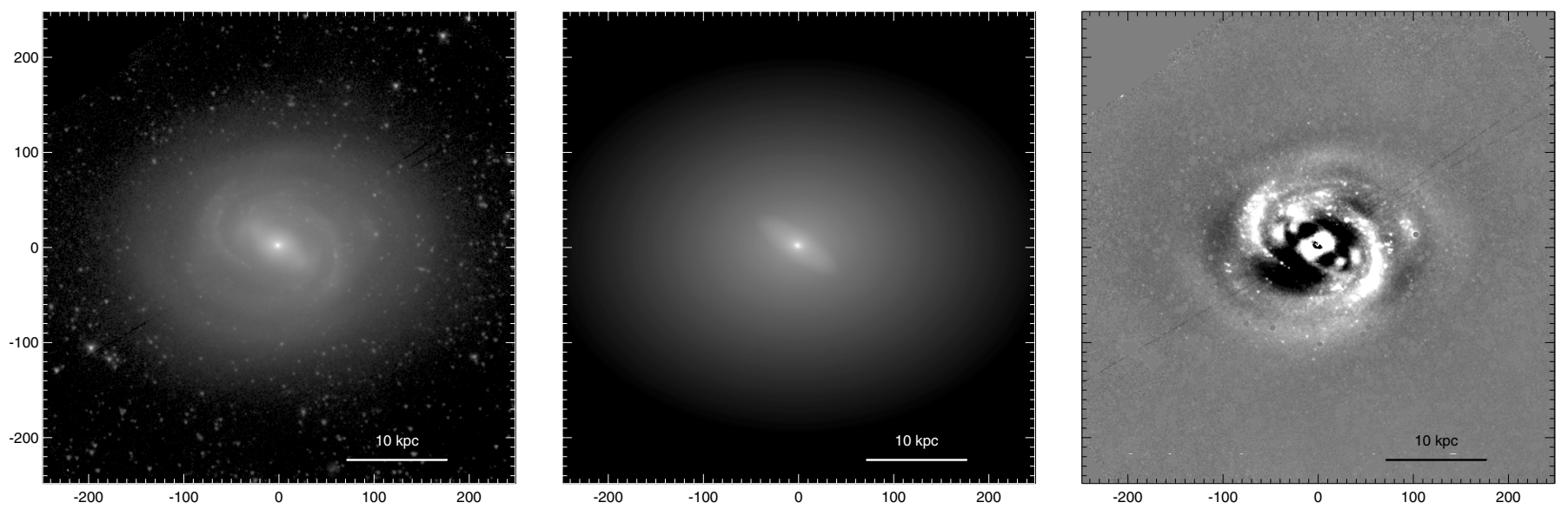

Fig. 6. Original image, P4 model, and model-subtracted image of NGC 4579. The model has three components, namely a bulge, a bar, and a disc. NGC 4579 is a galaxy classified as (RL, $\left.\mathrm{R}^{\prime}\right) \mathrm{SB}(\mathrm{rs})$ a by Buta et al. (in prep.). NGC 4579 has an exceedingly diffuse outer ring as seen in the first frame. The ring becomes more obvious in the model-subtracted image. The axis units are arcseconds.
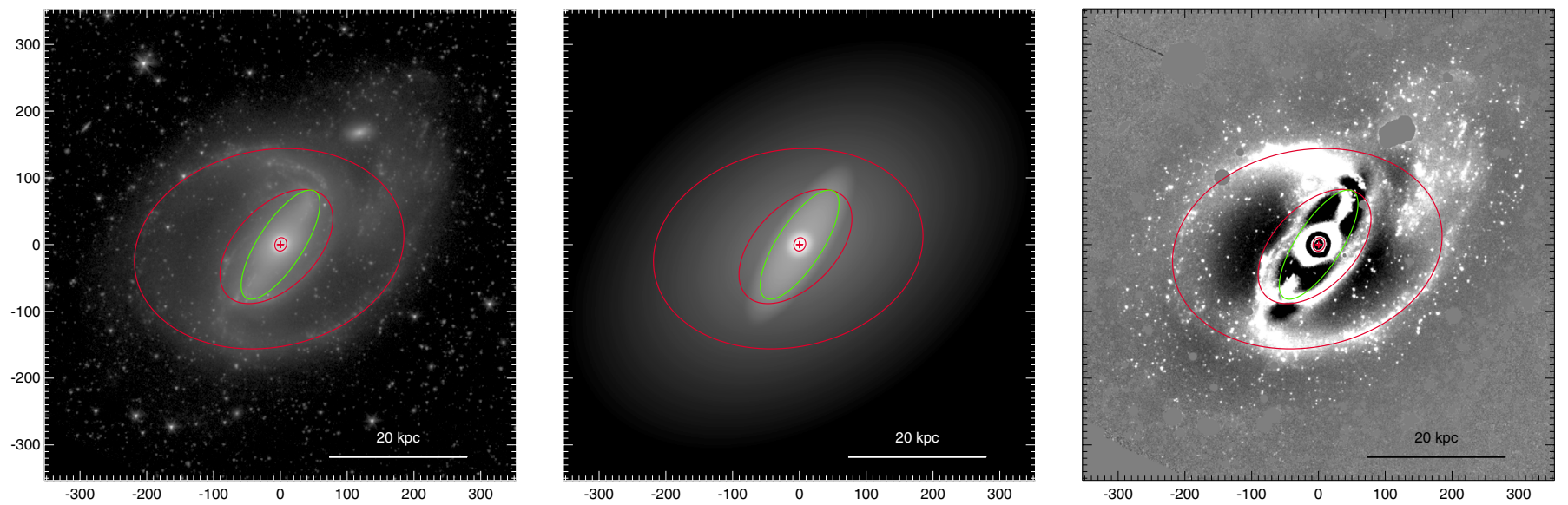

Fig. 7. Original image, P4 model, and model-subtracted image of NGC 1097, which is classified as an (R')SB(rs,nr)ab pec galaxy in Buta et al. (in prep.). The red ellipses indicate in an outside-in order the average of the two ellipse fits made to the outer, the inner, and the nuclear rings. The green ellipses are the result of an ellipse fit at the radius of the highest bar ellipticity. The red crosses indicate the centre of the galaxy. The axis units are arcseconds.

by marking by hand bright star-forming patches belonging to the feature or stellar emission that probably belonged to the ring, as was done in Grouchy et al. (2010). All the ring features were measured by the same person (S. Comerón). This procedure was repeated twice for each ring, typically with several days to a few months between the two measurements.

The points marking the outline of the rings for each of the two sets of measurements were fitted with an ellipse using a least-squares fitting algorithm. The output of the fits was the ring diameter in the major axis direction $D_{\mathrm{r}}$, the diameter along the minor axis direction $d_{\mathrm{r}}$, and the major axis PA $\left(\mathrm{PA}_{\mathrm{r}}\right)$. The data used for the statistics in this paper are presented in Appendix A and were obtained by averaging the $D_{\mathrm{r}}, d_{\mathrm{r}}$, and $\mathrm{PA}_{\mathrm{r}}$ values obtained from the fits to the two sets of measurements for each ring. Ellipses made from these averaged values are overlayed on the atlas images and in the example shown in Fig. 7.

\subsection{Accuracy of the measured projected ring properties}

To study the accuracy of the $D_{\mathrm{r}}, d_{\mathrm{r}}$, and $\mathrm{PA}_{\mathrm{r}}$ measurements, we examined the residuals between the values of these parameters obtained for each of the two fits and the averaged values, that is, $d_{\mathrm{r}}, D_{\mathrm{r}}-\left\langle d_{\mathrm{r}}, D_{\mathrm{r}}\right\rangle$ and $\mathrm{PA}_{\mathrm{r}}-\left\langle\mathrm{PA}_{\mathrm{r}}\right\rangle$. These residuals can be considered as a rough estimate of internal errors caused by the observer's judgement (Fig. 8). However, we warn that these error estimates do not include those associated with the use of a given method when measuring ring properties. A different ring measurement method may yield values differing from those presented here by many times the error estimate that we discuss.

The top-left panel in Fig. 8 shows how the error in the major and minor diameters tends to grow with ring radii: for small diameters, the $d_{\mathrm{r}}, D_{\mathrm{r}}-\left\langle d_{\mathrm{r}}, D_{\mathrm{r}}\right\rangle$ never exceeds 0.2 , but for larger diameters, it can increase up to $0{ }^{\prime} 6$. The four points with larger $d_{\mathrm{r}}, D_{\mathrm{r}}-\left\langle d_{\mathrm{r}}, D_{\mathrm{r}}\right\rangle$ correspond, from left to right, to the major axis diameters of the outer features of NGC 4736 and NGC 4258. The outer ring of NGC 4736 appears to be double in its south-eastern side. Our choice in selecting the outline of the ring changed between the two measurements, which explains the large error. NGC 4258 is a galaxy for which the $S^{4} G$ frame does not fully cover the disc, thus a significant fraction of the outer ring lies outside the frame and cannot be studied, causing a large uncertainty in the $D_{\mathrm{r}}$ measurement. The range of $d_{\mathrm{r}}, D_{\mathrm{r}}-\left\langle d_{\mathrm{r}}, D_{\mathrm{r}}\right\rangle$ values is similar to that found in the catalog of southern ringed galaxies (CSRG; Fig. 5a in Buta 1995). 

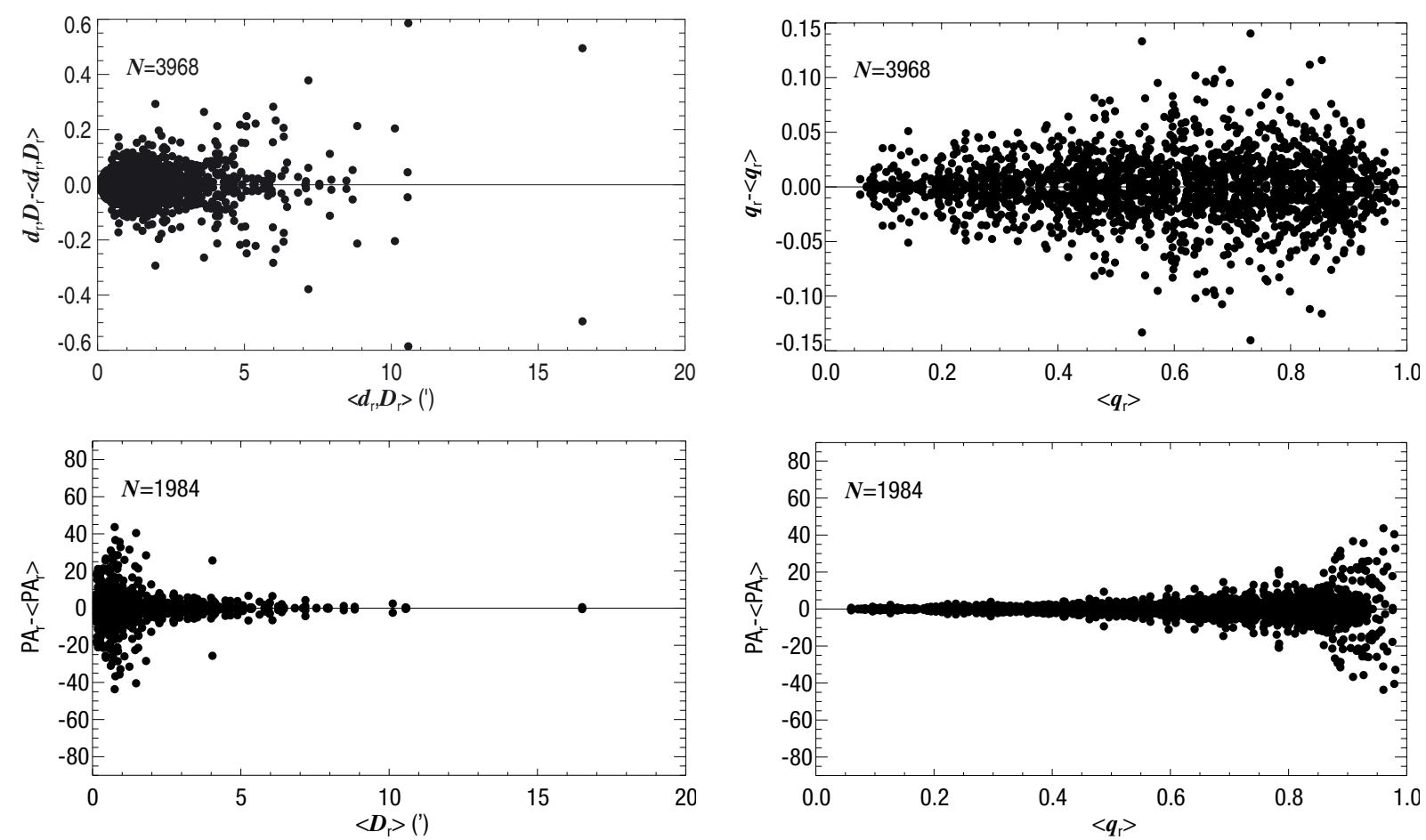

Fig. 8. Top-left panel: residuals of the ring diameters along the major and minor axes as a function of the averaged diameters, $\left\langle d_{\mathrm{r}}, D_{\mathrm{r}}\right\rangle . T o p-r i g h t$ panel: residuals of the ring axis ratios, $q_{\mathrm{r}}=d_{\mathrm{r}} / D_{\mathrm{r}}$, as a function of the mean axis ratios $\left\langle q_{\mathrm{r}}\right\rangle=\left\langle d_{\mathrm{r}}\right\rangle /\left\langle D_{\mathrm{r}}\right\rangle$. Bottom-left panel: residuals of the ring position angles as a function of the averaged ring major axis $\left\langle D_{\mathrm{r}}\right\rangle$. Bottom-right panel: residuals of the ring position angles as a function of the mean ring axis ratios. $N$ refers to the number of points in each plot.

The errors in the measured ring axis ratios are presented in the top-right panel of Fig. 8. We found that the average error is $\left|\Delta q_{\mathrm{r}}\right|=0.02$.

The $\mathrm{PA}_{\mathrm{r}}$ uncertainty is larger for smaller and/or rounder rings, as seen in the bottom panels of Fig. 8; this is natural since for a round ring $\mathrm{PA}_{\mathrm{r}}$ is undefined. The range of $\mathrm{PA}_{\mathrm{r}}-\left\langle\mathrm{PA}_{\mathrm{r}}\right\rangle$ in our study and its behaviour as a function of $\left\langle D_{\mathrm{r}}\right\rangle$ and $\left\langle q_{\mathrm{r}}\right\rangle=$ $1-\epsilon_{\mathrm{r}}=\left\langle d_{\mathrm{r}}\right\rangle /\left\langle D_{\mathrm{r}}\right\rangle$ is qualitatively similar to that in Figs. 5c, d of the CSRG.

Because the CSRG is the largest ring catalogue with an internal error estimate, it can be compared with our results. The CSRG data come from analogically measuring diameters and PAs in photographic plates, but here we present data measured by digital means, which may yield significantly different accuracies.

The CSRG presents two internal error functions that we reproduce here with $S^{4} G$ data. The internal error function for the diameter residuals was obtained by sorting the residuals according to their mean diameter and then calculating the standard deviation for groups of 200 residuals, $\sigma\left(d_{\mathrm{r}}, D_{\mathrm{r}}\right)$ (top panel in Fig. 9). As in the CSRG, we linearly fitted the points and obtained

$\sigma\left(d_{\mathrm{r}}, D_{\mathrm{r}}\right)=0.009+0.023\left\langle d_{\mathrm{r}}, D_{\mathrm{r}}\right\rangle$

Since in ARRAKIS the error grows slower with increasing $d_{\mathrm{r}}, D_{\mathrm{r}}$, our accuracy is slightly better than that obtained in the CSRG $\left(\sigma\left(d_{\mathrm{r}}, D_{\mathrm{r}}\right)=0.009+0.029\left\langle d_{\mathrm{r}}, D_{\mathrm{r}}\right\rangle\right)$. The $95 \%$ confidence bands in Fig. 9 were calculated with the assumption that the errors are normally distributed. The statistics for the confidence bands corresponding to the CSRG were obtained by measuring the position of the data points in a magnified version of Fig. 5e in the CSRG.
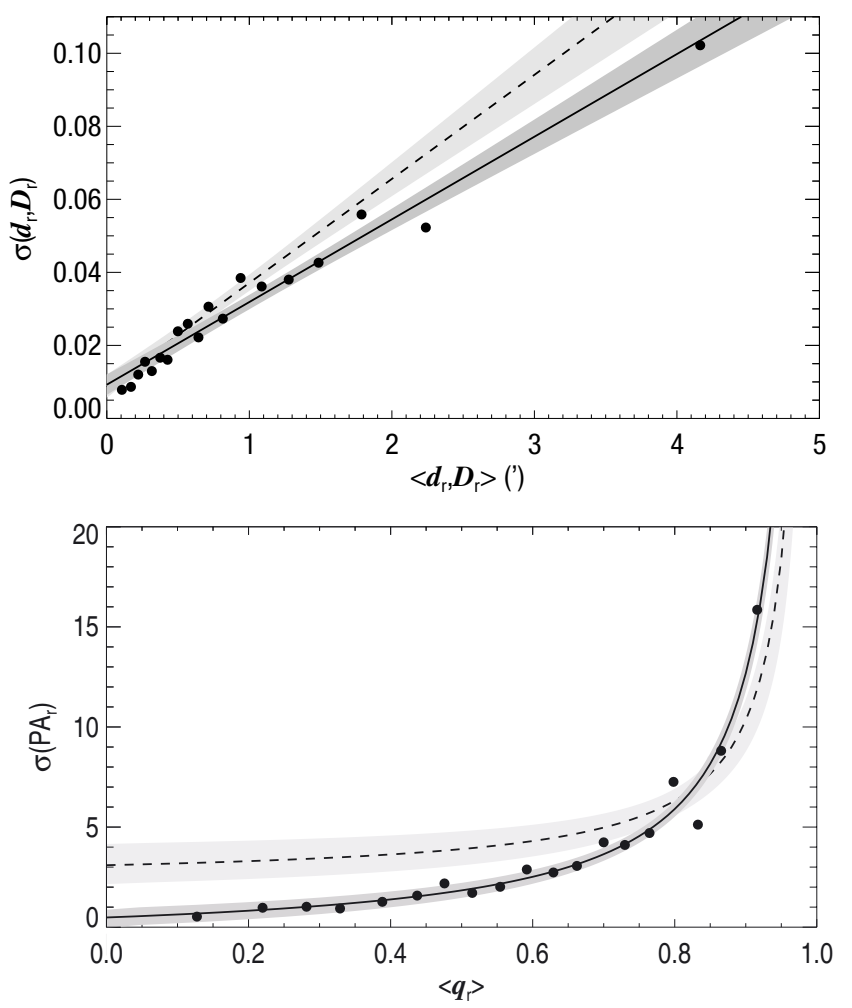

Fig. 9. Top panel: internal errors of $d_{\mathrm{r}}, D_{\mathrm{r}}$ as a function of $\left\langle d_{\mathrm{r}}, D_{\mathrm{r}}\right\rangle$. Bottom panel: internal errors of $\mathrm{PA}_{\mathrm{r}}$ as a function of $\left\langle q_{\mathrm{r}}\right\rangle$. The solid lines represent the fits described in Eqs. (1) and (2) and the dashed lines correspond to the same expressions as presented in the CSRG. Darker grey areas indicate the $95 \%$ confidence interval of the fit for ARRAKIS and lighter grey indicates the same for the CSRG. 
The internal errors for the ring orientations were calculated by sorting the $\mathrm{PA}_{\mathrm{r}}$ residuals according to their $q_{\mathrm{r}}$ and then calculating the standard deviation for groups of 100 residuals (bottom panel in Fig. 9). As in the CSRG, we produced a fit that scales inversely with the fitted ellipticity

$\sigma\left(\mathrm{PA}_{\mathrm{r}}\right)=-0.87+1.35 /\left(1-\left\langle q_{\mathrm{r}}\right\rangle\right)$,

and thus our $\mathrm{PA}_{\mathrm{r}}$ determinations are better than those in the CSRG for $q_{\mathrm{r}}<0.83$ (in the CSRG $\sigma\left(\mathrm{PA}_{\mathrm{r}}\right)=2^{\circ} .29+0.81 /(1-$ $\left.\left\langle q_{\mathrm{r}}\right\rangle\right)$ ). Our $\mathrm{PA}_{\mathrm{r}}$ measurements are at least twice as precise than those in the CSRG for $q_{\mathrm{r}}<0.53$. However, perhaps surprisingly, the manual $\mathrm{PA}_{\mathrm{r}}$ determination in the CSRG seems to have fewer internal errors than ARRAKIS for rings that are nearly round $\left(q_{\mathrm{r}}>0.83\right)$. This is no problem because $\mathrm{PA}_{\mathrm{r}}$ becomes meaningless for almost round rings. We calculated the confidence bands for this fit in the same way as for the top panel. For the CSRG fit, we made the statistics based on the information on CSRG's Fig. 5f.

We can thus conclude that the internal errors in ARRAKIS are the smallest for such a large dataset of rings so far and that this can be attributed to the use of digital deep images.

\subsection{Obtaining the projected bar parameters}

Simulations by Schwarz $(1981,1984)$ and the flux tube manifold orbital calculations (Athanassoula et al. 2009b,a) predict $\mathrm{R}_{1}$ rings to be elongated and perpendicular to bars, $\mathrm{R}_{2}$ rings to be elongated and parallel to bars, and inner rings to be elongated and parallel to bars. It is thus interesting to know the bar PA in galaxies with rings. We also measured the highest bar ellipticity $\epsilon_{\mathrm{b}}=1-q_{\mathrm{b}}=1-d_{\mathrm{b}} / D_{\mathrm{b}}\left(D_{\mathrm{b}}\right.$ and $d_{\mathrm{b}}$ are the bar major and minor diameters and $q_{\mathrm{b}}$ is the bar axis ratio). $\epsilon_{\mathrm{d}}$ roughly scales with more sophisticated bar strength indicators such as the bar torque (Block et al. 2001; Laurikainen et al. 2002).

The bar data were obtained by studying the ellipticity fits from $S^{4} G$ 's $P 4$. We searched for the peak ellipticity within the radius of the bar, regardless of how big the drop in the ellipticity after the maximum was or how the PA varied with radius. We considered the PA at that radius to be representative of the bar orientation. Moreover, the maximum ellipticity radius is typically a lower estimate of the bar length (Erwin 2005), except in galaxies in which the bar merges with rings and/or spiral arms. In the catalogue of Appendix A, we present the diameters along the major and minor axes of the ellipse fits at the position of the bar maximum ellipticity $\left(D_{\mathrm{b}}\right.$ and $d_{\mathrm{b}}$ ) and the bar PA at the same position, $\left(\mathrm{PA}_{\mathrm{b}}\right)$. We checked that each individual $\mathrm{PA}_{\mathrm{b}}$ roughly corresponds to what would be expected from visual inspection. If it did not, we labelled it as unsuitable for bar analysis, as discussed at the end of Sect. 4.5.

\subsection{Obtaining the intrinsic ring and bar shape and orientation}

ARRAKIS is the first large ring catalogue that provides intrinsic ring shapes and orientations. The reason is that deprojecting rings requires knowing, within a few percent, the disc orientation parameters, that is, the ellipticity, $\epsilon_{\mathrm{d}}=1-q_{\mathrm{d}}$, and the position angle, $\mathrm{PA}_{\mathrm{d}}$. Under the assumption of discs being intrinsically circular, one needs very deep images to measure $\epsilon_{\mathrm{d}}$ and $\mathrm{PA}_{d}$ in the outer disc, a region where the effect of perturbing non-axisymmetries such as bars is most likely weak. This was not possible for the CSRG because it was produced using photographic plates, but was performed in Knapen et al. (2002), Comerón et al. (2010), and Grouchy et al. (2010).
The $\mathrm{S}^{4} \mathrm{G}$ P4 makes use of deprojection parameters obtained from isophote fits at low surface brightness (for more details see Salo et al., in prep.). These are the deprojection parameters that we used here for obtaining intrinsic ring and bar shapes $\left(q_{\mathrm{r}, 0}\right.$ and $q_{\mathrm{b}, 0}$ ) except for the few ARRAKIS galaxies not in the $\mathrm{S}^{4} \mathrm{G}$ sample (but appearing in $\mathrm{S}^{4} \mathrm{G}$ frames) and NGC 4698. For this last galaxy our interpretation of the deprojection parameters is very different from that in $\mathrm{P} 4$. $\mathrm{P} 4$ deprojection parameters were also used to calculate the counter-clockwise angular distance between the line of nodes and the ring and bar major axis $\left(\theta_{\mathrm{r}}\right.$ and $\left.\theta_{\mathrm{b}}\right)$, under the assumptions that the outer parts of discs are circular and that rings and bars are roughly elliptical. The equations that we used for deprojection can be found in Appendix A of Gadotti et al. (2007). The deprojected $D$ and $d$ values for both rings and bars are presented in the catalogue of Appendix A. The angle difference between the deprojected major axis and the line of nodes of the deprojection is also indicated. The only rings for which we have not included deprojected parameters are the polar rings, because they are known not to lie in the galaxy disc plane.

\subsection{Reliability of the deprojected bar parameters}

The presence of a bar can be recognised in very inclined galaxies and sometimes also in edge-on galaxies (see, e.g., how box/peanut bulges are related to bars in Kuijken \& Merrifield 1995). However, obtaining their orientation and ellipticity from ellipse fits can be very difficult. Indeed, the ellipse deprojection would give precise bar parameters for isolated infinitely thin bars. But real bars have some amount of vertical thickening and are embedded in galaxies with discs and bulges. As a natural consequence of that, the more inclined a galaxy, the harder it is to obtain accurate bar intrinsic parameters.

To estimate how precise our bar measurements are, we prepared a set of four toy model galaxies with an exponential disc, a classical spherical bulge, and a Ferrers bar (Ferrers 1877). Assuming that the centre of the galaxy is located at $x=0, y=0$, and $z=0$ and that the disc lies in the $x-y$ plane, this simplified disc can be described as

$\rho_{\mathrm{d}}(R, z)=\rho_{\mathrm{d}, 0} \mathrm{e}^{-R / h_{R}} \mathrm{e}^{-|z| / h_{z}}$,

where $R$ is the radius in the plane of the galaxy $\left(R=\sqrt{x^{2}+y^{2}}\right)$, $\rho_{\mathrm{d}, 0}$ stands for the disc central mass density, $h_{R}$ for the disc scalelength, and $h_{z}$ for the disc scale-height.

To describe the bulge we used a Jaffe profile (Jaffe 1983) which, when projected onto a plane, results approximately in a de Vaucouleurs profile (de Vaucouleurs 1948):

$\rho_{\text {bulge }}(r)=\rho_{\text {bulge }, 0}\left(\frac{r}{r_{0}}\right)^{-2}\left(1+\frac{r}{r_{0}}\right)^{-2}$,

where $r$ is the $3 \mathrm{D}$ radius $\left(r=\sqrt{x^{2}+y^{2}+z^{2}}\right), \rho_{\text {bulge }, 0}$ controls the bulge mass, and $r_{0}$ controls its size.

Finally, the bar was described as

$\rho_{\mathrm{bar}}(m)=\rho_{\mathrm{b}, 0}\left(1-m^{2}\right)$ for $m \leq 1, \rho_{\mathrm{b}}=0$ for $m>1$,

where $\rho_{\mathrm{bar}, 0}$ is the central bar mass density and

$m^{2}(x, y, z)=\frac{x^{2}}{a^{2}}+\frac{y^{2}}{b^{2}}+\frac{z^{2}}{c^{2}}$

Here, $a$ is the major axis, $b$ is the minor axis, and $c$ is the vertical axis of the bar. 


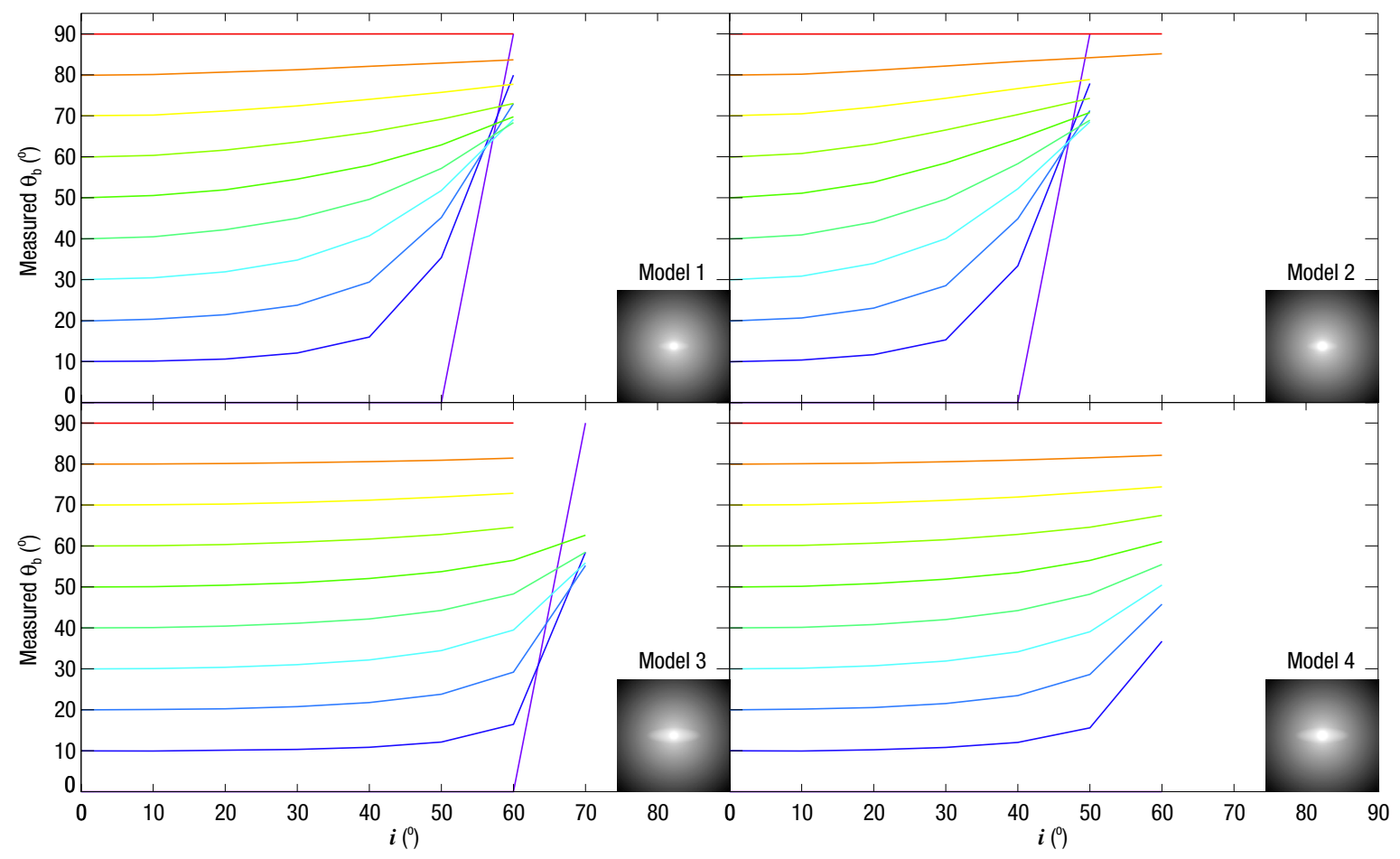

Fig. 10. Measured orientation of the bar with respect to the line of nodes of the galaxy $\left(\theta_{\mathrm{b}}\right)$ as a function of the galaxy inclination $(i)$ for each of our four galaxy models described in the text and Table 2 . Each coloured line represents a single real $\theta_{\mathrm{b}}$ value, which is the one corresponding to the position at which the line crosses the $i=0^{\circ}$ axis, that is, from purple to red (bottom to top), $\theta_{\mathrm{b}}=0^{\circ}, \theta_{\mathrm{b}}=10^{\circ}, \theta_{\mathrm{b}}=20^{\circ}, \theta_{\mathrm{b}}=30^{\circ}, \theta_{\mathrm{b}}=40^{\circ}$, $\theta_{\mathrm{b}}=50^{\circ}, \theta_{\mathrm{b}}=60^{\circ}, \theta_{\mathrm{b}}=70^{\circ}, \theta_{\mathrm{b}}=80^{\circ}$, and $\theta_{\mathrm{b}}=90^{\circ}$. The images at the bottom-right corner of each panel represent the corresponding galaxy when $\theta_{\mathrm{b}}=0^{\circ}$ and $i=0^{\circ}$.

The four model galaxies had (in arbitrary units) $\rho_{\mathrm{d}, 0}=1$, $\rho_{\mathrm{bar}, 0}=1, h_{R}=30, h_{z}=5$, and $r_{0}=5$. The other parameters were model-dependent and are listed in Table 2. Table 2 also presents the fraction of mass in the bulge and in the bar for each model. As seen there, the fraction of mass in bars is rather low and thus our fit to models is a worst-case study.

We decided that the $x$ axis would be the line of nodes of our rotation. We first rotated the bar by a given angle $\theta_{\mathrm{b}}$ with respect to the line of nodes and then inclined the galaxy by an angle $i$. We defined $\theta_{\mathrm{b}}$ to vary from $\theta_{\mathrm{b}}=0^{\circ}$ to $\theta_{\mathrm{b}}=90^{\circ}$ in steps of $10^{\circ}$. We explored the space of galaxy inclinations from $i=0^{\circ}$ to $i=80^{\circ}$ in steps of $10^{\circ}$. The rotated and inclined galaxies were then projected onto $2 \mathrm{D}$ fits images. We used these images to measure the bar properties by fitting ellipticity profiles. We considered that a bar ellipticity and PA could be measured when a peak in ellipticity with prominence larger than 0.01 occurred within the bar radius. We used the ellipticity fit at the maximum ellipticity peak to obtain the deprojected bar parameters, as was done for observed bars in Sect. 4.4. The results on the recovery of the original bar PA with respect to the line of nodes, $\theta_{\mathrm{b}}$, and the original bar ellipticity, $\epsilon_{\mathrm{b}, 0}=1-q_{\mathrm{b}, 0}$, are presented in Figs. 10 and 11. In several cases in highly inclined galaxies, no clear maximum in ellipticity was found, therefore no deprojection of the bar properties was obtained.

The angle $\theta_{\mathrm{b}}$ is typically well recovered for galaxies with $i \leq 40^{\circ}$ when the bar is small compared to the bulge size (models 1 and 2 in Fig. 10). For galaxies with longer bars (models 3 and 4), $\theta_{\mathrm{b}}$ is well recovered for $i \leq 60^{\circ}$. The reason for this is that the bulge, which is intrinsically spherical, and to some extent also the disc, soften the isophotes, which makes them less elliptical. In some cases, for low real $\theta_{\mathrm{b}}$ values and high disc inclinations, this effect is so strong that the measured projected ellipticity becomes lower than the ellipticity of the projected
Table 2. Parameters of the model galaxies used to study the reliability of the bar deprojected parameters.

\begin{tabular}{lcccccc}
\hline \hline Model & $\rho_{\text {bulge }, 0}$ & $a$ & $b$ & $c$ & $\begin{array}{c}\text { Bulge/ } \\
\text { total }\end{array}$ & $\begin{array}{c}\text { Bar/ } \\
\text { total }\end{array}$ \\
\hline Model 1 & 5 & 30 & 10 & 3 & $11 \%$ & $3 \%$ \\
Model 2 & 10 & 30 & 10 & 3 & $20 \%$ & $2 \%$ \\
Model 3 & 5 & 50 & 15 & 4 & $11 \%$ & $8 \%$ \\
Model 4 & 10 & 50 & 15 & 4 & $19 \%$ & $7 \%$ \\
\hline
\end{tabular}

Notes. Bulge/total stands for the bulge-to-total mass ratio and Bar/total stands for the bar-to-total mass ratio.

disc, causing the measured $\theta_{\mathrm{b}}$ to shift by some $90^{\circ}$ from the real value.

The measured intrinsic bar ellipticity $\epsilon_{\mathrm{b}, 0}=1-q_{\mathrm{b}, 0}$ is highly dependent on the real $\theta_{\mathrm{b}}$. When the bar is parallel to the line of nodes (real $\left.\theta_{\mathrm{b}} \sim 0^{\circ}\right), 1-q_{\mathrm{b}, 0}$ becomes increasingly underestimated for increasing $i$. For bars oriented perpendicular to the line of nodes (real $\theta_{\mathrm{b}} \sim 90^{\circ}$ ), the effect is reversed.

Because the $\mathrm{S}^{4} \mathrm{G}$ sample is under-abundant in early-type galaxies, bulges are in general not expected to play a significant role when producing ellipticity profiles. Therefore, we considered the parameters of the bars in galaxies with $i \leq 60^{\circ}$ (corresponding to a disc ellipticity $\epsilon_{\mathrm{d}}=1-q_{\mathrm{d}} \leq 0.5$ ) to be reliably measured. The properties of bars in such discs almost always appear in the catalogue in Appendix A and the ellipse fit to the highest bar ellipticity is overlayed on the images of the atlas in Appendix B.

However, even for galaxies with $i \leq 60^{\circ}$ six bar fits were found to be unreliable and were excluded from the appendices. The reason for this is that the bar fit is obviously 


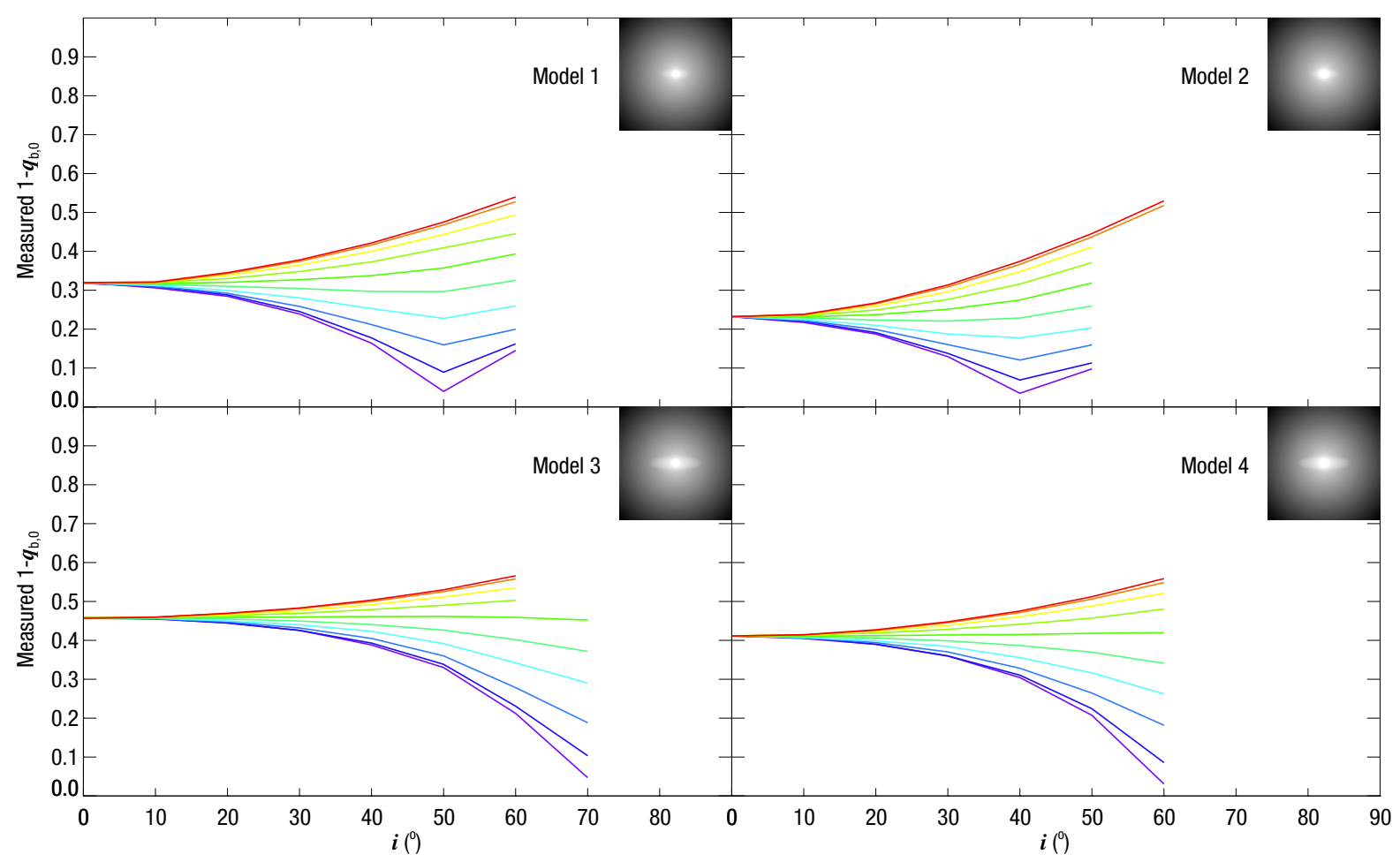

Fig. 11. Measured bar ellipticity (measured $1-q_{\mathrm{b}, 0}$ ) as a function the galaxy inclination $(i)$ for each of our four galaxy models. Each coloured line represents a single $\theta_{\mathrm{b}}$ value colour-coded in the same way as in Fig. 10 (also ordered here from bottom to top). The intrinsic $1-q_{\mathrm{b}, 0}$ value is that corresponding to $i=0^{\circ}$. The images at the top-right corner of each panel represent the corresponding galaxy when $\theta_{\mathrm{b}}=0^{\circ}$ and $i=0^{\circ}$.

wrong in galaxies with bars with a low contrast (NGC 2552) or that are influenced by surrounding bright regions such as a ring (NGC 2967, NGC 4351, NGC 5595, NGC 5744, and NGC 6923). For five additional galaxies with $\epsilon_{\mathrm{d}} \leq 0.5$ classified as barred by Buta et al. (in prep.), ESO 443-80, NGC 2460, NGC 5633, NGC 6278, and UGC 5814, we were unable to distinguish a peak corresponding to a bar in the ellipticity profiles. Finally, the centre of NGC 4108B is affected by an image artefact that made impossible to measure the bar properties. For galaxies with $i>60^{\circ}$, we did not include the bar properties in the appendices even for those that are obviously barred.

Moreover, even though inner rings are defined to be surrounding the bar, for 73 galaxies the maximum in ellipticity that we fitted as an estimate for the bar radius is slightly larger than the inner ring radius in projection and/or in deprojection. This is caused by our very simple approach at describing bars. In most of these cases the bar merges with the ring and the junction points have strong star-forming regions, ansæ, and/or the beginning of spiral arms, which affect the ellipticity fits in such a way as to move the ellipticity maximum outwards. In other cases, the reason that the bar is larger than the fitted ring diameter may be that some rings slightly deviate from an elliptical shape in such a way that the bar fits within them. These deviations are not captured by our simple ring-fitting approach. In none of the cases considered for the statistics in this paper would this change the bar orientation by more than a few degrees from what would be measured visually.

\subsection{Reliability of the deprojected ellipse parameters in discs with a finite thickness}

The reliability of the deprojected ring parameters depends on the quality of the $\mathrm{P} 4$ measured disc deprojection parameters.
One source of error when deprojecting is the assumption that discs are infinitely thin. Indeed, the thicker a galaxy disc, the more the measured galaxy inclination, $i$, will differ from the real inclination, $i^{\prime}$.

The thickness of discs has been studied by de Grijs (1998). He found that the ratio between the disc scale-height and the disc scale-length $\left(h_{z}\right.$ and $h_{R}$, respectively) decrease monotonically with Hubble stage. The average $h_{z} / h_{R}$ is around $1 / 3$ for S0 galaxies and decreases to $1 / 9$ for Sd galaxies. However, for a given stage the scatter in $h_{z} / h_{R}$ is large, so the relationship described by de Grijs (1998) cannot be used for correcting the finite thickness effects in deprojected parameters without adding a substantial error. Instead, in this section we adopt the approach of quantifying possible biases in the deprojected parameters.

Hubble (1926) described the formalism for finding inclination of an oblate ellipsoid,

$\cos ^{2} i^{\prime}=\frac{q_{\mathrm{d}}^{2}-q_{z}^{2}}{1-q_{z}^{2}}$,

where $q_{\mathrm{d}}$ is the observed galaxy disc axis ratio and $q_{z}$ is the galaxy flattening, that is, $h_{z} / h_{R}$. From this, and knowing that $\cos i=q_{\mathrm{d}}$, we can derive the formula for calculating the $i$ corresponding to a given $i^{\prime}$ :

$i=\arccos \left(\sqrt{q_{z}^{2}+\left(1+q_{z}^{2}\right) \cos ^{2} i^{\prime}}\right)$.

From this expression we can deduce that the maximum bias at finding a disc inclination comes from the galaxies with thicker discs, namely S0s with $h_{z} / h_{R} \sim 1 / 3$. Most of our results in Sect. 6 are based on galaxies with $i \leq 60^{\circ}$. For an S0 galaxy with $i^{\prime}=60^{\circ} \Delta i=i^{\prime}-i \sim 5^{\circ}$. Accordingly, for most of the galaxies with $i \leq 60^{\circ}, i \sim i^{\prime}$. 

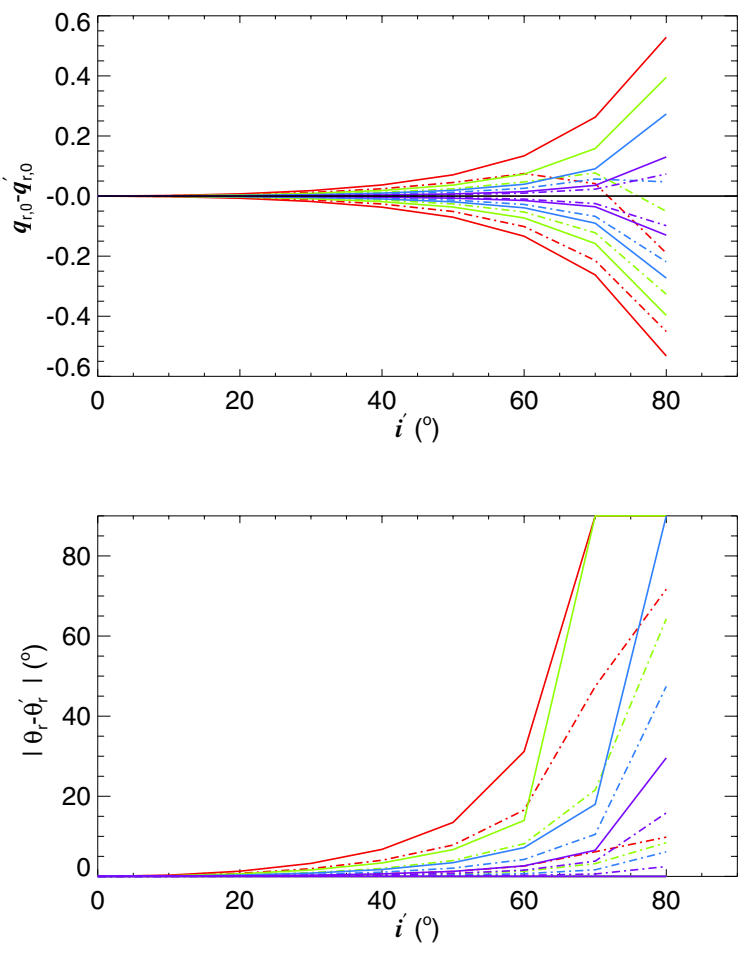

Fig. 12. Top panel: difference between the measured intrinsic axis ratio of a ring, $q_{\mathrm{r} 0}$, and the real one, $q_{\mathrm{r}, 0}^{\prime}$, as a function of the real galaxy inclination, $i^{\prime}$, for four disc scale-height to scale-length ratios. The colour code corresponds in an outside-in order to $h_{z} / h_{R}=1 / 3$ (red), $h_{z} / h_{R}=1 / 5$ (green), $h_{z} / h_{R}=1 / 7$ (blue), and $h_{z} / h_{R}=1 / 10$ (purple). Bottom panel: difference of the measured angle difference between the orientation of the major axis of a ring with respect to the line of nodes, $\theta_{\mathrm{r}}$, with respect to the real one, $\theta_{\mathrm{r}}^{\prime}$, as a function of the real galaxy inclination. In both panels, the solid lines indicate the maximum and the minimum $q_{\mathrm{r}, 0}-q_{\mathrm{r}, 0}^{\prime}\left(\left|\theta_{\mathrm{r}}-\theta_{\mathrm{r}}^{\prime}\right|\right)$ for a given $i^{\prime}$ colour-coded as in the top panel. The dashed lines indicate the limits of the region enclosing $68.2 \%$ of $q_{\mathrm{r}, 0}-q_{\mathrm{r}, 0}^{\prime}\left(\left|\theta_{\mathrm{r}}-\theta_{\mathrm{r}}^{\prime}\right|\right)$ values at a given $i^{\prime}$.

To examine how the biased $i$ values calculated from Eq. (8) would affect our ring deprojection values, we created four model galaxies with $h_{z} / h_{R}=1 / 3, h_{z} / h_{R}=1 / 5, h_{z} / h_{R}=1 / 7$, and $h_{z} / h_{R}=1 / 10$. We then inclined each of them with angles in the range $i^{\prime}=0^{\circ}$ to $i^{\prime}=80^{\circ}$ with $10^{\circ}$ intervals.

We checked how the underestimated inclination measurements would affect the measured intrinsic ring ellipticities, $q_{\mathrm{r}, 0}$, and the angle between their major axes and the lines of nodes of the deprojection, $\theta_{\mathrm{r}}$. To do this, we considered a population of rings with a Gaussian distribution of intrinsic axis ratios, $q_{\mathrm{r}, 0}^{\prime}$, with the centre of the Gaussian at $q_{\mathrm{r}, 0}^{\prime}=0.8$ and a dispersion of $\sigma^{\prime}=0.13$. These values are representative of the measured axis ratio distribution of inner rings as seen in Sect. 6.3. For each galaxy model with a given $h_{z} / h_{R}$ and for each of the studied inclinations, we obtained 1000 random ring axis ratios. The intrinsic axis ratio of each of these rings was recovered after projecting it using the real galaxy inclination, $i^{\prime}$, and deprojecting it using the measured galaxy inclination, $i$. This was repeated for 19 angle differences between the ring major axis and the line of nodes used for inclining the model galaxy. These angles ranged from $\theta_{\mathrm{r}}^{\prime}=0^{\circ}$ to $\theta_{\mathrm{r}}^{\prime}=90^{\circ}$ in steps of $5^{\circ}$.

In the top panel in Fig. 12 we study the accuracy of the measured ring axis ratios. If, as in our results section, we focus on galaxies with $i^{\prime} \leq 60^{\circ}$, the errors at measuring $q_{\mathrm{r}, 0}$ are always below $\left|\Delta q_{\mathrm{r}, 0}\right|=0.05$ except for galaxies with $h_{z} / h_{R}=1 / 3$ and a few cases with $h_{z} / h_{R}=1 / 5$. Except in exceptional cases for

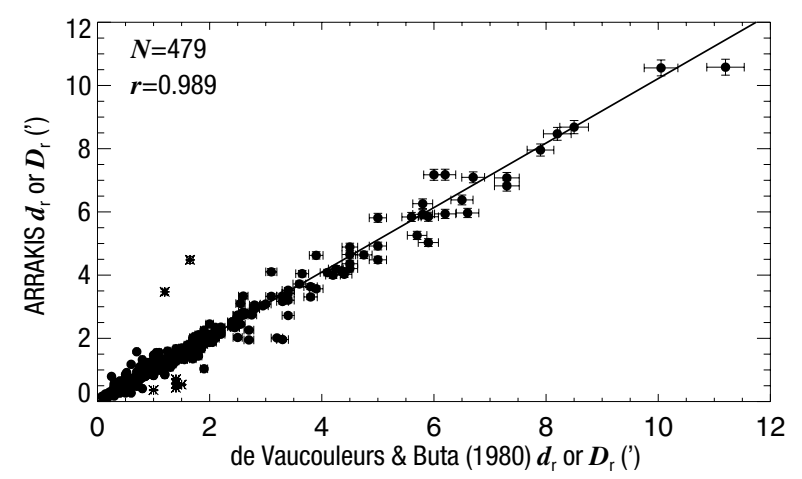

Fig. 13. Comparison of the angular diameters of ARRAKIS rings with those in VB80. The line corresponds to a linear fit to the data points in the plot. The outliers due to the wrong identification of the resonance to which a ring is linked in VB80 are marked with asterisks and are excluded from the fit. The numbers in the top-left corner indicate the number of points considered in the fit and their correlation coefficient.

$h_{z} / h_{R}=1 / 3,\left|\Delta q_{\mathrm{r}, 0}\right| \leq 0.10$. For $i^{\prime} \leq 40^{\circ}$, the uncertainties in $q_{0}$ due to the finite thickness of discs are always rather small and in the order of the uncertainty in the measurement of $q_{\mathrm{r}}$ $\left(\left|\Delta q_{\mathrm{r}}\right|=0.02\right)$ as measured in Sect. 4.2.

In the bottom panel in Fig. 12 we study the accuracy of the measured ring intrinsic orientation. We find that for galaxies with $i^{\prime} \leq 60^{\circ}$, the maximum $\left|\theta_{\mathrm{r}}-\theta_{\mathrm{r}}^{\prime}\right|$ is always below $10^{\circ}$ except, again, for galaxies with $h_{z} / h_{R}=1 / 3$ and in a few cases in those with $h_{z} / h_{R}=1 / 5$.

We conclude this section by saying that as found for bars in Sect. 4.5, results from deprojected parameters seem in general reliable for galaxies with $i \leq 60^{\circ}$. The exception are the moderately inclined galaxies $\left(i=40-60^{\circ}\right)$ with a larger disc thickness relative to the scale-length ( $\mathrm{S} 0$ galaxies), whose deprojected ring parameters are in some cases not very accurate.

\section{Comparison of ring properties in ARRAKIS with the data in the literature}

In this section we compare the measured properties of rings with those reported in a set of significant papers in the literature that have samples in common with ours.

In several cases, significant discrepancies have been found between the ring diameters in ARRAKIS and those in the literature. In most of them, we found that this is because the features are classified in a different way in ARRAKIS and in the literature. For example, the feature with an ARRAKIS major diameter of 1.48 in NGC 4736 was classified as an inner ring by de Vaucouleurs \& Buta (1980). Here, this feature is considered as a nuclear ring because it is found inside a very broad bar/oval that was unnoticed by de Vaucouleurs \& Buta (1980). The broad bar in NGC 4736 is here recognized as being surrounded by a subtle ring-lens 4.48 in major diameter that was considered to be an outer lens in de Vaucouleurs \& Buta (1980). Therefore, when comparing the inner ring in de Vaucouleurs \& Buta (1980) with that in ARRAKIS, we are actually comparing two different features. Such features with a wrong classification appear as outliers in Figs. 13-17 and have not been considered for the statistics in this section.

\subsection{ARRAKIS and de Vaucouleurs \& Buta (1980)}

De Vaucouleurs \& Buta (1980, hereafter VB80) compiled a catalogue that includes the diameter of outer and inner rings 


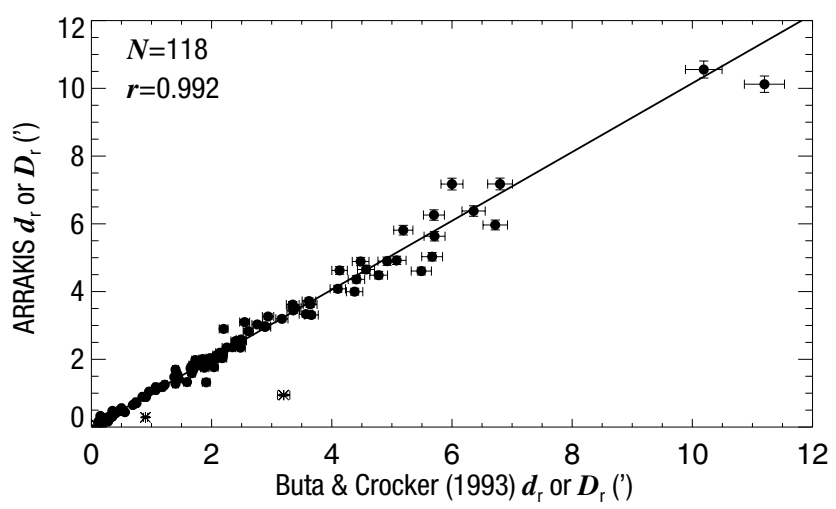

Fig. 14. As in Fig. 13, but with data from BC93.

in 532 bright galaxies. The $\mathrm{S}^{4} \mathrm{G}$ and the VB80 samples have 303 galaxies in common.

We compared the sizes of the rings listed both in ARRAKIS and VB80. For this, we performed a linear least-squares fit of the major and minor axis diameter measurements in the two surveys. The fit takes into account the errors both in the ARRAKIS and the VB80 measurements. The ARRAKIS diameter errors were considered to follow the internal error function in Eq. (1). VB80 did not quantify their errors. Since their data are collected from photographic plates, we considered that their errors are probably similar to those in the CSRG, and therefore we used the CSRG internal error function for their diameters.

The fit we obtained is

$$
\begin{aligned}
d_{\mathrm{r}}, D_{\mathrm{r}}(\text { ARRAKIS })= & 0.013 \pm 0^{\prime} 006 \\
& +(1.021 \pm 0.003)\left(d_{\mathrm{r}}, D_{\mathrm{r}}\right)(\mathrm{VB} 80)
\end{aligned}
$$

with a correlation factor $r=0.989$. This means that the agreement between the measurements in ARRAKIS and in VB80 is very good.

\subsection{ARRAKIS and Buta \& Crocker (1993)}

Buta \& Crocker (1993, hereafter BC93) studied galaxies with nuclear components, mostly nuclear rings, but also lenses and spirals. In BC93, galaxies with nuclear features also had their inner and outer features classified. The BC3 catalogue lists 64 galaxies, 37 of which are included in the $\mathrm{S}^{4} \mathrm{G}$. Figure $14 \mathrm{com}$ pares the sizes of the features measured in ARRAKIS with those in BC93.

As with the VB80 data, we performed a least-squares fit to compare the BC93 data with ours. Again, the CSRG internal error function was used to describe the errors in the diameters in BC3. The fit gives

$$
\begin{aligned}
d_{\mathrm{r}}, D_{\mathrm{r}}(\text { ARRAKIS })= & -0.008 \pm 0.016 \\
& +(1.016 \pm 0.006)\left(d_{\mathrm{r}}, D_{\mathrm{r}}\right)(\mathrm{BC} 93)
\end{aligned}
$$

with a correlation factor $r=0.992$.

\subsection{ARRAKIS and the CSRG}

The CSRG includes 3692 galaxies that host outer and inner rings and lenses south of declination $\delta=-17^{\circ}$. The CSRG has 120 galaxies in common with the $\mathrm{S}^{4} \mathrm{G}$.

In the top-left panel of Fig. 15 we compare the size of a feature when it is identified in both the CSRG and ARRAKIS. A linear fit to the data accounting for the ARRAKIS and CSRG interal error functions at measuring diameters yields

$$
\begin{aligned}
d_{\mathrm{r}}, D_{\mathrm{r}}(\text { ARRAKIS })= & -0.018 \pm 0.011 \\
& +(0.994 \pm 0.005)\left(d_{\mathrm{r}}, D_{\mathrm{r}}\right)(\mathrm{CSRG}),
\end{aligned}
$$

and a correlation factor $r=0.992$, again indicating excellent agreement.

The CSRG describes the projected ring orientation as the PA offset between the bar and the ring major axis. ARRAKIS has such data only for galaxies with $q_{\mathrm{d}}>0.5$ because we considered more inclined galaxies to have unreliable bar axis ratio determinations (Sect. 4.5). The correlation between the ring orientation values for the CSRG and ARRAKIS is not as tight as it is for the angular sizes (top-right panel in Fig. 15). This is probably due to the analogue procedure used for building the CSRG. Indeed, it is conceivable to obtain accurate results on ring diameters using a magnifying device, but the determination of the major axis of bars and especially rings is doomed to be much more subjective.

The difference between the CSRG PA $\mathrm{r}-\mathrm{PA}_{\mathrm{b}}$ values and ours is plotted in the bottom-left and bottom-right panels of Fig. 15 as a function of the ring size and the ring axis ratio. The differences in $\mathrm{PA}_{\mathrm{r}}-\mathrm{PA}_{\mathrm{b}}$ are larger than our internal error for $\mathrm{PA}_{\mathrm{r}}$, which is generally below $10^{\circ}$ for rings with $D_{\mathrm{r}}>3^{\prime}$ and/or $q_{\mathrm{r}}<0.7$ (Fig. 8).

\subsection{ARRAKIS and AINUR}

AINUR contains the most complete catalogue of nuclear rings to date, mostly relying on Hubble Space Telescope images that in some cases have an angular resolution as high as $\sim 0{ }^{\prime} 1$. Because of that, it contains detailed information of angularly small rings that cannot be expected to be described this thoroughly in ARRAKIS. Sixty out of 107 galaxies in AINUR are in the $S^{4} \mathrm{G}$ sample. Of these 60 galaxies 26 are reported here to have a nuclear ring.

When comparing the major axis diameter of features classified as nuclear rings both in AINUR and ARRAKIS, we obtain

$d_{\mathrm{r}}, D_{\mathrm{r}}($ ARRAKIS $)=00^{\prime} 022 \pm 0.010$

$$
+(0.858 \pm 0.029)\left(d_{\mathrm{r}}, D_{\mathrm{r}}\right)(\mathrm{AINUR}),
$$

with a correlation factor $r=0.983$. Here, the internal error functions for the diameters are those in Eq. (1) both for AINUR and ARRAKIS. We chose this error function for AINUR because, as for ARRAKIS, the ring measurements were made on digital images. This fit is much poorer than those found for VAU80, BU93, CSRG, and NIRSOS. This is probably because of the difficulty found in measuring the properties of features that are often barely resolved in the $\mathrm{S}^{4} \mathrm{G}$. The difference may also reflect that the procedures used to measure the ring properties in the two works are different.

\subsection{ARRAKIS and NIRSOS}

NIRSOS is a ground-based survey of 206 nearby early-type disc galaxies with a sample dominated by S0s. The survey was produced using the $K_{\mathrm{s}}$ band. NIRSOS does not reach as deep as the $\mathrm{S}^{4} \mathrm{G}$ but has a better angular resolution (typical pixel resolution of $0{ }^{\prime} 3$ and typical seeing around $\left.1^{\prime \prime}\right)$. NIRSOS and the $S^{4} G$ have 93 galaxies in common.

The ring sizes measured in NIRSOS agree very well with ours (Fig. 17). When comparing the ring diameters from ARRAKIS and NIRSOS (in both cases we use the internal error function from Eq. (1) because in both cases the rings are 

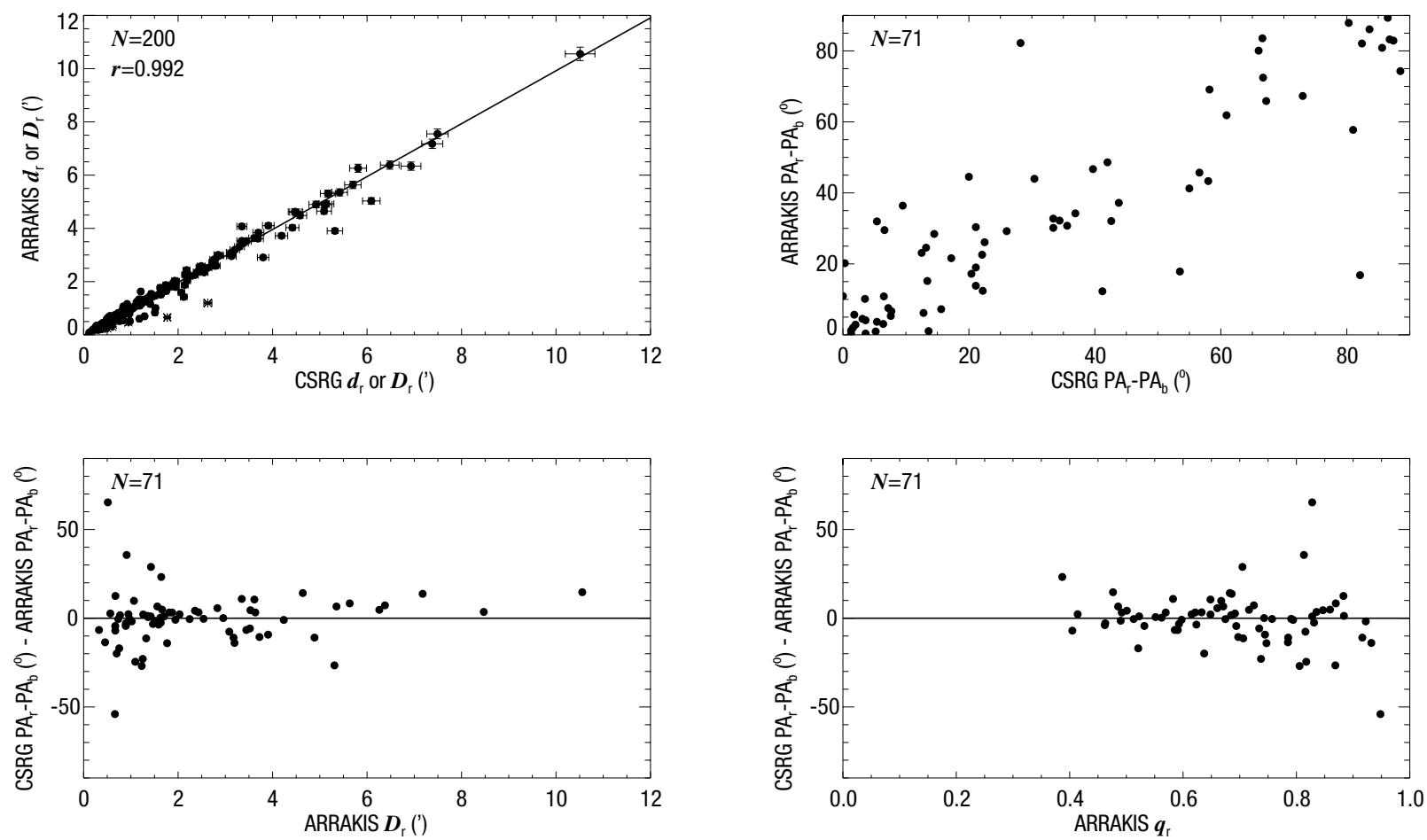

Fig. 15. Top-left panel: as in Fig. 13, but with data from the CSRG. Top-right panel: comparison of the PA offset between the major axis of bars and that of rings in the CSRG and ARRAKIS. Bottom panels: difference in the PA offsets between the bar and the ring major axis as a function of the ring size and of the ring axis ratio. In the three last panels, only points for barred galaxies with $\epsilon_{\mathrm{d}} \leq 0.5$ are presented.

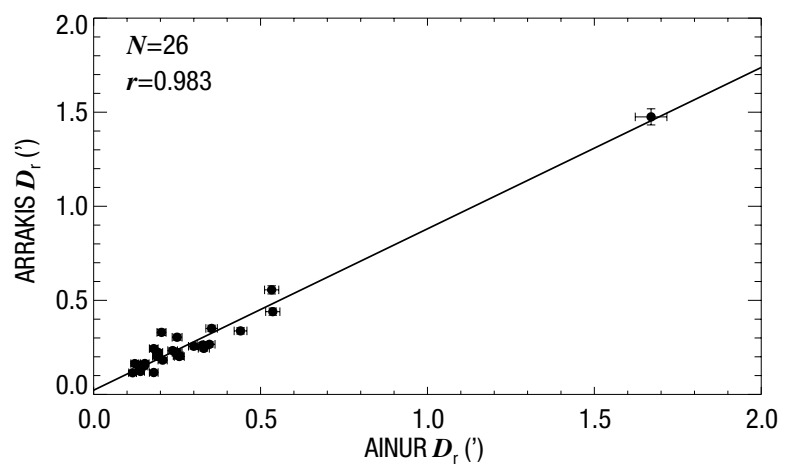

Fig. 16. As in Fig. 13, but with data from AINUR.

measured on digital images), we obtain the following excellent linear fit

$$
\begin{aligned}
d_{\mathrm{r}}, D_{\mathrm{r}}(\text { ARRAKIS })= & 0{ }^{\prime} 006 \pm 0.009 \\
& +(0.993 \pm 0.005)\left(d_{\mathrm{r}}, D_{\mathrm{r}}\right)(\mathrm{NIRS} 0 \mathrm{~S}),
\end{aligned}
$$

and the correlation factor is $r=0.988$.

Figure 17 also shows that the agreement in the measured position angle of the ring major axis is very good and much better than when comparing this paper with the CSRG. One possible reason is that NIRSOS is a digital survey where $\mathrm{PA}_{\mathrm{r}}$ can be measured more easily. The second reason is that in the CSRG the position angle difference between the ring and the bar major axis was measured. This measurement includes two errors: that related to the orientation of the ring, and that related to the orientation of the bar, and thus causes larger discrepancies than those found when comparing NIRSOS with this paper. As we found when we compared ARRAKIS with the CSRG and from our internal errors, the uncertainty in the orientation measurement increases for smaller and/or rounder rings.

\section{Results}

We recall that in this paper rings refers to the set including both open pseudorings and closed features. Here, the set including only closed features is referred to as closed rings.

\subsection{Fraction of rings as a function of the Hubble stage}

Stage classification is commonly described by a numerical code ranging from -5 to 10 corresponding to the sequence going from E to I galaxies. However, in some cases, a more detailed classification can be made and is then denoted with intermediate stages such as Sbc or S $\underline{c} d$, which in turn are codified as $T=4.5$ and $T=5.5$. In the few particular cases for which such a classification is provided, we rounded up the stage so that, for example, Sbc becomes Sc $(T=5)$ and $\underline{\operatorname{s}} \underline{\mathrm{c} d}$ becomes $\operatorname{Scd}(T=6)$.

Some galaxies in Buta et al. (in prep.) have not been classified into categories that fit in the traditional Hubble fork. Indeed, based on the van den Bergh (1976) hypothesis that S0 galaxies form a sequence from early to late galaxies just like normal disc galaxies do, some galaxies have been classified as $\mathrm{SO}_{\mathrm{b}}, \mathrm{SO}_{\mathrm{bc}}$, $\mathrm{SO}_{\mathrm{c}}, \mathrm{SO}_{\mathrm{cd}}, \mathrm{SO}_{\mathrm{d}}$, and $\mathrm{S}_{\mathrm{m}}$. This is true for $36 \mathrm{~S}^{4} \mathrm{G}$ galaxies, three of which host rings. These galaxies were included in the bin corresponding to their counterpart in the traditional sequence (e.g., $\mathrm{SO}_{\mathrm{b}}$ galaxies were grouped together with $\mathrm{Sb}$ galaxies).

A few galaxies (12) have a double stage (for details on double-stage galaxies see Buta et al. 2010), five of which are ringed. These galaxies were not included in the stage statistics. We also excluded the dwarf elliptical galaxies from the results in this subsection (34 galaxies, none of which is ringed). Finally, we also excluded $12 \mathrm{~S}^{4} \mathrm{G}$ galaxies that are so difficult to classify that there were assigned no stage. None of these galaxies is ringed. 

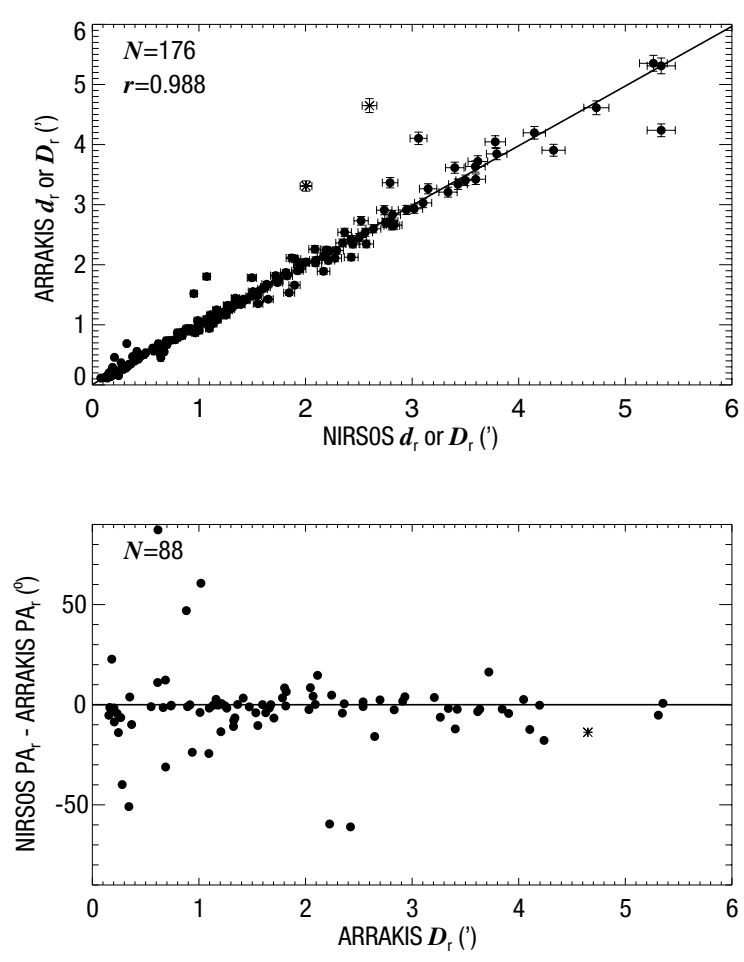

Fig. 17. As in Fig. 15, but with data from NIRSOS.

The distribution of resonant features varies greatly with the stage of the galaxies, as seen in Fig. 18. The bottom-row panels in that figure have $1-\sigma(68 \%)$ confidence levels calculated using binomial distribution statistics,

$\Delta(p)=\sqrt{\frac{p(1-p)}{n}}$,

where $p$ is the probability of a galaxy in a given stage bin to have a specific type of ring and $n$ the total number of galaxies in that stage bin. All the uncertainties given in this section were calculated using this expression. When the data in this section are quantitatively compared with the data in other papers, we verified that the uncertainties in those papers were computed in the same way as we did.

Inclination has to be taken into account before discussing in detail the stage distributions statistics. Indeed, it is more likely for inclined galaxies to have unidentified or misinterpreted rings. We found that of the whole sample of galaxies, $31 \%$ have some resonant feature, but that this fraction is increased to $41 \%$ for the subsample with $\epsilon_{\mathrm{d}} \leq 0.5$. These numbers indicate that as much as $\sim 50 \%$ of the resonant features are missed in inclined galaxies. This effect is especially pronounced for inner features, most likely because they are found in regions of galaxies that are hard to interpret because of bars, spiral arms, and/or intense star formation regions.

\subsubsection{Outer rings}

The stage distribution of outer rings based on the $S^{4} \mathrm{G}$ subsample with $\epsilon_{\mathrm{d}} \leq 0.5$ indicates the following (see also continuous blue lines in Fig. 18):

- They are found in $16 \pm 1 \%$ of $\mathrm{S}^{4} \mathrm{G}$ galaxies.

- They are mostly found at $-1 \leq T \leq 2$ where they appear in over $40 \%$ of galaxies. This number is similar to the $53 \pm 5 \%$
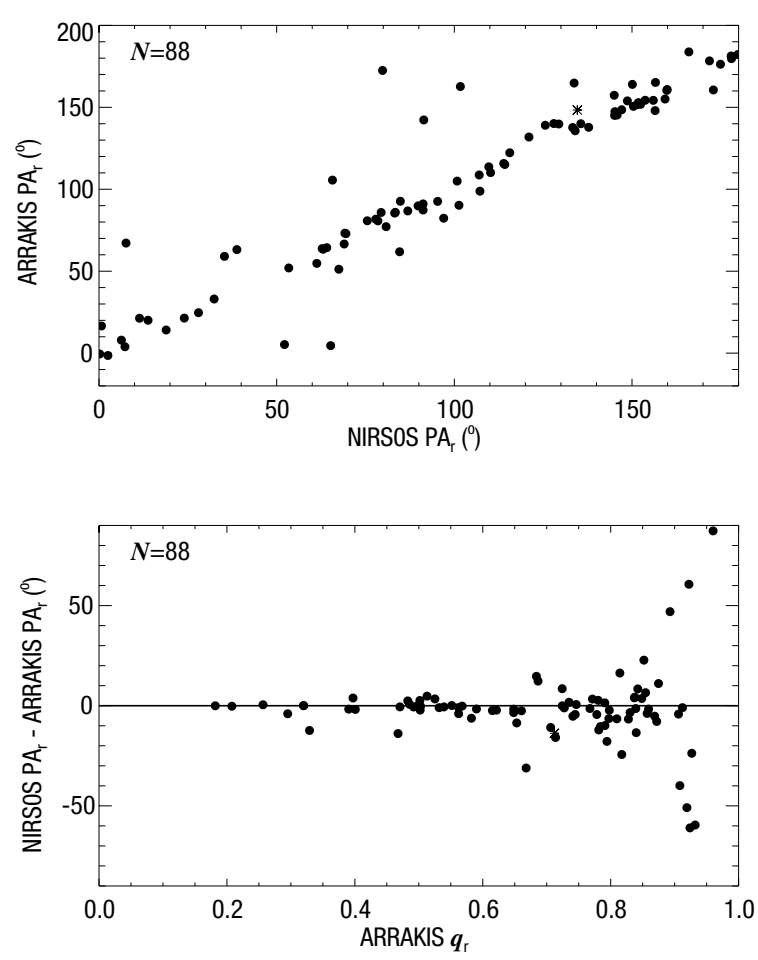

fraction of outer features found in NIRSOS for stages $-1 \leq$ $T \leq 1$ (Laurikainen et al. 2011).

- For $T \geq 4$ they are quite rare, and their frequency drops below $10 \%$.

- Outer closed rings (continuous light blue lines) are most frequent for $T \leq 0$, with a peak frequency of $\sim 50 \%$ at $T=-1$. Above $T=0$ their frequency drops fast with increasing stage. This behaviour is also seen in NIRSOS.

- Outer closed rings and closed ring-lenses account for $100 \%$ of the outer features for $T \leq-1$. This is a difference with NIRS0S: the fraction of pseudorings for $T \leq 1$ NIRSOS galaxies is non-zero, though still rather small, $19 \pm 7 \%$. Of the five NIRSOS galaxies with $T \leq-1$ and with an outer pseudoring, two are included in the $\mathrm{S}^{4} \mathrm{G}$ sample. These two galaxies have been classified with the same stage in NIRSOS and Buta et al. (in prep.), which means that the difference between NIRSOS and ARRAKIS probably caused by the different appearance of outer features at different wavelengths and not by problems in the stage classification.

Buta \& Combes (1996) found qualitatively similar results using RC3 data. Using data from VB80, Elmegreen et al. (1992) also found that earlier-type galaxies are more likely to have outer rings.

\subsubsection{Inner rings}

The stage distribution of inner rings based on the $S^{4} \mathrm{G}$ subsample with $\epsilon_{\mathrm{d}} \leq 0.5$ indicates the following (see also dashed green lines in Fig. 18):

- They are found in $35 \pm 1 \%$ of $\mathrm{S}^{4} \mathrm{G}$ galaxies.

- They have a frequency of over $40 \%$ for stages $-1 \leq T \leq 6$. The distribution is not very peaked and has its maximum for $-1 \leq T \leq 3$ (more than $60 \%$ of these galaxies have inner features). 


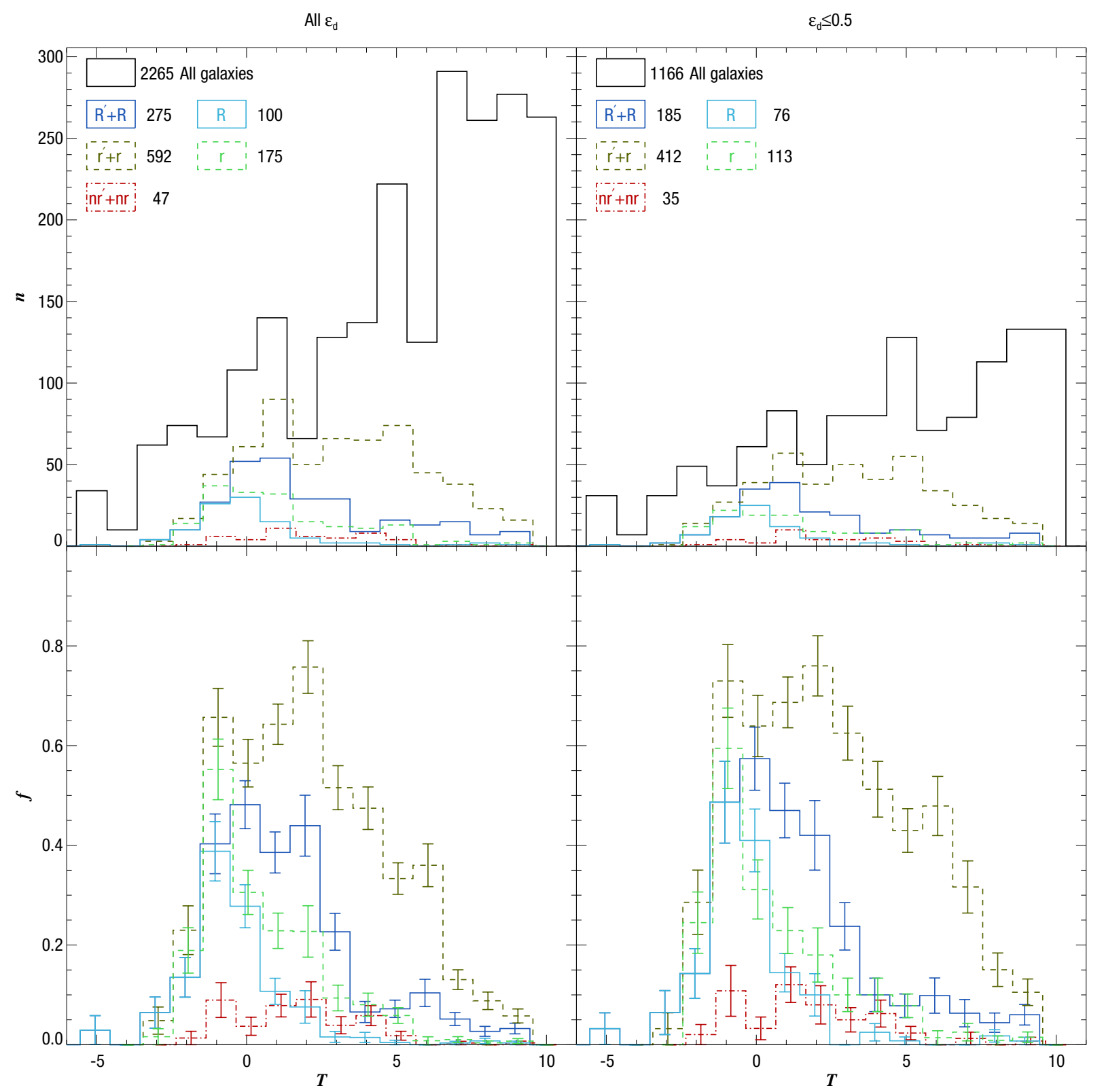

Fig. 18. Top panels: stage distribution of the $\mathrm{S}^{4} \mathrm{G}$ sample (black line), of galaxies hosting outer rings (continuous darker blue line), inner rings (dashed darker green line), and nuclear rings (dash-point red line). Lighter blue and green lines indicate histograms considering only galaxies hosting closed outer and closed inner rings, respectively. Bottom panels: fraction of galaxies with resonant features for a given stage colour-coded as in the two top panels. Left panels are for the whole sample and right panels for galaxies with a disc ellipticity $\epsilon_{\mathrm{d}} \leq 0.5$. The numbers in the top panels indicate the number of galaxies included in the histograms that corresponds to the colour and line pattern of the adjacent box.

- Their frequency drops below $20 \%$ for galaxies with $T \leq-3$ and $T \geq 8$. They are therefore very frequent in most disc galaxy stages.

- The peak in the inner closed ring frequency is found at $T=$ -1 (light green lines) as also found in NIRS0S. The inner closed ring frequency distribution is shifted towards earlier types than that of the inner ring distribution.

- Inner closed rings are more frequent than inner pseudorings for $T \leq 1$. This agrees very well with the results in NIRSOS. There the fraction of inner features that are inner closed rings in galaxies with $-3 \leq T \leq-1$ is $75 \pm 7 \%$, we found $81 \pm 6 \%$. For galaxies with types $0 \leq T \leq 1$ NIRSOS reports a fraction of inner closed rings among inner features of $38 \pm 8 \%$, we found $40 \pm 5 \%$.
These results differ from those presented in Buta \& Combes (1996), which were based on RC3 data. These authors found the inner closed ring fraction to be roughly constant for all types of disc galaxies with $T \leq 5$ (here the distribution is peaked at $T=-1$ ) and also that they are more frequent than pseudorings for $T \leq 3$ (here, this only occurs for $T \leq-1$ ). These differences are too large to be a consequence of the one stage shift caused by classifying galaxies in $3.6 \mu \mathrm{m}$ instead of the $B$ band.

The stage distribution of outer and inner rings is quite different. While the outer ring distribution drops between types $T=3$ and $T=4$, the inner rings distribution does so between types $T=7$ and $T=8$. 


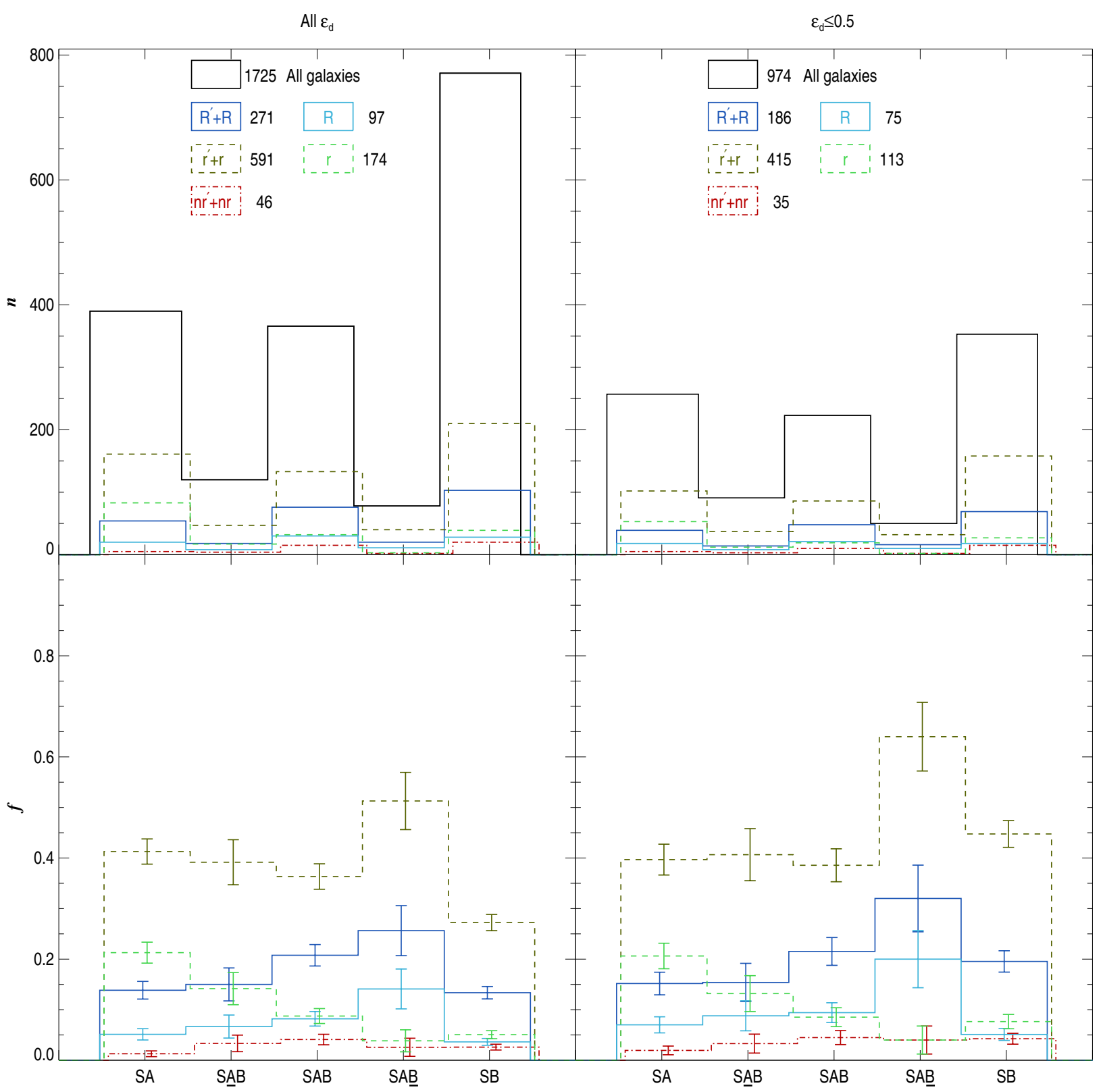

Fig. 19. Top panels: family distribution of the $S^{4} \mathrm{G}$ sample and ringed galaxies colour- and line-coded as in Fig. 18. Bottom panels: fraction of galaxies with rings for a given family colour- and line-coded as in the two top panels. Left panels are for the whole sample and right panels for galaxies with a disc ellipticity $\epsilon_{\mathrm{d}} \leq 0.5$. The numbers in the top panels indicate the number of galaxies included in the histogram that corresponds to the colour- and line-pattern of the adjacent box.

\subsubsection{Nuclear rings}

Nuclear rings (dash-point red lines in Fig. 18) are found in galaxies with $-1 \leq T \leq 6$, which is similar to that obtained in AINUR $(-3 \leq T \leq 7)$. However, the peak in the distribution $(T=2)$ does not coincide with those in AINUR $(T=-1$ and $T=4)$. These differences are probably not significant, because the number of nuclear rings here is a factor of three smaller than in AINUR.

In AINUR nuclear rings were found in $\sim 20 \%$ of galaxies with stages $-3 \leq T \leq 7$. A similar fraction was given by Knapen (2005). However, the fraction of low inclination $\left(\epsilon_{\mathrm{d}} \leq 0.5\right) \mathrm{S}^{4} \mathrm{G}$ galaxies that host nuclear rings is $5 \pm 1 \%$ in the same range of stages. The reason for the discrepancy is that, because of angular resolution problems, many of the smaller rings are undetected or are classified as nuclear lenses in the $\mathrm{S}^{4} \mathrm{G}$.

\subsection{Fraction of rings as a function of the galaxy family}

Of the $2348 \mathrm{~S}^{4} \mathrm{G}$ galaxies that have been classified in Buta et al. (in prep.), 1725 are disc galaxies $(-3 \leq T \leq 9)$ to which a family has been assigned (SA, SAB, SAB, SA $\underline{B}, \mathrm{SB})$. Some disc galaxies (233) have no assigned family and are mostly close to edgeon galaxies without obvious signatures of edge-on bars such as boxy inner isophotes. The ring distribution with family is shown in Fig. 19.

\subsubsection{Outer rings}

The fraction of galaxies with $\epsilon_{\mathrm{d}} \leq 0.5$ that host outer rings (blue continuous lines in Fig. 19) increases steadily from SA $(15 \pm 2 \%)$ to $\mathrm{SA} \underline{\mathrm{B}}(32 \pm 7 \%)$. For stronger bars (SB), it drops to $20 \pm 2 \%$. 
The distribution of outer closed rings (light blue continuous lines) is qualitatively similar: the fraction of galaxies that host closed rings rises from $7 \pm 2 \%$ to $20 \pm 6 \%$ between SA and SAB and then drops down to $5 \pm 1 \%$ for the SB family.

\subsubsection{Inner rings}

The inner ring family distribution is quite different from that for the outer rings (green dashed lines in Fig. 19). The fraction of galaxies that host inner rings remains roughly constant from SA to $\mathrm{SAB}$ at a level of $\sim 40 \%$ and then suddenly increases to $64 \pm 7 \%$ for $\mathrm{SAB}$ galaxies. The fraction of SB galaxies that host inner rings is similar to that in $\mathrm{SA}$ to $\mathrm{SAB}$ galaxies.

We also find that galaxies with no bars or weak bars (SA and $\mathrm{SAB}$ ) have a higher fraction of inner closed rings (light green dashed lines) than galaxies with stronger bars.

\subsubsection{Nuclear rings}

The number of nuclear rings in ARRAKIS is small and thus the validity of the statistics is uncertain. However, we can see that the nuclear feature fraction increases from families SA to $\mathrm{SAB}$ and then remains roughly constant (dash-point red lines in Fig. 19).

\subsection{Ring shapes}

\subsubsection{Projected ring shapes}

The top-left panel in Fig. 20 shows the projected axis ratio of resonance rings, under the assumption that they can be described by an ellipse coplanar with the disc of the galaxy. It is qualitatively similar to that found in the CSRG (top panels of its Fig. 7): the distribution grows gently from zero and has a maximum for quite round rings $\left(0.8<q_{\mathrm{r}} \leq 0.9\right.$ for inner and outer rings here and $0.7<q_{\mathrm{r}} \leq 0.8$ in the CSRG). It then drops abruptly for even rounder rings.

The statistical expectation is that if rings were intrinsically circular and sitting in perfectly circular discs, with their rotation axis with random orientations, the projected ring axis ratio probability density function would be uniform. As explained in the CSRG, the reason to justify the lack of rings with a low apparent axis ratio is that they become increasingly harder to identify in inclined galaxies. It is interesting to see that just like in the CSRG, for the axis ratios close to $q_{\mathrm{r}}=0$, outer rings show a behaviour closer to the statistical expectation than inner rings. This is because they are found in outer parts of the galaxy that are less messy and easier to interpret than inner regions when seen close to edge-on. We do not observe the distribution to be uniform near $q_{\mathrm{r}}=1$ because there is a dip in the observed distribution of rings. The reason why we do not see more rings that appear nearly round in projection is that they are not intrinsically circular, as described in the next subsubsection.

\subsubsection{Deprojected ring shapes}

One of the improvements of this study over the largest systematic study of rings conducted so far, the CSRG, is that the $S^{4} G$ $\mathrm{P} 4$ provides us with reliable orientations of the galaxies. This allows us to obtained intrinsic ring shapes and orientations, as explained in Sect. 4.4.

In the CSRG, the deprojected ring axis ratio distribution was studied under several assumptions and using a statistical treatment. One of the assumptions was that the actual shape of the deprojected axis ratio distribution is Gaussian, with a peak at $\left\langle q_{\mathrm{r}, 0}\right\rangle$ and a dispersion $\sigma$. With our deprojected data we do not need this assumption.

The deprojected ring distribution is shown in the bottom panels of Fig. 20. We overlay Gaussian fits to show how well the Gaussian distribution hypothesis works. The parameters of the Gaussian fits in this section can be found in Table 3. Although the fitted Gaussians are nearly the same when fitting rings in all galaxies and when fitting them only for galaxies with $\epsilon_{\mathrm{d}} \leq 0.5$, the distributions are slightly different in the low $-q_{\mathrm{r}}$ wing; indeed, when considering all galaxies, the left wing for outer and inner rings extends farther than when considering only galaxies with $\epsilon_{\mathrm{d}} \leq 0.5$. This is because for close to edge-on galaxies deprojections based on the shape of outer isophotes of discs become unreliable due to the non-zero thickness of discs (see Sect. 4.6) and/or the presence of extended bulges and haloes. Thus, for some galaxies, the obtained deprojection parameters can be considered to be tentative at best and may cause the calculated deprojected ring shapes to be unrealistically stretched. A notorious case is NGC 4594 (the Sombrero galaxy), where outer isophotes have an ellipticity of about $\epsilon_{\mathrm{d}}=0.58$ even though the real figure is probably closer to $\epsilon_{\mathrm{d}}=0.83$ (see, e.g., Jardel et al. 2011). All but one ring with deprojected axis ratio $q_{\mathrm{r}, 0} \leq 0.4$ are found in galaxies with fitted disc ellipticity $\epsilon_{\mathrm{d}}>0.5$ (IC 5264, NGC 4594, NGC 5078, NGC 5746, NGC 5777, and NGC 5900). The exception is NGC 986, a galaxy with $\epsilon_{\mathrm{d}}=0.1$ and a peculiar elongated nuclear ring. Because this effect may also occur (although in a less obvious way) in other inclined galaxies, we continue our analysis of intrinsic ring shapes based only on galaxies with $\epsilon_{\mathrm{d}} \leq 0.5$.

Based on the fitted $\left\langle q_{\mathrm{r}, 0}\right\rangle$, we find that outer rings are generally rounder than the inner ones. More specifically, for the ARRAKIS sample we find $\left\langle q_{\mathrm{r}, 0}(\mathrm{O})\right\rangle=0.88$ and $\left\langle q_{\mathrm{r}, 0}(\mathrm{I})\right\rangle=0.80$, respectively. Outer closed rings have the same average $\left\langle q_{\mathrm{r}, 0}(\mathrm{O})\right\rangle$ as outer features in general, but inner closed rings are on average slightly rounder than the average for inner features.

Rings become systematically more elliptical when moving along the family sequence from SA to SB (Fig. 21). However, the dispersion in each of the Gaussian distributions is always wide enough to allow very round rings to exist, even for SB galaxies. Fits for outer rings in SA galaxies are not shown because they yield that the centre of the Gaussian would be located at $\left.\left\langle q_{\mathrm{r}, 0}(\mathrm{O})\right\rangle\right\rangle 1$.

Inner rings tend to become more elliptical with increasing galaxy stage. This trend is not found for the outer features, although this might be explained by the low number of them found at stages $T \geq 4$.

\subsection{Ring orientations}

In this subsection we describe the orientations of the rings with respect to bars. We focus only on barred galaxies for which we are able to accurately measure their orientation (that is, most of those in galaxies with $\epsilon_{\mathrm{d}} \leq 0.5$ ). We compared the PA of the major axis of the ring, $\mathrm{PA}_{\mathrm{r}}$, and the bar, $\mathrm{PA}_{\mathrm{b}}$. We denote the difference between those major axis as $\mathrm{PA}_{\mathrm{r}}-\mathrm{PA}_{\mathrm{b}}$. However, because we define it to be in the first quadrant, it should be formally written as minimum $\left(\left|\mathrm{PA}_{\mathrm{r}}-\mathrm{PA}_{\mathrm{b}}\right|,\left|\mathrm{PA}_{\mathrm{b}}-\mathrm{PA}_{\mathrm{r}}\right|\right)$. Since $\mathrm{PA}$ is illdefined for rings that are almost round in projection, we only studied features with a projected axis ratio $q_{\mathrm{r}} \leq 0.85$.

Because for deprojected rings and bars the concept of PA is no longer applicable, we defined the deprojected orientation of a ring or a bar to be the counter-clockwise difference in angle between the orientation of its major axis and the line of nodes, 


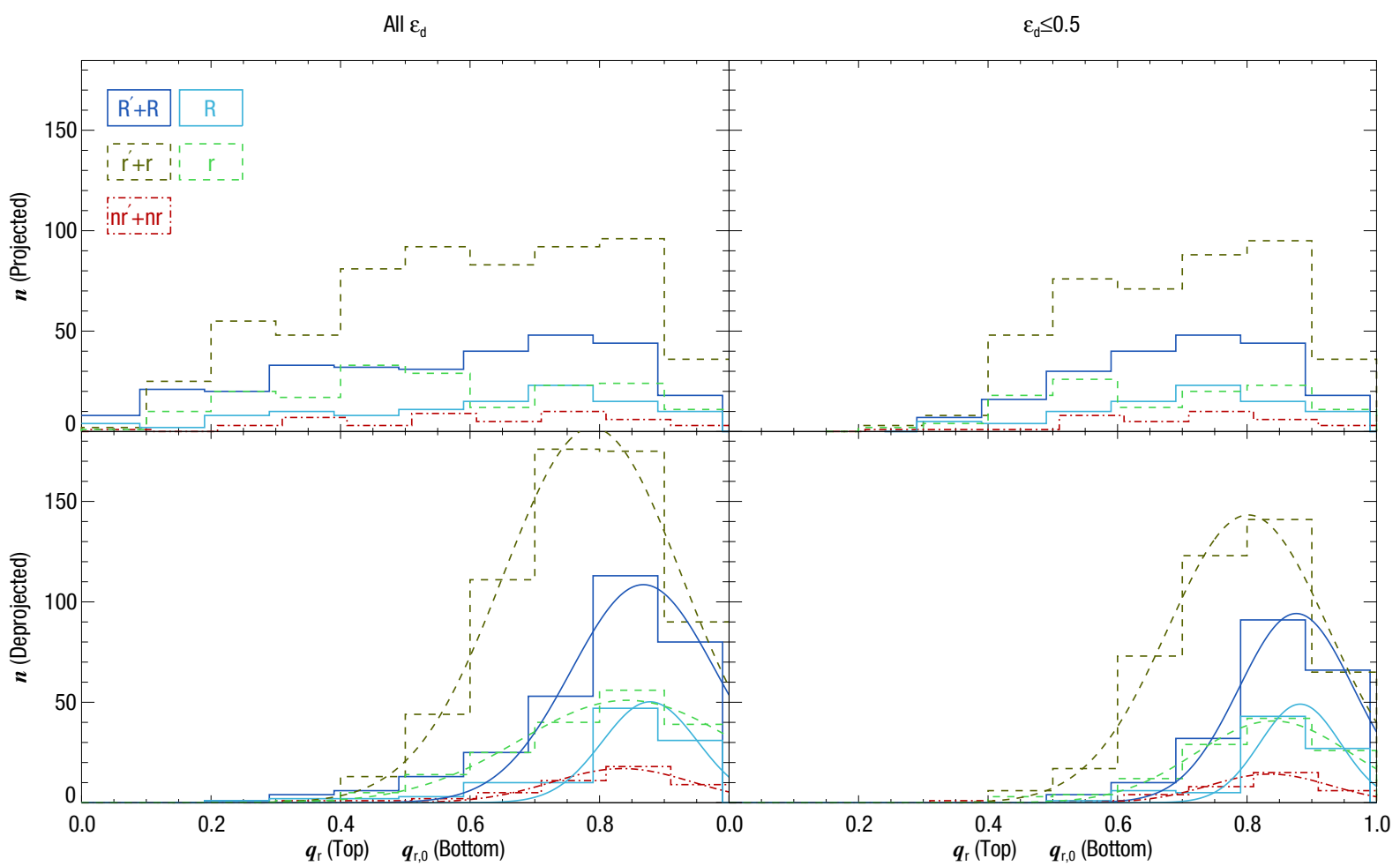

Fig. 20. Distribution of the resonance ring axis ratios colour- and line-coded as in Fig. 18. The left-hand plots are for the whole ARRAKIS sample and the right-hand plots correspond to rings in host galaxies with $\epsilon_{\mathrm{d}} \leq 0.5$. The top-row plots show the distribution of projected axis ratios, the bottom row shows the distribution of deprojected ones.

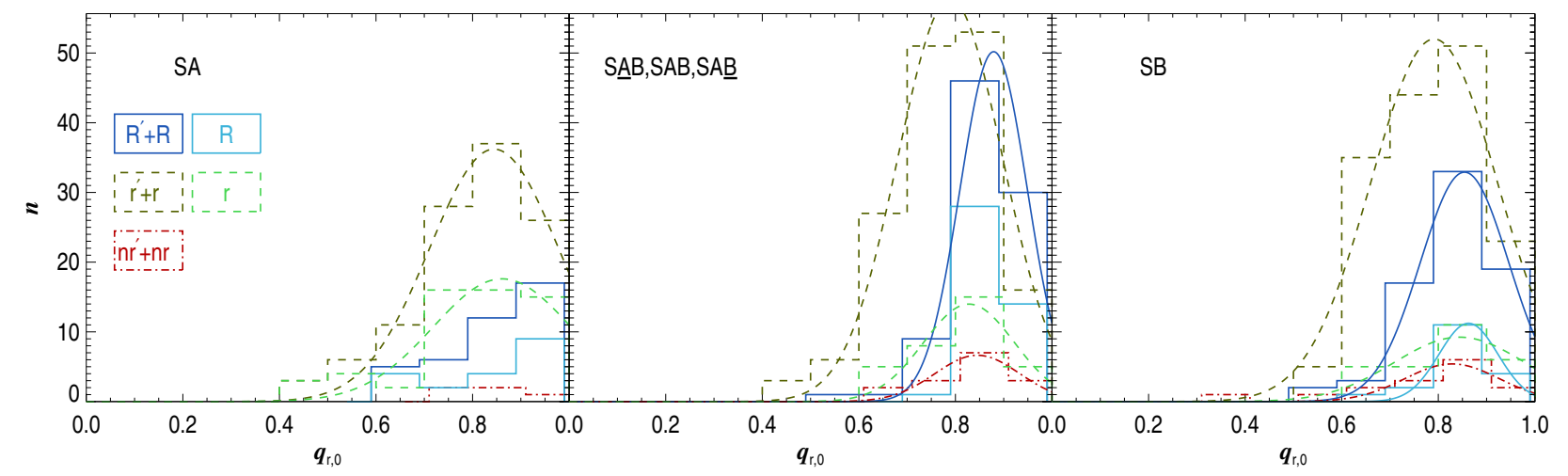

Fig. 21. Distribution of the resonance ring deprojected axis ratios colour- and line-coded as in Fig. 18 for different galaxy families and for $\epsilon_{\mathrm{d}} \leq 0.5$. The left panel shows SA galaxies, the middle one SAB to SAB galaxies, the right panel SB galaxies.

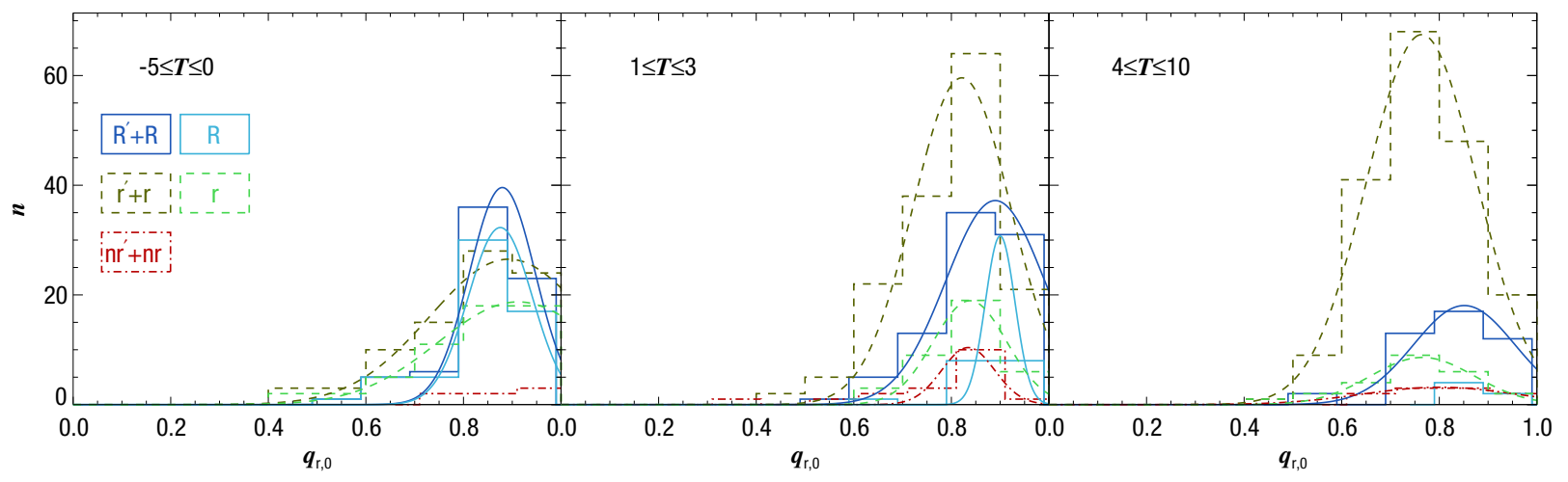

Fig. 22. Distribution of the resonance ring deprojected axis ratios colour- and line-coded as in Fig. 18 for different galaxy stage ranges and for $\epsilon_{\mathrm{d}} \leq 0.5$. The left panel represents galaxies with $-5 \leq T \leq 0$, the middle panel galaxies with $1 \leq T \leq 3$, the right panel galaxies with $4 \leq T \leq 10$. 
Table 3. Number of galaxies in the histograms in Figs. 20-22 and parameters of the Gaussian fits to the $q_{\mathrm{r}, 0}$ distributions.

\begin{tabular}{|c|c|c|c|c|}
\hline Galaxy subsample & Feature type & $n$ & $\left\langle q_{\mathrm{r}, 0}\right\rangle$ & $\sigma$ \\
\hline \multirow{5}{*}{ All galaxies } & Outer rings & 295 & $0.87(0.01)$ & $0.11(0.01)$ \\
\hline & Inner rings & 610 & $0.79(0.00)$ & $0.14(0.00)$ \\
\hline & Nuclear rings & 47 & $0.84(0.01)$ & $0.11(0.01)$ \\
\hline & Outer closed rings & 106 & $0.88(0.01)$ & $0.07(0.01)$ \\
\hline & Inner closed rings & 180 & $0.84(0.02)$ & $0.17(0.02)$ \\
\hline \multirow{5}{*}{$\epsilon_{\mathrm{d}} \leq 0.5$} & Outer rings & 203 & $0.88(0.01)$ & $0.09(0.01)$ \\
\hline & Inner rings & 425 & $0.80(0.01)$ & $0.12(0.01)$ \\
\hline & Nuclear rings & 35 & $0.84(0.01)$ & $0.09(0.01)$ \\
\hline & Outer closed rings & 82 & $0.88(0.01)$ & $0.06(0.01)$ \\
\hline & Inner closed rings & 116 & $0.84(0.01)$ & $0.12(0.01)$ \\
\hline \multirow{3}{*}{$\epsilon_{\mathrm{d}} \leq 0.5$} & Outer rings & 40 & - & - \\
\hline & Inner rings & 111 & $0.84(0.01)$ & $0.13(0.01)$ \\
\hline & Nuclear rings & 5 & - & - \\
\hline \multirow{2}{*}{ SA } & Outer closed rings & 19 & - & - \\
\hline & Inner closed rings & 56 & $0.86(0.04)$ & $0.14(0.04)$ \\
\hline \multirow{3}{*}{$\epsilon_{\mathrm{d}} \leq 0.5$} & Outer rings & 87 & $0.88(0.00)$ & $0.07(0.00)$ \\
\hline & Inner rings & 156 & $0.79(0.01)$ & $0.11(0.01)$ \\
\hline & Nuclear rings & 15 & $0.85(0.03)$ & $0.09(0.03)$ \\
\hline \multirow{2}{*}{$\mathrm{S} \underline{\mathrm{AB}}, \mathrm{SAB}, \mathrm{SA} \underline{\mathrm{B}}$} & Outer closed rings & 43 & - & - \\
\hline & Inner closed rings & 33 & $0.83(0.03)$ & $0.09(0.03)$ \\
\hline \multirow{3}{*}{$\epsilon_{\mathrm{d}} \leq 0.5$} & Outer rings & 74 & $0.85(0.00)$ & $0.09(0.00)$ \\
\hline & Inner rings & 158 & $0.79(0.02)$ & $0.13(0.02)$ \\
\hline & Nuclear rings & 15 & $0.83(0.02)$ & $0.10(0.02)$ \\
\hline \multirow{2}{*}{ SB } & Outer closed rings & 18 & $0.86(0.01)$ & $0.06(0.01)$ \\
\hline & Inner closed rings & 27 & $0.84(0.06)$ & $0.14(0.07)$ \\
\hline \multirow{3}{*}{$\epsilon_{\mathrm{d}} \leq 0.5$} & Outer rings & 71 & $0.88(0.01)$ & $0.07(0.01)$ \\
\hline & Inner rings & 83 & $0.89(0.03)$ & $0.16(0.03)$ \\
\hline & Nuclear rings & 7 & - & - \\
\hline \multirow{2}{*}{$-5 \leq T \leq 0$} & Outer closed rings & 58 & $0.88(0.01)$ & $0.07(0.01)$ \\
\hline & Inner closed rings & 56 & $0.91(0.02)$ & $0.16(0.02)$ \\
\hline \multirow{3}{*}{$\epsilon_{\mathrm{d}} \leq 0.5$} & Outer rings & 85 & $0.89(0.01)$ & $0.10(0.01)$ \\
\hline & Inner rings & 152 & $0.82(0.02)$ & $0.10(0.02)$ \\
\hline & Nuclear rings & 18 & $0.83(0.01)$ & $0.05(0.01)$ \\
\hline \multirow{2}{*}{$1 \leq T \leq 3$} & Outer closed rings & 17 & $0.90(0.00)$ & $0.03(2.97)$ \\
\hline & Inner closed rings & 37 & $0.84(0.01)$ & $0.08(0.01)$ \\
\hline \multirow{3}{*}{$\epsilon_{\mathrm{d}} \leq 0.5$} & Outer rings & 44 & $0.85(0.02)$ & $0.10(0.02)$ \\
\hline & Inner rings & 187 & $0.76(0.00)$ & $0.11(0.00)$ \\
\hline & Nuclear rings & 10 & $0.80(0.00)$ & $0.16(0.00)$ \\
\hline \multirow{2}{*}{$4 \leq T \leq 10$} & Outer closed rings & 6 & - & - \\
\hline & Inner closed rings & 24 & $0.77(0.01)$ & $0.11(0.01)$ \\
\hline
\end{tabular}

Notes. $\left\langle q_{\mathrm{r}, 0}\right\rangle$ stands for the fitted centre of the Gaussian distribution and $\sigma$ for its width. The numbers in brackets indicate 1-sigma errors of the fits.

$\theta_{\mathrm{r}}$ and $\theta_{\mathrm{b}}$. Thus, for deprojected galaxies we denote the orientation difference between a ring and the bar as $\theta_{\mathrm{r}}-\theta_{\mathrm{b}}$ [although it is defined as minimum $\left.\left(\left|\theta_{\mathrm{r}}-\theta_{\mathrm{b}}\right|,\left|\theta_{\mathrm{b}}-\theta_{\mathrm{r}}\right|\right)\right]$. Because $\theta_{r}$ is illdefined for rings that are almost round in deprojection, we only studied features with a deprojected axis ratio $q_{\mathrm{r}, 0} \leq 0.85$. In Fig. 23 we present the distributions of $\mathrm{PA}_{\mathrm{r}}-\mathrm{PA}_{\mathrm{b}}$ and $\theta_{\mathrm{r}}-\theta_{\mathrm{b}}$.

For the outer rings $\mathrm{PA}_{\mathrm{r}}(\mathrm{O})-\mathrm{PA}_{\mathrm{b}}$ there is some hint that the distribution has two mild peaks at $\mathrm{PA}_{\mathrm{r}}(\mathrm{O})-\mathrm{PA}_{\mathrm{b}}=0^{\circ}$ and $\mathrm{PA}_{\mathrm{r}}(\mathrm{O})-\mathrm{PA}_{\mathrm{b}}=90^{\circ}$ divided by a shallow valley. This behaviour is similar to that found in the CSRG, although there the peak at $\mathrm{PA}_{\mathrm{r}}(\mathrm{O})-\mathrm{PA}_{\mathrm{b}}=0^{\circ}$ has more contrast; this may be due to our relatively low number of outer features (our plot has five times fewer objects than Fig. $7 \mathrm{~g}$ in the CSRG).

The distribution for inner rings has a large peak at $\mathrm{PA}_{\mathrm{r}}(\mathrm{I})-$ $\mathrm{PA}_{\mathrm{b}}=0^{\circ}$ and the distribution drops until it stabilises between $\mathrm{PA}_{\mathrm{r}}(\mathrm{I})-\mathrm{PA}_{\mathrm{b}}=50^{\circ}$ and $\mathrm{PA}_{\mathrm{r}}(\mathrm{I})-\mathrm{PA}_{\mathrm{b}}=90^{\circ}$.

In deprojected galaxies, the outer rings have two preferred orientations, namely parallel and perpendicular to bars
$\left[\theta_{\mathrm{r}}(\mathrm{O})-\theta_{\mathrm{b}}=0^{\circ}\right.$ and $\left.\theta_{\mathrm{r}}(\mathrm{O})-\theta_{\mathrm{b}}=90^{\circ}\right]$, which can be associated with the $R_{2}$ and $R_{1}$ ring types in the simulations of Schwarz $(1981,1984)$ as well as with the predictions from nondeprojected data in the CSRG.

The intrinsic inner ring orientation distribution has a clear peak parallel to the bar $\left[\theta_{\mathrm{r}}(\mathrm{I})-\theta_{\mathrm{b}}=0^{\circ}\right]$, as was previously hinted at in other works (e.g., the CSRG, de Vaucouleurs et al. 1964; Schwarz 1981, 1984; Buta 1986b; Athanassoula et al. 2009b). However, we also found that a significant fraction of rings have random orientations with respect to the bar, as indicated by the flat distribution for $\theta_{\mathrm{r}}(\mathrm{I})-\theta_{\mathrm{b}}>30^{\circ}$. Such a randomly oriented inner ring fraction, although sometimes suspected on the basis of a few individual cases of obvious bar/ring misalignment [ESO 565-11 (Buta et al. 1995), NGC 309 (Buta et al. 2007), NGC 4319 (Sulentic \& Arp 1987), and NGC 6300 (Buta 1987)] has never been described before.

We fitted the outer ring $\theta_{\mathrm{r}}(\mathrm{O})-\theta_{\mathrm{b}}$ distribution with the superposition of two Gaussian curves centred at $\theta_{\mathrm{r}}(\mathrm{O})-\theta_{\mathrm{b}}=0^{\circ}$ and 


\section{S. Comerón et al.: ARRAKIS}

Table 4. Number of galaxies in the histograms in Figs. 23-25 and parameters of the Gaussian fits to the $q_{\mathrm{r}, 0}$ distributions.

\begin{tabular}{|c|c|c|c|c|c|c|c|}
\hline Galaxy subsample & Feature type & $n$ & $\sigma_{0^{\circ}}\left({ }^{\circ}\right)$ & $w_{0^{\circ}}$ & $\sigma_{90^{\circ}}\left({ }^{\circ}\right)$ & $w_{90^{\circ}}$ & $w_{\mathrm{c}}$ \\
\hline \multirow{3}{*}{$\epsilon_{\mathrm{d}} \leq 0.5$} & Outer rings & 65 & $25(3)$ & $0.35(0.18)$ & $17(2)$ & $0.65(0.39)$ & - \\
\hline & Inner rings & 215 & $11(1)$ & $0.48(0.12)$ & - & - & $0.52(0.10)$ \\
\hline & Nuclear rings & 18 & - & - & - & - & - \\
\hline \multirow{2}{*}{ Barred } & Outer closed rings & 23 & $53(4)$ & $0.39(0.11)$ & $18(1)$ & $0.61(0.20)$ & - \\
\hline & Inner closed rings & 33 & $11(1)$ & $0.59(0.18)$ & - & - & $0.41(0.16)$ \\
\hline \multirow{3}{*}{$\epsilon_{\mathrm{d}} \leq 0.5$} & Outer rings & 31 & $27(2)$ & $0.32(0.08)$ & $16(1)$ & $0.68(0.19)$ & - \\
\hline & Inner rings & 103 & $17(1)$ & $0.51(0.11)$ & - & - & $0.49(0.09)$ \\
\hline & Nuclear rings & 8 & - & - & - & - & - \\
\hline \multirow{2}{*}{ 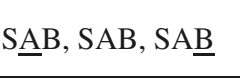 } & Outer closed rings & 16 & $37(8)$ & $0.34(0.27)$ & $18(1)$ & $0.66(0.59)$ & - \\
\hline & Inner closed rings & 17 & $14(1)$ & $0.58(0.27)$ & - & - & $0.42(0.30)$ \\
\hline \multirow{3}{*}{$\epsilon_{\mathrm{d}} \leq 0.5$} & Outer rings & 34 & $22(2)$ & $0.35(0.17)$ & $19(1)$ & $0.65(0.44)$ & - \\
\hline & Inner rings & 110 & $9(1)$ & $0.53(0.18)$ & - & - & $0.47(0.15)$ \\
\hline & Nuclear rings & 10 & - & - & - & - & - \\
\hline \multirow{2}{*}{ SB } & Outer closed rings & 7 & - & - & - & - & - \\
\hline & Inner closed rings & 16 & $7(1)$ & $0.53(0.17)$ & - & - & $0.47(0.22)$ \\
\hline \multirow{3}{*}{$\epsilon_{\mathrm{d}} \leq 0.5$} & Outer rings & 21 & $20(1)$ & $0.32(0.11)$ & $16(0)$ & $0.68(0.36)$ & - \\
\hline & Inner rings & 26 & $10(1)$ & $0.72(0.41)$ & - & - & $0.28(0.28)$ \\
\hline & Nuclear rings & 2 & - & - & - & - & - \\
\hline \multirow{2}{*}{$-5 \leq T \leq 0$} & Outer closed rings & 18 & $60(8)$ & $0.33(0.15)$ & $16(1)$ & $0.67(0.31)$ & - \\
\hline & Inner closed rings & 13 & $11(2)$ & $0.50(0.35)$ & - & - & $0.50(0.50)$ \\
\hline \multirow{3}{*}{$\epsilon_{\mathrm{d}} \leq 0.5$} & Outer rings & 26 & $13(0)$ & $0.19(0.03)$ & $15(0)$ & $0.81(0.21)$ & - \\
\hline & Inner rings & 70 & $12(2)$ & $0.69(0.33)$ & - & - & $0.31(0.20)$ \\
\hline & Nuclear rings & 9 & - & - & - & - & - \\
\hline \multirow{2}{*}{$1 \leq T \leq 3$} & Outer closed rings & 3 & - & - & - & - & - \\
\hline & Inner closed rings & 14 & $12(1)$ & $0.69(0.18)$ & - & - & $0.31(0.16)$ \\
\hline \multirow{3}{*}{$\epsilon_{\mathrm{d}} \leq 0.5$} & Outer rings & 16 & - & - & - & - & - \\
\hline & Inner rings & 116 & $11(1)$ & $0.25(0.06)$ & - & - & $0.75(0.10)$ \\
\hline & Nuclear rings & 7 & - & - & - & - & - \\
\hline \multirow{2}{*}{$4 \leq T \leq 10$} & Outer closed rings & 0 & - & - & - & - & - \\
\hline & Inner closed rings & 6 & - & - & - & - & - \\
\hline
\end{tabular}

Notes. $\sigma_{0^{\circ}}$ and $\sigma_{90^{\circ}}$ stand for the fitted widths of the Gaussian components set to be centred at $\theta_{\mathrm{r}}-\theta_{\mathrm{b}}=0^{\circ}$ and $\theta_{\mathrm{r}}-\theta_{\mathrm{b}}=90^{\circ}$. $w_{0^{\circ}}, w_{90^{\circ}}$, and $w_{\mathrm{c}}$ stand for the fitted fraction of rings found to be in one of the two Gaussian components and that in the component of rings randomly orientated with respect to the bar. The numbers in brackets indicate 1-sigma errors of the fit.

$\theta_{\mathrm{r}}(\mathrm{O})-\theta_{\mathrm{b}}=90^{\circ}$, as assumed in the CSRG. For the inner rings, we fitted the superposition of a Gaussian centred at $\theta_{\mathrm{r}}(\mathrm{I})-\theta_{\mathrm{b}}=0^{\circ}$, describing the population of inner features parallel to the bar and a constant distribution representing the population of rings oriented at random with respect to the bar. The fitted parameters and the number of galaxies in each histogram are tabulated in Table 4. The fit also provides the fraction of rings that belong to the components parallel and anti-parallel to the bar for outer rings $\left(w_{0^{\circ}}\right.$ and $\left.w_{90^{\circ}}\right)$ and parallel and oriented at random with respect to the bar for inner rings $\left(w_{0}\right.$ and $\left.w_{\mathrm{c}}\right)$. We found that the fraction of outer rings aligned parallel to the bar is $35 \pm 18 \%$ and that of features oriented perpendicular to the bar is $65 \pm 39 \%$. For inner rings, we found that some $48 \pm 12 \%$ are aligned parallel to the bar and the other $52 \pm 10 \%$ have random orientations.

The distribution of the intrinsic orientations of outer features does not change much when looking separately at weakly and strongly barred galaxies (Fig. 24). The inner ring distribution changes more, since the width of the distribution of galaxies parallel to the bar decreases from $17 \pm 1^{\circ}$ for galaxies with families $\mathrm{S} \underline{\mathrm{AB}}$ to $\mathrm{SA} \underline{\mathrm{B}}$ to only $9 \pm 1^{\circ}$ for $\mathrm{SB}$ galaxies.

The outer ring orientation distribution is also independent of the galaxy stage (Fig. 25). But again, this is not the case for the inner ring distribution. Indeed, the later the stage, the larger the fraction of inner rings oriented at random with respect to the bar. This fraction increases from $28 \pm 28 \%(-5 \leq T \leq 0)$ and $31 \pm 20 \%(1 \leq T \leq 3)$ to $75 \pm 10 \%$ for galaxies with $4 \leq T \leq 10$.
For inner rings in late-type stages there is even some hint of a preferred orientation perpendicular to the bar (right panel in Fig. 25).

\subsection{Absolute ring sizes}

Absolute ring diameters were obtained using the average of the redshift-independent distances provided by NED as the host galaxy distance. If such a distance measurement was not available, we used the NED Hubble-Lemaitre flow distance corrected for the effects of the Virgo cluster, the Great Attractor, and the Shapley cluster (Hubble-Lemaître constant $H_{0}=73 \mathrm{~km} \mathrm{~s}^{-1} \mathrm{Mpc}^{-1}$ ). Because the $\mathrm{S}^{4} \mathrm{G}$ sample selection was based on uncorrected flow distances, this means that some of the galaxy distances listed in Appendix A exceed $41 \mathrm{Mpc}$. Of these galaxies with rings, only six are farther away than $60 \mathrm{Mpc}$ (ESO 576-1, NGC 4722, NGC 5600, PGC 47721, and UGC 5814). Such high distances might indicate either inaccurate redshift-based or redshift-independent distance determinations. However, since these galaxies are only a few, we did not exclude them from the study of absolute ring sizes.

Here, we define the size of a ring to be its major axis diameter. That is, $D_{\mathrm{r}}$ in the projected case and $D_{\mathrm{r}, 0}$ in the deprojected case. Because rings are in general almost circular, their absolute size distribution does not vary much when comparing the projected and the deprojected sizes (Fig. 26). Moreover, because 


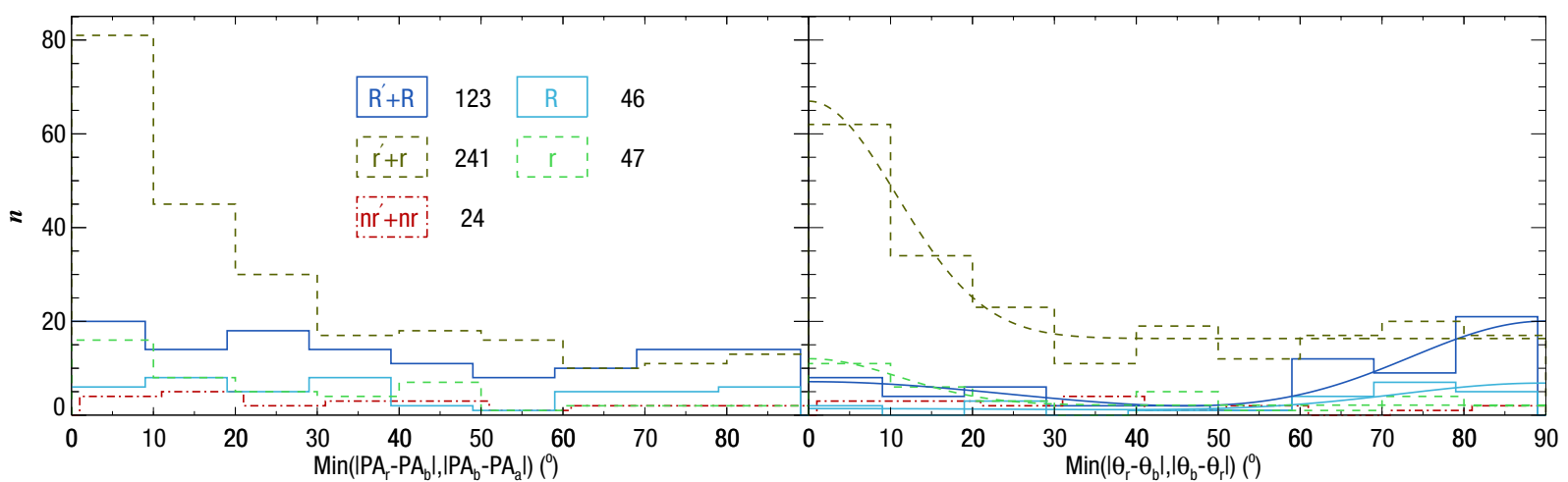

Fig. 23. Distribution of the projected resonance ring PA differences with respect to the bar (left panel) and ring orientation with respect to the bar in the deprojected galaxies (right panel). The histograms are colour- and line-coded as in Fig. 18 . The plots only include rings with $q_{\mathrm{r}} \leq 0.85$ (left panel) and $q_{\mathrm{r}, 0} \leq 0.85$ (right panel) and in host galaxies with $\epsilon_{\mathrm{d}} \leq 0.5$. The numbers in the left panel indicate the number of galaxies included in the histogram corresponding to the colour of the adjacent box.

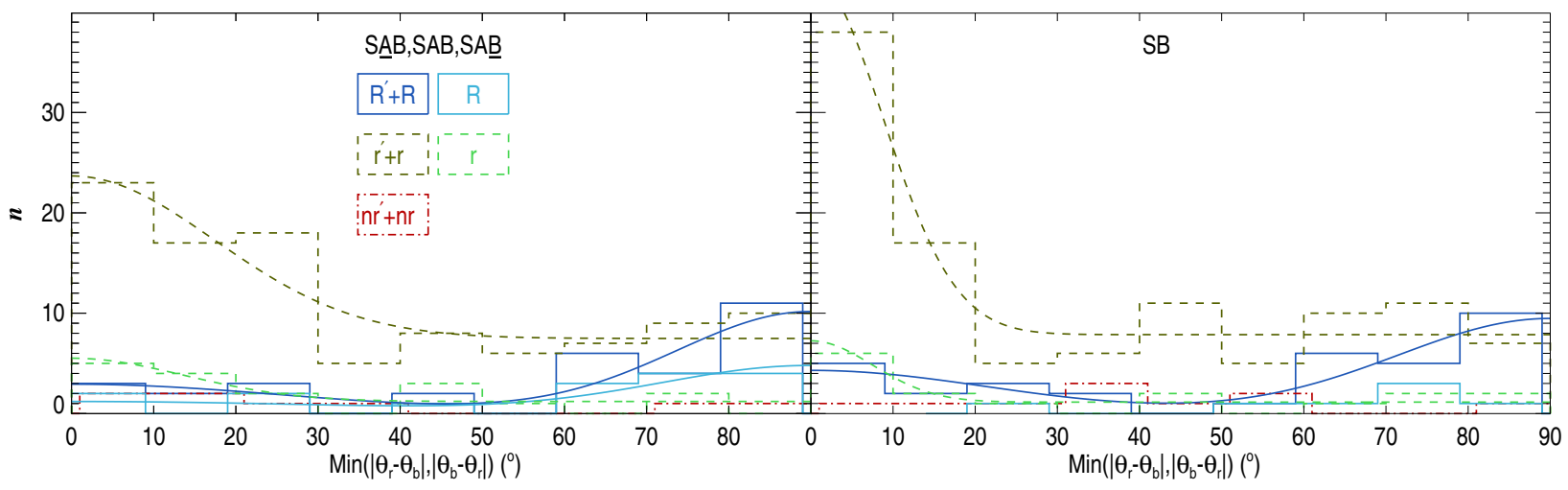

Fig. 24. Distribution of the resonance ring deprojected major axis angle difference with the bar colour- and line-coded as in Fig. 18 for different galaxy families and for $\epsilon_{\mathrm{d}} \leq 0.5$. The left panel is for $\mathrm{S} \underline{\mathrm{B}}$ to $\mathrm{SAB}$ galaxies, the right panel for $\mathrm{SB}$ galaxies. The plots only include rings with $q_{\mathrm{r}, 0} \leq 0.85$.

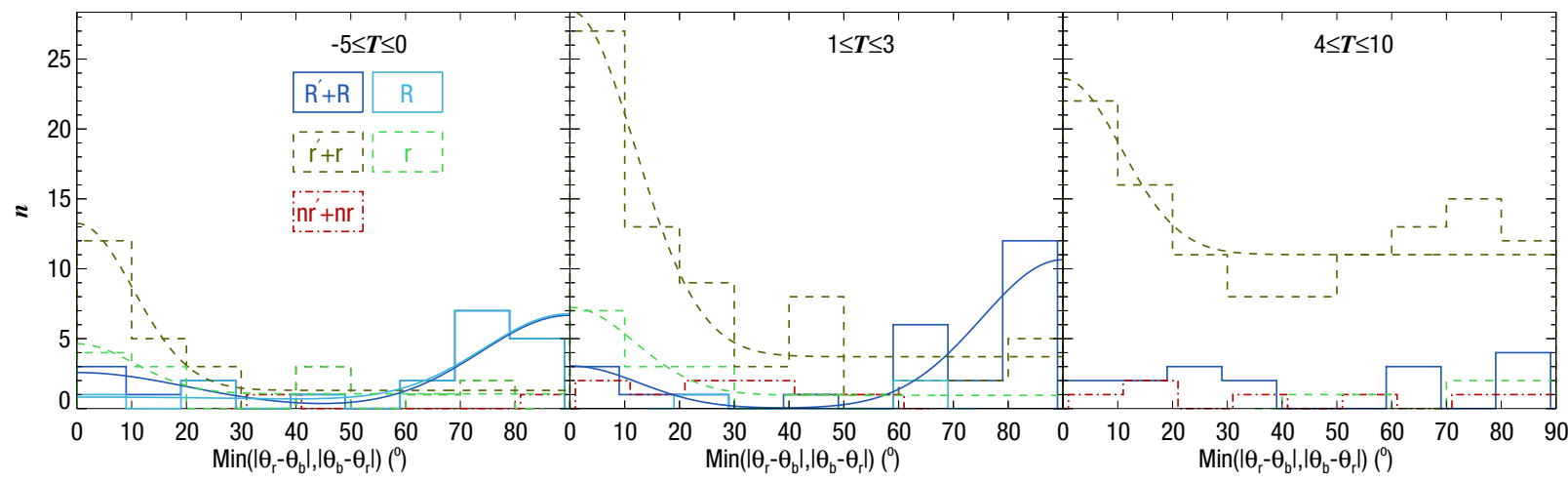

Fig. 25. Distribution of the resonance ring deprojected major axis angle difference with the bar colour- and line-coded as in Fig. 18 for different galaxy stage ranges and for $\epsilon_{\mathrm{d}} \leq 0.5$. The left panel represents galaxies with $-5 \leq T \leq 0$, the middle panel galaxies with $1 \leq T \leq 3$, the right panel galaxies with $4 \leq T \leq 10$. The plots only include rings with $q_{\mathrm{r}, 0} \leq 0.85$.

the ring size is easier to measure than its orientation, the size distribution does not vary much when studying our whole sample or a subsample restricted by disc ellipticity. The averages and the standard deviations of each deprojected distribution in the plots are listed in Table 5.

The average absolute size for each of the three ring flavours is nearly independent of galaxy family (Fig. 27 and Table 5). It is noteworthy that the dispersion in the size of inner rings in SA galaxies is higher than that in barred galaxies. This high dispersion can also be seen by examining the smallest and largest rings in the top panel in Fig. 27; the smallest resonance feature in SA galaxies is an inner pseudoring. This is also the case for the third-largest feature in this panel. Such a behaviour is not seen in barred galaxies, where the small- and large-size tails are dominated by nuclear and outer rings, respectively. A possible explanation of this high dispersion in the size of inner features found in SA galaxies is that a fraction of outer and nuclear resonant features have been misclassified as inner rings.

Outer and inner rings in galaxies with stages $4 \leq T \leq 10$ are on average much smaller than those found in earlier stages 
Table 5. Number of galaxies in the histograms in Figs. 26-28 and average and standard deviation of the absolute ring deprojected diameters for several ARRAKIS subsamples.

\begin{tabular}{|c|c|c|c|c|}
\hline Galaxy subsample & Feature type & $n$ & $\left\langle D_{\mathrm{r}, 0}\right\rangle(\mathrm{kpc})$ & $\sigma\left(D_{\mathrm{r}, 0}\right)(\mathrm{kpc})$ \\
\hline \multirow{5}{*}{ All galaxies } & Outer rings & 295 & 17.0 & 10.3 \\
\hline & Inner rings & 610 & 7.1 & 4.7 \\
\hline & Nuclear rings & 47 & 1.4 & 0.6 \\
\hline & Outer closed rings & 106 & 18.1 & 10.2 \\
\hline & Inner closed rings & 180 & 7.2 & 5.3 \\
\hline \multirow{5}{*}{$\epsilon_{\mathrm{d}} \leq 0.5$} & Outer rings & 203 & 16.5 & 10.3 \\
\hline & Inner rings & 425 & 6.7 & 4.6 \\
\hline & Nuclear rings & 35 & 1.4 & 0.6 \\
\hline & Outer closed rings & 82 & 17.8 & 10.3 \\
\hline & Inner closed rings & 116 & 6.7 & 4.9 \\
\hline \multirow{3}{*}{$\epsilon_{\mathrm{d}} \leq 0.5$} & Outer rings & 40 & 16.0 & 12.1 \\
\hline & Inner rings & 111 & 6.0 & 5.9 \\
\hline & Nuclear rings & 5 & 1.4 & 0.6 \\
\hline \multirow{2}{*}{ SA } & Outer closed rings & 19 & 16.5 & 12.7 \\
\hline & Inner closed rings & 56 & 5.9 & 5.8 \\
\hline \multirow{3}{*}{$\epsilon_{\mathrm{d}} \leq 0.5$} & Outer rings & 87 & 16.4 & 9.1 \\
\hline & Inner rings & 156 & 6.7 & 3.7 \\
\hline & Nuclear rings & 15 & 1.3 & 0.4 \\
\hline \multirow{2}{*}{ SAㅡ, SAB, SA믐 } & Outer closed rings & 43 & 18.0 & 9.4 \\
\hline & Inner closed rings & 33 & 6.7 & 3.8 \\
\hline \multirow{3}{*}{$\epsilon_{\mathrm{d}} \leq 0.5$} & Outer rings & 74 & 16.5 & 10.8 \\
\hline & Inner rings & 158 & 7.4 & 4.3 \\
\hline & Nuclear rings & 15 & 1.4 & 0.8 \\
\hline \multirow{2}{*}{ SB } & Outer closed rings & 18 & 17.8 & 9.9 \\
\hline & Inner closed rings & 27 & 8.2 & 3.7 \\
\hline \multirow{3}{*}{$\epsilon_{\mathrm{d}} \leq 0.5$} & Outer rings & 71 & 18.5 & 10.4 \\
\hline & Inner rings & 83 & 7.3 & 4.6 \\
\hline & Nuclear rings & 7 & 1.2 & 0.5 \\
\hline \multirow{2}{*}{$-5 \leq T \leq 0$} & Outer closed rings & 58 & 17.9 & 10.5 \\
\hline & Inner closed rings & 55 & 7.1 & 4.7 \\
\hline \multirow{3}{*}{$\epsilon_{\mathrm{d}} \leq 0.5$} & Outer rings & 85 & 17.0 & 9.4 \\
\hline & Inner rings & 152 & 8.4 & 5.6 \\
\hline & Nuclear rings & 18 & 1.4 & 0.8 \\
\hline \multirow{2}{*}{$1 \leq T \leq 3$} & Outer closed rings & 17 & 20.2 & 10.6 \\
\hline & Inner closed rings & 37 & 7.7 & 5.7 \\
\hline \multirow{3}{*}{$\epsilon_{\mathrm{d}} \leq 0.5$} & Outer rings & 44 & 12.5 & 11.2 \\
\hline & Inner rings & 184 & 5.1 & 2.9 \\
\hline & Nuclear rings & 9 & 1.3 & 0.3 \\
\hline \multirow{2}{*}{$4 \leq T \leq 10$} & Outer closed rings & 6 & 10.7 & 5.2 \\
\hline & Inner closed rings & 22 & 3.9 & 2.2 \\
\hline
\end{tabular}

(Fig. 28). This effect is not seen for nuclear features, but this may well be because some of them are too small to be resolved in the $\mathrm{S}^{4} \mathrm{G}$.

\subsection{Ring sizes relative to the size of the galaxy}

One of the outputs of the $S^{4} \mathrm{G}$ pipeline 3 (Muñoz-Mateos et al., in prep.) is the diameter at the $\mu_{3.6 \mu \mathrm{m}}=25.5 \mathrm{mag} \operatorname{arcsec}^{-2}$ level, $D_{25.5}$. This diameter can be used as an indicator of the size of the disc, although in a few cases it may be overestimating its true size because of the effect of bright extended spheroidal components (an obvious case probably is the Sombrero galaxy). Since $D_{25.5}$ is located in the outskirts of the galaxy, in the region where its orientation is typically measured, it does not need to be deprojected. $D_{25.5}$ is available for all sample galaxies but two, NGC 253 and NGC 4647. For NGC 253 this is because the galaxy is tightly fitted in the $\mathrm{S}^{4} \mathrm{G}$ frame and the $D_{25.5}$ diameter is close to the limits of the frame or even beyond. For NGC 4647 this is because the galaxy isophotes are distorted because of a close galaxy in the same frame.

The distribution of projected and deprojected relative radii of the rings, $D_{\mathrm{r}} / D_{25.5}$ and $D_{\mathrm{r}, 0} / D_{25.5}$, (Fig. 29) shows that these magnitudes are much more useful than $D_{\mathrm{r}}$ and $D_{\mathrm{r}, 0}$ for separating the three flavours of resonance features. The overlap in radius of the different types of features is much smaller than that found when looking at absolute radii because distributions are much more peaked. Indeed, comparing Tables 5 and 6 shows that for outer rings in galaxies with $\epsilon_{\mathrm{d}} \leq 0.5$ the ratio between the peak value of the distribution and its dispersion is $\left[D_{\mathrm{r}, 0}(\mathrm{O}) / D_{25.5}\right] / \sigma\left[D_{\mathrm{r}, 0}(\mathrm{O}) / D_{25.5}\right]=3.2$, which is almost twice higher than $D_{\mathrm{r}, 0}(\mathrm{O}) / \sigma\left[D_{\mathrm{r}, 0}(\mathrm{O})\right]=1$.6. For inner rings the values are $\left[D_{\mathrm{r}, 0}(\mathrm{I}) / D_{25.5}\right] / \sigma\left[D_{\mathrm{r}, 0}(\mathrm{I}) / D_{25.5}\right]=2.2$ and $D_{\mathrm{r}, 0}(\mathrm{I}) / \sigma\left[D_{\mathrm{r}, 0}(\mathrm{I})\right]=1.5$.

This effect is not seen for nuclear rings; a reason may be that outer and inner rings are associated to a single resonance or manifold structure that is found at a given radius relative to the bar (with small variations due to the bar pattern speed and 


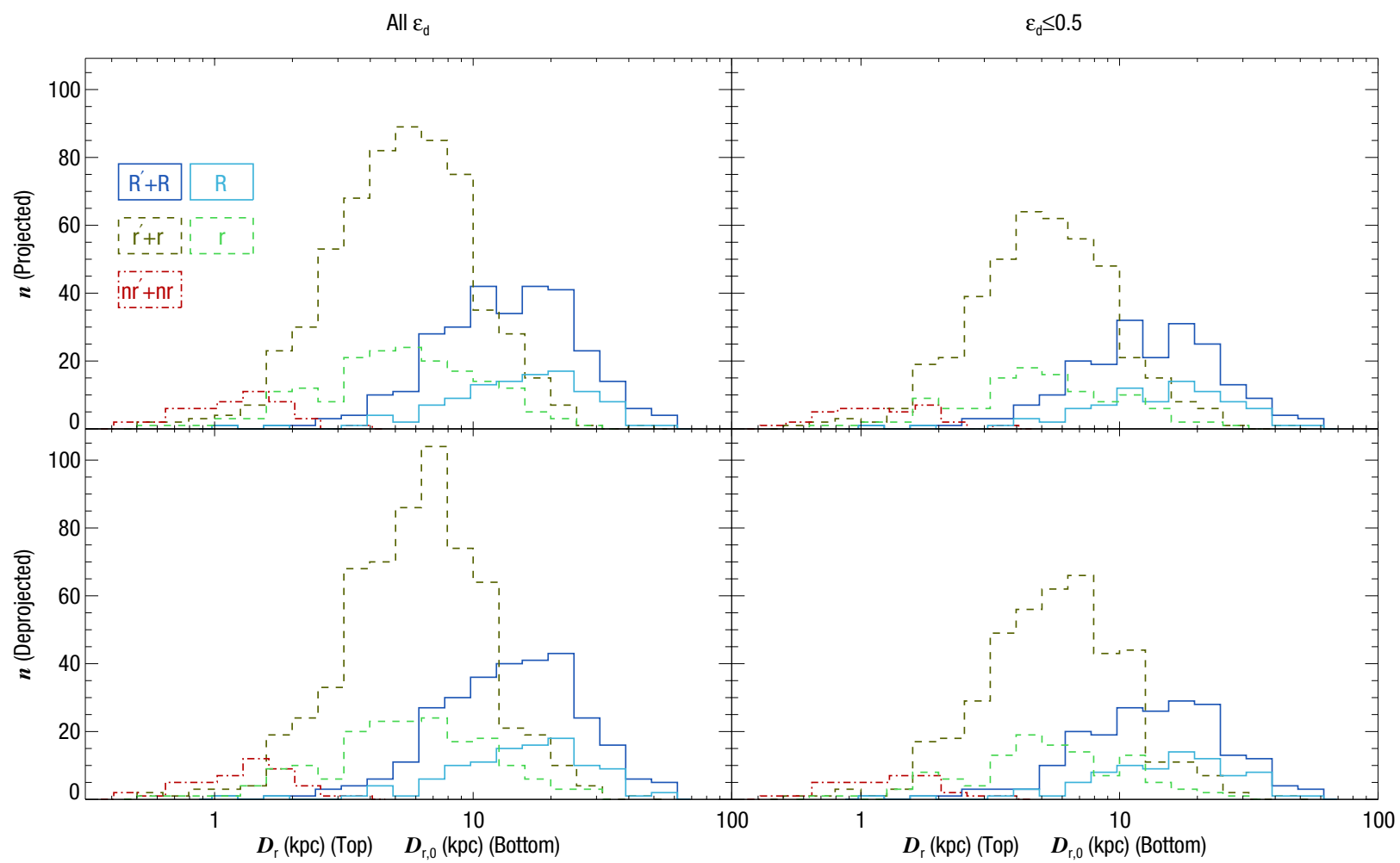

Fig. 26. Distribution of the resonance ring absolute diameters. The top row shows the projected diameters and the bottom row the deprojected ones. The left column shows all galaxies, the right column galaxies with $\epsilon_{\mathrm{d}} \leq 0.5$. The histograms are colour- and line-coded as in Fig. 18 .

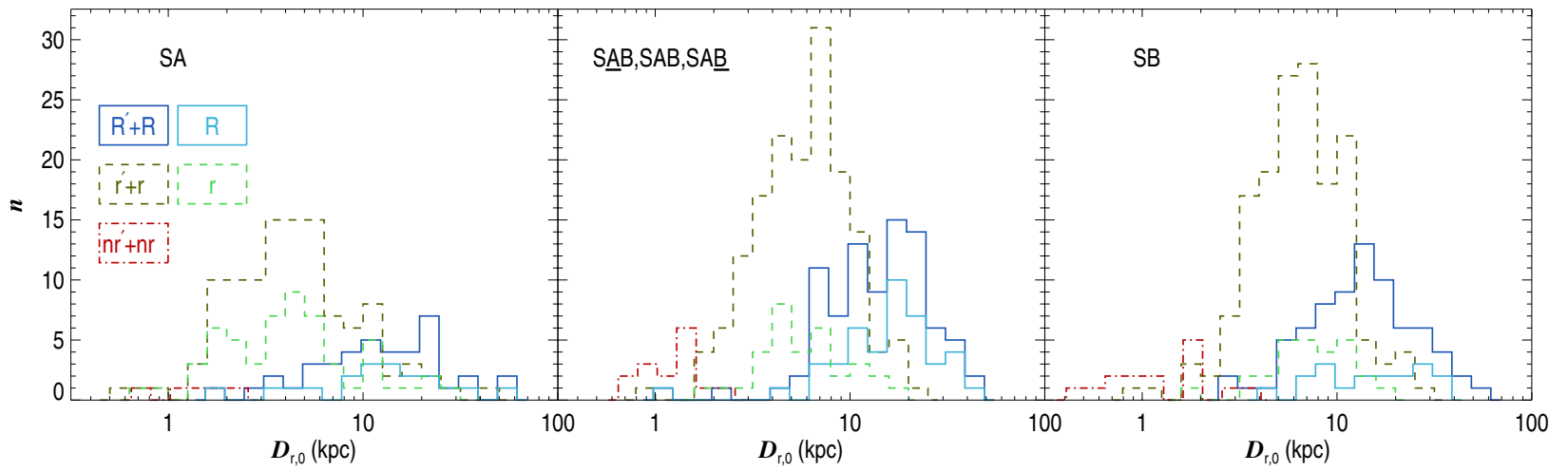

Fig. 27. Distribution of the resonance ring deprojected major axis absolute diameter colour- and line-coded as in Fig. 18 for different galaxy families and for $\epsilon_{\mathrm{d}} \leq 0.5$. The left panel shows SA galaxies, the middle one $\underline{\mathrm{SAB}}$ to SA $\underline{\mathrm{B}}$ galaxies, the right panel SB galaxies.

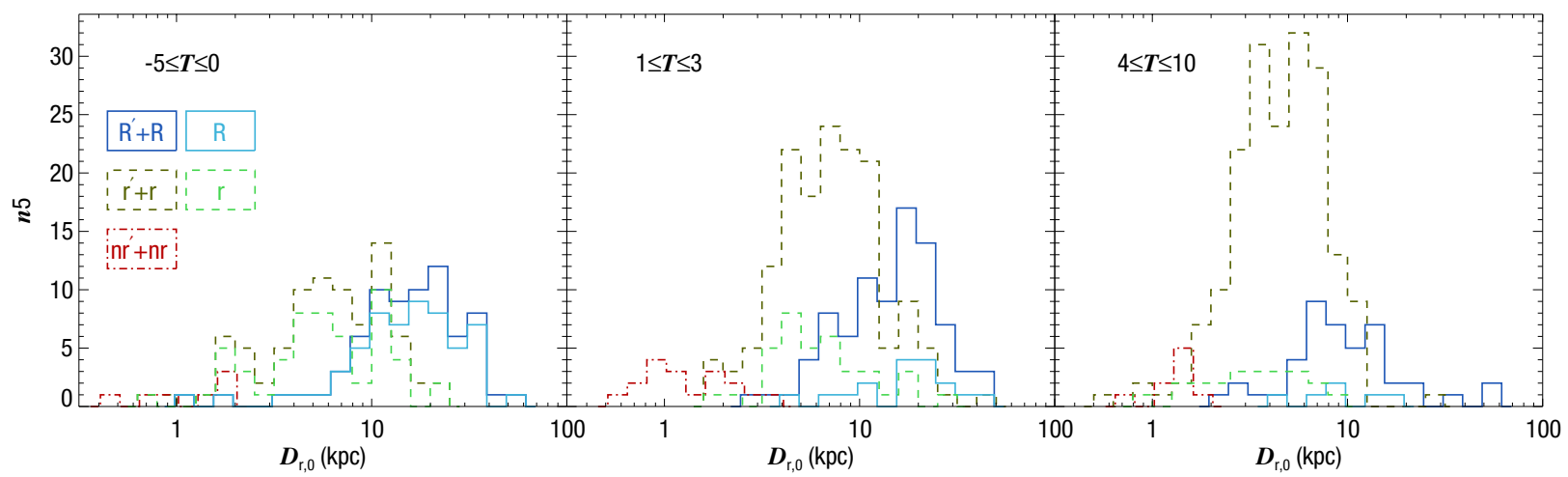

Fig. 28. Distribution of the resonance ring deprojected major axis absolute diameter colour- and line-coded as in Fig. 18 for different galaxy stage ranges and for $\epsilon_{\mathrm{d}} \leq 0.5$. The left panel represents galaxies with $-5 \leq T \leq 0$ galaxies, the middle panel galaxies with $1 \leq T \leq 3$, the right panel galaxies with $4 \leq T \leq 10$. 
the galaxy rotation curve). Instead, nuclear rings are associated to the range of radii between the two ILRs, or to the range of radii between the centre and the ILR, in case of galaxies with only one such resonance. A complementary explanation is that we are missing all nuclear features below a given angular size. If enough galaxies of a possible $D_{\mathrm{r}, 0}(\mathrm{~N}) / D_{25.5}$ peak were smaller than that threshold, the scatter of the nuclear ring absolute size distribution would not be reduced by much when using the ring diameters normalised with $D_{25.5}$.

When segregating galaxies according to their family (Fig. 30), we found a large overlap between the range of relative radii occupied by the inner and nuclear rings in SA galaxies. Such a large overlap is not seen in barred galaxies and may indicate, as commented before when looking at the absolute radii of rings, that some nuclear rings may have been classified as inner features in unbarred galaxies. We also found that the peak in the inner ring relative size distribution increases systematically with increasing bar strength. This is probably because of a slight tendency of stronger bars (SB) to be longer on average relative to galaxy size than weaker bars (SAB, SAB, SAB), at least for late-type galaxies. This was originally shown by Erwin (2005), based on the data of Martin (1995). Erwin (2005) used the RC3 $R_{25}$ measurement as the galaxy size.

Finally, we find that the relative ring size distributions are not sensitive to galaxy stage (Fig. 31).

\subsection{Outer ring sizes relative to those of inner rings}

Our sample contains 163 galaxies with both outer and inner features. We calculated the ratio of the major axis of these features, $D_{\mathrm{r}, 0}(\mathrm{O}) / D_{\mathrm{r}, 0}(\mathrm{I})$. Our sample has 19 galaxies with outer and inner features that also have more than one outer ring and/or more than one inner ring. When that was the case, the average of the major axis diameters of the outer (inner) features was used as the diameter of the outer (inner) feature.

The resulting diameter ratio distribution is represented in Fig. 32, colour- and line-coded according to different host galaxy families and stages. One galaxy, NGC 5033, has $D_{\mathrm{r}, 0}(\mathrm{O}) / D_{\mathrm{r}, 0}(\mathrm{I})=17.5$ and is beyond the horizontal limits of the plots. This high ratio may indicate that the innermost resonance feature in this unbarred galaxy is a nuclear and not an inner ring. In fact, this innermost feature in NGC 5033 is classified as a nuclear ring in AINUR.

In Fig. 32, the histograms for barred galaxies and those for stages $-5 \leq T \leq 3$ have a shape that can be roughly approximated by a Gaussian, therefore we fitted them with that function. Other histograms (SA galaxies and galaxies with stages $4 \leq T \leq 10$ ) have too few galaxies to be fitted. The histograms for SA galaxies are very flat and have no distinct peak. The results of the fits and the number of galaxies included in each histogram, are listed in Table 7.

We found that $D_{\mathrm{r}, 0}(\mathrm{O}) / D_{\mathrm{r}, 0}(\mathrm{I})$ is larger for weaker bars and also for earlier-type galaxies.

The peaks of the fitted distributions in Fig. 32 are always about $D_{\mathrm{r}, 0}(\mathrm{O}) / D_{\mathrm{r}, 0}(\mathrm{I})=2$, which is consistent with the CSRG, Kormendy (1979), (Athanassoula et al. 1982), and (Buta 1986a).

\section{Discussion}

\subsection{Why is the stage distribution of inner and outer resonance features different?}

In Sect. 6.1 we found that the distribution of stages (morphological type) for inner and outer rings is significantly different.
Indeed, the outer ring fraction is high (above 20\%) for $-1 \leq T \leq$ 3 before dropping at later stages, but the inner ring distribution only drops at $T=6$ or $T=7$ (see a schematic view of this in Table 8 where the stages with a frequency of rings one fourth that of the peak for that ring flavour are considered to rarely host rings). For stages below $T=3$ outer rings are at least $40 \%$ as abundant as inner rings, but this ratio decreases for later stages. Thus, there is a significant under-abundance of outer rings for late-type galaxies (Fig. 33).

A possible justification for this effect can be found in the simulations by Combes \& Elmegreen (1993), who compared models designed to mimic both early- and late-type spirals distinguished by steeply and slowly rising inner rotation curves, respectively. However, to what extent their galaxy models are realistic is a matter of debate, since their $t=0$ models were sharply truncated at a radius of only a few disc scale-lengths (as little as 1.5 scale-lengths in some cases). Therefore, any explanation based on these simulations has to be taken with some caution. Combes \& Elmegreen (1993) found that for early-type galaxies the $\mathrm{CR}$ radius is well inside the optical disc of the galaxy, but for later-type galaxies the CR is moved farther out. As a consequence, for a late-type galaxy, the OLR radius is typically found in a low-density region, and very little material is rearranged into a ring shape. Eventually, an outer feature forms when particles near the CR obtain angular momentum and are moved outwards, but this is a secular process that is even slower in late-type galaxies because regions near the CR have a lower particle density than that in earlier-type galaxies. Our results show that for this particular problem, the transition between an early- and a latetype disc galaxy is at $T=3$. The connection discussed here between simulations and observational data was previously made by Combes \& Elmegreen (1993).

\subsection{Why is the family distribution of inner and outer resonance features different?}

In Sect. 6.2 we showed that the outer and inner ring distributions differ in the range of families from SA to SAB. In that range, the fraction of galaxies that host inner rings is constant within the error bars, but that of galaxies that host outer features increases steadily $(15 \pm 2 \%$ for SA galaxies, $15 \pm 4 \%$ for SABB galaxies, and $22 \pm 3 \%$ for SAB galaxies). A straightforward explanation for this effect is that outer rings do not form easily in unbarred galaxies. Indeed, weaker bars are likely to be less effective at redistributing material and angular momentum. It is therefore reasonable to assume that some have not yet had time to bring enough material from the $\mathrm{CR}$ region to the $\mathrm{OR}$ and, as a consequence, there is not enough material there to build an outer ring.

\subsection{Rings in unbarred galaxies}

Based on the information in Fig. 19 we found that $15 \pm 2 \%$ of SA galaxies have an outer ring. This fraction increases to $21 \pm 2 \%$ for galaxies with bars (SABB, SAB, SAB, $\mathrm{SB})$. For inner rings the values are $40 \pm 3 \%$ and $\overline{44} \pm 2 \%$, respectively.

In the range $-1 \leq T \leq 3$, which is where outer features are frequently found according to Table 8 , outer rings are found in $29 \pm 5 \%$ of the SA galaxies and in $49 \pm 3 \%$ of the barred galaxies. In the range $-1 \leq T \leq 7$, where most inner features are found, the fraction of SA galaxies with inner rings is $46 \pm 4 \%$ and that of barred ones hosting them is $59 \pm 2 \%$. 


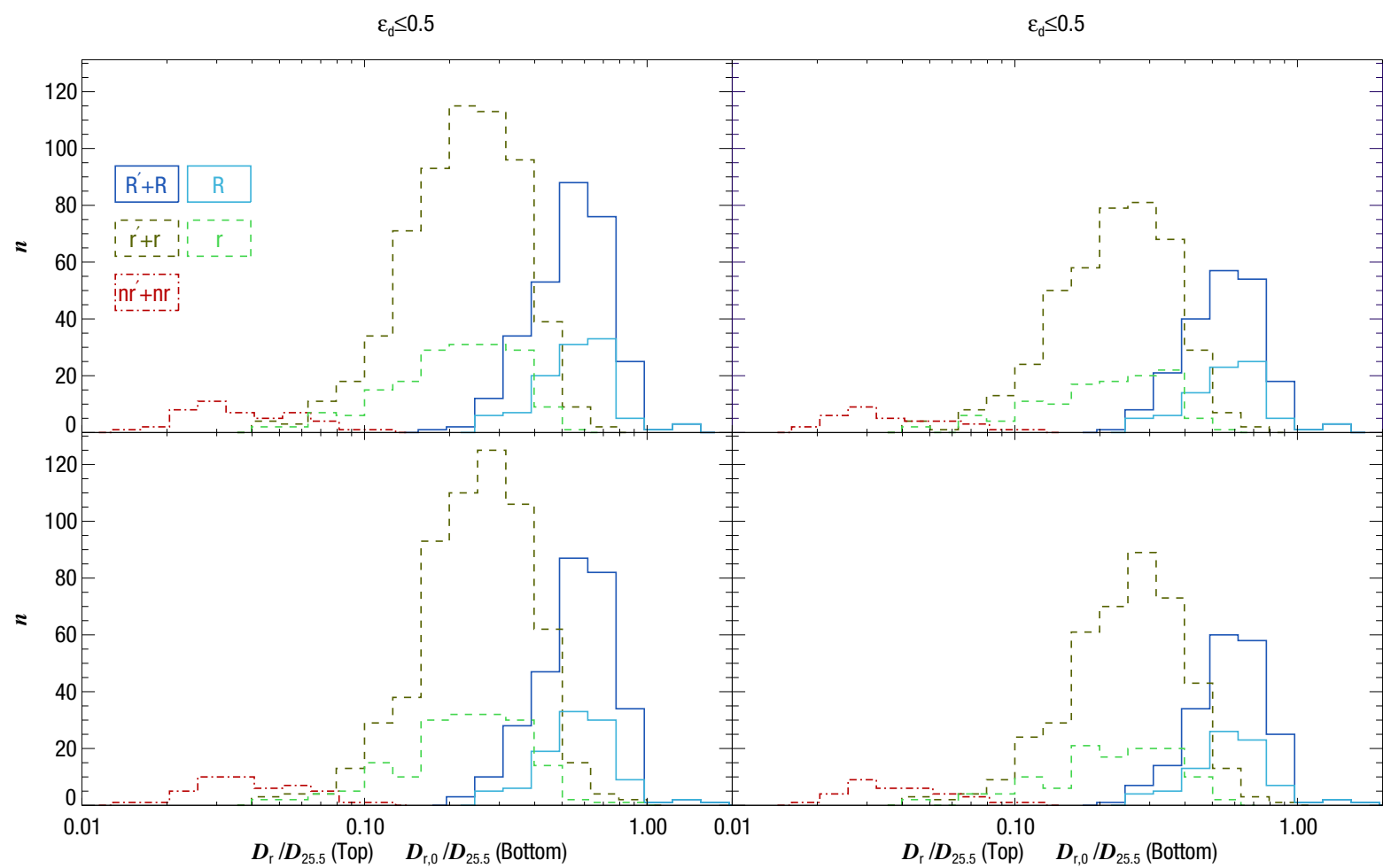

Fig. 29. Distribution of the resonance ring deprojected major axis diameters relative to $D_{25.5}$. The left column shows all galaxies, the right column galaxies with $\epsilon_{\mathrm{d}} \leq 0.5$. The histograms are colour and line-coded as in Fig. 18 .

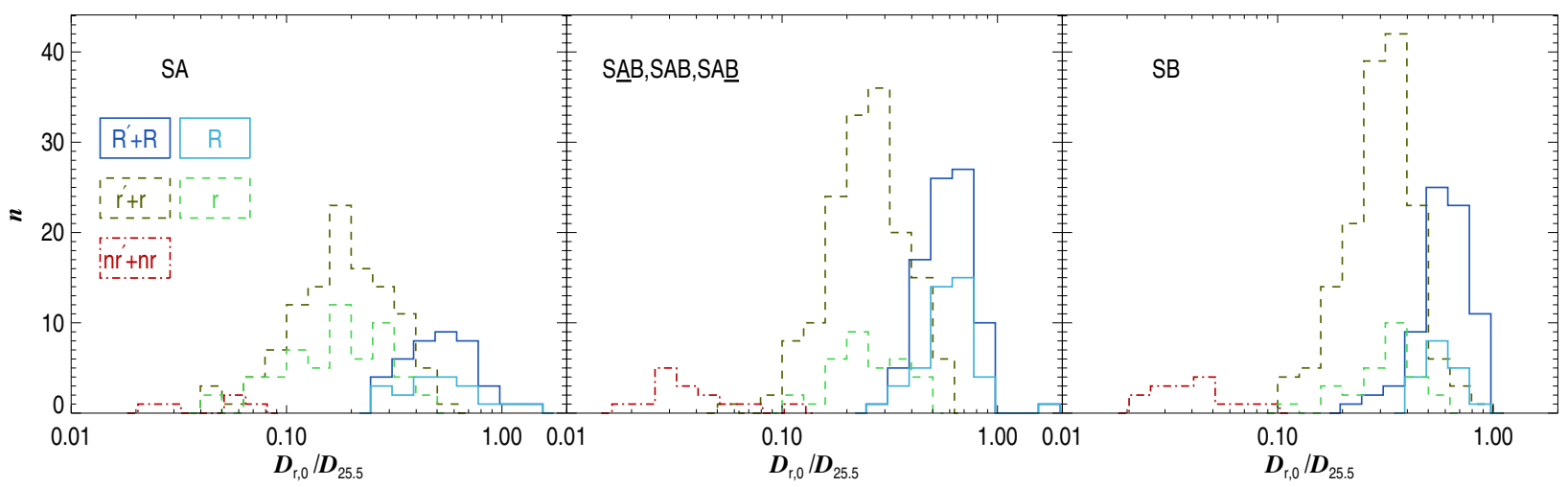

Fig. 30. Distribution of the resonance ring deprojected major axis relative to $D_{25.5}$ colour- and line-coded as in Fig. 18 for different galaxy families and for $\epsilon_{\mathrm{d}} \leq 0.5$. The left panel shows SA galaxies, the middle one S므 to SA $\underline{B}$ galaxies, the right panel SB galaxies.

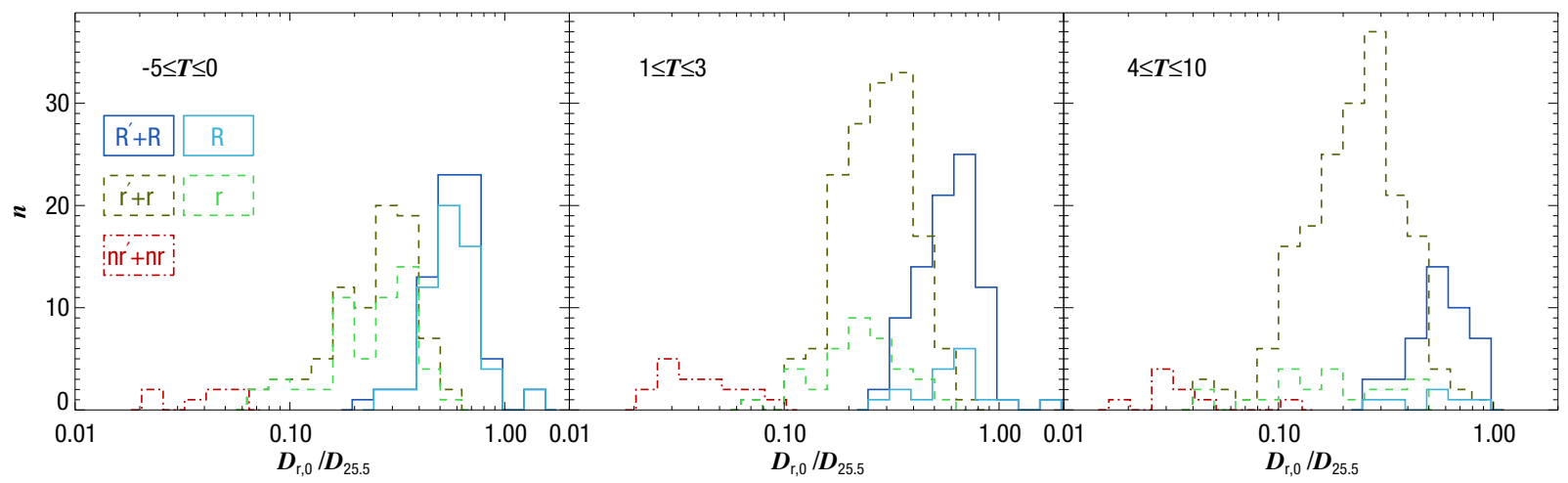

Fig. 31. Distribution of the resonance ring deprojected major axis diameter relative to $D_{25.5}$ colour and line-coded as in Fig. 18 for different galaxy stage ranges and for $\epsilon_{\mathrm{d}} \leq 0.5$. The left panel is for $-5 \leq T \leq 0$ galaxies, the middle panel is for $1 \leq T \leq 3$ galaxies, and the right panel is for $4 \leq T \leq 10$ galaxies. 
Table 6. Number of galaxies in the histograms in Figs. 29-31 and average and standard deviation of the ring deprojected diameters relative to $D_{25.5}$ for several ARRAKIS subsamples.

\begin{tabular}{|c|c|c|c|c|}
\hline Galaxy subsample & Feature type & $n$ & $\left\langle D_{\mathrm{r}, 0} / D_{25.5}\right\rangle$ & $\sigma\left(D_{\mathrm{r}, 0} / D_{25.5}\right)$ \\
\hline \multirow{5}{*}{ All galaxies } & Outer rings & 295 & 0.60 & 0.19 \\
\hline & Inner rings & 608 & 0.27 & 0.12 \\
\hline & Nuclear rings & 47 & 0.04 & 0.02 \\
\hline & Outer closed rings & 106 & 0.63 & 0.22 \\
\hline & Inner closed rings & 180 & 0.25 & 0.12 \\
\hline \multirow{5}{*}{$\epsilon_{\mathrm{d}} \leq 0.5$} & Outer rings & 203 & 0.62 & 0.20 \\
\hline & Inner rings & 424 & 0.27 & 0.12 \\
\hline & Nuclear rings & 35 & 0.04 & 0.02 \\
\hline & Outer closed rings & 82 & 0.64 & 0.24 \\
\hline & Inner closed rings & 116 & 0.24 & 0.11 \\
\hline \multirow{3}{*}{$\epsilon_{\mathrm{d}} \leq 0.5$} & Outer rings & 40 & 0.58 & 0.24 \\
\hline & Inner rings & 111 & 0.20 & 0.10 \\
\hline & Nuclear rings & 5 & 0.05 & 0.02 \\
\hline \multirow{2}{*}{ SA } & Outer closed rings & 19 & 0.60 & 0.30 \\
\hline & Inner closed rings & 56 & 0.19 & 0.10 \\
\hline \multirow{3}{*}{$\epsilon_{\mathrm{d}} \leq 0.5$} & Outer rings & 87 & 0.63 & 0.19 \\
\hline & Inner rings & 155 & 0.27 & 0.11 \\
\hline & Nuclear rings & 15 & 0.04 & 0.02 \\
\hline \multirow{2}{*}{$\mathrm{S} \underline{\mathrm{AB}}, \mathrm{SAB}, \mathrm{SA} \underline{\mathrm{B}}$} & Outer closed rings & 43 & 0.65 & 0.22 \\
\hline & Inner closed rings & 33 & 0.26 & 0.09 \\
\hline \multirow{3}{*}{$\epsilon_{\mathrm{d}} \leq 0.5$} & Outer rings & 74 & 0.62 & 0.16 \\
\hline & Inner rings & 158 & 0.32 & 0.12 \\
\hline & Nuclear rings & 15 & 0.04 & 0.02 \\
\hline \multirow{2}{*}{ SB } & Outer closed rings & 18 & 0.60 & 0.14 \\
\hline & Inner closed rings & 27 & 0.33 & 0.10 \\
\hline \multirow{3}{*}{$\epsilon_{\mathrm{d}} \leq 0.5$} & Outer rings & 71 & 0.62 & 0.20 \\
\hline & Inner rings & 83 & 0.27 & 0.11 \\
\hline & Nuclear rings & 7 & 0.04 & 0.01 \\
\hline \multirow{2}{*}{$-5 \leq T \leq 0$} & Outer closed rings & 58 & 0.62 & 0.20 \\
\hline & Inner closed rings & 55 & 0.26 & 0.11 \\
\hline \multirow{3}{*}{$\epsilon_{\mathrm{d}} \leq 0.5$} & Outer rings & 85 & 0.63 & 0.21 \\
\hline & Inner rings & 152 & 0.29 & 0.11 \\
\hline & Nuclear rings & 18 & 0.04 & 0.02 \\
\hline \multirow{2}{*}{$1 \leq T \leq 3$} & Outer closed rings & 17 & 0.70 & 0.33 \\
\hline & Inner closed rings & 37 & 0.25 & 0.10 \\
\hline \multirow{3}{*}{$\epsilon_{\mathrm{d}} \leq 0.5$} & Outer rings & 44 & 0.60 & 0.18 \\
\hline & Inner rings & 183 & 0.25 & 0.13 \\
\hline & Nuclear rings & 9 & 0.04 & 0.02 \\
\hline \multirow{2}{*}{$4 \leq T \leq 10$} & Outer closed rings & 6 & 0.59 & 0.25 \\
\hline & Inner closed rings & 22 & 0.20 & 0.13 \\
\hline
\end{tabular}

These numbers indicate that outer rings are 1.7 times more frequent in the SAB to SB families than in the SA family. For inner rings the fraction in barred galaxies is only larger by a factor of about 1.3 than that in SA galaxies.

Our results are again mostly similar to those that can be obtained from the NIRSOS galaxy classifications (Laurikainen et al. 2011), which include disc galaxies in the range of stages $-3 \leq T \leq 1$. For outer features, we (they) found that the fraction of SA galaxies hosting them is $27 \pm 5 \%(15 \pm 5 \%)$ and that the fraction of barred galaxies hosting them is $49 \pm 4 \%(47 \pm 5 \%)$. For inner features in that range of stages, we (they) found that the fraction of SA galaxies hosting them is $51 \pm 5 \%(24 \pm 6 \%)$ and that the fraction of barred galaxies hosting them is $60 \pm 4 \%$ $(56 \pm 5 \%)$. The NIRSOS statistics were calculated here excluding the galaxies labelled as spindle in Laurikainen et al. (2011) and ours were calculated with galaxies with $\epsilon_{\mathrm{d}} \leq 0.5$.

The fact that outer features are more sensitive to the presence of a bar than inner features fits naturally with a scenario in which the rings in SA galaxies have formed due to broad oval distortions or long-lasting spiral modes (Rautiainen \& Salo 2000) or with a model in which bars have been destroyed or dissolved after forming the rings. In the first case, simulations show that outer resonance features take longer to form than their inner counterparts. In the second case, the deficit of outer rings in unbarred galaxies can be explained because they can be destroyed easily by the interaction with companion galaxies (Elmegreen et al. 1992). Alternatively, this effect can be attributed to the fact that a significant fraction of outer rings may have been misclassified as inner rings in unbarred galaxies (Sect. 6.5).

Quantifying the link between resonance features and ovals or spiral patterns requires studying parameters such as the nonaxisymmetric torque, $Q$. This was defined by Combes \& Sanders (1981) and indicates the strength of the non-axisymmetries of the galactic potential. This parameter can be local $\left(Q_{\mathrm{r}}\right.$, the local non-axisymmetric torque) or global $\left(Q_{\mathrm{g}}\right.$, the maximum of all $Q_{\mathrm{r}}$ ). In a study that contained 16 unbarred and 31 barred 


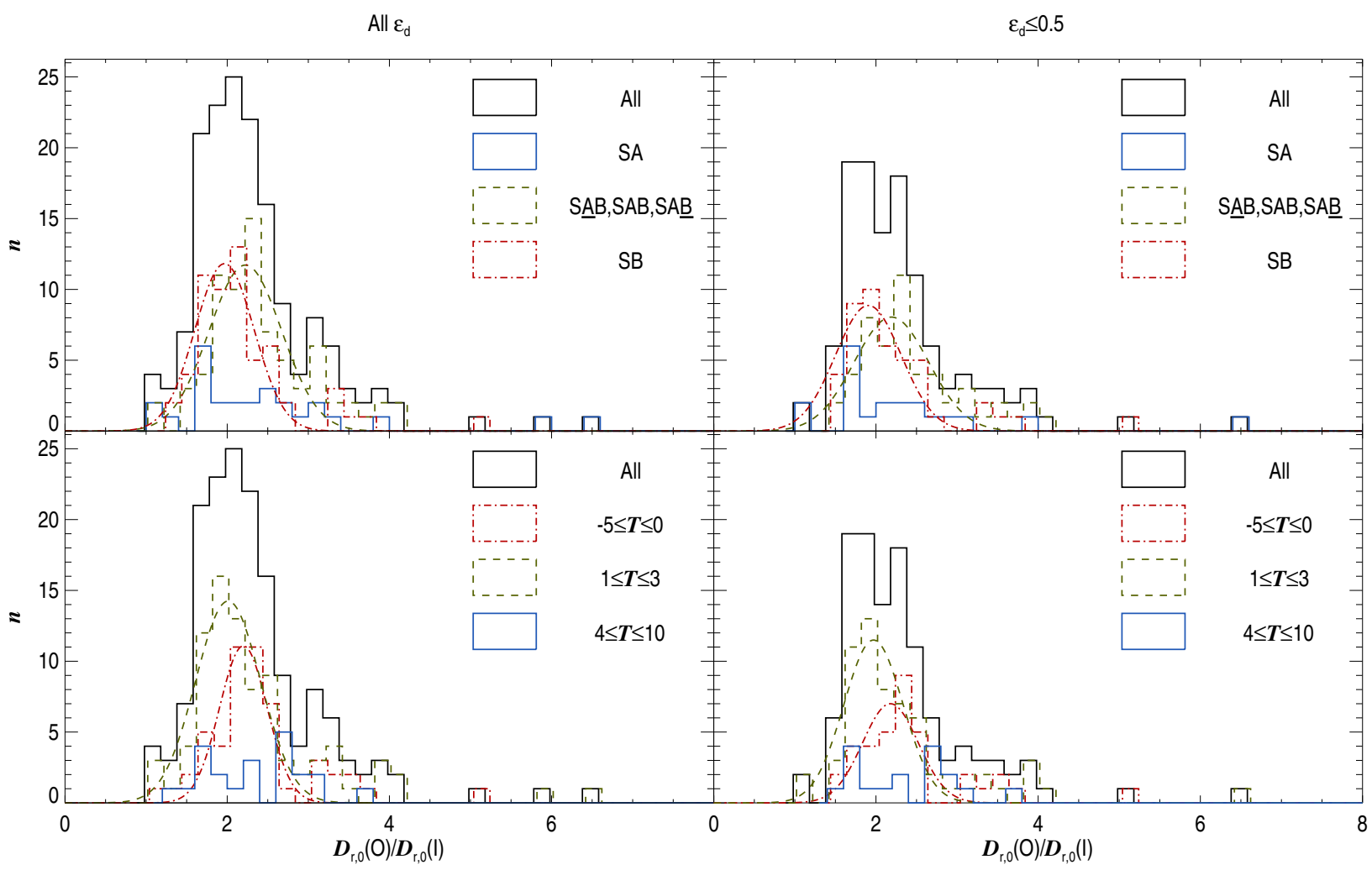

Fig. 32. Distribution of the ratios of the diameters of outer and inner rings. The left column shows all galaxies, the right column galaxies with $\epsilon_{\mathrm{d}} \leq 0.5$. The of histograms are colour- and line-coded according to the host galaxy family (top panels) and stage (bottom panels). One galaxy, NGC 5033, has $D_{\mathrm{r}, 0}(\mathrm{O}) / D_{\mathrm{r}, 0}(\mathrm{I})=17.5$ and is beyond the horizontal limits of the plots.

Table 7. Number of galaxies in the histograms in Fig. 32 and parameters of the Gaussian fits to the $D_{\mathrm{r}, 0}(\mathrm{O}) / D_{\mathrm{r}, 0}(\mathrm{I})$ distributions.

\begin{tabular}{ccccc}
\hline \hline $\begin{array}{c}\text { Galaxy } \\
\text { inclinations }\end{array}$ & Subsample & $n$ & $\left\langle D_{\mathrm{r}, 0}(\mathrm{O}) / D_{\mathrm{r}, 0}(\mathrm{I})\right\rangle$ & $\sigma$ \\
\hline & $\mathrm{SA}$ & 29 & - & - \\
& $\mathrm{S} \underline{\mathrm{AB}}, \mathrm{SAB}, \mathrm{SAB} \underline{-}$ & 75 & $2.22(0.06)$ & $0.45(0.06)$ \\
All $\epsilon_{\mathrm{d}}$ & $\mathrm{SB}$ & 59 & $1.97(0.04)$ & $0.37(0.04)$ \\
& $-5 \leq T \leq 0$ & 52 & $2.20(0.03)$ & $0.29(0.03)$ \\
& $1 \leq T \leq 3$ & 87 & $2.01(0.03)$ & $0.40(0.03)$ \\
& $4 \leq T \leq 10$ & 23 & - & - \\
\hline & $\mathrm{SA}$ & 22 & - & - \\
$\epsilon_{\mathrm{d}} \leq 0.5$ & $\mathrm{SAB}, \mathrm{SAB}, \mathrm{SAB}$ & 51 & $2.19(0.08)$ & $0.46(0.08)$ \\
& $\mathrm{SB}$ & 45 & $1.91(0.04)$ & $0.40(0.05)$ \\
& $-5 \leq T \leq 0$ & 36 & $2.18(0.05)$ & $0.34(0.06)$ \\
& $1 \leq T \leq 3$ & 63 & $1.97(0.03)$ & $0.36(0.03)$ \\
& $4 \leq T \leq 10$ & 18 & - & - \\
\hline
\end{tabular}

Notes. $\left\langle D_{\mathrm{r}, 0}(\mathrm{O}) / D_{\mathrm{r}, 0}(\mathrm{I})\right\rangle$ stands for the fitted centre of the Gaussian distribution and $\sigma$ for its width. The numbers in brackets indicate 1-sigma errors of the fits.

Table 8. Simplified behaviour of the probability of finding outer and inner rings for different galaxy stage ranges.

\begin{tabular}{llcccc}
\hline \hline & & $-5 \leq T \leq-2$ & $-1 \leq T \leq 3$ & $4 \leq T \leq 7$ & $8 \leq T \leq 10$ \\
\hline \multirow{2}{*}{ Can one frequently find...? } & Outer rings & NO & YES & NO & NO \\
& Inner rings & NO & YES & YES & NO \\
\hline
\end{tabular}

galaxies, Grouchy et al. (2010) pointed out a correlation between the ring axis ratio and the $Q_{\mathrm{r}}$ at its radius independently of whether the host galaxy is barred or not. This shows that, indeed, not only bars are responsible for shaping the rings, but also other non-axisymmetries in the galactic potential such as those caused by ovals and spiral arms. Further investigation will require analysing larger samples, which may be drawn from the $\mathrm{S}^{4} \mathrm{G}$. 


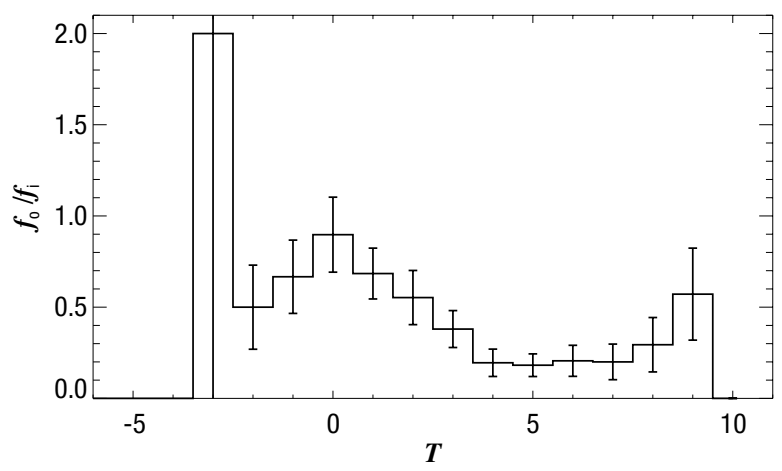

Fig. 33. Fraction of galaxies with $\epsilon_{\mathrm{d}} \leq 0.5$ with outer rings divided by that with inner rings, as a function of the galaxy stage.

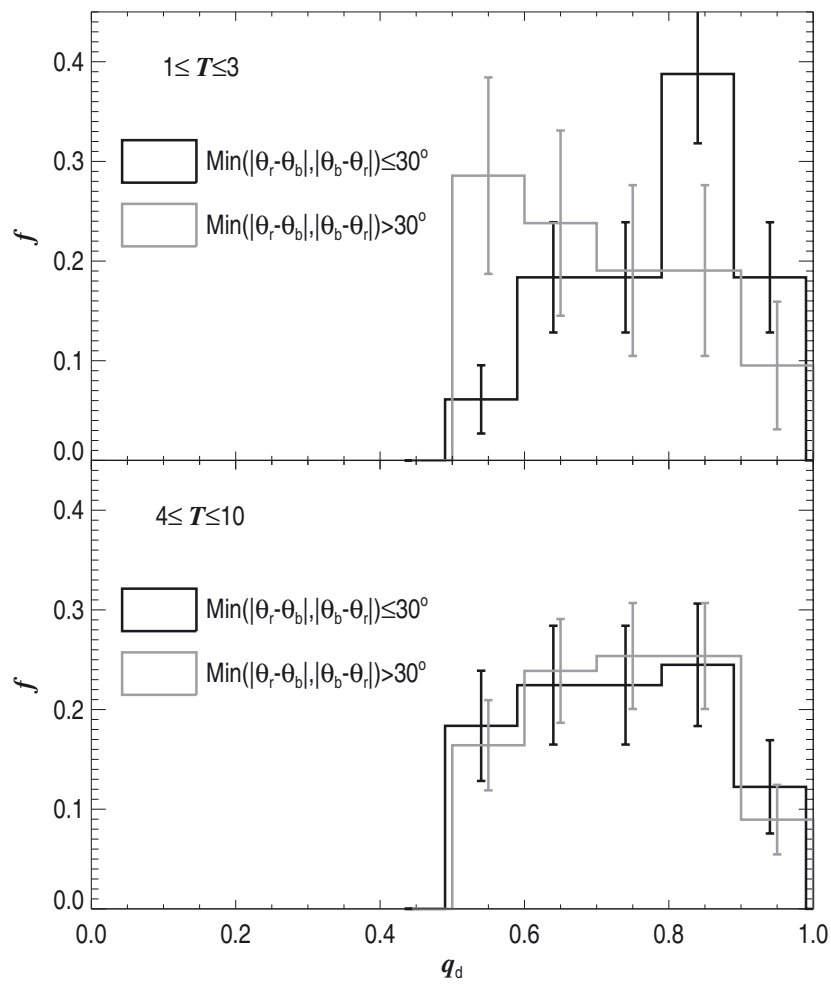

Fig. 34. Fraction of galaxies with inner rings aligned with the bar $\left(\theta_{\mathrm{r}}-\right.$ $\theta_{\mathrm{b}} \leq 30^{\circ}$; in black) and inner rings misaligned with the bar $\left(\theta_{\mathrm{r}}-\theta_{\mathrm{b}}>30^{\circ}\right.$; in grey) that have a given disc axis ratio. The top panel shows galaxies with stages $1 \leq T \leq 3$, the bottom panel galaxies with stages $4 \leq T \leq$ 10. Only galaxies with deprojected ring axis ratios $q_{\mathrm{r}, 0}(\mathrm{I})<0.85$ and disc axis ratios $q_{\mathrm{d}} \leq 0.5$ are included. The error bars are calculated using binomial statistics.

\subsection{Why is the inner ring orientation dependent on the host galaxy stage?}

In Sect. 6.4 we found that inner rings prefer to be aligned in parallel with the bar major axis. However, we also found that a significant fraction of inner rings $(\sim 50 \%)$ is oriented at random with respect to the bar. The fraction of rings with random orientations is low (20-30\%) for early-type galaxies, but increases to $\sim 70 \%$ for galaxies with $T \geq 4$.

We investigated whether this effect might be related to a high galaxy inclination, which would prevent the accurate measurement of bar and ring orientations. To do this, we divided the galaxies that host inner rings into two groups: those whose inner rings are roughly aligned with the $\operatorname{bar}\left(\theta_{\mathrm{r}}-\theta_{\mathrm{b}} \leq 30^{\circ}\right)$, and those that are misaligned with it $\left(\theta_{\mathrm{r}}-\theta_{\mathrm{b}}>30^{\circ}\right)$. For each of these

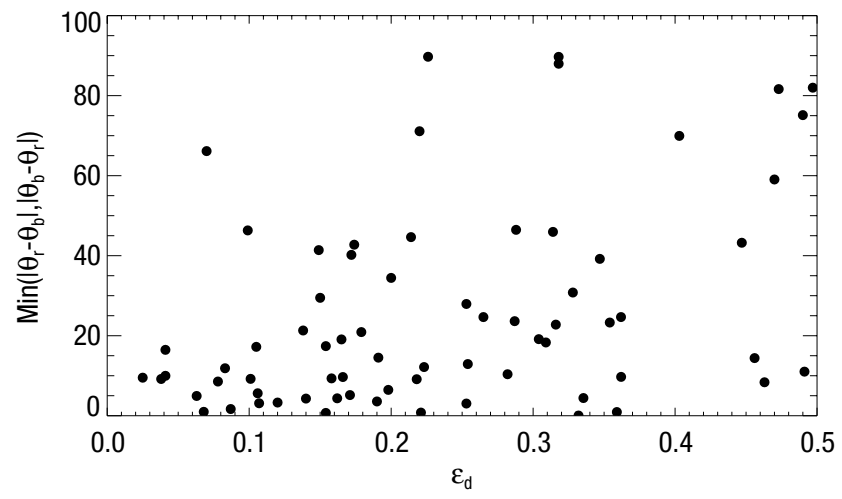

Fig. 35. Ring deprojected major axis angle difference with the bar as a function of the galaxy disc ellipticity for $1 \leq T \leq 3$ and $\epsilon \leq 0.5$.

groups we plotted the fraction of galaxies that can be found at a given disc axis ratio, restricting ourselves to $\epsilon \leq 0.5$. The expectation for circular discs with their rotation axis pointing at random is that the distribution is uniform. We calculated this for galaxies with $1 \leq T \leq 3$ and $4 \leq T \leq 10$ (top and bottom panels in Fig. 34 respectively). Earlier-type galaxies have nearly no misaligned inner rings.

The top panel in Fig. 34 suggests that misaligned rings tend to be found more frequently in the bins with more highly inclined galaxies, and the reversed trend is seen for galaxies oriented parallel with the ring (if we except the bin centred at $q_{\mathrm{d}}=0.95$ ). To verify whether this trend is statistically significant, we plotted in Fig. 35 the intrinsic ring/bar misalignments as a function of the disc ellipticity for all galaxies in the top panel of Fig. 34. We can see that misaligned rings have a preference for inclined galaxies. We calculated the Spearman coefficient of the data in Fig. 35 and found that $\rho=0.37$ and that the probability of the variables in the two axes to be uncorrelated is $p=0.002$. It is therefore very likely that at least some of the misalignments are caused by incorrect deprojections. We see no such effect for galaxies with $4 \leq T \leq 10$ (bottom panel in Fig. 34), which suggests that these deprojection problems might be related to the thickness of the discs. Indeed, as discussed in Sect. 4.6, earliertype galaxies have thicker discs.

For late-type discs, the distributions in Fig. 34 differ from the uniform expectation in the sense that the bin for rounder discs is less populated than the others. This effect is well known for disc galaxies in general and has been attributed to genuine disc deviations from circularity of the order of $b / a=0.9$ or to the presence of substructure in the discs (Lambas et al. 1992). We examined whether these deviations from perfectly circular discs might be responsible for rings misaligned with their bars if we were wrongly assuming purely circular discs. We did this by projecting several thousand elliptical discs with random orientations. These discs had a bar also oriented at random and rings with an orientation differing from that of the bar following a normal distribution with a dispersion of the order of $10^{\circ}$. Then, we deprojected the galaxies, but this time we assumed that they were circular. We found that for moderate disc intrinsic ellipticities $(b / a \gtrsim 0.8)$, this did not cause a fraction of $\sim 70 \%$ rings apparently oriented at random with respect to the bar, as we observe for the late spirals. However, the fraction of rings oriented at random with respect to the bar for galaxies with $1 \leq T \leq 3$ can be explained in this way. This is natural since, as we already argued, at least some ring/bar misalignments in early spirals might be caused by errors in the deprojections. 


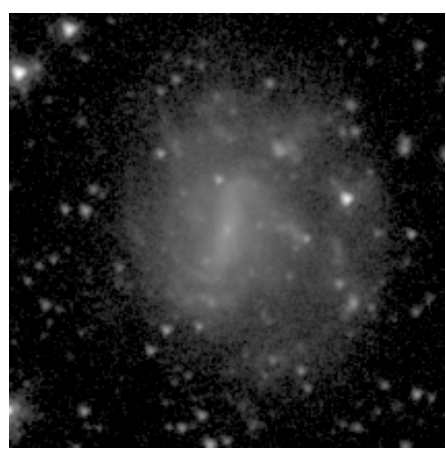

IC 4536 SB(rs) cd $\left|\theta_{\mathrm{r}}-\theta_{\mathrm{b}}\right|=71^{\circ}$

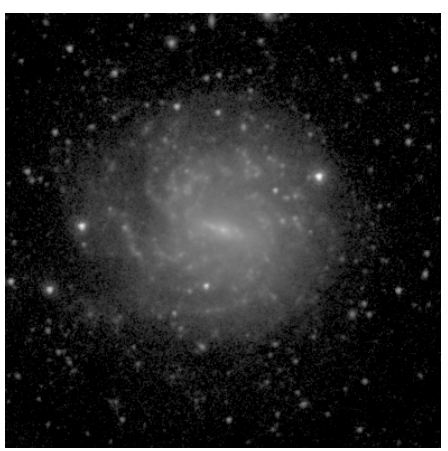

NGC $1493 \quad$ SB(rs)c

$\left|\theta_{\mathrm{r}}-\theta_{\mathrm{b}}\right|=38^{\circ}$

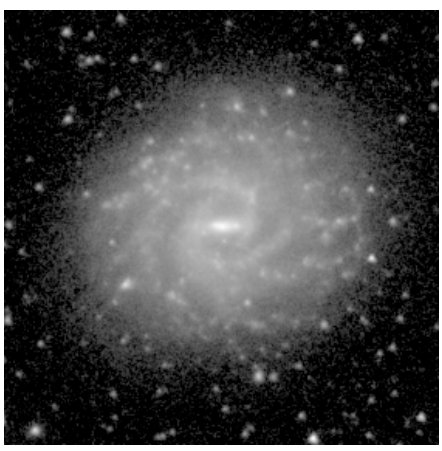

NGC $3346 \quad$ SB(rs)cd

$\left|\theta_{\mathrm{r}}-\theta_{\mathrm{b}}\right|=74^{\circ}$

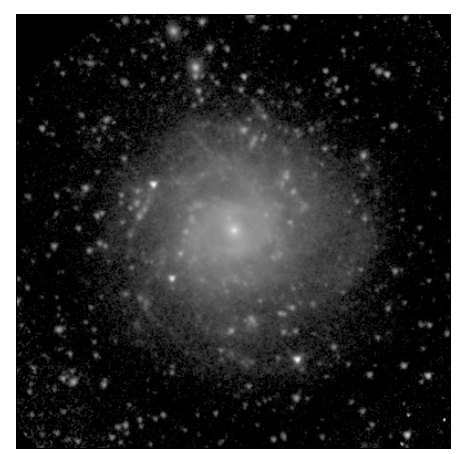

NGC $4136 \quad$ SABB(rs)bc

$\left|\theta_{\mathrm{r}}-\theta_{\mathrm{b}}\right|=\overline{8} 4^{\circ}$

Fig. 36. Selection of close to face-on $\mathrm{S}^{4} \mathrm{G}$ galaxies with stages $4 \leq T \leq 10$ that host inner pseudorings misaligned with the bar. The images have the same properties as those in Fig. 2. The name of the galaxies, their morphological classification, and the bar-pseudoring intrinsic misalignment can be found below each image.

Therefore our results indicate that at least for late-type galaxies misalignments are not caused by uncertainties in bar and ring properties measured in highly inclined discs or because discs might not exactly be circular, as assumed when calculating the deprojections. Therefore, a significant fraction of inner rings are intrinsically misaligned, especially those in late-type galaxies. A selection of very low-inclination galaxies with such misaligned inner rings is shown in Fig. 36.

The existence of misaligned inner features was also shown in simulations by Rautiainen \& Salo (2000). In their study, this misalignment appears because a spiral mode with a pattern speed lower than that of the bar dominates the Fourier amplitude spectrum at the radius of the inner ring. This means that our results would be consistent with spiral modes rotating with a pattern speed different from that of the bar and with a significant contribution at the I4R radius for late-type galaxies. Alternatively, this is caused by a rotation of the Lagrangian points from their usual position, in the direction of the bar major axis, by an angle that increases with increasing spiral amplitude (e.g., Athanassoula et al. 2010). A way to explore these possibilities would be to compare the amplitude of the bar and spiral arm torque at the ring radius in our sample galaxies as reported, for instance, by Block et al. (2004) and Salo et al. (2010). The latter authors described that the spiral density amplitude correlates well with the local bar torque as long as it is measured within 1.5 times the bar length. Since bars in late-type galaxies can significantly shorter than their CR radius (see, e.g., Combes \& Elmegreen 1993), a correlation between the bar and the spiral torque at the inner ring radius may not be found.

An alternative possibility is that the apparent random orientation between bars and rings is caused by errors in measuring the inner feature position angle. This is because the later a galaxy is, the more ill-defined its rings become. Also, inner pseudorings in late-type galaxies tend to be more spiral-like than those in early-type galaxies, which partly invalidates the approximation made when fitting them with ellipses.

\section{Summary and conclusions}

We presented ARRAKIS, the atlas of resonant rings as known in the $S^{4} \mathrm{G}$, and a catalogue of the ring and pseudoring properties. The preliminary analysis of the data contained in the catalogue (Appendix A) and the atlas (Appendix B) was also presented. In this paper rings and pseudorings have been collectively termed rings.

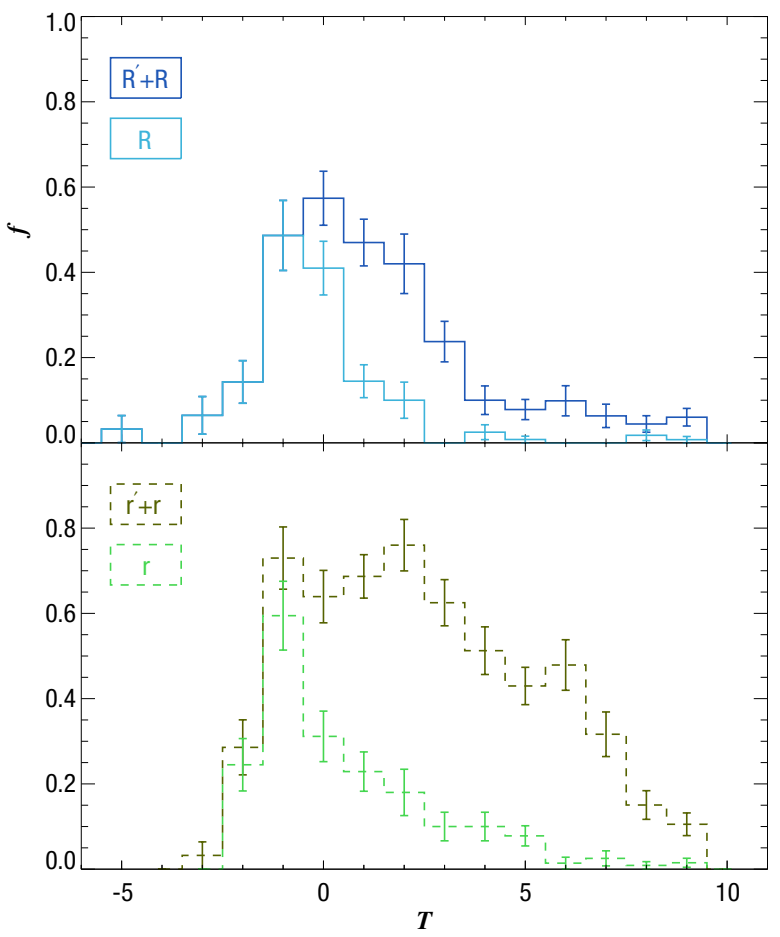

Fig. 37. Fraction of galaxies with outer rings (top panel) and inner rings (bottom panel) for a given stage. The plots only include galaxies with a disc ellipticity $\epsilon_{\mathrm{d}} \leq 0.5$. In each of the panels, both rings as a whole and closed rings are indicated.

The most common rings are resonance rings. These rings are thought to be due to gathering of gas, which can later be transformed into stars, at or close to the radii of the resonances caused by non-axisymmetries such as bars, ovals, and strong spiral patterns (e.g., Schwarz 1981, 1984; Sellwood \& Wilkinson 1993; Byrd et al. 1994; Rautiainen \& Salo 2000). They come in three flavours (outer, inner, and nuclear), depending on their radius relative to that of the bar. Outer features are thought to be related to the OLR or occasionally to the O4R, inner features to the I4R, and nuclear features to the ILRs. Inner and outer rings might also be based on flux tube manifolds triggered by the bar or oval distortion (Romero-Gómez et al. 2006, 2007; Athanassoula et al. 2009b,a, 2010; Athanassoula 2012b). Either way, rings are tracers of the underlying galactic potential, which makes them 


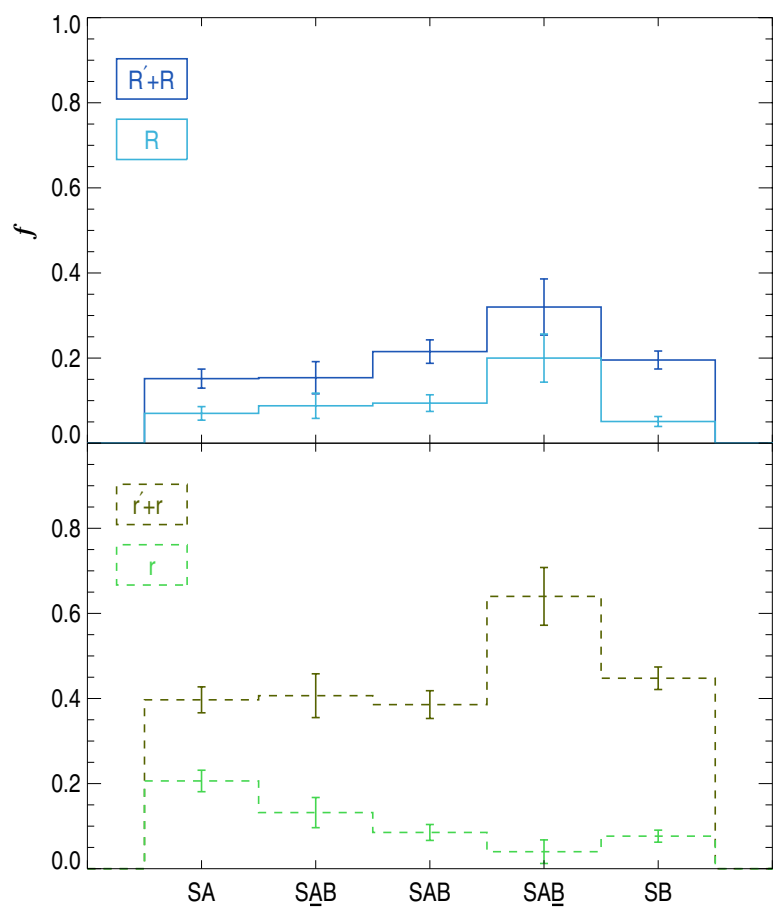

Fig. 38. As in Fig. 37, but now with the bar family instead of stage.

important clues for the understanding of the secular evolution of disc galaxies.

The $S^{4} \mathrm{G}$ is a survey of 2352 galaxies representative of the local Universe observed at 3.6 and $4.5 \mu \mathrm{m}$ wavelengths. The galaxies in the $S^{4} \mathrm{G}$ were classified by R. J. Buta, who also identified the rings (Buta et al., in prep.). We studied all the rings in the $S^{4} G$ frames, but our focus was on resonance rings in the $S^{4} G$ galaxy sample. Because dust obscuration is weak in the mid-infrared, the chances that rings are hidden by dust are much lower than at optical wavelengths. This makes the $S^{4} \mathrm{G}$ a very good tool for studying rings.

We described the ring shape and orientation by marking their contours and fitting ellipses to them. To do this, we used modelsubtracted images of the galaxies produced by the $P 4$ of the $S^{4} G$ (Salo et al., in prep.). We obtained intrinsic ring shapes and orientations by deprojecting the fitted ellipses. The deprojection parameters were obtained from the $\mathrm{P} 4$ ellipse fits to the outer parts of $\mathrm{S}^{4} \mathrm{G}$ galaxies. Bar orientations and ellipticities (a rough indicator of bar strength) were measured by looking at the ellipse fit corresponding to the radius where the highest ellipticity was found within the bar. They were deprojected in the same way as for rings.

We summarise our findings as follows:

- Section 6.1 and Figs. 18 and 37: outer rings are found in $16 \pm 1 \%$ of the $S^{4} \mathrm{G}$ galaxies and are more frequent for stages $-1 \leq T \leq 2$ (over $40 \%$ frequency). Outer closed rings account for $100 \%$ of outer features with $T \leq-1$. Inner rings are the most frequent resonance feature in the local Universe ( $35 \pm 1 \%$ frequency in the $\mathrm{S}^{4} \mathrm{G}$ sample). They are typically found in the range $-1 \leq T \leq 6$ (over $40 \%$ frequency) and their frequency peaks at stages $-1 \leq T \leq 3$ (over $60 \%$ frequency). The inner closed ring distribution is shifted to earlier stages than that of inner features.

- Sections 6.2 and 7.2 and Figs. 19 and 38: the outer ring frequency increases from $15 \pm 2 \%$ to $32 \pm 7 \%$ along the family sequence from $\mathrm{SA}$ to $\mathrm{SAB}$, and decreases again to $20 \pm 2 \%$ for SB galaxies. The inner ring frequency is qualitatively different, since it is roughly constant at $\sim 40 \%$ for all families except for a peak at $64 \pm 7 \%$ frequency for SAB galaxies. The reason might be that outer rings take a longer time than inner rings to be built in galaxies with weaker non-axisymmetries. We also found that inner closed rings have a preference for unbarred and weakly barred galaxies.

- Section 7.3: barred galaxies have outer rings 1.7 times more often than unbarred ones. Barred galaxies have 1.3 more inner rings than their unbarred counterparts. This indicates that although they are stimulated by bars, rings do not need them to exist. Simulations suggest (e.g., Salo et al. 1999; Rautiainen \& Salo 2000) that rings in unbarred galaxies may be related to weak ovals and/or long-lived spiral modes. Alternatively, they may have formed because of bars that have since been destroyed or have dissolved.

- Sections 6.1 and 7.3: we confirmed the results of the NIRSOS survey regarding the frequencies of outer and inner rings as a function of the stage and the family for galaxies with stages $-3 \leq T \leq 1$.

- Section 6.3 and Figs. 20, 21, and 22: the axis ratio distribution of each of the three ring flavours - outer, inner, and nuclear - can be fitted with a Gaussian curve. Their intrinsic axis ratios typically range from $q_{\mathrm{r}, 0}=0.6$ to $q_{\mathrm{r}, 0}=1.0$. Inner rings are in general more elliptical than their outer counterparts. We found that outer and inner rings become on average more elliptical when the bar strength increases (when the galaxy family changes from SA to SB) and that inner rings are more elliptical in late-stage galaxies than in earlier-stage galaxies.

- Section 6.4 and Fig. 23: we confirmed that outer rings have two preferred orientations, namely parallel and perpendicular to the bar. Of the outer rings in barred galaxies $35 \pm 18 \%$ are parallel to the bar and $65 \pm 39 \%$ are oriented perpendicular to it.

- Sections 6.4 and 7.4 and Figs. 23 and 25: we found that many inner rings have their major axes oriented parallel to the bar, as predicted in simulations and as previously reported from observations. However, we also found that maybe as much as $50 \%$ of inner rings have random orientations with respect to the bar. These misaligned inner rings are mostly found in late-type spirals $(T \geq 4)$. We speculate that this may be because the Fourier amplitude spectrum at the radius of the I4R is dominated by spiral modes with a pattern speed different from that of the bar late-type galaxies. Alternatively, this can be due to the increased difficulty of measuring inner ring properties when they become ill-defined at late stages.

- Sections 6.5 and 6.6 and Figs. 26, 27, 29, and 30: the size of a ring relative to that of the galaxy is a much better indicator of its flavour (outer, inner, or nuclear) than its absolute size. The wings of both the absolute and relative size distributions of inner rings are more extended in unbarred galaxies than for those with bars. This may indicate that several outer and nuclear rings have been misclassified as inner features in unbarred galaxies.

- Section 6.7: several of our sample galaxies have both an outer and an inner ring. The distribution of their diameter ratio peaks at $D_{\mathrm{r}, 0}(\mathrm{O}) / D_{\mathrm{r}, 0}(\mathrm{I}) \sim 2$, which agrees with the literature.

\subsection{Open questions}

We discussed two very interesting questions on rings which still have no clear solution. Why are some rings found in apparently unbarred galaxies? And why do inner rings in late-type galaxies 
have a higher tendency to be oriented at random with respect to the bar than those in earlier-type galaxies?

As developed in the discussion in Sect. 7, galaxies with these properties have occasionally been found in simulations. However, to test whether the mechanism creating rings in unbarred galaxies and the one shaping inner rings that are misaligned with the bar is the same in nature and in simulations, one has to study each galaxy at a deeper level of detail than we did here. Indeed, visual identification of the bar and a rough estimate of its orientation may be insufficient and one may need to perform a Fourier analysis to unveil the effects of nonaxisymmetries induced by spiral arms or broad weak ovals. Again, the $S^{4} \mathrm{G}$ is the right tool for this type of research because the mid-infrared is an excellent tracer of the stellar mass and thus of the baryonic matter contribution to the galactic potential.

Acknowledgements. We thank our anonymous referee, who very carefully read this paper. The authors wish to thank the entire $S^{4} \mathrm{G}$ team for their efforts in this project. We thank Pertti Rautiainen for useful discussions on the ring formation in his simulations, Simón Díaz-García for helping with fundamental statistical concepts, and Glenn van de Ven for his useful comments. We acknowledge financial support to the DAGAL network from the People Programme (Marie Curie Actions) of the European Union's Seventh Framework Programme FP7/20072013/ under REA grant agreement number PITN-GA-2011-289313. E.A. and A.B. acknowledge financial support from the CNES (Centre National d'Études Spatiales - France). K.S., J.-C.M.-M., T.K., and T.M. acknowledge support from the National Radio Observatory, which is a facility of the National Radio Astronomy Observatory operated under cooperative agreement by Associated Universities, Inc. S.C., H.S., E.L., M.H.-H., and J.L. acknowledge support from the Academy of Finland. This work is based on observations and archival data made with the Spitzer Space Telescope, which is operated by the Jet Propulsion Laboratory, California Institute of Technology under a contract with NASA. We are grateful to the dedicated staff at the Spitzer Science Center for their help and support in planning and execution of this Exploration Science program. We also gratefully acknowledge support from NASA JPL/Spitzer grant RSA 1374189 provided for the $S^{4} G$ project. This research has made use of SAOImage DS9, developed by Smithsonian Astrophysical Observatory. This research has made use of the NASA/IPAC Extragalactic Database (NED) which is operated by the Jet Propulsion Laboratory, California Institute of Technology, under contract with the National Aeronautics and Space Administration.

\section{References}

Appleton, P. N., \& Struck-Marcell, C. 1996, Fund. Cosmic Phys., 16, 111

Athanassoula, E. 1996, in IAU Colloq. 157: Barred Galaxies, eds. R. Buta, D. A. Crocker, \& B. G. Elmegreen, ASP Conf. Ser., 91, 309

Athanassoula, E. 2012a, Proc. XXIII Canary Islands Winter School of Astrophysics: Secular Evolution of Galaxies, eds. J. Falcon-Barroso, \& J. H. Knapen (Cambridge: University Press) [arXiv: 1211.6752]

Athanassoula, E. 2012b, MNRAS, 426, L46

Athanassoula, E., Bosma, A., Creze, M., \& Schwarz, M. P. 1982, A\&A, 107, 101 Athanassoula, E., Romero-Gómez, M., Bosma, A., \& Masdemont, J. J. 2009a, MNRAS, 400, 1706

Athanassoula, E., Romero-Gómez, M., \& Masdemont, J. J. 2009b, MNRAS, 394, 67

Athanassoula, E., Romero-Gómez, M., Bosma, A., \& Masdemont, J. J. 2010, MNRAS, 407, 1433

Athanassoula, E., Machado, R. E. G., \& Rodionov, S. A. 2013, MNRAS, 429, 1949

Berentzen, I., Athanassoula, E., Heller, C. H., \& Fricke, K. J. 2003, MNRAS, 341,343

Berentzen, I., Shlosman, I., Martinez-Valpuesta, I., \& Heller, C. H. 2007, ApJ, 666,189

Bettoni, D., Galletta, G., García-Burillo, S., \& Rodríguez-Franco, A. 2001, A\&A, 374, 421

Block, D. L., Puerari, I., Knapen, J. H., et al. 2001, A\&A, 375, 761

Block, D. L., Buta, R., Knapen, J. H., et al. 2004, AJ, 128, 183

Bournaud, F., \& Combes, F. 2002, A\&A, 392, 83

Bournaud, F., Combes, F., \& Semelin, B. 2005, MNRAS, 364, L18

Buta, R. 1986a, ApJS, 61, 609

Buta, R. 1986b, ApJS, 61, 631

Buta, R. 1987, ApJS, 64, 383

Buta, R. 1991, ApJ, 370, 130
Buta, R. 1995, ApJS, 96, 39 (CSRG)

Buta, R. 2002, in Disks of Galaxies: Kinematics, Dynamics and Peturbations, eds. E. Athanassoula, A. Bosma, \& R. Mujica, ASP Conf. Ser., 275, 185

Buta, R., \& Combes, F. 1996, Fund. Cosmic Phys., 17, 95

Buta, R., \& Crocker, D. A. 1993, AJ, 105, 1344 (BC93)

Buta, R., Purcell, G. B., \& Crocker, D. A. 1995, AJ, 110, 1588

Buta, R. J. 2013, in Galaxy Morphology, eds. T. D. Oswalt, \& W. C. Keel (Dordrech: Springer Science + Business Media), 1

Buta, R. J., Corwin, H. G., \& Odewahn, S. C. 2007, The de Vaucouleurs Altlas of Galaxies (Cambridge University Press)

Buta, R. J., Sheth, K., Regan, M., et al. 2010, ApJS, 190, 147

Byrd, G., Rautiainen, P., Salo, H., Buta, R., \& Crocher, D. A. 1994, AJ, 108, 476

Byrd, G. G., Ousley, D., \& dalla Piazza, C. 1998, MNRAS, 298, 78

Combes, F., \& Elmegreen, B. G. 1993, A\&A, 271, 391

Combes, F., \& Sanders, R. H. 1981, A\&A, 96, 164

Comerón, S. 2013, A\&A, 555, L4

Comerón, S., Knapen, J. H., \& Beckman, J. E. 2008a, J. Phys. Conf. Ser., 131, 012046

Comerón, S., Knapen, J. H., \& Beckman, J. E. 2008b, A\&A, 485, 695

Comerón, S., Knapen, J. H., Beckman, J. E., \& Shlosman, I. 2008c, A\&A, 478 403

Comerón, S., Knapen, J. H., Beckman, J. E., et al. 2010, MNRAS, 402, 2462

Corsini, E. M., Pizzella, A., Funes, J. G., Vega Beltran, J. C., \& Bertola, F. 1998 A\&A, 337, 80

Crocker, D. A., Baugus, P. D., \& Buta, R. 1996, ApJS, 105, 353

Curtis, H. D. 1918, Publ. Lick Obs., 13, 9

Debattista, V. P., Carollo, C. M., Mayer, L., \& Moore, B. 2004, ApJ, 604, L93

Debattista, V. P., Mayer, L., Carollo, C. M., et al. 2006, ApJ, 645, 209

de Grijs, R. 1998, MNRAS, 299, 595

de Vaucouleurs, G. 1948, Ann. Astrophys., 11, 247

de Vaucouleurs, G. 1959, Handbuch der Physik (Springer Verlag), 53, 275

de Vaucouleurs, G. 1963, ApJS, 8, 31

de Vaucouleurs, G., \& Buta, R. 1980, AJ, 85, 637 (vB80)

de Vaucouleurs, G. H., de Vaucouleurs, A., \& Shapley, H. 1964, Reference catalogue of bright galaxies (Austin: University of Texas press)

de Vaucouleurs, G., de Vaucouleurs, A., Corwin, Jr., H. G., et al. 1991, Third Reference Catalogue of Bright Galaxies (RC3), Vol. I: Explanations and references. Vol. II: Data for galaxies between $0^{\mathrm{h}}$ and $12^{\mathrm{h}}$. Vol. III: Data for galaxies between $12^{\mathrm{h}}$ and $24^{\mathrm{h}}$ (New York: Springer)

Dreyer, J. L. E. 1888, MmRAS, 49, 1

Dreyer, J. L. E. 1895, MmRAS, 51, 185

Eliche-Moral, M. C., González-García, A. C., Balcells, M., et al. 2011, A\&A, 533, A104

Elmegreen, D. M., Elmegreen, B. G., Combes, F., \& Bellin, A. D. 1992, A\&A, 257, 17

Englmaier, P., \& Gerhard, O. 1997, MNRAS, 287, 57

Erwin, P. 2005, MNRAS, 364, 283

Erwin, P., \& Sparke, L. S. 1999, ApJ, 521, L37

Erwin, P., \& Sparke, L. S. 2002, AJ, 124, 65

Fazio, G. G., Hora, J. L., Allen, L. E., et al. 2004, ApJS, 154, 10

Ferrers, N. M. 1877, Q. J. Pure Appl. Math., 14, 1

Friedli, D., \& Benz, W. 1993, A\&A, 268, 65

Gadotti, D. A., Athanassoula, E., Carrasco, L., et al. 2007, MNRAS, 381, 943

Grouchy, R. D., Buta, R. J., Salo, H., \& Laurikainen, E. 2010, AJ, 139, 2465

Hubble, E. P. 1926, ApJ, 64, 321

Jaffe, W. 1983, MNRAS, 202, 995

Jardel, J. R., Gebhardt, K., Shen, J., et al. 2011, ApJ, 739, 21

Jore, K. P., Broeils, A. H., \& Haynes, M. P. 1996, AJ, 112, 438

Joye, W. A., \& Mandel, E. 2003, in Astronomical Data Analysis Software and Systems XII, eds. H. E. Payne, R. I. Jedrzejewski, \& R. N. Hook, ASP Conf. Ser., 295, 489

Jungwiert, B., \& Palous, J. 1996, A\&A, 311, 397

Keeler, J. E. 1908, Publ. Lick Obs., 8, 1

Kim, W.-T., Seo, W.-Y., Stone, J. M., Yoon, D., \& Teuben, P. J. 2012, ApJ, 747, 60

Knapen, J. H. 2005, A\&A, 429, 141

Knapen, J. H., Beckman, J. E., Heller, C. H., Shlosman, I., \& de Jong, R. S. 1995, ApJ, 454, 623

Knapen, J. H., Pérez-Ramírez, D., \& Laine, S. 2002, MNRAS, 337, 808

Knapen, J. H., Whyte, L. F., de Blok, W. J. G., \& van der Hulst, J. M. 2004, A\&A, 423, 481

Kormendy, J. 1979, ApJ, 227, 714

Kormendy, J., \& Kennicutt, Jr., R. C. 2004, ARA\&A, 42, 603

Kuijken, K., \& Merrifield, M. R. 1995, ApJ, 443, L13

Lambas, D. G., Maddox, S. J., \& Loveday, J. 1992, MNRAS, 258, 404

Laurikainen, E., Salo, H., \& Rautiainen, P. 2002, MNRAS, 331, 880

Laurikainen, E., Salo, H., Buta, R., \& Knapen, J. H. 2011, MNRAS, 418, 1452

Lynden-Bell, D., \& Kalnajs, A. J. 1972, MNRAS, 157, 1 


\section{S. Comerón et al.: ARRAKIS}

Lynds, R., \& Toomre, A. 1976, ApJ, 209, 382

Marochnik, L. S., Mishurov, Y. N., \& Suchkov, A. A. 1972, Ap\&SS, 19, 285

Martin, P. 1995, AJ, 109, 2428

Martinez-Valpuesta, I., \& Shlosman, I. 2004, ApJ, 613, L29

Mazzuca, L. M., Sarzi, M., Knapen, J. H., Veilleux, S., \& Swaters, R. 2006, ApJ, 649, L79

Parsons, C. 1880, Scientific Trans. Roy. Dublin Soc., II

Paturel, G., Petit, C., Prugniel, P., et al. 2003, A\&A, 412, 45

Peng, C. Y., Ho, L. C., Impey, C. D., \& Rix, H.-W. 2002, AJ, 124, 266

Peng, C. Y., Ho, L. C., Impey, C. D., \& Rix, H.-W. 2010, AJ, 139, 2097

Perrine, C. D. 1922, MNRAS, 82, 486

Raha, N., Sellwood, J. A., James, R. A., \& Kahn, F. D. 1991, Nature, 352, 411

Rautiainen, P., \& Salo, H. 2000, A\&A, 362, 465

Regan, M. W., \& Teuben, P. J. 2004, ApJ, 600, 595

Romero-Gómez, M., Masdemont, J. J., Athanassoula, E., \& García-Gómez, C. 2006, A\&A, 453, 39

Romero-Gómez, M., Athanassoula, E., Masdemont, J. J., \& García-Gómez, C. 2007, A\&A, 472, 63

Salo, H., Rautiainen, P., Buta, R., et al. 1999, AJ, 117, 792

Salo, H., Laurikainen, E., Buta, R., \& Knapen, J. H. 2010, ApJ, 715, L56

Schommer, R. A., \& Sullivan, III, W. T. 1976, Astrophys. Lett., 17, 191

Schwarz, M. P. 1981, ApJ, 247, 77

Schwarz, M. P. 1984, MNRAS, 209, 93
Schweizer, F., Whitmore, B. C., \& Rubin, V. C. 1983, AJ, 88, 909 Schweizer, F., van Gorkom, J. H., \& Seitzer, P. 1989, ApJ, 338, 770 Sellwood, J. A. 1981, A\&A, 99, 362

Sellwood, J. A., \& Wilkinson, A. 1993, Rep. Prog. Phys., 56, 173

Sheth, K., Regan, M. W., Vogel, S. N., \& Teuben, P. J. 2000, ApJ, 532, 221

Sheth, K., Regan, M., Hinz, J. L., et al. 2010, PASP, 122, 1397

Shlosman, I. 1999, in The Evolution of Galaxies on Cosmological Timescales, eds. J. E. Beckman, \& T. J. Mahoney, ASP Conf. Ser., 187, 100

Sil'chenko, O. K., \& Moiseev, A. V. 2006, AJ, 131, 1336

Sulentic, J. W., \& Arp, H. C. 1987, ApJ, 319, 687

Thakar, A. R., Ryden, B. S., Jore, K. P., \& Broeils, A. H. 1997, ApJ, 479, 702

Theys, J. C., \& Spiegel, E. A. 1977, ApJ, 212, 616

Tody, D. 1986, in SPIE Conf. Ser., 627, ed. D. L. Crawford, 733

Tody, D. 1993, in Astronomical Data Analysis Software and Systems II, eds.

R. J. Hanisch, R. J. V. Brissenden, \& J. Barnes, ASP Conf. Ser., 52, 173

Toomre, A. 1977, in Evolution of Galaxies and Stellar Populations, eds. B. M.

Tinsley, \& R. B. G. Larson, D. Campbell, 401

Treuthardt, P., Salo, H., Rautiainen, P., \& Buta, R. 2008, AJ, 136, 300

Tutukov, A. V., \& Fedorova, A. V. 2006, Astron. Rep., 50, 785

van den Bergh, S. 1976, ApJ, 206, 883

van Driel, W., Combes, F., Casoli, F., et al. 1995, AJ, 109, 942

Werner, M. W., Roellig, T. L., Low, F. J., et al. 2004, ApJS, 154, 1

Whitmore, B. C., Lucas, R. A., McElroy, D. B., et al. 1990, AJ, 100, 1489 


\section{Appendix A: Catalogue}

The catalogue contains two tables. One for galaxies included in the $\mathrm{S}^{4} \mathrm{G}$ sample and a second one for galaxies that appear in $\mathrm{S}^{4} \mathrm{G}$ frames, but are not included in the original sample. The information in the tables in the catalogue is organized as follows:

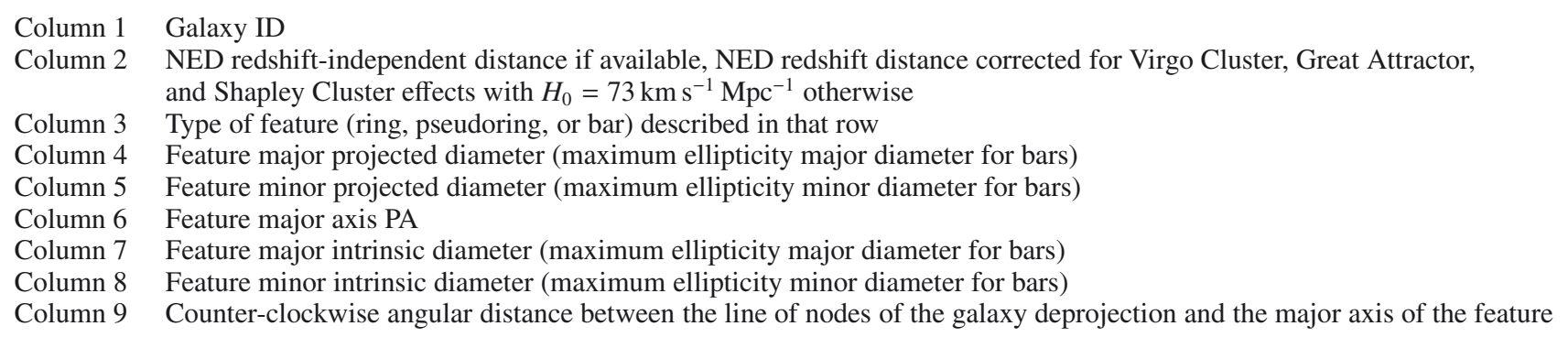

Deprojected ring parameters are not given for polar rings.

\section{Appendix B: Atlas}

The atlas contains two sets of galaxies. One for galaxies included in the $\mathrm{S}^{4} \mathrm{G}$ sample and a second one for galaxies that appear in $\mathrm{S}^{4} \mathrm{G}$ frames, but are not included in the original sample. The information in the atlas is organized as follows:

- Line 1: Galaxy ID

- Line 2: Galaxy classification according to Buta et al. (in prep.).

- $\mathrm{S}^{4} \mathrm{G}$ original galaxy image and P4 model-subtracted image (Salo et al. in prep.). The red ellipses indicate the rings in the galaxy. The green ellipses are the result of an ellipse fit at the radius of the maximum bar ellipticity. The red crosses indicate the centre of the galaxy. The axis units are arcseconds. North is up and east is left.

- Below this information we present a table showing the information on the projected and deprojected information on the rings, pseudorings, and bars of that galaxy as shown in the catalogue in Appendix A. 


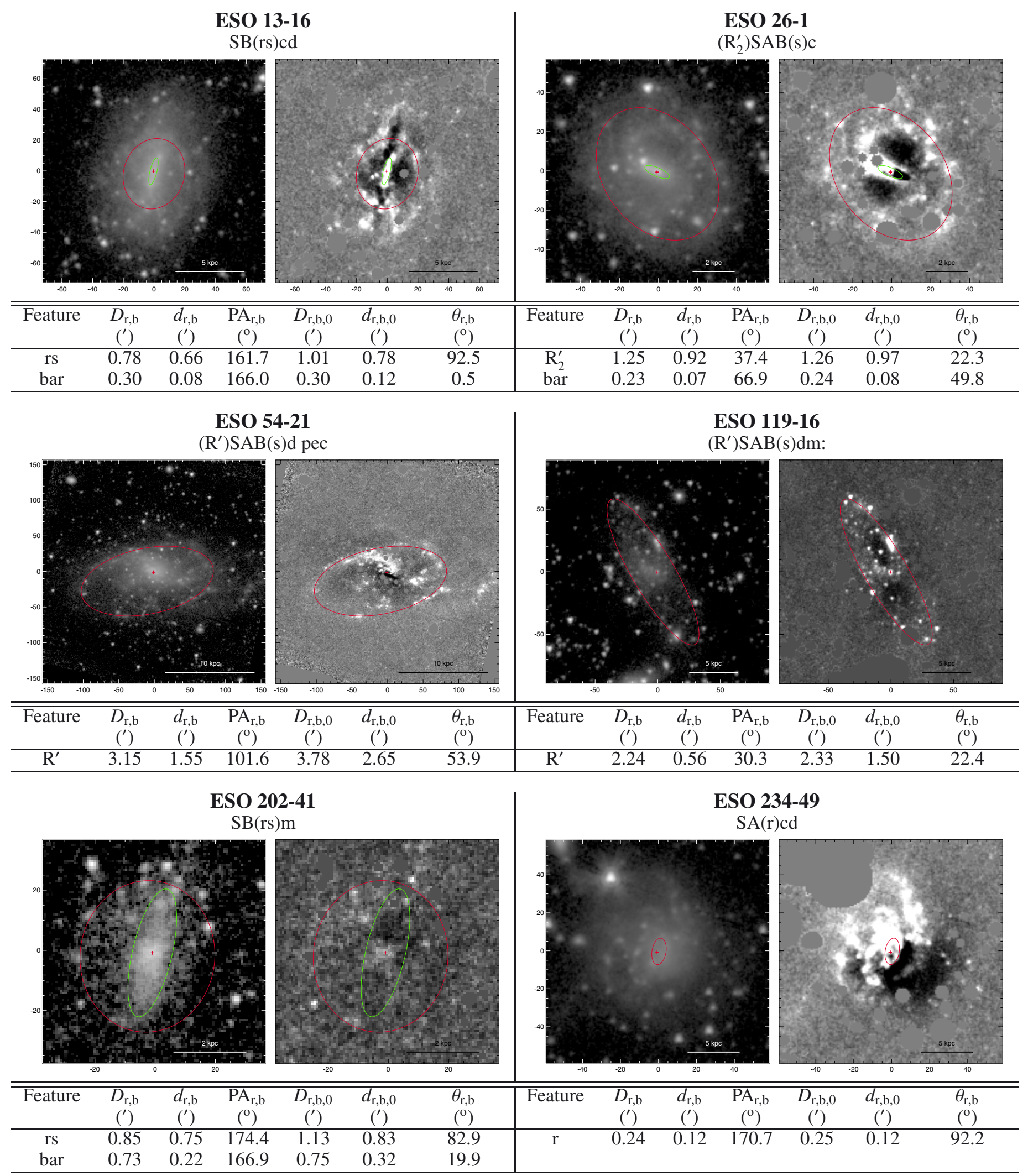




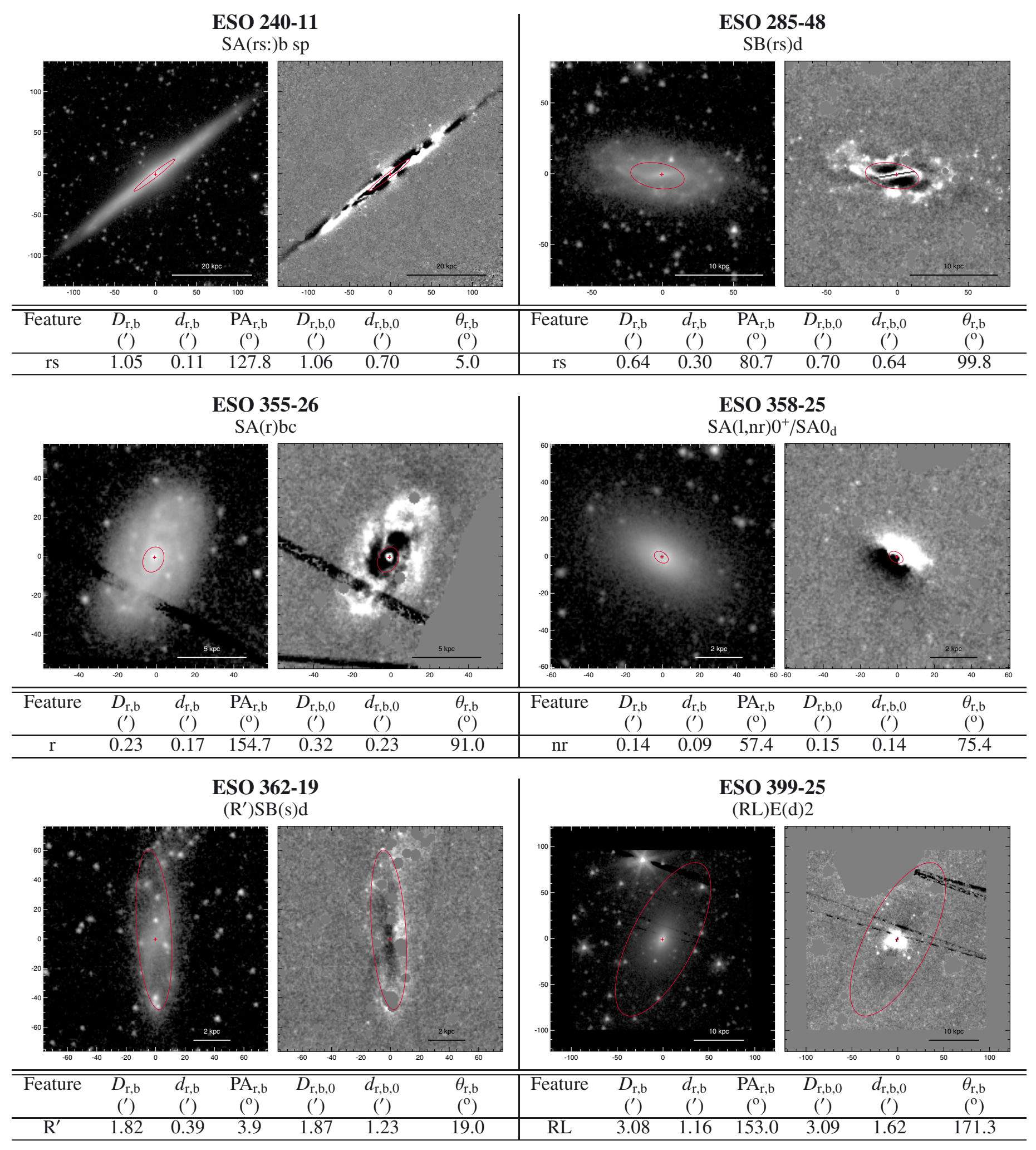


ESO 402-26

$\left(\mathrm{R}^{\prime}\right) \mathrm{SAB}(\mathrm{r}, \mathrm{nl}) \mathrm{ab}$
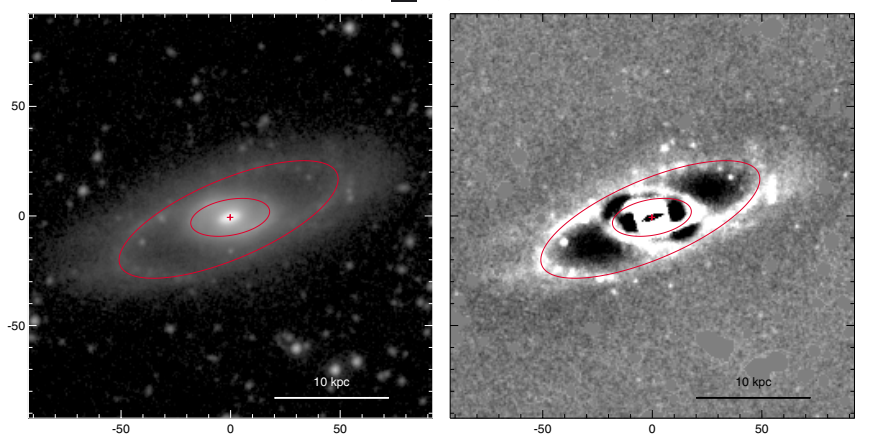

\begin{tabular}{|c|c|c|c|c|c|c|c|c|c|c|c|c|c|}
\hline Feature & $\begin{array}{c}D_{\mathrm{r}, \mathrm{b}} \\
\left({ }^{\prime}\right)\end{array}$ & $\begin{array}{c}d_{\mathrm{r}, \mathrm{b}} \\
\left({ }^{\prime}\right)\end{array}$ & $\begin{array}{c}\mathrm{PA}_{\mathrm{r}, \mathrm{b}} \\
\left({ }^{\circ}\right)\end{array}$ & $\begin{array}{c}D_{\mathrm{r}, \mathrm{b}, 0} \\
\left(^{\prime}\right)\end{array}$ & $\begin{array}{c}d_{\mathrm{r}, \mathrm{b}, 0} \\
\left(^{\prime}\right)\end{array}$ & $\begin{array}{l}\theta_{\mathrm{r}, \mathrm{b}} \\
\left({ }^{\circ}\right)\end{array}$ & Feature & $\begin{array}{c}D_{\mathrm{r}, \mathrm{b}} \\
\left({ }^{\prime}\right)\end{array}$ & $\begin{array}{c}d_{\mathrm{r}, \mathrm{b}} \\
\left({ }^{\prime}\right)\end{array}$ & $\begin{array}{c}\mathrm{PA}_{\mathrm{r}, \mathrm{b}} \\
\left(^{\circ}\right)\end{array}$ & $\begin{array}{c}D_{\mathrm{r}, \mathrm{b}, 0} \\
\left(^{\prime}\right)\end{array}$ & $\begin{array}{c}d_{\mathrm{r}, \mathrm{b}, 0} \\
\left(^{\prime}\right)\end{array}$ & $\begin{array}{l}\theta_{\mathrm{r}, \mathrm{b}} \\
\left({ }^{\circ}\right)\end{array}$ \\
\hline$\overline{\mathrm{R}^{\prime}}$ & 1.80 & 0.61 & 113.0 & 1.91 & 1.59 & 38.9 & rs & 0.18 & 0.07 & 134.4 & 0.18 & 0.12 & 171.4 \\
\hline$r$ & 0.61 & 0.27 & 100.4 & 0.83 & 0.56 & 111.4 & & & & & & & \\
\hline
\end{tabular}

ESO 404-12

$\mathrm{SAB}(\mathrm{rs}) \mathrm{b}$
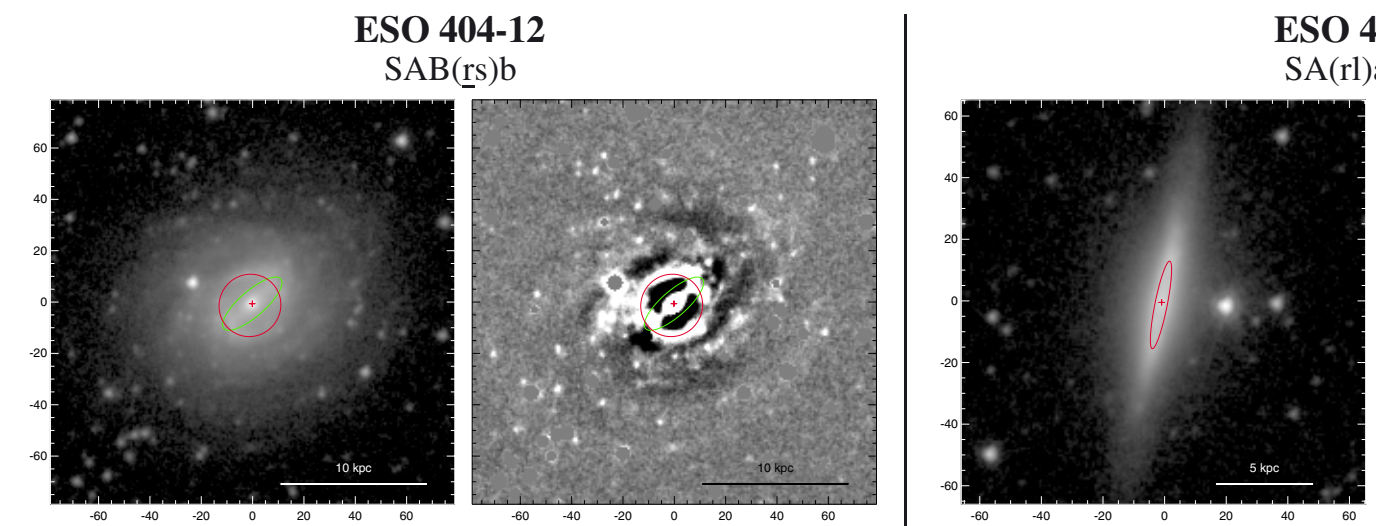

ESO 407-7

SA(rl)a: sp

\begin{tabular}{|c|c|c|c|c|c|c|c|c|c|c|c|c|c|}
\hline Feature & $\begin{array}{c}D_{\mathrm{r}, \mathrm{b}} \\
\left({ }^{\prime}\right)\end{array}$ & $\begin{array}{c}d_{\mathrm{r}, \mathrm{b}} \\
(')\end{array}$ & $\begin{array}{c}\mathrm{PA}_{\mathrm{r}, \mathrm{b}} \\
\left({ }^{\circ}\right)\end{array}$ & $\begin{array}{c}D_{\mathrm{r}, \mathrm{b}, 0} \\
\left(^{\prime}\right)\end{array}$ & $\begin{array}{c}d_{\mathrm{r}, \mathrm{b}, 0} \\
\left({ }^{\prime}\right)\end{array}$ & $\begin{array}{l}\theta_{\mathrm{r}, \mathrm{b}} \\
\left({ }^{\circ}\right)\end{array}$ & Feature & $\begin{array}{c}D_{\mathrm{r}, \mathrm{b}} \\
\left({ }^{\prime}\right)\end{array}$ & $\begin{array}{c}d_{\mathrm{r}, \mathrm{b}} \\
\left({ }^{\prime}\right)\end{array}$ & $\begin{array}{c}\mathrm{PA}_{\mathrm{r}, \mathrm{b}} \\
\left({ }^{\circ}\right)\end{array}$ & $\begin{array}{c}D_{\mathrm{r}, \mathrm{b}, 0} \\
\left(^{\prime}\right)\end{array}$ & $\begin{array}{c}d_{\mathrm{r}, \mathrm{b}, 0} \\
\left(^{\prime}\right)\end{array}$ & $\begin{array}{l}\theta_{\mathrm{r}, \mathrm{b}} \\
\left({ }^{\circ}\right)\end{array}$ \\
\hline bar & 0.49 & 0.17 & 131.1 & 0.50 & 0.19 & 15.1 & $\mathrm{rl}$ & 0.49 & 0.07 & 168.7 & 0.49 & 0.34 & 4.8 \\
\hline rs & 0.41 & 0.40 & 145.6 & 0.47 & 0.41 & 86.3 & & & & & & & \\
\hline
\end{tabular}

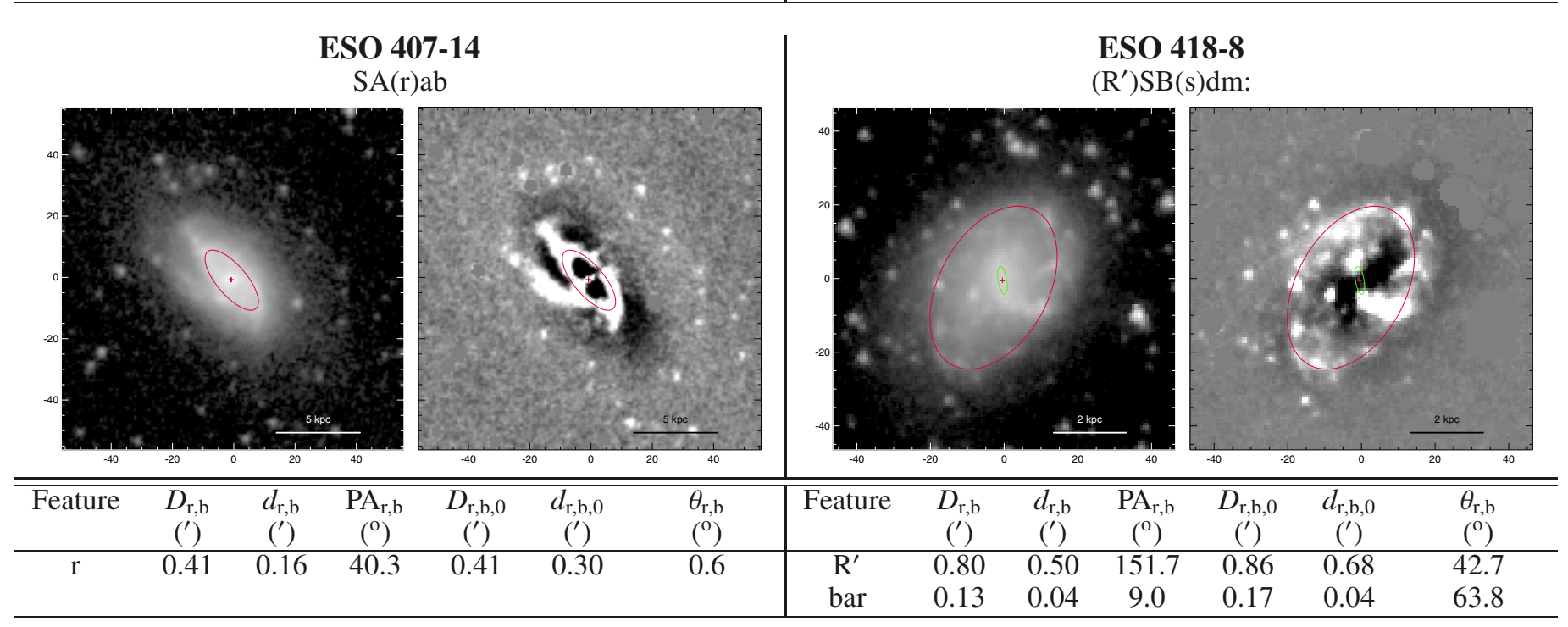


ESO 420-9

$\mathrm{SAB}(\mathrm{rs}) \mathrm{c}$
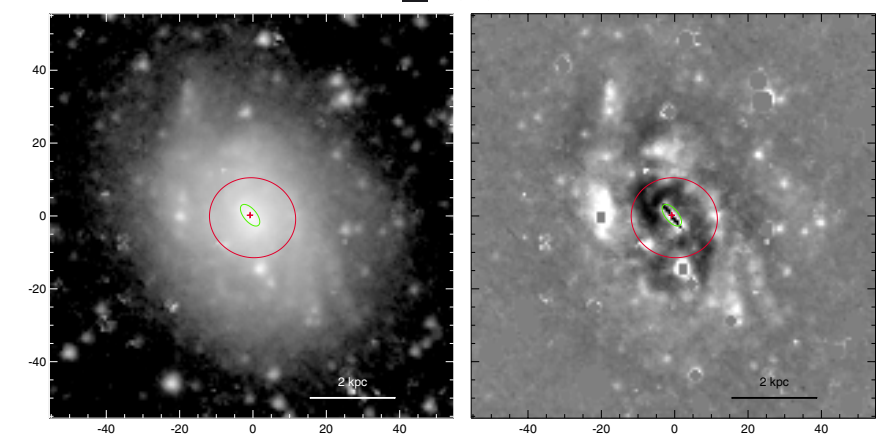

\begin{tabular}{|c|c|c|c|c|c|c|c|c|c|c|c|c|c|}
\hline Feature & $\begin{array}{c}D_{\mathrm{r}, \mathrm{b}} \\
\left(^{\prime}\right)\end{array}$ & $\begin{array}{c}d_{\mathrm{r}, \mathrm{b}} \\
\left(^{\prime}\right)\end{array}$ & $\begin{array}{c}\mathrm{PA}_{\mathrm{r}, \mathrm{b}} \\
\left({ }^{\circ}\right)\end{array}$ & $\begin{array}{c}D_{\mathrm{r}, \mathrm{b}, 0} \\
\left(^{\prime}\right)\end{array}$ & $\begin{array}{c}d_{\mathrm{r}, \mathrm{b}, 0} \\
\left(^{\prime}\right)\end{array}$ & $\begin{array}{c}\theta_{\mathrm{r}, \mathrm{b}} \\
\left({ }^{\circ}\right)\end{array}$ & Feature & $\begin{array}{c}D_{\mathrm{r}, \mathrm{b}} \\
\left({ }^{\prime}\right)\end{array}$ & $\begin{array}{c}d_{\mathrm{r}, \mathrm{b}} \\
\left(^{\prime}\right)\end{array}$ & $\begin{array}{c}\mathrm{PA}_{\mathrm{r}, \mathrm{b}} \\
\left({ }^{\circ}\right)\end{array}$ & $\begin{array}{c}D_{\mathrm{r}, \mathrm{b}, 0} \\
\left(^{\prime}\right)\end{array}$ & $\begin{array}{c}d_{\mathrm{r}, \mathrm{b}, 0} \\
\left({ }^{\prime}\right)\end{array}$ & $\begin{array}{c}\theta_{\mathrm{r}, \mathrm{b}} \\
\left({ }^{\circ}\right)\end{array}$ \\
\hline rs & 0.40 & 0.36 & 76.9 & 0.50 & 0.38 & 81.2 & rs & 0.47 & 0.40 & 51.0 & 0.58 & 0.44 & 74.9 \\
\hline bar & 0.12 & 0.06 & 39.8 & 0.12 & 0.08 & 13.0 & bār & 0.25 & 0.13 & 79.5 & 0.32 & 0.14 & 69.2 \\
\hline
\end{tabular}

ESO 422-41

$\left(\mathrm{R}^{\prime}\right) \mathrm{SA}(1) \mathrm{c}$ :
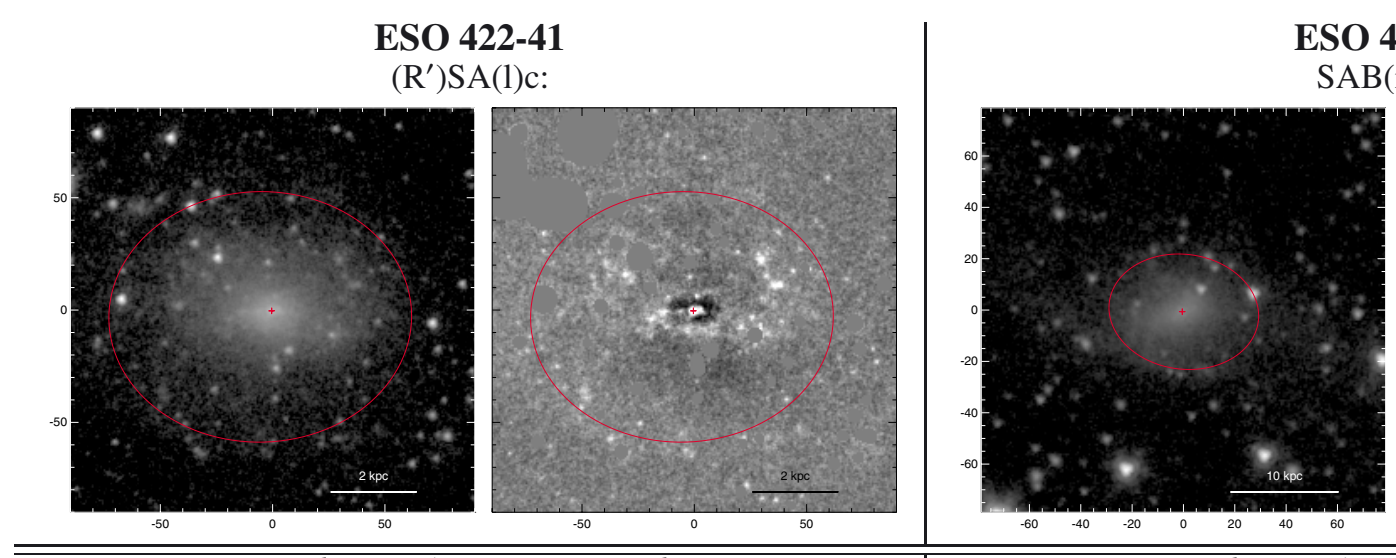

ESO 440-4

$\mathrm{SAB}(\mathrm{rl}) \mathrm{d}$

\begin{tabular}{|c|c|c|c|c|c|c|c|c|c|c|c|c|c|}
\hline Feature & $\begin{array}{c}D_{\mathrm{r}, \mathrm{b}} \\
\left({ }^{\prime}\right)\end{array}$ & $\begin{array}{c}d_{\mathrm{r}, \mathrm{b}} \\
\left(^{\prime}\right)\end{array}$ & $\begin{array}{c}\mathrm{PA}_{\mathrm{r}, \mathrm{b}} \\
\left({ }^{\circ}\right)\end{array}$ & $\begin{array}{c}D_{\mathrm{r}, \mathrm{b}, 0} \\
\left(^{\prime}\right)\end{array}$ & $\begin{array}{c}d_{\mathrm{r}, \mathrm{b}, 0} \\
\left({ }^{\prime}\right)\end{array}$ & $\begin{array}{l}\theta_{\mathrm{r}, \mathrm{b}} \\
\left({ }^{\circ}\right)\end{array}$ & Feature & $\begin{array}{c}D_{\mathrm{r}, \mathrm{b}} \\
\left({ }^{\prime}\right)\end{array}$ & $\begin{array}{c}d_{\mathrm{r}, \mathrm{b}} \\
\left(^{\prime}\right)\end{array}$ & $\begin{array}{c}\mathrm{PA}_{\mathrm{r}, \mathrm{b}} \\
\left({ }^{\circ}\right)\end{array}$ & $\begin{array}{c}D_{\mathrm{r}, \mathrm{b}, 0} \\
\left(^{\prime}\right)\end{array}$ & $\begin{array}{c}d_{\mathrm{r}, \mathrm{b}, 0} \\
{ }^{\prime}()\end{array}$ & $\begin{array}{c}\theta_{\mathrm{r}, \mathrm{b}} \\
\left({ }^{\circ}\right)\end{array}$ \\
\hline$\overline{\mathrm{R}^{\prime}}$ & 2.26 & 1.87 & 91.1 & 2.39 & 2.15 & 55.8 & $\mathrm{rl}$ & 0.98 & 0.75 & 82.4 & 1.80 & 0.98 & 87.7 \\
\hline
\end{tabular}

ESO 440-11

$\mathrm{SB}(\mathrm{rs}) \mathrm{cd}$
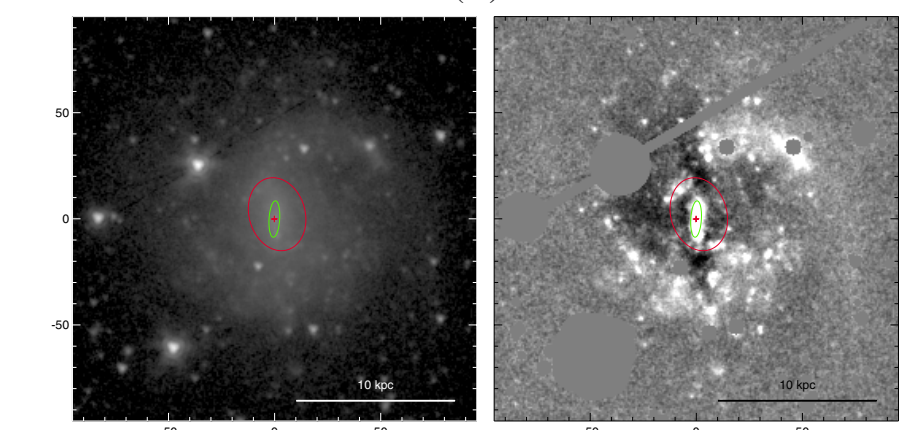

\begin{tabular}{|c|c|c|c|c|c|c|c|c|c|c|c|c|c|}
\hline Feature & $\begin{array}{c}D_{\mathrm{r}, \mathrm{b}} \\
\left(^{\prime}\right)\end{array}$ & $\begin{array}{c}d_{\mathrm{r}, \mathrm{b}} \\
\left({ }^{\prime}\right)\end{array}$ & $\begin{array}{c}\mathrm{PA}_{\mathrm{r}, \mathrm{b}} \\
\left({ }^{\circ}\right)\end{array}$ & 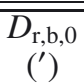 & $\begin{array}{c}d_{\mathrm{r}, \mathrm{b}, 0} \\
\left(^{\prime}\right)\end{array}$ & $\begin{array}{l}\theta_{\mathrm{r}, \mathrm{b}} \\
\left({ }^{\circ}\right)\end{array}$ & Feature & $\begin{array}{c}D_{\mathrm{r}, \mathrm{b}} \\
\left(^{\prime}\right)\end{array}$ & $\begin{array}{c}d_{\mathrm{r}, \mathrm{b}} \\
\left(^{\prime}\right)\end{array}$ & $\begin{array}{c}\mathrm{PA}_{\mathrm{r}, \mathrm{b}} \\
\left({ }^{\circ}\right)\end{array}$ & $\begin{array}{c}D_{\mathrm{r}, \mathrm{b}, 0} \\
\text { (') }^{\prime}\end{array}$ & $\begin{array}{c}d_{\mathrm{r}, \mathrm{b}, 0} \\
\left(^{\prime}\right)\end{array}$ & $\begin{array}{l}\theta_{\mathrm{r}, \mathrm{b}} \\
\left({ }^{\circ}\right)\end{array}$ \\
\hline rs & 0.58 & 0.44 & 15.9 & 0.59 & 0.48 & 154.5 & rs & 0.67 & 0.59 & 172.9 & 0.73 & 0.61 & 67.1 \\
\hline bar & 0.29 & 0.08 & 176.5 & 0.30 & 0.09 & 140.3 & bar & 0.52 & 0.18 & 174.0 & 0.56 & 0.19 & 53.2 \\
\hline
\end{tabular}

ESO 422-5

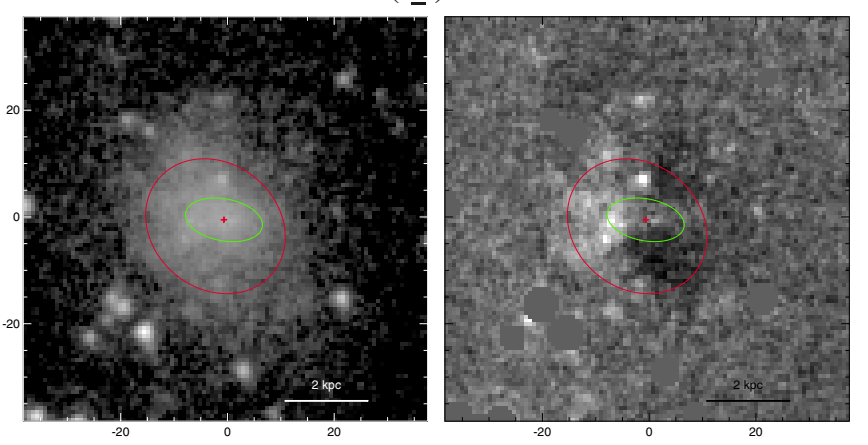

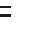


ESO 443-80

$\mathrm{Pec} / \mathrm{RG}$ ? or $\mathrm{SAB}(\mathrm{s}) \mathrm{m}$ ?
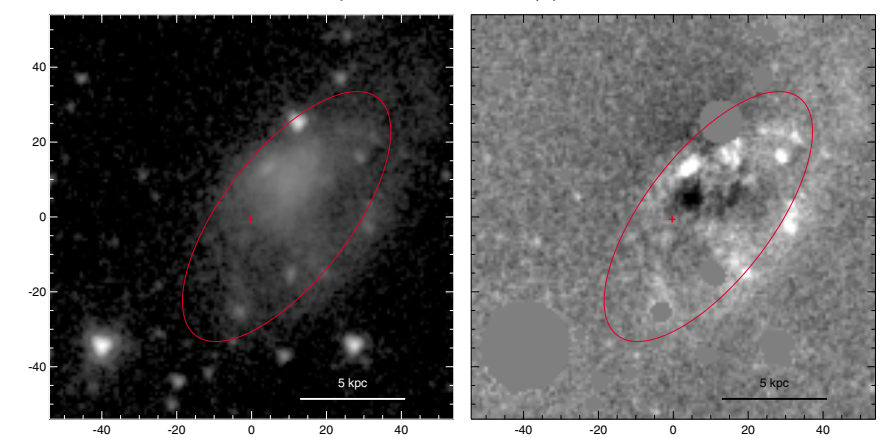

ESO 479-4

$\mathrm{SB}(\mathrm{rs}) \mathrm{d}$

\begin{tabular}{ccccccc|ccccccc}
\hline \hline Feature & $\begin{array}{c}D_{\mathrm{r}, \mathrm{b}} \\
\left({ }^{\prime}\right)\end{array}$ & $\begin{array}{c}d_{\mathrm{r}, \mathrm{b}} \\
\left({ }^{\prime}\right)\end{array}$ & $\begin{array}{c}\mathrm{PA}_{\mathrm{r}, \mathrm{b}} \\
\left({ }^{\circ}\right)\end{array}$ & $\begin{array}{c}D_{\mathrm{r}, \mathrm{b}, 0} \\
\left({ }^{\prime}\right)\end{array}$ & $\begin{array}{c}d_{\mathrm{r}, \mathrm{b}, 0} \\
\left({ }^{\prime}\right)\end{array}$ & $\begin{array}{c}\theta_{\mathrm{r}, \mathrm{b}} \\
\left({ }^{\circ}\right)\end{array}$ & Feature & $\begin{array}{c}D_{\mathrm{r}, \mathrm{b}} \\
\left({ }^{\prime}\right)\end{array}$ & $\begin{array}{c}d_{\mathrm{r}, \mathrm{b}} \\
\left({ }^{\prime}\right)\end{array}$ & $\begin{array}{c}\mathrm{PA}_{\mathrm{r}, \mathrm{b}} \\
\left({ }^{\circ}\right)\end{array}$ & $\begin{array}{c}D_{\mathrm{r}, \mathrm{b}, 0} \\
\left({ }^{\prime}\right)\end{array}$ & $\begin{array}{c}d_{\mathrm{r}, \mathrm{b}, 0} \\
\left({ }^{\prime}\right)\end{array}$ & $\begin{array}{c}\theta_{\mathrm{r}, \mathrm{b}} \\
\left({ }^{\circ}\right)\end{array}$ \\
\hline $\mathrm{RG}$ & 1.34 & 0.57 & 142.5 & 1.37 & 0.79 & 162.1 & $\mathrm{rs}$ & 0.84 & 0.49 & 59.3 & 0.92 & 0.83 & 72.1 \\
& & & & & & & $\mathrm{bar}$ & 0.20 & 0.06 & 37.4 & 0.23 & 0.09 \\
\hline
\end{tabular}

ESO 482-35

$\mathrm{SB}(\mathrm{rs}) \mathrm{b}$

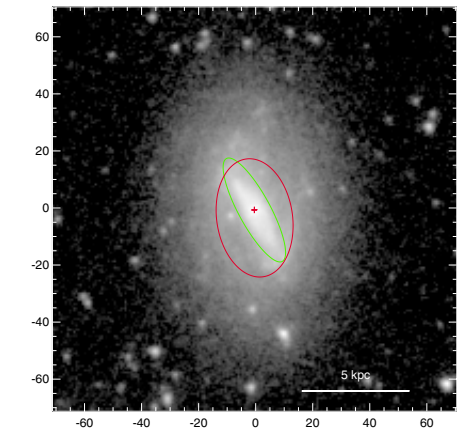

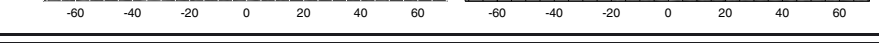

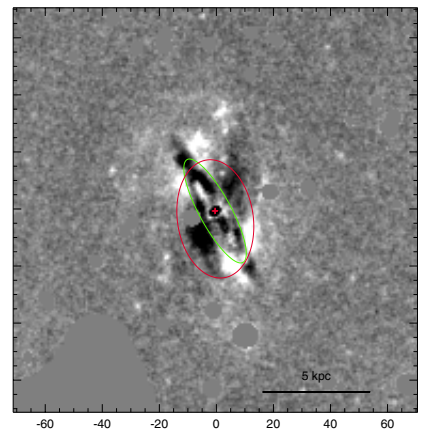

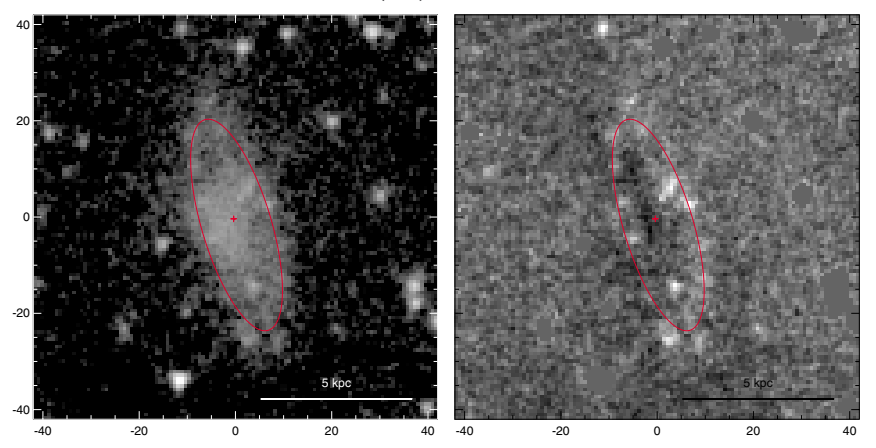

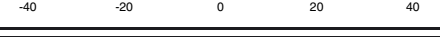

\section{ESO 507-65
$\left(\mathrm{R}^{\prime}\right) \mathrm{Sm} ?$ \\ ESO 507-65
$\left(\mathrm{R}^{\prime}\right) \mathrm{Sm} ?$}

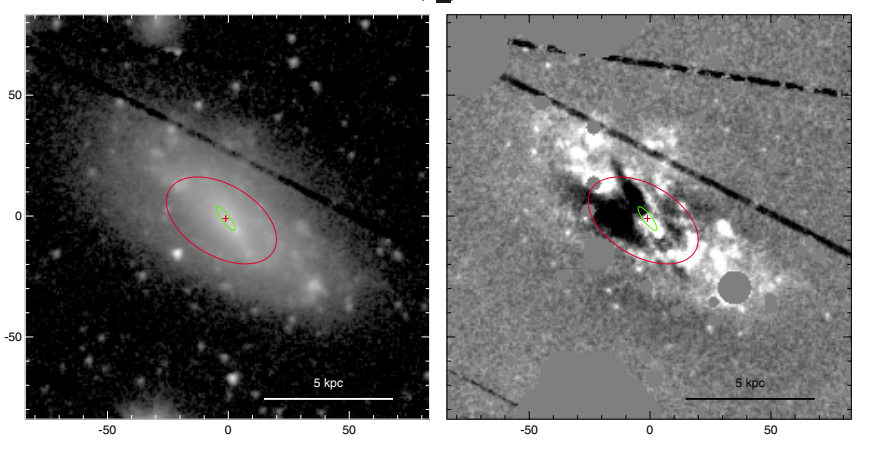

\begin{tabular}{|c|c|c|c|c|c|c|c|c|c|c|c|c|c|}
\hline Feature & $\begin{array}{c}D_{\mathrm{r}, \mathrm{b}} \\
\left({ }^{\prime}\right)\end{array}$ & $\begin{array}{c}d_{\mathrm{r}, \mathrm{b}} \\
\left(^{\prime}\right)\end{array}$ & $\begin{array}{c}\mathrm{PA}_{\mathrm{r}, \mathrm{b}} \\
\left({ }^{\circ}\right)\end{array}$ & $\begin{array}{c}D_{\mathrm{r}, \mathrm{b}, 0} \\
\text { (') }^{\prime}\end{array}$ & $\begin{array}{c}d_{\mathrm{r}, \mathrm{b}, 0} \\
\left({ }^{\prime}\right)\end{array}$ & $\begin{array}{l}\theta_{\mathrm{r}, \mathrm{b}} \\
\left({ }^{\circ}\right)\end{array}$ & Feature & $\begin{array}{c}D_{\mathrm{r}, \mathrm{b}} \\
\left({ }^{\prime}\right)\end{array}$ & $\begin{array}{c}d_{\mathrm{r}, \mathrm{b}} \\
\left({ }^{\prime}\right)\end{array}$ & $\begin{array}{c}\mathrm{PA}_{\mathrm{r}, \mathrm{b}} \\
\left({ }^{\circ}\right)\end{array}$ & $\begin{array}{c}D_{\mathrm{r}, \mathrm{b}, 0} \\
\left(^{\prime}\right)\end{array}$ & $\begin{array}{c}d_{\mathrm{r}, \mathrm{b}, 0} \\
\left({ }^{\prime}\right)\end{array}$ & $\begin{array}{c}\theta_{\mathrm{r}, \mathrm{b}} \\
\left({ }^{\circ}\right)\end{array}$ \\
\hline rs & 0.70 & 0.45 & 8.3 & 0.75 & 0.66 & 50.4 & $\overline{\mathrm{R}^{\prime}}$ & 0.77 & 0.24 & 16.8 & 0.77 & 0.56 & 3.4 \\
\hline bar & 0.69 & 0.19 & 28.5 & 0.80 & 0.25 & 42.3 & & & & & & & \\
\hline
\end{tabular}

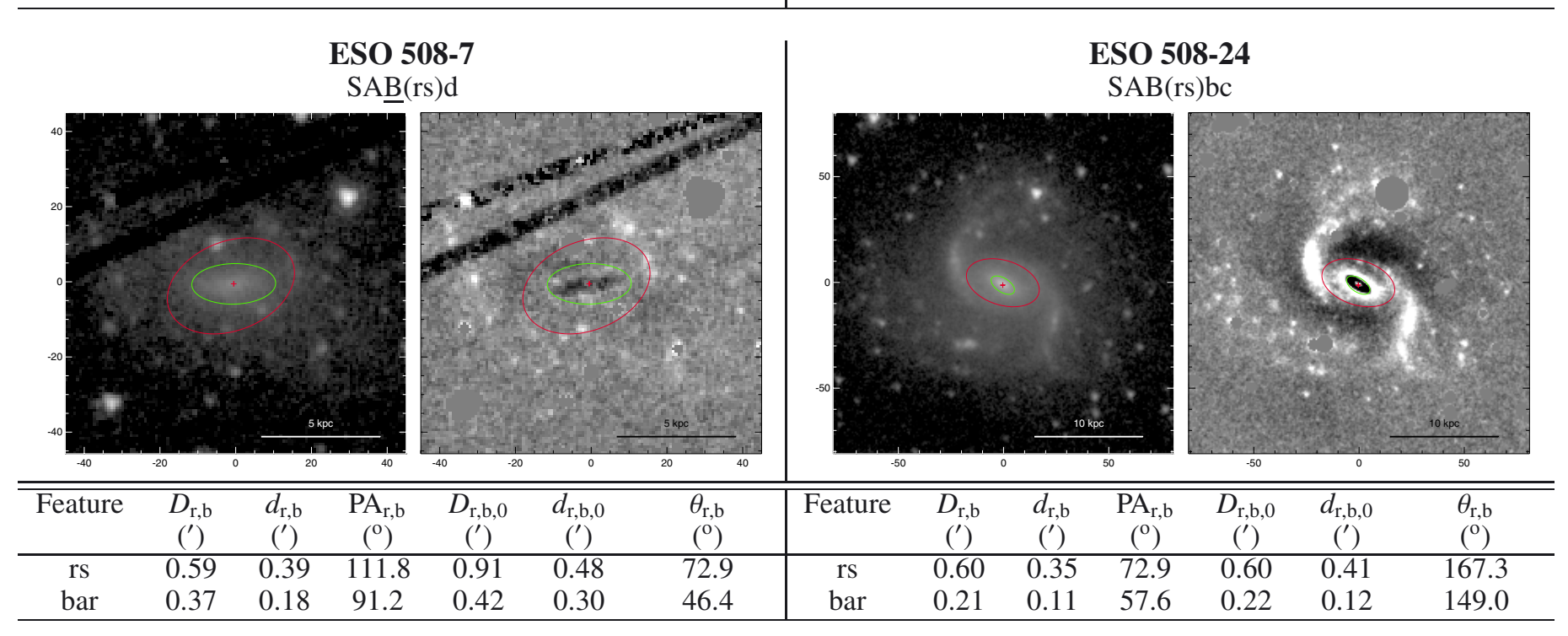


ESO 509-74

$\mathrm{S}$ (nd or nr)bc sp
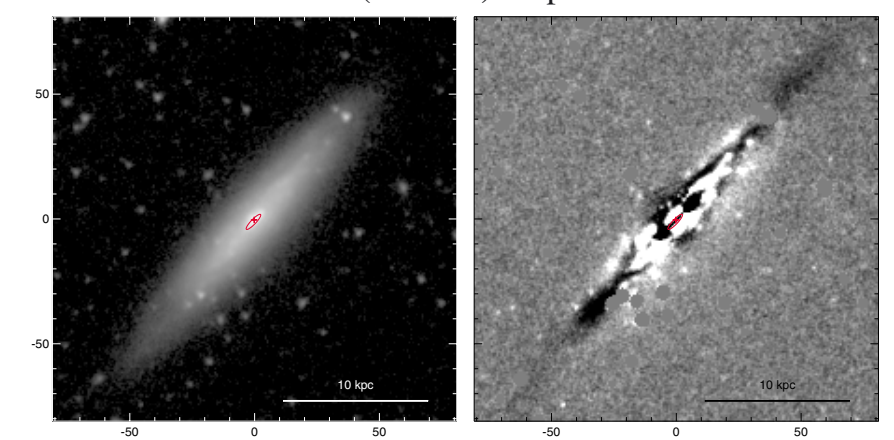

\begin{tabular}{|c|c|c|c|c|c|c|c|c|c|c|c|c|c|}
\hline Feature & $\begin{array}{c}D_{\mathrm{r}, \mathrm{b}} \\
\left({ }^{\prime}\right)\end{array}$ & $\begin{array}{c}d_{\mathrm{r}, \mathrm{b}} \\
\left({ }^{\prime}\right)\end{array}$ & $\begin{array}{c}\mathrm{PA}_{\mathrm{r}, \mathrm{b}} \\
\left({ }^{\mathrm{O}}\right)\end{array}$ & $\begin{array}{c}D_{\mathrm{r}, \mathrm{b}, 0} \\
\left(^{\prime}\right)\end{array}$ & $\begin{array}{c}d_{\mathrm{r}, \mathrm{b}, 0} \\
\left({ }^{\prime}\right)\end{array}$ & $\begin{array}{l}\theta_{\mathrm{r}, \mathrm{b}} \\
\left({ }^{\circ}\right)\end{array}$ & Feature & $\begin{array}{c}D_{\mathrm{r}, \mathrm{b}} \\
\left({ }^{\prime}\right)\end{array}$ & $\begin{array}{c}d_{\mathrm{r}, \mathrm{b}} \\
\left({ }^{\prime}\right)\end{array}$ & $\begin{array}{c}\mathrm{PA}_{\mathrm{r}, \mathrm{b}} \\
\left({ }^{\mathrm{O}}\right)\end{array}$ & $\begin{array}{c}D_{\mathrm{r}, \mathrm{b}, 0} \\
\left(^{\prime}\right)\end{array}$ & $\begin{array}{c}d_{\mathrm{r}, \mathrm{b}, 0} \\
{ }^{\prime}\left({ }^{\prime}\right)\end{array}$ & $\begin{array}{l}\theta_{\mathrm{r}, \mathrm{b}} \\
\left({ }^{\circ}\right)\end{array}$ \\
\hline $\mathrm{nr}$ & 0.14 & 0.03 & 136.2 & 0.14 & 0.12 & 151.3 & $\begin{array}{l}\mathrm{R}^{\prime} \\
\text { bar }\end{array}$ & $\begin{array}{l}0.79 \\
0.35\end{array}$ & $\begin{array}{l}0.48 \\
0.23\end{array}$ & $\begin{array}{c}5.9 \\
161.1\end{array}$ & $\begin{array}{l}0.82 \\
0.38\end{array}$ & $\begin{array}{l}0.69 \\
0.31\end{array}$ & $\begin{array}{c}36.4 \\
128.9\end{array}$ \\
\hline
\end{tabular}

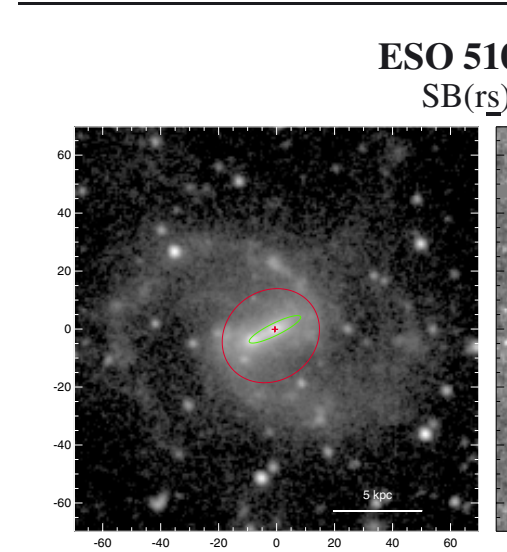

$\mathrm{SB}(\mathrm{rs}) \mathrm{c}$

\begin{tabular}{|c|c|c|c|c|c|c|c|c|c|c|c|c|c|}
\hline Feature & $\begin{array}{c}D_{\mathrm{r}, \mathrm{b}} \\
\left(^{\prime}\right)\end{array}$ & $\begin{array}{c}d_{\mathrm{r}, \mathrm{b}} \\
\left(^{\prime}\right)\end{array}$ & $\begin{array}{c}\mathrm{PA}_{\mathrm{r}, \mathrm{b}} \\
\left({ }^{\circ}\right)\end{array}$ & $\begin{array}{c}D_{\mathrm{r}, \mathrm{b}, 0} \\
\left(^{\prime}\right)\end{array}$ & $\begin{array}{c}d_{\mathrm{r}, \mathrm{b}, 0} \\
\left({ }^{\prime}\right)\end{array}$ & $\begin{array}{c}\theta_{\mathrm{r}, \mathrm{b}} \\
\left({ }^{\circ}\right)\end{array}$ & Feature & $\begin{array}{c}D_{\mathrm{r}, \mathrm{b}} \\
\left(^{\prime}\right)\end{array}$ & $\begin{array}{c}d_{\mathrm{r}, \mathrm{b}} \\
\left(^{\prime}\right)\end{array}$ & $\begin{array}{c}\mathrm{PA}_{\mathrm{r}, \mathrm{b}} \\
\left({ }^{\circ}\right)\end{array}$ & $\begin{array}{c}D_{\mathrm{r}, \mathrm{b}, 0} \\
\left(^{\prime}\right)\end{array}$ & $\begin{array}{c}d_{\mathrm{r}, \mathrm{b}, 0} \\
\left(^{\prime}\right)\end{array}$ & $\begin{array}{c}\theta_{\mathrm{r}, \mathrm{b}} \\
\left(^{\circ}\right)\end{array}$ \\
\hline rs & 0.59 & 0.51 & 127.8 & 0.89 & 0.54 & 82.3 & rs & 0.51 & 0.42 & 0.5 & 0.71 & 0.46 & 77.6 \\
\hline bār & 0.33 & 0.07 & 116.2 & 0.41 & 0.09 & 49.8 & bār & 0.45 & 0.13 & 17.3 & 0.63 & 0.13 & 70.6 \\
\hline
\end{tabular}

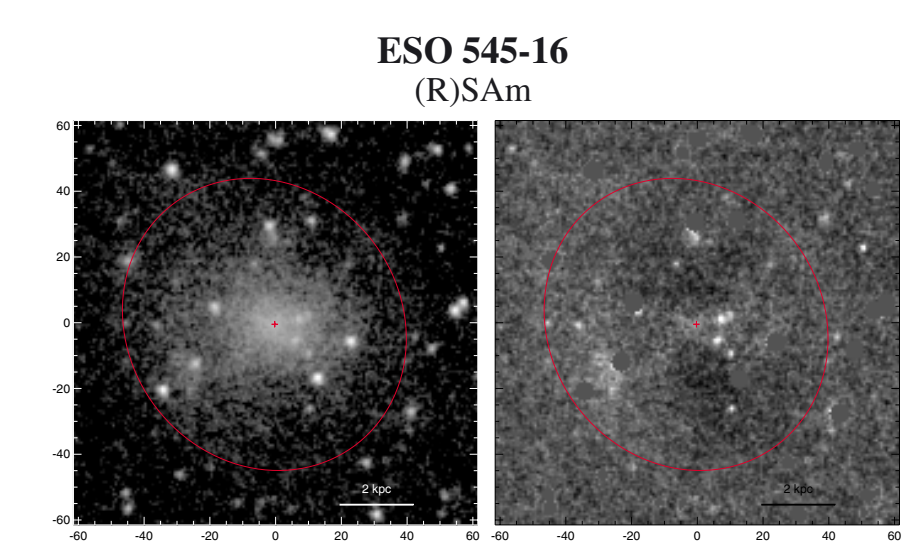

\section{ESO 532-22 \\ $\mathrm{SB}(\mathrm{rs}) \mathrm{d}$}
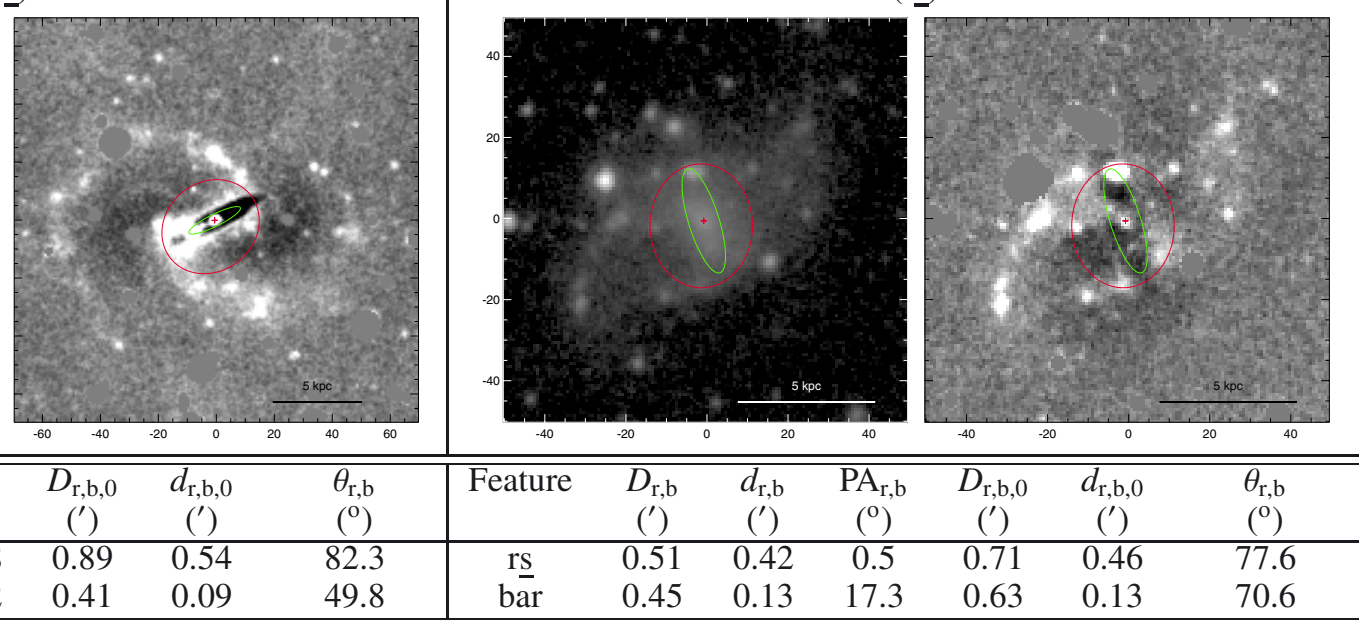

$\left(\mathrm{R}^{\prime}\right) \mathrm{SAB}(\mathrm{s}, \mathrm{nb}) \mathrm{m}$ or $\mathrm{RG}$ ?

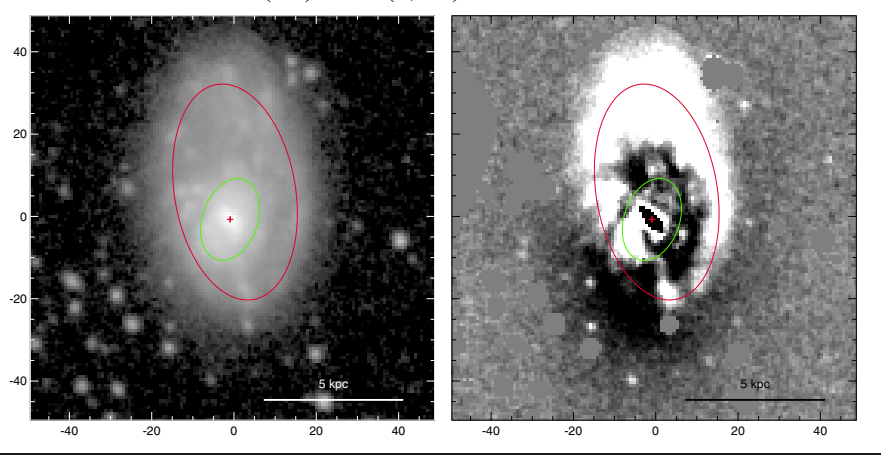

\section{.}


ESO 548-5

$\mathrm{SAB}(\mathrm{rs}) \mathrm{dm}$
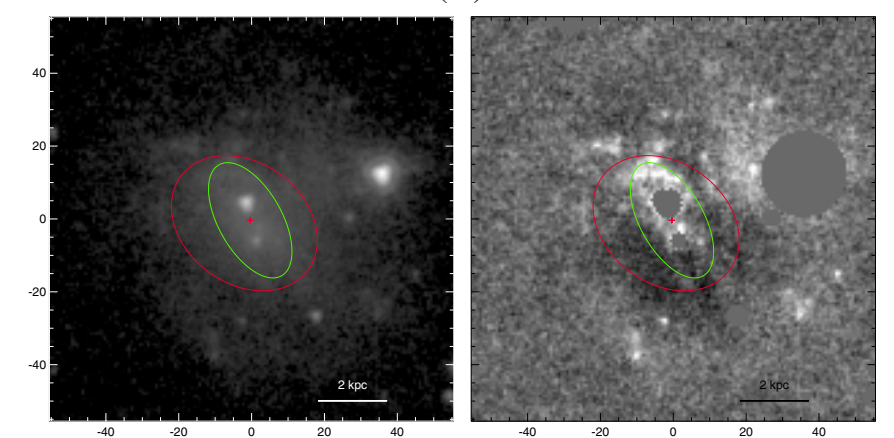

\begin{tabular}{|c|c|c|c|c|c|c|c|c|c|c|c|c|c|}
\hline Feature & $\begin{array}{c}D_{\mathrm{r}, \mathrm{b}} \\
\left({ }^{\prime}\right)\end{array}$ & $\begin{array}{c}d_{\mathrm{r}, \mathrm{b}} \\
\left({ }^{\prime}\right)\end{array}$ & $\begin{array}{c}\overline{\mathrm{PA}_{\mathrm{r}, \mathrm{b}}} \\
\left({ }^{\circ}\right)\end{array}$ & $\begin{array}{c}D_{\mathrm{r}, \mathrm{b}, 0} \\
\left({ }^{\prime}\right)\end{array}$ & $\begin{array}{c}d_{\mathrm{r}, \mathrm{b}, 0} \\
\left(^{\prime}\right)\end{array}$ & $\begin{array}{l}\theta_{\mathrm{r}, \mathrm{b}} \\
\left({ }^{\circ}\right)\end{array}$ & Feature & $\begin{array}{c}D_{\mathrm{r}, \mathrm{b}} \\
\left({ }^{\prime}\right)\end{array}$ & $\begin{array}{c}d_{\mathrm{r}, \mathrm{b}} \\
\left({ }^{\prime}\right)\end{array}$ & $\begin{array}{c}\mathrm{PA}_{\mathrm{r}, \mathrm{b}} \\
\left({ }^{\circ}\right)\end{array}$ & $\begin{array}{c}D_{\mathrm{r}, \mathrm{b}, 0} \\
\left({ }^{\prime}\right)\end{array}$ & $\begin{array}{c}d_{\mathrm{r}, \mathrm{b}, 0} \\
\left({ }^{\prime}\right)\end{array}$ & $\begin{array}{c}\theta_{\mathrm{r}, \mathrm{b}} \\
\left({ }^{\circ}\right)\end{array}$ \\
\hline rs & 0.73 & 0.56 & 53.7 & 0.73 & 0.65 & 19.9 & RL & 0.66 & 0.35 & 24.3 & 0.68 & 0.66 & 70.0 \\
\hline bar & 0.59 & 0.29 & 29.4 & 0.60 & 0.34 & 159.1 & & & & & & & \\
\hline
\end{tabular}

ESO 548-25

(R'L)SB(s)a/d
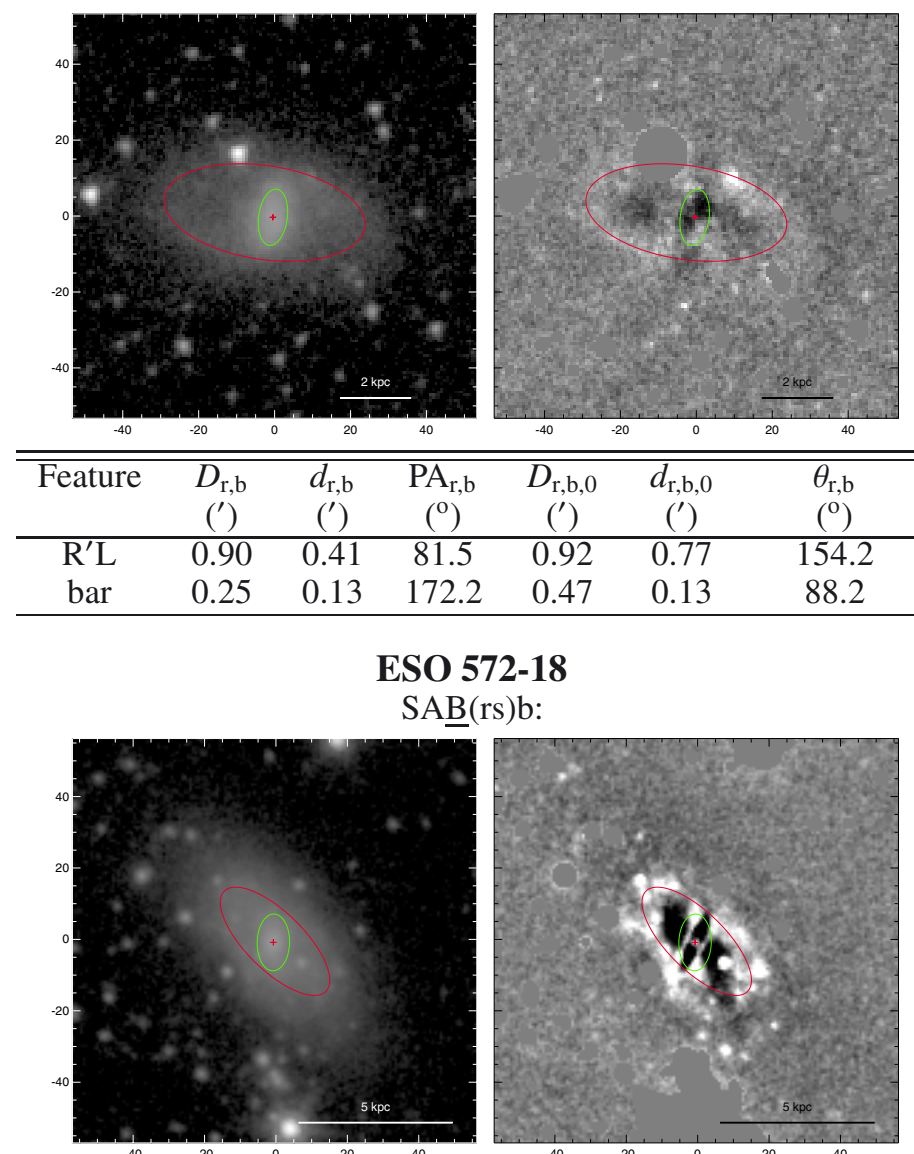

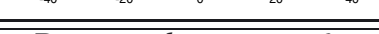

$\begin{array}{cc}D_{\mathrm{r}, \mathrm{b}, 0} & d_{\mathrm{r}, \mathrm{b}, 0} \\ \left({ }^{\prime}\right) & \left({ }^{\prime}\right)\end{array}$

$\theta_{\mathrm{r}, \mathrm{b}}$
$\left({ }^{\mathrm{o}}\right)$

$0.47 \quad 0.13$
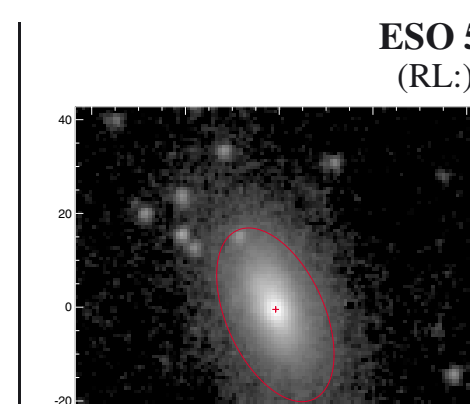

SO 548-23

(RL:)SA0 $0^{-}$

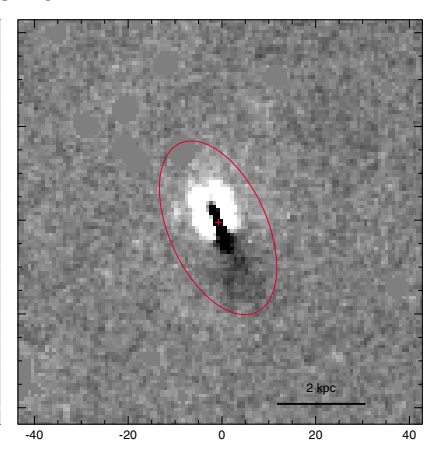

ESO 549-18

$\left(\mathrm{L}, \mathrm{R}_{2}^{\prime}\right) \mathrm{SAB}(\mathrm{s}) \mathrm{a} / \mathrm{c}$

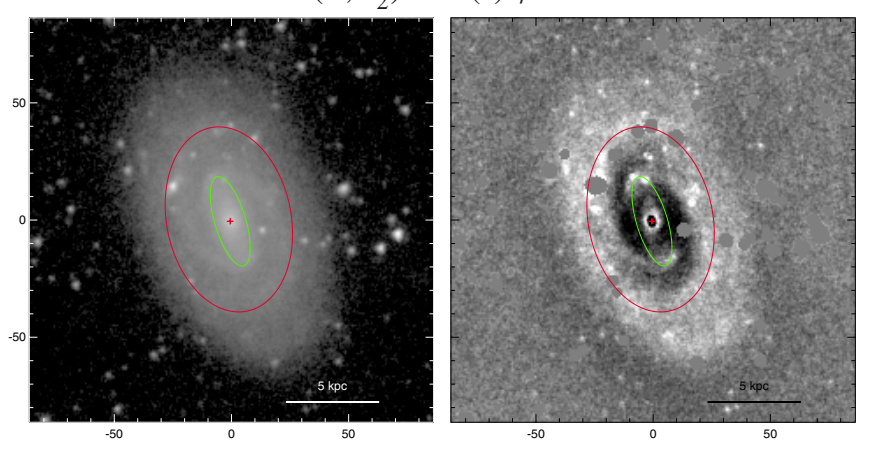

$\begin{array}{lllllll}\text { Feature } & D_{\mathrm{r}, \mathrm{b}} & d_{\mathrm{r}, \mathrm{b}} & \mathrm{PA}_{\mathrm{r}, \mathrm{b}} & D_{\mathrm{r}, \mathrm{b}, 0} & d_{\mathrm{r}, \mathrm{b}, 0} & \theta_{\mathrm{r}, \mathrm{b}}\end{array}$

\begin{tabular}{ccccccc} 
& $\left({ }^{\prime}\right)$ & $\left({ }^{\prime}\right)$ & $\left(^{\circ}\right)$ & $\left(^{\prime}\right)$ & $\left(^{\prime}\right)$ & $\left(^{\circ}\right)$ \\
\hline $\mathrm{R}_{2}^{\prime}$ & 1.34 & 0.89 & 11.2 & 1.51 & 1.29 & 112.9 \\
bar & 0.66 & 0.22 & 16.8 & 0.66 & 0.36 & 175.2 \\
\hline
\end{tabular}

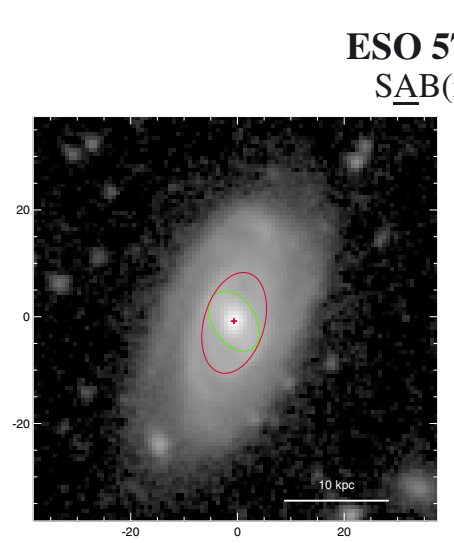

ESO 576-1

$\mathrm{S}$ AB $(r) \mathrm{a}$

\begin{tabular}{ccccccc|ccccccc}
\hline \hline Feature & $\begin{array}{c}D_{\mathrm{r}, \mathrm{b}} \\
\left({ }^{\prime}\right)\end{array}$ & $\begin{array}{c}d_{\mathrm{r}, \mathrm{b}} \\
\left({ }^{\prime}\right)\end{array}$ & $\begin{array}{c}\mathrm{PA}_{\mathrm{r}, \mathrm{b}} \\
\left({ }^{\circ}\right)\end{array}$ & $\begin{array}{c}D_{\mathrm{r}, \mathrm{b}, 0} \\
\left({ }^{\prime}\right)\end{array}$ & $\begin{array}{c}d_{\mathrm{r}, \mathrm{b}, 0} \\
\left({ }^{\prime}\right)\end{array}$ & $\begin{array}{c}\theta_{\mathrm{r}, \mathrm{b}} \\
\left({ }^{\circ}\right)\end{array}$ & Feature & $\begin{array}{c}D_{\mathrm{r}, \mathrm{b}} \\
\left({ }^{\prime}\right)\end{array}$ & $\begin{array}{c}d_{\mathrm{r}, \mathrm{b}} \\
\left({ }^{\prime}\right)\end{array}$ & $\begin{array}{c}\mathrm{PA}_{\mathrm{r}, \mathrm{b}} \\
\left({ }^{\circ}\right)\end{array}$ & $\begin{array}{c}D_{\mathrm{r}, \mathrm{b}, 0} \\
\left({ }^{\prime}\right)\end{array}$ & $\begin{array}{c}d_{\mathrm{r}, \mathrm{b}, 0} \\
\left({ }^{\prime}\right)\end{array}$ & $\begin{array}{c}\theta_{\mathrm{r}, \mathrm{b}} \\
\left({ }^{\circ}\right)\end{array}$ \\
\hline rs & 0.67 & 0.27 & 45.4 & 0.70 & 0.52 & 27.4 & $\mathrm{r}$ & 0.33 & 0.19 & 164.1 & 0.39 & 0.31 & 68.2 \\
$\mathrm{bar}$ & 0.27 & 0.15 & 178.7 & 0.43 & 0.19 & 109.4 & $\mathrm{bar}$ & 0.21 & 0.14 & 32.7 & 0.38 & 0.15 & 79.2 \\
\hline
\end{tabular}


ESO 576-3

$\mathrm{SB}(\mathrm{rs}) \mathrm{dm}$
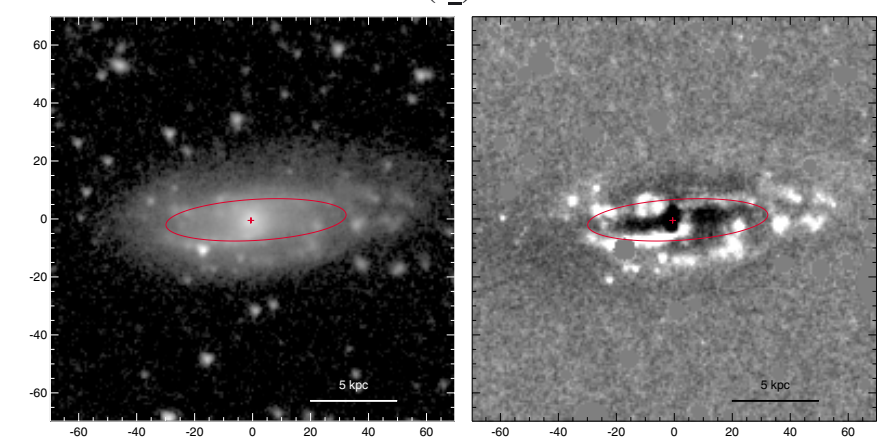

\begin{tabular}{|c|c|c|c|c|c|c|c|c|c|c|c|c|c|}
\hline Feature & $\begin{array}{c}D_{\mathrm{r}, \mathrm{b}} \\
\left({ }^{\prime}\right)\end{array}$ & $\begin{array}{c}d_{\mathrm{r}, \mathrm{b}} \\
\left({ }^{\prime}\right)\end{array}$ & $\begin{array}{c}\mathrm{PA}_{\mathrm{r}, \mathrm{b}} \\
\left({ }^{\circ}\right)\end{array}$ & $\begin{array}{c}D_{\mathrm{r}, \mathrm{b}, 0} \\
\left({ }^{\prime}\right)\end{array}$ & $\begin{array}{c}d_{\mathrm{r}, \mathrm{b}, 0} \\
\left({ }^{\prime}\right)\end{array}$ & $\begin{array}{c}\theta_{\mathrm{r}, \mathrm{b}} \\
\left({ }^{\circ}\right)\end{array}$ & Feature & $\begin{array}{c}D_{\mathrm{r}, \mathrm{b}} \\
\left({ }^{\prime}\right)\end{array}$ & $\begin{array}{c}d_{\mathrm{r}, \mathrm{b}} \\
\left({ }^{\prime}\right)\end{array}$ & $\begin{array}{c}\mathrm{PA}_{\mathrm{r}, \mathrm{b}} \\
\left({ }^{\circ}\right)\end{array}$ & $\begin{array}{c}D_{\mathrm{r}, \mathrm{b}, 0} \\
\left(^{\prime}\right)\end{array}$ & $\begin{array}{c}d_{\mathrm{r}, \mathrm{b}, 0} \\
\left.\text { (') }^{\prime}\right)\end{array}$ & $\begin{array}{l}\theta_{\mathrm{r}, \mathrm{b}} \\
\left({ }^{\circ}\right) \\
\end{array}$ \\
\hline ris & 1.04 & 0.24 & 93.4 & 1.05 & 0.61 & 169.0 & $\begin{array}{c}\text { bar } \\
\text { rs }\end{array}$ & $\begin{array}{l}0.69 \\
0.67\end{array}$ & $\begin{array}{l}0.17 \\
0.46\end{array}$ & $\begin{array}{l}153.2 \\
131.6\end{array}$ & $\begin{array}{l}0.73 \\
0.68\end{array}$ & $\begin{array}{l}0.23 \\
0.65\end{array}$ & $\begin{array}{c}26.7 \\
149.0\end{array}$ \\
\hline
\end{tabular}

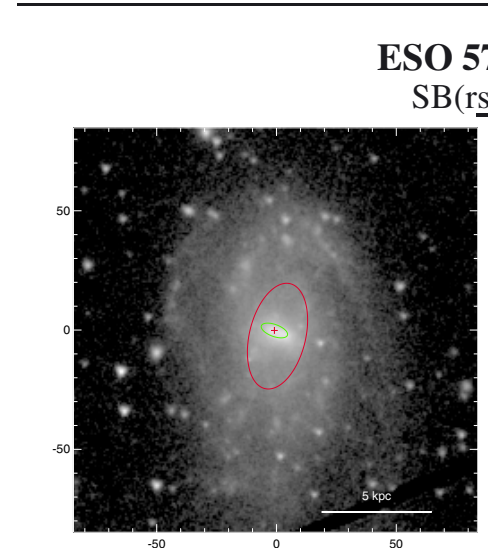

SO 576-50

$\mathrm{SB}(\mathrm{rs}) \mathrm{cd}$

\begin{tabular}{|c|c|c|c|c|c|c|c|c|c|c|c|c|c|}
\hline Feature & $\begin{array}{c}D_{\mathrm{r}, \mathrm{b}} \\
\left({ }^{\prime}\right)\end{array}$ & $\begin{array}{c}d_{\mathrm{r}, \mathrm{b}} \\
\left({ }^{\prime}\right)\end{array}$ & $\begin{array}{c}\mathrm{PA}_{\mathrm{r}, \mathrm{b}} \\
\left({ }^{\circ}\right)\end{array}$ & $\begin{array}{c}\overline{D_{\mathrm{r}, \mathrm{b}, 0}, 0} \\
\left({ }^{\prime}\right)\end{array}$ & $\begin{array}{c}d_{\mathrm{r}, \mathrm{b}, 0} \\
\left(^{\prime}\right)\end{array}$ & $\begin{array}{c}\theta_{\mathrm{r}, \mathrm{b}} \\
\left({ }^{\circ}\right)\end{array}$ & Feature & $\begin{array}{c}D_{\mathrm{r}, \mathrm{b}} \\
(')\end{array}$ & $\begin{array}{c}d_{\mathrm{r}, \mathrm{b}} \\
\left({ }^{\prime}\right)\end{array}$ & $\begin{array}{c}\mathrm{PA}_{\mathrm{r}, \mathrm{b}} \\
\left({ }^{\mathrm{o}}\right)\end{array}$ & $\begin{array}{c}D_{\mathrm{r}, \mathrm{b}, 0} \\
\left(^{\prime}\right)\end{array}$ & $\begin{array}{c}d_{\mathrm{r}, \mathrm{b}, 0}, 0 \\
\left({ }^{\prime}\right)\end{array}$ & $\begin{array}{c}\theta_{\mathrm{r}, \mathrm{b}} \\
\left({ }^{\circ}\right)\end{array}$ \\
\hline$\underline{\mathrm{rs}}$ & 0.75 & 0.39 & 166.4 & 0.84 & 0.56 & 137.4 & rs & 0.76 & 0.09 & 71.6 & 0.77 & 0.49 & 168.2 \\
\hline bär & 0.19 & 0.08 & 70.0 & 0.29 & 0.09 & 75.6 & & & & & & & \\
\hline
\end{tabular}
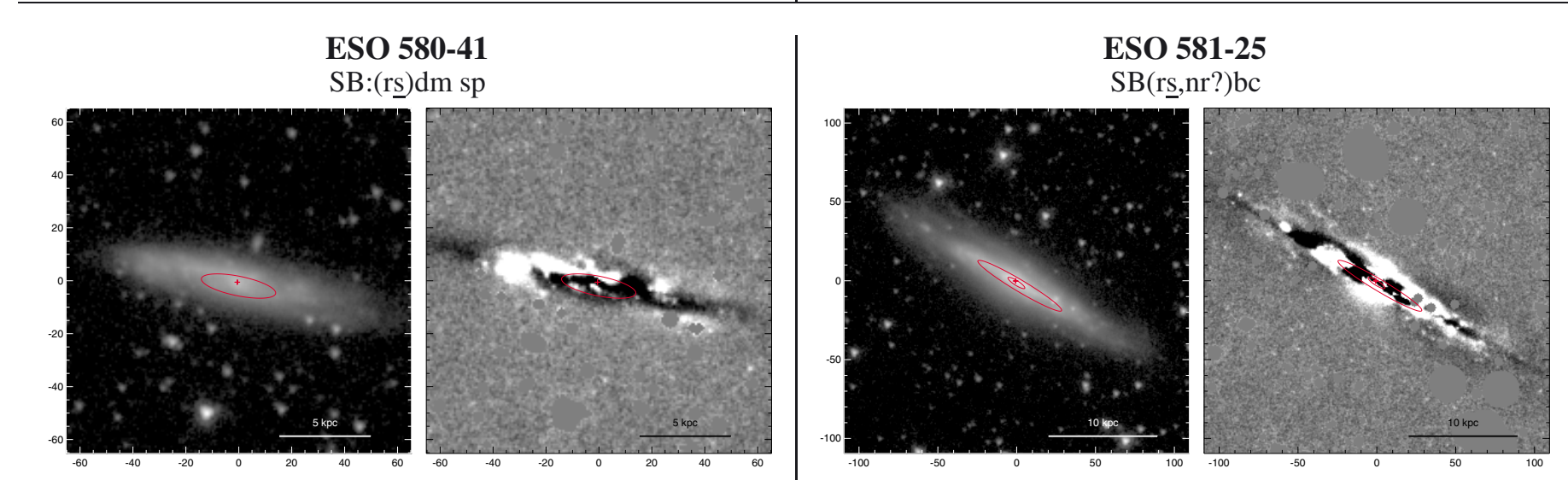

\begin{tabular}{|c|c|c|c|c|c|c|c|c|c|c|c|c|c|}
\hline Feature & $\begin{array}{c}D_{\mathrm{r}, \mathrm{b}} \\
\left({ }^{\prime}\right)\end{array}$ & $\begin{array}{c}d_{\mathrm{r}, \mathrm{b}} \\
\left({ }^{\prime}\right)\end{array}$ & $\begin{array}{c}\mathrm{PA}_{\mathrm{r}, \mathrm{b}} \\
\left({ }^{\circ}\right)\end{array}$ & $\begin{array}{c}D_{\mathrm{r}, \mathrm{b}, 0} \\
\left(^{\prime}\right)\end{array}$ & $\begin{array}{c}d_{\mathrm{r}, \mathrm{b}, 0} \\
\left({ }^{\prime}\right)\end{array}$ & $\begin{array}{c}\theta_{\mathrm{r}, \mathrm{b}} \\
\left({ }^{\circ}\right)\end{array}$ & "Feature & $\begin{array}{c}D_{\mathrm{r}, \mathrm{b}} \\
\left({ }^{\prime}\right)\end{array}$ & $\begin{array}{c}d_{\mathrm{r}, \mathrm{b}} \\
\left({ }^{\prime}\right)\end{array}$ & $\begin{array}{c}\mathrm{PA}_{\mathrm{r}, \mathrm{b}} \\
\left({ }^{\circ}\right)\end{array}$ & $\begin{array}{c}D_{\mathrm{r}, \mathrm{b}, 0} \\
\left(^{\prime}\right)\end{array}$ & $\begin{array}{c}d_{\mathrm{r}, \mathrm{b}, 0} \\
\left(^{\prime}\right)\end{array}$ & $\begin{array}{c}\theta_{\mathrm{r}, \mathrm{b}} \\
\left({ }^{\circ}\right)\end{array}$ \\
\hline rs & 0.48 & 0.12 & 78.6 & 0.63 & 0.47 & 77.1 & $\frac{\mathrm{rs}}{\mathrm{nr}}$ & $\begin{array}{l}1.03 \\
0.21\end{array}$ & $\begin{array}{l}0.12 \\
0.05\end{array}$ & $\begin{array}{l}59.2 \\
57.6\end{array}$ & $\begin{array}{l}1.03 \\
0.26\end{array}$ & $\begin{array}{l}0.62 \\
0.20\end{array}$ & $\begin{array}{l}176.8 \\
108.0\end{array}$ \\
\hline
\end{tabular}


S. Comerón et al.: ARRAKIS

ESO 602-30

$\mathrm{SB}(\mathrm{rs}) \mathrm{d}$
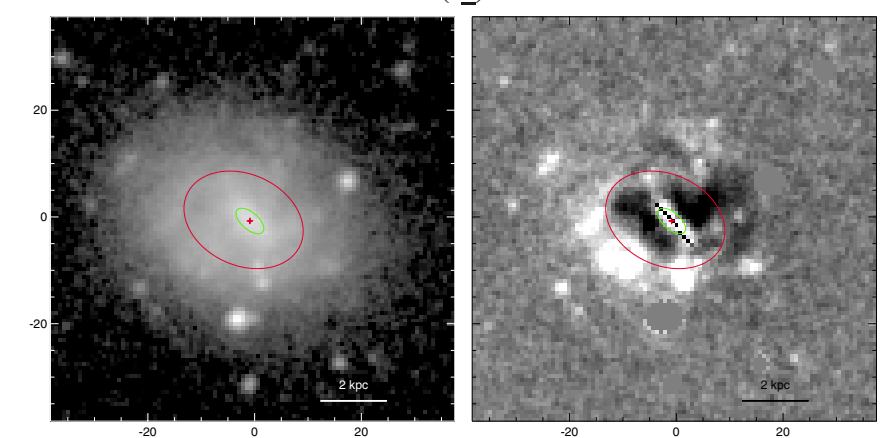

IC 51

PRG

\begin{tabular}{ccccccc|ccccccc}
\hline \hline Feature & $\begin{array}{c}D_{\mathrm{r}, \mathrm{b}} \\
\left({ }^{\prime}\right)\end{array}$ & $\begin{array}{c}d_{\mathrm{r}, \mathrm{b}} \\
\left({ }^{\prime}\right)\end{array}$ & $\begin{array}{c}\mathrm{PA}_{\mathrm{r}, \mathrm{b}} \\
\left({ }^{\circ}\right)\end{array}$ & $\begin{array}{c}D_{\mathrm{r}, \mathrm{b}, 0} \\
\left({ }^{\prime}\right)\end{array}$ & $\begin{array}{c}d_{\mathrm{r}, \mathrm{b}, 0} \\
\left({ }^{\prime}\right)\end{array}$ & $\begin{array}{c}\theta_{\mathrm{r}, \mathrm{b}} \\
\left({ }^{\circ}\right)\end{array}$ & Feature & $\begin{array}{c}D_{\mathrm{r}, \mathrm{b}} \\
\left({ }^{\prime}\right)\end{array}$ & $\begin{array}{c}d_{\mathrm{r}, \mathrm{b}} \\
\left({ }^{\prime}\right)\end{array}$ & $\begin{array}{c}\mathrm{PA}_{\mathrm{r}, \mathrm{b}} \\
\left({ }^{\circ}\right)\end{array}$ & $\begin{array}{c}D_{\mathrm{r}, \mathrm{b}, 0} \\
\left({ }^{\prime}\right)\end{array}$ & $\begin{array}{c}d_{\mathrm{r}, \mathrm{b}, 0} \\
\left({ }^{\prime}\right)\end{array}$ & $\begin{array}{c}\theta_{\mathrm{r}, \mathrm{b}} \\
\left({ }^{\circ}\right)\end{array}$ \\
\hline$\underline{\mathrm{r}}$ & 0.39 & 0.29 & 65.6 & 0.40 & 0.38 & 140.9 & PRG & 0.50 & 0.10 & 113.9 & - & - & - \\
$\mathrm{bar}$ & 0.11 & 0.05 & 49.1 & 0.11 & 0.07 & 145.4 & & & & & & & - \\
\hline
\end{tabular}

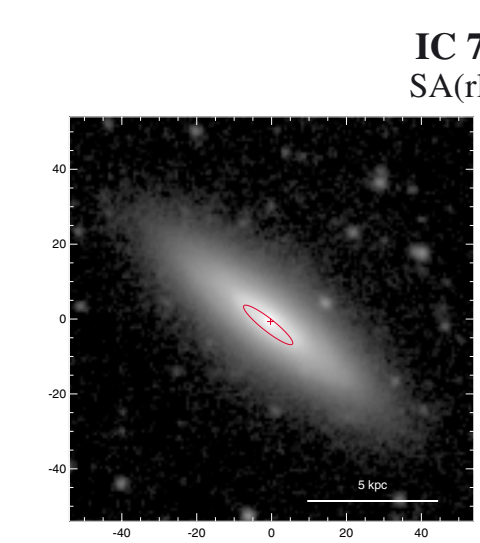

IC 719

IC 749

$\mathrm{SB}(\mathrm{rs}) \mathrm{cd}$
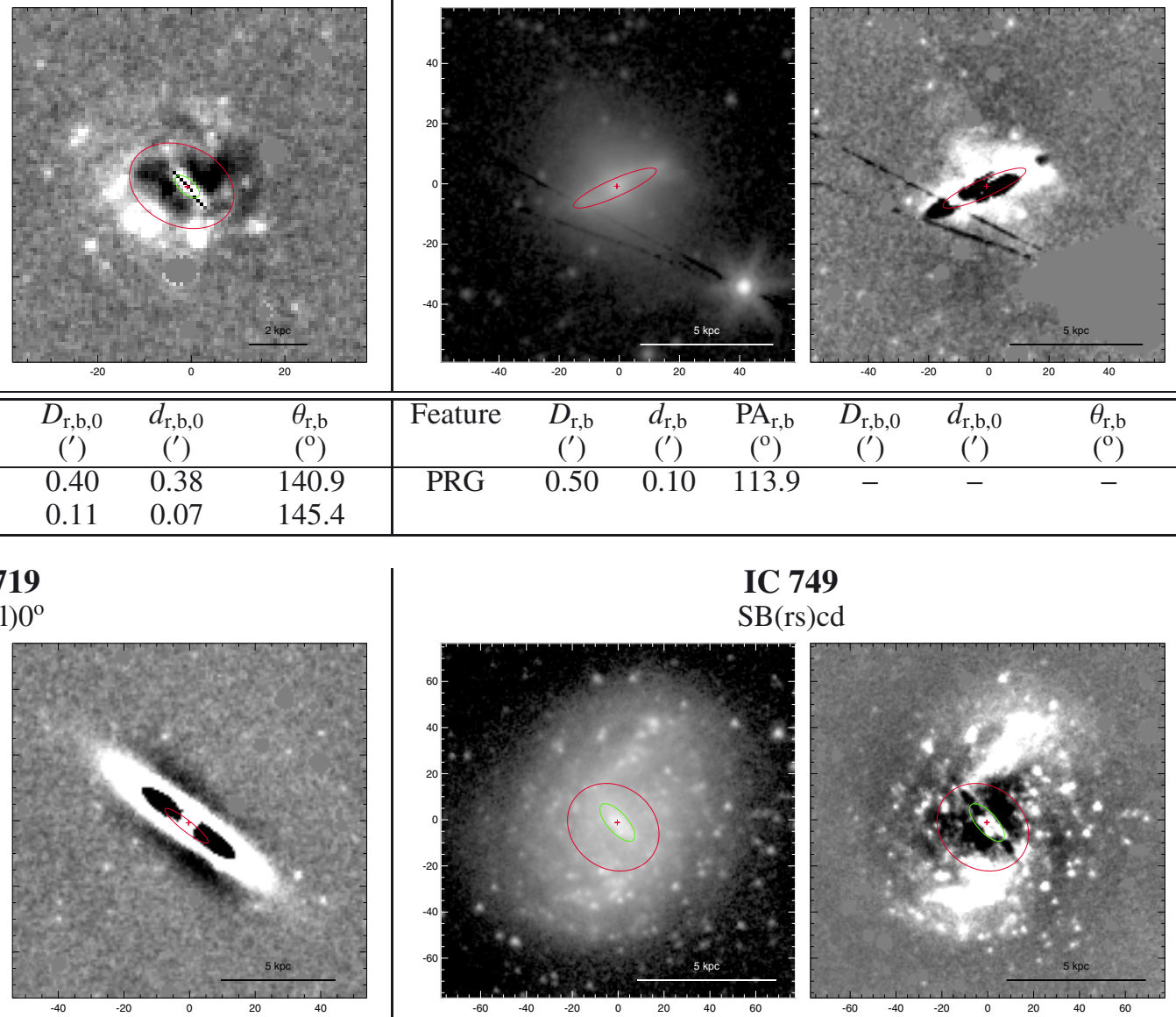

\begin{tabular}{|c|c|c|c|c|c|c|c|c|c|c|c|c|c|}
\hline Feature & $\begin{array}{c}D_{\mathrm{r}, \mathrm{b}} \\
\left({ }^{\prime}\right)\end{array}$ & $\begin{array}{c}d_{\mathrm{r}, \mathrm{b}} \\
\left({ }^{\prime}\right)\end{array}$ & $\begin{array}{c}\mathrm{PA}_{\mathrm{r}, \mathrm{b}} \\
\left(^{\mathrm{O}}\right)\end{array}$ & $\begin{array}{c}D_{\mathrm{r}, \mathrm{b}, 0} \\
\left(^{\prime}\right)\end{array}$ & $\begin{array}{c}d_{\mathrm{r}, \mathrm{b}, 0} \\
\left(^{\prime}\right)\end{array}$ & $\begin{array}{l}\theta_{\mathrm{r}, \mathrm{b}} \\
\left({ }^{\circ}\right)\end{array}$ & Feature & $\begin{array}{c}D_{\mathrm{r}, \mathrm{b}} \\
\left({ }^{\prime}\right) \\
\end{array}$ & $\begin{array}{c}d_{\mathrm{r}, \mathrm{b}} \\
\left(^{\prime}\right) \\
\end{array}$ & $\begin{array}{c}\mathrm{PA}_{\mathrm{r}, \mathrm{b}} \\
\left(^{\circ}\right)\end{array}$ & $\begin{array}{c}D_{\mathrm{r}, \mathrm{b}, 0} \\
\left({ }^{\prime}\right)\end{array}$ & $\begin{array}{c}d_{\mathrm{r}, \mathrm{b}, 0} \\
\left({ }^{\prime}\right)\end{array}$ & $\begin{array}{l}\theta_{\mathrm{r}, \mathrm{b}} \\
\left({ }^{\circ}\right)\end{array}$ \\
\hline $\mathrm{rl}$ & 0.28 & 0.06 & 51.8 & 0.28 & 0.14 & 4.5 & $\begin{array}{c}\mathrm{rs} \\
\text { bar }\end{array}$ & $\begin{array}{l}0.70 \\
0.35\end{array}$ & $\begin{array}{l}0.60 \\
0.15\end{array}$ & $\begin{array}{l}53.1 \\
42.1\end{array}$ & $\begin{array}{l}0.78 \\
0.39\end{array}$ & $\begin{array}{l}0.61 \\
0.15\end{array}$ & $\begin{array}{l}106.4 \\
104.7\end{array}$ \\
\hline
\end{tabular}

IC 863

$\left(\mathrm{R}^{\prime}\right) \mathrm{SB}(\mathrm{rs}) \mathrm{b}$

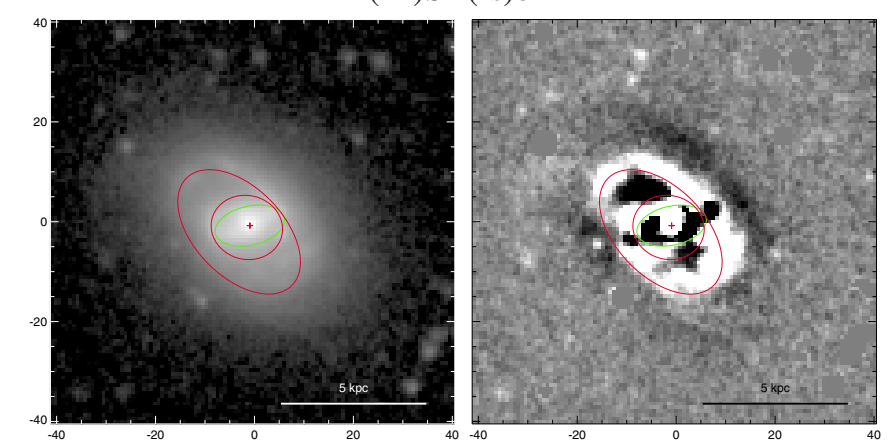

IC 1014

$\mathrm{SB}(\mathrm{r}) \mathrm{m}$

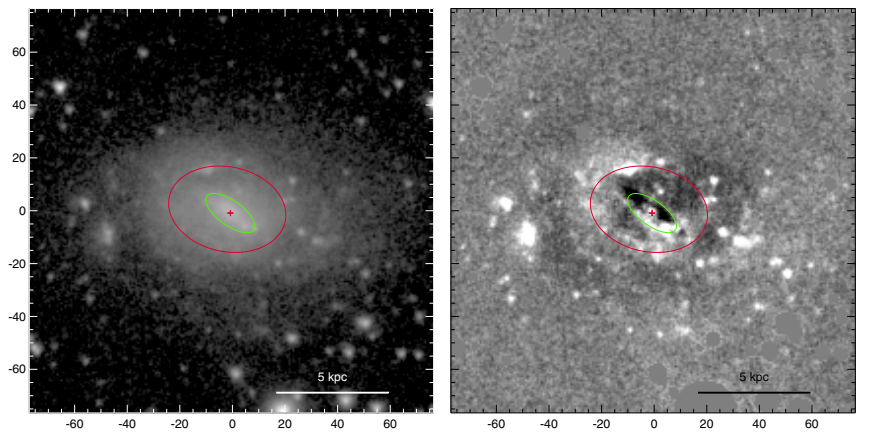

\begin{tabular}{ccccccc|ccccccc}
\hline \hline Feature & $\begin{array}{c}D_{\mathrm{r}, \mathrm{b}} \\
\left({ }^{\prime}\right)\end{array}$ & $\begin{array}{c}d_{\mathrm{r}, \mathrm{b}} \\
\left({ }^{\prime}\right)\end{array}$ & $\begin{array}{c}\mathrm{PA}_{\mathrm{r}, \mathrm{b}} \\
\left({ }^{\circ}\right)\end{array}$ & $\begin{array}{c}D_{\mathrm{r}, \mathrm{b}, 0} \\
\left({ }^{\prime}\right)\end{array}$ & $\begin{array}{c}d_{\mathrm{r}, \mathrm{b}, 0} \\
\left({ }^{\prime}\right)\end{array}$ & $\begin{array}{c}\theta_{\mathrm{r}, \mathrm{b}} \\
\left({ }^{\circ}\right)\end{array}$ & Feature & $\begin{array}{c}D_{\mathrm{r}, \mathrm{b}} \\
\left({ }^{\prime}\right)\end{array}$ & $\begin{array}{c}d_{\mathrm{r}, \mathrm{b}} \\
\left({ }^{\prime}\right)\end{array}$ & $\begin{array}{c}\mathrm{PA}_{\mathrm{r}, \mathrm{b}} \\
\left({ }^{\circ}\right)\end{array}$ & $\begin{array}{c}D_{\mathrm{r}, \mathrm{b}, 0} \\
\left({ }^{\prime}\right)\end{array}$ & $\begin{array}{c}d_{\mathrm{r}, \mathrm{b}, 0} \\
\left({ }^{\prime}\right)\end{array}$ & $\begin{array}{c}\theta_{\mathrm{r}, \mathrm{b}} \\
\left({ }^{\circ}\right)\end{array}$ \\
\hline $\mathrm{R}^{\prime}$ & 0.51 & 0.30 & 44.1 & 0.51 & 0.40 & 167.6 & $\mathrm{r}$ & 0.75 & 0.54 & 78.2 & 0.83 & 0.73 & 116.6 \\
$\mathrm{rs}$ & 0.24 & 0.22 & 76.6 & 0.30 & 0.24 & 78.7 & bar & 0.37 & 0.16 & 54.2 & 0.43 & 0.20 & 130.3 \\
$\mathrm{bar}$ & 0.24 & 0.13 & 102.8 & 0.30 & 0.14 & 65.8 & & & & & & & \\
\hline
\end{tabular}


IC 1029

$\left(\mathrm{R}^{\prime}\right) \mathrm{SB}_{\mathrm{a}}$ (s,nd)a
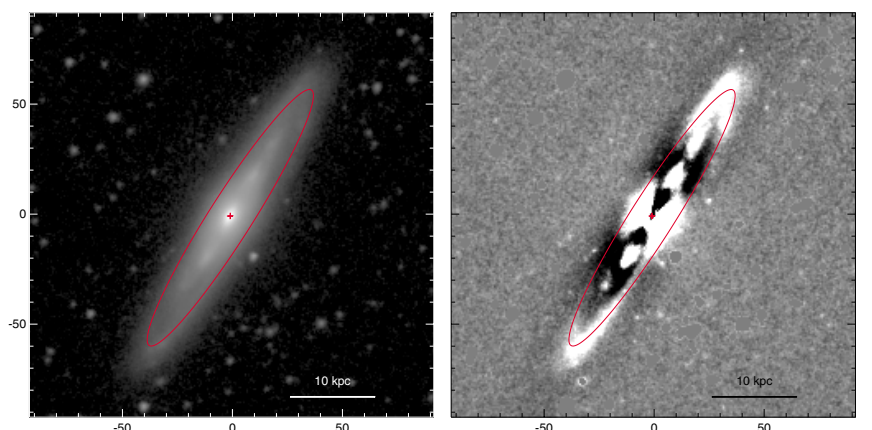

\section{IC 1048}

$\left(\mathrm{R}^{\prime}\right) \mathrm{SB}(\mathrm{s}) \mathrm{d} \mathrm{sp}$

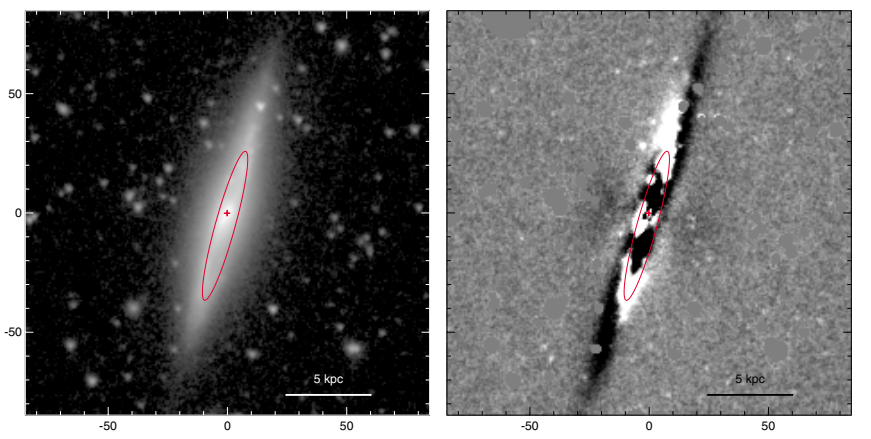

\begin{tabular}{|c|c|c|c|c|c|c|c|c|c|c|c|c|c|}
\hline Feature & $\begin{array}{c}D_{\mathrm{r}, \mathrm{b}} \\
\left({ }^{\prime}\right)\end{array}$ & $\begin{array}{c}d_{\mathrm{r}, \mathrm{b}} \\
\left({ }^{\prime}\right)\end{array}$ & $\begin{array}{c}\mathrm{PA}_{\mathrm{r}, \mathrm{b}} \\
\left({ }^{\mathrm{O}}\right)\end{array}$ & $\begin{array}{c}D_{\mathrm{r}, \mathrm{b}, 0} \\
\left(^{\prime}\right)\end{array}$ & $\begin{array}{c}d_{\mathrm{r}, \mathrm{b}, 0} \\
\left.\text { (') }^{\prime}\right)\end{array}$ & $\begin{array}{l}\theta_{\mathrm{r}, \mathrm{b}} \\
\left({ }^{\circ}\right)\end{array}$ & Feature & $\begin{array}{c}D_{\mathrm{r}, \mathrm{b}} \\
\left({ }^{\prime}\right)\end{array}$ & $\begin{array}{c}d_{\mathrm{r}, \mathrm{b}} \\
\left({ }^{\prime}\right)\end{array}$ & $\begin{array}{c}\mathrm{PA}_{\mathrm{r}, \mathrm{b}} \\
\left({ }^{\circ}\right)\end{array}$ & $\begin{array}{c}D_{\mathrm{r}, \mathrm{b}, 0} \\
\left(^{\prime}\right)\end{array}$ & $\begin{array}{c}d_{\mathrm{r}, \mathrm{b}, 0} \\
\left(^{\prime}\right)\end{array}$ & $\begin{array}{c}\theta_{\mathrm{r}, \mathrm{b}} \\
\left({ }^{\circ}\right)\end{array}$ \\
\hline $\mathrm{R}^{\prime}$ & 2.30 & 0.33 & 147.6 & 2.34 & 1.41 & 167.1 & $\overline{\mathrm{R}^{\prime}}$ & 1.08 & 0.14 & 164.6 & 1.11 & 0.62 & 15.7 \\
\hline
\end{tabular}

IC 1055

$\left(\mathrm{R}^{\prime}\right) \mathrm{SA}(\mathrm{rs}, \mathrm{r}) \mathrm{ab}$

\section{IC 1067}

SB $(r) b$
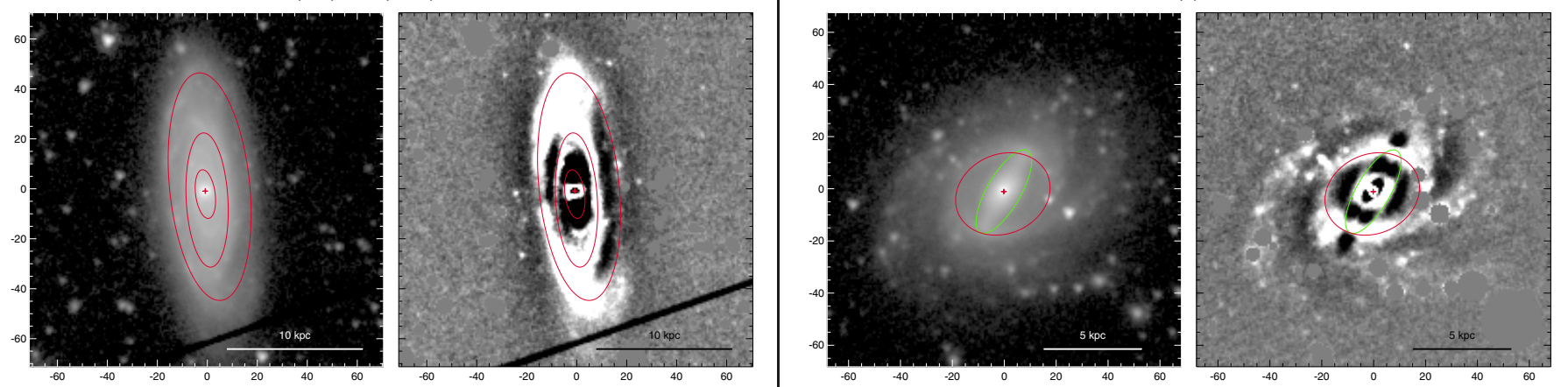

\begin{tabular}{ccccccc|ccccccc}
\hline \hline Feature & $\begin{array}{c}D_{\mathrm{r}, \mathrm{b}} \\
\left({ }^{\prime}\right)\end{array}$ & $\begin{array}{c}d_{\mathrm{r}, \mathrm{b}} \\
\left({ }^{\prime}\right)\end{array}$ & $\begin{array}{c}\mathrm{PA}_{\mathrm{r}, \mathrm{b}} \\
\left({ }^{\circ}\right)\end{array}$ & $\begin{array}{c}D_{\mathrm{r}, \mathrm{b}, 0} \\
\left({ }^{\prime}\right)\end{array}$ & $\begin{array}{c}d_{\mathrm{r}, \mathrm{b}, 0} \\
\left({ }^{\prime}\right)\end{array}$ & $\begin{array}{c}\theta_{\mathrm{r}, \mathrm{b}} \\
\left({ }^{\circ}\right)\end{array}$ & Feature & $\begin{array}{c}D_{\mathrm{r}, \mathrm{b}} \\
\left({ }^{\prime}\right)\end{array}$ & $\begin{array}{c}d_{\mathrm{r}, \mathrm{b}} \\
\left({ }^{\prime}\right)\end{array}$ & $\begin{array}{c}\mathrm{PA}_{\mathrm{r}, \mathrm{b}} \\
\left({ }^{\circ}\right)\end{array}$ & $\begin{array}{c}D_{\mathrm{r}, \mathrm{b}, 0} \\
\left({ }^{\prime}\right)\end{array}$ & $\begin{array}{c}d_{\mathrm{r}, \mathrm{b}, 0} \\
\left({ }^{\prime}\right)\end{array}$ & $\begin{array}{c}\theta_{\mathrm{r}, \mathrm{b}} \\
\left({ }^{\circ}\right)\end{array}$ \\
\hline $\mathrm{R}^{\prime}$ & 1.53 & 0.54 & 5.7 & 1.54 & 1.33 & 6.3 & $\mathrm{r}$ & 0.63 & 0.51 & 114.0 & 0.65 & 0.63 & 106.0 \\
$\mathrm{rs}$ & 0.90 & 0.28 & 3.2 & 0.91 & 0.69 & 170.7 & $\mathrm{bar}$ & 0.61 & 0.22 & 150.0 & 0.67 & 0.25 & 41.4 \\
$\mathrm{r}$ & 0.33 & 0.13 & 7.6 & 0.34 & 0.31 & 42.4 & & & & & & \\
\hline
\end{tabular}

IC 1158

$\mathrm{S} \underline{\mathrm{AB}}$ (rss)cd

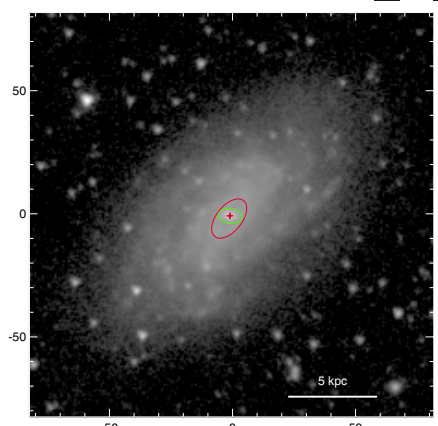

IC 1210

SAB(r)a: pec
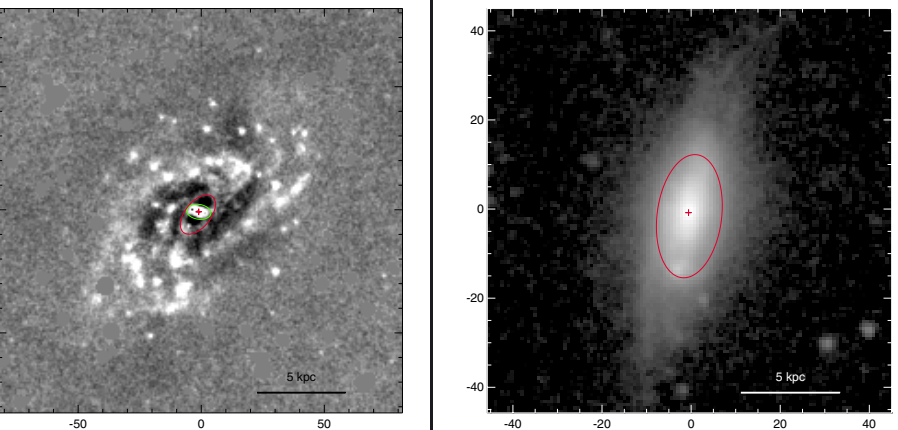

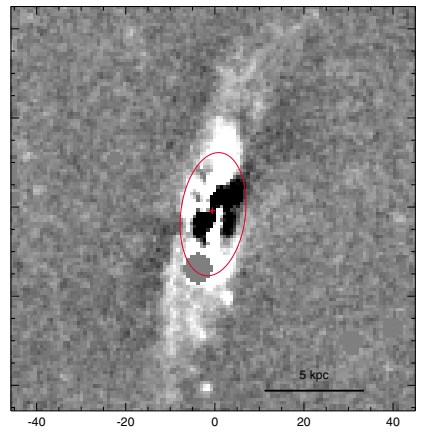

(3)

Feature $D$

\begin{tabular}{|c|c|c|c|c|c|c|c|c|c|c|c|c|c|}
\hline Feature & $\begin{array}{c}D_{\mathrm{r}, \mathrm{b}} \\
\left({ }^{\prime}\right)\end{array}$ & $\begin{array}{c}d_{\mathrm{r}, \mathrm{b}} \\
\left({ }^{\prime}\right)\end{array}$ & $\begin{array}{c}\mathrm{PA}_{\mathrm{r}, \mathrm{b}} \\
\left({ }^{\circ}\right)\end{array}$ & $\begin{array}{c}D_{\mathrm{r}, \mathrm{b}, 0} \\
\left(^{\prime}\right)\end{array}$ & $\begin{array}{c}d_{\mathrm{r}, \mathrm{b}, 0} \\
\left.\text { (') }^{\prime}\right)\end{array}$ & $\begin{array}{l}\theta_{\mathrm{r}, \mathrm{b}} \\
\left({ }^{\circ}\right)\end{array}$ & Feature & $\begin{array}{c}D_{\mathrm{r}, \mathrm{b}} \\
\left({ }^{\prime}\right)\end{array}$ & $\begin{array}{c}d_{\mathrm{r}, \mathrm{b}} \\
\left({ }^{\prime}\right)\end{array}$ & $\begin{array}{c}\mathrm{PA}_{\mathrm{r}, \mathrm{b}} \\
\left({ }^{\circ}\right)\end{array}$ & $\begin{array}{c}D_{\mathrm{r}, \mathrm{b}, 0} \\
\left(^{\prime}\right)\end{array}$ & $\begin{array}{c}d_{\mathrm{r}, \mathrm{b}, 0} \\
\left(^{\prime}\right)\end{array}$ & $\begin{array}{l}\theta_{\mathrm{r}, \mathrm{b}} \\
\left({ }^{\circ}\right)\end{array}$ \\
\hline rs & 0.31 & 0.17 & 141.2 & 0.33 & 0.29 & 39.9 & $\overline{\mathrm{r}}$ & 0.47 & 0.24 & 172.9 & 0.54 & 0.45 & 64.6 \\
\hline$\overline{b a r}$ & 0.17 & 0.10 & 81.8 & 0.26 & 0.11 & 105.7 & & & & & & & \\
\hline
\end{tabular}




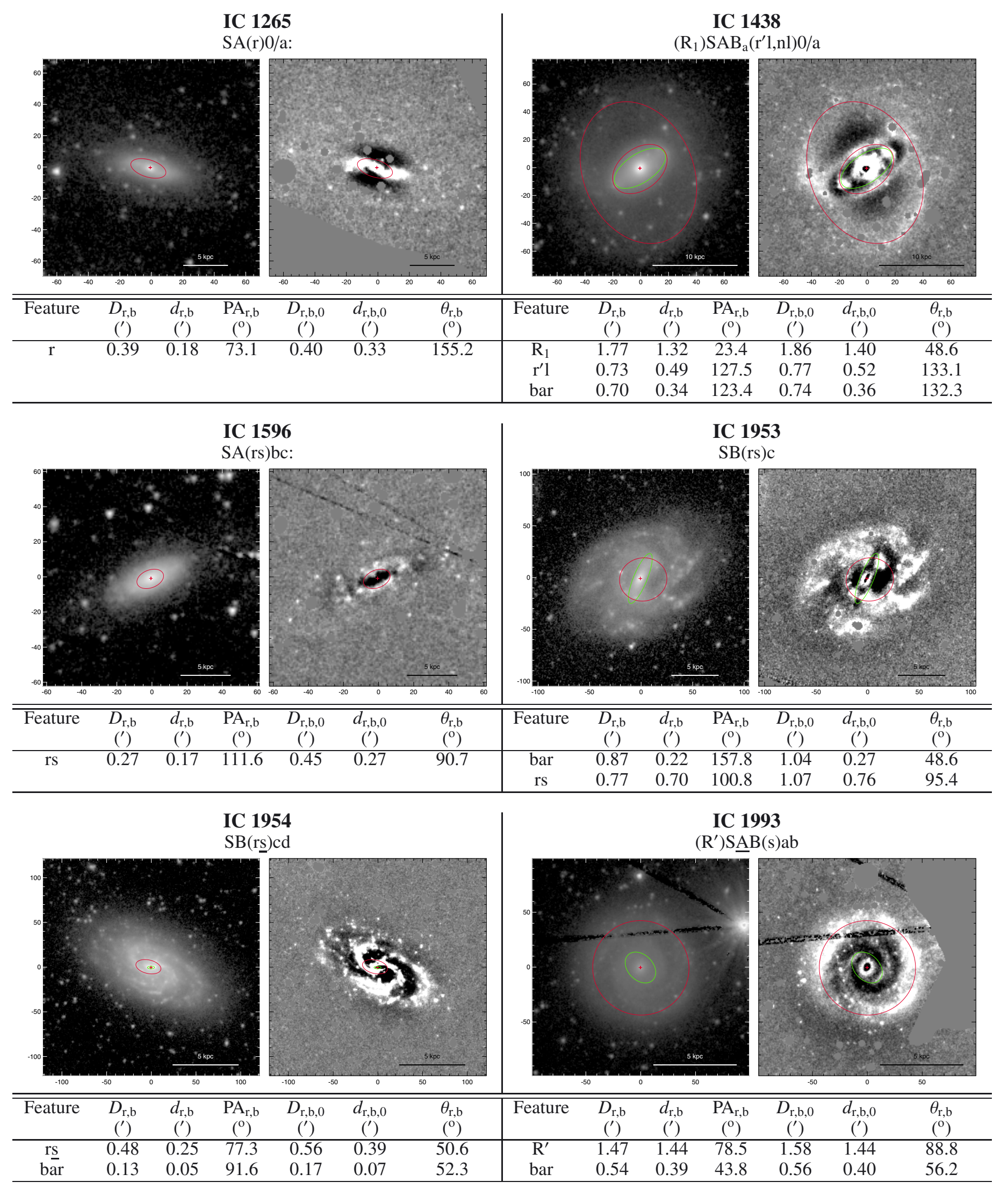


IC 2051

$\mathrm{SB}(\mathrm{rs}) \mathrm{b}$
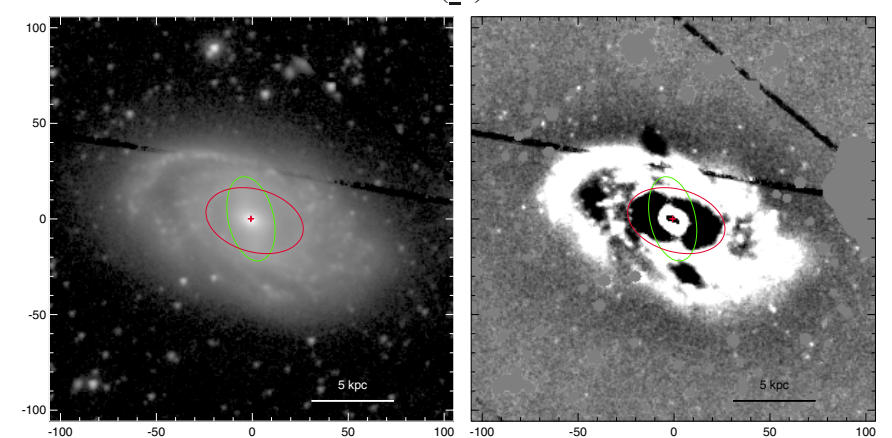

\begin{tabular}{|c|c|c|c|c|c|c|c|c|c|c|c|c|c|}
\hline Feature & $\begin{array}{c}D_{\mathrm{r}, \mathrm{b}} \\
\left({ }^{\prime}\right)\end{array}$ & $\begin{array}{c}d_{\mathrm{r}, \mathrm{b}} \\
(')\end{array}$ & $\begin{array}{c}\mathrm{PA}_{\mathrm{r}, \mathrm{b}} \\
\left({ }^{\circ}\right)\end{array}$ & $\begin{array}{c}D_{\mathrm{r}, \mathrm{b}, 0} \\
\left(^{\prime}\right)\end{array}$ & $\begin{array}{c}d_{\mathrm{r}, \mathrm{b}, 0} \\
\left({ }^{\prime}\right)\end{array}$ & $\begin{array}{l}\theta_{\mathrm{r}, \mathrm{b}} \\
\left(^{\circ}\right)\end{array}$ & Feature & $\begin{array}{c}D_{\mathrm{r}, \mathrm{b}} \\
\left({ }^{\prime}\right)\end{array}$ & $\begin{array}{c}d_{\mathrm{r}, \mathrm{b}} \\
\left({ }^{\prime}\right)\end{array}$ & $\begin{array}{c}\mathrm{PA}_{\mathrm{r}, \mathrm{b}} \\
\left(^{\circ}\right)\end{array}$ & $\begin{array}{c}D_{\mathrm{r}, \mathrm{b}, 0} \\
\left(^{\prime}\right)\end{array}$ & $\begin{array}{c}d_{\mathrm{r}, \mathrm{b}, 0} \\
\left(^{\prime}\right)\end{array}$ & $\begin{array}{c}\theta_{\mathrm{r}, \mathrm{b}} \\
\left({ }^{\circ}\right)\end{array}$ \\
\hline$\underline{\mathrm{rS}}$ & 0.87 & 0.54 & 74.0 & 0.91 & 0.86 & 71.7 & r $\underline{s}$ & 0.27 & 0.23 & 114.5 & 0.31 & 0.24 & 94.5 \\
\hline bar & 0.75 & 0.40 & 12.8 & 1.16 & 0.43 & 105.2 & & & & & & & \\
\hline
\end{tabular}

IC 2361

$\mathrm{SA}(\mathrm{rs}) \mathrm{bc}$
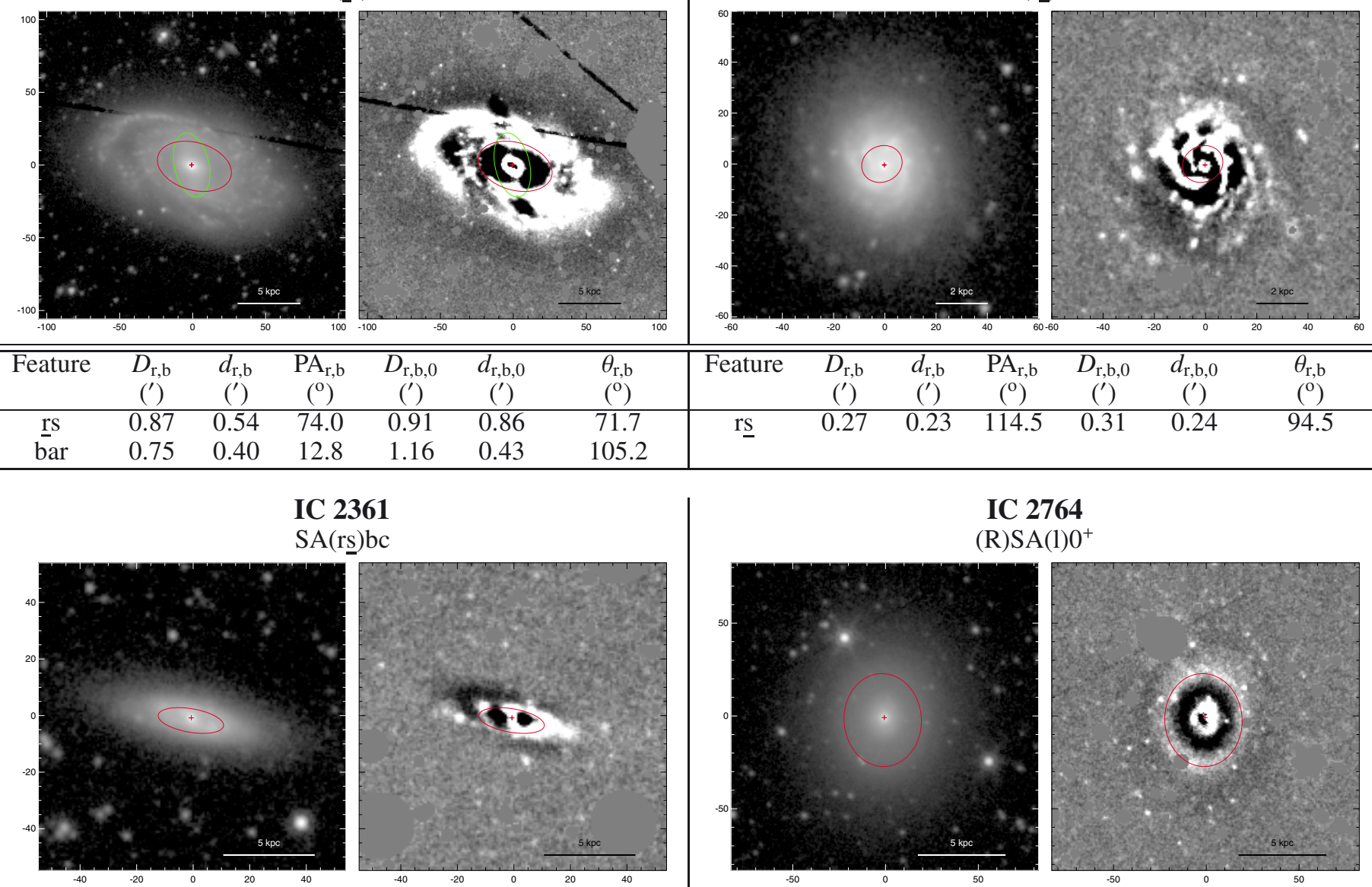

IC 2056

$\mathrm{SA}(\mathrm{rs}) \mathrm{bc}$

IC 2764

(R) SA(1) $0^{+}$

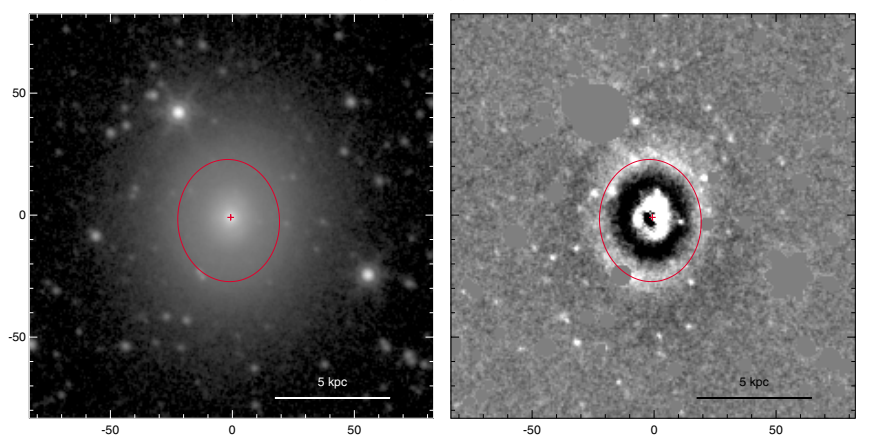

\begin{tabular}{|c|c|c|c|c|c|c|c|c|c|c|c|c|c|}
\hline Feature & $\begin{array}{c}D_{\mathrm{r}, \mathrm{b}} \\
\left(^{\prime}\right)\end{array}$ & $\begin{array}{c}d_{\mathrm{r}, \mathrm{b}} \\
\left(^{\prime}\right)\end{array}$ & $\begin{array}{c}\mathrm{PA}_{\mathrm{r}, \mathrm{b}} \\
\left({ }^{\circ}\right)\end{array}$ & $\begin{array}{c}D_{\mathrm{r}, \mathrm{b}, 0} \\
\left(^{\prime}\right)\end{array}$ & $\begin{array}{c}d_{\mathrm{r}, \mathrm{b}, 0} \\
\left(^{\prime}\right)\end{array}$ & $\begin{array}{c}\theta_{\mathrm{r}, \mathrm{b}} \\
\left(^{\circ}\right)\end{array}$ & Feature & $\begin{array}{c}D_{\mathrm{r}, \mathrm{b}} \\
\left(^{\prime}\right)\end{array}$ & $\begin{array}{c}d_{\mathrm{r}, \mathrm{b}} \\
\left(^{\prime}\right)\end{array}$ & $\begin{array}{c}\mathrm{PA}_{\mathrm{r}, \mathrm{b}} \\
\left({ }^{\circ}\right)\end{array}$ & $\begin{array}{c}D_{\mathrm{r}, \mathrm{b}, 0} \\
\left(^{\prime}\right)\end{array}$ & $\begin{array}{c}d_{\mathrm{r}, \mathrm{b}, 0} \\
\left({ }^{\prime}\right)\end{array}$ & $\begin{array}{c}\theta_{\mathrm{r}, \mathrm{b}} \\
\left({ }^{\circ}\right)\end{array}$ \\
\hline$r s$ & 0.39 & 0.14 & 80.4 & 0.43 & 0.36 & 46.0 & $\overline{\mathrm{R}}$ & 0.84 & 0.70 & 3.3 & 0.84 & 0.82 & 9.6 \\
\hline
\end{tabular}

IC 2969

$\mathrm{SA}(\mathrm{r}) \mathrm{d}$
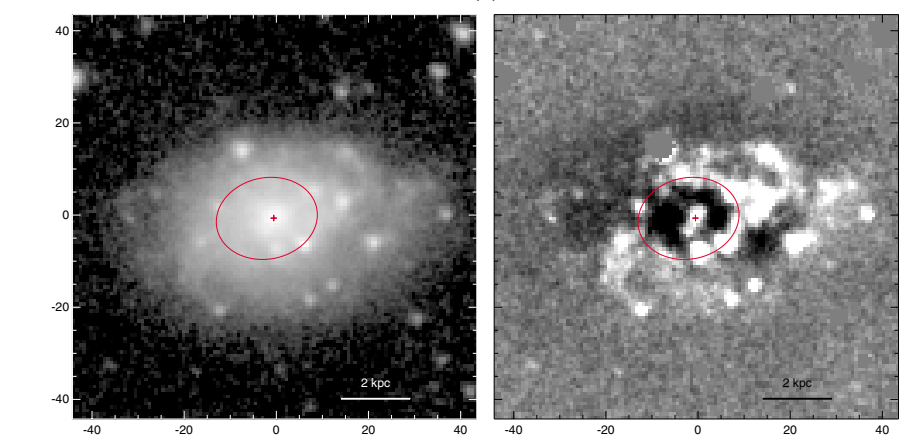

IC 3102

( $\left.\mathrm{R}^{\prime} \mathrm{L}\right) \mathrm{SAB}(\underline{\mathrm{rs}}) 0 / \mathrm{a}$

\begin{tabular}{|c|c|c|c|c|c|c|c|c|c|c|c|c|c|}
\hline Feature & $\begin{array}{c}D_{\mathrm{r}, \mathrm{b}} \\
\left({ }^{\prime}\right)\end{array}$ & $\begin{array}{c}d_{\mathrm{r}, \mathrm{b}} \\
\left({ }^{\prime}\right)\end{array}$ & $\begin{array}{c}\mathrm{PA}_{\mathrm{r}, \mathrm{b}} \\
\left({ }^{\circ}\right)\end{array}$ & $\begin{array}{c}D_{\mathrm{r}, \mathrm{b}, 0} \\
\left(^{\prime}\right)\end{array}$ & $\begin{array}{c}d_{\mathrm{r}, \mathrm{b}, 0} \\
\left({ }^{\prime}\right)\end{array}$ & $\begin{array}{c}\theta_{\mathrm{r}, \mathrm{b}} \\
\left({ }^{\circ}\right)\end{array}$ & Feature & $\begin{array}{c}D_{\mathrm{r}, \mathrm{b}} \\
\left({ }^{\prime}\right)\end{array}$ & $\begin{array}{c}d_{\mathrm{r}, \mathrm{b}} \\
\left({ }^{\prime}\right)\end{array}$ & $\begin{array}{c}\mathrm{PA}_{\mathrm{r}, \mathrm{b}} \\
\left({ }^{\circ}\right)\end{array}$ & $\begin{array}{c}D_{\mathrm{r}, \mathrm{b}, 0} \\
\left(^{\prime}\right)\end{array}$ & $\begin{array}{c}d_{\mathrm{r}, \mathrm{b}, 0} \\
\left(^{\prime}\right)\end{array}$ & $\begin{array}{c}\theta_{\mathrm{r}, \mathrm{b}} \\
\left({ }^{\circ}\right)\end{array}$ \\
\hline \multirow[t]{3}{*}{$\mathrm{r}$} & 0.37 & 0.30 & 101.5 & 0.45 & 0.37 & 80.4 & $\mathrm{R}^{\prime} \mathrm{L}$ & 2.76 & 1.45 & 119.9 & 2.77 & 2.33 & 8.0 \\
\hline & & & & & & & $\underline{\text { rs }}$ & 1.55 & 0.81 & 117.6 & 1.55 & 1.30 & 178.8 \\
\hline & & & & & & & bar & 0.78 & 0.38 & 128.0 & 0.81 & 0.59 & 27.7 \\
\hline
\end{tabular}




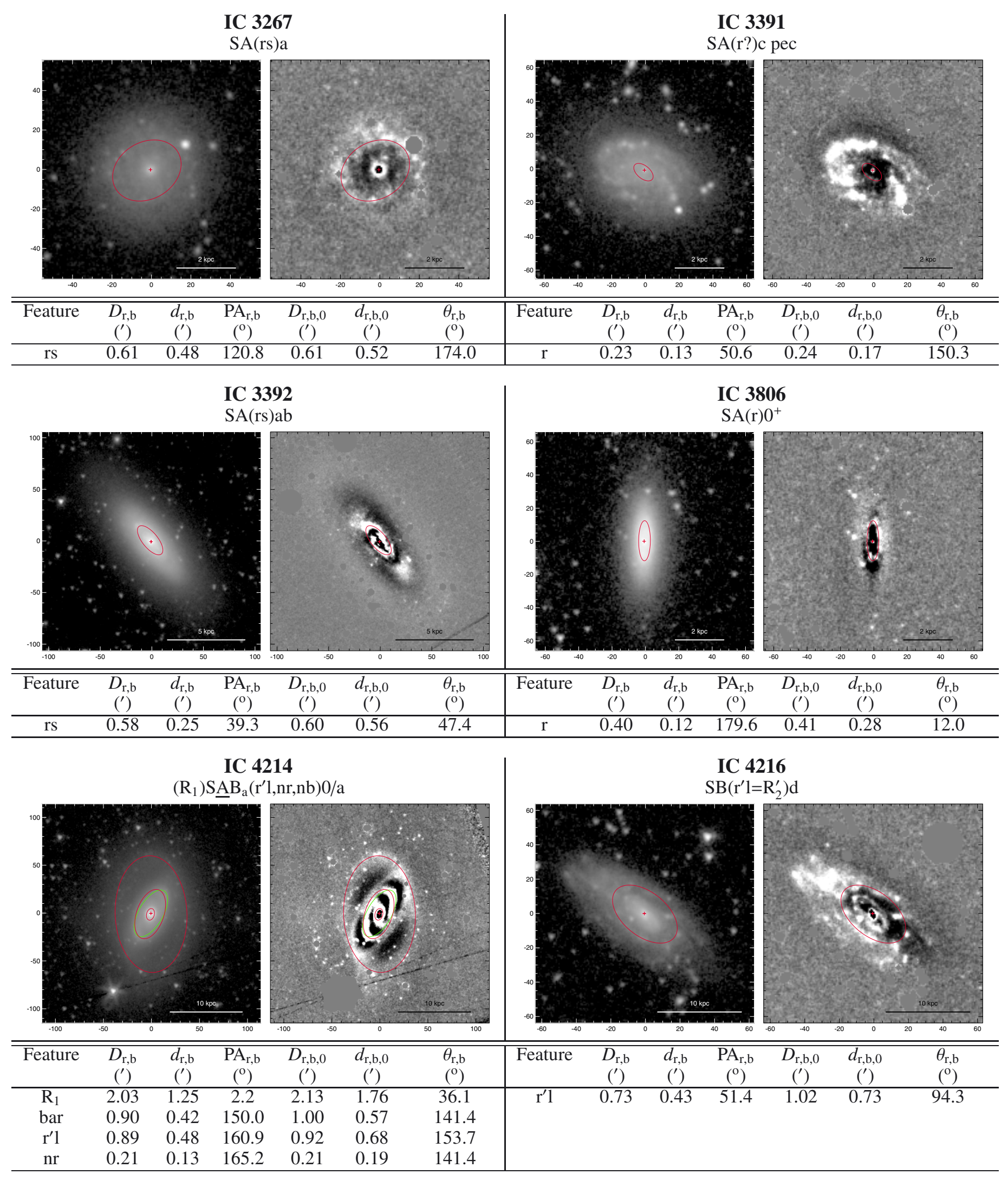









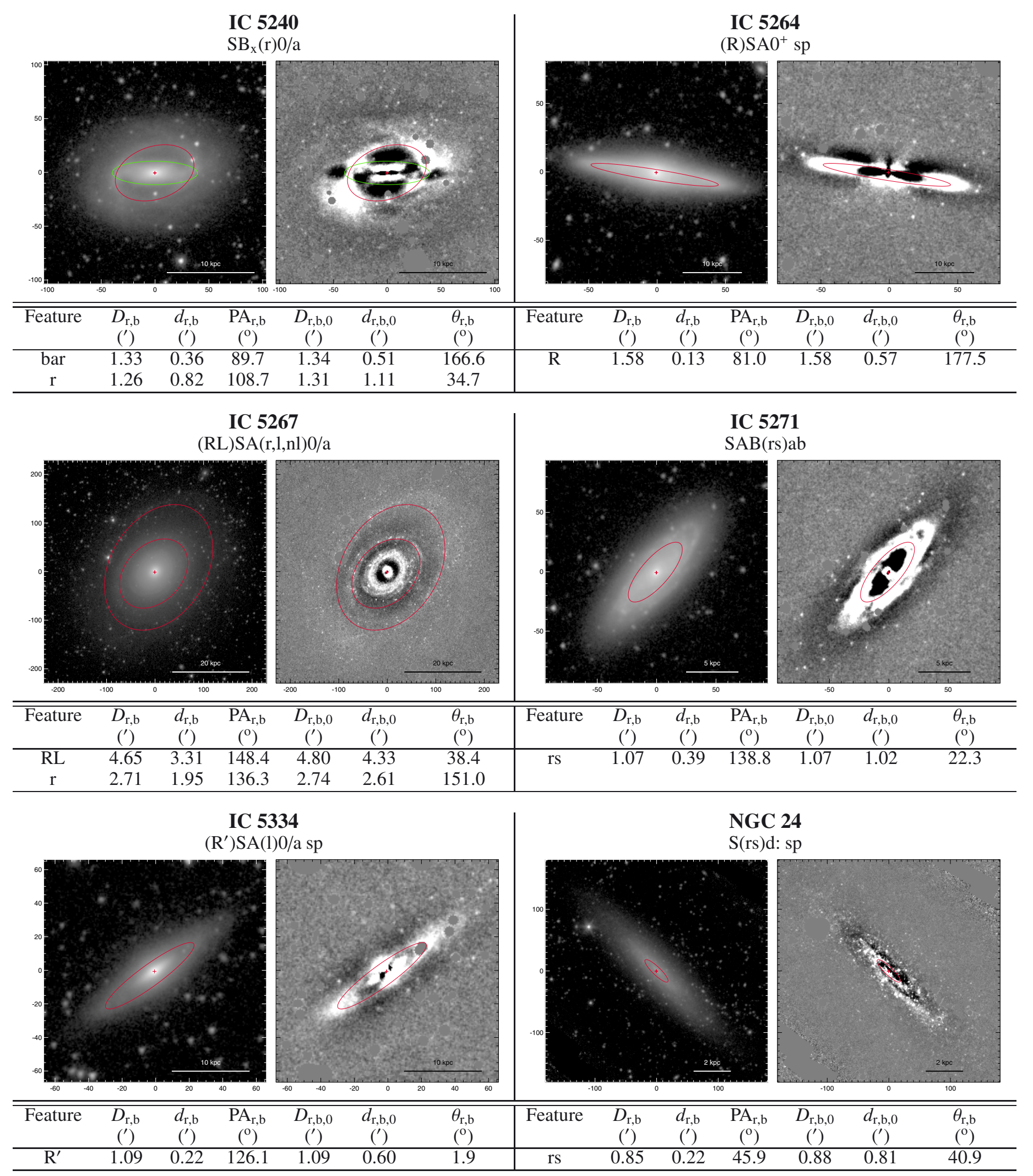


NGC 131

SAB(rs)b:
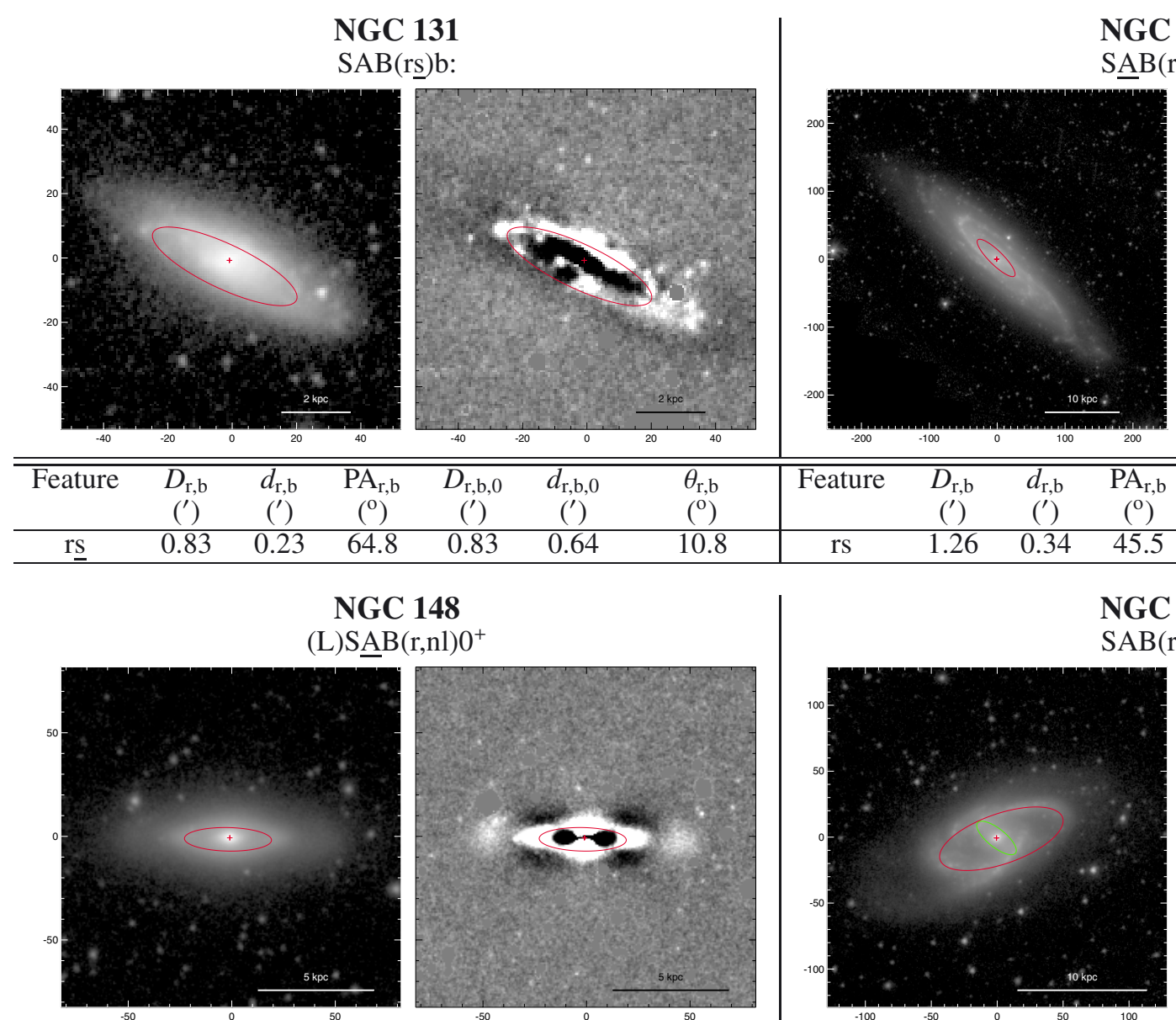

NGC 134

$\mathrm{SAB}(\mathrm{rs}) \mathrm{bc}$
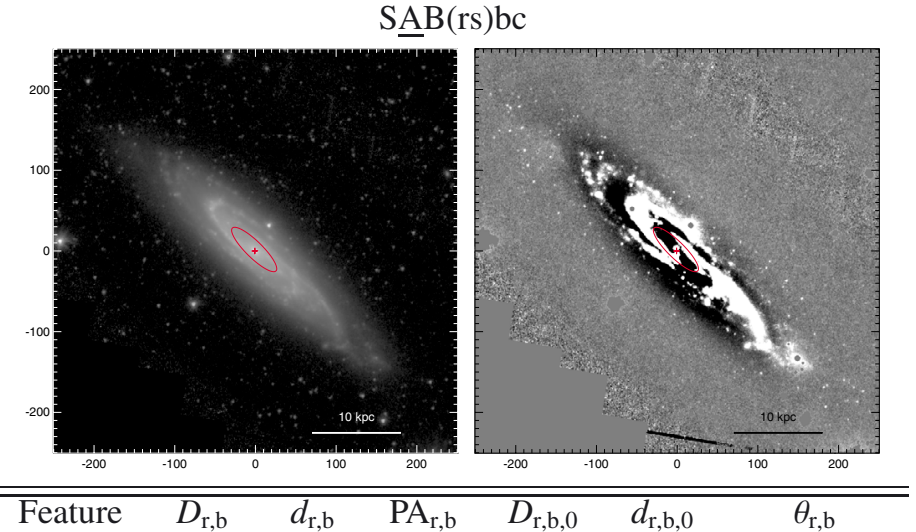

$\begin{array}{ccc}\left({ }^{\prime}\right) & \left({ }^{\prime}\right) & \left(^{\circ}\right) \\ 0.83 & 0.64 & 10.8\end{array}$

\begin{tabular}{l|l}
()$^{\prime}$ & \\
\hline 10.8 & rs
\end{tabular}

$\begin{array}{ccc}\left({ }^{\prime}\right) & \left({ }^{\prime}\right) & \left(^{\circ}\right) \\ 1.26 & 0.34 & 45.5\end{array}$

NGC 150

$\mathrm{SAB}(\mathrm{rs}) \mathrm{ab}$
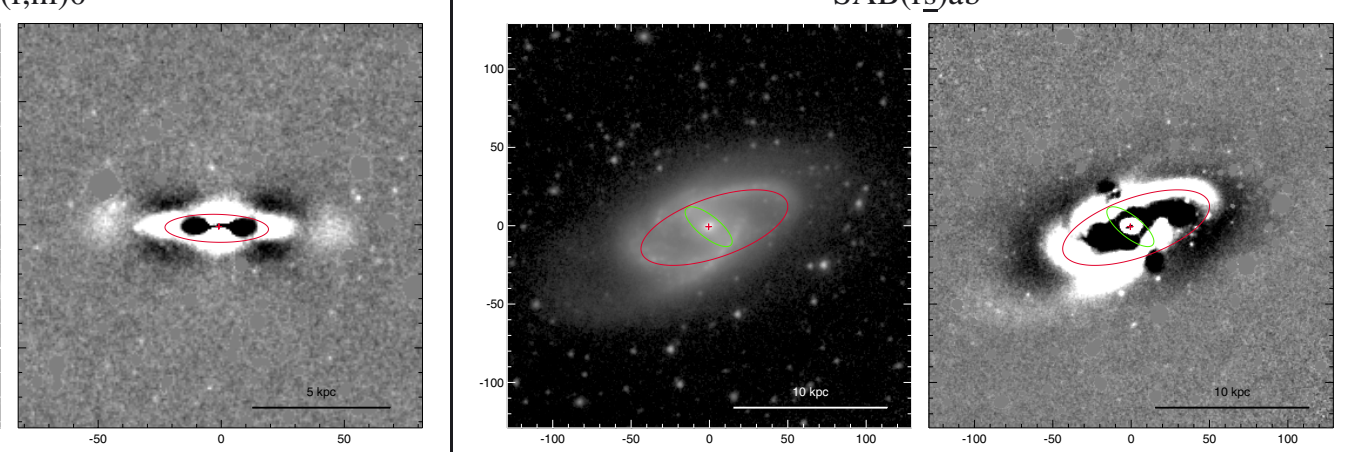

\begin{tabular}{|c|c|c|c|c|c|c|c|c|c|c|c|c|c|}
\hline Feature & $\begin{array}{c}D_{\mathrm{r}, \mathrm{b}} \\
\left({ }^{\prime}\right)\end{array}$ & $\begin{array}{c}d_{\mathrm{r}, \mathrm{b}} \\
\left({ }^{\prime}\right)\end{array}$ & $\begin{array}{c}\mathrm{PA}_{\mathrm{r}, \mathrm{b}} \\
\left({ }^{\circ}\right)\end{array}$ & $\begin{array}{c}D_{\mathrm{r}, \mathrm{b}, 0} \\
\left(^{\prime}\right)\end{array}$ & $\begin{array}{c}d_{\mathrm{r}, \mathrm{b}, 0} \\
\left({ }^{\prime}\right)\end{array}$ & $\begin{array}{l}\theta_{\mathrm{r}, \mathrm{b}} \\
\left({ }^{\circ}\right)\end{array}$ & Feature & $\begin{array}{c}D_{\mathrm{r}, \mathrm{b}} \\
\left({ }^{\prime}\right)\end{array}$ & $\begin{array}{c}d_{\mathrm{r}, \mathrm{b}} \\
\left(^{\prime}\right)\end{array}$ & $\begin{array}{c}\mathrm{PA}_{\mathrm{r}, \mathrm{b}} \\
\left({ }^{\circ}\right)\end{array}$ & $\begin{array}{c}D_{\mathrm{r}, \mathrm{b}, 0} \\
\left(^{\prime}\right)\end{array}$ & $\begin{array}{c}d_{\mathrm{r}, \mathrm{b}, 0} \\
\left(^{\prime}\right)\end{array}$ & $\begin{array}{l}\theta_{\mathrm{r}, \mathrm{b}} \\
\left({ }^{\circ}\right)\end{array}$ \\
\hline$r$ & 0.70 & 0.19 & 88.9 & 0.71 & 0.49 & 4.3 & bars & $\begin{array}{l}1.64 \\
0.62\end{array}$ & $\begin{array}{l}0.64 \\
0.23\end{array}$ & $\begin{array}{c}109.1 \\
51.3\end{array}$ & $\begin{array}{l}1.64 \\
1.08\end{array}$ & $\begin{array}{l}1.25 \\
0.26\end{array}$ & $\begin{array}{c}0.8 \\
105.7\end{array}$ \\
\hline
\end{tabular}

NGC 210

$\left(\mathrm{R}_{1}^{\prime}, \mathrm{R} 2^{\prime} \mathrm{L}\right) \mathrm{SAB}\left(\mathrm{r}^{\prime} 1, \mathrm{nr}\right) \mathrm{ab}$
NGC 244

(L)SA(rs:)m
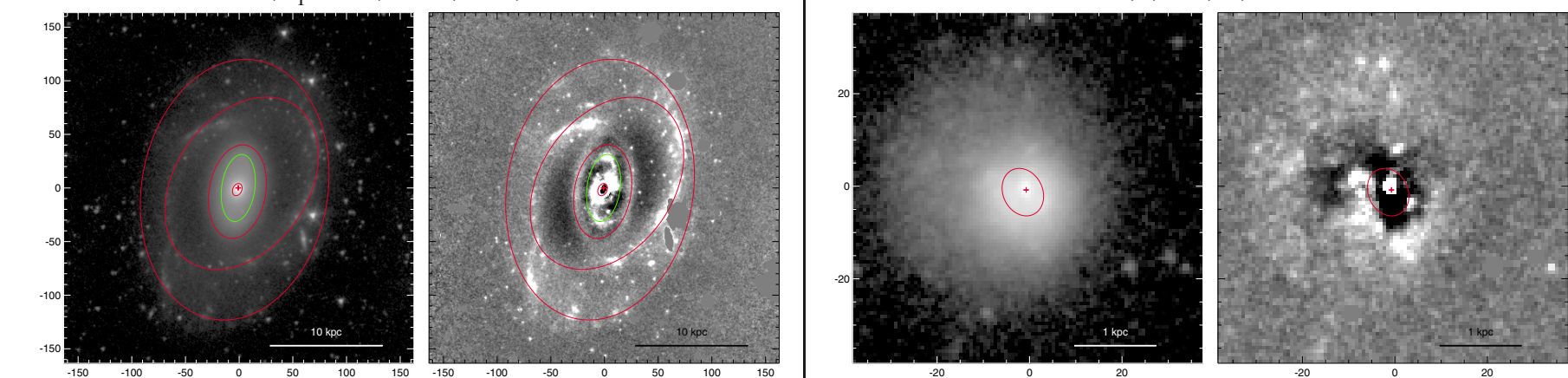

\begin{tabular}{|c|c|c|c|c|c|c|c|c|c|c|c|c|c|}
\hline$\overline{\text { Feature }}$ & $\begin{array}{c}D_{\mathrm{r}, \mathrm{b}} \\
\left(^{\prime}\right)\end{array}$ & $\begin{array}{c}d_{\mathrm{r}, \mathrm{b}} \\
\left(^{\prime}\right)\end{array}$ & $\begin{array}{c}\mathrm{PA}_{\mathrm{r}, \mathrm{b}} \\
\left({ }^{\circ}\right)\end{array}$ & $\begin{array}{c}D_{\mathrm{r}, \mathrm{b}, 0} \\
\left(^{\prime}\right)\end{array}$ & $\begin{array}{c}d_{\mathrm{r}, \mathrm{b}, 0} \\
\left(^{\prime}\right)\end{array}$ & $\begin{array}{l}\theta_{\mathrm{r}, \mathrm{b}} \\
\left({ }^{\circ}\right)\end{array}$ & Feature & $\begin{array}{c}D_{\mathrm{r}, \mathrm{b}} \\
\left(^{\prime}\right)\end{array}$ & $\begin{array}{c}d_{\mathrm{r}, \mathrm{b}} \\
\left(^{\prime}\right)\end{array}$ & $\begin{array}{c}\mathrm{PA}_{\mathrm{r}, \mathrm{b}} \\
\left({ }^{\circ}\right)\end{array}$ & $\begin{array}{c}D_{\mathrm{r}, \mathrm{b}, 0} \\
\left(^{\prime}\right)\end{array}$ & $\begin{array}{c}d_{\mathrm{r}, \mathrm{b}, 0} \\
\left(^{\prime}\right)\end{array}$ & $\begin{array}{l}\theta_{\mathrm{r}, \mathrm{b}} \\
\left({ }^{\circ}\right)\end{array}$ \\
\hline$\overline{\mathrm{R}_{2}^{\prime} \mathrm{L}}$ & 4.08 & 2.90 & 170.4 & 4.56 & 4.00 & 70.1 & rs & 0.18 & 0.14 & 26.1 & 0.18 & 0.15 & 138.2 \\
\hline $\mathrm{R}_{1}^{\prime}$ & 2.94 & 2.06 & 144.8 & 3.49 & 2.67 & 116.9 & & & & & & & \\
\hline $\mathrm{r}^{\prime} 1$ & 1.47 & 0.88 & 169.9 & 1.51 & 1.32 & 30.8 & & & & & & & \\
\hline bar & 1.05 & 0.52 & 172.0 & 1.08 & 0.78 & 21.4 & & & & & & & \\
\hline $\mathrm{nr}$ & 0.20 & 0.14 & 155.5 & 0.22 & 0.19 & 112.7 & & & & & & & \\
\hline
\end{tabular}


NGC 253

$\mathrm{SB}(\underline{\mathrm{r} s}) \mathrm{bc} \mathrm{sp}$
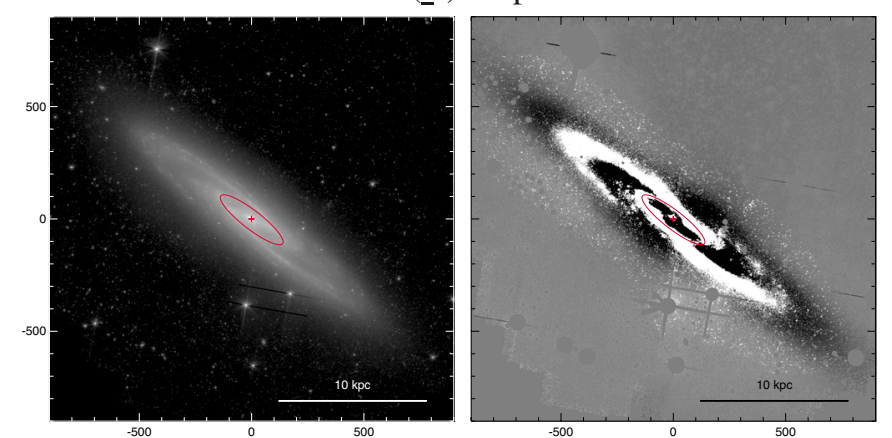

\begin{tabular}{ccccccc|ccccccc}
\hline \hline Feature & $\begin{array}{c}D_{\mathrm{r}, \mathrm{b}} \\
\left({ }^{\prime}\right)\end{array}$ & $\begin{array}{c}d_{\mathrm{r}, \mathrm{b}} \\
\left({ }^{\prime}\right)\end{array}$ & $\begin{array}{c}\mathrm{PA}_{\mathrm{r}, \mathrm{b}} \\
\left({ }^{\circ}\right)\end{array}$ & $\begin{array}{c}D_{\mathrm{r}, \mathrm{b}, 0} \\
\left({ }^{\prime}\right)\end{array}$ & $\begin{array}{c}d_{\mathrm{r}, \mathrm{b}, 0} \\
\left({ }^{\prime}\right)\end{array}$ & $\begin{array}{c}\theta_{\mathrm{r}, \mathrm{b}} \\
\left({ }^{\circ}\right)\end{array}$ & Feature & $\begin{array}{c}D_{\mathrm{r}, \mathrm{b}} \\
\left({ }^{\prime}\right)\end{array}$ & $\begin{array}{c}d_{\mathrm{r}, \mathrm{b}} \\
\left({ }^{\prime}\right)\end{array}$ & $\begin{array}{c}\mathrm{PA}_{\mathrm{r}, \mathrm{b}} \\
\left({ }^{\circ}\right)\end{array}$ & $\begin{array}{c}D_{\mathrm{r}, \mathrm{b}, 0} \\
\left({ }^{\prime}\right)\end{array}$ & $\begin{array}{c}d_{\mathrm{r}, \mathrm{b}, 0} \\
\left({ }^{\prime}\right)\end{array}$ & $\begin{array}{c}\theta_{\mathrm{r}, \mathrm{b}} \\
\left({ }^{\circ}\right)\end{array}$ \\
\hline$\underline{\mathrm{rs}}$ & 5.80 & 1.38 & 52.4 & 7.22 & 5.76 & 80.7 & $\mathrm{R}$ & 1.59 & 1.25 & 139.5 & 1.91 & 1.57 & 79.9 \\
\end{tabular}

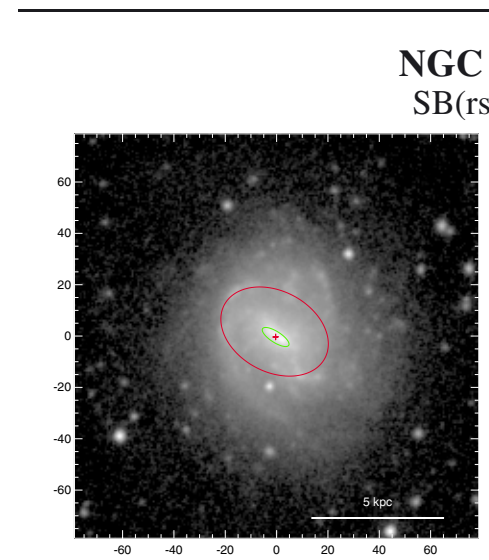

NGC 255

$\mathrm{SB}(\mathrm{rs}) \mathrm{cd}$

\begin{tabular}{|c|c|c|c|c|c|c|c|c|c|c|c|c|c|}
\hline Feature & $\begin{array}{c}D_{\mathrm{r}, \mathrm{b}} \\
\left({ }^{\prime}\right)\end{array}$ & $\begin{array}{c}d_{\mathrm{r}, \mathrm{b}} \\
(')\end{array}$ & $\begin{array}{c}\mathrm{PA}_{\mathrm{r}, \mathrm{b}} \\
\left({ }^{\circ}\right)\end{array}$ & $\begin{array}{c}D_{\mathrm{r}, \mathrm{b}, 0} \\
\left(^{\prime}\right)\end{array}$ & $\begin{array}{c}d_{\mathrm{r}, \mathrm{b}, 0} \\
\left({ }^{\prime}\right)\end{array}$ & $\begin{array}{l}\theta_{\mathrm{r}, \mathrm{b}} \\
\left({ }^{\circ}\right)\end{array}$ & Feature & $\begin{array}{c}D_{\mathrm{r}, \mathrm{b}} \\
\left({ }^{\prime}\right)\end{array}$ & $\begin{array}{c}d_{\mathrm{r}, \mathrm{b}} \\
\left(^{\prime}\right)\end{array}$ & $\begin{array}{c}\mathrm{PA}_{\mathrm{r}, \mathrm{b}} \\
\left({ }^{\circ}\right)\end{array}$ & $\begin{array}{c}D_{\mathrm{r}, \mathrm{b}, 0} \\
\left({ }^{\prime}\right)\end{array}$ & $\begin{array}{c}d_{\mathrm{r}, \mathrm{b}, 0} \\
\left(^{\prime}\right)\end{array}$ & $\begin{array}{l}\theta_{\mathrm{r}, \mathrm{b}} \\
\left({ }^{\circ}\right)\end{array}$ \\
\hline rs & 0.74 & 0.53 & 62.0 & 0.81 & 0.53 & 71.7 & $\mathrm{R}$ & 0.57 & 0.51 & 167.4 & 0.59 & 0.52 & 56.4 \\
\hline bar & 0.20 & 0.07 & 57.4 & 0.22 & 0.07 & 64.9 & & & & & & & \\
\hline
\end{tabular}

NGC 289

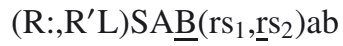
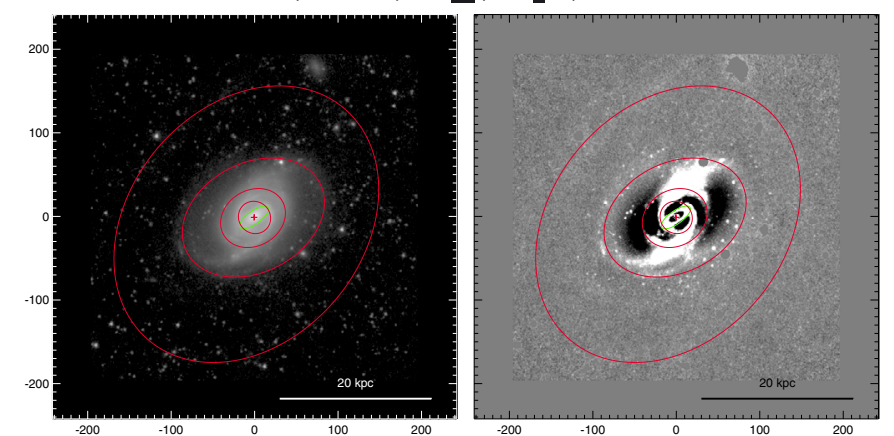

\begin{tabular}{|c|c|c|c|c|c|c|c|c|c|c|c|c|c|}
\hline Feature & $\begin{array}{c}D_{\mathrm{r}, \mathrm{b}} \\
\left(^{\prime}\right)\end{array}$ & $\begin{array}{c}d_{\mathrm{r}, \mathrm{b}} \\
\left({ }^{\prime}\right)\end{array}$ & $\begin{array}{c}\mathrm{PA}_{\mathrm{r}, \mathrm{b}} \\
\left({ }^{\circ}\right)\end{array}$ & $\begin{array}{c}D_{\mathrm{r}, \mathrm{b}, 0} \\
\left(^{\prime}\right)\end{array}$ & $\begin{array}{c}d_{\mathrm{r}, \mathrm{b}, 0} \\
\left(^{\prime}\right)\end{array}$ & $\begin{array}{l}\theta_{\mathrm{r}, \mathrm{b}} \\
\left({ }^{\circ}\right)\end{array}$ & Feature & $\begin{array}{c}D_{\mathrm{r}, \mathrm{b}} \\
\left(^{\prime}\right)\end{array}$ & $\begin{array}{c}d_{\mathrm{r}, \mathrm{b}} \\
\left(^{\prime}\right)\end{array}$ & $\begin{array}{c}\mathrm{PA}_{\mathrm{r}, \mathrm{b}} \\
\left({ }^{\circ}\right)\end{array}$ & $\begin{array}{c}D_{\mathrm{r}, \mathrm{b}, 0} \\
\left(^{\prime}\right)\end{array}$ & $\begin{array}{c}d_{\mathrm{r}, \mathrm{b}, 0} \\
\left(^{\prime}\right)\end{array}$ & $\begin{array}{c}\theta_{\mathrm{r}, \mathrm{b}} \\
\left({ }^{\circ}\right)\end{array}$ \\
\hline $\mathrm{R}$ & 6.07 & 4.68 & 139.9 & 7.19 & 5.79 & 69.9 & rs & 1.23 & 0.69 & 145.3 & 1.43 & 1.13 & 121.6 \\
\hline $\mathrm{R}^{\prime} \mathrm{L}$ & 3.00 & 2.18 & 117.6 & 3.26 & 2.95 & 111.6 & bar & 0.76 & 0.34 & 12.0 & 1.10 & 0.45 & 62.5 \\
\hline rs & 1.35 & 1.11 & 120.4 & 1.63 & 1.35 & 93.4 & & & & & & & \\
\hline bar & 0.69 & 0.26 & 125.4 & 0.69 & 0.38 & 3.1 & & & & & & & \\
\hline rs & 0.66 & 0.63 & 43.2 & 0.97 & 0.63 & 91.1 & & & & & & & \\
\hline
\end{tabular}

NGC 254

(R)SAB(l) $0^{+}$

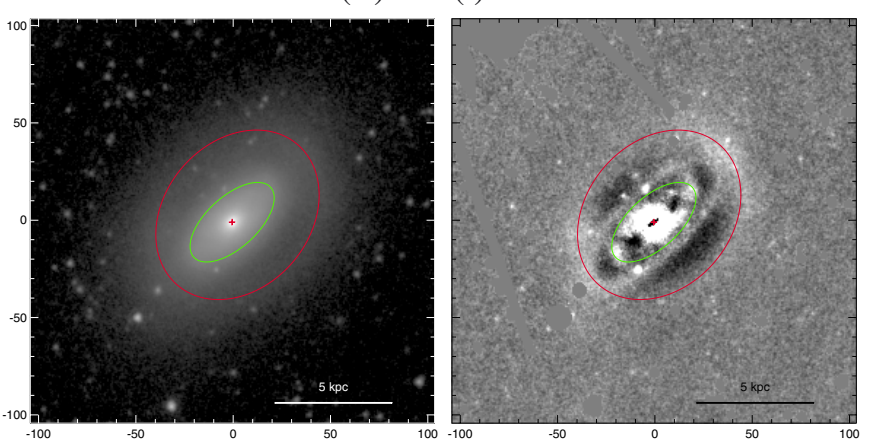

NGC 274

(R)SA(1)0 $0^{-}$

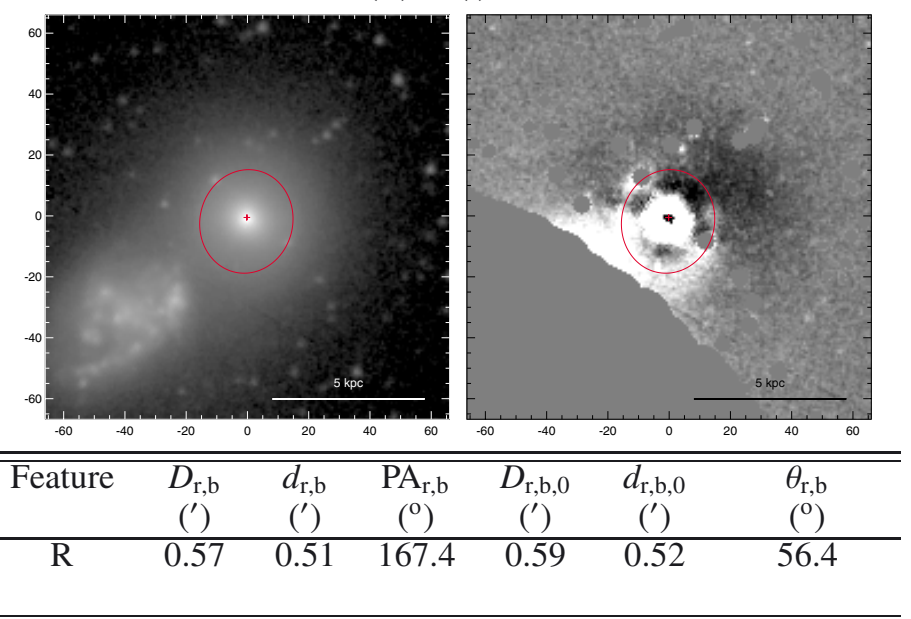

NGC 470

$\mathrm{S} \underline{\mathrm{AB}}(\mathrm{rs}) \mathrm{ab}$

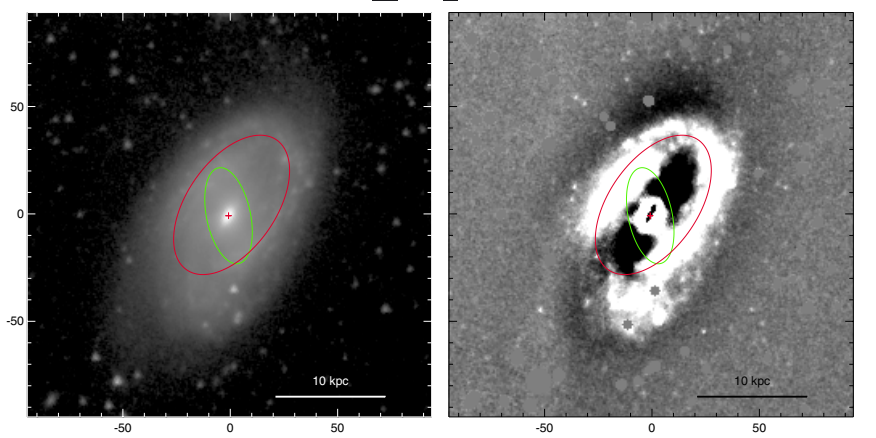


NGC 473

(L)SA(r) $0^{+}$
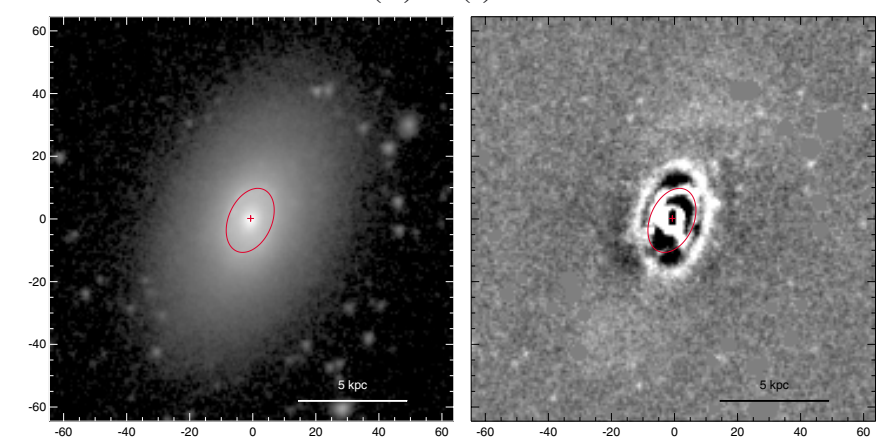

\begin{tabular}{|c|c|c|c|c|c|c|c|c|c|c|c|c|c|}
\hline Feature & $\begin{array}{c}D_{\mathrm{r}, \mathrm{b}} \\
\left(^{\prime}\right)\end{array}$ & $\begin{array}{c}d_{\mathrm{r}, \mathrm{b}} \\
\left({ }^{\prime}\right)\end{array}$ & $\begin{array}{c}\mathrm{PA}_{\mathrm{r}, \mathrm{b}} \\
\left(^{\mathrm{O}}\right)\end{array}$ & $\begin{array}{c}D_{\mathrm{r}, \mathrm{b}, 0} \\
\left(^{\prime}\right)\end{array}$ & $\begin{array}{c}d_{\mathrm{r}, \mathrm{b}, 0} \\
\left(^{\prime}\right)\end{array}$ & $\begin{array}{c}\theta_{\mathrm{r}, \mathrm{b}} \\
\left({ }^{\circ}\right)\end{array}$ & Feature & $\begin{array}{c}D_{\mathrm{r}, \mathrm{b}} \\
\left(^{\prime}\right)\end{array}$ & $\begin{array}{c}d_{\mathrm{r}, \mathrm{b}} \\
\left(^{\prime}\right)\end{array}$ & $\begin{array}{c}\mathrm{PA}_{\mathrm{r}, \mathrm{b}} \\
\left(^{\circ}\right)\end{array}$ & $\begin{array}{c}D_{\mathrm{r}, \mathrm{b}, 0} \\
\left(^{\prime}\right)\end{array}$ & $\begin{array}{c}d_{\mathrm{r}, \mathrm{b}, 0} \\
\left(^{\prime}\right)\end{array}$ & $\begin{array}{l}\theta_{\mathrm{r}, \mathrm{b}} \\
\left({ }^{\circ}\right)\end{array}$ \\
\hline $\mathrm{r}$ & 0.36 & 0.23 & 156.4 & 0.36 & 0.34 & 29.0 & $\begin{array}{l}\mathrm{R}^{\prime} \\
\text { bar }\end{array}$ & $\begin{array}{l}2.23 \\
0.81 \\
\end{array}$ & $\begin{array}{l}2.07 \\
0.63 \\
\end{array}$ & $\begin{array}{l}67.2 \\
16.8\end{array}$ & $\begin{array}{l}2.28 \\
0.84 \\
\end{array}$ & $\begin{array}{l}2.21 \\
0.66 \\
\end{array}$ & $\begin{array}{c}62.5 \\
130.3\end{array}$ \\
\hline
\end{tabular}

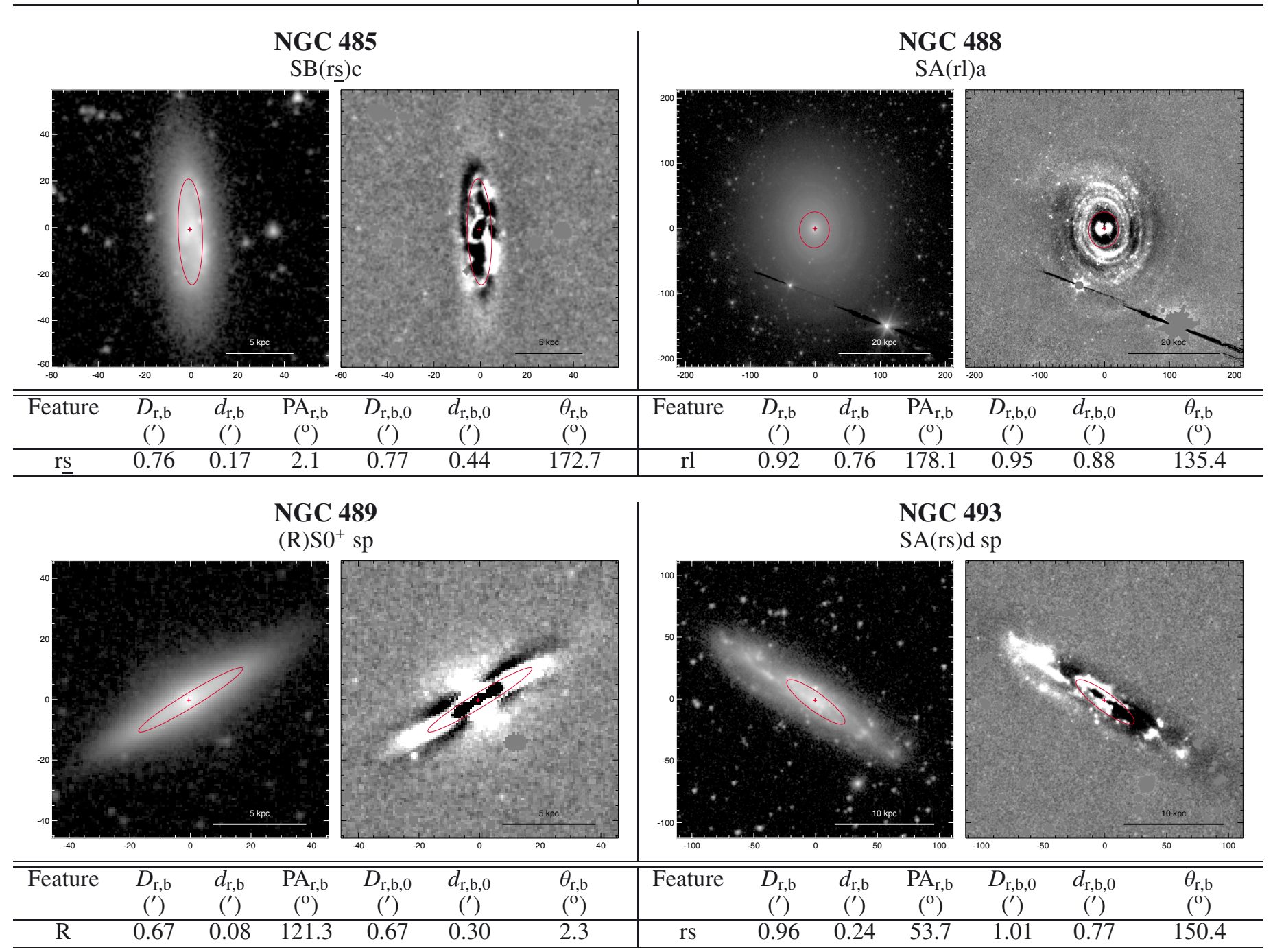

NGC 474

( $\left.\mathrm{R}^{\prime}\right) \mathrm{SAB}(\mathrm{l}) 0 / \mathrm{a}$ (shells/ripples)

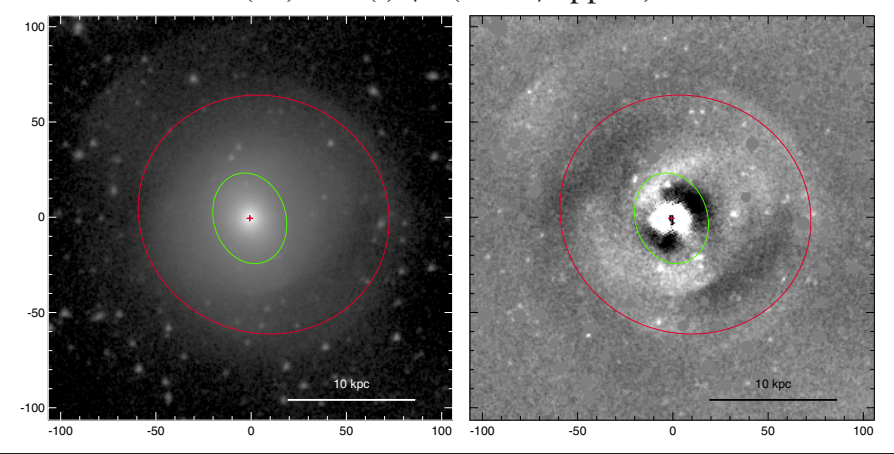

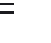


S. Comerón et al.: ARRAKIS

NGC 532

$\mathrm{SAB}_{\mathrm{xa}}(\mathrm{r}) 0^{+}$
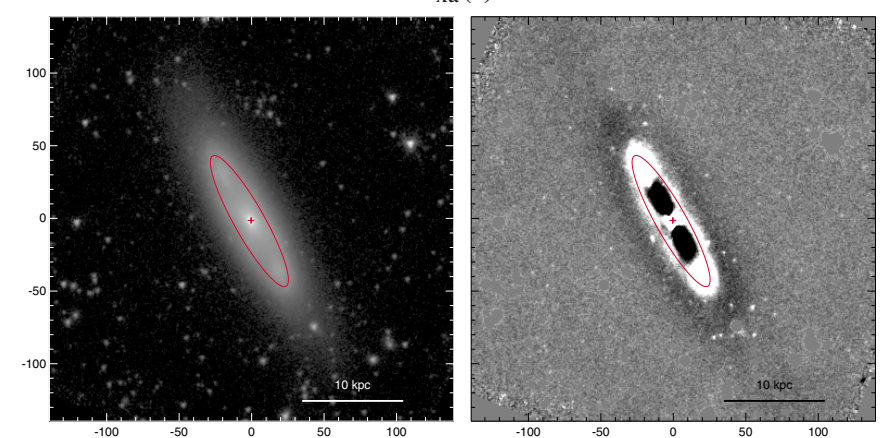

\begin{tabular}{|c|c|c|c|c|c|c|c|c|c|c|c|c|c|}
\hline Feature & $\begin{array}{c}D_{\mathrm{r}, \mathrm{b}} \\
\left({ }^{\prime}\right)\end{array}$ & $\begin{array}{c}d_{\mathrm{r}, \mathrm{b}} \\
\left({ }^{\prime}\right)\end{array}$ & $\begin{array}{c}\mathrm{PA}_{\mathrm{r}, \mathrm{b}} \\
\left({ }^{\circ}\right)\end{array}$ & $\begin{array}{c}D_{\mathrm{r}, \mathrm{b}, 0} \\
\left(^{\prime}\right)\end{array}$ & $\begin{array}{c}d_{\mathrm{r}, \mathrm{b}, 0} \\
\left(^{\prime}\right)\end{array}$ & $\begin{array}{l}\theta_{\mathrm{r}, \mathrm{b}} \\
\left({ }^{\circ}\right)\end{array}$ & Feature & $\begin{array}{c}D_{\mathrm{r}, \mathrm{b}} \\
\left({ }^{\prime}\right)\end{array}$ & $\begin{array}{c}d_{\mathrm{r}, \mathrm{b}} \\
\left({ }^{\prime}\right)\end{array}$ & $\begin{array}{c}\mathrm{PA}_{\mathrm{r}, \mathrm{b}} \\
\left({ }^{\circ}\right)\end{array}$ & $\begin{array}{c}D_{\mathrm{r}, \mathrm{b}, 0} \\
\left(^{\prime}\right)\end{array}$ & $\begin{array}{c}d_{\mathrm{r}, \mathrm{b}, 0} \\
\left(^{\prime}\right)\end{array}$ & $\begin{array}{l}\theta_{\mathrm{r}, \mathrm{b}} \\
\left({ }^{\circ}\right)\end{array}$ \\
\hline $\mathrm{r}$ & 1.72 & 0.35 & 29.4 & 1.72 & 1.24 & 1.4 & $\stackrel{\text { rs }}{\text { bar }}$ & $\begin{array}{l}1.07 \\
0.23\end{array}$ & $\begin{array}{l}0.76 \\
0.04\end{array}$ & $\begin{array}{l}76.0 \\
16.9\end{array}$ & $\begin{array}{l}1.09 \\
0.24\end{array}$ & $\begin{array}{l}0.81 \\
0.04\end{array}$ & $\begin{array}{c}35.4 \\
147.7\end{array}$ \\
\hline
\end{tabular}
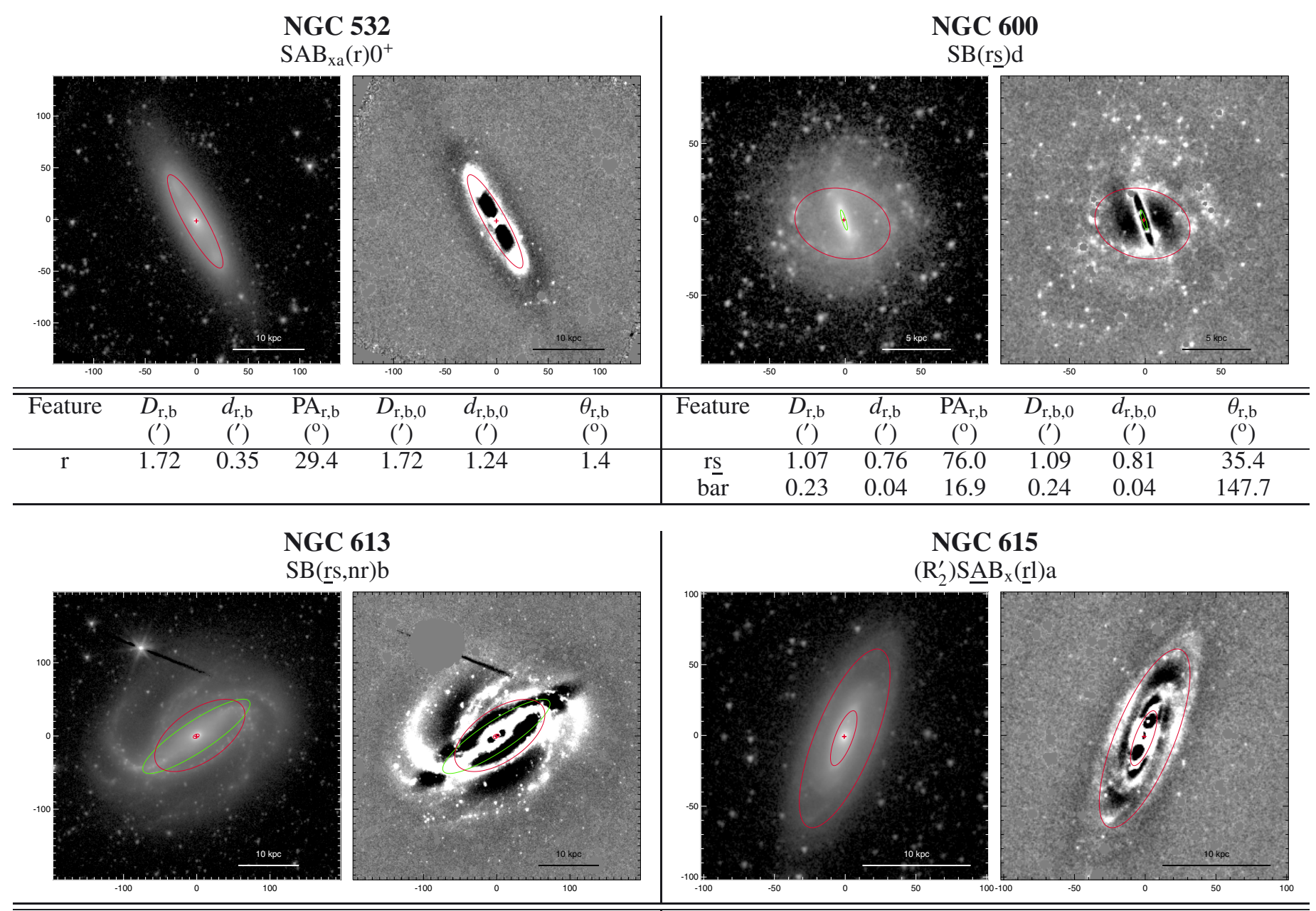

NGC 615

$\left.\mathrm{R}_{2}^{\prime}\right) \underline{S A B}_{\mathrm{x}}(\underline{\mathrm{rl}}) \mathrm{a}$

\begin{tabular}{|c|c|c|c|c|c|c|c|c|c|c|c|c|c|}
\hline Feature & $\begin{array}{c}D_{\mathrm{r}, \mathrm{b}} \\
\left({ }^{\prime}\right)\end{array}$ & $\begin{array}{c}d_{\mathrm{r}, \mathrm{b}} \\
\left(^{\prime}\right)\end{array}$ & $\begin{array}{c}\mathrm{PA}_{\mathrm{r}, \mathrm{b}} \\
\left({ }^{\mathrm{O}}\right)\end{array}$ & $\begin{array}{c}D_{\mathrm{r}, \mathrm{b}, 0} \\
\left(^{\prime}\right)\end{array}$ & $\begin{array}{c}d_{\mathrm{r}, \mathrm{b}, 0} \\
\left({ }^{\prime}\right)\end{array}$ & $\begin{array}{l}\theta_{\mathrm{r}, \mathrm{b}} \\
\left({ }^{\circ}\right)\end{array}$ & Feature & $\begin{array}{c}D_{\mathrm{r}, \mathrm{b}} \\
\left({ }^{\prime}\right)\end{array}$ & $\begin{array}{c}d_{\mathrm{r}, \mathrm{b}} \\
(')\end{array}$ & $\begin{array}{c}\mathrm{PA}_{\mathrm{r}, \mathrm{b}} \\
\left({ }^{\circ}\right)\end{array}$ & $\begin{array}{c}D_{\mathrm{r}, \mathrm{b}, 0} \\
\left(^{\prime}\right)\end{array}$ & $\begin{array}{c}d_{\mathrm{r}, \mathrm{b}, 0} \\
\left(^{\prime}\right)\end{array}$ & $\begin{array}{l}\theta_{\mathrm{r}, \mathrm{b}} \\
\left({ }^{\circ}\right)\end{array}$ \\
\hline bar & 2.90 & 0.70 & 123.3 & 2.90 & 0.90 & 176.6 & $\overline{\mathrm{R}_{2}^{\prime}}$ & 2.26 & 0.70 & 157.8 & 2.26 & 1.72 & 177.3 \\
\hline$\underline{r} s$ & 2.36 & 1.18 & 124.3 & 2.36 & 1.52 & 177.4 & $\underline{\mathrm{rl}}$ & 0.69 & 0.21 & 159.0 & 0.69 & 0.51 & 3.2 \\
\hline $\mathrm{nr}$ & 0.15 & 0.08 & 114.9 & 0.15 & 0.11 & 160.9 & & & & & & & \\
\hline
\end{tabular}

NGC 658

$\mathrm{SA}(\mathrm{rs}) \mathrm{bc}$
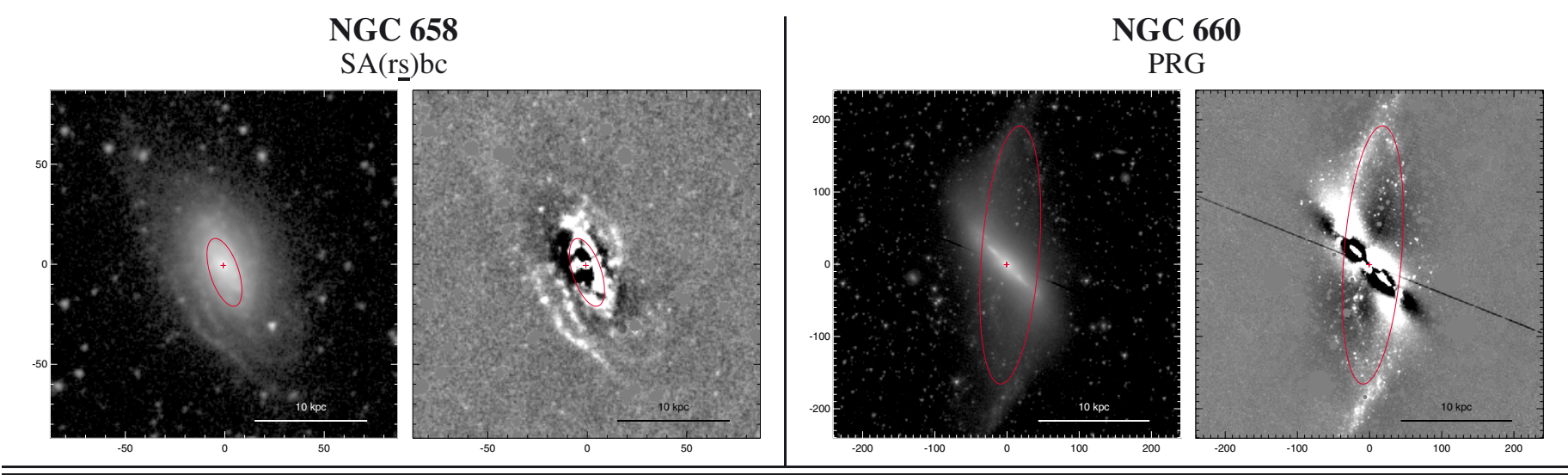

\begin{tabular}{|c|c|c|c|c|c|c|c|c|c|c|c|c|c|}
\hline Feature & $\begin{array}{c}D_{\mathrm{r}, \mathrm{b}} \\
\left(^{\prime}\right)\end{array}$ & $\begin{array}{c}d_{\mathrm{r}, \mathrm{b}} \\
\left(^{\prime}\right)\end{array}$ & $\begin{array}{c}\mathrm{PA}_{\mathrm{r}, \mathrm{b}} \\
\left({ }^{\circ}\right)\end{array}$ & $\begin{array}{c}D_{\mathrm{r}, \mathrm{b}, 0} \\
\left({ }^{\prime}\right)\end{array}$ & $\begin{array}{c}d_{\mathrm{r}, \mathrm{b}, 0} \\
\left(^{\prime}\right)\end{array}$ & $\begin{array}{l}\theta_{\mathrm{r}, \mathrm{b}} \\
\left({ }^{\circ}\right)\end{array}$ & Feature & $\begin{array}{c}D_{\mathrm{r}, \mathrm{b}} \\
\left({ }^{\prime}\right)\end{array}$ & $\begin{array}{c}d_{\mathrm{r}, \mathrm{b}} \\
\left({ }^{\prime}\right)\end{array}$ & $\begin{array}{c}\mathrm{PA}_{\mathrm{r}, \mathrm{b}} \\
\left(^{\circ}\right)\end{array}$ & $\begin{array}{c}D_{\mathrm{r}, \mathrm{b}, 0} \\
\left(^{\prime}\right)\end{array}$ & $\begin{array}{c}d_{\mathrm{r}, \mathrm{b}, 0} \\
\left(^{\prime}\right)\end{array}$ & $\begin{array}{l}\theta_{\mathrm{r}, \mathrm{b}} \\
\left({ }^{\circ}\right)\end{array}$ \\
\hline rs & 0.60 & 0.24 & 18.4 & 0.62 & 0.39 & 157.6 & $\overline{\mathrm{PRG}}$ & 5.98 & 1.33 & 175.5 & - & - & - \\
\hline
\end{tabular}


NGC 672

$\left(\mathrm{R}^{\prime}\right) \mathrm{SB}(\mathrm{s}) \mathrm{d}$
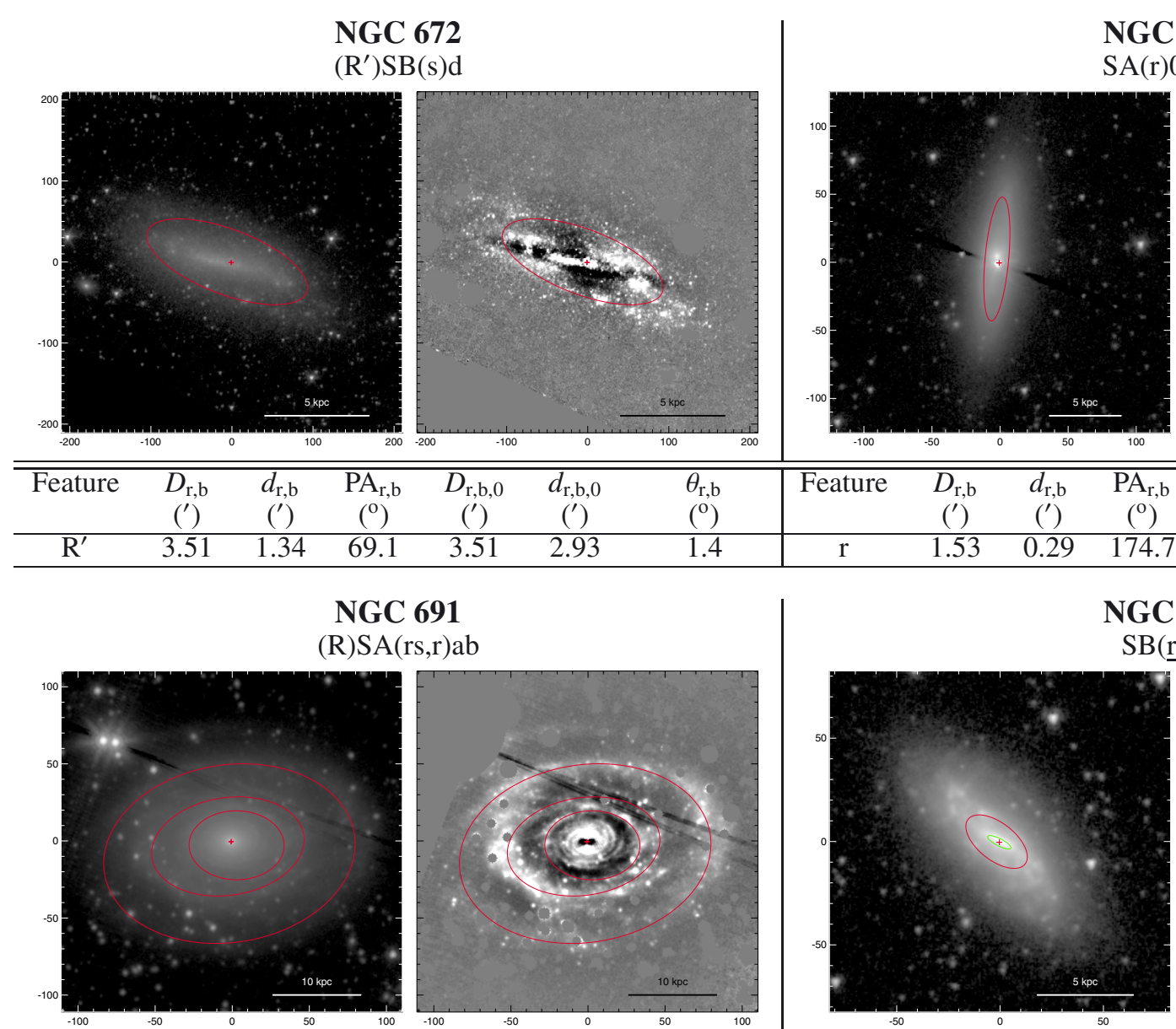

NGC 676

$\mathrm{SA}(\mathrm{r}) 0^{+} \mathrm{sp}$

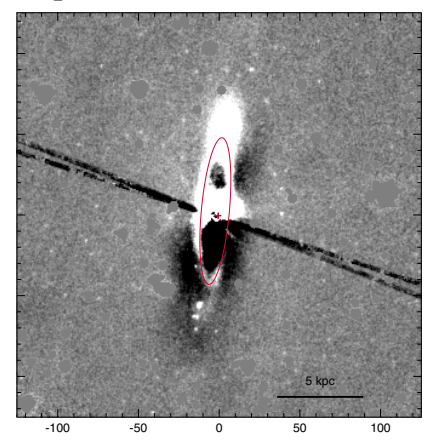

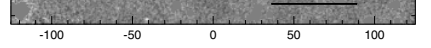

\begin{tabular}{|c|c|c|c|c|c|c|c|c|c|c|c|c|c|}
\hline Feature & $\begin{array}{c}D_{\mathrm{r}, \mathrm{b}} \\
\left({ }^{\prime}\right)\end{array}$ & $\begin{array}{c}d_{\mathrm{r}, \mathrm{b}} \\
\left(^{\prime}\right)\end{array}$ & $\begin{array}{c}\mathrm{PA}_{\mathrm{r}, \mathrm{b}} \\
\left({ }^{\circ}\right)\end{array}$ & $\begin{array}{c}D_{\mathrm{r}, \mathrm{b}, 0} \\
\left({ }^{\prime}\right)\end{array}$ & $\begin{array}{c}d_{\mathrm{r}, \mathrm{b}, 0} \\
\left(^{\prime}\right)\end{array}$ & $\begin{array}{l}\theta_{\mathrm{r}, \mathrm{b}} \\
\left({ }^{\circ}\right)\end{array}$ & Feature & $\begin{array}{c}D_{\mathrm{r}, \mathrm{b}} \\
\left({ }^{\prime}\right)\end{array}$ & $\begin{array}{c}d_{\mathrm{r}, \mathrm{b}} \\
\left({ }^{\prime}\right)\end{array}$ & $\begin{array}{c}\mathrm{PA}_{\mathrm{r}, \mathrm{b}} \\
\left(^{\circ}\right)\end{array}$ & $\begin{array}{c}D_{\mathrm{r}, \mathrm{b}, 0} \\
\left({ }^{\prime}\right)\end{array}$ & $\begin{array}{c}d_{\mathrm{r}, \mathrm{b}, 0} \\
\left(^{\prime}\right)\end{array}$ & $\begin{array}{l}\theta_{\mathrm{r}, \mathrm{b}} \\
\left({ }^{\circ}\right)\end{array}$ \\
\hline$\overline{\mathrm{R}^{\prime}}$ & 3.51 & 1.34 & 69.1 & 3.51 & 2.93 & 1.4 & $\mathrm{r}$ & 1.53 & 0.29 & 174.7 & 1.56 & 0.87 & 15.5 \\
\hline
\end{tabular}

NGC 691

SA(rs,r)ab

\begin{tabular}{|c|c|c|c|c|c|c|c|c|c|c|c|c|c|}
\hline$\overline{\text { Feature }}$ & $\begin{array}{c}D_{\mathrm{r}, \mathrm{b}} \\
\left({ }^{\prime}\right)\end{array}$ & $\begin{array}{c}d_{\mathrm{r}, \mathrm{b}} \\
\left(^{\prime}\right)\end{array}$ & $\begin{array}{c}\mathrm{PA}_{\mathrm{r}, \mathrm{b}} \\
\left({ }^{\circ}\right)\end{array}$ & $\begin{array}{c}D_{\mathrm{r}, \mathrm{b}, 0} \\
\left(^{\prime}\right)\end{array}$ & $\begin{array}{c}d_{\mathrm{r}, \mathrm{b}, 0} \\
\left({ }^{\prime}\right)\end{array}$ & $\begin{array}{l}\theta_{\mathrm{r}, \mathrm{b}} \\
\left({ }^{\circ}\right)\end{array}$ & Feature & $\begin{array}{c}D_{\mathrm{r}, \mathrm{b}} \\
\left({ }^{\prime}\right)\end{array}$ & $\begin{array}{c}d_{\mathrm{r}, \mathrm{b}} \\
\left(^{\prime}\right)\end{array}$ & $\begin{array}{c}\mathrm{PA}_{\mathrm{r}, \mathrm{b}} \\
\left({ }^{\circ}\right)\end{array}$ & $\begin{array}{c}D_{\mathrm{r}, \mathrm{b}, 0} \\
\left({ }^{\prime}\right)\end{array}$ & $\begin{array}{c}d_{\mathrm{r}, \mathrm{b}, 0} \\
\left({ }^{\prime}\right)\end{array}$ & $\begin{array}{l}\theta_{\mathrm{r}, \mathrm{b}} \\
\left({ }^{\circ}\right)\end{array}$ \\
\hline$\overline{\mathrm{R}}$ & 2.74 & 1.93 & 98.8 & 2.79 & 2.55 & 29.0 & rs & 0.59 & 0.30 & 51.9 & 0.64 & 0.53 & 47.5 \\
\hline rs & 1.67 & 1.06 & 98.6 & 1.68 & 1.41 & 17.0 & bar & 0.21 & 0.06 & 63.0 & 0.24 & 0.10 & 37.4 \\
\hline $\mathrm{r}$ & 1.03 & 0.75 & 89.5 & 1.04 & 1.00 & 151.7 & & & & & & & \\
\hline
\end{tabular}

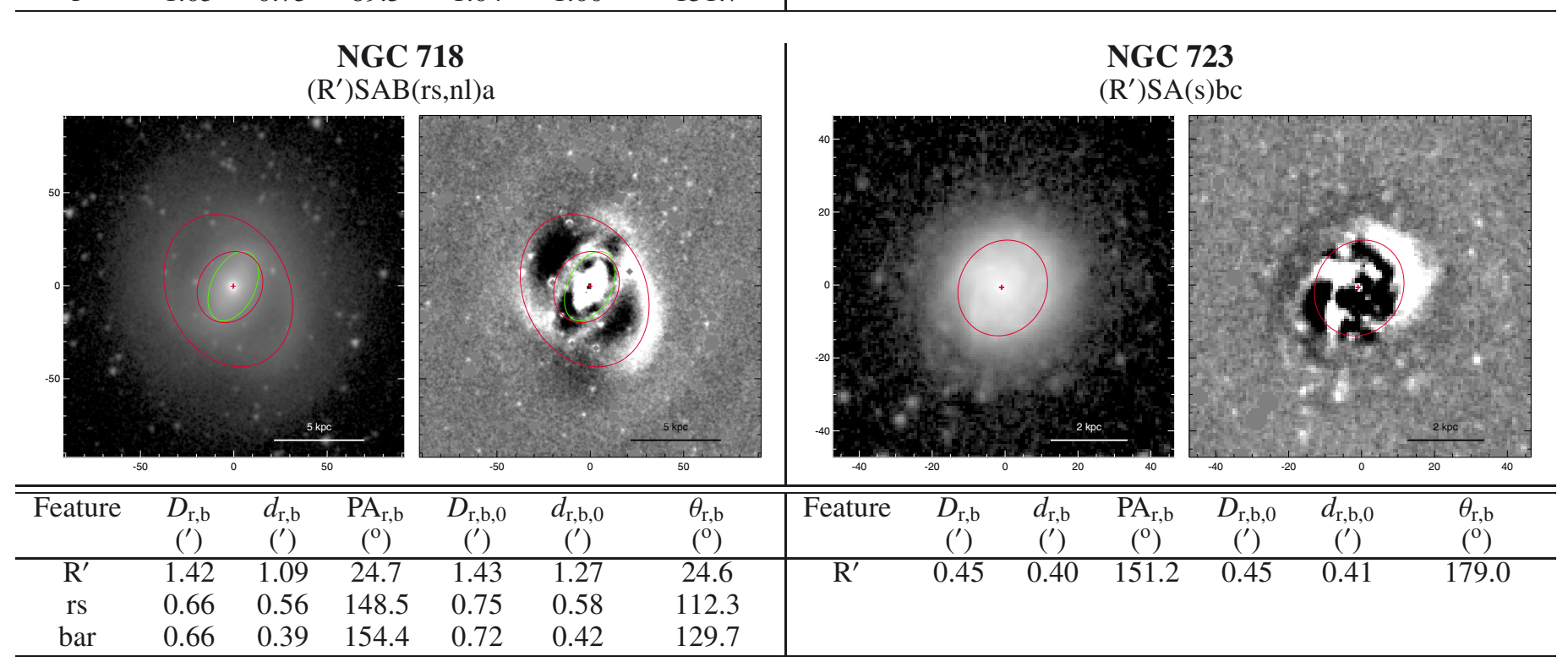


NGC 755

$\mathrm{SB}(\mathrm{rs}) \mathrm{d}$
NGC 779

$(\mathrm{L}) \mathrm{SAB}_{\mathrm{x}}(\mathrm{rs}) \mathrm{a}$
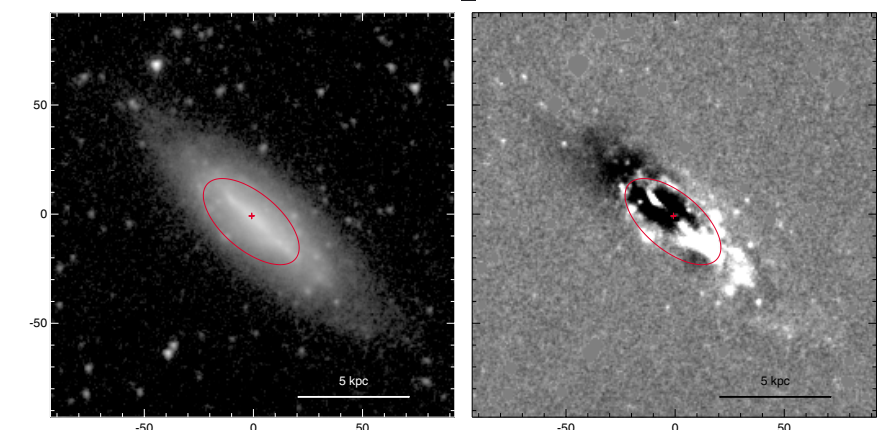

\begin{tabular}{ccccccc|ccccccc}
\hline \hline Feature & $\begin{array}{c}D_{\mathrm{r}, \mathrm{b}} \\
\left({ }^{\prime}\right)\end{array}$ & $\begin{array}{c}d_{\mathrm{r}, \mathrm{b}} \\
\left({ }^{\prime}\right)\end{array}$ & $\begin{array}{c}\mathrm{PA}_{\mathrm{r}, \mathrm{b}} \\
\left({ }^{\circ}\right)\end{array}$ & $\begin{array}{c}D_{\mathrm{r}, \mathrm{b}, 0} \\
\left({ }^{\prime}\right)\end{array}$ & $\begin{array}{c}d_{\mathrm{r}, \mathrm{b}, 0} \\
\left({ }^{\prime}\right)\end{array}$ & $\begin{array}{c}\theta_{\mathrm{r}, \mathrm{b}} \\
\left({ }^{\circ}\right)\end{array}$ & Feature & $\begin{array}{c}D_{\mathrm{r}, \mathrm{b}} \\
\left({ }^{\prime}\right)\end{array}$ & $\begin{array}{c}d_{\mathrm{r}, \mathrm{b}} \\
\left({ }^{\prime}\right)\end{array}$ & $\begin{array}{c}\mathrm{PA}_{\mathrm{r}, \mathrm{b}} \\
\left({ }^{\circ}\right)\end{array}$ & $\begin{array}{c}D_{\mathrm{r}, \mathrm{b}, 0} \\
\left({ }^{\prime}\right)\end{array}$ & $\begin{array}{c}d_{\mathrm{r}, \mathrm{b}, 0} \\
\left({ }^{\prime}\right)\end{array}$ & $\begin{array}{c}\theta_{\mathrm{r}, \mathrm{b}} \\
\left({ }^{\circ}\right)\end{array}$ \\
\hline $\mathrm{r} \underline{\mathrm{r}}$ & 0.89 & 0.44 & 50.0 & 1.18 & 0.89 & 89.5 & $\mathrm{rs}$ & 0.94 & 0.30 & 163.4 & 0.98 & 0.85 & 33.8 \\
\hline
\end{tabular}

NGC 864

$\mathrm{SA} \underline{\mathrm{B}}(\mathrm{rs}) \mathrm{bc}$
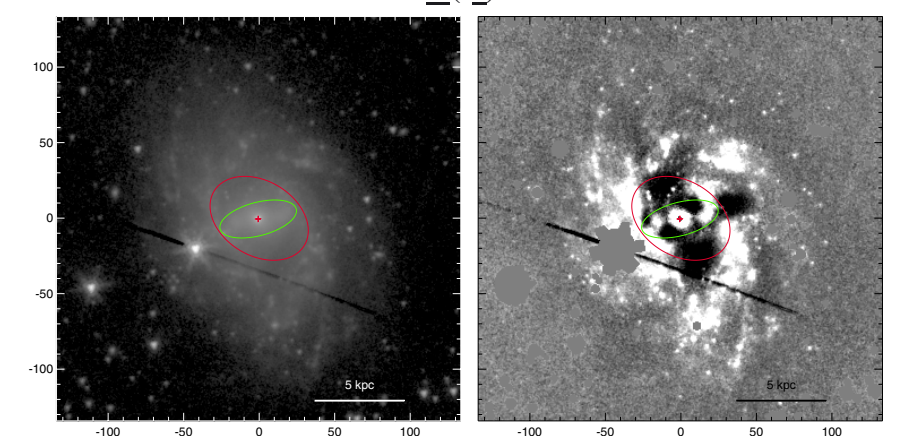

\begin{tabular}{ccccccc|ccccccc}
\hline \hline Feature & $\begin{array}{c}D_{\mathrm{r}, \mathrm{b}} \\
\left({ }^{\prime}\right)\end{array}$ & $\begin{array}{c}d_{\mathrm{r}, \mathrm{b}} \\
\left({ }^{\prime}\right)\end{array}$ & $\begin{array}{c}\mathrm{PA}_{\mathrm{r}, \mathrm{b}} \\
\left({ }^{\circ}\right)\end{array}$ & $\begin{array}{c}D_{\mathrm{r}, \mathrm{b}, 0} \\
\left({ }^{\prime}\right)\end{array}$ & $\begin{array}{c}d_{\mathrm{r}, \mathrm{b}, 0} \\
\left({ }^{\prime}\right)\end{array}$ & $\begin{array}{c}\theta_{\mathrm{r}, \mathrm{b}} \\
\left({ }^{\circ}\right)\end{array}$ & Feature & $\begin{array}{c}D_{\mathrm{r}, \mathrm{b}} \\
\left({ }^{\prime}\right)\end{array}$ & $\begin{array}{c}d_{\mathrm{r}, \mathrm{b}} \\
\left({ }^{\prime}\right)\end{array}$ & $\begin{array}{c}\mathrm{PA}_{\mathrm{r}, \mathrm{b}} \\
\left({ }^{\circ}\right)\end{array}$ & $\begin{array}{c}D_{\mathrm{r}, \mathrm{b}, 0} \\
\left({ }^{\prime}\right)\end{array}$ & $\begin{array}{c}d_{\mathrm{r}, \mathrm{b}, 0} \\
\left({ }^{\prime}\right)\end{array}$ & $\begin{array}{c}\theta_{\mathrm{r}, \mathrm{b}} \\
\left({ }^{\circ}\right)\end{array}$ \\
\hline $\mathrm{rs}$ & 1.17 & 0.83 & 59.2 & 1.46 & 0.97 & 64.8 & $\underline{\mathrm{rs}}$ & 0.29 & 0.19 & 94.6 & 0.32 & 0.24 & 138.9 \\
$\mathrm{bar}$ & 0.87 & 0.37 & 104.8 & 1.27 & 0.37 & 83.1 & bar & 0.14 & 0.08 & 158.9 & 0.17 & 0.09 & 64.4 \\
\hline
\end{tabular}

NGC 908

$\mathrm{SA}(\mathrm{rs}) \mathrm{b}$
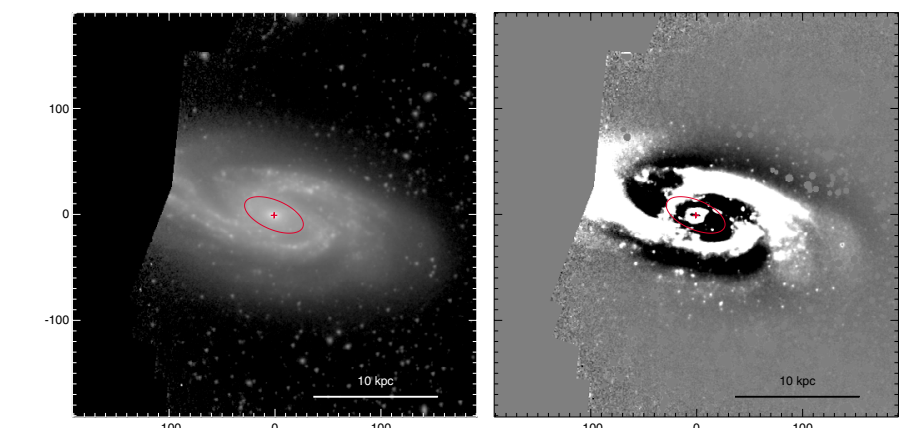

\begin{tabular}{|c|c|c|c|c|c|c|c|c|c|c|c|c|c|}
\hline Feature & $\begin{array}{c}D_{\mathrm{r}, \mathrm{b}} \\
\left({ }^{\prime}\right)\end{array}$ & $\begin{array}{c}d_{\mathrm{r}, \mathrm{b}} \\
\left(^{\prime}\right)\end{array}$ & $\begin{array}{c}\mathrm{PA}_{\mathrm{r}, \mathrm{b}} \\
\left({ }^{\mathrm{o}}\right)\end{array}$ & $\begin{array}{c}D_{\mathrm{r}, \mathrm{b}, 0} \\
\left(^{\prime}\right)\end{array}$ & $\begin{array}{c}d_{\mathrm{r}, \mathrm{b}, 0} \\
\left({ }^{\prime}\right)\end{array}$ & $\begin{array}{l}\theta_{\mathrm{r}, \mathrm{b}} \\
\left({ }^{\circ}\right)\end{array}$ & Feature & $\begin{array}{c}D_{\mathrm{r}, \mathrm{b}} \\
\left({ }^{\prime}\right)\end{array}$ & $\begin{array}{c}d_{\mathrm{r}, \mathrm{b}} \\
\left({ }^{\prime}\right)\end{array}$ & $\begin{array}{c}\mathrm{PA}_{\mathrm{r}, \mathrm{b}} \\
\left({ }^{\circ}\right)\end{array}$ & $\begin{array}{c}D_{\mathrm{r}, \mathrm{b}, 0} \\
\left(^{\prime}\right)\end{array}$ & $\begin{array}{c}d_{\mathrm{r}, \mathrm{b}, 0} \\
\left(^{\prime}\right)\end{array}$ & $\begin{array}{c}\theta_{\mathrm{r}, \mathrm{b}} \\
\left({ }^{\circ}\right)\end{array}$ \\
\hline $\mathrm{rs}$ & 0.99 & 0.46 & 66.8 & 1.08 & 0.80 & 140.6 & $\begin{array}{l}\text { rs } \\
\text { bar }\end{array}$ & $\begin{array}{l}1.55 \\
1.33\end{array}$ & $\begin{array}{l}.22 \\
0.69\end{array}$ & $\begin{array}{c}139.8 \\
81.2\end{array}$ & $\begin{array}{l}1.71 \\
1.61\end{array}$ & $\begin{array}{l}1.49 \\
0.77\end{array}$ & $\begin{array}{c}64.1 \\
120.6\end{array}$ \\
\hline
\end{tabular}

NGC 936

(L) $\mathrm{SB}(\underline{\mathrm{rs}}, \mathrm{bl}) 0^{+}$
NGC 895

$\mathrm{SAB}(\underline{\mathrm{r} s}) \mathrm{c}$

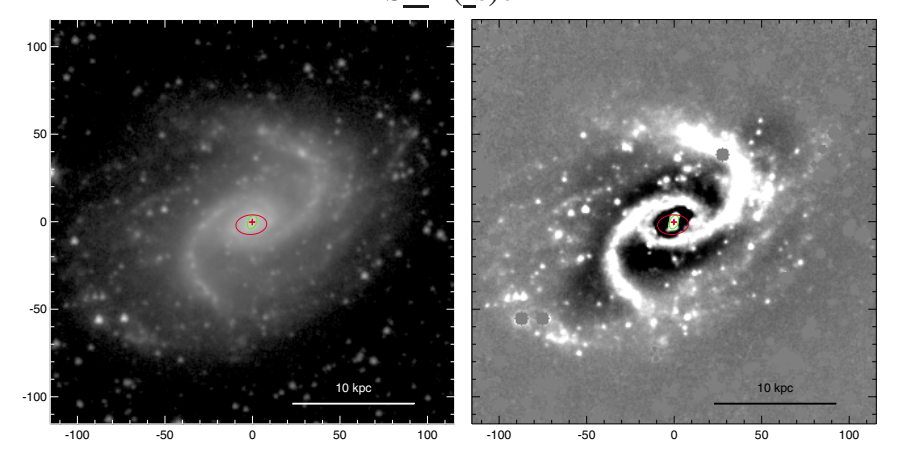




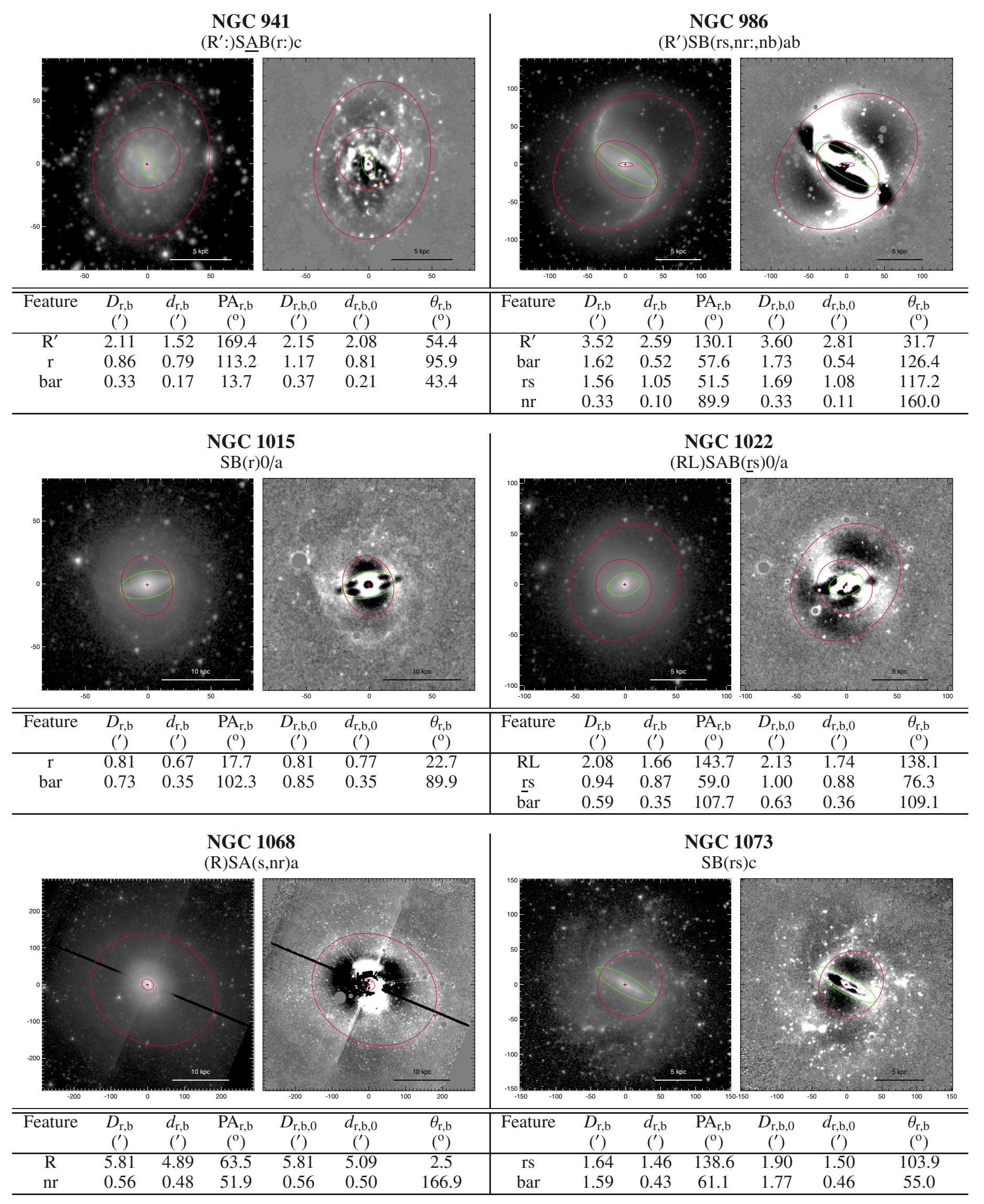


NGC 1079

(L) $\mathrm{SAB}_{\mathrm{a}}(\mathrm{rs}, \mathrm{bl}) 0^{+}$
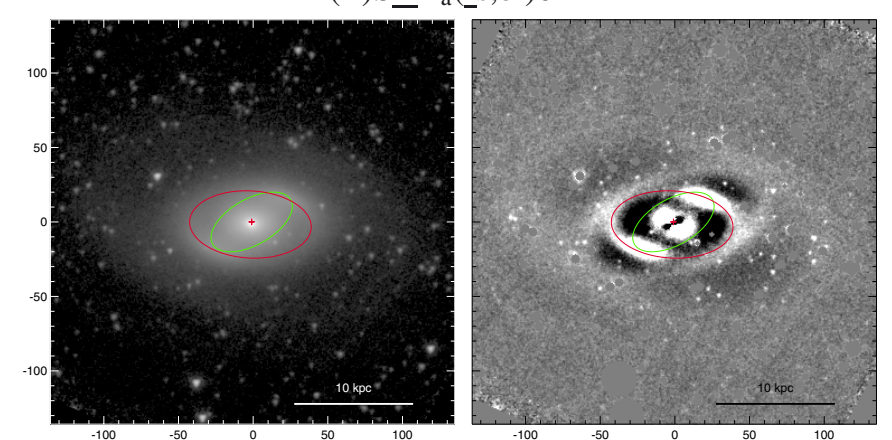

NGC 1087

SB(rs)d

\begin{tabular}{ccccccc|ccccccc}
\hline \hline Feature & $\begin{array}{c}D_{\mathrm{r}, \mathrm{b}} \\
\left({ }^{\prime}\right)\end{array}$ & $\begin{array}{c}d_{\mathrm{r}, \mathrm{b}} \\
\left({ }^{\prime}\right)\end{array}$ & $\begin{array}{c}\mathrm{PA}_{\mathrm{r}, \mathrm{b}} \\
\left({ }^{\mathrm{O}}\right)\end{array}$ & $\begin{array}{c}D_{\mathrm{r}, \mathrm{b}, 0} \\
\left({ }^{\prime}\right)\end{array}$ & $\begin{array}{c}d_{\mathrm{r}, \mathrm{b}, 0} \\
\left({ }^{\prime}\right)\end{array}$ & $\begin{array}{c}\theta_{\mathrm{r}, \mathrm{b}} \\
\left({ }^{\circ}\right)\end{array}$ & Feature & $\begin{array}{c}D_{\mathrm{r}, \mathrm{b}} \\
\left({ }^{\prime}\right)\end{array}$ & $\begin{array}{c}d_{\mathrm{r}, \mathrm{b}} \\
\left({ }^{\prime}\right)\end{array}$ & $\begin{array}{c}\mathrm{PA}_{\mathrm{r}, \mathrm{b}} \\
\left({ }^{\circ}\right)\end{array}$ & $\begin{array}{c}D_{\mathrm{r}, \mathrm{b}, 0} \\
\left({ }^{\prime}\right)\end{array}$ & $\begin{array}{c}d_{\mathrm{r}, \mathrm{b}, 0} \\
\left({ }^{\prime}\right)\end{array}$ & $\begin{array}{c}\theta_{\mathrm{r}, \mathrm{b}} \\
\left({ }^{\circ}\right)\end{array}$ \\
\hline$\underline{\mathrm{rs}}$ & 1.36 & 0.75 & 86.8 & 1.42 & 1.18 & 32.8 & $\mathrm{rs}$ & 0.62 & 0.49 & 14.1 & 0.79 & 0.61 & 78.2 \\
bar & 1.01 & 0.51 & 119.5 & 1.37 & 0.62 & 62.7 & $\mathrm{bar}$ & 0.50 & 0.22 & 128.5 & 0.71 & 0.25 & 111.9 \\
\hline
\end{tabular}

NGC 1090

$\mathrm{SAB}\left(\mathrm{s}, \mathrm{nr} \mathrm{r}^{\prime} \mathrm{l}\right) \mathrm{bc}$
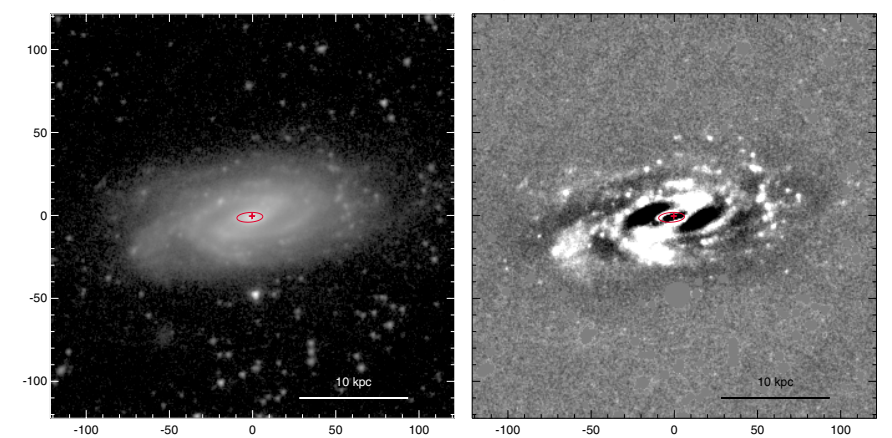

NGC 1097

$\left(\mathrm{R}^{\prime}\right) \mathrm{SB}(\mathrm{rs}, \mathrm{nr}) \mathrm{ab}$ pec

\begin{tabular}{|c|c|c|c|c|c|c|c|c|c|c|c|c|c|}
\hline Feature & $\begin{array}{c}D_{\mathrm{r}, \mathrm{b}} \\
\left({ }^{\prime}\right)\end{array}$ & $\begin{array}{c}d_{\mathrm{r}, \mathrm{b}} \\
\left({ }^{\prime}\right)\end{array}$ & $\begin{array}{c}\overline{\mathrm{PA}_{\mathrm{r}, \mathrm{b}}} \\
\left({ }^{\circ}\right)\end{array}$ & $\begin{array}{c}D_{\mathrm{r}, \mathrm{b}, 0} \\
\left(^{\prime}\right)\end{array}$ & $\begin{array}{c}d_{\mathrm{r}, \mathrm{b}, 0} \\
\left({ }^{\prime}\right)\end{array}$ & $\begin{array}{l}\theta_{\mathrm{r}, \mathrm{b}} \\
\left({ }^{\circ}\right)\end{array}$ & Feature & $\begin{array}{c}D_{\mathrm{r}, \mathrm{b}} \\
\left({ }^{\prime}\right)\end{array}$ & $\begin{array}{c}d_{\mathrm{r}, \mathrm{b}} \\
\left({ }^{\prime}\right)\end{array}$ & $\begin{array}{c}\mathrm{PA}_{\mathrm{r}, \mathrm{b}} \\
\left({ }^{\circ}\right)\end{array}$ & $\begin{array}{c}D_{\mathrm{r}, \mathrm{b}, 0} \\
\left(^{\prime}\right)\end{array}$ & $\begin{array}{c}d_{\mathrm{r}, \mathrm{b}, 0} \\
\left({ }^{\prime}\right)\end{array}$ & $\begin{array}{l}\overline{\theta_{\mathrm{r}, \mathrm{b}}} \\
\left({ }^{\circ}\right)\end{array}$ \\
\hline \multirow[t]{4}{*}{$\overline{n r^{\prime} 1}$} & 0.26 & 0.10 & 93.7 & 0.27 & 0.21 & 155.4 & $\overline{\mathrm{R}^{\prime}}$ & 7.18 & 4.92 & 105.7 & 8.38 & 6.31 & 121.4 \\
\hline & & & & & & & rs & 3.44 & 2.03 & 136.9 & 3.60 & 2.91 & 33.2 \\
\hline & & & & & & & bar & 3.17 & 1.12 & 147.0 & 3.44 & 1.55 & 33.3 \\
\hline & & & & & & & $\mathrm{nr}$ & 0.34 & 0.29 & 167.3 & 0.47 & 0.31 & 80.1 \\
\hline
\end{tabular}

NGC 1163

$\mathrm{SA}(\mathrm{r}:) 0^{+} \mathrm{sp}$
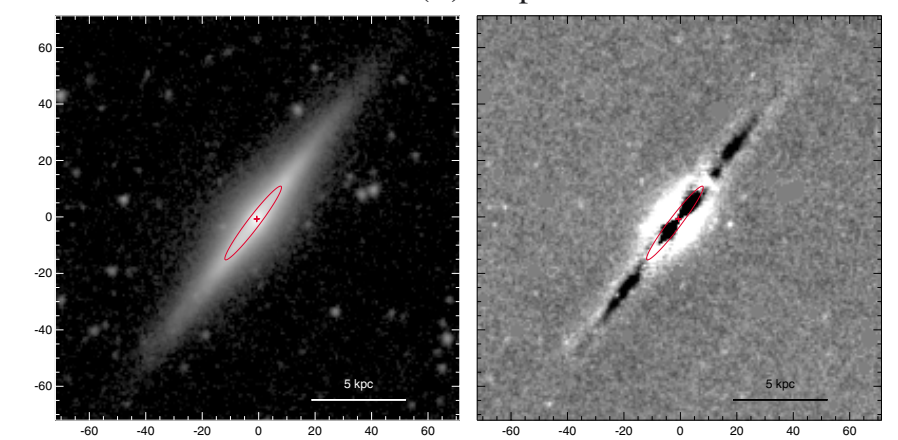

NGC 1179

$\mathrm{SAB}$ (rs)cd

\begin{tabular}{|c|c|c|c|c|c|c|c|c|c|c|c|c|c|}
\hline Feature & $\begin{array}{c}D_{\mathrm{r}, \mathrm{b}} \\
\left({ }^{\prime}\right)\end{array}$ & $\begin{array}{c}d_{\mathrm{r}, \mathrm{b}} \\
\left({ }^{\prime}\right)\end{array}$ & $\begin{array}{c}\mathrm{PA}_{\mathrm{r}, \mathrm{b}} \\
\left({ }^{\mathrm{O}}\right)\end{array}$ & $\begin{array}{c}D_{\mathrm{r}, \mathrm{b}, 0} \\
\left(^{\prime}\right)\end{array}$ & $\begin{array}{c}d_{\mathrm{r}, \mathrm{b}, 0} \\
\left(^{\prime}\right)\end{array}$ & $\begin{array}{l}\theta_{\mathrm{r}, \mathrm{b}} \\
\left({ }^{\circ}\right)\end{array}$ & Feature & $\begin{array}{c}D_{\mathrm{r}, \mathrm{b}} \\
\left({ }^{\prime}\right)\end{array}$ & $\begin{array}{c}d_{\mathrm{r}, \mathrm{b}} \\
\left({ }^{\prime}\right)\end{array}$ & $\begin{array}{c}\mathrm{PA}_{\mathrm{r}, \mathrm{b}} \\
\left({ }^{\circ}\right)\end{array}$ & $\begin{array}{c}D_{\mathrm{r}, \mathrm{b}, 0} \\
\left(^{\prime}\right)\end{array}$ & $\begin{array}{c}d_{\mathrm{r}, \mathrm{b}, 0} \\
\left(^{\prime}\right)\end{array}$ & $\begin{array}{l}\theta_{\mathrm{r}, \mathrm{b}} \\
\left({ }^{\circ}\right)\end{array}$ \\
\hline$r$ & 0.55 & 0.07 & 142.7 & 0.55 & 0.30 & 178.3 & bars & $\begin{array}{l}1.18 \\
0.44\end{array}$ & $\begin{array}{l}1.10 \\
0.21\end{array}$ & $\begin{array}{c}46.0 \\
154.4\end{array}$ & $\begin{array}{l}1.33 \\
0.53\end{array}$ & $\begin{array}{l}1.18 \\
0.21\end{array}$ & $\begin{array}{r}92.0 \\
101.7\end{array}$ \\
\hline
\end{tabular}


NGC 1187

$\mathrm{SAB}$ (rs)bc
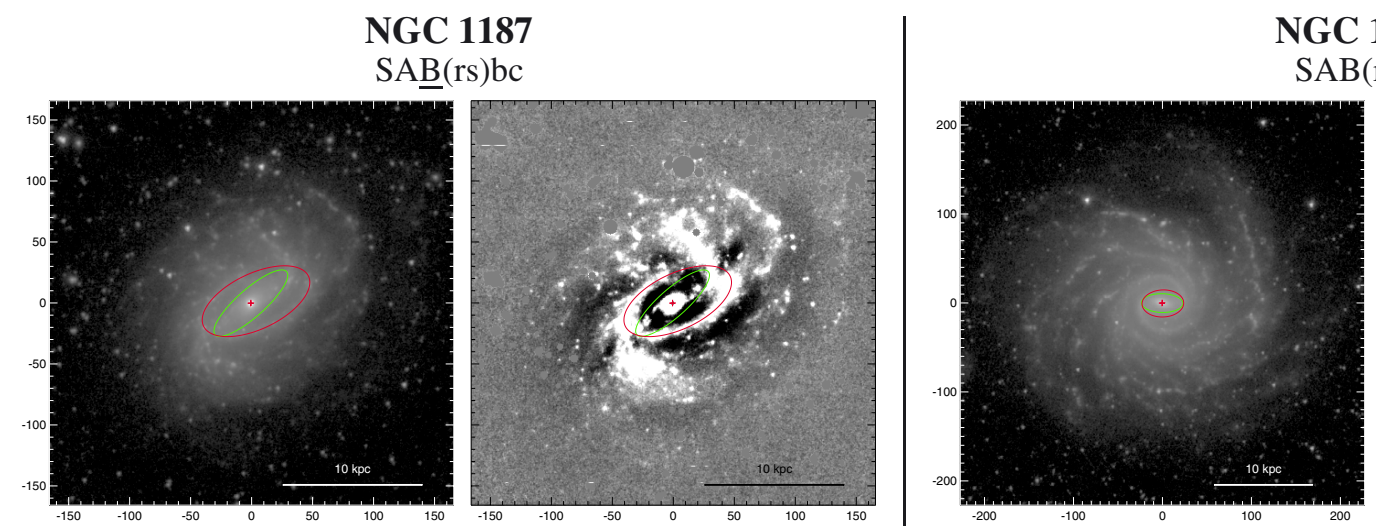

NGC 1232

$\mathrm{SAB}(\mathrm{rs}) \mathrm{c}$

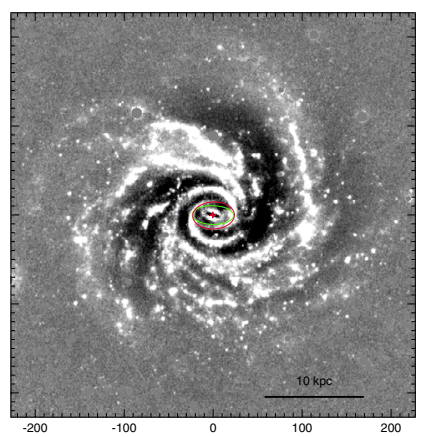

\begin{tabular}{|c|c|c|c|c|c|c|c|c|c|c|c|c|c|}
\hline Feature & $\begin{array}{c}D_{\mathrm{r}, \mathrm{b}} \\
\left({ }^{\prime}\right)\end{array}$ & $\begin{array}{c}d_{\mathrm{r}, \mathrm{b}} \\
(')\end{array}$ & $\begin{array}{c}\mathrm{PA}_{\mathrm{r}, \mathrm{b}} \\
\left(^{\mathrm{O}}\right)\end{array}$ & $\begin{array}{c}D_{\mathrm{r}, \mathrm{b}, 0} \\
\left(^{\prime}\right)\end{array}$ & $\begin{array}{c}d_{\mathrm{r}, \mathrm{b}, 0} \\
\left(^{\prime}\right)\end{array}$ & $\begin{array}{l}\theta_{\mathrm{r}, \mathrm{b}} \\
\left({ }^{\circ}\right)\end{array}$ & Feature & $\begin{array}{c}D_{\mathrm{r}, \mathrm{b}} \\
\left({ }^{\prime}\right)\end{array}$ & $\begin{array}{c}d_{\mathrm{r}, \mathrm{b}} \\
\left({ }^{\prime}\right)\end{array}$ & $\begin{array}{c}\mathrm{PA}_{\mathrm{r}, \mathrm{b}} \\
\left(^{\circ}\right)\end{array}$ & $\begin{array}{c}D_{\mathrm{r}, \mathrm{b}, 0} \\
\left(^{\prime}\right)\end{array}$ & $\begin{array}{c}d_{\mathrm{r}, \mathrm{b}, 0} \\
\left(^{\prime}\right)\end{array}$ & $\begin{array}{l}\theta_{\mathrm{r}, \mathrm{b}} \\
\left(^{\circ}\right)\end{array}$ \\
\hline rs & 1.59 & 0.76 & 115.5 & 1.60 & 1.02 & 167.4 & rs & 0.78 & 0.51 & 92.2 & 0.78 & 0.59 & 177.0 \\
\hline bar & 1.31 & 0.38 & 131.5 & 1.32 & 0.51 & 12.9 & bar & 0.78 & 0.35 & 88.9 & 0.78 & 0.41 & 173.2 \\
\hline
\end{tabular}

\section{NGC 1249}

SB(rs:)m
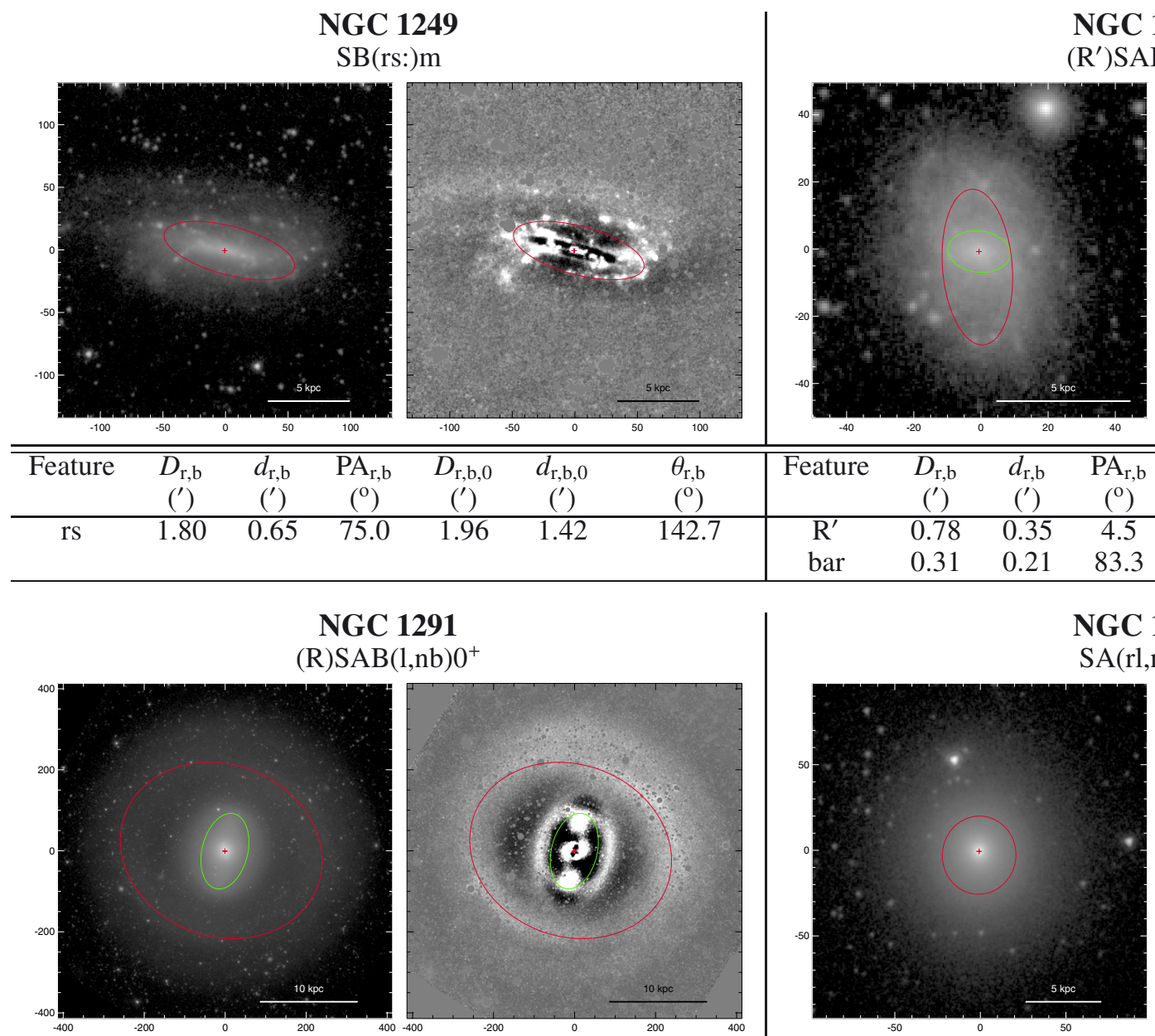

NGC 1258

$\left(\mathrm{R}^{\prime}\right) \mathrm{SAB}(\mathrm{s}) \mathrm{a}$ :
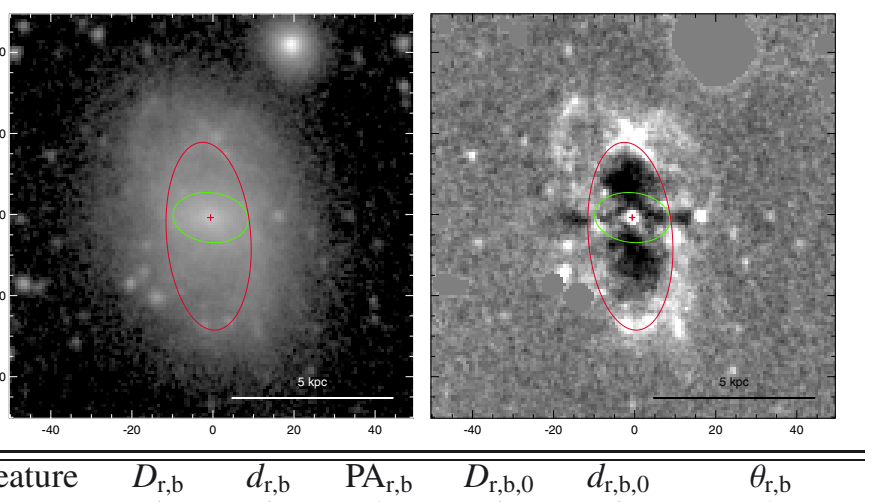

\begin{tabular}{|c|c|c|c|c|c|}
\hline $\begin{array}{l}c_{\text {rob }} \\
\left({ }^{\prime}\right)\end{array}$ & $\begin{array}{c}u_{\mathrm{r}, \mathrm{b}} \\
\left({ }^{\prime}\right)\end{array}$ & $\left({ }^{\circ}\right)$ & $\begin{array}{l}\mathrm{r}, \mathrm{b}, \mathrm{u} \\
\left({ }^{\prime}\right)\end{array}$ & $\begin{array}{c}a_{\mathrm{r}, \mathrm{b}, 0} \\
\left({ }^{\prime}\right)\end{array}$ & $\begin{array}{l}\mathrm{r}_{\mathrm{r}, \mathrm{b}} \\
\left({ }^{\circ}\right)\end{array}$ \\
\hline 0.78 & 0.35 & 4.5 & 0.80 & 0.47 & 160.0 \\
\hline 0.31 & 0.21 & 83.3 & 0.42 & 0.21 & 77.3 \\
\hline
\end{tabular}

NGC 1297

$\mathrm{SA}(\mathrm{rl}, \mathrm{nl}) 0^{\circ}$

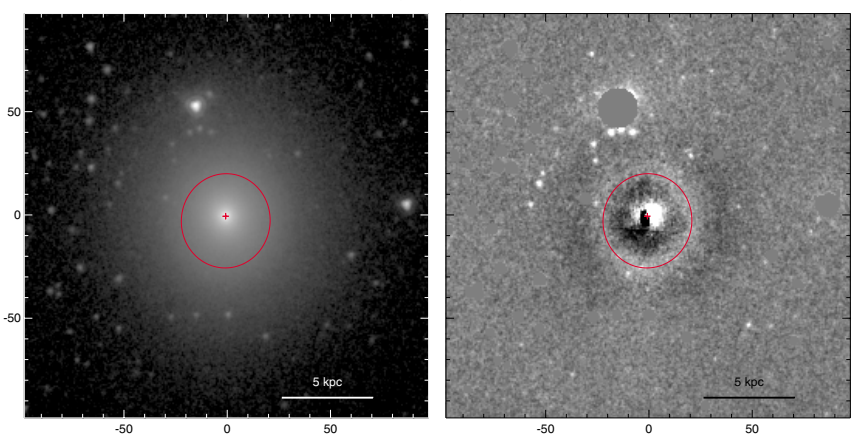

\begin{tabular}{|c|c|c|c|c|c|c|c|c|c|c|c|c|c|}
\hline Feature & $\begin{array}{c}D_{\mathrm{r}, \mathrm{b}} \\
\left({ }^{\prime}\right)\end{array}$ & $\begin{array}{c}d_{\mathrm{r}, \mathrm{b}} \\
\left(^{\prime}\right)\end{array}$ & $\begin{array}{c}\mathrm{PA}_{\mathrm{r}, \mathrm{b}} \\
\left({ }^{\circ}\right)\end{array}$ & $\begin{array}{c}D_{\mathrm{r}, \mathrm{b}, 0} \\
\left(^{\prime}\right)\end{array}$ & $\begin{array}{c}d_{\mathrm{r}, \mathrm{b}, 0} \\
\left(^{\prime}\right)\end{array}$ & $\begin{array}{l}\theta_{\mathrm{r}, \mathrm{b}} \\
\left(^{\circ}\right)\end{array}$ & Feature & $\begin{array}{c}D_{\mathrm{r}, \mathrm{b}} \\
\left({ }^{\prime}\right)\end{array}$ & $\begin{array}{c}d_{\mathrm{r}, \mathrm{b}} \\
\left({ }^{\prime}\right)\end{array}$ & $\begin{array}{c}\mathrm{PA}_{\mathrm{r}, \mathrm{b}} \\
\left(^{\circ}\right)\end{array}$ & $\begin{array}{c}D_{\mathrm{r}, \mathrm{b}, 0} \\
\left.\text { (') }^{\prime}\right)\end{array}$ & $\begin{array}{c}d_{\mathrm{r}, \mathrm{b}, 0} \\
\left(^{\prime}\right)\end{array}$ & $\begin{array}{l}\theta_{\mathrm{r}, \mathrm{b}} \\
\left(^{\circ}\right)\end{array}$ \\
\hline$\overline{\mathrm{R}}$ & 8.47 & 7.08 & 69.6 & 8.51 & 7.10 & 53.1 & $\mathrm{rl}$ & 0.76 & 0.72 & 174.1 & 0.78 & 0.75 & 121.4 \\
\hline bar & 3.17 & 1.88 & 166.3 & 3.18 & 1.90 & 148.2 & & & & & & & \\
\hline
\end{tabular}


NGC 1300

$\left(\mathrm{R}^{\prime}\right) \mathrm{SB}(\mathrm{s}, \mathrm{nrl}) \mathrm{b}$
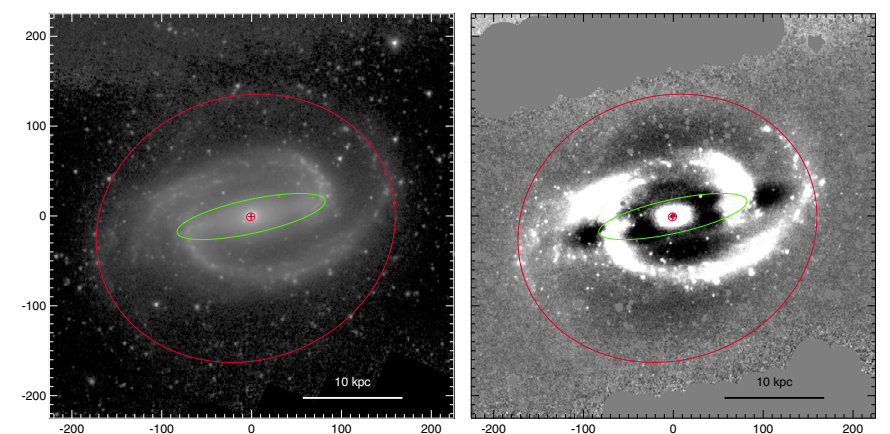

NGC 1302

(RoRi)SAB(r) $0^{+}$

\begin{tabular}{ccccccc|ccccccc}
\hline \hline Feature & $\begin{array}{c}D_{\mathrm{r}, \mathrm{b}} \\
\left({ }^{\prime}\right)\end{array}$ & $\begin{array}{c}d_{\mathrm{r}, \mathrm{b}} \\
\left({ }^{\prime}\right)\end{array}$ & $\begin{array}{c}\mathrm{PA}_{\mathrm{r}, \mathrm{b}} \\
\left({ }^{\circ}\right)\end{array}$ & $\begin{array}{c}D_{\mathrm{r}, \mathrm{b}, 0} \\
\left({ }^{\prime}\right)\end{array}$ & $\begin{array}{c}d_{\mathrm{r}, \mathrm{b}, 0} \\
\left({ }^{\prime}\right)\end{array}$ & $\begin{array}{c}\theta_{\mathrm{r}, \mathrm{b}} \\
\left({ }^{\circ}\right)\end{array}$ & Feature & $\begin{array}{c}D_{\mathrm{r}, \mathrm{b}} \\
\left({ }^{\prime}\right)\end{array}$ & $\begin{array}{c}d_{\mathrm{r}, \mathrm{b}} \\
\left({ }^{\prime}\right)\end{array}$ & $\begin{array}{c}\mathrm{PA}_{\mathrm{r}, \mathrm{b}} \\
\left({ }^{\circ}\right)\end{array}$ & $\begin{array}{c}D_{\mathrm{r}, \mathrm{b}, 0} \\
\left({ }^{\prime}\right)\end{array}$ & $\begin{array}{c}d_{\mathrm{r}, \mathrm{b}, 0} \\
\left({ }^{\prime}\right)\end{array}$ & $\begin{array}{c}\theta_{\mathrm{r}, \mathrm{b}} \\
\left({ }^{\circ}\right)\end{array}$ \\
\hline $\mathrm{R}^{\prime}$ & 5.63 & 4.90 & 109.5 & 5.88 & 5.62 & 79.1 & $\mathrm{R}$ & 3.19 & 2.98 & 17.8 & 3.20 & 3.09 & 13.0 \\
$\mathrm{bar}$ & 2.82 & 0.63 & 102.3 & 2.82 & 0.75 & 175.3 & $\mathrm{R}$ & 2.19 & 1.95 & 60.7 & 2.24 & 1.98 & 57.7 \\
$\mathrm{nrl}$ & 0.15 & 0.14 & 114.0 & 0.17 & 0.15 & 84.0 & $\mathrm{r}$ & 1.02 & 0.94 & 4.6 & 1.02 & 0.97 & 167.1 \\
& & & & & & & bar & 0.93 & 0.62 & 169.4 & 0.93 & 0.64 & 155.7 \\
\hline
\end{tabular}

\section{NGC 1310}

$\mathrm{SB}(\mathrm{rs}) \mathrm{cd}$

\section{NGC 1326}

$\left(\mathrm{R}_{1}\right) \mathrm{SAB}_{\mathrm{a}}(\mathrm{r}, \mathrm{bl}, \mathrm{nr}) 0^{+}$
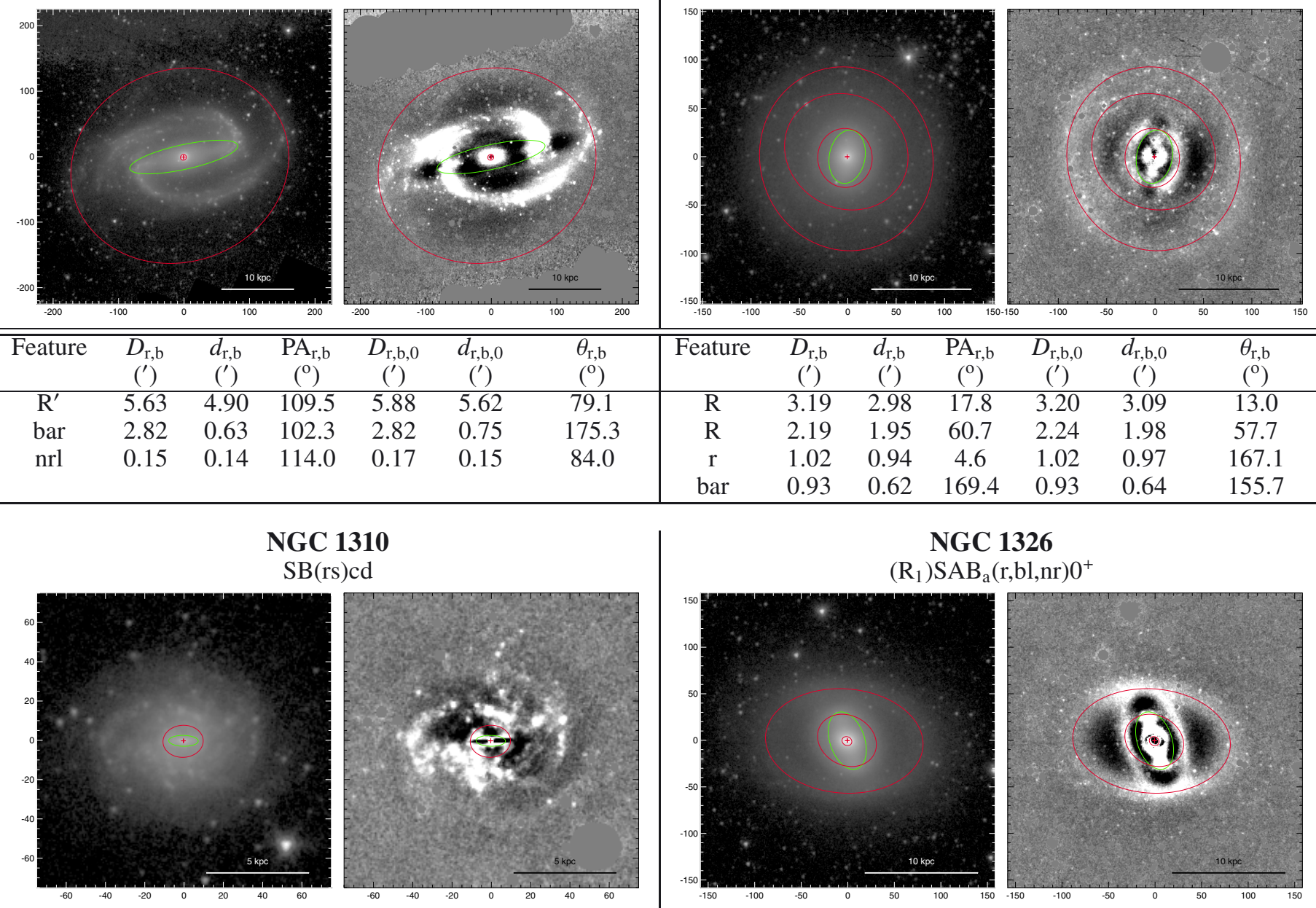

$$
\begin{array}{lllllll}
-60 & -40 & -20 & 0 & 20 & 40 & { }^{60} \\
\hline \hline \text { Feature } & &
\end{array}
$$
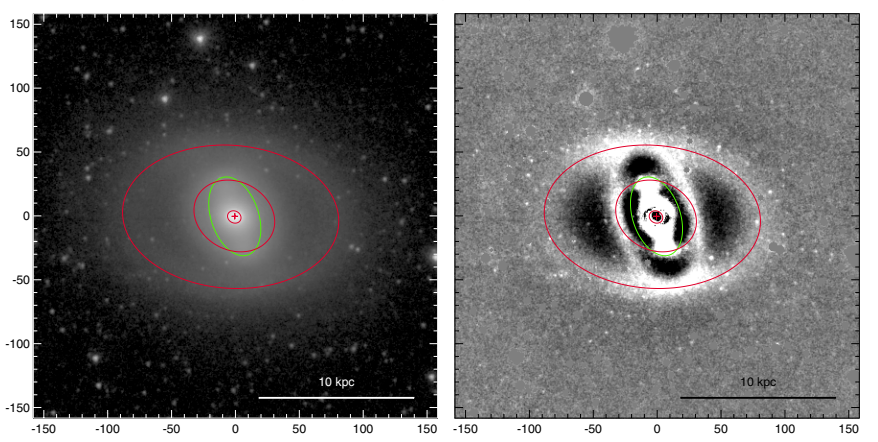

\begin{tabular}{ccccccc|ccccccc}
\hline \hline Feature & $\begin{array}{c}D_{\mathrm{r}, \mathrm{b}} \\
\left({ }^{\prime}\right)\end{array}$ & $\begin{array}{c}d_{\mathrm{r}, \mathrm{b}} \\
\left({ }^{\prime}\right)\end{array}$ & $\begin{array}{c}\mathrm{PA}_{\mathrm{r}, \mathrm{b}} \\
\left({ }^{\circ}\right)\end{array}$ & $\begin{array}{c}D_{\mathrm{r}, \mathrm{b}, 0} \\
\left({ }^{\prime}\right)\end{array}$ & $\begin{array}{c}d_{\mathrm{r}, \mathrm{b}, 0} \\
\left({ }^{\prime}\right)\end{array}$ & $\begin{array}{c}\theta_{\mathrm{r}, \mathrm{b}} \\
\left({ }^{(}\right)\end{array}$ & Feature & $\begin{array}{c}D_{\mathrm{r}, \mathrm{b}} \\
\left({ }^{\prime}\right)\end{array}$ & $\begin{array}{c}d_{\mathrm{r}, \mathrm{b}} \\
\left({ }^{\prime}\right)\end{array}$ & $\begin{array}{c}\mathrm{PA}_{\mathrm{r}, \mathrm{b}} \\
\left({ }^{\circ}\right)\end{array}$ & $\begin{array}{c}D_{\mathrm{r}, \mathrm{b}, 0} \\
\left({ }^{\prime}\right)\end{array}$ & $\begin{array}{c}d_{\mathrm{r}, \mathrm{b}, 0} \\
\left({ }^{\prime}\right)\end{array}$ & $\begin{array}{c}\theta_{\mathrm{r}, \mathrm{b}} \\
\left({ }^{\circ}\right)\end{array}$ \\
\hline rs & 0.34 & 0.27 & 93.1 & 0.36 & 0.32 & 42.8 & $\mathrm{R}_{1}$ & 2.83 & 1.87 & 85.9 & 2.84 & 2.34 & 12.2 \\
bar & 0.25 & 0.09 & 89.0 & 0.25 & 0.11 & 12.6 & $\mathrm{r}$ & 1.09 & 0.89 & 63.2 & 1.18 & 1.04 & 120.9 \\
& & & & & & & $\mathrm{bar}$ & 1.07 & 0.62 & 18.6 & 1.29 & 0.65 & 109.5 \\
& & & & & & & $\mathrm{nr}$ & 0.18 & 0.16 & 61.9 & 0.20 & 0.18 & 112.1 \\
\hline
\end{tabular}

\section{NGC 1341}

$\left(\mathrm{R}^{\prime}\right) \mathrm{SB}(\mathrm{s}) \mathrm{d}$ pec

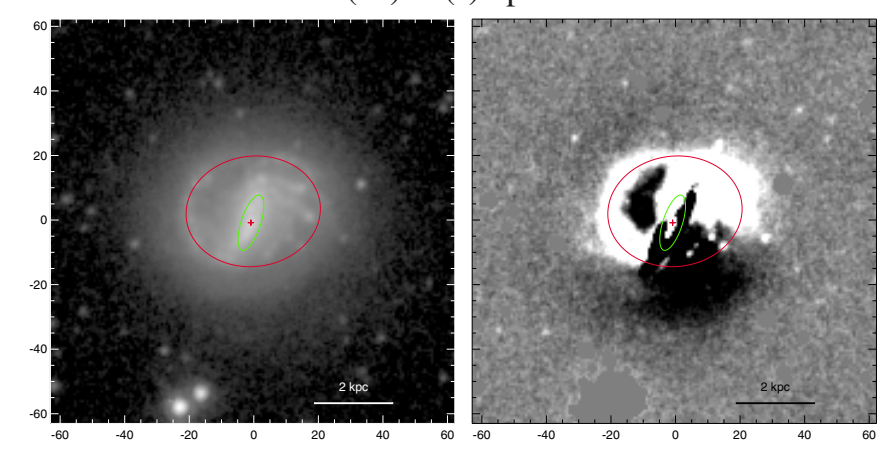

\section{NGC 1350}

(R)SAB $(r, b l) 0 / a$

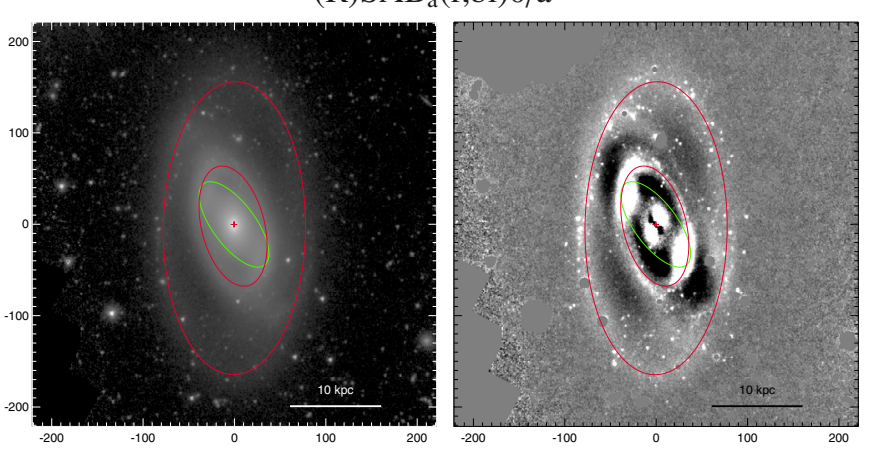

\begin{tabular}{ccccccc|ccccccc}
\hline \hline Feature & $\begin{array}{c}D_{\mathrm{r}, \mathrm{b}} \\
\left({ }^{\prime}\right)\end{array}$ & $\begin{array}{c}d_{\mathrm{r}, \mathrm{b}} \\
\left({ }^{\prime}\right)\end{array}$ & $\begin{array}{c}\mathrm{PA}_{\mathrm{r}, \mathrm{b}} \\
\left({ }^{\circ}\right)\end{array}$ & $\begin{array}{c}D_{\mathrm{r}, \mathrm{b}, 0} \\
\left({ }^{\prime}\right)\end{array}$ & $\begin{array}{c}d_{\mathrm{r}, \mathrm{b}, 0} \\
\left({ }^{\prime}\right)\end{array}$ & $\begin{array}{c}\theta_{\mathrm{r}, \mathrm{b}} \\
\left({ }^{(}\right)\end{array}$ & Feature & $\begin{array}{c}D_{\mathrm{r}, \mathrm{b}} \\
\left({ }^{\prime}\right)\end{array}$ & $\begin{array}{c}d_{\mathrm{r}, \mathrm{b}} \\
\left({ }^{\prime}\right)\end{array}$ & $\begin{array}{c}\mathrm{PA}_{\mathrm{r}, \mathrm{b}} \\
\left({ }^{\circ}\right)\end{array}$ & $\begin{array}{c}D_{\mathrm{r}, \mathrm{b}, 0} \\
\left({ }^{\prime}\right)\end{array}$ & $\begin{array}{c}d_{\mathrm{r}, \mathrm{b}, 0} \\
\left({ }^{\prime}\right)\end{array}$ & $\begin{array}{c}\theta_{\mathrm{r}, \mathrm{b}} \\
\left({ }^{\circ}\right)\end{array}$ \\
\hline $\mathrm{R}^{\prime}$ & 0.70 & 0.57 & 96.8 & 0.73 & 0.57 & 80.2 & $\mathrm{R}$ & 5.35 & 2.60 & 179.5 & 5.56 & 4.75 & 147.5 \\
$\mathrm{bar}$ & 0.30 & 0.10 & 161.9 & 0.30 & 0.10 & 141.9 & $\mathrm{r}$ & 2.25 & 1.15 & 14.1 & 2.49 & 1.97 & 47.4 \\
& & & & & & & bar & 1.86 & 0.78 & 36.7 & 2.56 & 1.08 & 58.3 \\
\hline
\end{tabular}


NGC 1353

SAB(rs,nl)a
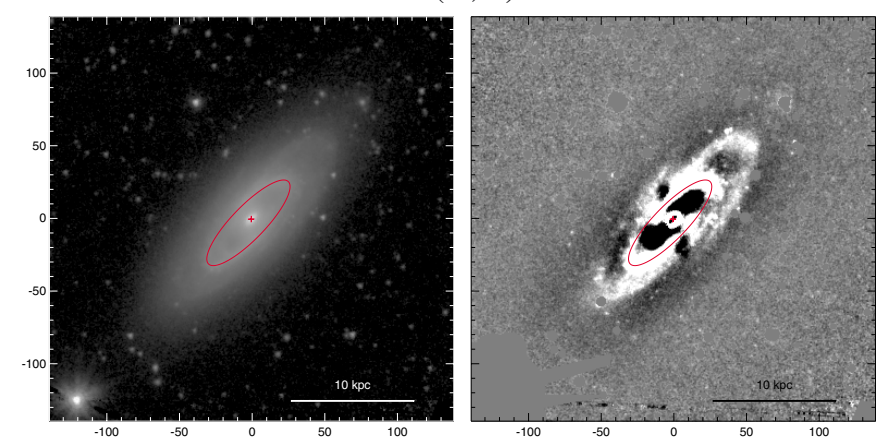

\begin{tabular}{|c|c|c|c|c|c|c|c|c|c|c|c|c|c|}
\hline Feature & $\begin{array}{c}D_{\mathrm{r}, \mathrm{b}} \\
\left({ }^{\prime}\right)\end{array}$ & $\begin{array}{c}d_{\mathrm{r}, \mathrm{b}} \\
\left({ }^{\prime}\right)\end{array}$ & $\begin{array}{c}\mathrm{PA}_{\mathrm{r}, \mathrm{b}} \\
\left({ }^{\mathrm{O}}\right)\end{array}$ & $\begin{array}{c}D_{\mathrm{r}, \mathrm{b}, 0} \\
\left(^{\prime}\right)\end{array}$ & $\begin{array}{c}d_{\mathrm{r}, \mathrm{b}, 0} \\
\left({ }^{\prime}\right)\end{array}$ & $\begin{array}{l}\theta_{\mathrm{r}, \mathrm{b}} \\
\left({ }^{\circ}\right)\end{array}$ & Feature & $\begin{array}{c}D_{\mathrm{r}, \mathrm{b}} \\
\left({ }^{\prime}\right)\end{array}$ & $\begin{array}{c}d_{\mathrm{r}, \mathrm{b}} \\
\left({ }^{\prime}\right)\end{array}$ & $\begin{array}{c}\mathrm{PA}_{\mathrm{r}, \mathrm{b}} \\
\left(^{\circ}\right)\end{array}$ & $\begin{array}{c}D_{\mathrm{r}, \mathrm{b}, 0} \\
\left({ }^{\prime}\right)\end{array}$ & $\begin{array}{c}d_{\mathrm{r}, \mathrm{b}, 0} \\
{ }^{\prime}\left({ }^{\prime}\right)\end{array}$ & $\begin{array}{l}\theta_{\mathrm{r}, \mathrm{b}} \\
\left({ }^{\circ}\right)\end{array}$ \\
\hline rs & 1.32 & 0.38 & 135.9 & 1.32 & 0.91 & 174.4 & $\begin{array}{l}\mathrm{R}^{\prime} \mathrm{L} \\
\mathrm{rs}\end{array}$ & $\begin{array}{l}2.74 \\
1.05\end{array}$ & $\begin{array}{l}2.20 \\
0.86\end{array}$ & $\begin{array}{l}69.5 \\
69.4\end{array}$ & $\begin{array}{l}2.89 \\
1.12\end{array}$ & $\begin{array}{l}2.64 \\
1.02\end{array}$ & $\begin{array}{l}124.6 \\
119.3\end{array}$ \\
\hline
\end{tabular}

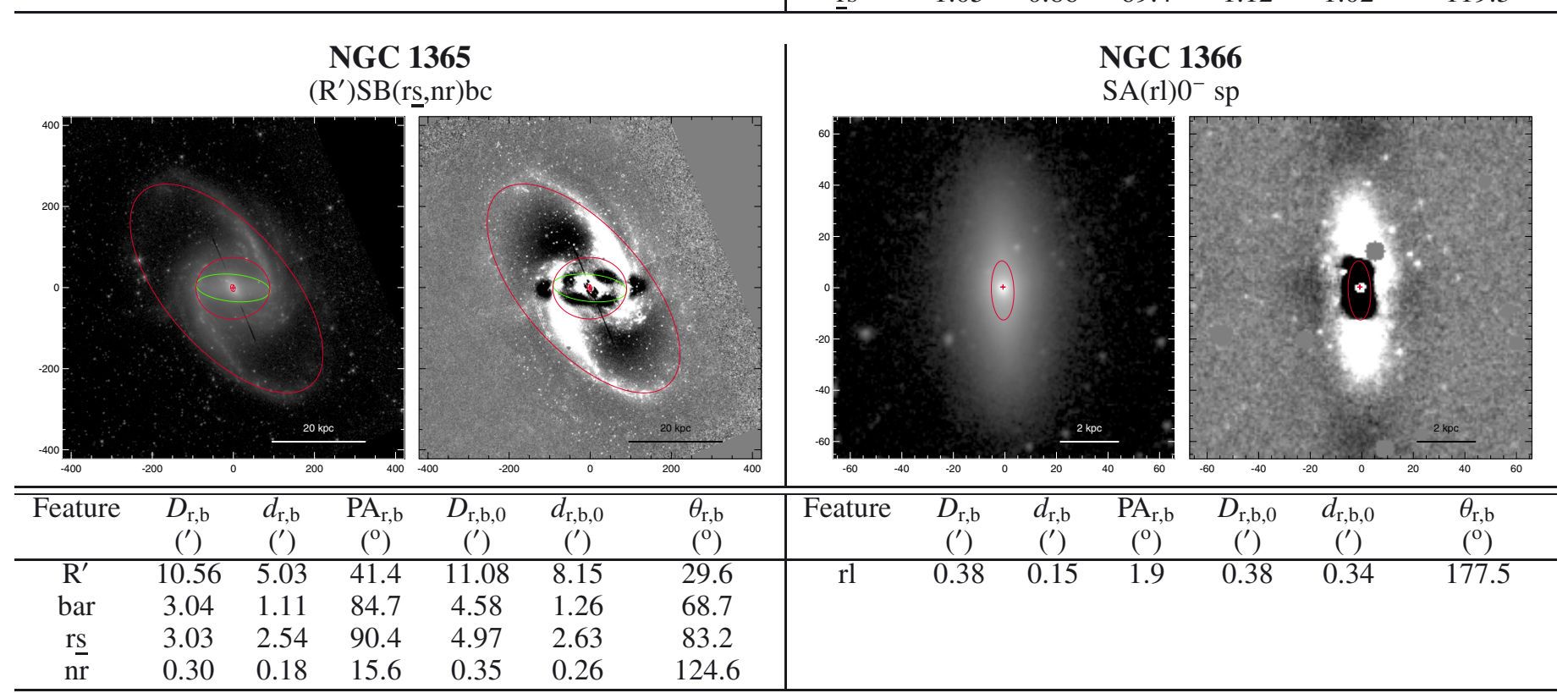

NGC 1367

(RL,RL)SAB(rs)0/a
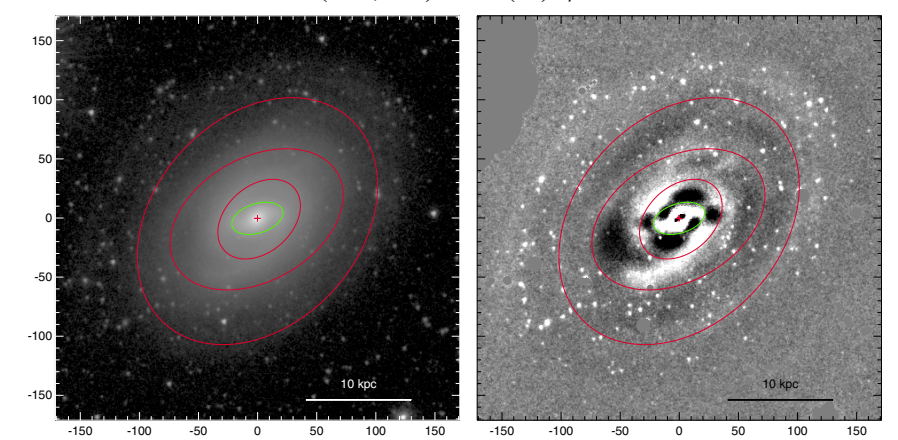

NGC 1385

$\mathrm{SB}(\mathrm{rs}) \mathrm{dm}$ pec /Sph

\begin{tabular}{|c|c|c|c|c|c|c|c|c|c|c|c|c|c|}
\hline Feature & $\begin{array}{c}D_{\mathrm{r}, \mathrm{b}} \\
\left({ }^{\prime}\right)\end{array}$ & $\begin{array}{c}d_{\mathrm{r}, \mathrm{b}} \\
\left({ }^{\prime}\right)\end{array}$ & $\begin{array}{c}\mathrm{PA}_{\mathrm{r}, \mathrm{b}} \\
\left({ }^{\mathrm{O}}\right)\end{array}$ & $\begin{array}{c}D_{\mathrm{r}, \mathrm{b}, 0} \\
\left(^{\prime}\right)\end{array}$ & $\begin{array}{c}d_{\mathrm{r}, \mathrm{b}, 0} \\
\left.\text { (') }^{\prime}\right)\end{array}$ & $\begin{array}{l}\theta_{\mathrm{r}, \mathrm{b}} \\
\left({ }^{\circ}\right)\end{array}$ & Feature & $\begin{array}{c}D_{\mathrm{r}, \mathrm{b}} \\
\left({ }^{\prime}\right)\end{array}$ & $\begin{array}{c}d_{\mathrm{r}, \mathrm{b}} \\
\left({ }^{\prime}\right)\end{array}$ & $\begin{array}{c}\mathrm{PA}_{\mathrm{r}, \mathrm{b}} \\
\left({ }^{\circ}\right)\end{array}$ & $\begin{array}{c}D_{\mathrm{r}, \mathrm{b}, 0} \\
\left(^{\prime}\right)\end{array}$ & $\begin{array}{c}d_{\mathrm{r}, \mathrm{b}, 0} \\
\left({ }^{\prime}\right)\end{array}$ & $\begin{array}{c}\theta_{\mathrm{r}, \mathrm{b}} \\
\left({ }^{\circ}\right)\end{array}$ \\
\hline $\mathrm{RL}$ & 3.91 & 2.91 & 137.7 & 4.26 & 3.88 & 75.9 & rs & 0.68 & 0.60 & 39.5 & 0.99 & 0.63 & 82.6 \\
\hline RL & 2.63 & 1.74 & 119.5 & 2.85 & 2.34 & 133.0 & bār & 0.20 & 0.06 & 78.5 & 0.31 & 0.06 & 87.6 \\
\hline rs & 1.32 & 0.94 & 131.9 & 1.36 & 1.32 & 107.7 & & & & & & & \\
\hline bar & 0.75 & 0.42 & 107.3 & 0.85 & 0.53 & 132.5 & & & & & & & \\
\hline
\end{tabular}

NGC 1357

( $\left.\mathrm{R}^{\prime} \mathrm{L}\right) \mathrm{SA}(\underline{\mathrm{rs}}) 0 / \mathrm{a}$

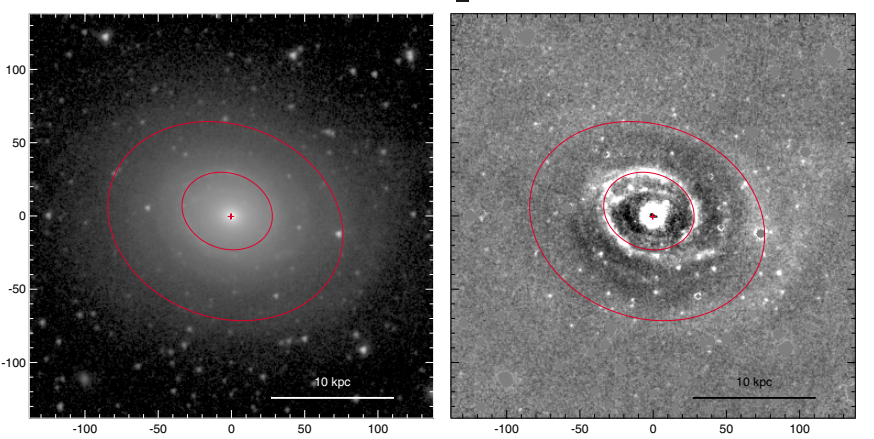

.


NGC 1386

$\left(\mathrm{R}^{\prime}\right) \mathrm{SA}(\mathrm{r}) 0 / \mathrm{a}$
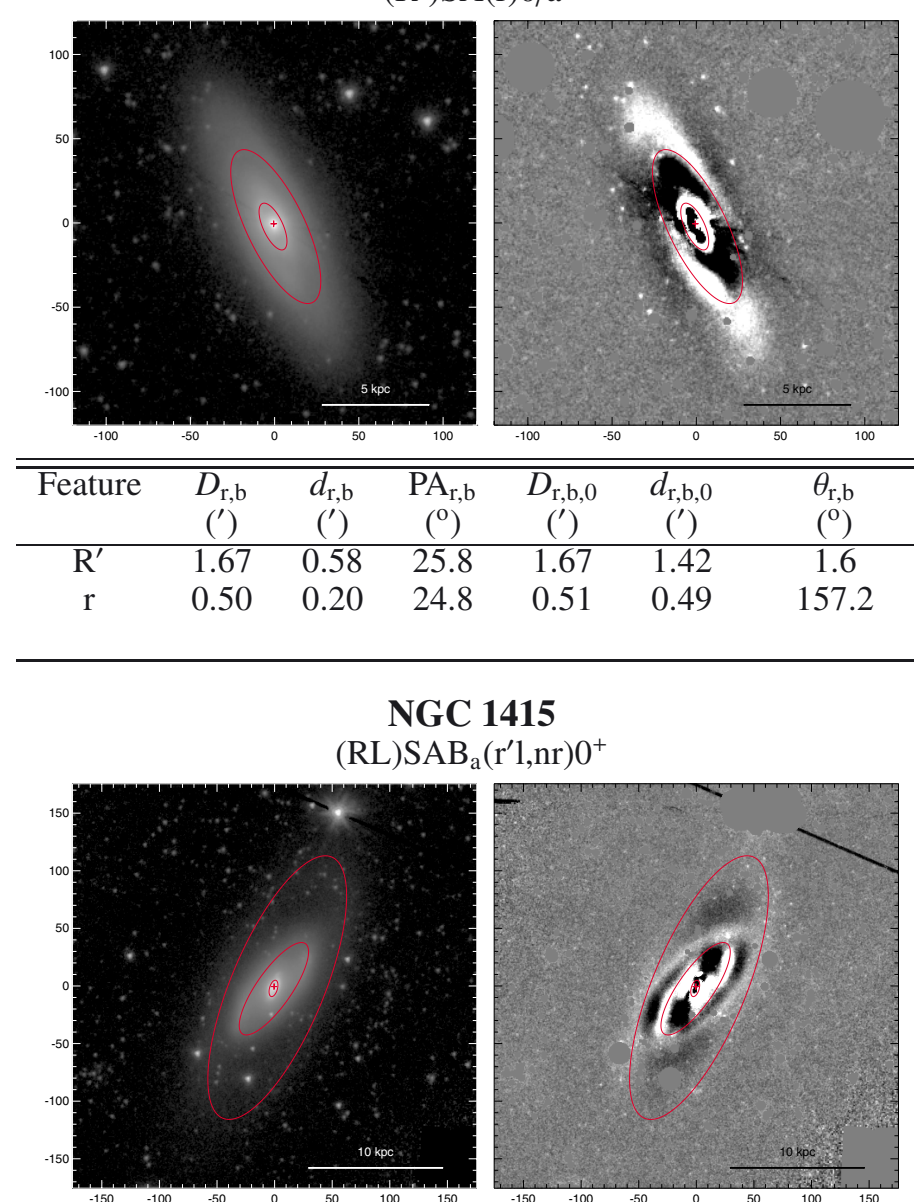

\section{$D_{\mathrm{r}, \mathrm{b}, 0} d_{\mathrm{r}, \mathrm{b}, \mathrm{O}}$}

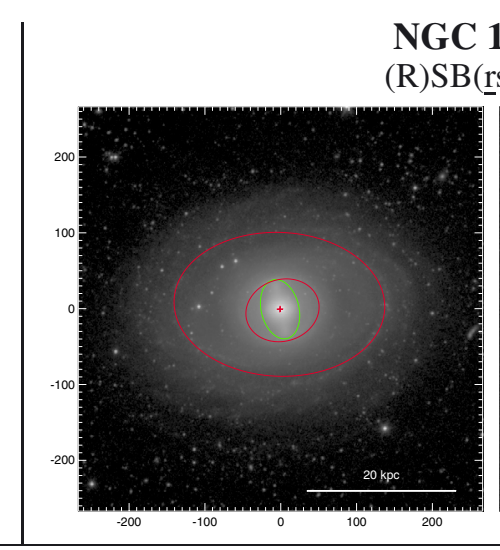

GC 1398

R) $\mathrm{SB}(\underline{\mathrm{rs}}, \mathrm{bl}) \mathrm{a}$
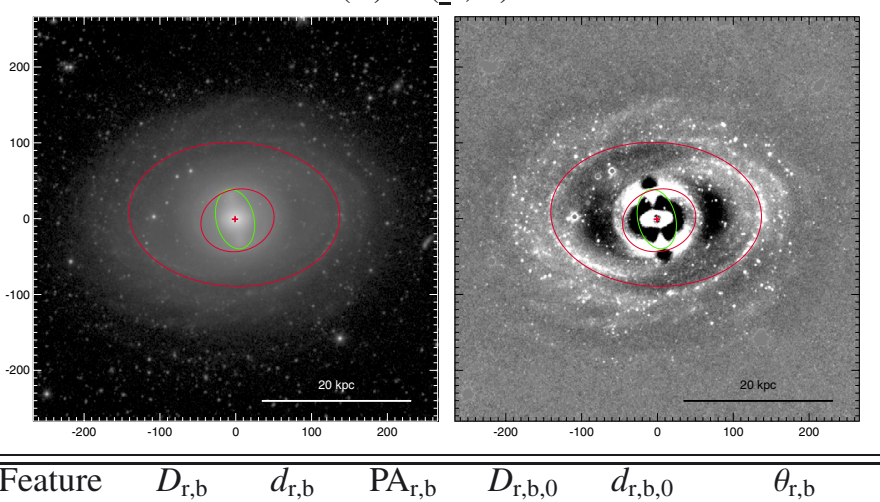

$\begin{array}{ccc}\left({ }^{\prime}\right) & \left({ }^{\prime}\right) & \left(^{\circ}\right) \\ 1.67 & 1.42 & 1.6\end{array}$

$\begin{array}{ll}0.51 & 0.49\end{array}$

\begin{tabular}{ccccccc} 
& $\left({ }^{\prime}\right)$ & $\left({ }^{\prime}\right)$ & $\left({ }^{\circ}\right)$ & $\left(^{\prime}\right)$ & $\left({ }^{\prime}\right)$ & $\left(^{\circ}\right)$ \\
\hline R & 4.64 & 3.17 & 87.7 & 4.70 & 4.22 & 156.8 \\
rs & 1.63 & 1.36 & 107.3 & 1.88 & 1.60 & 73.9 \\
bar & 1.33 & 0.83 & 13.4 & 1.78 & 0.84 & 95.7 \\
\hline
\end{tabular}

\section{(1) \\ (1)}

NGC 1425

$\left(\mathrm{R}^{\prime} \mathrm{R}^{\prime}\right) \mathrm{SA}(\mathrm{rl}) \mathrm{b}$
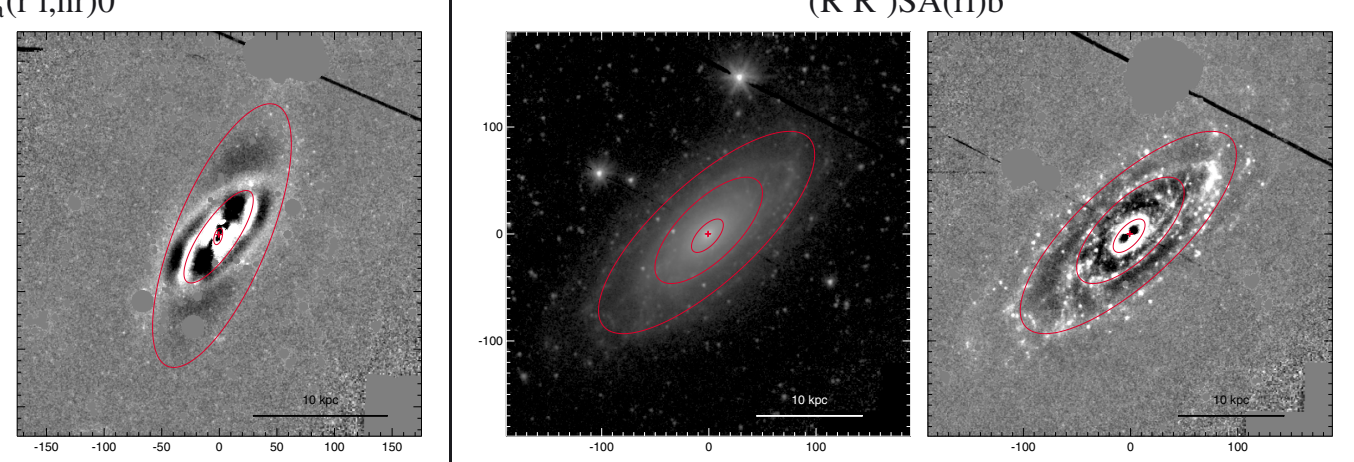

\begin{tabular}{ccccccc|ccccccc}
\hline \hline Feature & $\begin{array}{c}D_{\mathrm{r}, \mathrm{b}} \\
\left({ }^{\prime}\right)\end{array}$ & $\begin{array}{c}d_{\mathrm{r}, \mathrm{b}} \\
\left({ }^{\prime}\right)\end{array}$ & $\begin{array}{c}\mathrm{PA}_{\mathrm{r}, \mathrm{b}} \\
\left({ }^{\circ}\right)\end{array}$ & $\begin{array}{c}D_{\mathrm{r}, \mathrm{b}, 0} \\
\left({ }^{\prime}\right)\end{array}$ & $\begin{array}{c}d_{\mathrm{r}, \mathrm{b}, 0} \\
\left({ }^{\prime}\right)\end{array}$ & $\begin{array}{c}\theta_{\mathrm{r}, \mathrm{b}} \\
\left({ }^{\circ}\right)\end{array}$ & Feature & $\begin{array}{c}D_{\mathrm{r}, \mathrm{b}} \\
\left({ }^{\prime}\right)\end{array}$ & $\begin{array}{c}d_{\mathrm{r}, \mathrm{b}} \\
\left({ }^{\prime}\right)\end{array}$ & $\begin{array}{c}\mathrm{PA}_{\mathrm{r}, \mathrm{b}} \\
\left({ }^{\circ}\right)\end{array}$ & $\begin{array}{c}D_{\mathrm{r}, \mathrm{b}, 0} \\
\left({ }^{\prime}\right)\end{array}$ & $\begin{array}{c}d_{\mathrm{r}, \mathrm{b}, 0} \\
\left({ }^{\prime}\right)\end{array}$ & $\begin{array}{c}\theta_{\mathrm{r}, \mathrm{b}} \\
\left({ }^{\circ}\right)\end{array}$ \\
\hline $\mathrm{RL}$ & 4.10 & 1.35 & 157.4 & 4.29 & 3.21 & 27.0 & $\mathrm{R}^{\prime}$ & 4.34 & 1.56 & 132.6 & 4.35 & 3.51 & 6.0 \\
$\mathrm{r} 1$ & 1.60 & 0.51 & 145.1 & 1.72 & 1.18 & 147.8 & $\mathrm{R}^{\prime}$ & 2.17 & 0.91 & 134.6 & 2.24 & 2.00 & 33.1 \\
$\mathrm{nr}$ & 0.24 & 0.11 & 164.0 & 0.32 & 0.22 & 63.4 & $\mathrm{rl}$ & 0.65 & 0.30 & 137.1 & 0.73 & 0.61 & 57.5 \\
\hline
\end{tabular}

NGC 1433

$\left(\mathrm{R}_{1}^{\prime}\right) \mathrm{SB}(\mathrm{r}, \mathrm{nr}, \mathrm{nb}) \mathrm{a}$
NGC 1436

$\left(\mathrm{R}^{\prime}\right) \mathrm{SAB}(\mathrm{s}) \mathrm{ab} / \mathrm{Sph}$

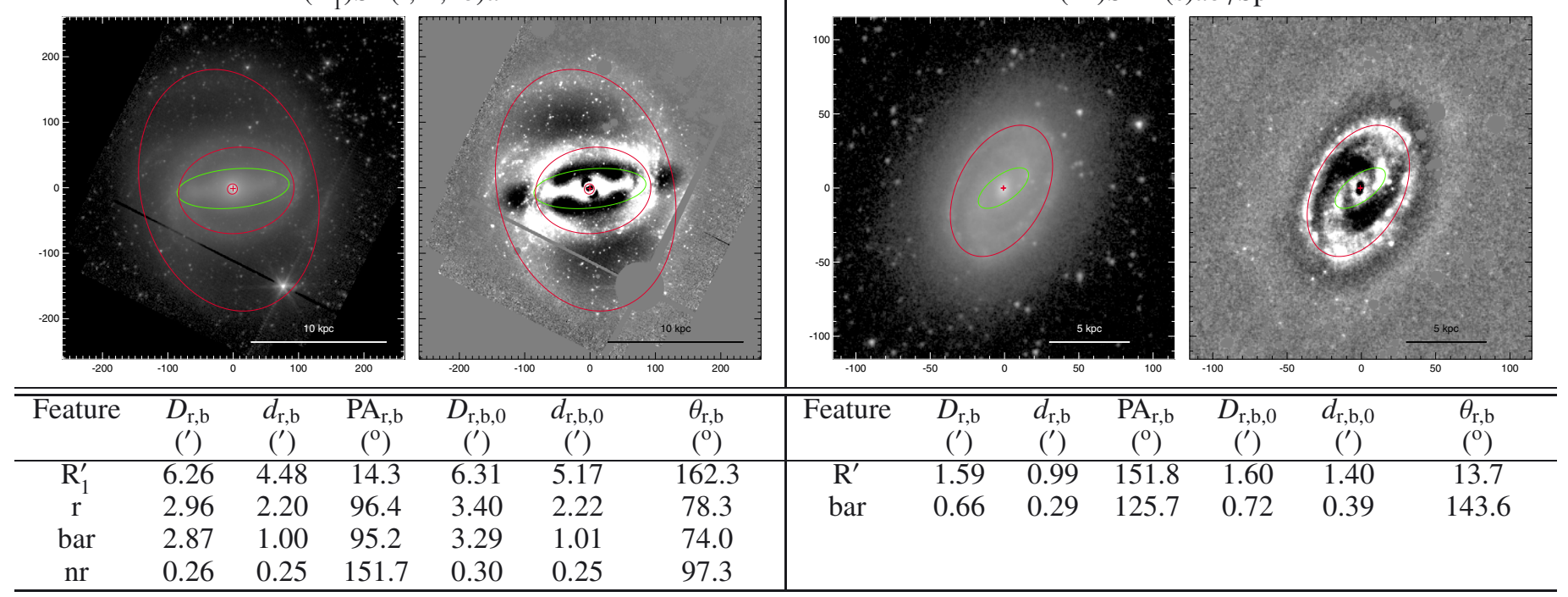


NGC 1438

$\mathrm{SAB}(\mathrm{r}) 0 / \mathrm{a}$

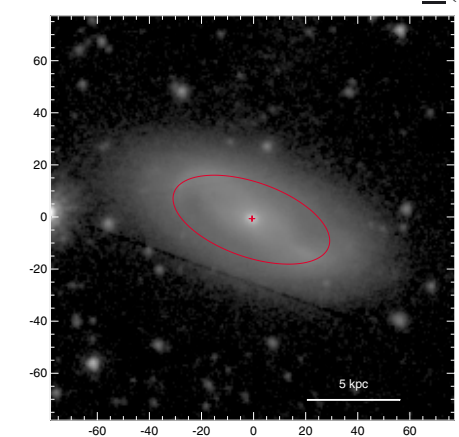

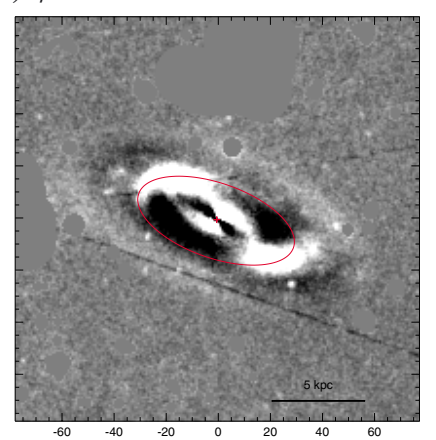

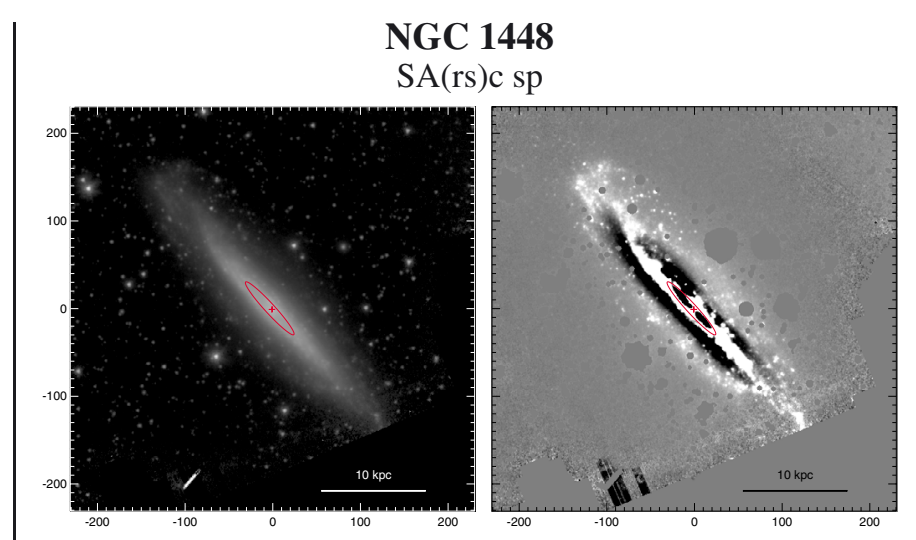

\begin{tabular}{ccccccc|ccccccc}
\hline \hline Feature & $\begin{array}{c}D_{\mathrm{r}, \mathrm{b}} \\
\left({ }^{\prime}\right)\end{array}$ & $\begin{array}{c}d_{\mathrm{r}, \mathrm{b}}\left({ }^{\prime}\right) \\
\left({ }^{\circ}\right)\end{array}$ & $\begin{array}{c}\mathrm{PA}_{\mathrm{r}, \mathrm{b}} \\
\left({ }^{\circ}\right)\end{array}$ & $\begin{array}{c}D_{\mathrm{r}, \mathrm{b}, 0} \\
\left({ }^{\prime}\right)\end{array}$ & $\begin{array}{c}d_{\mathrm{r}, \mathrm{b}, 0} \\
\left({ }^{\prime}\right)\end{array}$ & $\begin{array}{c}\theta_{\mathrm{r}, \mathrm{b}} \\
\left({ }^{\circ}\right)\end{array}$ & Feature & $\begin{array}{c}D_{\mathrm{r}, \mathrm{b}} \\
\left({ }^{\prime}\right)\end{array}$ & $\begin{array}{c}d_{\mathrm{r}, \mathrm{b}} \\
\left({ }^{\prime}\right)\end{array}$ & $\begin{array}{c}\mathrm{PA}_{\mathrm{r}, \mathrm{b}} \\
\left({ }^{\circ}\right)\end{array}$ & $\begin{array}{c}D_{\mathrm{r}, \mathrm{b}, 0} \\
\left({ }^{\prime}\right)\end{array}$ & $\begin{array}{c}d_{\mathrm{r}, \mathrm{b}, 0} \\
\left({ }^{\prime}\right)\end{array}$ & $\begin{array}{c}\theta_{\mathrm{r}, \mathrm{b}} \\
\left({ }^{\circ}\right)\end{array}$ \\
\hline $\mathrm{r}$ & 1.05 & 0.48 & 70.7 & 1.06 & 1.00 & 21.1 & $\mathrm{rs}$ & 1.36 & 0.19 & 42.5 & 1.36 & 0.97 & 177.6 \\
\hline
\end{tabular}

NGC 1452

(RL)SB(r,bl)0/a
NGC 1483

$\left(\mathrm{R}^{\prime}\right) \mathrm{SB}(\mathrm{s}) \mathrm{d}$
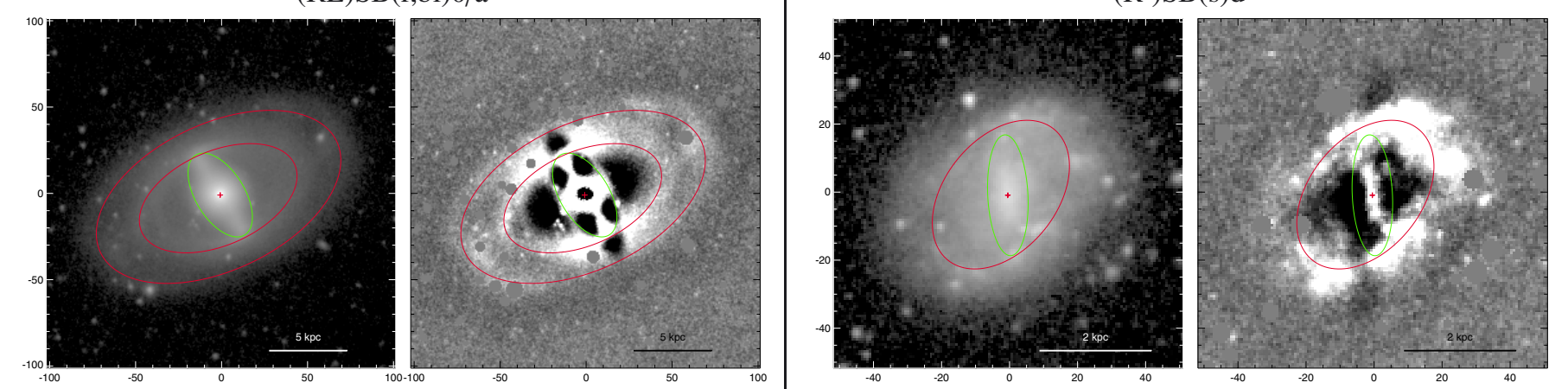

\begin{tabular}{ccccccc|ccccccc}
\hline \hline Feature & $\begin{array}{c}D_{\mathrm{r}, \mathrm{b}} \\
\left({ }^{\prime}\right)\end{array}$ & $\begin{array}{c}d_{\mathrm{r}, \mathrm{b}}\left({ }^{\prime}\right) \\
\mathrm{PA}_{\mathrm{r}, \mathrm{b}} \\
\left({ }^{\circ}\right)\end{array}$ & $\begin{array}{c}D_{\mathrm{r}, \mathrm{b}, 0} \\
\left({ }^{\prime}\right)\end{array}$ & $\begin{array}{c}d_{\mathrm{r}, \mathrm{b}, 0} \\
\left({ }^{\prime}\right)\end{array}$ & $\begin{array}{c}\theta_{\mathrm{r}, \mathrm{b}} \\
\left({ }^{(}\right)\end{array}$ & Feature & $\begin{array}{c}D_{\mathrm{r}, \mathrm{b}} \\
\left({ }^{\prime}\right)\end{array}$ & $\begin{array}{c}d_{\mathrm{r}, \mathrm{b}} \\
\left({ }^{\prime}\right)\end{array}$ & $\begin{array}{c}\mathrm{PA}_{\mathrm{r}, \mathrm{b}} \\
\left({ }^{\circ}\right)\end{array}$ & $\begin{array}{c}D_{\mathrm{r}, \mathrm{b}, 0} \\
\left({ }^{\prime}\right)\end{array}$ & $\begin{array}{c}d_{\mathrm{r}, \mathrm{b}, 0} \\
\left({ }^{\prime}\right)\end{array}$ & $\begin{array}{c}\theta_{\mathrm{r}, \mathrm{b}} \\
\left({ }^{\circ}\right)\end{array}$ \\
\hline $\mathrm{RL}$ & 2.54 & 1.43 & 115.1 & 2.55 & 2.38 & 13.0 & $\mathrm{R}^{\prime}$ & 0.83 & 0.55 & 141.0 & 0.85 & 0.66 & 28.3 \\
$\mathrm{r}$ & 1.63 & 0.91 & 113.7 & 1.63 & 1.53 & 179.8 & bar & 0.60 & 0.20 & 2.9 & 0.70 & 0.21 & 63.5 \\
$\mathrm{bar}$ & 0.91 & 0.48 & 31.6 & 1.52 & 0.48 & 93.8 & & & & & & & \\
\hline
\end{tabular}

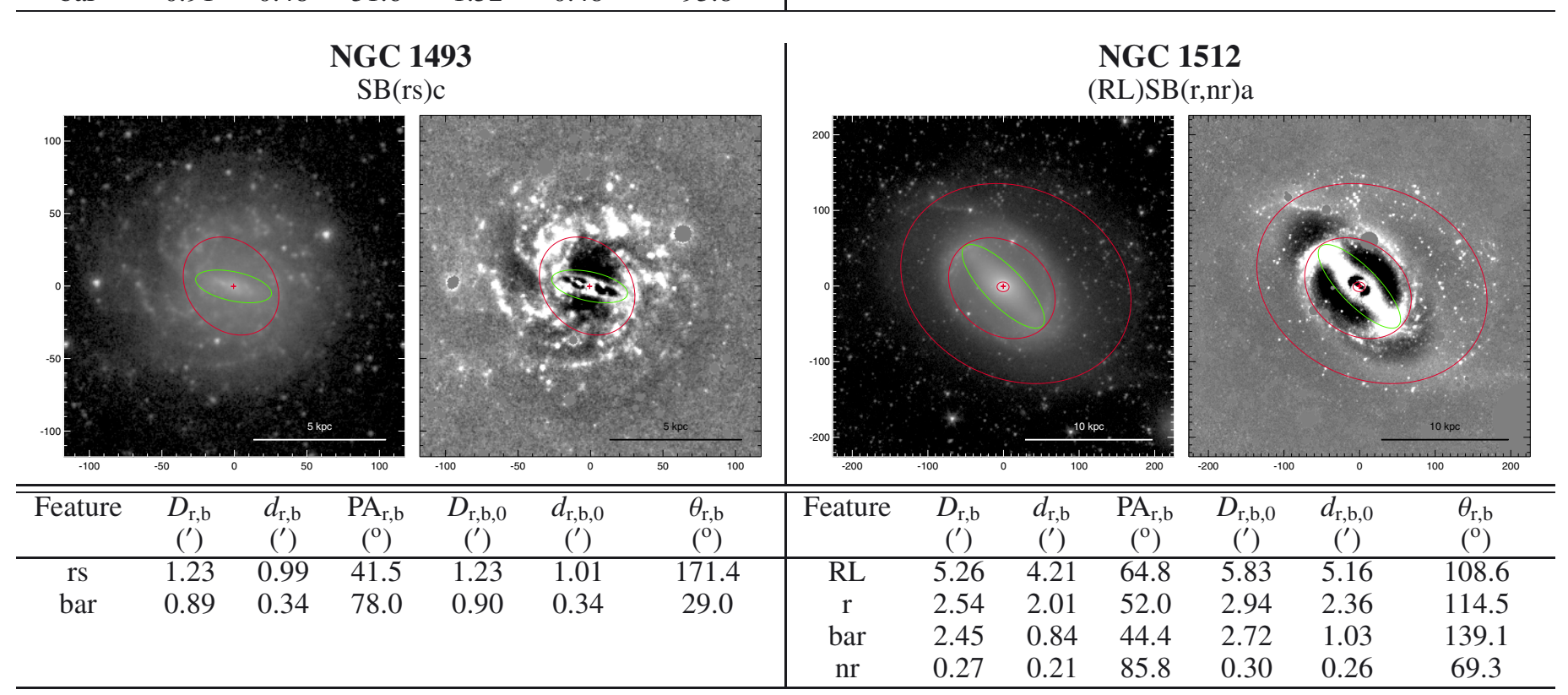


NGC 1515

(L)SAㅡㅁ $\left(\underline{r s}, n b_{a}\right) 0 / a \mathrm{sp}$

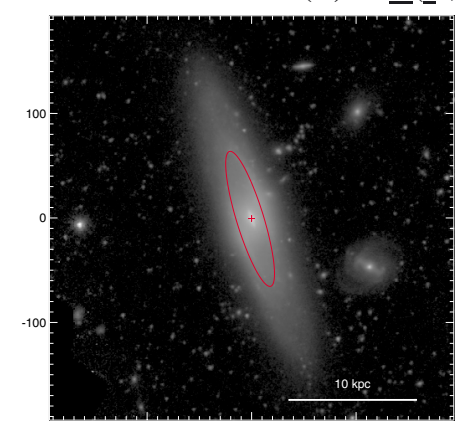

NGC 1532

$\mathrm{SAB}(\mathrm{rs}, \mathrm{nd})$ ab sp pec
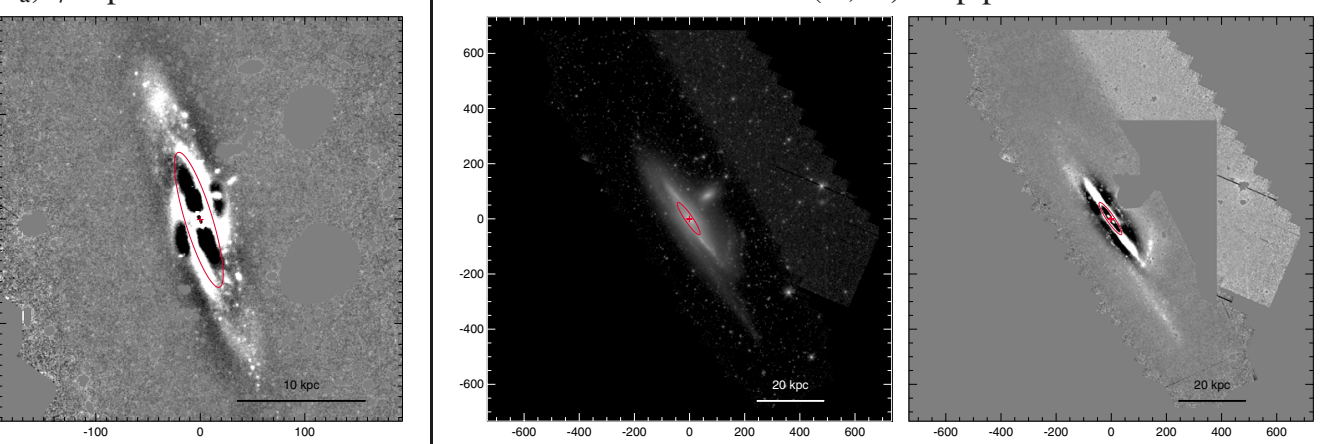

\begin{tabular}{ccccccc|ccccccc}
\hline \hline Feature & $\begin{array}{c}D_{\mathrm{r}, \mathrm{b}} \\
\left({ }^{\prime}\right)\end{array}$ & $\begin{array}{c}d_{\mathrm{r}, \mathrm{b}} \\
\left({ }^{\prime}\right)\end{array}$ & $\begin{array}{c}\mathrm{PA}_{\mathrm{r}, \mathrm{b}} \\
\left({ }^{\circ}\right)\end{array}$ & $\begin{array}{c}D_{\mathrm{r}, \mathrm{b}, 0} \\
\left({ }^{\prime}\right)\end{array}$ & $\begin{array}{c}d_{\mathrm{r}, \mathrm{b}, 0} \\
\left({ }^{\prime}\right)\end{array}$ & $\begin{array}{c}\theta_{\mathrm{r}, \mathrm{b}} \\
\left({ }^{\circ}\right)\end{array}$ & Feature & $\begin{array}{c}D_{\mathrm{r}, \mathrm{b}} \\
\left({ }^{\prime}\right)\end{array}$ & $\begin{array}{c}d_{\mathrm{r}, \mathrm{b}} \\
\left({ }^{\prime}\right)\end{array}$ & $\begin{array}{c}\mathrm{PA}_{\mathrm{r}, \mathrm{b}} \\
\left({ }^{\circ}\right)\end{array}$ & $\begin{array}{c}D_{\mathrm{r}, \mathrm{b}, 0} \\
\left({ }^{\prime}\right)\end{array}$ & $\begin{array}{c}d_{\mathrm{r}, \mathrm{b}, 0} \\
\left({ }^{\prime}\right)\end{array}$ & $\begin{array}{c}\theta_{\mathrm{r}, \mathrm{b}} \\
\left({ }^{\circ}\right)\end{array}$ \\
\hline$\underline{\mathrm{r} s}$ & 2.25 & 0.44 & 17.0 & 2.26 & 1.68 & 174.3 & rs & 2.34 & 0.46 & 34.4 & 2.34 & 1.45 & 0.4 \\
\hline
\end{tabular}

NGC 1533

(RL)SB0 ${ }^{\circ}$
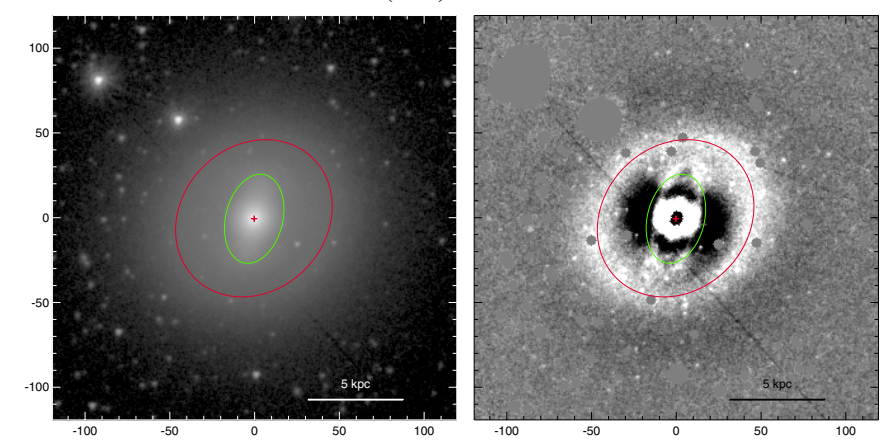

NGC 1546

$\mathrm{E}(\mathrm{b}) 3 /\left(\mathrm{R}^{\prime}\right) \mathrm{SA}(\mathrm{r}) \mathrm{ab}$

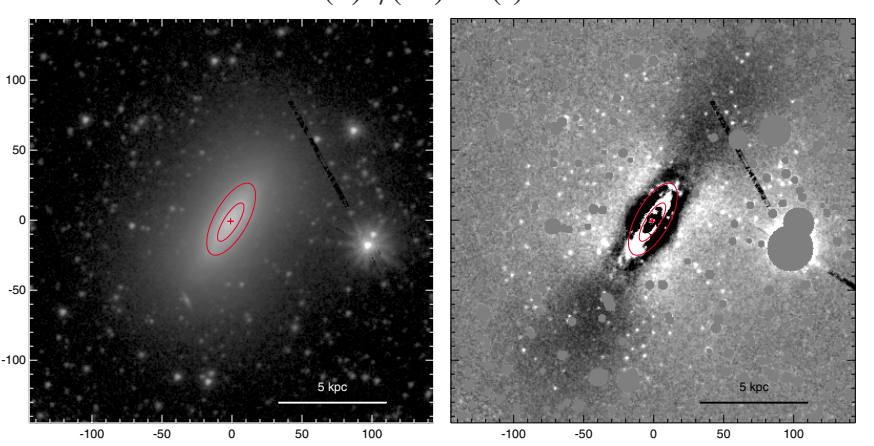

\begin{tabular}{ccccccc|ccccccc}
\hline \hline Feature & $\begin{array}{c}D_{\mathrm{r}, \mathrm{b}} \\
\left({ }^{\prime}\right)\end{array}$ & $\begin{array}{c}d_{\mathrm{r}, \mathrm{b}} \\
\left({ }^{\prime}\right)\end{array}$ & $\begin{array}{c}\mathrm{PA}_{\mathrm{r}, \mathrm{b}} \\
\left({ }^{\circ}\right)\end{array}$ & $\begin{array}{c}D_{\mathrm{r}, \mathrm{b}, 0} \\
\left({ }^{\prime}\right)\end{array}$ & $\begin{array}{c}d_{\mathrm{r}, \mathrm{b}, 0} \\
\left({ }^{\prime}\right)\end{array}$ & $\begin{array}{c}\theta_{\mathrm{r}, \mathrm{b}} \\
\left({ }^{\circ}\right)\end{array}$ & Feature & $\begin{array}{c}D_{\mathrm{r}, \mathrm{b}} \\
\left({ }^{\prime}\right)\end{array}$ & $\begin{array}{c}d_{\mathrm{r}, \mathrm{b}} \\
\left({ }^{\prime}\right)\end{array}$ & $\begin{array}{c}\mathrm{PA}_{\mathrm{r}, \mathrm{b}} \\
\left({ }^{\circ}\right)\end{array}$ & $\begin{array}{c}D_{\mathrm{r}, \mathrm{b}, 0} \\
\left({ }^{\prime}\right)\end{array}$ & $\begin{array}{c}d_{\mathrm{r}, \mathrm{b}, 0} \\
\left({ }^{\prime}\right)\end{array}$ & $\begin{array}{c}\theta_{\mathrm{r}, \mathrm{b}} \\
\left({ }^{\circ}\right)\end{array}$ \\
\hline $\mathrm{RL}$ & 1.66 & 1.43 & 135.7 & 1.66 & 1.48 & 11.5 & $\mathrm{R}^{\prime}$ & 0.96 & 0.40 & 150.8 & 0.99 & 0.87 & 33.8 \\
$\mathrm{bar}$ & 0.89 & 0.56 & 166.4 & 0.91 & 0.58 & 41.9 & $\mathrm{r}$ & 0.53 & 0.17 & 148.0 & 0.53 & 0.39 & 2.9 \\
\hline
\end{tabular}

NGC 1553

$\mathrm{SA}\left(\mathrm{rl}, \mathrm{nr}^{\prime} \mathrm{l}\right) 0^{+}$
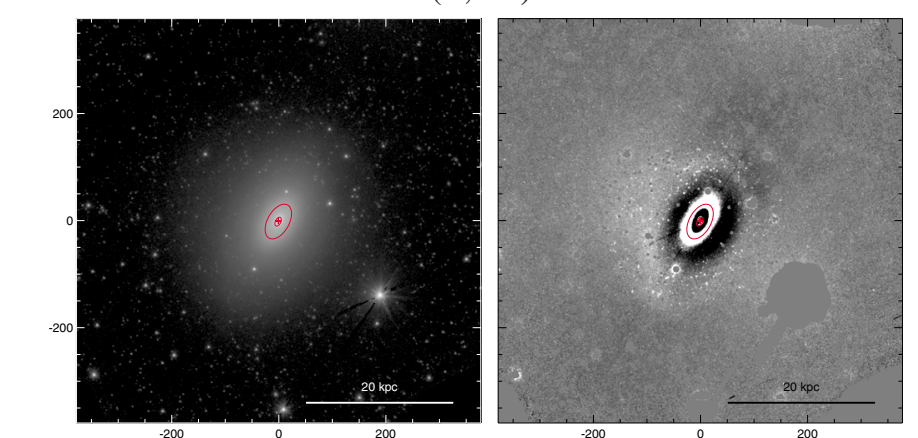

NGC 1566

$\left(\mathrm{R}_{1}^{\prime}\right) \mathrm{SAB}\left(\mathrm{r}^{\prime} 1 \mathrm{l}, \mathrm{s}, \mathrm{nb}\right) \mathrm{b}$

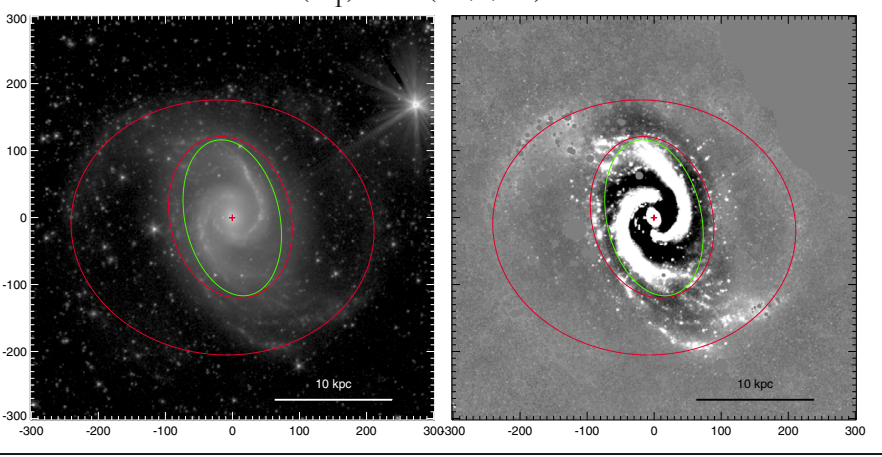

\begin{tabular}{ccccccc|ccccccc}
\hline \hline Feature & $\begin{array}{c}D_{\mathrm{r}, \mathrm{b}} \\
\left({ }^{\prime}\right)\end{array}$ & $\begin{array}{c}d_{\mathrm{r}, \mathrm{b}} \\
\left({ }^{\prime}\right)\end{array}$ & $\begin{array}{c}\mathrm{PA}_{\mathrm{r}, \mathrm{b}} \\
\left({ }^{\circ}\right)\end{array}$ & $\begin{array}{c}D_{\mathrm{r}, \mathrm{b}, 0} \\
\left({ }^{\prime}\right)\end{array}$ & $\begin{array}{c}d_{\mathrm{r}, \mathrm{b}, 0} \\
\left({ }^{\prime}\right)\end{array}$ & $\begin{array}{c}\theta_{\mathrm{r}, \mathrm{b}} \\
\left({ }^{(}\right)\end{array}$ & Feature & $\begin{array}{c}D_{\mathrm{r}, \mathrm{b}} \\
\left({ }^{\prime}\right)\end{array}$ & $\begin{array}{c}d_{\mathrm{r}, \mathrm{b}} \\
\left({ }^{\prime}\right)\end{array}$ & $\begin{array}{c}\mathrm{PA}_{\mathrm{r}, \mathrm{b}} \\
\left({ }^{\circ}\right)\end{array}$ & $\begin{array}{c}D_{\mathrm{r}, \mathrm{b}, 0} \\
\left({ }^{\prime}\right)\end{array}$ & $\begin{array}{c}d_{\mathrm{r}, \mathrm{b}, 0} \\
\left({ }^{\prime}\right)\end{array}$ & $\begin{array}{c}\theta_{\mathrm{r}, \mathrm{b}} \\
\left({ }^{\circ}\right)\end{array}$ \\
\hline $\mathrm{rl}$ & 1.18 & 0.67 & 150.6 & 1.18 & 0.93 & 174.3 & $\mathrm{R}_{1}^{\prime}$ & 7.55 & 6.34 & 85.3 & 7.55 & 7.49 & 167.7 \\
$\mathrm{nr}^{\prime} 1$ & 0.30 & 0.16 & 153.7 & 0.30 & 0.23 & 1.8 & $\mathrm{r}^{\prime} 1$ & 4.07 & 3.00 & 14.1 & 4.76 & 3.04 & 101.6 \\
& & & & & & & bar & 3.94 & 2.35 & 12.6 & 4.61 & 2.38 & 102.3 \\
\hline
\end{tabular}


NGC 1640

$\left(\mathrm{R}^{\prime}\right) \mathrm{SB}_{\mathrm{a}}(\mathrm{r}, \mathrm{bl}) \mathrm{a}$
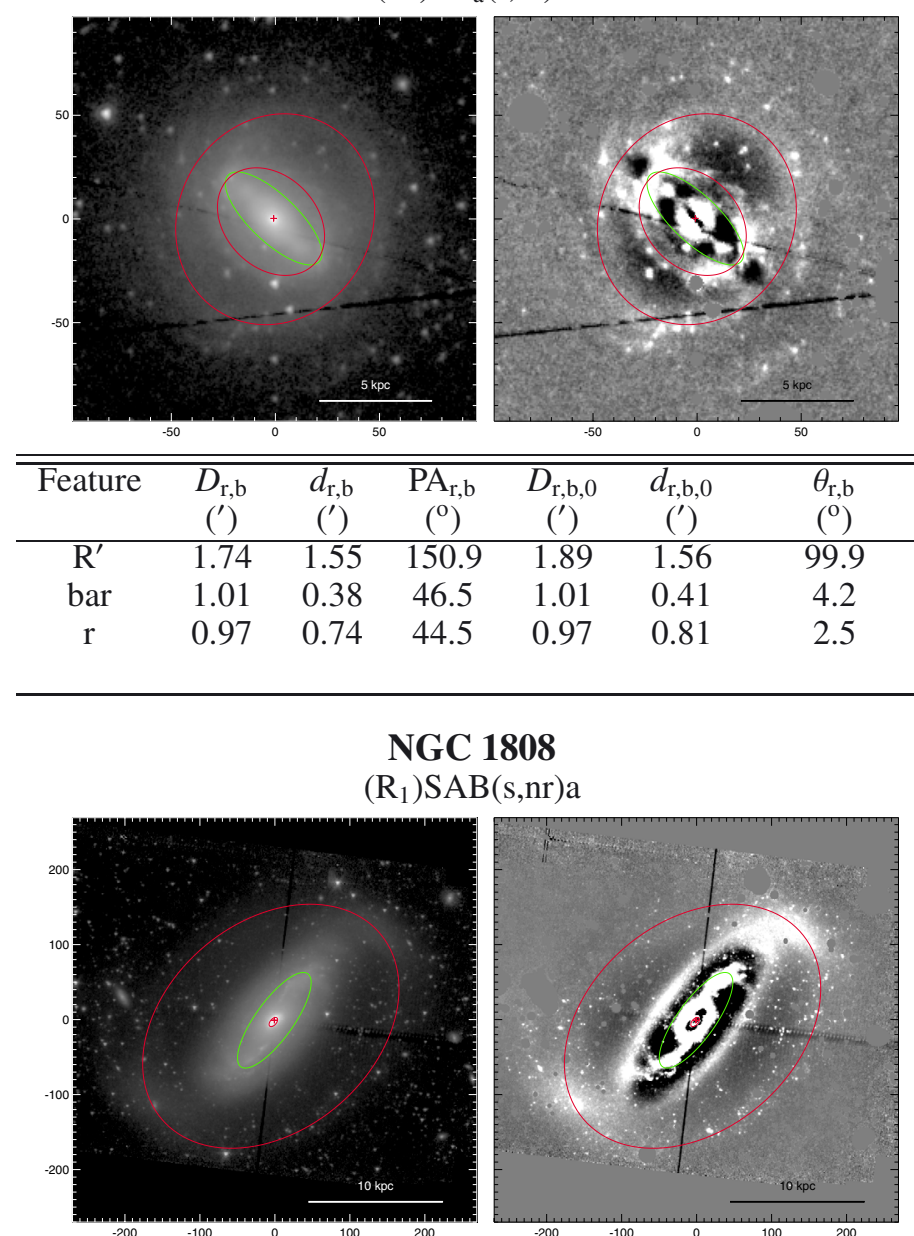

NGC 1808

$\left(\mathrm{R}_{1}\right) \mathrm{SAB}(\mathrm{s}, \mathrm{nr}) \mathrm{a}$

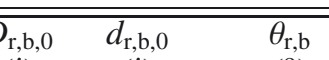

$\begin{array}{ccc}\left({ }^{\prime}\right) & \left({ }^{\prime}\right) & \left({ }^{\circ}\right) \\ 1.89 & 1.56 & 99 .\end{array}$

$\begin{array}{lll}0.97 & 0.81 \quad 2.5\end{array}$

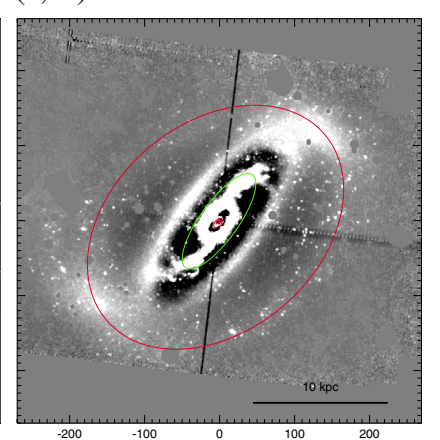

NGC 1672

$\left(\mathrm{R}^{\prime}\right) \mathrm{SAB}(\mathrm{rs}, \mathrm{nr}) \mathrm{b}$
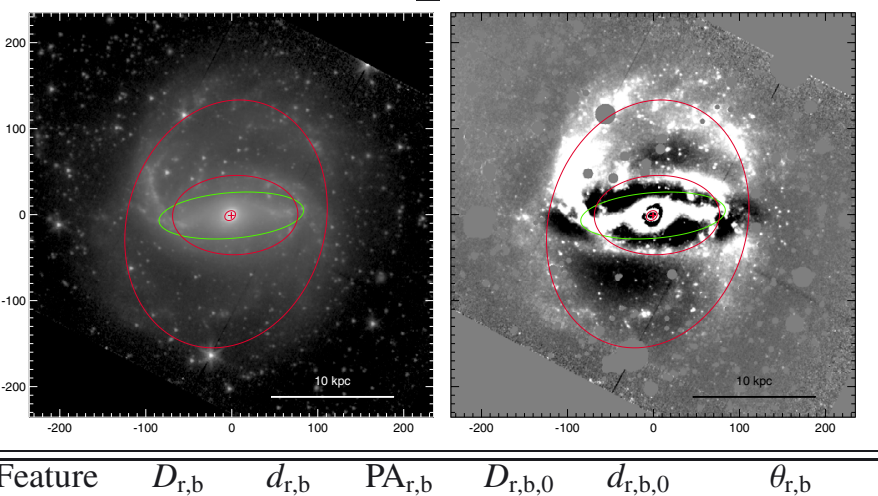

\begin{tabular}{c|c}
$\left({ }^{\circ}\right)$ & $\left({ }^{\prime}\right)$ \\
\hline 99.9 & $\mathrm{R}^{\prime}$
\end{tabular}

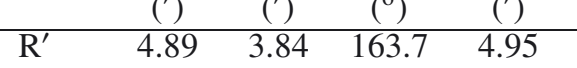

$\begin{array}{lllll}\text { bar } & 2.82 & 0.89 & 94.5 & 3.04\end{array}$

$\begin{array}{lllll}\text { rs } & 2.43 & 1.54 & 91.5 & 2.65\end{array}$

$\begin{array}{lllll}\mathrm{nr} & 0.22 & 0.17 & 124.3 & 0.23\end{array}$

4.24

0.92

$\left.{ }^{\mathrm{o}}\right)$

(6.0

NGC 2460

$\mathrm{SAB}(\mathrm{rs}) \mathrm{a}$

\begin{tabular}{|c|c|c|c|c|c|c|c|c|c|c|c|c|c|}
\hline Feature & $\begin{array}{c}D_{\mathrm{r}, \mathrm{b}} \\
\left(^{\prime}\right) \\
\end{array}$ & $\begin{array}{c}d_{\mathrm{r}, \mathrm{b}} \\
\left({ }^{\prime}\right)\end{array}$ & $\begin{array}{c}\mathrm{PA}_{\mathrm{r}, \mathrm{b}} \\
\left({ }^{\circ}\right)\end{array}$ & $\begin{array}{c}D_{\mathrm{r}, \mathrm{b}, 0} \\
\left(^{\prime}\right)\end{array}$ & $\begin{array}{c}d_{\mathrm{r}, \mathrm{b}, 0} \\
\left(^{\prime}\right)\end{array}$ & $\begin{array}{c}\theta_{\mathrm{r}, \mathrm{b}} \\
\left({ }^{\circ}\right)\end{array}$ & Feature & $\begin{array}{c}D_{\mathrm{r}, \mathrm{b}} \\
\left({ }^{\prime}\right)\end{array}$ & $\begin{array}{c}d_{\mathrm{r}, \mathrm{b}} \\
\left({ }^{\prime}\right)\end{array}$ & $\begin{array}{c}\mathrm{PA}_{\mathrm{r}, \mathrm{b}} \\
\left({ }^{\circ}\right)\end{array}$ & $\begin{array}{c}D_{\mathrm{r}, \mathrm{b}, 0} \\
\left(^{\prime}\right)\end{array}$ & $\begin{array}{c}d_{\mathrm{r}, \mathrm{b}, 0} \\
\left(^{\prime}\right)\end{array}$ & $\begin{array}{l}\theta_{\mathrm{r}, \mathrm{b}} \\
\left({ }^{\circ}\right)\end{array}$ \\
\hline$\overline{\mathrm{R}_{1}}$ & 6.38 & 4.62 & 130.5 & 6.52 & 6.18 & 42.2 & rs & 0.37 & 0.29 & 21.3 & 0.45 & 0.34 & 110.1 \\
\hline bar & 2.55 & 0.84 & 144.3 & 2.67 & 1.10 & 26.8 & & & & & & & \\
\hline $\mathrm{nr}$ & 0.24 & 0.14 & 138.8 & 0.25 & 0.18 & 26.1 & & & & & & & \\
\hline
\end{tabular}
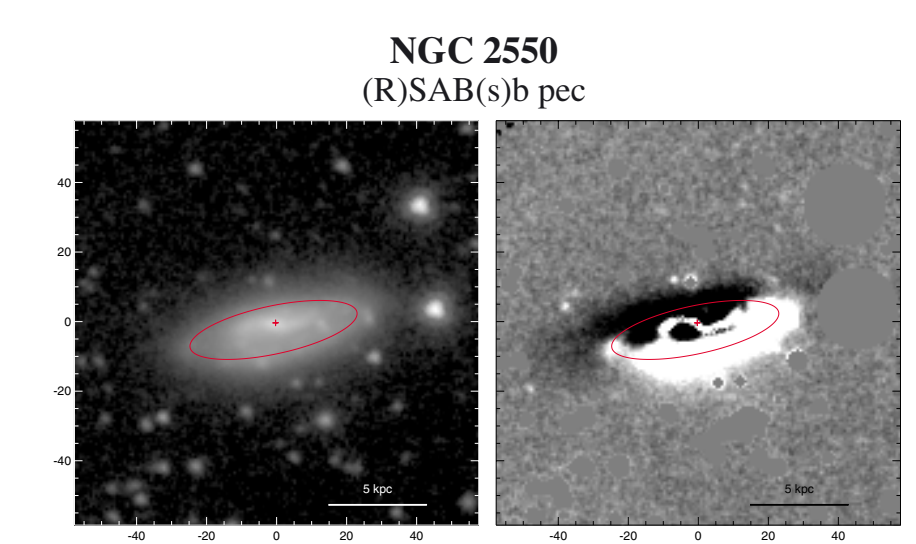

NGC 2552

$\left(\mathrm{R}^{\prime}\right) \mathrm{SAB}(\mathrm{s}) \mathrm{m}$

\begin{tabular}{ccccccc|ccccccc}
\hline \hline Feature & $\begin{array}{c}D_{\mathrm{r}, \mathrm{b}} \\
\left({ }^{\prime}\right)\end{array}$ & $\begin{array}{c}d_{\mathrm{r}, \mathrm{b}} \\
\left({ }^{\prime}\right)\end{array}$ & $\begin{array}{c}\mathrm{PA}_{\mathrm{r}, \mathrm{b}} \\
\left({ }^{\circ}\right)\end{array}$ & $\begin{array}{c}D_{\mathrm{r}, \mathrm{b}, 0} \\
\left({ }^{\prime}\right)\end{array}$ & $\begin{array}{c}d_{\mathrm{r}, \mathrm{b}, 0} \\
\left({ }^{\prime}\right)\end{array}$ & $\begin{array}{c}\theta_{\mathrm{r}, \mathrm{b}} \\
\left({ }^{\circ}\right)\end{array}$ & Feature & $\begin{array}{c}D_{\mathrm{r}, \mathrm{b}} \\
\left({ }^{\prime}\right)\end{array}$ & $\begin{array}{c}d_{\mathrm{r}, \mathrm{b}} \\
\left({ }^{\prime}\right)\end{array}$ & $\begin{array}{c}\mathrm{PA}_{\mathrm{r}, \mathrm{b}} \\
\left({ }^{\circ}\right)\end{array}$ & $\begin{array}{c}D_{\mathrm{r}, \mathrm{b}, 0} \\
\left({ }^{\prime}\right)\end{array}$ & $\begin{array}{c}d_{\mathrm{r}, \mathrm{b}, 0} \\
\left({ }^{\prime}\right)\end{array}$ & $\begin{array}{c}\theta_{\mathrm{r}, \mathrm{b}} \\
\left({ }^{\circ}\right)\end{array}$ \\
\hline $\mathrm{R}$ & 0.82 & 0.23 & 102.0 & 0.82 & 0.54 & 177.6 & $\mathrm{R}^{\prime}$ & 1.88 & 1.22 & 53.6 & 1.96 & 1.67 & 35.9 \\
\hline
\end{tabular}




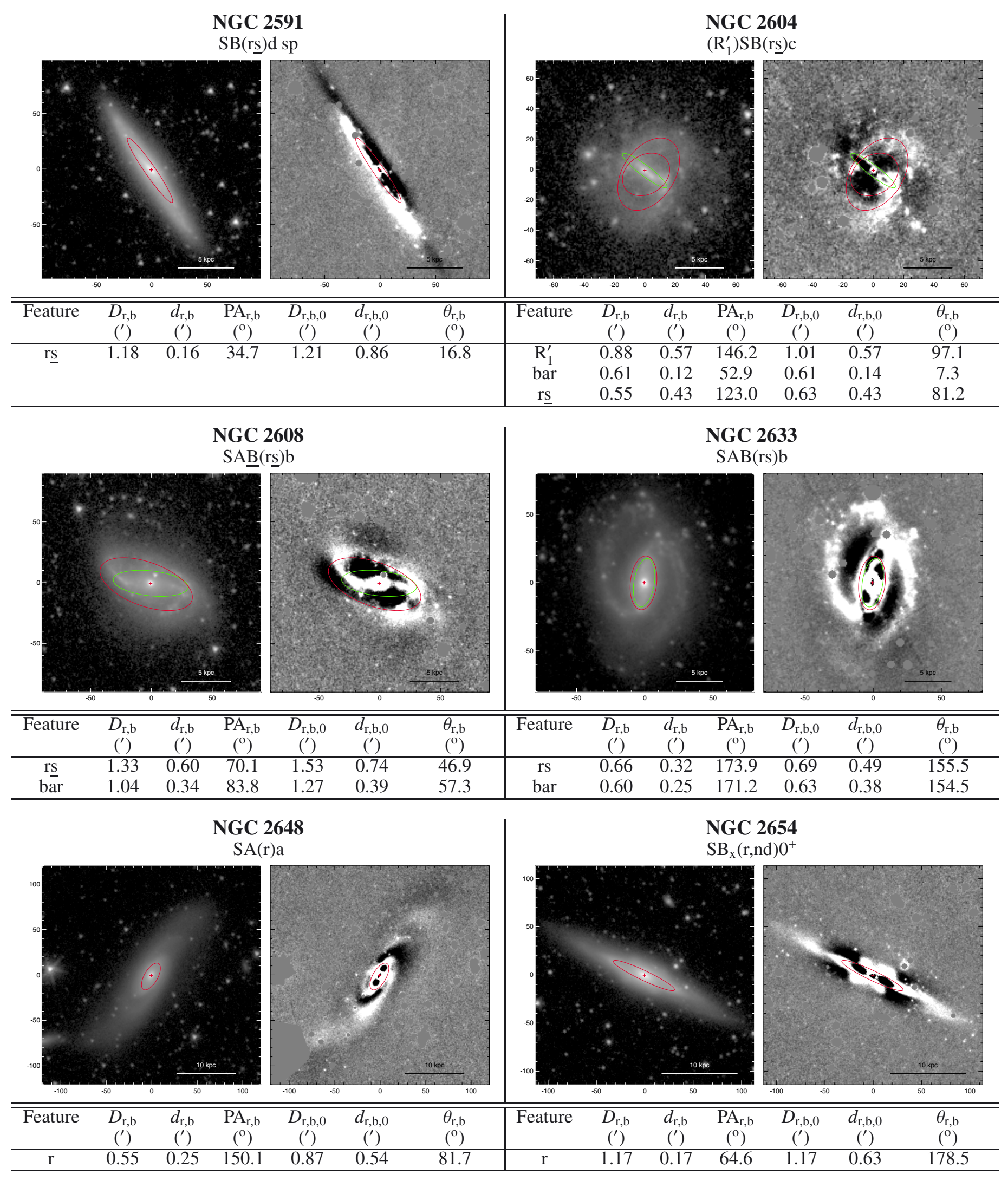


NGC 2681

(R)SAB(rs)0/a
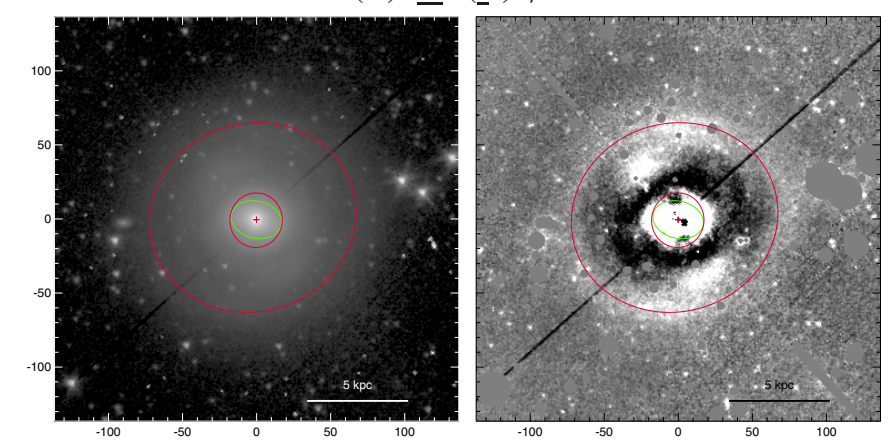

\begin{tabular}{|c|c|c|c|c|c|c|c|c|c|c|c|c|c|}
\hline Feature & $\begin{array}{c}D_{\mathrm{r}, \mathrm{b}} \\
\left({ }^{\prime}\right)\end{array}$ & $\begin{array}{c}d_{\mathrm{r}, \mathrm{b}} \\
\left(^{\prime}\right) \\
\end{array}$ & $\begin{array}{c}\mathrm{PA}_{\mathrm{r}, \mathrm{b}} \\
\left({ }^{\circ}\right)\end{array}$ & $\begin{array}{c}D_{\mathrm{r}, \mathrm{b}, 0} \\
\left({ }^{\prime}\right)\end{array}$ & $\begin{array}{c}d_{\mathrm{r}, \mathrm{b}, 0} \\
\left(^{\prime}\right)\end{array}$ & $\begin{array}{c}\theta_{\mathrm{r}, \mathrm{b}} \\
\left({ }^{\circ}\right) \\
\end{array}$ & Feature & $\begin{array}{c}D_{\mathrm{r}, \mathrm{b}} \\
\left({ }^{\prime}\right)\end{array}$ & $\begin{array}{c}d_{\mathrm{r}, \mathrm{b}} \\
\left(^{\prime}\right) \\
\end{array}$ & $\begin{array}{c}\mathrm{PA}_{\mathrm{r}, \mathrm{b}} \\
\left({ }^{\mathrm{O}}\right)\end{array}$ & $\begin{array}{c}D_{\mathrm{r}, \mathrm{b}, 0} \\
\left({ }^{\prime}\right)\end{array}$ & $\begin{array}{c}d_{\mathrm{r}, \mathrm{b}, 0} \\
\left(^{\prime}\right)\end{array}$ & $\begin{array}{l}\theta_{\mathrm{r}, \mathrm{b}} \\
\left({ }^{\circ}\right)\end{array}$ \\
\hline $\bar{R}$ & 2.35 & 2.13 & 105.0 & 2.35 & 2.16 & 165.7 & $\mathrm{RL}$ & 6.82 & 0.91 & 43.8 & 6.83 & 3.50 & 3.8 \\
\hline$\underline{r s}$ & 0.62 & 0.59 & 172.5 & 0.62 & 0.59 & 66.1 & $\underline{\mathrm{rs}}$ & 3.34 & 0.39 & 43.3 & 3.34 & 1.48 & 0.8 \\
\hline bar & 0.58 & 0.42 & 76.8 & 0.58 & 0.42 & 138.4 & & & & & & & \\
\hline
\end{tabular}

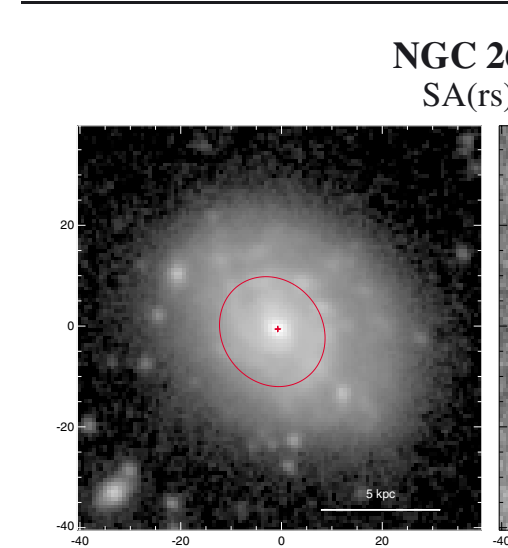

GC 2684

$\mathrm{SA}(\mathrm{rs}) \mathrm{b}$

\begin{tabular}{ccccccc|ccccccc}
\hline \hline Feature & $\begin{array}{c}D_{\mathrm{r}, \mathrm{b}} \\
\left({ }^{\prime}\right)\end{array}$ & $\begin{array}{c}d_{\mathrm{r}, \mathrm{b}} \\
\left({ }^{\prime}\right)\end{array}$ & $\begin{array}{c}\mathrm{PA}_{\mathrm{r}, \mathrm{b}} \\
\left({ }^{\circ}\right)\end{array}$ & $\begin{array}{c}D_{\mathrm{r}, \mathrm{b}, 0} \\
\left({ }^{\prime}\right)\end{array}$ & $\begin{array}{c}d_{\mathrm{r}, \mathrm{b}, 0} \\
\left({ }^{\prime}\right)\end{array}$ & $\begin{array}{c}\theta_{\mathrm{r}, \mathrm{b}} \\
\left({ }^{\circ}\right)\end{array}$ & Feature & $\begin{array}{c}D_{\mathrm{r}, \mathrm{b}} \\
\left({ }^{\prime}\right)\end{array}$ & $\begin{array}{c}d_{\mathrm{r}, \mathrm{b}} \\
\left({ }^{\prime}\right)\end{array}$ & $\begin{array}{c}\mathrm{PA}_{\mathrm{r}, \mathrm{b}} \\
\left({ }^{\circ}\right)\end{array}$ & $\begin{array}{c}D_{\mathrm{r}, \mathrm{b}, 0} \\
\left({ }^{\prime}\right)\end{array}$ & $\begin{array}{c}d_{\mathrm{r}, \mathrm{b}, 0} \\
\left({ }^{\prime}\right)\end{array}$ & $\begin{array}{c}\theta_{\mathrm{r}, \mathrm{b}} \\
\left({ }^{\circ}\right)\end{array}$ \\
\hline $\mathrm{rs}$ & 0.38 & 0.34 & 35.7 & 0.42 & 0.38 & 105.4 & $\mathrm{R}$ & 4.08 & 1.80 & 33.9 & 4.39 & 2.65 & 147.0 \\
& & & & & & & $\mathrm{PRG}$ & 1.36 & 0.70 & 114.0 & - & - & - \\
\hline
\end{tabular}

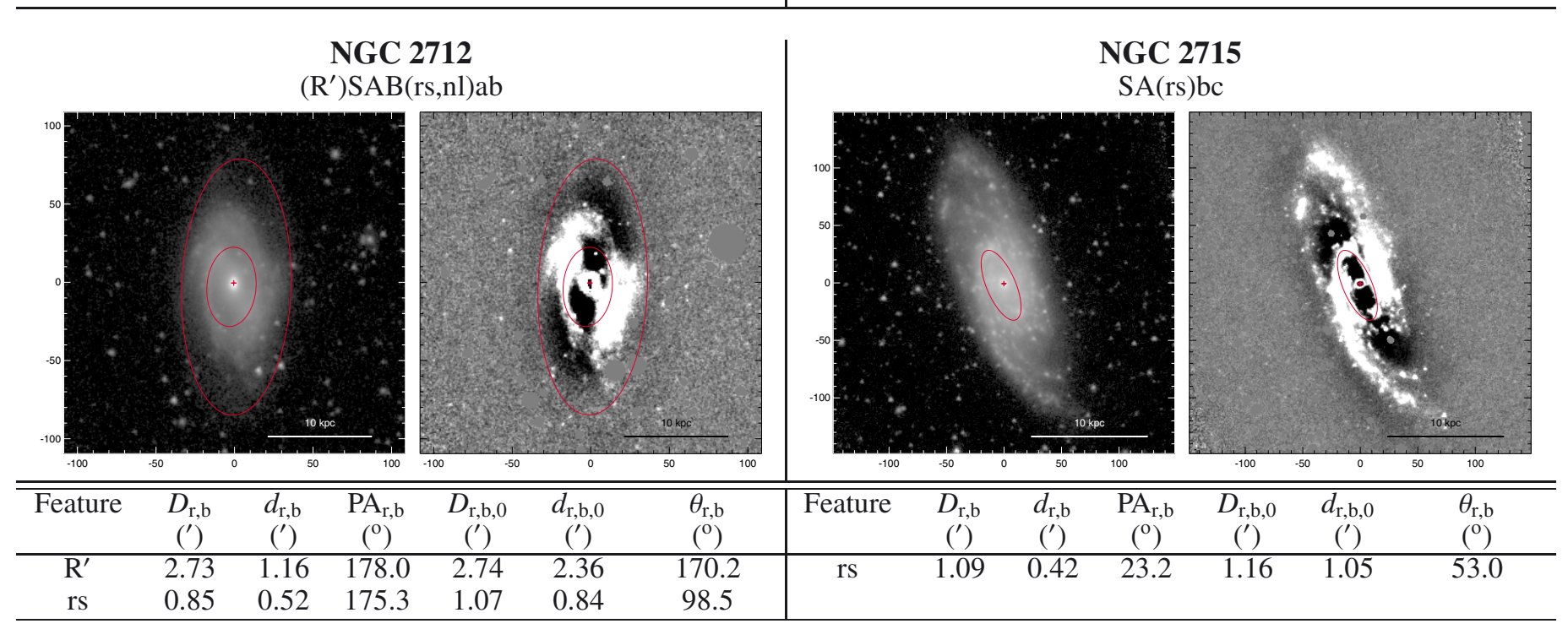


S. Comerón et al.: ARRAKIS

NGC 2726

SB?(rs)0/a
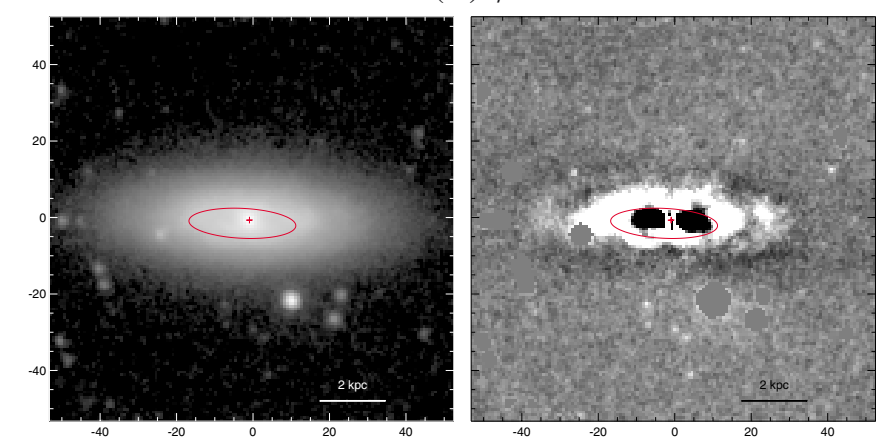

\begin{tabular}{|c|c|c|c|c|c|c|c|c|c|c|c|c|c|}
\hline$\overline{\text { Feature }}$ & $\begin{array}{c}D_{\mathrm{r}, \mathrm{b}} \\
\left({ }^{\prime}\right)\end{array}$ & $\begin{array}{c}d_{\mathrm{r}, \mathrm{b}} \\
\left({ }^{\prime}\right)\end{array}$ & $\begin{array}{c}\mathrm{PA}_{\mathrm{r}, \mathrm{b}} \\
\left({ }^{\circ}\right)\end{array}$ & $\begin{array}{c}D_{\mathrm{r}, \mathrm{b}, 0} \\
\left(^{\prime}\right)\end{array}$ & $\begin{array}{c}d_{\mathrm{r}, \mathrm{b}, 0} \\
\left({ }^{\prime}\right)\end{array}$ & $\begin{array}{l}\theta_{\mathrm{r}, \mathrm{b}} \\
\left({ }^{\circ}\right)\end{array}$ & Feature & $\begin{array}{c}D_{\mathrm{r}, \mathrm{b}} \\
\left({ }^{\prime}\right)\end{array}$ & $\begin{array}{c}d_{\mathrm{r}, \mathrm{b}} \\
\left({ }^{\prime}\right)\end{array}$ & $\begin{array}{c}\mathrm{PA}_{\mathrm{r}, \mathrm{b}} \\
\left({ }^{\circ}\right)\end{array}$ & $\begin{array}{c}D_{\mathrm{r}, \mathrm{b}, 0} \\
\left(^{\prime}\right)\end{array}$ & $\begin{array}{c}d_{\mathrm{r}, \mathrm{b}, 0} \\
\left({ }^{\prime}\right)\end{array}$ & $\begin{array}{l}\theta_{\mathrm{r}, \mathrm{b}} \\
\left(^{\circ}\right)\end{array}$ \\
\hline rs & 0.47 & 0.13 & 87.3 & 0.47 & 0.34 & 175.3 & $\mathrm{r}$ & 0.45 & 0.13 & 102.9 & 0.51 & 0.33 & 37.7 \\
\hline
\end{tabular}

NGC 2743

$\mathrm{SB}(\mathrm{rs}) \mathrm{m}$

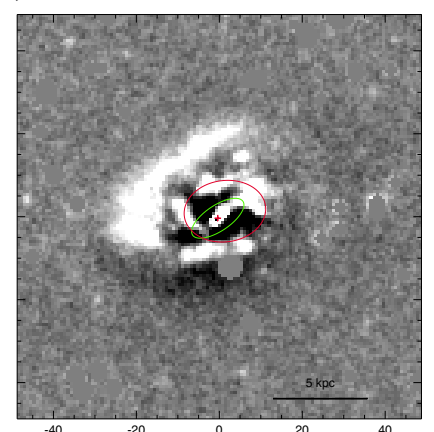

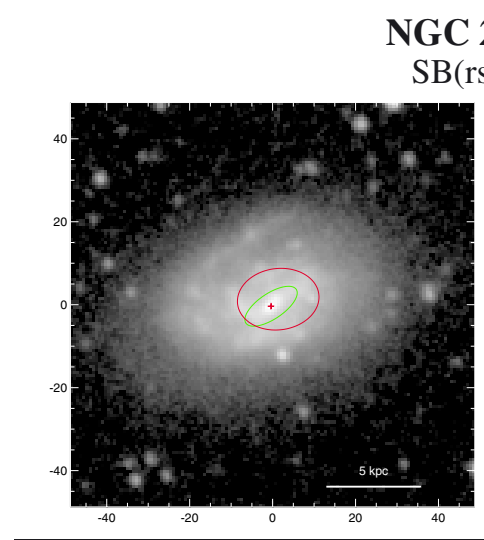

NGC 2748

$\left(\mathrm{R}^{\prime}\right) \mathrm{SAB}(\mathrm{r}) \mathrm{bc}+\mathrm{PR}$ ?
NGC 2735

SA:(r)0/a sp pec

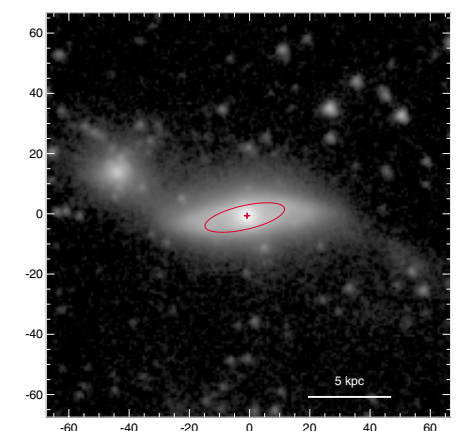

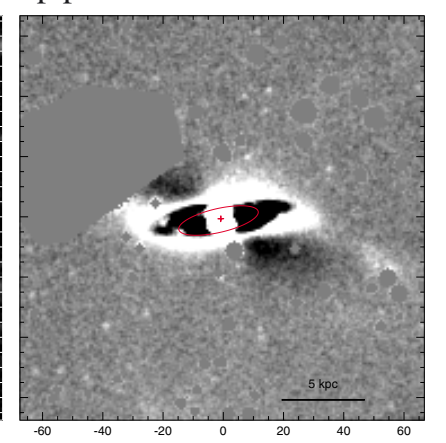

(o)
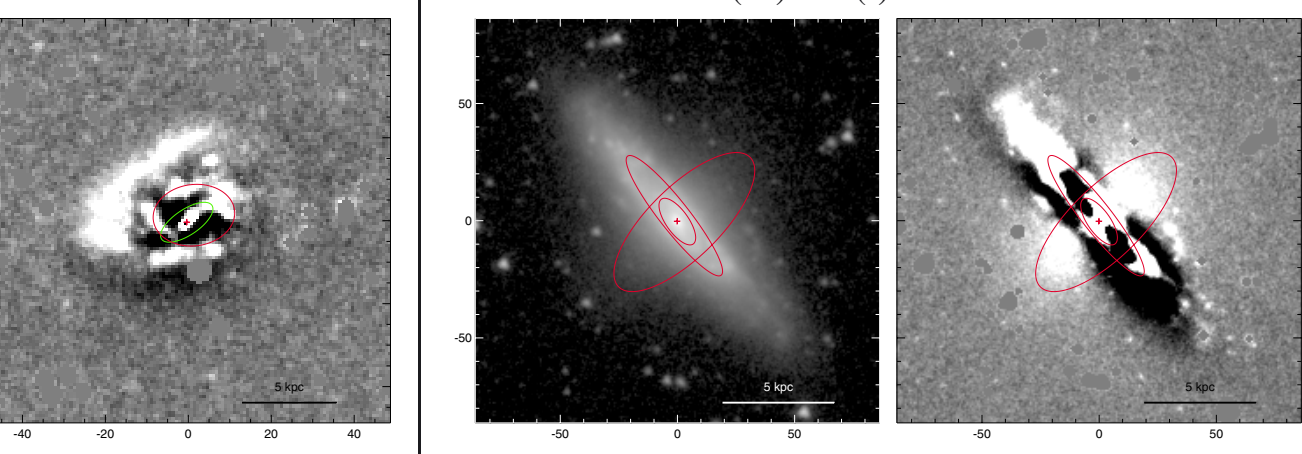

\begin{tabular}{ccccccc|ccccccc}
\hline \hline Feature & $\begin{array}{c}D_{\mathrm{r}, \mathrm{b}} \\
\left({ }^{\prime}\right)\end{array}$ & $\begin{array}{c}d_{\mathrm{r}, \mathrm{b}} \\
\left({ }^{\prime}\right)\end{array}$ & $\begin{array}{c}\mathrm{PA}_{\mathrm{r}, \mathrm{b}} \\
\left({ }^{\circ}\right)\end{array}$ & $\begin{array}{c}D_{\mathrm{r}, \mathrm{b}, 0} \\
\left(^{\prime}\right)\end{array}$ & $\begin{array}{c}d_{\mathrm{r}, \mathrm{b}, 0} \\
\left(^{\prime}\right)\end{array}$ & $\begin{array}{c}\theta_{\mathrm{r}, \mathrm{b}} \\
\left({ }^{\circ}\right)\end{array}$ & Feature & $\begin{array}{c}D_{\mathrm{r}, \mathrm{b}} \\
\left({ }^{\prime}\right)\end{array}$ & $\begin{array}{c}d_{\mathrm{r}, \mathrm{b}} \\
\left(^{\prime}\right)\end{array}$ & $\begin{array}{c}\mathrm{PA}_{\mathrm{r}, \mathrm{b}} \\
\left({ }^{\circ}\right)\end{array}$ & $\begin{array}{c}D_{\mathrm{r}, \mathrm{b}, 0} \\
\left({ }^{\prime}\right)\end{array}$ & $\begin{array}{c}d_{\mathrm{r}, \mathrm{b}, 0} \\
\left({ }^{\prime}\right)\end{array}$ & $\begin{array}{c}\theta_{\mathrm{r}, \mathrm{b}} \\
\left({ }^{\circ}\right)\end{array}$ \\
\hline rs & 0.33 & 0.25 & 96.9 & 0.39 & 0.32 & 106.6 & $\mathrm{PRG}$ & 1.32 & 0.51 & 134.5 & - & - & - \\
$\mathrm{bar}$ & 0.25 & 0.10 & 123.8 & 0.26 & 0.15 & 31.0 & $\mathrm{R}$ & 1.08 & 0.21 & 38.2 & 1.11 & 0.70 & 163.5 \\
& & & & & & & $\mathrm{r}$ & 0.39 & 0.16 & 35.3 & 0.57 & 0.38 & 104.5 \\
\hline
\end{tabular}
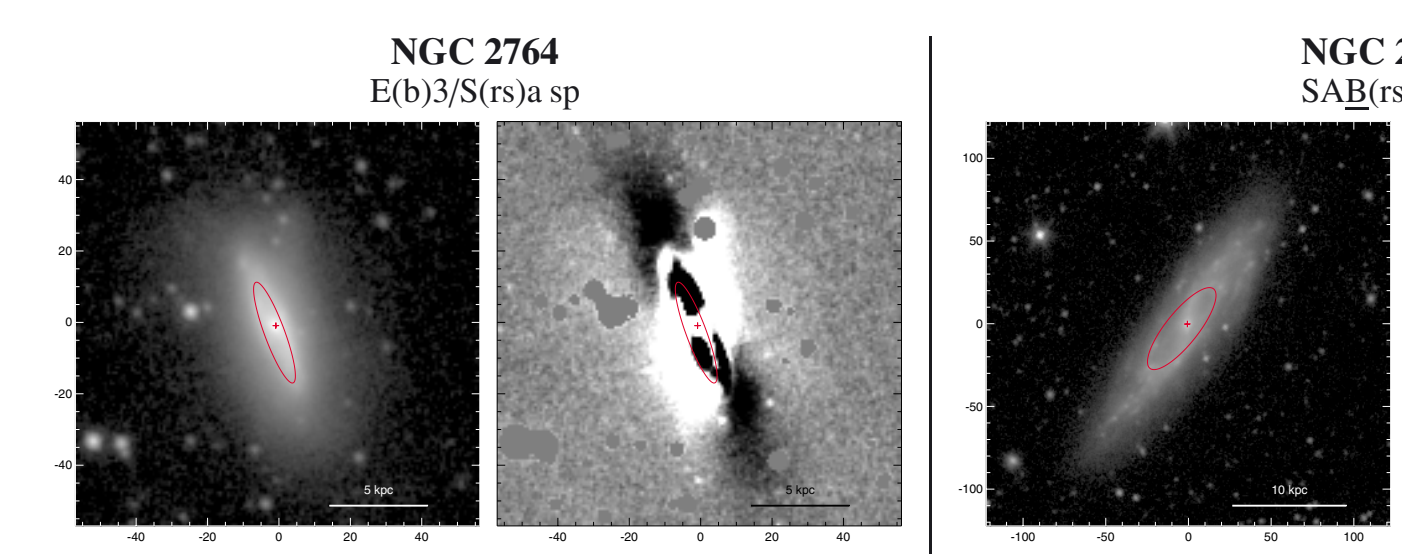

NGC 2770

$\mathrm{SA} \underline{B}(\mathrm{rs}) \mathrm{c} \mathrm{sp}$

\begin{tabular}{|c|c|c|c|c|c|c|c|c|c|c|c|c|c|}
\hline Feature & $\begin{array}{c}D_{\mathrm{r}, \mathrm{b}} \\
\left(^{\prime}\right)\end{array}$ & $\begin{array}{c}d_{\mathrm{r}, \mathrm{b}} \\
\left(^{\prime}\right)\end{array}$ & $\begin{array}{c}\mathrm{PA}_{\mathrm{r}, \mathrm{b}} \\
\left({ }^{\circ}\right)\end{array}$ & $\begin{array}{c}D_{\mathrm{r}, \mathrm{b}, 0} \\
\left(^{\prime}\right)\end{array}$ & $\begin{array}{c}d_{\mathrm{r}, \mathrm{b}, 0} \\
\left(^{\prime}\right)\end{array}$ & $\begin{array}{l}\theta_{\mathrm{r}, \mathrm{b}} \\
\left({ }^{\circ}\right)\end{array}$ & Feature & $\begin{array}{c}D_{\mathrm{r}, \mathrm{b}} \\
\left(^{\prime}\right)\end{array}$ & $\begin{array}{c}d_{\mathrm{r}, \mathrm{b}} \\
\left(^{\prime}\right)\end{array}$ & $\begin{array}{c}\mathrm{PA}_{\mathrm{r}, \mathrm{b}} \\
\left({ }^{\circ}\right)\end{array}$ & $\begin{array}{c}D_{\mathrm{r}, \mathrm{b}, 0} \\
\left(^{\prime}\right)\end{array}$ & $\begin{array}{c}d_{\mathrm{r}, \mathrm{b}, 0} \\
\left({ }^{\prime}\right)\end{array}$ & $\begin{array}{l}\theta_{\mathrm{r}, \mathrm{b}} \\
\left({ }^{\circ}\right)\end{array}$ \\
\hline $\mathrm{rs}$ & 0.50 & 0.10 & 20.3 & 0.51 & 0.29 & 172.8 & rs & 1.03 & 0.33 & 141.3 & 1.22 & 0.96 & 119.0 \\
\hline
\end{tabular}




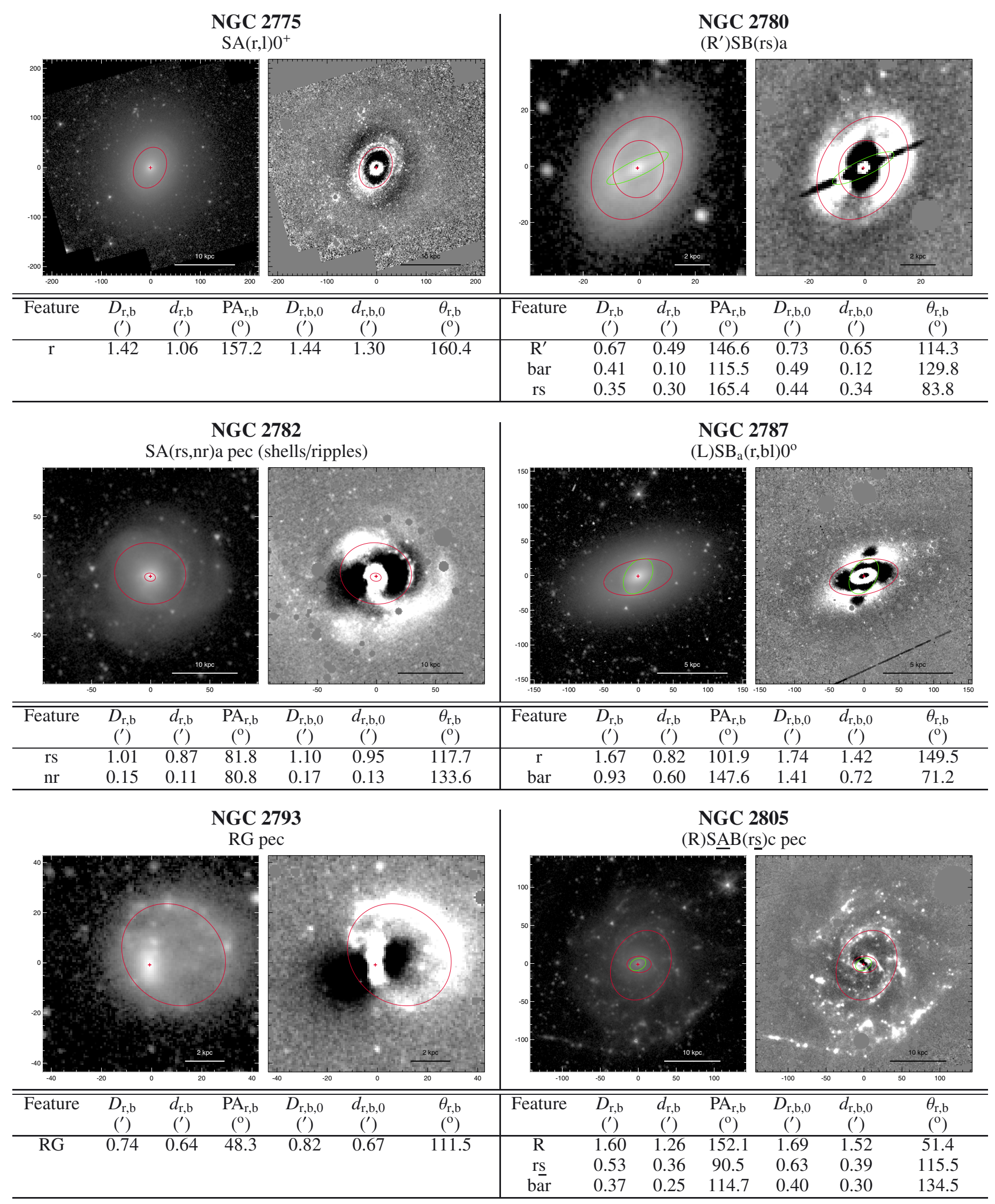




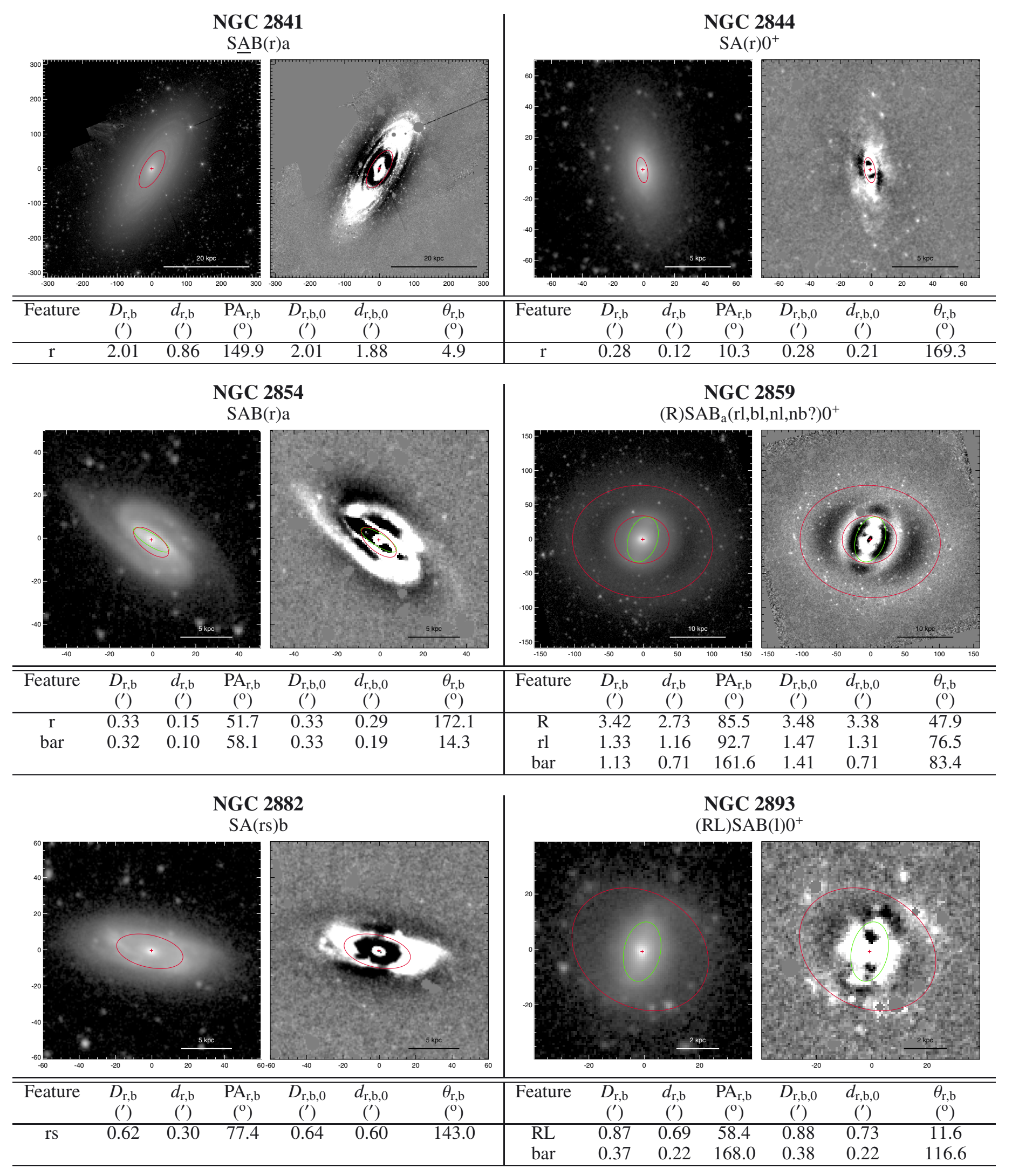


NGC 2894

(R:)SAB:0-
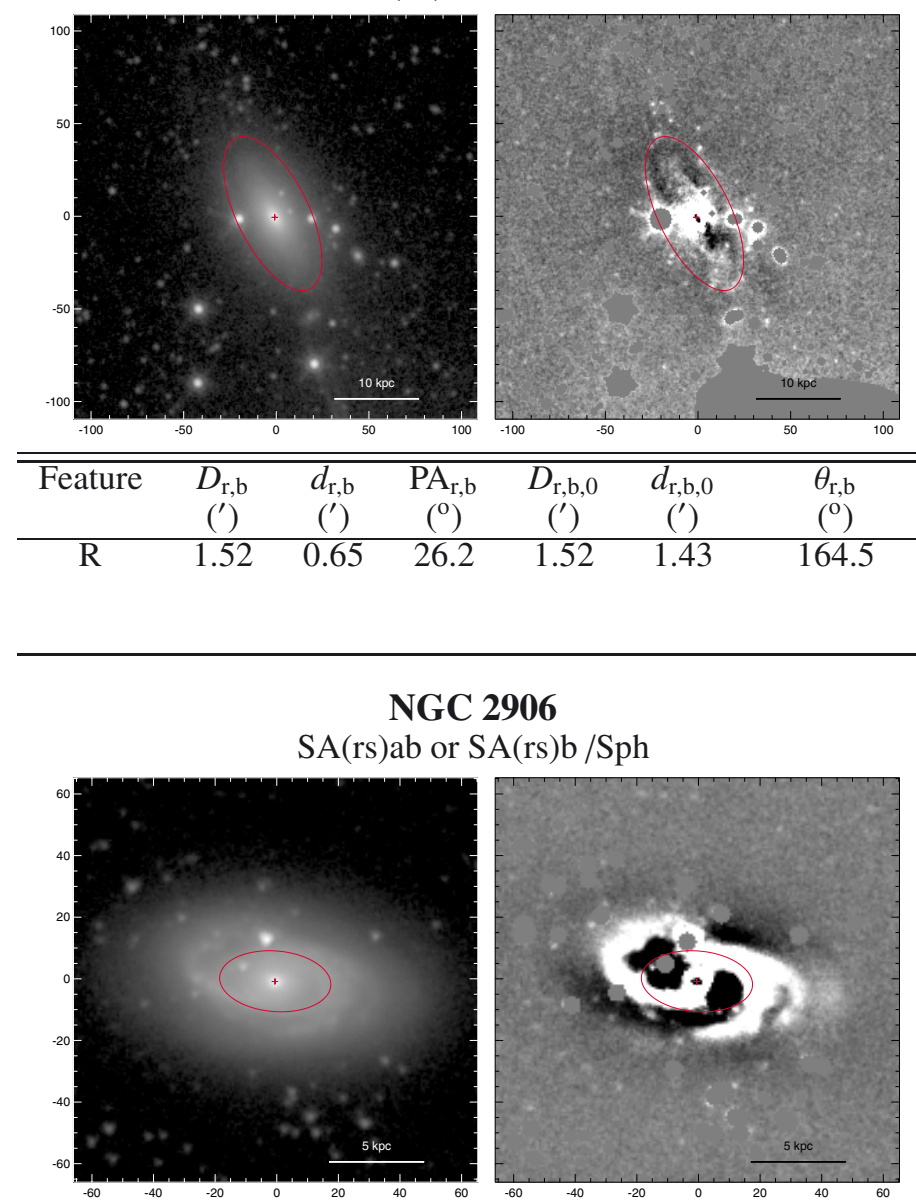

NGC 2906

$\mathrm{SA}(\mathrm{rs}) \mathrm{ab}$ or $\mathrm{SA}(\mathrm{rs}) \mathrm{b} / \mathrm{Sph}$
NGC 2903

$\left(\mathrm{R}^{\prime}\right) \mathrm{SB}(\mathrm{rs}, \mathrm{nr}) \mathrm{b}$

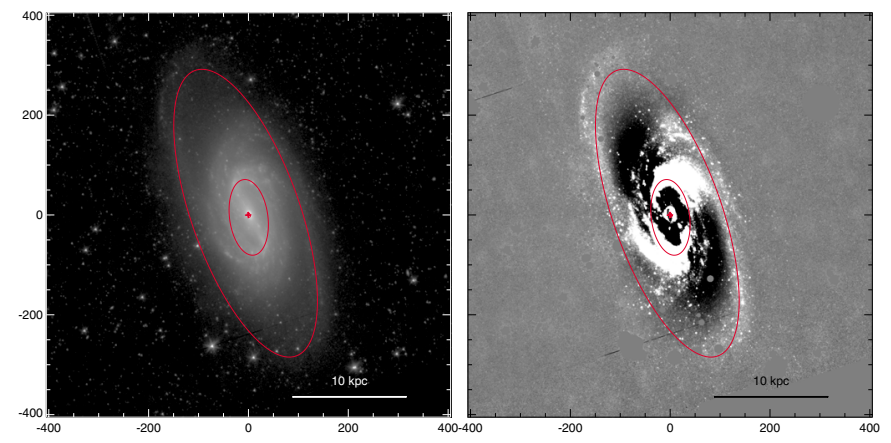

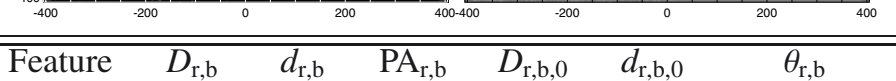

\begin{tabular}{ccc|ccccccc}
$\begin{array}{l}D_{\mathrm{r}, \mathrm{b}, 0} \\
\left({ }^{\prime}\right)\end{array}$ & $\begin{array}{c}d_{\mathrm{r}, \mathrm{b}, 0} \\
\left({ }^{\prime}\right)\end{array}$ & $\begin{array}{c}\theta_{\mathrm{r}, \mathrm{b}} \\
\left({ }^{\circ}\right)\end{array}$ & Feature & $\begin{array}{c}D_{\mathrm{r}, \mathrm{b}} \\
\left({ }^{\prime}\right)\end{array}$ & $\begin{array}{c}d_{\mathrm{r}, \mathrm{b}} \\
\left({ }^{\prime}\right)\end{array}$ & $\begin{array}{c}\mathrm{PA}_{\mathrm{r}, \mathrm{b}} \\
\left({ }^{\circ}\right)\end{array}$ & $\begin{array}{c}D_{\mathrm{r}, \mathrm{b}, 0} \\
\left({ }^{\prime}\right)\end{array}$ & $\begin{array}{c}d_{\mathrm{r}, \mathrm{b}, 0} \\
\left({ }^{\prime}\right)\end{array}$ & $\begin{array}{c}\theta_{\mathrm{r}, \mathrm{b}} \\
\left({ }^{\circ}\right)\end{array}$ \\
\hline .52 & 1.43 & 164.5 & $\mathrm{R}^{\prime}$ & 10.12 & 3.62 & 19.5 & 10.12 & 7.44 & 179.5 \\
& & & $\mathrm{rs}$ & 2.55 & 1.28 & 7.7 & 3.03 & 2.21 & 124.8 \\
& & $\mathrm{nr}$ & 0.16 & 0.09 & 24.2 & 0.19 & 0.16 & 70.2 \\
\hline
\end{tabular}

\begin{tabular}{|c|c|c|c|c|c|c|c|c|c|c|c|c|c|}
\hline Feature & $\begin{array}{c}D_{\mathrm{r}, \mathrm{b}} \\
(')\end{array}$ & $\begin{array}{c}d_{\mathrm{r}, \mathrm{b}} \\
\left({ }^{\prime}\right)\end{array}$ & $\begin{array}{c}\mathrm{PA}_{\mathrm{r}, \mathrm{b}} \\
\left({ }^{\circ}\right)\end{array}$ & $\begin{array}{c}D_{\mathrm{r}, \mathrm{b}, 0} \\
\left(^{\prime}\right)\end{array}$ & $\begin{array}{c}d_{\mathrm{r}, \mathrm{b}, 0} \\
\left(^{\prime}\right)\end{array}$ & $\begin{array}{c}\theta_{\mathrm{r}, \mathrm{b}} \\
\left({ }^{\circ}\right)\end{array}$ & Feature & $\begin{array}{c}D_{\mathrm{r}, \mathrm{b}} \\
\left(^{\prime}\right)\end{array}$ & $\begin{array}{c}d_{\mathrm{r}, \mathrm{b}} \\
\left(^{\prime}\right)\end{array}$ & $\begin{array}{c}\mathrm{PA}_{\mathrm{r}, \mathrm{b}} \\
\left(^{\circ}\right)\end{array}$ & $\begin{array}{c}D_{\mathrm{r}, \mathrm{b}, 0} \\
\left(^{\prime}\right)\end{array}$ & $\begin{array}{c}d_{\mathrm{r}, \mathrm{b}, 0} \\
\left(^{\prime}\right)\end{array}$ & $\begin{array}{l}\theta_{\mathrm{r}, \mathrm{b}} \\
\left({ }^{\circ}\right)\end{array}$ \\
\hline \multirow[t]{3}{*}{ rs } & 0.61 & 0.33 & 85.3 & 0.61 & 0.55 & 23.0 & $\overline{\mathrm{R}}$ & 2.13 & 1.42 & 7.5 & 2.18 & 2.12 & 108.1 \\
\hline & & & & & & & bar & 0.94 & 0.43 & 174.5 & 0.99 & 0.62 & 151.3 \\
\hline & & & & & & & $\mathrm{rl}$ & 0.93 & 0.55 & 2.7 & 0.95 & 0.82 & 155.6 \\
\hline
\end{tabular}

NGC 2964

$\mathrm{SAB}(\mathrm{rs}) \mathrm{b}$

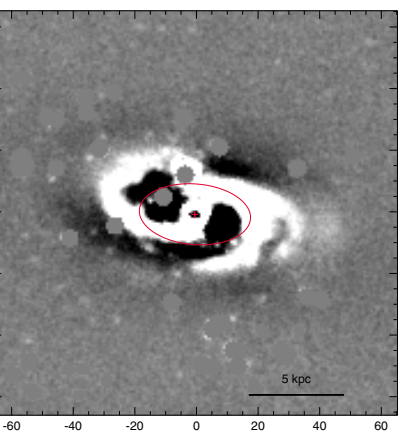

NGC 2962

(R)SAB ${ }_{\mathrm{a}}(\mathrm{rl}) 0^{+}$

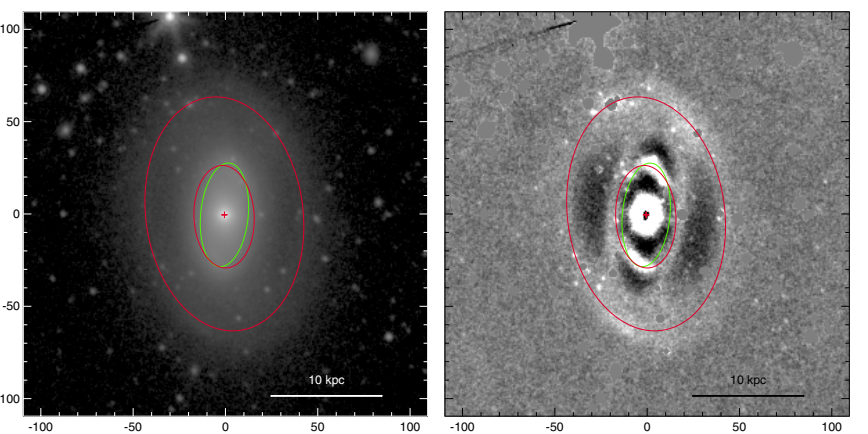

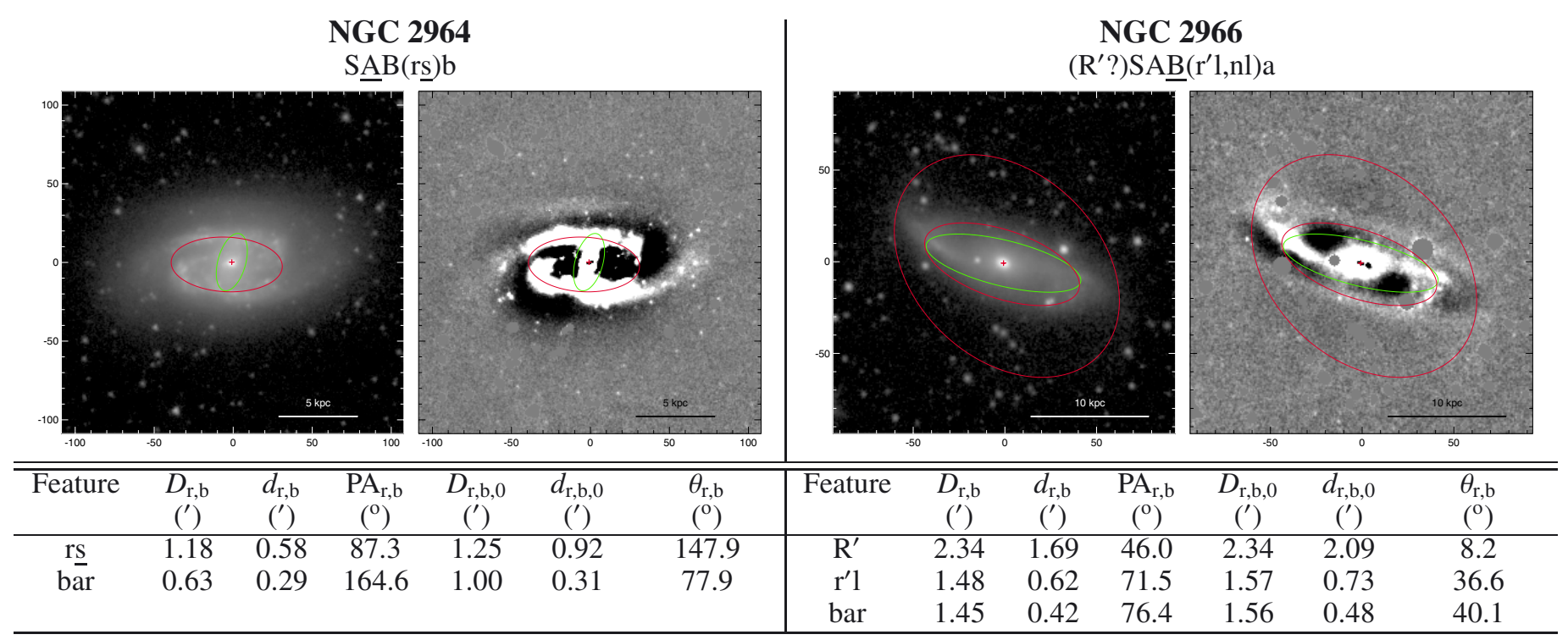


NGC 2967

$\left(\mathrm{R}^{\prime}\right) \mathrm{SAB}(\mathrm{rs}) \mathrm{c}$
NGC 2968

$\mathrm{SB}(\underline{\mathrm{rs}}) 0^{+}$
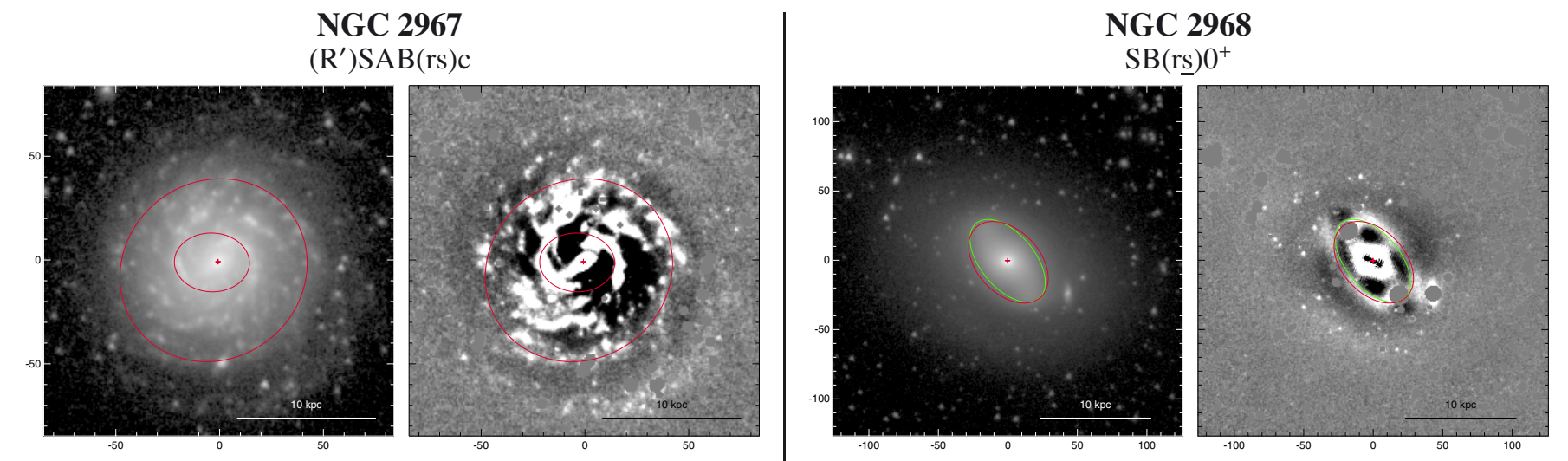

\begin{tabular}{|c|c|c|c|c|c|c|c|c|c|c|c|c|c|}
\hline Feature & $\begin{array}{c}D_{\mathrm{r}, \mathrm{b}} \\
\left({ }^{\prime}\right)\end{array}$ & $\begin{array}{c}d_{\mathrm{r}, \mathrm{b}} \\
\left({ }^{\prime}\right)\end{array}$ & $\begin{array}{c}\mathrm{PA}_{\mathrm{r}, \mathrm{b}} \\
\left({ }^{\mathrm{O}}\right)\end{array}$ & $\begin{array}{c}D_{\mathrm{r}, \mathrm{b}, 0} \\
\left(^{\prime}\right)\end{array}$ & $\begin{array}{c}d_{\mathrm{r}, \mathrm{b}, 0} \\
\left({ }^{\prime}\right)\end{array}$ & $\begin{array}{c}\theta_{\mathrm{r}, \mathrm{b}} \\
\left({ }^{\circ}\right)\end{array}$ & Feature & $\begin{array}{c}D_{\mathrm{r}, \mathrm{b}} \\
\left({ }^{\prime}\right)\end{array}$ & $\begin{array}{c}d_{\mathrm{r}, \mathrm{b}} \\
\left({ }^{\prime}\right)\end{array}$ & $\begin{array}{c}\mathrm{PA}_{\mathrm{r}, \mathrm{b}} \\
\left(^{\circ}\right)\end{array}$ & $\begin{array}{c}D_{\mathrm{r}, \mathrm{b}, 0} \\
\left(^{\prime}\right)\end{array}$ & $\begin{array}{c}d_{\mathrm{r}, \mathrm{b}, 0} \\
\left(^{\prime}\right)\end{array}$ & $\begin{array}{c}\theta_{\mathrm{r}, \mathrm{b}} \\
\left({ }^{\circ}\right)\end{array}$ \\
\hline$\overline{\mathrm{R}^{\prime}}$ & 1.55 & 1.43 & 126.4 & 1.61 & 1.44 & 71.6 & bar & 1.18 & 0.65 & 39.9 & 1.31 & 0.80 & 134.9 \\
\hline rs & 0.61 & 0.47 & 88.2 & 0.61 & 0.49 & 28.4 & rs & 1.14 & 0.75 & 43.1 & 1.28 & 0.93 & 130.4 \\
\hline
\end{tabular}

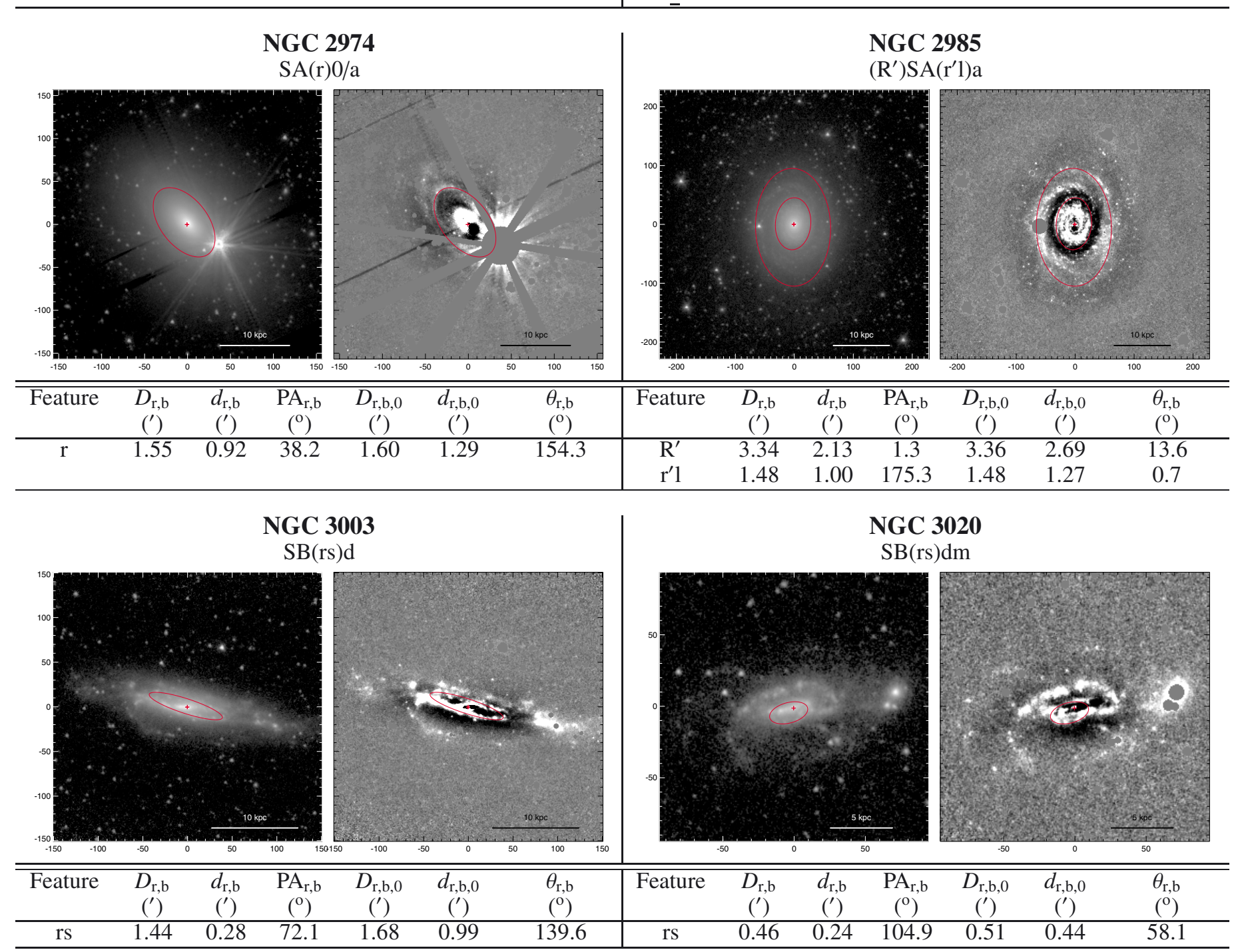


NGC 3031

$\mathrm{SA}(\mathrm{rs}, \mathrm{r}, \mathrm{nb}) \mathrm{a}$
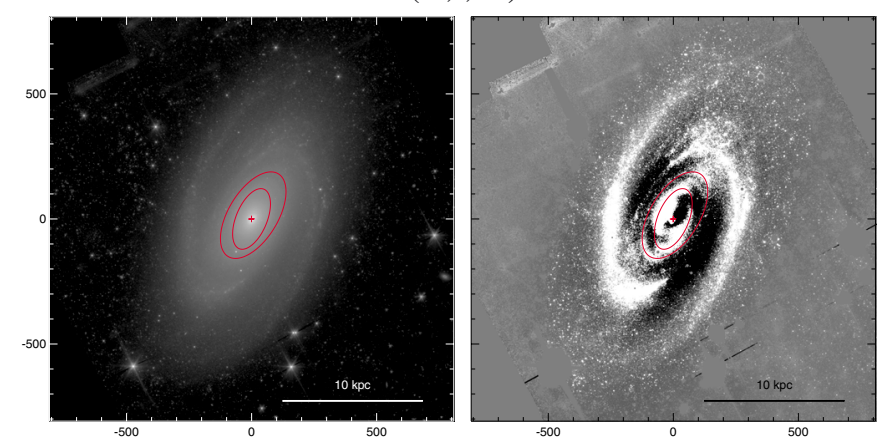

NGC 3032

$\mathrm{SA}(\mathrm{rl}, 1) 0^{\circ}$

\begin{tabular}{|c|c|c|c|c|c|c|c|c|c|c|c|c|c|}
\hline Feature & $\begin{array}{c}D_{\mathrm{r}, \mathrm{b}} \\
\left(^{\prime}\right)\end{array}$ & $\begin{array}{c}d_{\mathrm{r}, \mathrm{b}} \\
\left({ }^{\prime}\right)\end{array}$ & $\begin{array}{c}\mathrm{PA}_{\mathrm{r}, \mathrm{b}} \\
\left(^{\mathrm{O}}\right)\end{array}$ & $\begin{array}{c}D_{\mathrm{r}, \mathrm{b}, 0} \\
\left(^{\prime}\right)\end{array}$ & $\begin{array}{c}d_{\mathrm{r}, \mathrm{b}, 0} \\
\left(^{\prime}\right)\end{array}$ & $\begin{array}{l}\theta_{\mathrm{r}, \mathrm{b}} \\
\left({ }^{\circ}\right)\end{array}$ & Feature & $\begin{array}{c}D_{\mathrm{r}, \mathrm{b}} \\
\left(^{\prime}\right)\end{array}$ & $\begin{array}{c}d_{\mathrm{r}, \mathrm{b}} \\
\left({ }^{\prime}\right)\end{array}$ & $\begin{array}{c}\mathrm{PA}_{\mathrm{r}, \mathrm{b}} \\
\left(^{\circ}\right)\end{array}$ & $\begin{array}{c}D_{\mathrm{r}, \mathrm{b}, 0} \\
\left(^{\prime}\right)\end{array}$ & $\begin{array}{c}d_{\mathrm{r}, \mathrm{b}, 0} \\
\left(^{\prime}\right)\end{array}$ & $\begin{array}{l}\theta_{\mathrm{r}, \mathrm{b}} \\
\left({ }^{\circ}\right)\end{array}$ \\
\hline rs & 6.45 & 3.29 & 148.7 & 6.88 & 5.68 & 139.9 & $\mathrm{rl}$ & 0.61 & 0.54 & 90.2 & 0.68 & 0.61 & 98.3 \\
\hline $\mathrm{r}$ & 4.30 & 2.07 & 157.4 & 4.30 & 3.80 & 6.8 & & & & & & & \\
\hline
\end{tabular}

NGC 3041

$\mathrm{SA}(\mathrm{rs}) \mathrm{b}$
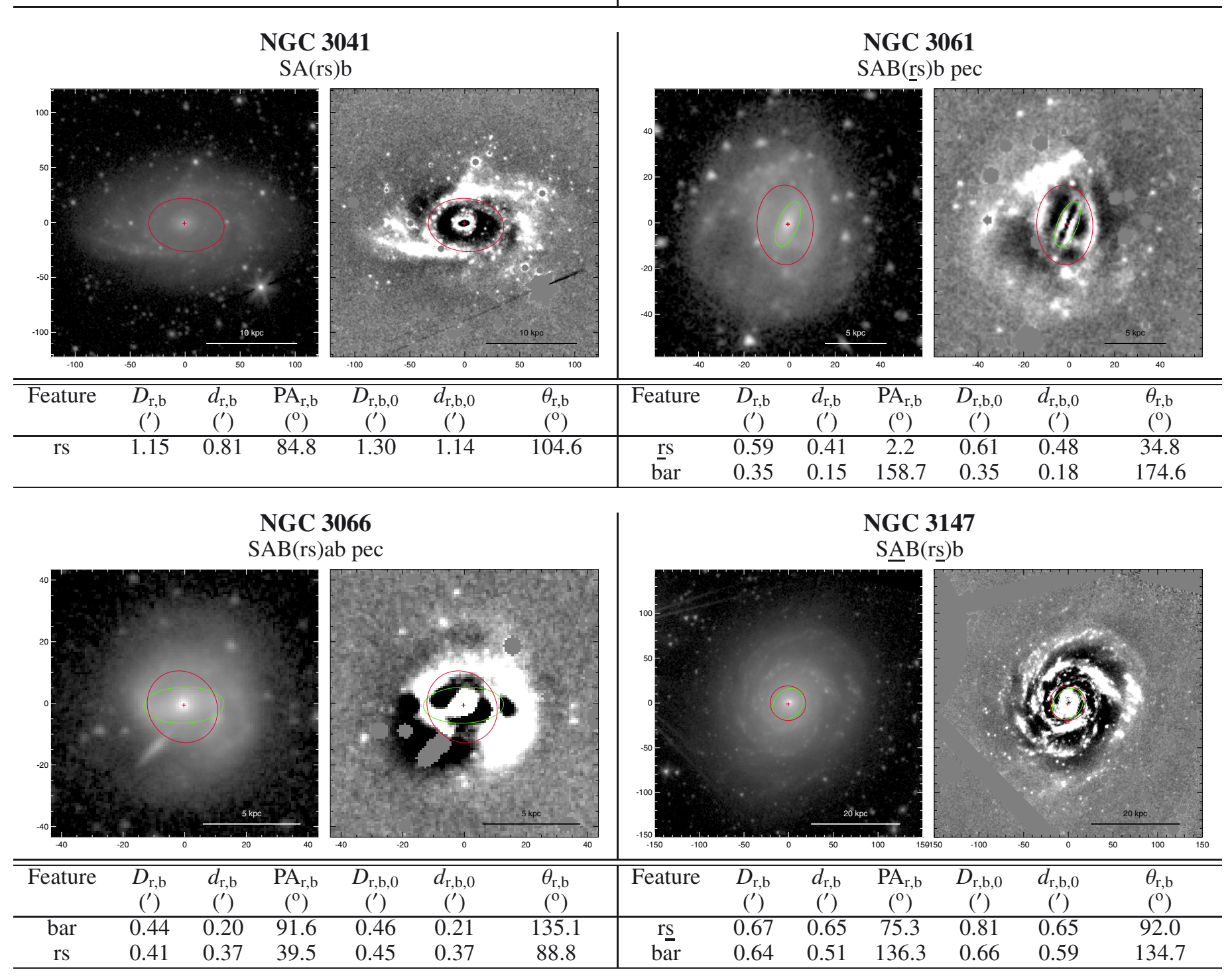

\begin{tabular}{|c|c|c|c|c|c|c|c|c|c|c|c|c|c|}
\hline Feature & $\begin{array}{c}D_{\mathrm{r}, \mathrm{b}} \\
\left({ }^{\prime}\right)\end{array}$ & $\begin{array}{c}d_{\mathrm{r}, \mathrm{b}} \\
\left({ }^{\prime}\right)\end{array}$ & $\begin{array}{c}\mathrm{PA}_{\mathrm{r}, \mathrm{b}} \\
\left(^{\mathrm{o}}\right)\end{array}$ & $\begin{array}{c}D_{\mathrm{r}, \mathrm{b}, 0} \\
\left(^{\prime}\right)\end{array}$ & $\begin{array}{c}d_{\mathrm{r}, \mathrm{b}, 0} \\
\left(^{\prime}\right)\end{array}$ & $\begin{array}{l}\theta_{\mathrm{r}, \mathrm{b}} \\
\left({ }^{\circ}\right)\end{array}$ & Feature & $\begin{array}{c}D_{\mathrm{r}, \mathrm{b}} \\
\left(^{\prime}\right)\end{array}$ & $\begin{array}{c}d_{\mathrm{r}, \mathrm{b}} \\
\left(^{\prime}\right)\end{array}$ & $\begin{array}{c}\mathrm{PA}_{\mathrm{r}, \mathrm{b}} \\
\left({ }^{\mathrm{o}}\right)\end{array}$ & $\begin{array}{c}D_{\mathrm{r}, \mathrm{b}, 0} \\
\left(^{\prime}\right)\end{array}$ & $\begin{array}{c}d_{\mathrm{r}, \mathrm{b}, 0} \\
\left(^{\prime}\right)\end{array}$ & $\begin{array}{l}\theta_{\mathrm{r}, \mathrm{b}} \\
\left(^{\mathrm{o}}\right)\end{array}$ \\
\hline rs & 1.15 & 0.81 & 84.8 & 1.30 & 1.14 & 104.6 & rs & 0.59 & 0.41 & 2.2 & 0.61 & 0.48 & 34.8 \\
\hline & & & & & & & bar & 0.35 & 0.15 & 158.7 & 0.35 & 0.18 & 174.6 \\
\hline
\end{tabular}
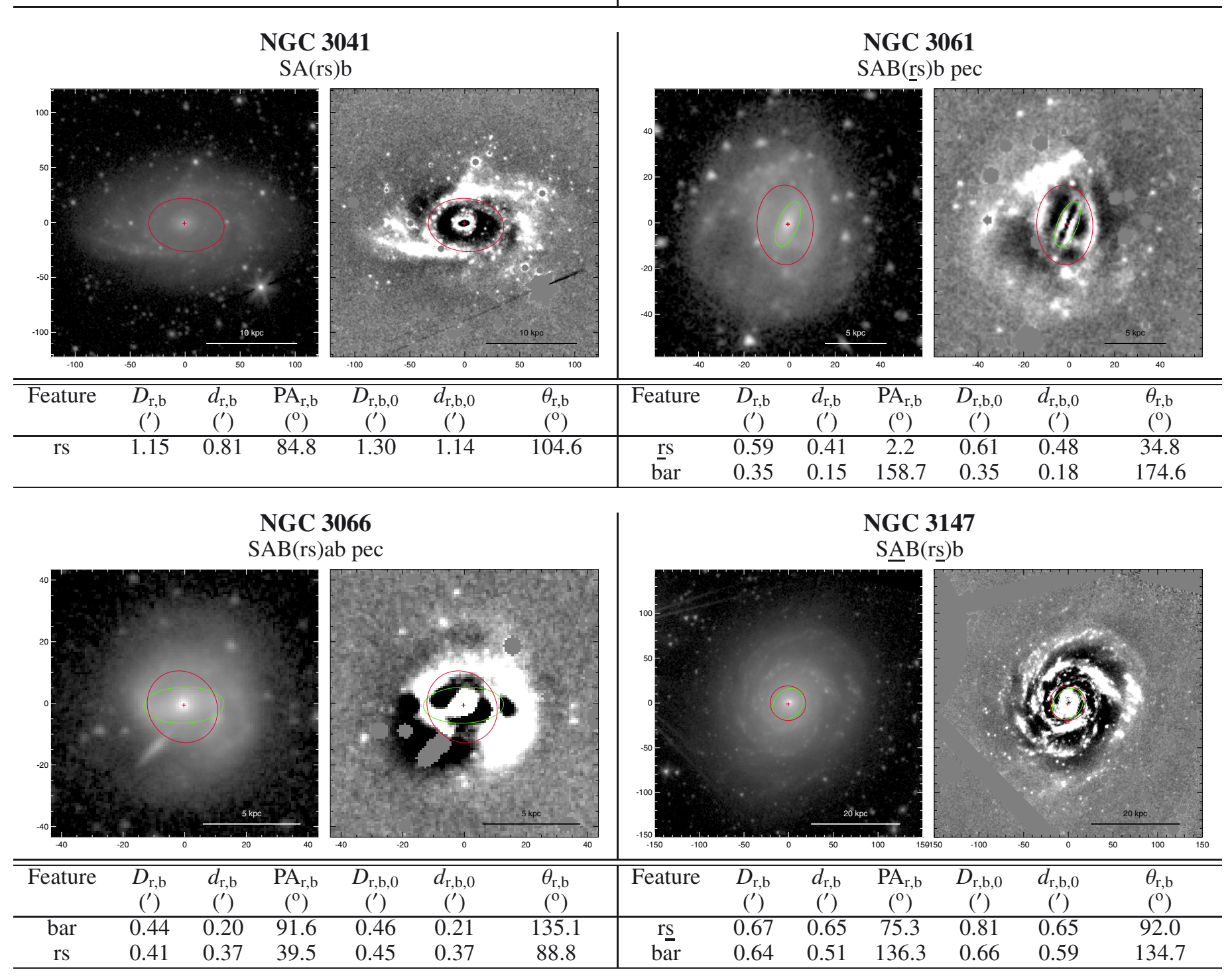

NGC 3061

$\mathrm{SAB}(\underline{\mathrm{rs}}) \mathrm{b}$ pec
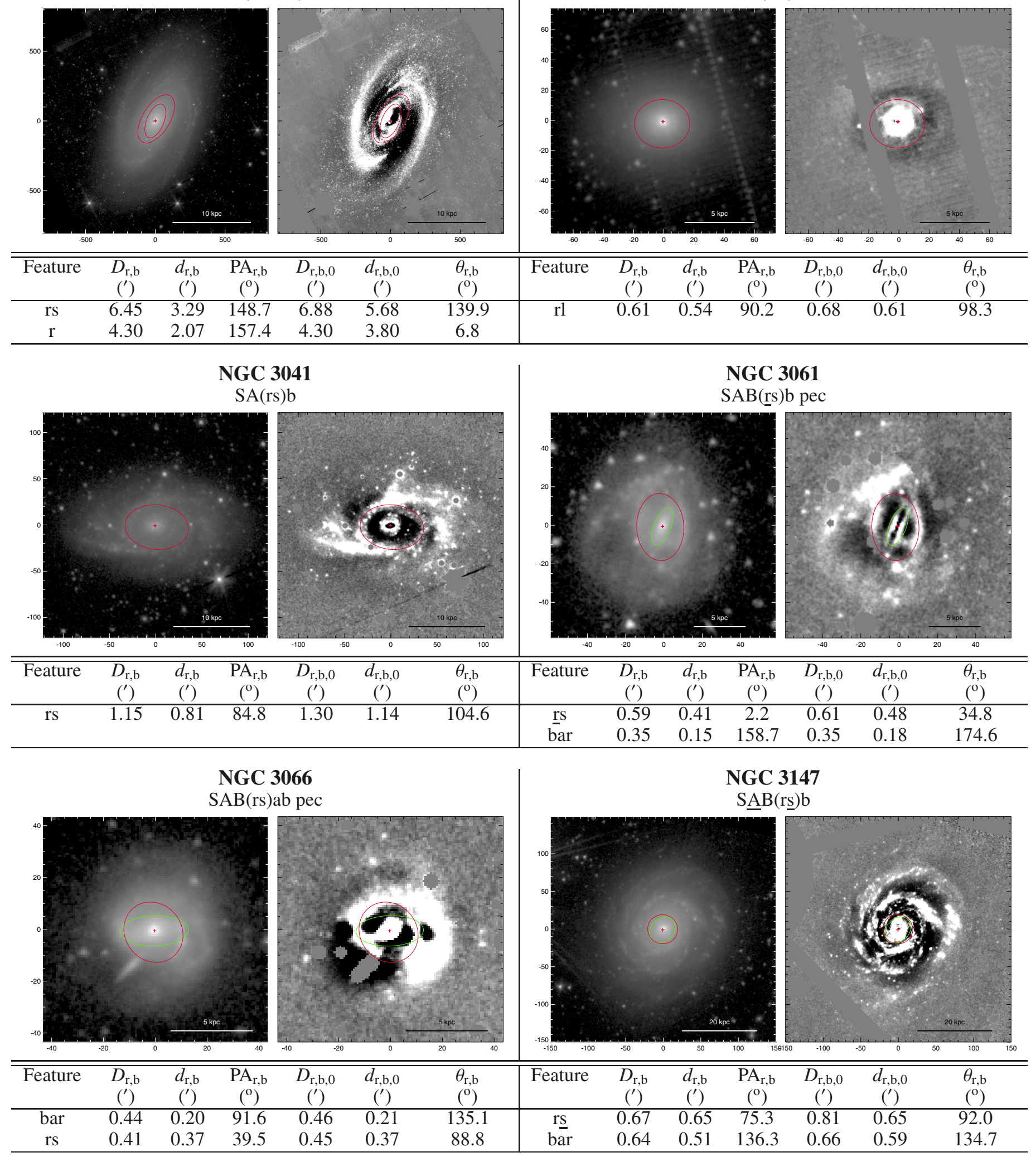


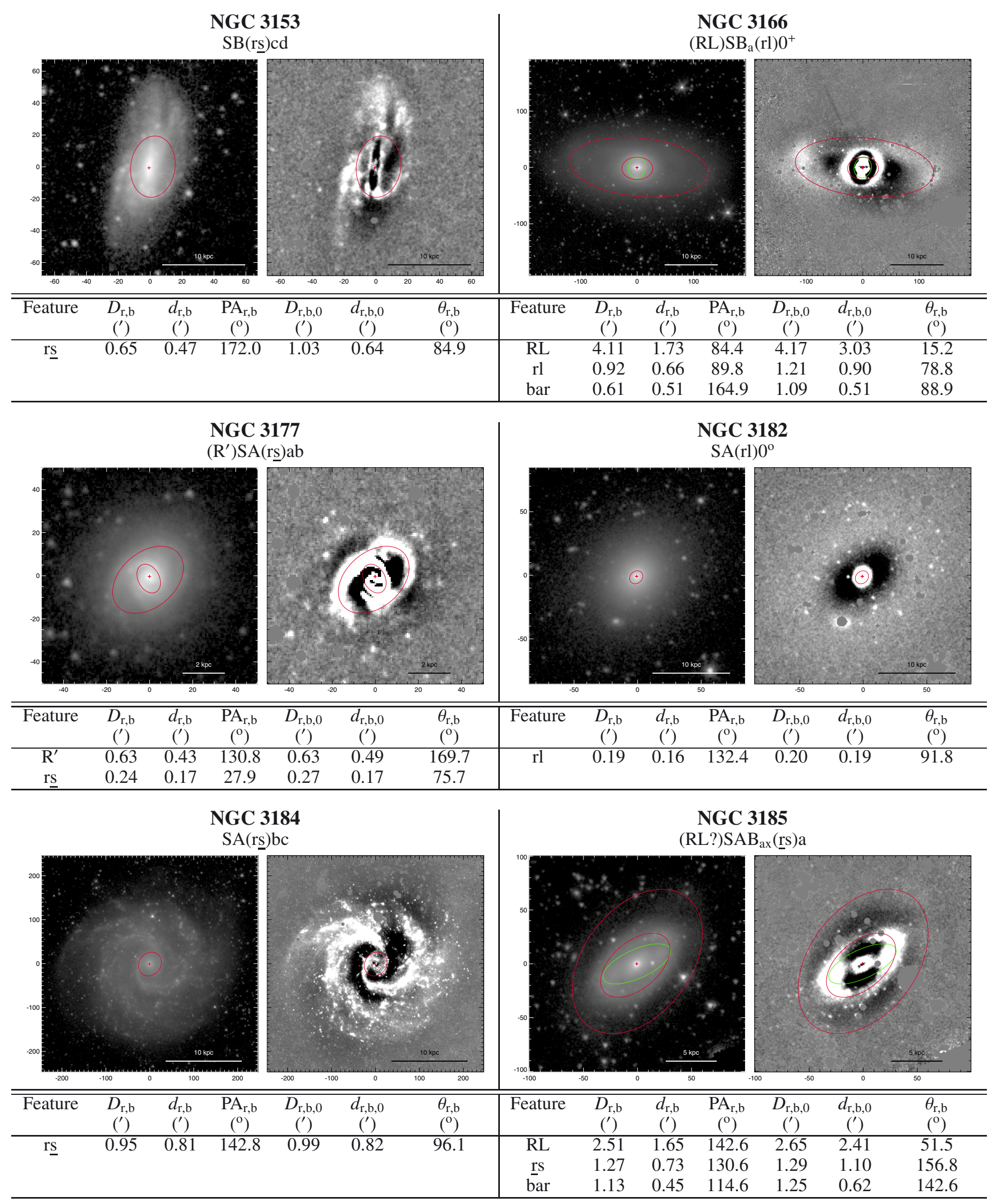




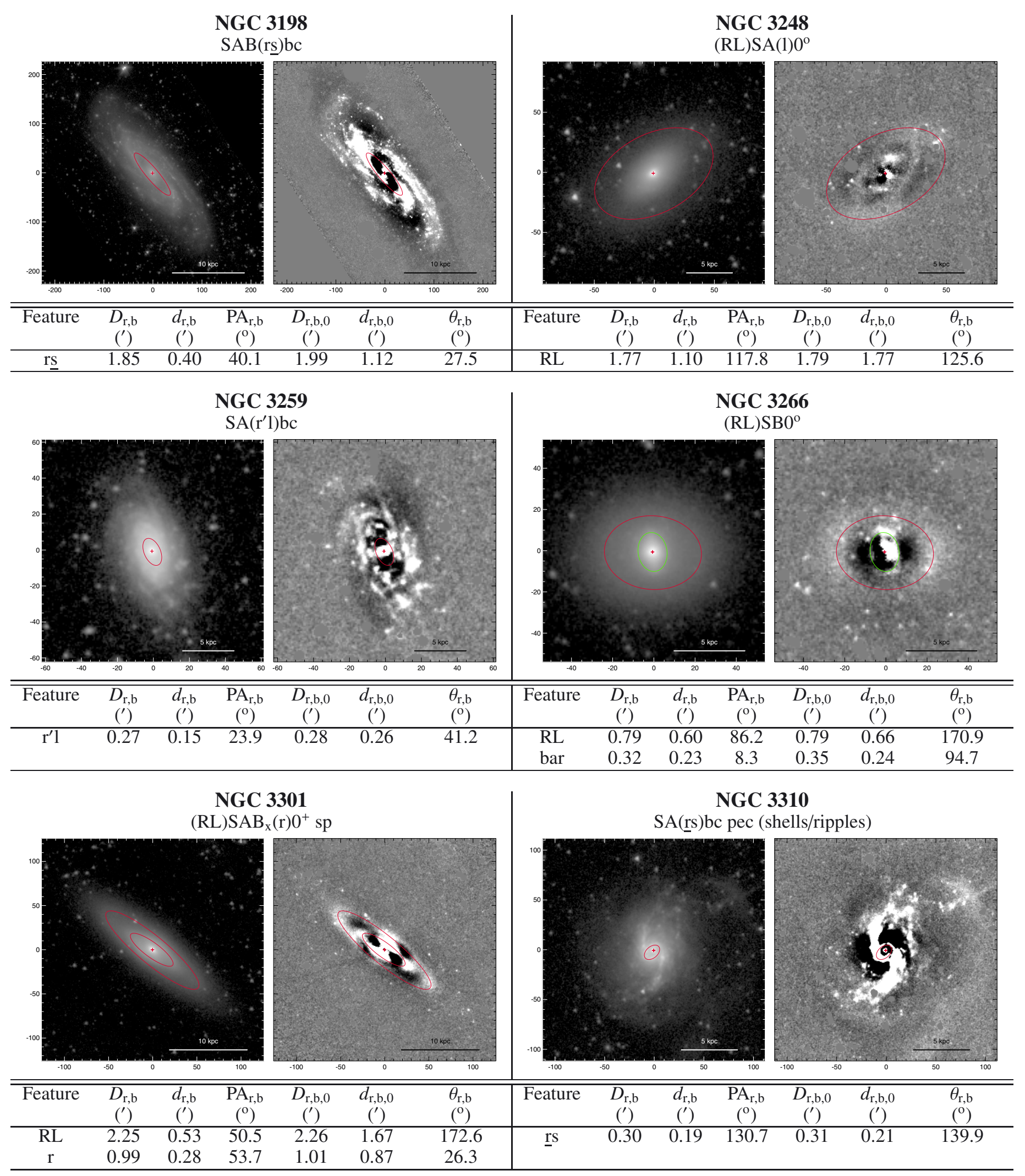


NGC 3319

$\left(\mathrm{R}^{\prime}\right) \mathrm{SB}(\mathrm{s}) \mathrm{cd}$
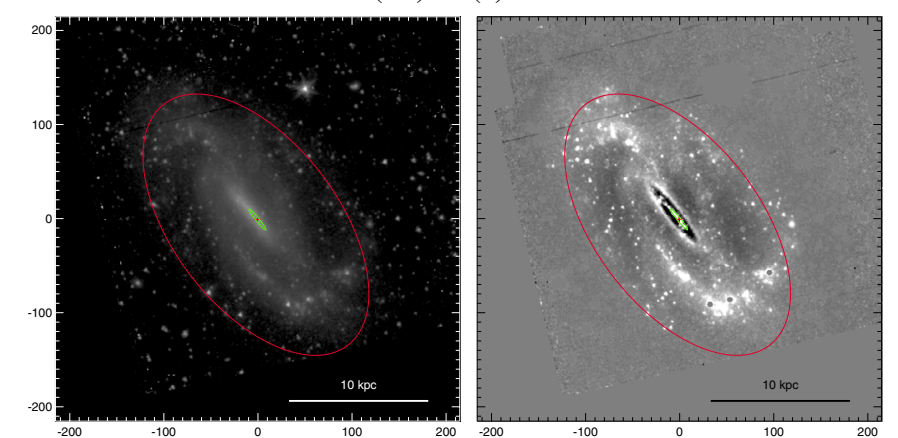

\begin{tabular}{ccccccc|ccccccc}
\hline \hline Feature & $\begin{array}{c}D_{\mathrm{r}, \mathrm{b}} \\
\left({ }^{\prime}\right)\end{array}$ & $\begin{array}{c}d_{\mathrm{r}, \mathrm{b}} \\
\left({ }^{\prime}\right)\end{array}$ & $\begin{array}{c}\mathrm{PA}_{\mathrm{r}, \mathrm{b}} \\
\left({ }^{\circ}\right)\end{array}$ & $\begin{array}{c}D_{\mathrm{r}, \mathrm{b}, 0} \\
\left({ }^{\prime}\right)\end{array}$ & $\begin{array}{c}d_{\mathrm{r}, \mathrm{b}, 0} \\
\left({ }^{\prime}\right)\end{array}$ & $\begin{array}{c}\theta_{\mathrm{r}, \mathrm{b}} \\
\left({ }^{\circ}\right)\end{array}$ & Feature & $\begin{array}{c}D_{\mathrm{r}, \mathrm{b}} \\
\left({ }^{\prime}\right)\end{array}$ & $\begin{array}{c}d_{\mathrm{r}, \mathrm{b}} \\
\left({ }^{\prime}\right)\end{array}$ & $\begin{array}{c}\mathrm{PA}_{\mathrm{r}, \mathrm{b}} \\
\left({ }^{\circ}\right)\end{array}$ & $\begin{array}{c}D_{\mathrm{r}, \mathrm{b}, 0} \\
\left({ }^{\prime}\right)\end{array}$ & $\begin{array}{c}d_{\mathrm{r}, \mathrm{b}, 0} \\
\left({ }^{\prime}\right)\end{array}$ & $\begin{array}{c}\theta_{\mathrm{r}, \mathrm{b}} \\
\left({ }^{\circ}\right)\end{array}$ \\
\hline $\mathrm{R}^{\prime}$ & 5.38 & 2.94 & 37.2 & 5.64 & 4.96 & 139.1 & $\underline{\mathrm{rs}}$ & 0.54 & 0.34 & 14.0 & 0.76 & 0.50 & 107.9 \\
$\mathrm{bar}$ & 0.48 & 0.06 & 39.0 & 0.48 & 0.11 & 172.7 & & & & & & & \\
\hline
\end{tabular}

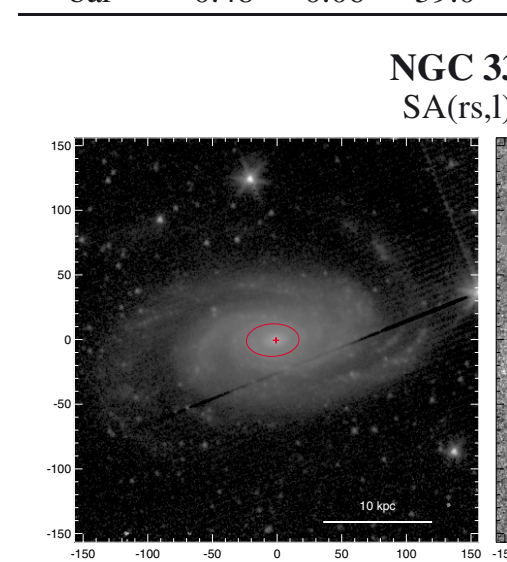

NGC 3338

$\mathrm{SA}(\mathrm{rs}, 1) \mathrm{bc}$

\begin{tabular}{|c|c|c|c|c|c|c|c|c|c|c|c|c|c|}
\hline "Feature & $\begin{array}{c}D_{\mathrm{r}, \mathrm{b}} \\
\left({ }^{\prime}\right)\end{array}$ & $\begin{array}{c}d_{\mathrm{r}, \mathrm{b}} \\
\left({ }^{\prime}\right)\end{array}$ & $\begin{array}{c}\mathrm{PA}_{\mathrm{r}, \mathrm{b}} \\
\left({ }^{\circ}\right)\end{array}$ & $\begin{array}{c}D_{\mathrm{r}, \mathrm{b}, 0} \\
\left({ }^{\prime}\right)\end{array}$ & $\begin{array}{c}d_{\mathrm{r}, \mathrm{b}, 0} \\
\left(^{\prime}\right)\end{array}$ & $\begin{array}{c}\theta_{\mathrm{r}, \mathrm{b}} \\
\left(^{\circ}\right)\end{array}$ & Feature & $\begin{array}{c}D_{\mathrm{r}, \mathrm{b}} \\
\left({ }^{\prime}\right)\end{array}$ & $\begin{array}{c}d_{\mathrm{r}, \mathrm{b}} \\
\left({ }^{\prime}\right)\end{array}$ & $\begin{array}{c}\mathrm{PA}_{\mathrm{r}, \mathrm{b}} \\
\left({ }^{\circ}\right)\end{array}$ & $\begin{array}{c}D_{\mathrm{r}, \mathrm{b}, 0} \\
\left({ }^{\prime}\right)\end{array}$ & $\begin{array}{c}d_{\mathrm{r}, \mathrm{b}, 0} \\
\left({ }^{\prime}\right)\end{array}$ & $\begin{array}{c}\theta_{\mathrm{r}, \mathrm{b}} \\
\left({ }^{\circ}\right)\end{array}$ \\
\hline $\mathrm{rs}$ & 0.68 & 0.42 & 92.5 & 0.87 & 0.67 & 98.7 & $\begin{array}{c}\mathrm{r} \\
\mathrm{bar}\end{array}$ & $\begin{array}{l}1.02 \\
0.84\end{array}$ & $\begin{array}{l}0.87 \\
0.52\end{array}$ & $\begin{array}{c}125.2 \\
4.5\end{array}$ & $\begin{array}{l}1.04 \\
0.89\end{array}$ & $\begin{array}{l}0.95 \\
0.55\end{array}$ & $\begin{array}{c}139.8 \\
49.2\end{array}$ \\
\hline
\end{tabular}
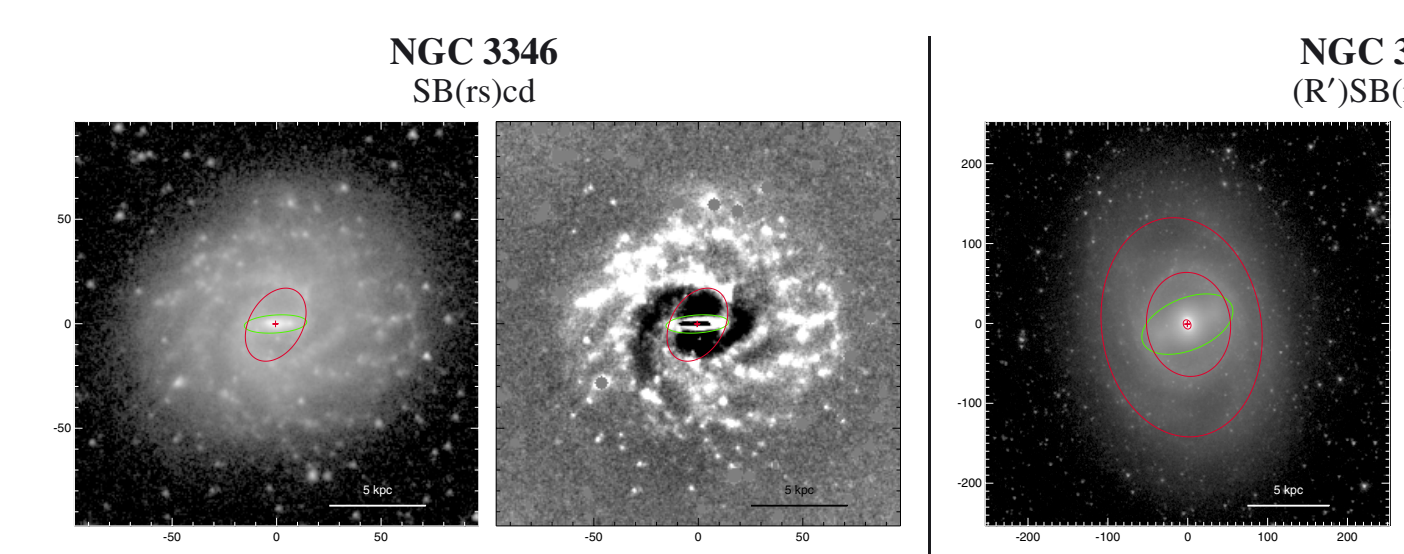

\begin{tabular}{ccccccc|ccccccc}
\hline \hline Feature & $\begin{array}{c}D_{\mathrm{r}, \mathrm{b}} \\
\left({ }^{\prime}\right)\end{array}$ & $\begin{array}{c}d_{\mathrm{r}, \mathrm{b}} \\
\left({ }^{\prime}\right)\end{array}$ & $\begin{array}{c}\mathrm{PA}_{\mathrm{r}, \mathrm{b}} \\
\left({ }^{\circ}\right)\end{array}$ & $\begin{array}{c}D_{\mathrm{r}, \mathrm{b}, 0} \\
\left({ }^{\prime}\right)\end{array}$ & $\begin{array}{c}d_{\mathrm{r}, \mathrm{b}, 0} \\
\left({ }^{\prime}\right)\end{array}$ & $\begin{array}{c}\theta_{\mathrm{r}, \mathrm{b}} \\
\left({ }^{\circ}\right)\end{array}$ & Feature & $\begin{array}{c}D_{\mathrm{r}, \mathrm{b}} \\
\left({ }^{\prime}\right)\end{array}$ & $\begin{array}{c}d_{\mathrm{r}, \mathrm{b}} \\
\left({ }^{\prime}\right)\end{array}$ & $\begin{array}{c}\mathrm{PA}_{\mathrm{r}, \mathrm{b}} \\
\left({ }^{\circ}\right)\end{array}$ & $\begin{array}{c}D_{\mathrm{r}, \mathrm{b}, 0} \\
()^{\prime}\end{array}$ & $\begin{array}{c}d_{\mathrm{r}, \mathrm{b}, 0} \\
\left({ }^{\prime}\right)\end{array}$ & $\begin{array}{c}\theta_{\mathrm{r}, \mathrm{b}} \\
\left({ }^{\circ}\right)\end{array}$ \\
\hline rs & 0.63 & 0.43 & 149.6 & 0.68 & 0.47 & 46.9 & $\mathrm{R}^{\prime}$ & 4.61 & 3.34 & 8.7 & 4.72 & 4.60 & 85.5 \\
bar & 0.50 & 0.14 & 93.7 & 0.52 & 0.17 & 152.9 & $\mathrm{r}$ & 2.18 & 1.75 & 6.2 & 2.48 & 2.18 & 93.7 \\
& & & & & & & $\mathrm{bar}$ & 2.01 & 1.08 & 112.5 & 2.81 & 1.10 & 98.3 \\
& & & & & & & $\mathrm{nr}$ & 0.20 & 0.15 & 26.1 & 0.23 & 0.19 & 63.4 \\
\hline
\end{tabular}

NGC 3351

$\left(\mathrm{R}^{\prime}\right) \mathrm{SB}(\mathrm{r}, \mathrm{nr}) \mathrm{a}$

$\mathrm{SA} \underline{\mathrm{B}}(\underline{\mathrm{rs}}) \mathrm{cd}$

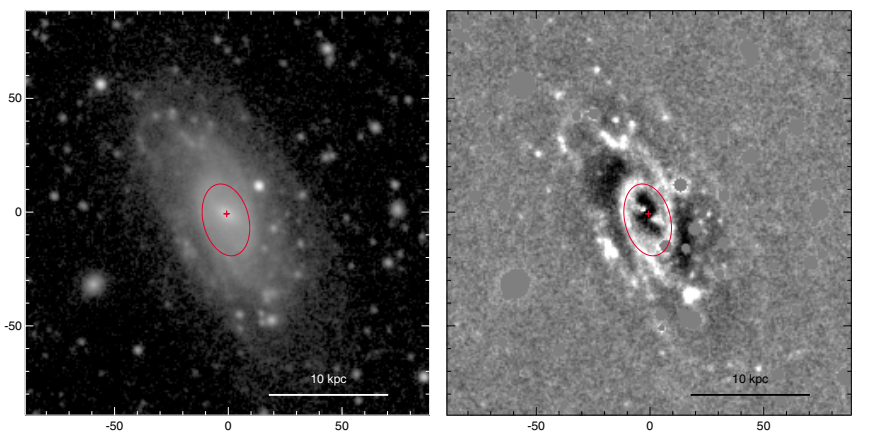

NGC 3344

$\mathrm{SAB}(\mathrm{r}) \mathrm{bc}$

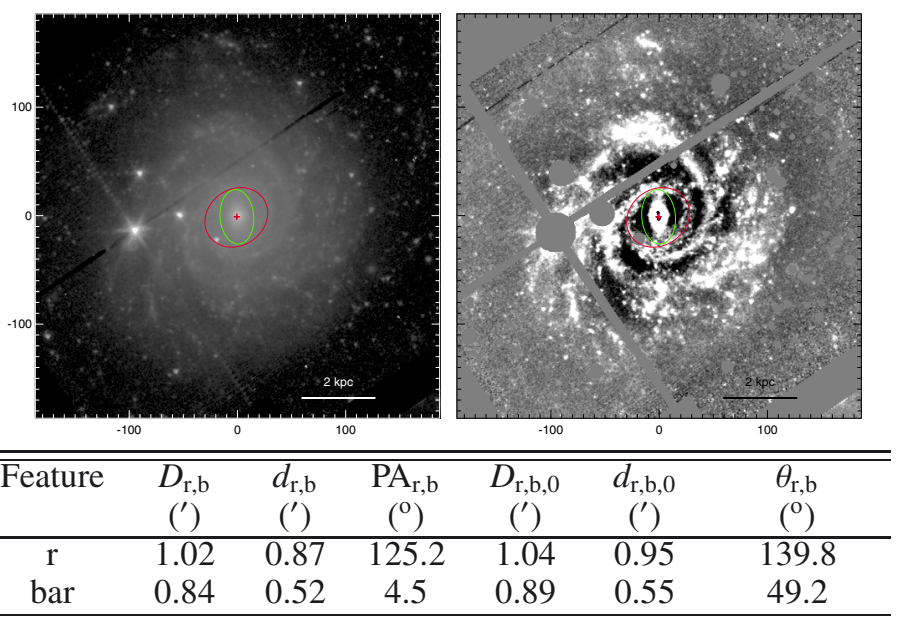

\section{काग}

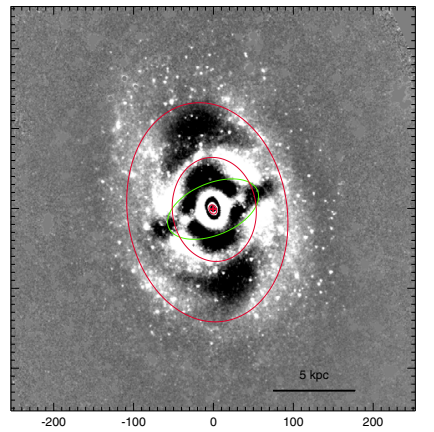

NGC 3321 


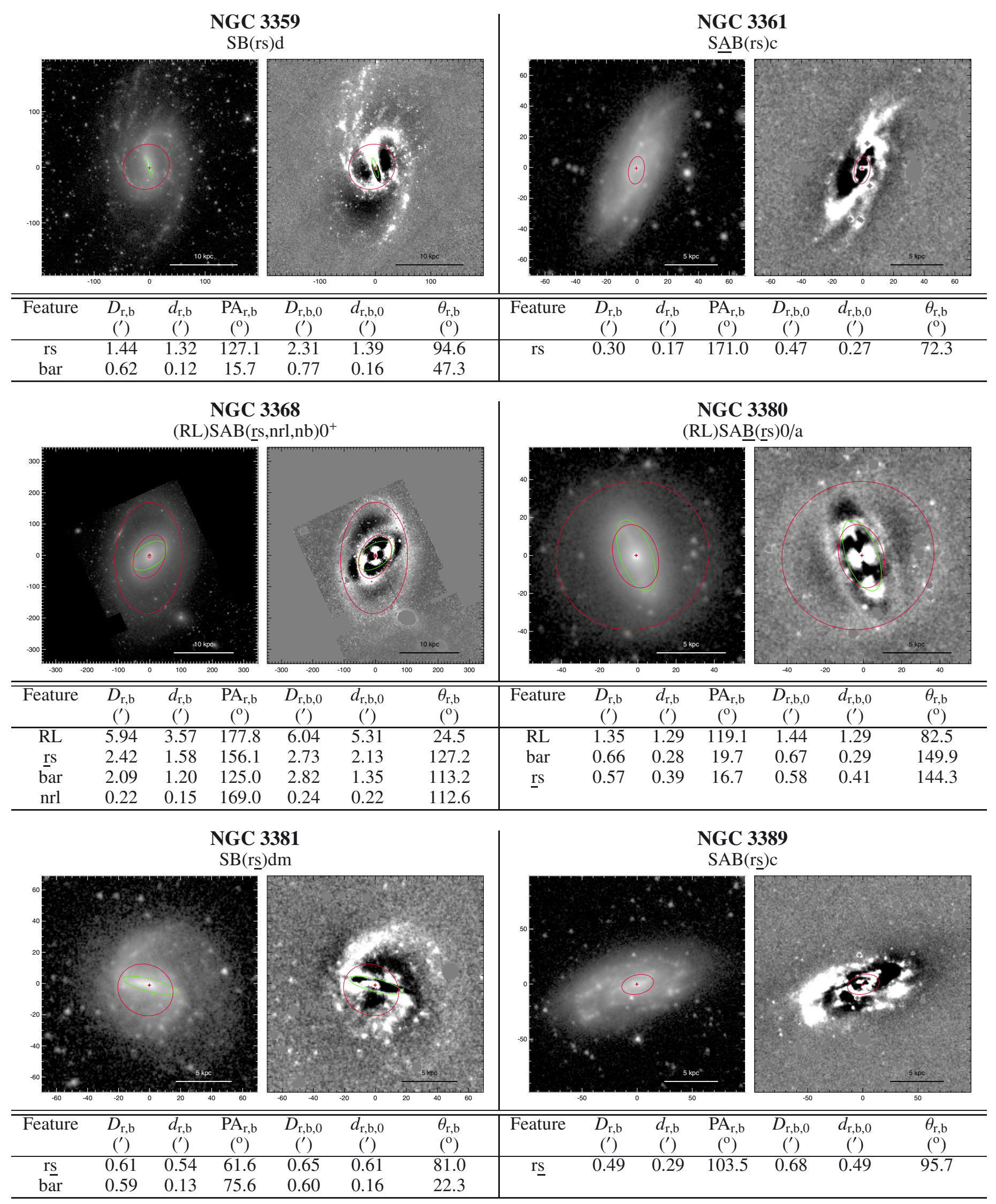




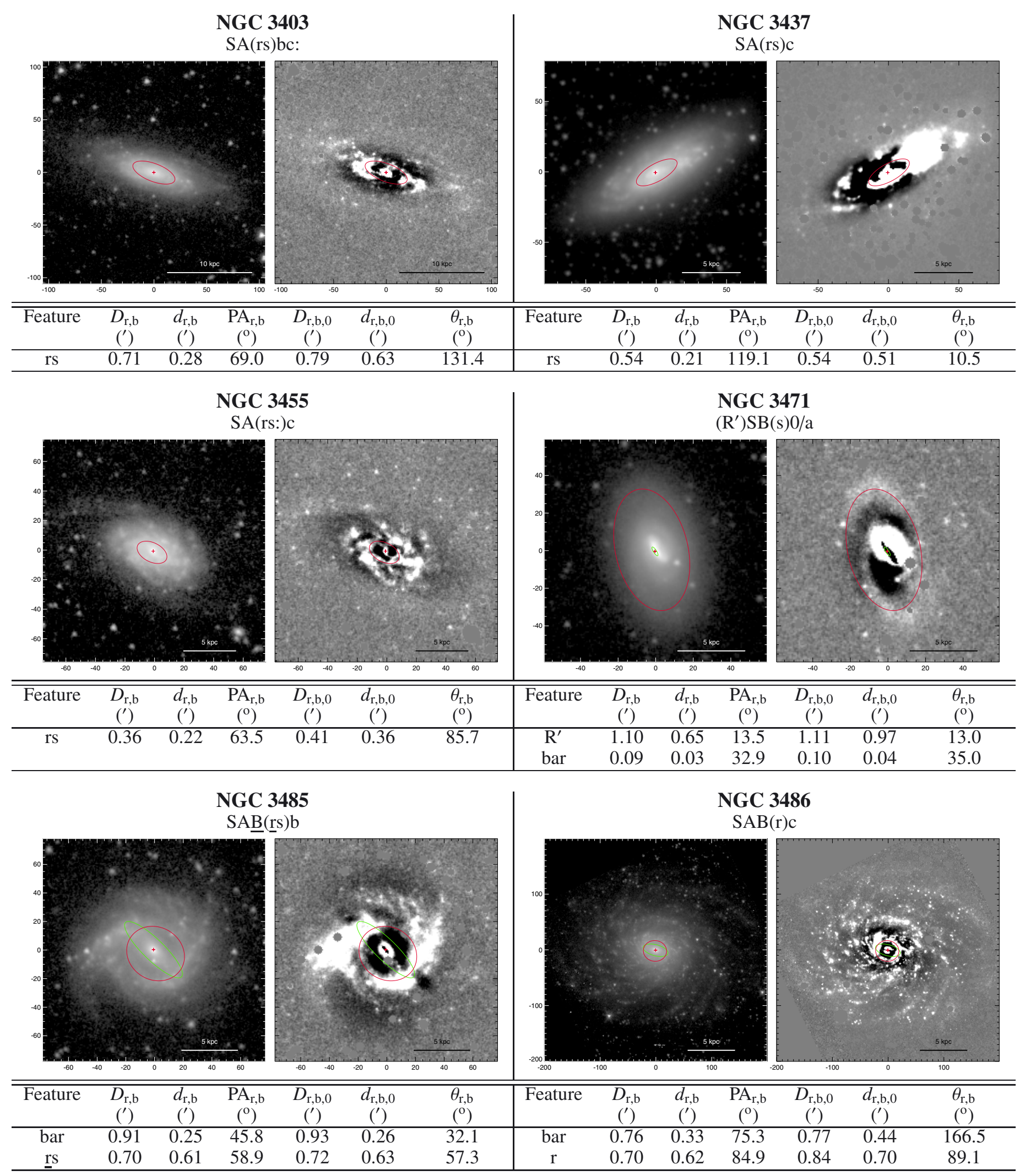


NGC 3489

(R) $\mathrm{SB}(\mathrm{r}:) 0^{\circ}$
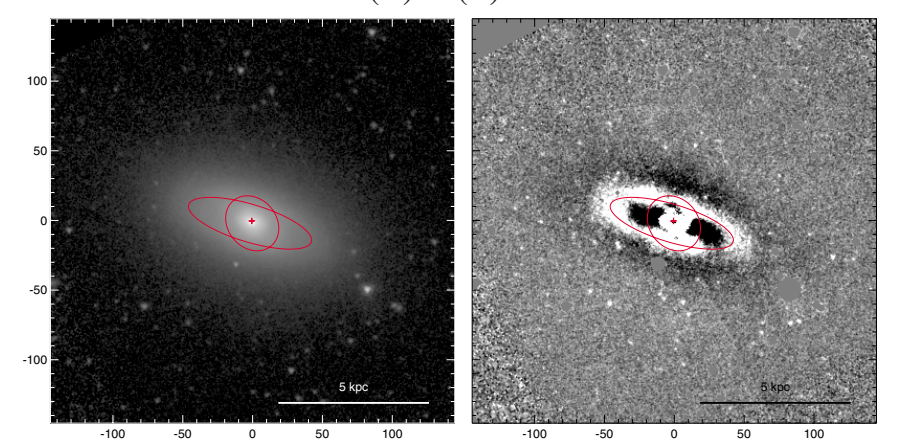

\begin{tabular}{|c|c|c|c|c|c|c|c|c|c|c|c|c|c|}
\hline Feature & $\begin{array}{l}D_{\mathrm{r}, \mathrm{b}} \\
\left({ }^{\prime}\right)\end{array}$ & $\begin{array}{l}d_{\mathrm{r}, \mathrm{b}} \\
\left({ }^{\prime}\right)\end{array}$ & $\begin{array}{c}\mathrm{PA}_{\mathrm{r}, \mathrm{b}} \\
\left({ }^{\circ}\right)\end{array}$ & $\begin{array}{c}\begin{array}{c}\mathrm{r}, \mathrm{b}, 0 \\
\left(^{\prime}\right)\end{array} \\
\end{array}$ & $\begin{array}{c}d_{\mathrm{r}, \mathrm{b}, 0} \\
\left({ }^{\prime}\right)\end{array}$ & $\begin{array}{l}\theta_{\mathrm{r}, \mathrm{b}} \\
\left({ }^{\circ}\right)\end{array}$ & Feature & $\begin{array}{c}D_{\mathrm{r}, \mathrm{b}} \\
\left({ }^{\prime}\right)\end{array}$ & $\begin{array}{c}d_{\mathrm{r}, \mathrm{b}} \\
\left({ }^{\prime}\right)\end{array}$ & $\begin{array}{c}\mathrm{PA}_{\mathrm{r}, \mathrm{b}} \\
\left({ }^{\circ}\right)\end{array}$ & $\begin{array}{c}D_{\mathrm{r}, \mathrm{b}, 0} \\
\left({ }^{\prime}\right)\end{array}$ & $\begin{array}{c}d_{\mathrm{r}, \mathrm{b}, 0} \\
\left(^{\prime}\right)\end{array}$ & $\begin{array}{l}\theta_{\mathrm{r}, \mathrm{b}} \\
\left({ }^{\circ}\right)\end{array}$ \\
\hline $\bar{R}$ & 1.53 & 0.45 & 73.2 & 1.54 & 0.90 & 6.4 & $\overline{\mathrm{R}^{\prime}}$ & 3.05 & 0.58 & 20.1 & 3.06 & 2.18 & 171.6 \\
\hline $\mathrm{r}$ & 0.71 & 0.58 & 39.7 & 1.25 & 0.66 & 97.3 & rs & 1.01 & 0.30 & 20.6 & 1.12 & 1.01 & 99.0 \\
\hline
\end{tabular}

NGC 3504

$\left(\mathrm{R}_{1}^{\prime}\right) \mathrm{SA} \underline{\mathrm{B}}(\underline{\mathrm{rs}}, \mathrm{nl}) \mathrm{a}$
NGC 3495

$\left(\mathrm{R}^{\prime}\right) \mathrm{SB}(\mathrm{rs}) \mathrm{c}$ :

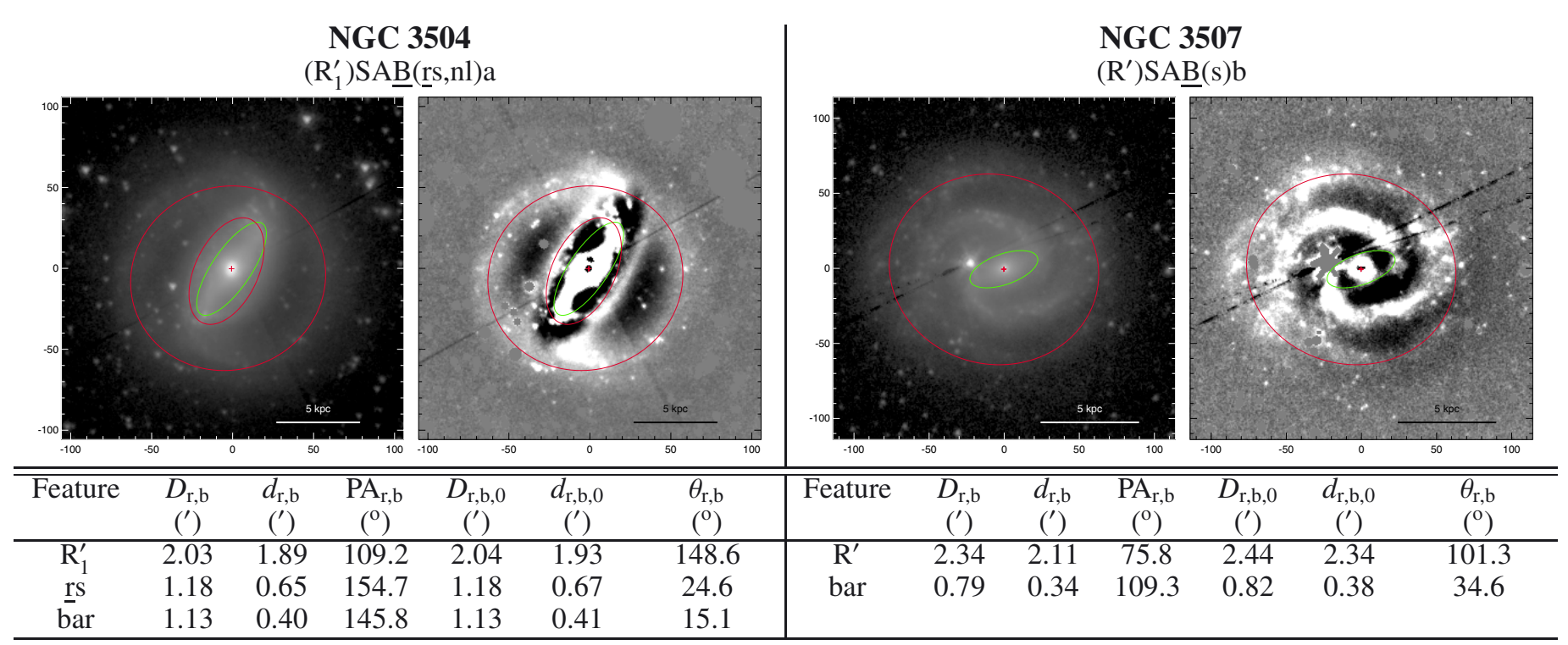

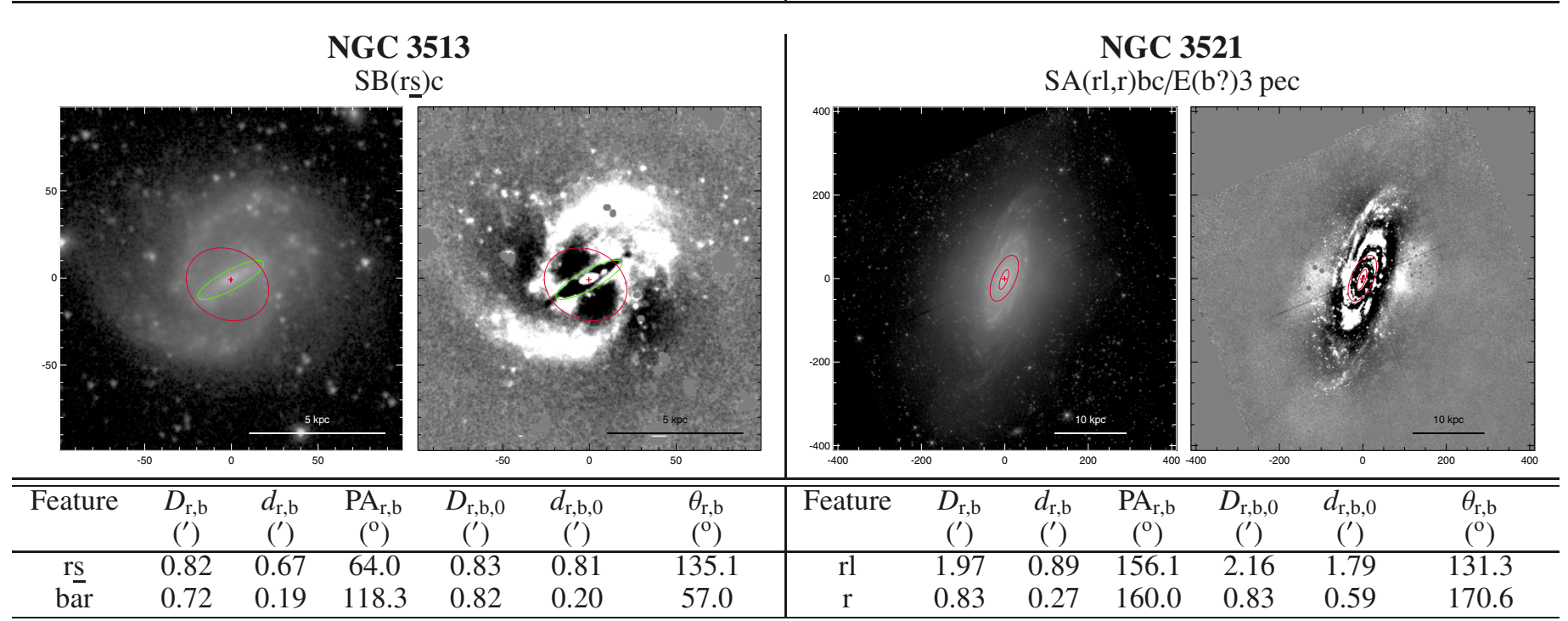




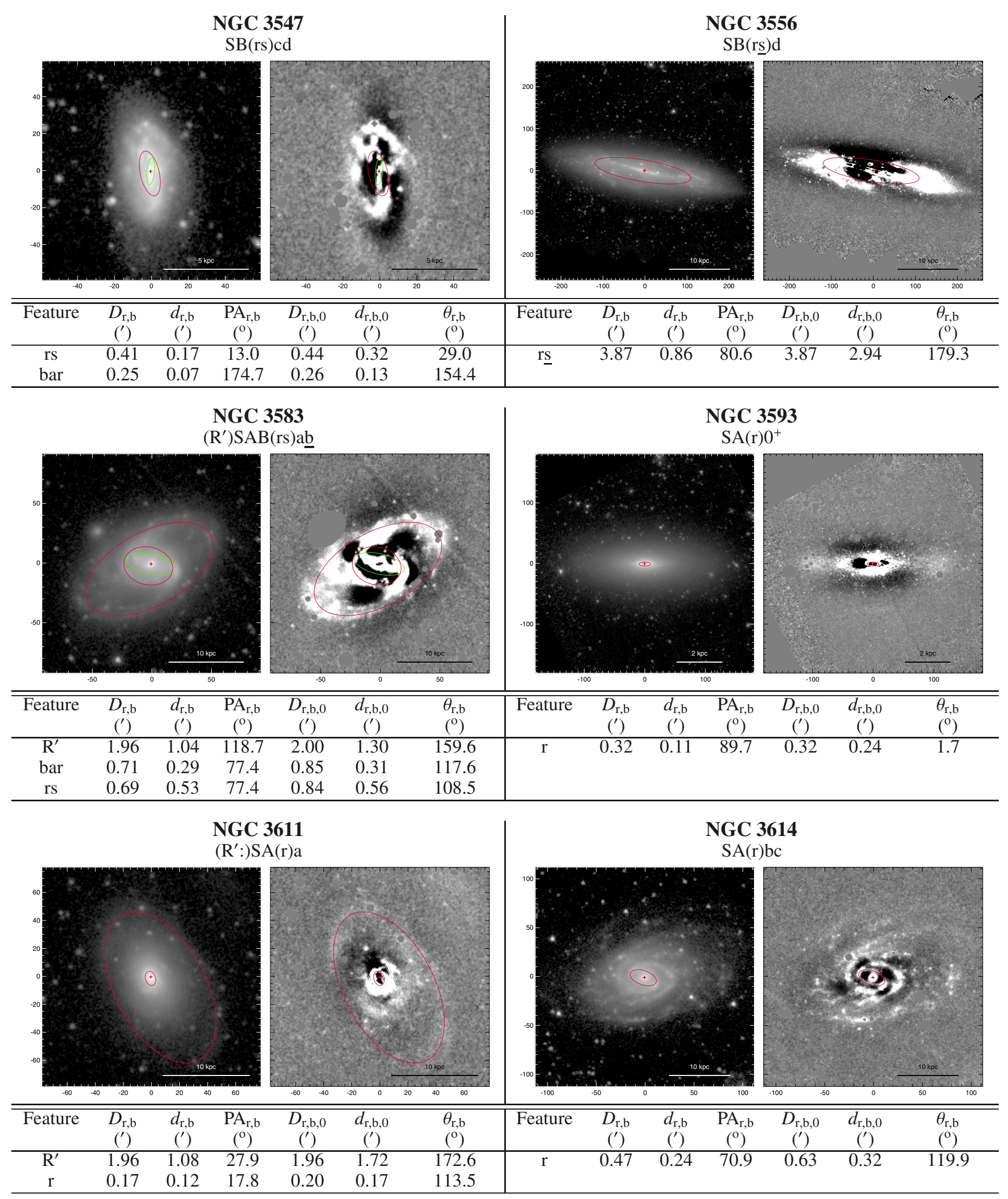


NGC 3623

$\left(\mathrm{R}^{\prime}\right) \mathrm{SAB}_{\mathrm{x}}(\mathrm{rs}) \mathrm{a}$
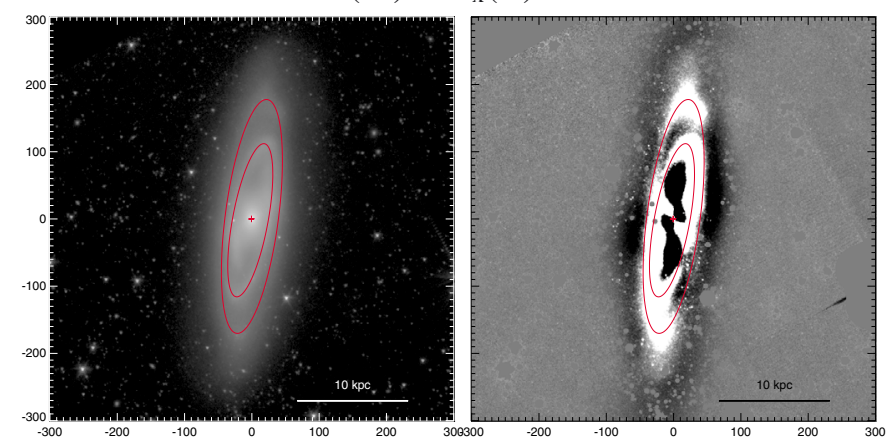

\begin{tabular}{|c|c|c|c|c|c|c|c|c|c|c|c|c|c|}
\hline Feature & $\begin{array}{c}D_{\mathrm{r}, \mathrm{b}} \\
\left({ }^{\prime}\right)\end{array}$ & $\begin{array}{c}d_{\mathrm{r}, \mathrm{b}} \\
\left(^{\prime}\right)\end{array}$ & $\begin{array}{c}\mathrm{PA}_{\mathrm{r}, \mathrm{b}} \\
\left({ }^{\circ}\right)\end{array}$ & $\begin{array}{c}D_{\mathrm{r}, \mathrm{b}, 0} \\
\left(^{\prime}\right)\end{array}$ & $\begin{array}{c}d_{\mathrm{r}, \mathrm{b}, 0} \\
\left({ }^{\prime}\right)\end{array}$ & $\begin{array}{l}\theta_{\mathrm{r}, \mathrm{b}} \\
\left({ }^{\circ}\right)\end{array}$ & Feature & $\begin{array}{c}D_{\mathrm{r}, \mathrm{b}} \\
\left({ }^{\prime}\right)\end{array}$ & $\begin{array}{c}d_{\mathrm{r}, \mathrm{b}} \\
\left({ }^{\prime}\right)\end{array}$ & $\begin{array}{c}\mathrm{PA}_{\mathrm{r}, \mathrm{b}} \\
\left({ }^{\circ}\right)\end{array}$ & $\begin{array}{c}D_{\mathrm{r}, \mathrm{b}, 0} \\
\left(^{\prime}\right)\end{array}$ & $\begin{array}{c}d_{\mathrm{r}, \mathrm{b}, 0} \\
{ }^{\prime}\left({ }^{\prime}\right)\end{array}$ & $\begin{array}{c}\theta_{\mathrm{r}, \mathrm{b}} \\
\left({ }^{\circ}\right)\end{array}$ \\
\hline$\overline{\mathrm{R}^{\prime}}$ & 5.85 & 1.32 & 172.5 & 5.90 & 4.65 & 166.7 & rs & 0.37 & 0.15 & 155.5 & 0.40 & 0.35 & 52.8 \\
\hline rs & 3.86 & 0.88 & 169.5 & 4.16 & 2.91 & 148.0 & & & & & & & \\
\hline
\end{tabular}

NGC 3626

(RL,R)SAB(rl,nl?)0/a
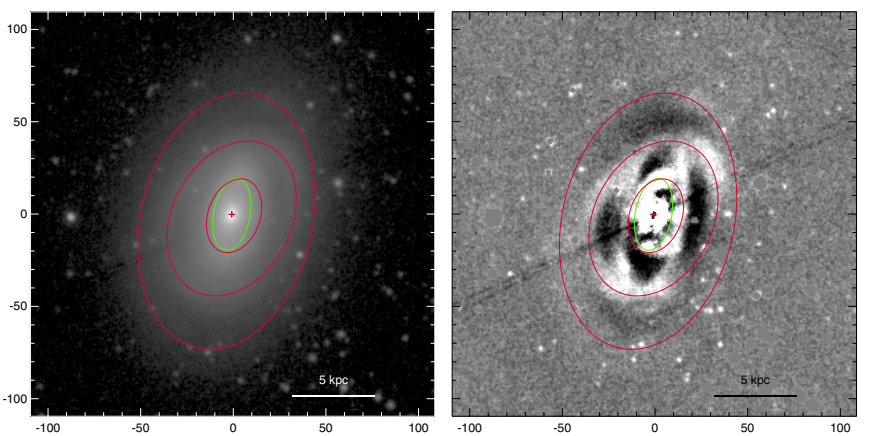

\begin{tabular}{|c|c|c|c|c|c|c|c|c|c|c|c|c|c|}
\hline 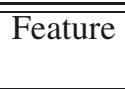 & $\begin{array}{c}D_{\mathrm{r}, \mathrm{b}} \\
\left({ }^{\prime}\right)\end{array}$ & $\begin{array}{c}d_{\mathrm{r}, \mathrm{b}} \\
\left({ }^{\prime}\right)\end{array}$ & $\begin{array}{c}\mathrm{PA}_{\mathrm{r}, \mathrm{b}} \\
\left({ }^{\circ}\right)\end{array}$ & $\begin{array}{c}D_{\mathrm{r}, \mathrm{b}, 0} \\
\left(^{\prime}\right)\end{array}$ & $\begin{array}{c}d_{\mathrm{r}, \mathrm{b}, 0} \\
\left({ }^{\prime}\right)\end{array}$ & $\begin{array}{l}\theta_{\mathrm{r}, \mathrm{b}} \\
\left({ }^{\circ}\right)\end{array}$ & Feature & $\begin{array}{c}D_{\mathrm{r}, \mathrm{b}} \\
\left({ }^{\prime}\right)\end{array}$ & $\begin{array}{c}d_{\mathrm{r}, \mathrm{b}} \\
\left({ }^{\prime}\right)\end{array}$ & $\begin{array}{c}\mathrm{PA}_{\mathrm{r}, \mathrm{b}} \\
\left({ }^{\circ}\right)\end{array}$ & $\begin{array}{c}D_{\mathrm{r}, \mathrm{b}, 0} \\
\left(^{\prime}\right)\end{array}$ & $\begin{array}{c}d_{\mathrm{r}, \mathrm{b}, 0} \\
\left(^{\prime}\right)\end{array}$ & $\begin{array}{c}\theta_{\mathrm{r}, \mathrm{b}} \\
\left({ }^{\circ}\right)\end{array}$ \\
\hline$\overline{R L}$ & 2.35 & 1.55 & 166.8 & 2.37 & 2.22 & 26.1 & $\mathrm{r}$ & 0.32 & 0.10 & 68.8 & 0.33 & 0.27 & 159.8 \\
\hline $\mathrm{R}$ & 1.47 & 1.07 & 152.9 & 1.62 & 1.41 & 118.6 & & & & & & & \\
\hline $\mathrm{rl}$ & 0.69 & 0.47 & 160.6 & 0.70 & 0.67 & 139.0 & & & & & & & \\
\hline bar & 0.66 & 0.33 & 169.8 & 0.66 & 0.47 & 14.8 & & & & & & & \\
\hline
\end{tabular}

NGC 3637

(RL)SB $(\mathrm{rl}, \mathrm{bl}) 0^{+}$
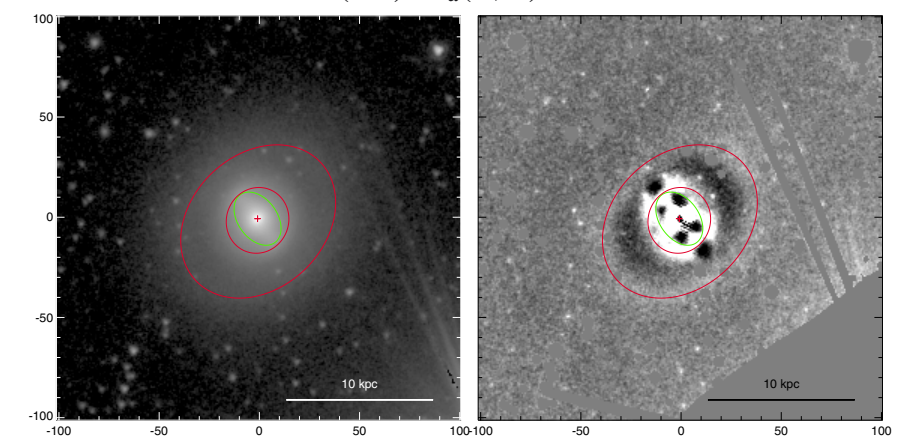

\begin{tabular}{|c|c|c|c|c|c|c|c|c|c|c|c|c|c|}
\hline Feature & $\begin{array}{c}D_{\mathrm{r}, \mathrm{b}} \\
\left({ }^{\prime}\right)\end{array}$ & $\begin{array}{c}d_{\mathrm{r}, \mathrm{b}} \\
\left({ }^{\prime}\right)\end{array}$ & $\begin{array}{c}\mathrm{PA}_{\mathrm{r}, \mathrm{b}} \\
\left({ }^{\mathrm{o}}\right)\end{array}$ & $\begin{array}{c}D_{\mathrm{r}, \mathrm{b}, 0} \\
\left({ }^{\prime}\right)\end{array}$ & $\begin{array}{c}d_{\mathrm{r}, \mathrm{b}, 0} \\
\left({ }^{\prime}\right)\end{array}$ & $\begin{array}{l}\theta_{\mathrm{r}, \mathrm{b}} \\
\left({ }^{\circ}\right)\end{array}$ & Feature & $\begin{array}{c}D_{\mathrm{r}, \mathrm{b}} \\
\left(^{\prime}\right)\end{array}$ & $\begin{array}{c}d_{\mathrm{r}, \mathrm{b}} \\
(')\end{array}$ & $\begin{array}{c}\mathrm{PA}_{\mathrm{r}, \mathrm{b}} \\
\left({ }^{\mathrm{o}}\right)\end{array}$ & $\begin{array}{c}D_{\mathrm{r}, \mathrm{b}, 0} \\
\left({ }^{\prime}\right)\end{array}$ & $\begin{array}{c}d_{\mathrm{r}, \mathrm{b}, 0} \\
\left({ }^{\prime}\right)\end{array}$ & $\begin{array}{l}\theta_{\mathrm{r}, \mathrm{b}} \\
\left({ }^{\mathrm{o}}\right)\end{array}$ \\
\hline $\mathrm{RL}$ & 1.44 & 1.12 & 133.7 & 1.44 & 1.25 & 171.9 & $\mathrm{rl}$ & 0.42 & 0.40 & 105.1 & 0.44 & 0.42 & 85.4 \\
\hline $\mathrm{rl}$ & 0.55 & 0.52 & 159.1 & 0.59 & 0.55 & 73.6 & & & & & & & \\
\hline bar & 0.49 & 0.33 & 35.7 & 0.55 & 0.33 & 80.5 & & & & & & & \\
\hline
\end{tabular}

NGC 3625

$\mathrm{SB}(\mathrm{rs}) \mathrm{cd}$

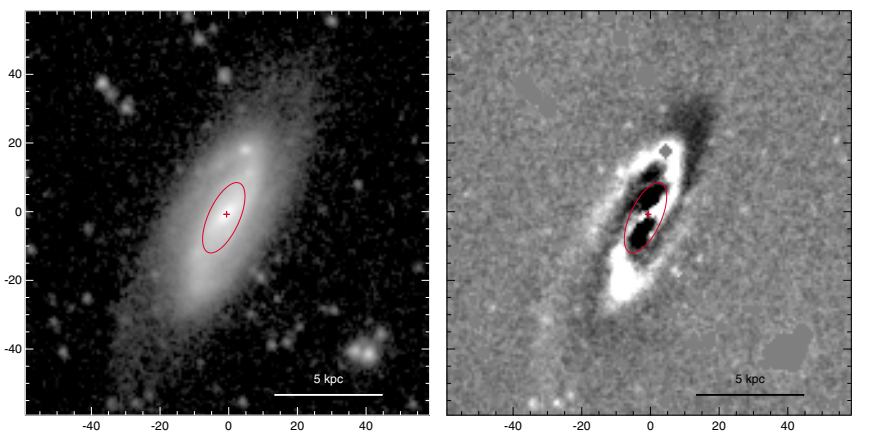

NGC 3633

SA(r)a

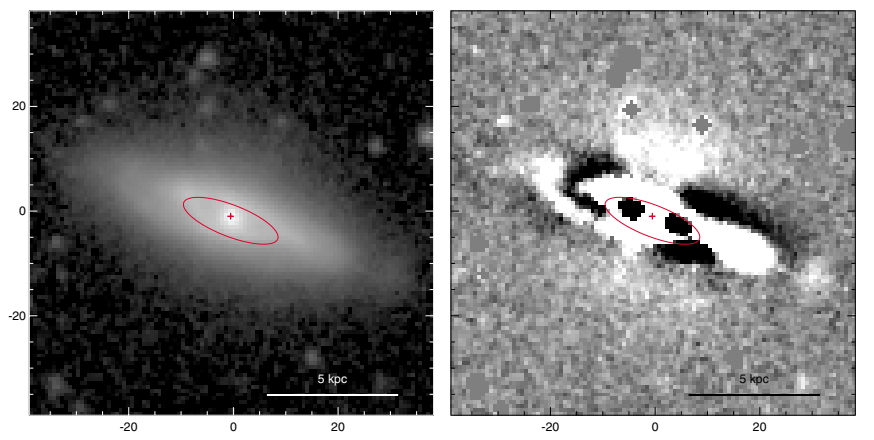

NGC 3642

$\mathrm{SA}(\mathrm{rl}) \mathrm{ab}$

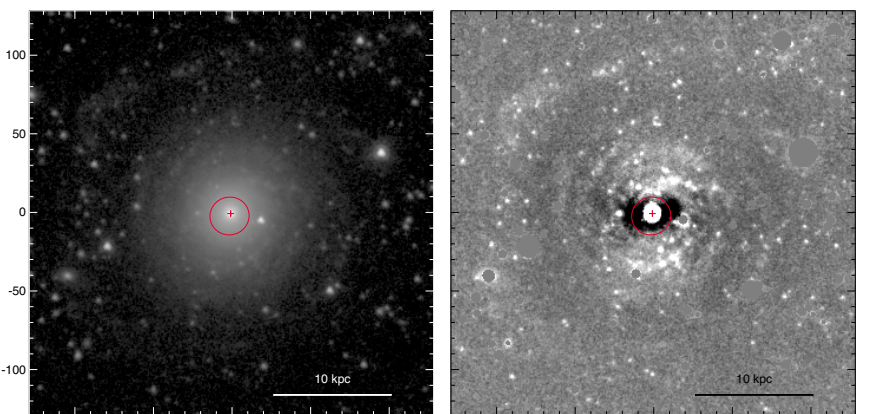


S. Comerón et al.: ARRAKIS

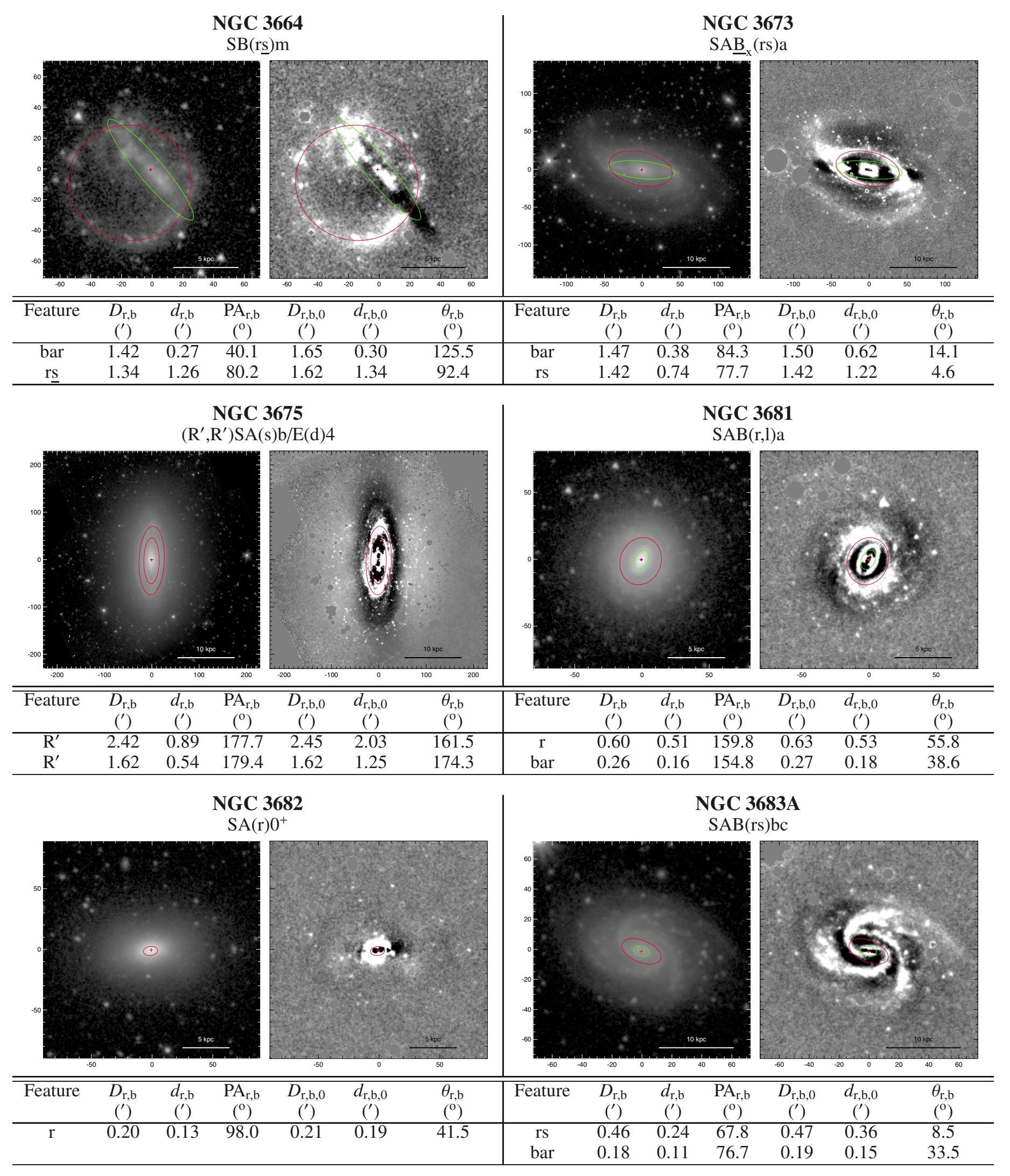


NGC 3684

$\left(\mathrm{R}^{\prime}\right) \mathrm{SAB}(\mathrm{s}) \mathrm{c}$
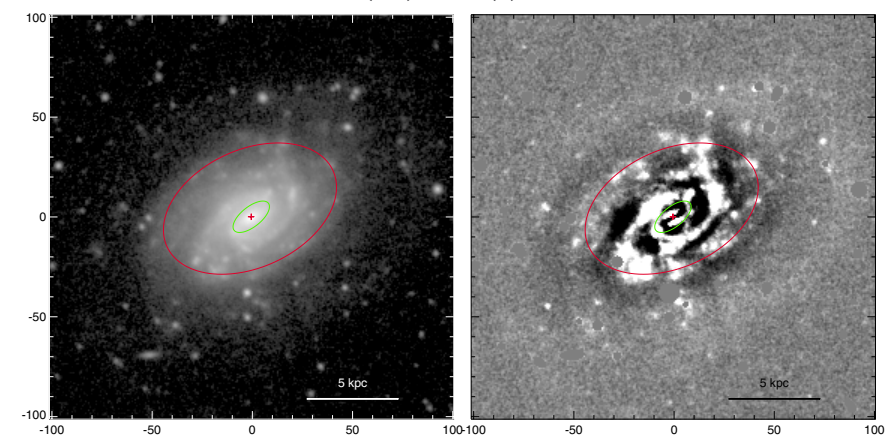

\begin{tabular}{ccccccc|ccccccc}
\hline \hline Feature & $\begin{array}{c}D_{\mathrm{r}, \mathrm{b}} \\
\left({ }^{\prime}\right)\end{array}$ & $\begin{array}{c}d_{\mathrm{r}, \mathrm{b}} \\
\left({ }^{\prime}\right)\end{array}$ & $\begin{array}{c}\mathrm{PA}_{\mathrm{r}, \mathrm{b}} \\
\left({ }^{\circ}\right)\end{array}$ & $\begin{array}{c}D_{\mathrm{r}, \mathrm{b}, 0} \\
\left({ }^{\prime}\right)\end{array}$ & $\begin{array}{c}d_{\mathrm{r}, \mathrm{b}, 0} \\
\left({ }^{\prime}\right)\end{array}$ & $\begin{array}{c}\theta_{\mathrm{r}, \mathrm{b}} \\
\left({ }^{\circ}\right)\end{array}$ & Feature & $\begin{array}{c}D_{\mathrm{r}, \mathrm{b}} \\
\left({ }^{\prime}\right)\end{array}$ & $\begin{array}{c}d_{\mathrm{r}, \mathrm{b}} \\
\left({ }^{\prime}\right)\end{array}$ & $\begin{array}{c}\mathrm{PA}_{\mathrm{r}, \mathrm{b}} \\
\left({ }^{\circ}\right)\end{array}$ & $\begin{array}{c}D_{\mathrm{r}, \mathrm{b}, 0} \\
\left({ }^{\prime}\right)\end{array}$ & $\begin{array}{c}d_{\mathrm{r}, \mathrm{b}, 0} \\
\left({ }^{\prime}\right)\end{array}$ & $\begin{array}{c}\theta_{\mathrm{r}, \mathrm{b}} \\
\left({ }^{\circ}\right)\end{array}$ \\
\hline $\mathrm{R}^{\prime}$ & 1.52 & 0.98 & 114.6 & 1.56 & 1.42 & 147.8 & $\mathrm{RL}$ & 1.43 & 1.34 & 164.6 & 1.49 & 1.35 & 108.7 \\
bar & 0.36 & 0.17 & 128.5 & 0.37 & 0.24 & 17.3 & rs & 0.44 & 0.41 & 123.7 & 0.46 & 0.41 & 85.5 \\
& & & & & & & bar & 0.43 & 0.21 & 174.8 & 0.44 & 0.22 & 130.4 \\
\hline
\end{tabular}
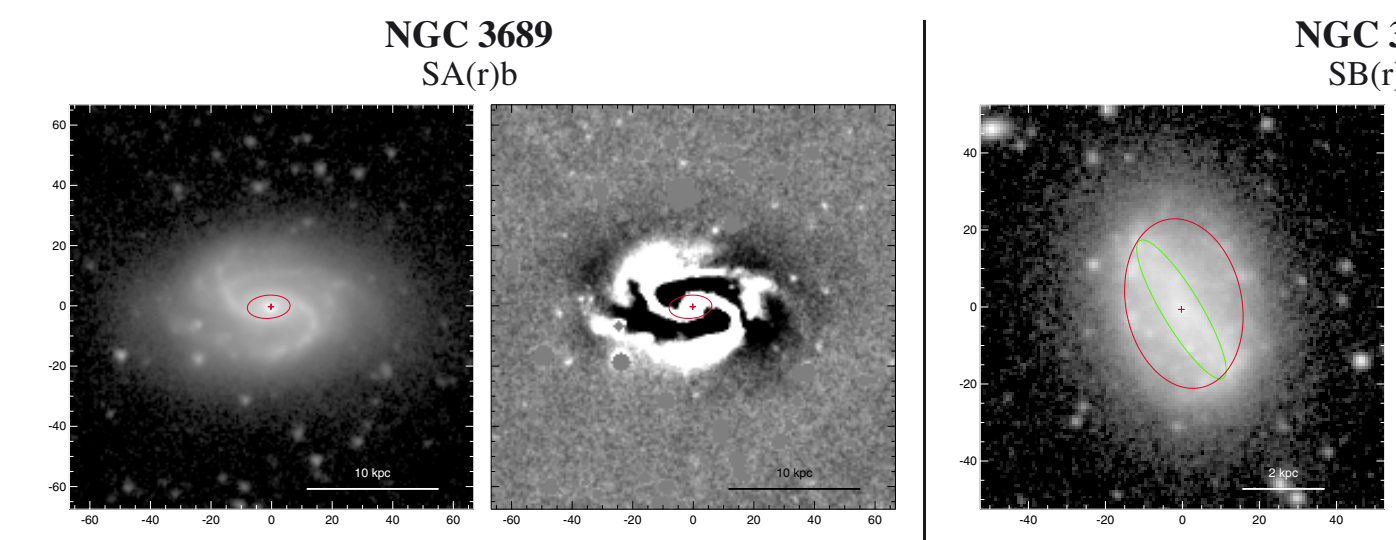

NGC 3691

$\mathrm{SB}(\mathrm{r}) \mathrm{m}$

\begin{tabular}{ccccccc|ccccccc}
\hline \hline Feature & $\begin{array}{c}D_{\mathrm{r}, \mathrm{b}} \\
\left({ }^{\prime}\right)\end{array}$ & $\begin{array}{c}d_{\mathrm{r}, \mathrm{b}} \\
\left({ }^{\prime}\right)\end{array}$ & $\begin{array}{c}\mathrm{PA}_{\mathrm{r}, \mathrm{b}} \\
\left({ }^{\circ}\right)\end{array}$ & $\begin{array}{c}D_{\mathrm{r}, \mathrm{b}, 0} \\
\left({ }^{\prime}\right)\end{array}$ & $\begin{array}{c}d_{\mathrm{r}, \mathrm{b}, 0} \\
\left({ }^{\prime}\right)\end{array}$ & $\begin{array}{c}\theta_{\mathrm{r}, \mathrm{b}} \\
\left({ }^{\circ}\right)\end{array}$ & Feature & $\begin{array}{c}D_{\mathrm{r}, \mathrm{b}} \\
\left({ }^{\prime}\right)\end{array}$ & $\begin{array}{c}d_{\mathrm{r}, \mathrm{b}} \\
\left({ }^{\prime}\right)\end{array}$ & $\begin{array}{c}\mathrm{PA}_{\mathrm{r}, \mathrm{b}} \\
\left({ }^{\circ}\right)\end{array}$ & $\begin{array}{c}D_{\mathrm{r}, \mathrm{b}, 0} \\
\left({ }^{\prime}\right)\end{array}$ & $\begin{array}{c}d_{\mathrm{r}, \mathrm{b}, 0} \\
\left({ }^{\prime}\right)\end{array}$ & $\begin{array}{c}\theta_{\mathrm{r}, \mathrm{b}} \\
\left({ }^{\circ}\right)\end{array}$ \\
\hline $\mathrm{r}$ & 0.24 & 0.13 & 94.3 & 0.24 & 0.21 & 15.6 & $\mathrm{r}$ & 0.75 & 0.51 & 11.4 & 0.77 & 0.65 & 150.9 \\
& & & & & & & $\mathrm{bar}$ & 0.70 & 0.17 & 31.2 & 0.71 & 0.22 & 13.1 \\
\hline
\end{tabular}

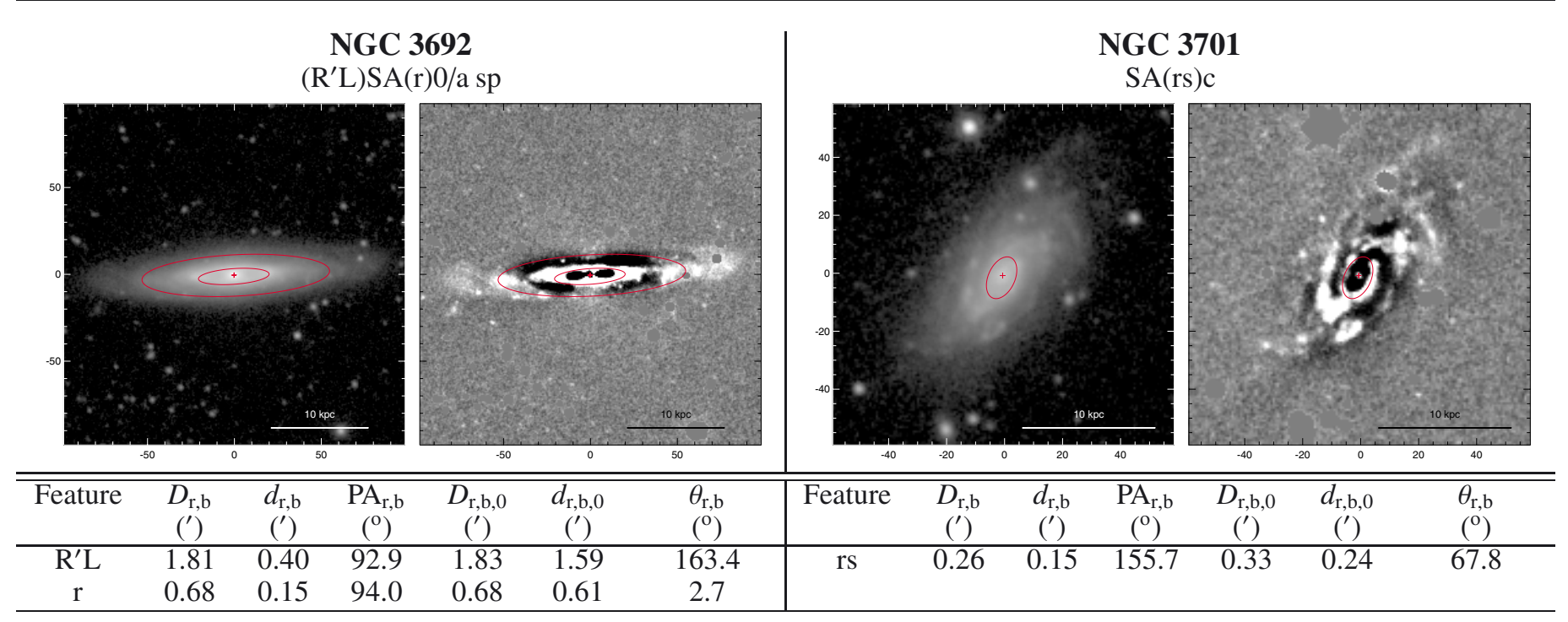


NGC 3705

$\left(\mathrm{R}^{\prime}\right) \mathrm{SAB}(\mathrm{rs}) \mathrm{b}$
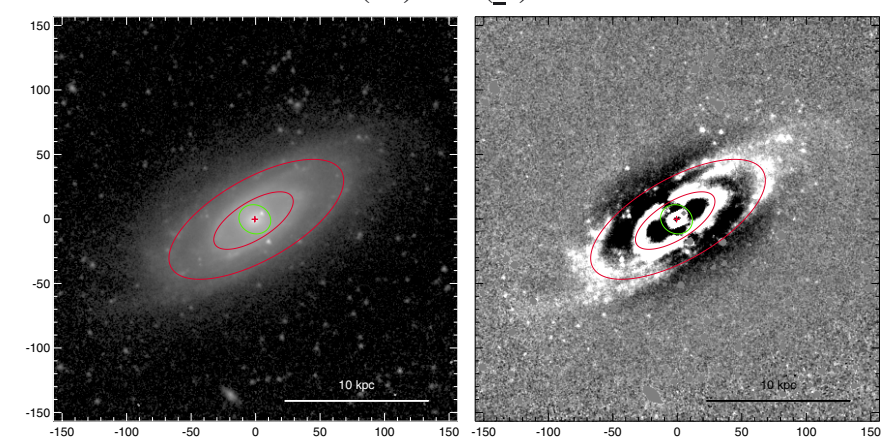

\begin{tabular}{ccccccc|ccccccc}
\hline \hline Feature & $\begin{array}{c}D_{\mathrm{r}, \mathrm{b}} \\
\left({ }^{\prime}\right)\end{array}$ & $\begin{array}{c}d_{\mathrm{r}, \mathrm{b}} \\
\left({ }^{\prime}\right)\end{array}$ & $\begin{array}{c}\mathrm{PA}_{\mathrm{r}, \mathrm{b}} \\
\left({ }^{\circ}\right)\end{array}$ & $\begin{array}{c}D_{\mathrm{r}, \mathrm{b}, 0} \\
\left({ }^{\prime}\right)\end{array}$ & $\begin{array}{c}d_{\mathrm{r}, \mathrm{b}, 0} \\
\left({ }^{\prime}\right)\end{array}$ & $\begin{array}{c}\theta_{\mathrm{r}, \mathrm{b}} \\
\left({ }^{\circ}\right)\end{array}$ & Feature & $\begin{array}{c}D_{\mathrm{r}, \mathrm{b}} \\
\left({ }^{\prime}\right)\end{array}$ & $\begin{array}{c}d_{\mathrm{r}, \mathrm{b}} \\
\left({ }^{\prime}\right)\end{array}$ & $\begin{array}{c}\mathrm{PA}_{\mathrm{r}, \mathrm{b}} \\
\left({ }^{\circ}\right)\end{array}$ & $\begin{array}{c}D_{\mathrm{r}, \mathrm{b}, 0} \\
\left({ }^{\prime}\right)\end{array}$ & $\begin{array}{c}d_{\mathrm{r}, \mathrm{b}, 0} \\
\left({ }^{\prime}\right)\end{array}$ & $\begin{array}{c}\theta_{\mathrm{r}, \mathrm{b}} \\
\left({ }^{\circ}\right)\end{array}$ \\
\hline $\mathrm{R}^{\prime}$ & 2.53 & 1.06 & 119.6 & 2.54 & 2.00 & 4.8 & $\mathrm{rs}$ & 0.32 & 0.27 & 112.0 & 0.35 & 0.30 & 119.6 \\
$\mathrm{rs}$ & 1.17 & 0.49 & 121.8 & 1.18 & 0.92 & 13.3 & bar & 0.25 & 0.18 & 75.8 & 0.30 & 0.18 & 108.1 \\
$\underline{\mathrm{bar}}$ & 0.42 & 0.37 & 65.5 & 0.77 & 0.38 & 94.9 & & & & & & & \\
\hline
\end{tabular}

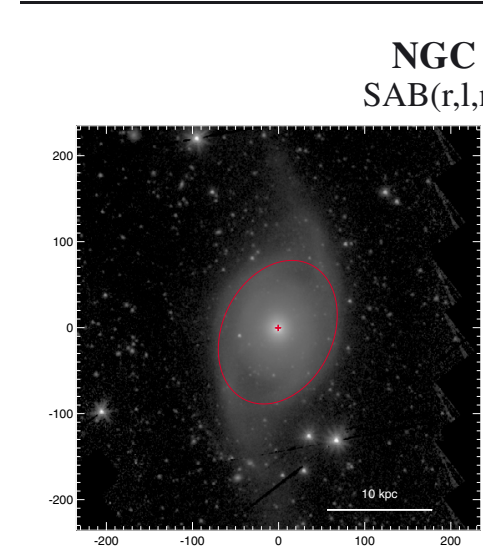

NGC 3718

SAB(r,l,nl)a pec

\begin{tabular}{ccccccc|ccccccc}
\hline \hline Feature & $\begin{array}{c}D_{\mathrm{r}, \mathrm{b}} \\
\left({ }^{\prime}\right)\end{array}$ & $\begin{array}{c}d_{\mathrm{r}, \mathrm{b}} \\
\left({ }^{\prime}\right)\end{array}$ & $\begin{array}{c}\mathrm{PA}_{\mathrm{r}, \mathrm{b}} \\
\left({ }^{\circ}\right)\end{array}$ & $\begin{array}{c}D_{\mathrm{r}, \mathrm{b}, 0} \\
\left({ }^{\prime}\right)\end{array}$ & $\begin{array}{c}d_{\mathrm{r}, \mathrm{b}, 0} \\
\left({ }^{\prime}\right)\end{array}$ & $\begin{array}{c}\theta_{\mathrm{r}, \mathrm{b}} \\
\left({ }^{\circ}\right)\end{array}$ & Feature & $\begin{array}{c}D_{\mathrm{r}, \mathrm{b}} \\
\left({ }^{\prime}\right)\end{array}$ & $\begin{array}{c}d_{\mathrm{r}, \mathrm{b}} \\
\left({ }^{\prime}\right)\end{array}$ & $\begin{array}{c}\mathrm{PA}_{\mathrm{r}, \mathrm{b}} \\
\left({ }^{\circ}\right)\end{array}$ & $\begin{array}{c}D_{\mathrm{r}, \mathrm{b}, 0} \\
\left({ }^{\prime}\right)\end{array}$ & $\begin{array}{c}d_{\mathrm{r}, \mathrm{b}, 0} \\
\left({ }^{\prime}\right)\end{array}$ & $\begin{array}{c}\theta_{\mathrm{r}, \mathrm{b}} \\
\left({ }^{\circ}\right)\end{array}$ \\
\hline $\mathrm{r}$ & 2.91 & 2.14 & 154.3 & 6.29 & 2.57 & 98.2 & $\mathrm{r}$ & 1.50 & 0.84 & 12.1 & 1.54 & 1.31 & 154.0 \\
& & & & & & & & & & & & &
\end{tabular}

NGC 3729

(L)SB(r)0/a + dwarf comp.
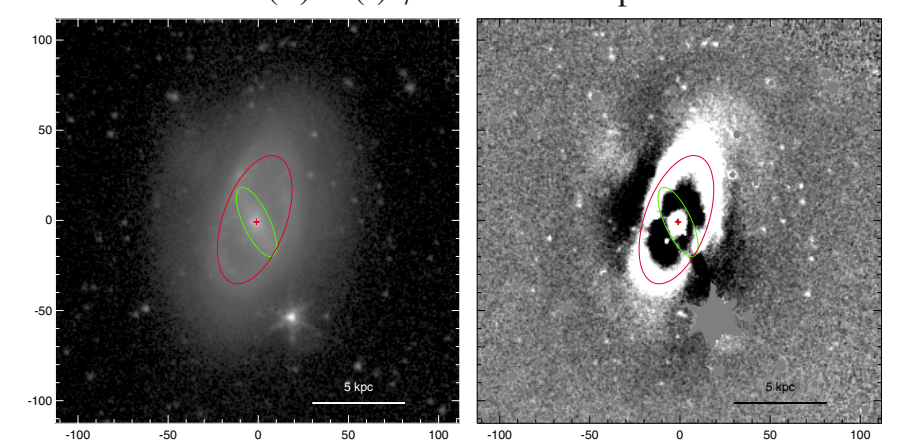

\begin{tabular}{|c|c|c|c|c|c|c|c|c|c|c|c|c|c|}
\hline Feature & $\begin{array}{c}D_{\mathrm{r}, \mathrm{b}} \\
\left({ }^{\prime}\right)\end{array}$ & $\begin{array}{c}d_{\mathrm{r}, \mathrm{b}} \\
(')\end{array}$ & $\begin{array}{c}\mathrm{PA}_{\mathrm{r}, \mathrm{b}} \\
\left({ }^{\circ}\right)\end{array}$ & $\begin{array}{c}D_{\mathrm{r}, \mathrm{b}, 0} \\
\left(^{\prime}\right)\end{array}$ & $\begin{array}{c}d_{\mathrm{r}, \mathrm{b}, 0} \\
\left({ }^{\prime}\right)\end{array}$ & $\begin{array}{c}\theta_{\mathrm{r}, \mathrm{b}} \\
\left({ }^{\circ}\right)\end{array}$ & 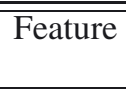 & $\begin{array}{c}D_{\mathrm{r}, \mathrm{b}} \\
\left({ }^{\prime}\right)\end{array}$ & $\begin{array}{c}d_{\mathrm{r}, \mathrm{b}} \\
\left({ }^{\prime}\right)\end{array}$ & $\begin{array}{c}\mathrm{PA}_{\mathrm{r}, \mathrm{b}} \\
\left({ }^{\circ}\right)\end{array}$ & $\begin{array}{c}D_{\mathrm{r}, \mathrm{b}, 0} \\
\left(^{\prime}\right)\end{array}$ & $\begin{array}{c}d_{\mathrm{r}, \mathrm{b}, 0} \\
\left({ }^{\prime}\right)\end{array}$ & $\begin{array}{c}\theta_{\mathrm{r}, \mathrm{b}} \\
\left({ }^{\circ}\right)\end{array}$ \\
\hline $\mathrm{r}$ & 1.25 & 0.59 & 160.4 & 1.28 & 0.80 & 158.1 & rs & 0.21 & 0.18 & 94.9 & 0.21 & 0.19 & 32.6 \\
\hline bar & 0.70 & 0.24 & 25.3 & 0.80 & 0.30 & 45.5 & & & & & & & \\
\hline
\end{tabular}

NGC 3715

$\mathrm{SAB}(\mathrm{rs}) \mathrm{ab}$

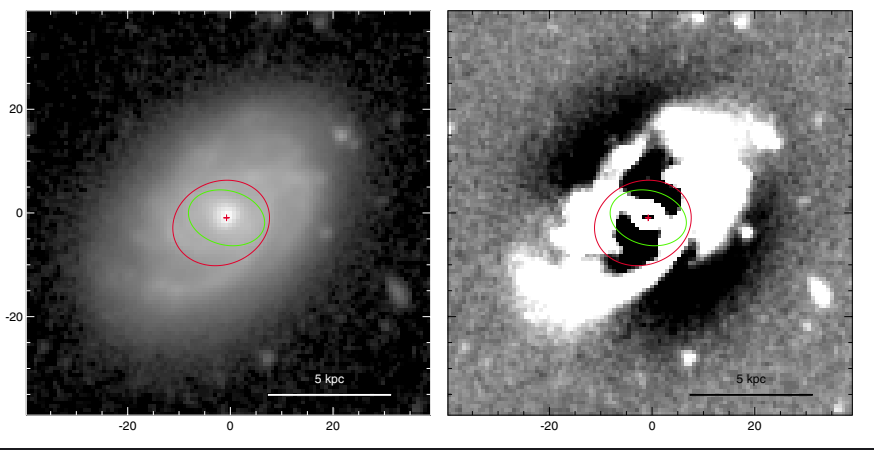

NGC 3726

$\mathrm{SA} \underline{\mathrm{B}}(\mathrm{r}) \mathrm{bc}$
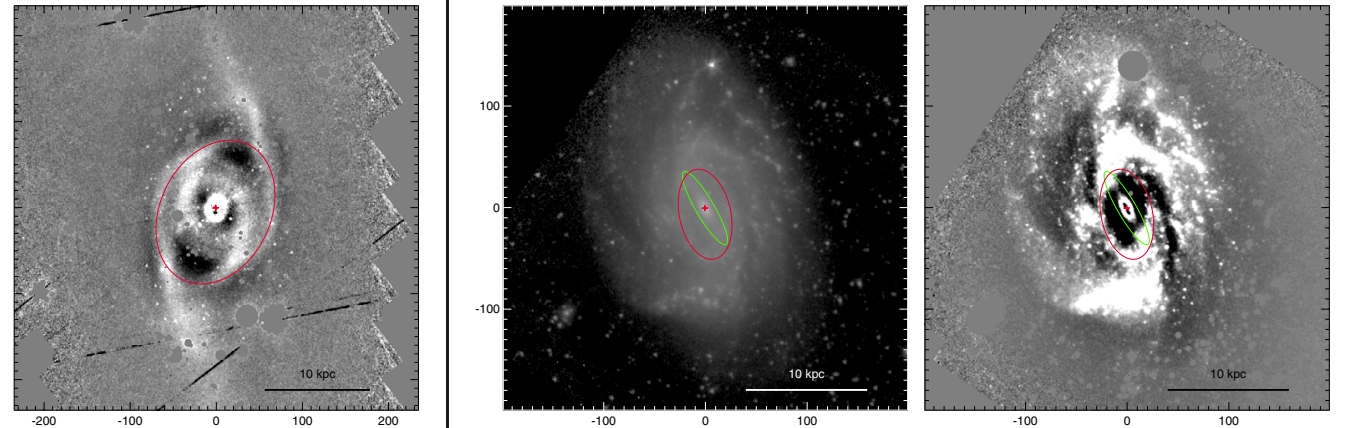

NGC 3730

SA(rs)a

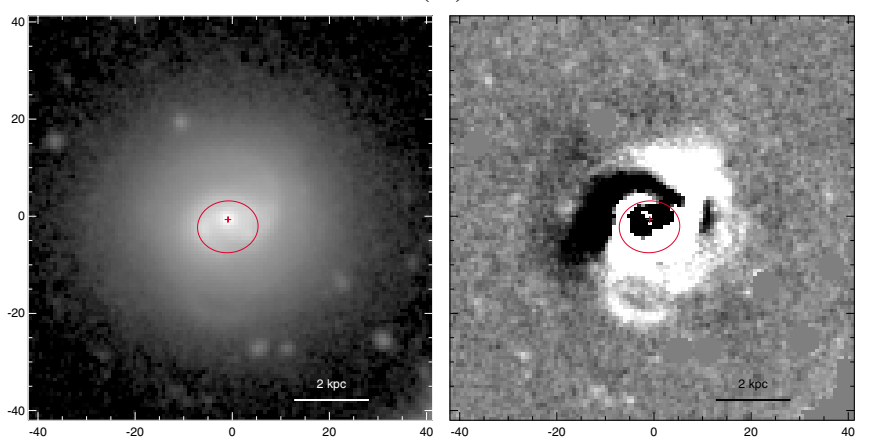


NGC 3735

$\mathrm{SAB}(\mathrm{rs}) \mathrm{c}$ : sp
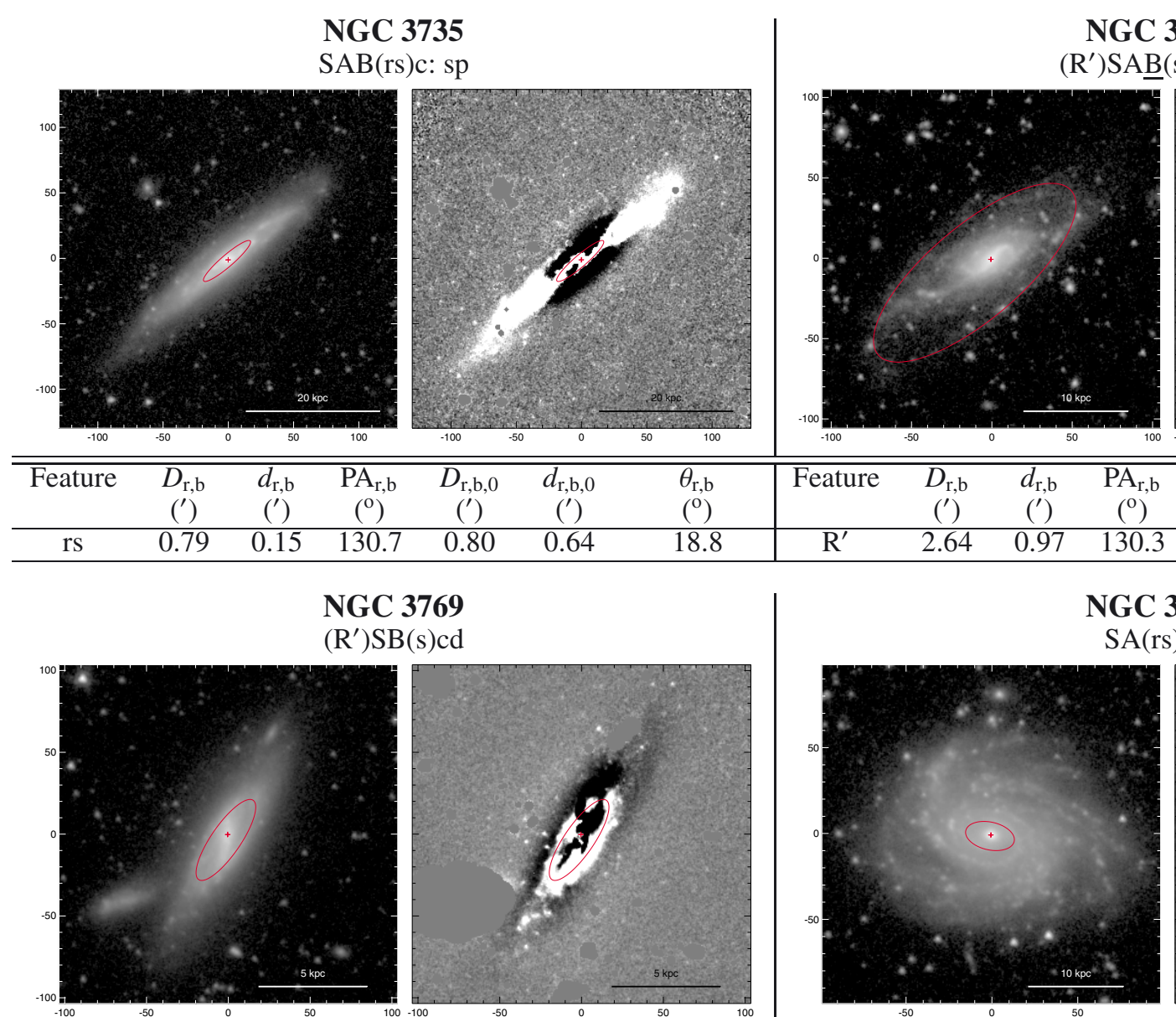

NGC 3755

$\left(\mathrm{R}^{\prime}\right) \mathrm{SA} \underline{\mathrm{B}}(\mathrm{s}) \mathrm{c}$ pec

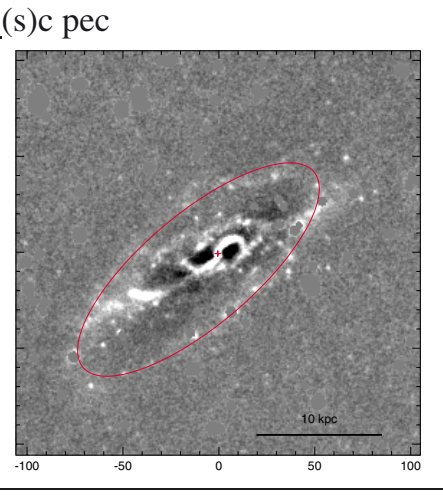

$\begin{array}{lllllll}\text { Feature } & D_{\mathrm{r}, \mathrm{b}} & d_{\mathrm{r}, \mathrm{b}} & \mathrm{PA}_{\mathrm{r}, \mathrm{b}} & D_{\mathrm{r}, \mathrm{b}, 0} & d_{\mathrm{r}, \mathrm{b}, 0} & \theta_{\mathrm{r}, \mathrm{b}}\end{array}$

\begin{tabular}{|c|c|c|c|c|c|c|c|c|c|c|c|c|c|}
\hline Feature & $\begin{array}{c}D_{\mathrm{r}, \mathrm{b}} \\
\left({ }^{\prime}\right)\end{array}$ & $\begin{array}{c}d_{\mathrm{r}, \mathrm{b}} \\
\left({ }^{\prime}\right) \\
\end{array}$ & $\begin{array}{c}\mathrm{PA}_{\mathrm{r}, \mathrm{b}} \\
\left({ }^{\circ}\right)\end{array}$ & $\begin{array}{c}D_{\mathrm{r}, \mathrm{b}, 0} \\
\left({ }^{\prime}\right)\end{array}$ & $\begin{array}{c}d_{\mathrm{r}, \mathrm{b}, 0} \\
\left({ }^{\prime}\right)\end{array}$ & $\begin{array}{c}\theta_{\mathrm{r}, \mathrm{b}} \\
\left({ }^{\circ}\right)\end{array}$ & Feature & $\begin{array}{c}D_{\mathrm{r}, \mathrm{b}} \\
\left({ }^{\prime}\right)\end{array}$ & $\begin{array}{c}d_{\mathrm{r}, \mathrm{b}} \\
\left({ }^{\prime}\right)\end{array}$ & $\begin{array}{c}\mathrm{PA}_{\mathrm{r}, \mathrm{b}} \\
\left({ }^{\circ}\right)\end{array}$ & $\begin{array}{c}D_{\mathrm{r}, \mathrm{b}, 0} \\
\left({ }^{\prime}\right)\end{array}$ & $\begin{array}{c}d_{\mathrm{r}, \mathrm{b}, 0} \\
\left(^{\prime}\right)\end{array}$ & $\begin{array}{c}\theta_{\mathrm{r}, \mathrm{b}} \\
\left({ }^{\circ}\right)\end{array}$ \\
\hline rs & 0.79 & 0.15 & 130.7 & 0.80 & 0.64 & 18.8 & $\overline{\mathrm{R}^{\prime}}$ & 2.64 & 0.97 & 130.3 & 2.71 & 2.59 & 52.2 \\
\hline
\end{tabular}

NGC 3769

$\left(\mathrm{R}^{\prime}\right) \mathrm{SB}(\mathrm{s}) \mathrm{cd}$

\begin{tabular}{|c|c|c|c|c|c|c|c|c|c|c|c|c|c|}
\hline Feature & $\begin{array}{c}D_{\mathrm{r}, \mathrm{b}} \\
\left({ }^{\prime}\right)\end{array}$ & $\begin{array}{c}d_{\mathrm{r}, \mathrm{b}} \\
\left({ }^{\prime}\right)\end{array}$ & $\begin{array}{c}\mathrm{PA}_{\mathrm{r}, \mathrm{b}} \\
\left({ }^{\circ}\right)\end{array}$ & $\begin{array}{c}D_{\mathrm{r}, \mathrm{b}, 0} \\
\left(^{\prime}\right)\end{array}$ & $\begin{array}{c}d_{\mathrm{r}, \mathrm{b}, 0} \\
\left(^{\prime}\right)\end{array}$ & $\begin{array}{c}\theta_{\mathrm{r}, \mathrm{b}} \\
\left({ }^{\circ}\right)\end{array}$ & Feature & $\begin{array}{c}D_{\mathrm{r}, \mathrm{b}} \\
\left({ }^{\prime}\right)\end{array}$ & $\begin{array}{c}d_{\mathrm{r}, \mathrm{b}} \\
\left({ }^{\prime}\right)\end{array}$ & $\begin{array}{c}\mathrm{PA}_{\mathrm{r}, \mathrm{b}} \\
\left({ }^{\circ}\right)\end{array}$ & $\begin{array}{c}D_{\mathrm{r}, \mathrm{b}, 0} \\
\left(^{\prime}\right)\end{array}$ & $\begin{array}{c}d_{\mathrm{r}, \mathrm{b}, 0} \\
\left(^{\prime}\right)\end{array}$ & $\begin{array}{l}\theta_{\mathrm{r}, \mathrm{b}} \\
\left({ }^{\circ}\right)\end{array}$ \\
\hline$\overline{\mathrm{R}^{\prime}}$ & 0.98 & 0.30 & 145.5 & 1.07 & 0.87 & 136.8 & $\mathrm{rs}$ & 0.48 & 0.27 & 77.5 & 0.48 & 0.35 & 171.7 \\
\hline
\end{tabular}

NGC 3782

$\mathrm{SB}(\mathrm{rs}) \mathrm{dm}$

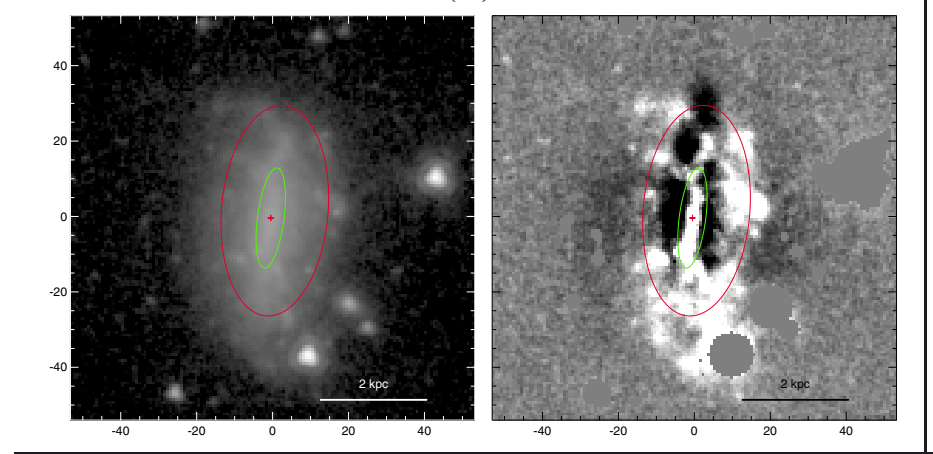

NGC 3780

$\mathrm{SA}(\mathrm{rs}) \mathrm{bc}$
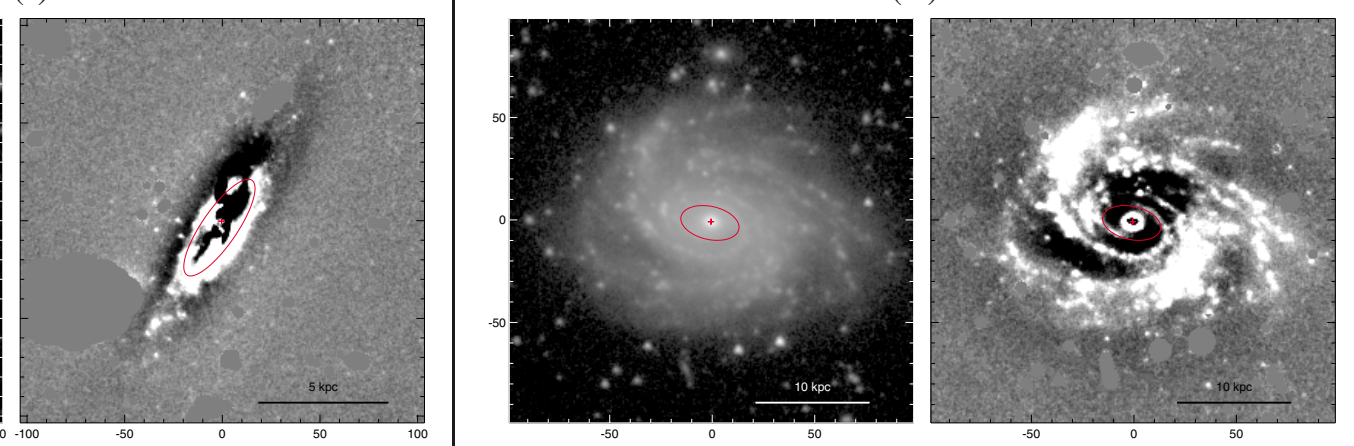

NGC 3786

(R)SA(r)0/a pec

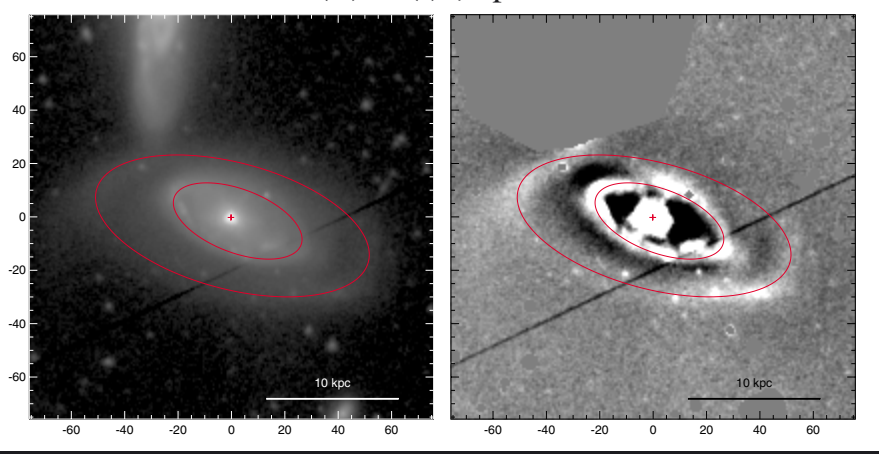

\begin{tabular}{ccccccc|ccccccc}
\hline \hline Feature & $\begin{array}{c}D_{\mathrm{r}, \mathrm{b}} \\
\left({ }^{\prime}\right)\end{array}$ & $\begin{array}{c}d_{\mathrm{r}, \mathrm{b}} \\
\left({ }^{\prime}\right)\end{array}$ & $\begin{array}{c}\mathrm{PA}_{\mathrm{r}, \mathrm{b}} \\
\left({ }^{\circ}\right)\end{array}$ & $\begin{array}{c}D_{\mathrm{r}, \mathrm{b}, 0} \\
\left({ }^{\prime}\right)\end{array}$ & $\begin{array}{c}d_{\mathrm{r}, \mathrm{b}, 0} \\
\left({ }^{\prime}\right)\end{array}$ & $\begin{array}{c}\theta_{\mathrm{r}, \mathrm{b}} \\
\left({ }^{(}\right)\end{array}$ & Feature & $\begin{array}{c}D_{\mathrm{r}, \mathrm{b}} \\
\left({ }^{\prime}\right)\end{array}$ & $\begin{array}{c}d_{\mathrm{r}, \mathrm{b}} \\
\left({ }^{\prime}\right)\end{array}$ & $\begin{array}{c}\mathrm{PA}_{\mathrm{r}, \mathrm{b}} \\
\left({ }^{\circ}\right)\end{array}$ & $\begin{array}{c}D_{\mathrm{r}, \mathrm{b}, 0} \\
\left({ }^{\prime}\right)\end{array}$ & $\begin{array}{c}d_{\mathrm{r}, \mathrm{b}, 0} \\
\left({ }^{\prime}\right)\end{array}$ & $\begin{array}{c}\theta_{\mathrm{r}, \mathrm{b}} \\
\left({ }^{\circ}\right)\end{array}$ \\
\hline $\mathrm{rs}$ & 0.93 & 0.47 & 175.2 & 0.94 & 0.57 & 170.3 & $\mathrm{R}$ & 1.77 & 0.79 & 75.1 & 1.79 & 1.43 & 17.4 \\
$\mathrm{bar}$ & 0.45 & 0.12 & 172.6 & 0.45 & 0.14 & 168.3 & $\mathrm{r}$ & 0.85 & 0.40 & 69.4 & 0.85 & 0.73 & 171.9 \\
\hline
\end{tabular}


NGC 3788

SA(r)a
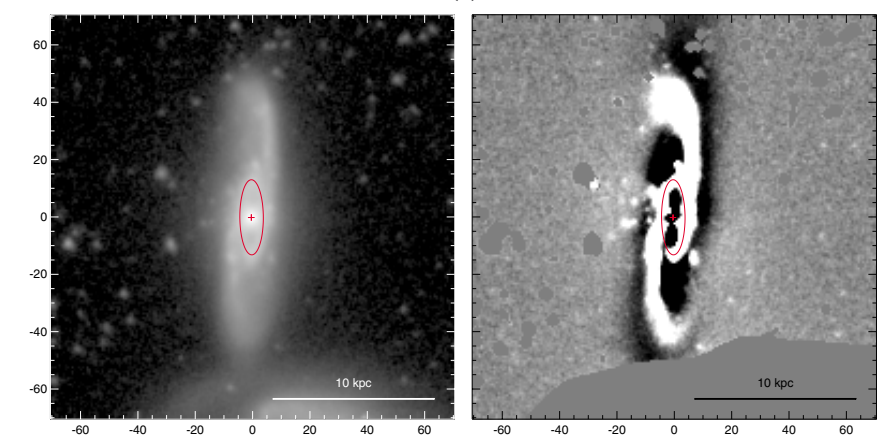

\begin{tabular}{ccccccc|ccccccc}
\hline \hline Feature & $\begin{array}{c}D_{\mathrm{r}, \mathrm{b}} \\
\left({ }^{\prime}\right)\end{array}$ & $\begin{array}{c}d_{\mathrm{r}, \mathrm{b}} \\
\left({ }^{\prime}\right)\end{array}$ & $\begin{array}{c}\mathrm{PA}_{\mathrm{r}, \mathrm{b}} \\
\left({ }^{\circ}\right)\end{array}$ & $\begin{array}{c}D_{\mathrm{r}, \mathrm{b}, 0} \\
\left({ }^{\prime}\right)\end{array}$ & $\begin{array}{c}d_{\mathrm{r}, \mathrm{b}, 0} \\
\left({ }^{\prime}\right)\end{array}$ & $\begin{array}{c}\theta_{\mathrm{r}, \mathrm{b}} \\
\left({ }^{\circ}\right)\end{array}$ & Feature & $\begin{array}{c}D_{\mathrm{r}, \mathrm{b}} \\
\left({ }^{\prime}\right)\end{array}$ & $\begin{array}{c}d_{\mathrm{r}, \mathrm{b}} \\
\left({ }^{\prime}\right)\end{array}$ & $\begin{array}{c}\mathrm{PA}_{\mathrm{r}, \mathrm{b}} \\
\left({ }^{\circ}\right)\end{array}$ & $\begin{array}{c}D_{\mathrm{r}, \mathrm{b}, 0} \\
\left({ }^{\prime}\right)\end{array}$ & $\begin{array}{c}d_{\mathrm{r}, \mathrm{b}, 0} \\
\left({ }^{\prime}\right)\end{array}$ & $\begin{array}{c}\theta_{\mathrm{r}, \mathrm{b}} \\
\left({ }^{\circ}\right)\end{array}$ \\
\hline $\mathrm{r}$ & 0.44 & 0.14 & 0.3 & 0.61 & 0.42 & 72.2 & $\underline{\mathrm{rs}}$ & 0.51 & 0.16 & 58.8 & 0.51 & 0.48 & 9.3 \\
\hline
\end{tabular}

NGC 3870

$\mathrm{SB}(\mathrm{rs}) 0^{\circ}$ ?
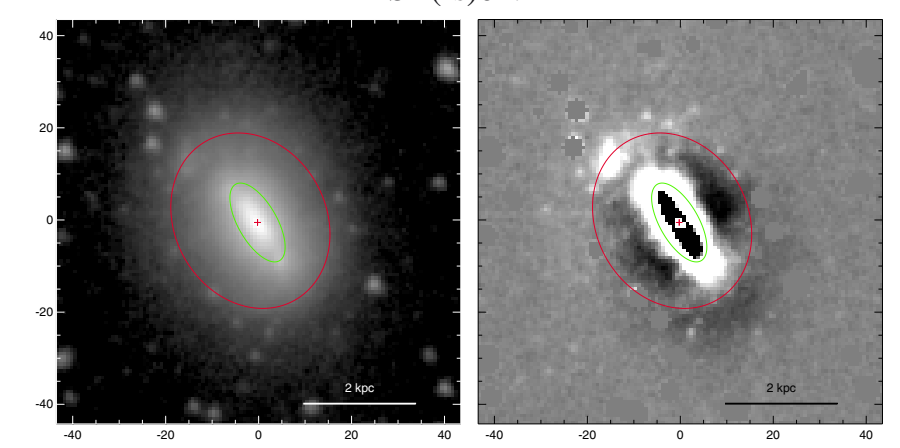

\begin{tabular}{ccccccc|ccccccc}
\hline \hline Feature & $\begin{array}{c}D_{\mathrm{r}, \mathrm{b}} \\
\left({ }^{\prime}\right)\end{array}$ & $\begin{array}{c}d_{\mathrm{r}, \mathrm{b}}\left({ }^{\prime}\right) \\
\left(\mathrm{PA}_{\mathrm{r}, \mathrm{b}}\right.\end{array}$ & $\begin{array}{c}D_{\mathrm{r}, \mathrm{b}, 0} \\
\left({ }^{\prime}\right)\end{array}$ & $\begin{array}{c}d_{\mathrm{r}, \mathrm{b}, 0} \\
\left({ }^{\prime}\right)\end{array}$ & $\begin{array}{c}\theta_{\mathrm{r}, \mathrm{b}} \\
\left({ }^{\circ}\right)\end{array}$ & Feature & $\begin{array}{c}D_{\mathrm{r}, \mathrm{b}} \\
\left({ }^{\prime}\right)\end{array}$ & $\begin{array}{c}d_{\mathrm{r}, \mathrm{b}} \\
\left({ }^{\prime}\right)\end{array}$ & $\begin{array}{c}\mathrm{PA}_{\mathrm{r}, \mathrm{b}} \\
\left({ }^{\circ}\right)\end{array}$ & $\begin{array}{c}D_{\mathrm{r}, \mathrm{b}, 0} \\
\left({ }^{\prime}\right)\end{array}$ & $\begin{array}{c}d_{\mathrm{r}, \mathrm{b}, 0} \\
\left({ }^{\prime}\right)\end{array}$ & $\begin{array}{c}\theta_{\mathrm{r}, \mathrm{b}} \\
\left({ }^{\circ}\right)\end{array}$ \\
\hline $\mathrm{rs}$ & 0.66 & 0.55 & 28.9 & 0.76 & 0.66 & 82.8 & RG & 0.56 & 0.39 & 110.0 & 0.68 & 0.55 & 74.0 \\
$\mathrm{bar}$ & 0.32 & 0.14 & 29.8 & 0.32 & 0.19 & 12.1 & & & & & & & \\
\hline
\end{tabular}

NGC 3885

(L) $\underline{\operatorname{ABB}}(\underline{\mathrm{r}}, \mathrm{nl}) 0^{+}$
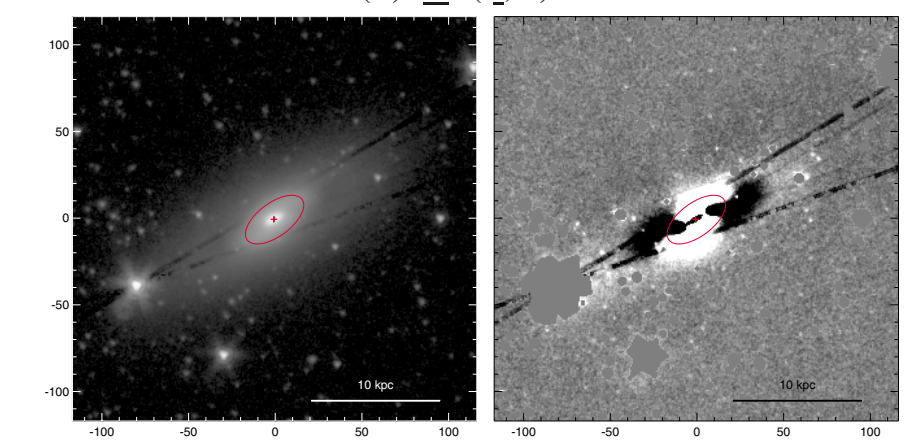

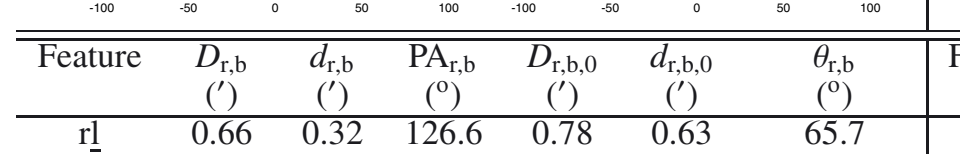

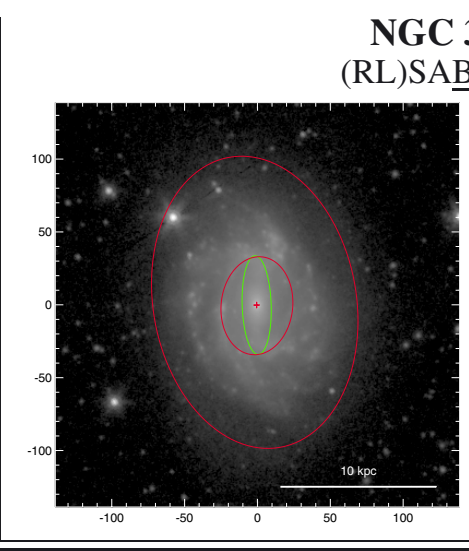

NGC 3887

$(\mathrm{RL}) \mathrm{SA} \underline{B}_{\mathrm{x}}(\mathrm{rs}) \mathrm{bc}$

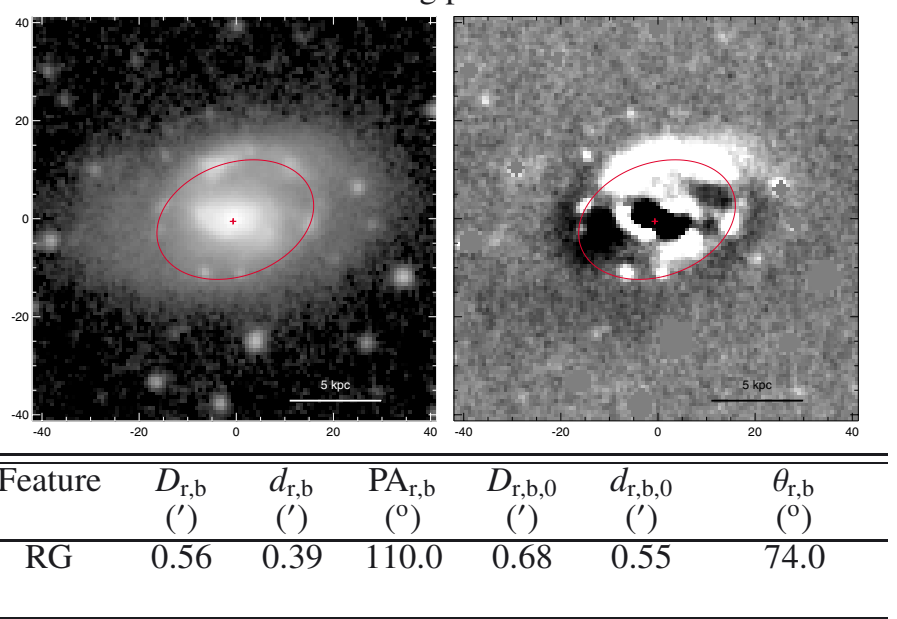

GC 3876

Ring pec

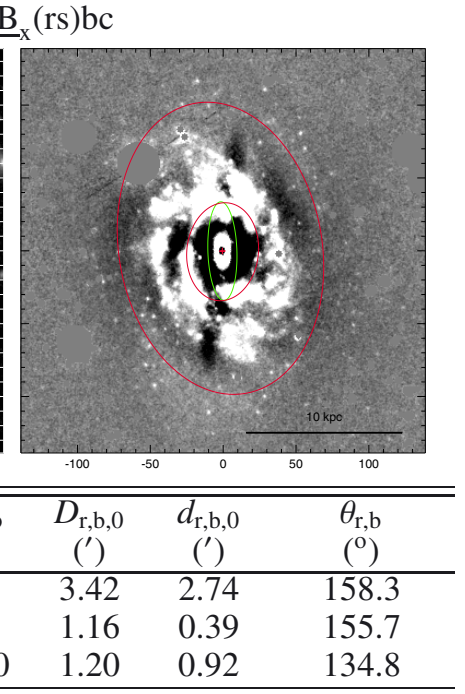




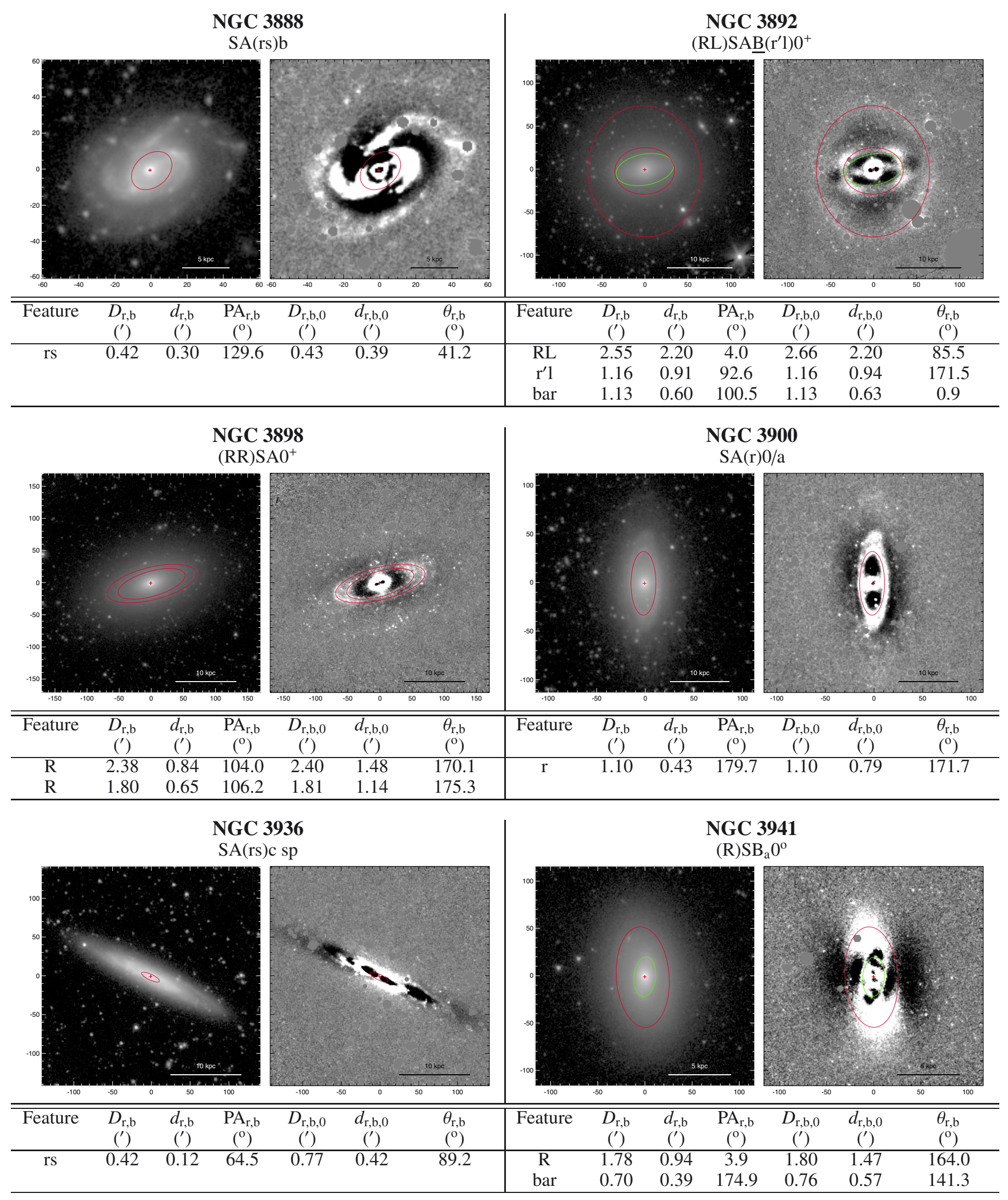


NGC 3949

$\mathrm{SAB}(\mathrm{rs}) \mathrm{c}$
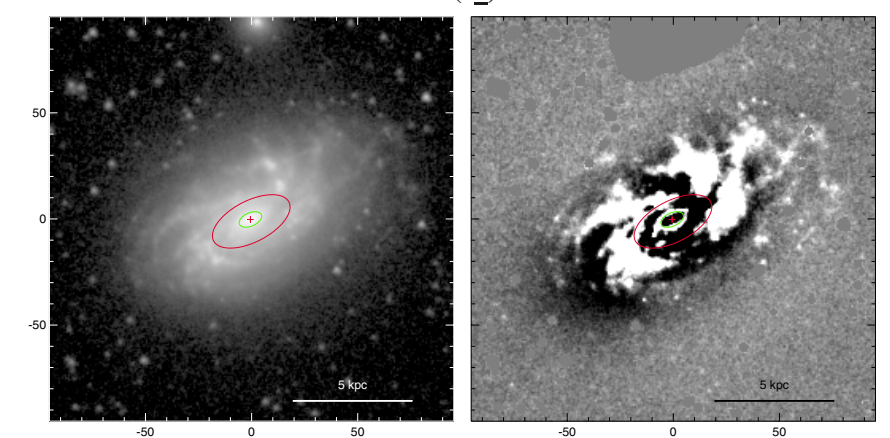

\begin{tabular}{|c|c|c|c|c|c|c|c|c|c|c|c|c|c|}
\hline Feature & $\begin{array}{c}D_{\mathrm{r}, \mathrm{b}} \\
\left(^{\prime}\right)\end{array}$ & $\begin{array}{c}d_{\mathrm{r}, \mathrm{b}} \\
\left(^{\prime}\right)\end{array}$ & $\begin{array}{c}\mathrm{PA}_{\mathrm{r}, \mathrm{b}} \\
\left({ }^{\circ}\right)\end{array}$ & $\begin{array}{c}D_{\mathrm{r}, \mathrm{b}, 0} \\
\left(^{\prime}\right)\end{array}$ & $\begin{array}{c}d_{\mathrm{r}, \mathrm{b}, 0} \\
\left(^{\prime}\right)\end{array}$ & $\begin{array}{l}\theta_{\mathrm{r}, \mathrm{b}} \\
\left({ }^{\circ}\right)\end{array}$ & Feature & $\begin{array}{c}D_{\mathrm{r}, \mathrm{b}} \\
\left({ }^{\prime}\right)\end{array}$ & $\begin{array}{c}d_{\mathrm{r}, \mathrm{b}} \\
\left(^{\prime}\right)\end{array}$ & $\begin{array}{c}\mathrm{PA}_{\mathrm{r}, \mathrm{b}} \\
\left({ }^{\circ}\right)\end{array}$ & $\begin{array}{c}D_{\mathrm{r}, \mathrm{b}, 0} \\
\left(^{\prime}\right)\end{array}$ & $\begin{array}{c}d_{\mathrm{r}, \mathrm{b}, 0} \\
\left(^{\prime}\right)\end{array}$ & $\begin{array}{l}\theta_{\mathrm{r}, \mathrm{b}} \\
\left({ }^{\circ}\right)\end{array}$ \\
\hline $\mathrm{rs}$ & 0.67 & 0.33 & 117.3 & 0.68 & 0.53 & 164.6 & $\mathrm{r}$ & 1.60 & 0.73 & 6.6 & 1.68 & 1.33 & 147.0 \\
\hline bār & 0.19 & 0.10 & 113.5 & 0.20 & 0.17 & 143.2 & bar & 0.91 & 0.42 & 47.3 & 1.29 & 0.57 & 61.4 \\
\hline
\end{tabular}

NGC 3955

$\mathrm{SB}(\mathrm{rs}) \mathrm{bc} / \mathrm{S}^{-} / \mathrm{Sph}$

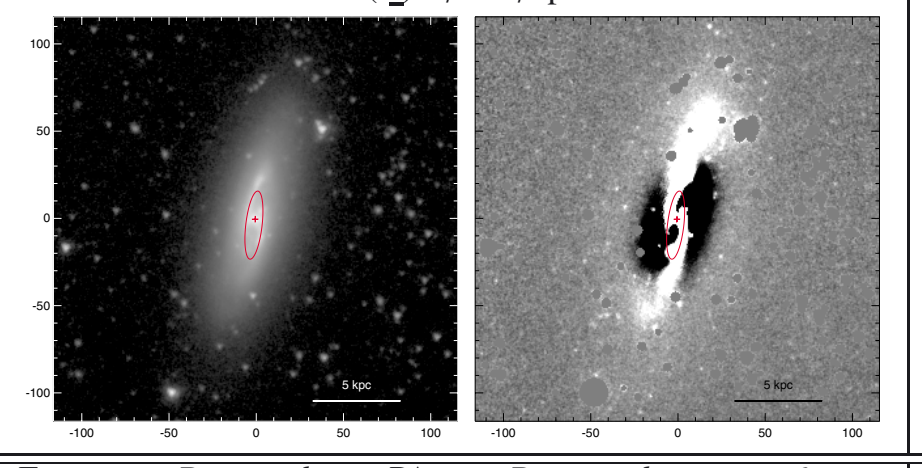

\begin{tabular}{|c|c|c|c|c|c|c|c|c|c|c|c|c|c|}
\hline Feature & $\begin{array}{c}D_{\mathrm{r}, \mathrm{b}} \\
\left({ }^{\prime}\right)\end{array}$ & $\begin{array}{c}d_{\mathrm{r}, \mathrm{b}} \\
\left({ }^{\prime}\right)\end{array}$ & $\begin{array}{c}\mathrm{PA}_{\mathrm{r}, \mathrm{b}} \\
\left({ }^{\circ}\right)\end{array}$ & $\begin{array}{c}D_{\mathrm{r}, \mathrm{b}, 0} \\
\left(^{\prime}\right)\end{array}$ & $\begin{array}{c}d_{\mathrm{r}, \mathrm{b}, 0} \\
\left(^{\prime}\right)\end{array}$ & $\begin{array}{l}\theta_{\mathrm{r}, \mathrm{b}} \\
\left({ }^{\circ}\right)\end{array}$ & Feature & $\begin{array}{c}D_{\mathrm{r}, \mathrm{b}} \\
\left({ }^{\prime}\right)\end{array}$ & $\begin{array}{c}d_{\mathrm{r}, \mathrm{b}} \\
\left({ }^{\prime}\right)\end{array}$ & $\begin{array}{c}\mathrm{PA}_{\mathrm{r}, \mathrm{b}} \\
\left({ }^{\circ}\right)\end{array}$ & $\begin{array}{c}D_{\mathrm{r}, \mathrm{b}, 0} \\
\left({ }^{\prime}\right)\end{array}$ & $\begin{array}{c}d_{\mathrm{r}, \mathrm{b}, 0} \\
\left(^{\prime}\right)\end{array}$ & $\begin{array}{l}\theta_{\mathrm{r}, \mathrm{b}} \\
\left({ }^{\circ}\right)\end{array}$ \\
\hline rs & 0.66 & 0.16 & 173.3 & 0.71 & 0.42 & 30.0 & rs & 0.78 & 0.21 & 54.7 & 0.93 & 0.57 & 134. \\
\hline
\end{tabular}

NGC 3976

$\mathrm{SAB}(\mathrm{rs}) \underline{\mathrm{b} c}$
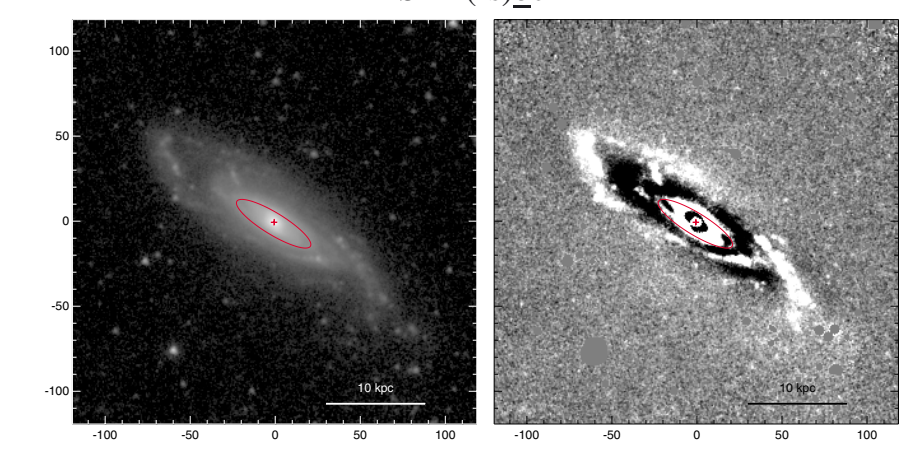

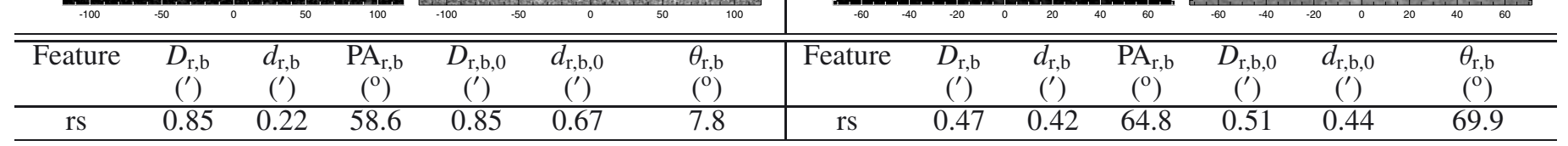

NGC 3953

SB(r) $\underline{b c}$

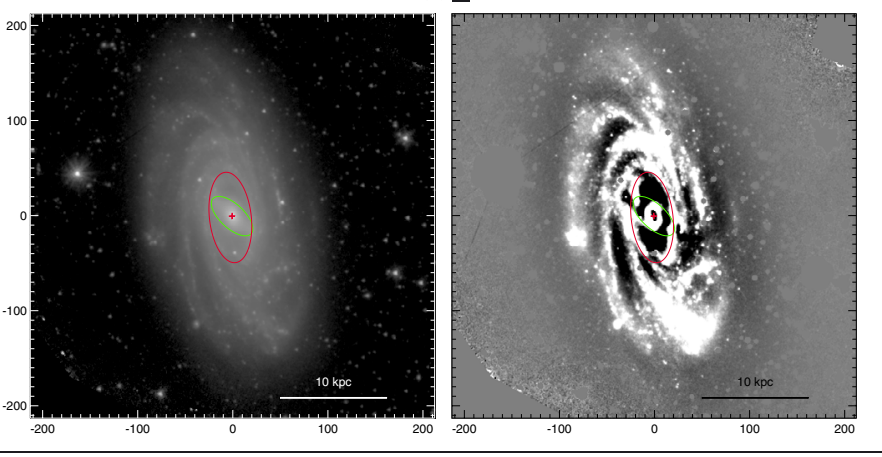

NGC 3956

$\mathrm{SAB}(\mathrm{rs}) \mathrm{cd}$

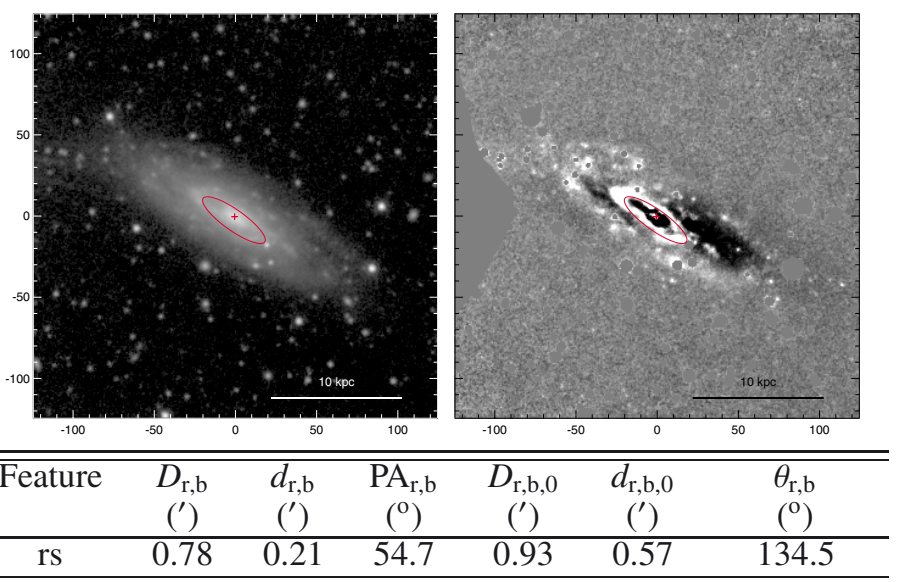

NGC 3982

$\mathrm{SA}(\mathrm{rs}) \mathrm{b}$

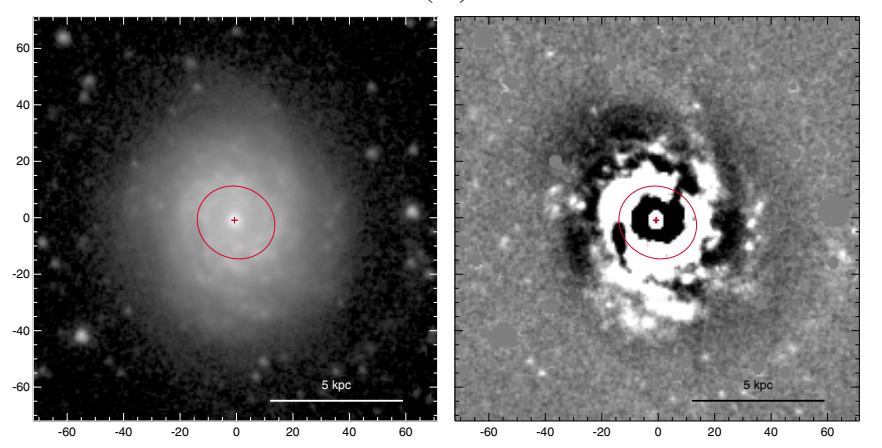

rs 


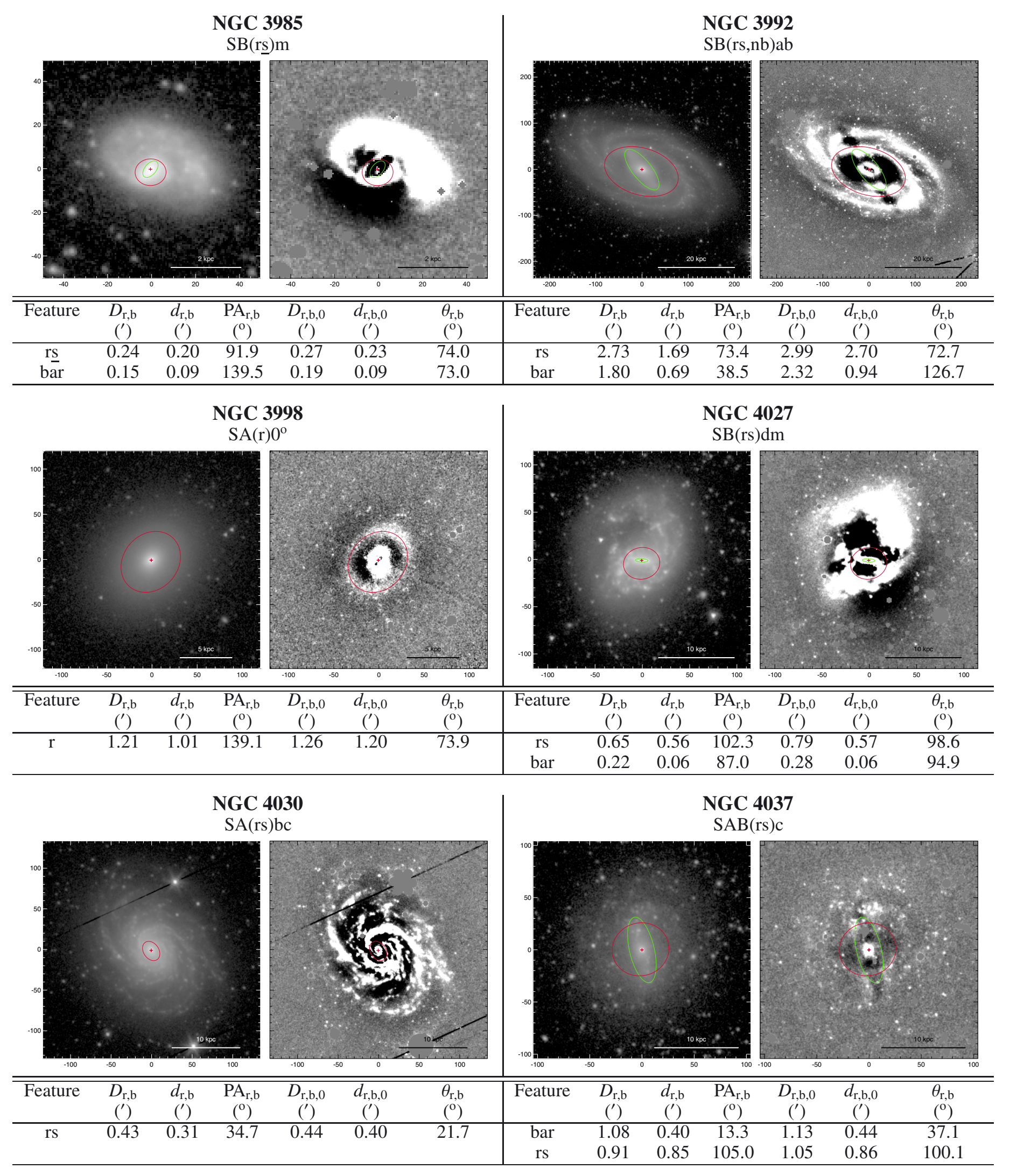


NGC 4041

$\mathrm{SAB}(\mathrm{rs}) \mathrm{c}$

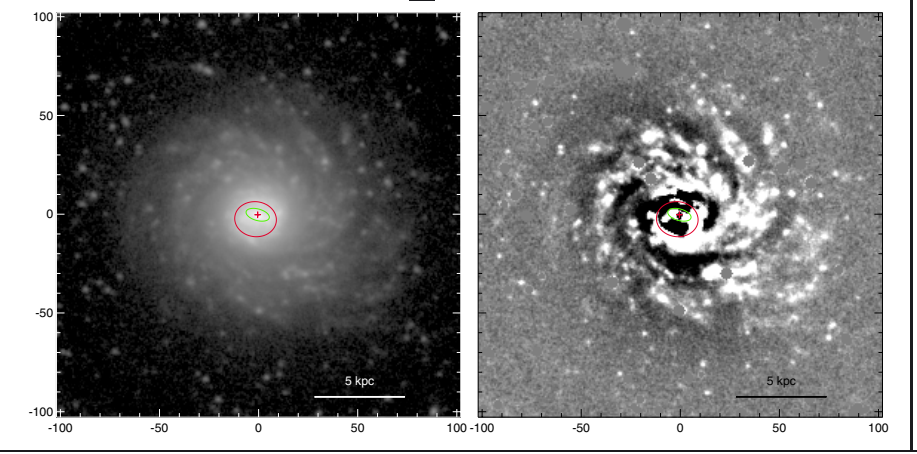

\begin{tabular}{ccccccc|ccccccc}
\hline \hline Feature & $\begin{array}{c}D_{\mathrm{r}, \mathrm{b}} \\
\left({ }^{\prime}\right)\end{array}$ & $\begin{array}{c}d_{\mathrm{r}, \mathrm{b}}\left({ }^{\prime}\right) \\
\left(\mathrm{PA}_{\mathrm{r}, \mathrm{b}}\right.\end{array}$ & $\begin{array}{c}D_{\mathrm{r}, \mathrm{b}, 0} \\
\left({ }^{\prime}\right)\end{array}$ & $\begin{array}{c}d_{\mathrm{r}, \mathrm{b}, 0} \\
\left({ }^{\prime}\right)\end{array}$ & $\begin{array}{c}\theta_{\mathrm{r}, \mathrm{b}} \\
\left({ }^{(}\right)\end{array}$ & Feature & $\begin{array}{c}D_{\mathrm{r}, \mathrm{b}} \\
\left({ }^{\prime}\right)\end{array}$ & $\begin{array}{c}d_{\mathrm{r}, \mathrm{b}} \\
\left({ }^{\prime}\right)\end{array}$ & $\begin{array}{c}\mathrm{PA}_{\mathrm{r}, \mathrm{b}} \\
\left({ }^{\circ}\right)\end{array}$ & $\begin{array}{c}D_{\mathrm{r}, \mathrm{b}, 0} \\
\left({ }^{\prime}\right)\end{array}$ & $\begin{array}{c}d_{\mathrm{r}, \mathrm{b}, 0} \\
\left({ }^{\prime}\right)\end{array}$ & $\begin{array}{c}\theta_{\mathrm{r}, \mathrm{b}} \\
\left({ }^{\circ}\right)\end{array}$ \\
\hline $\mathrm{rs}$ & 0.35 & 0.30 & 83.6 & 0.37 & 0.31 & 55.9 & $\mathrm{R}_{1}^{\prime} \mathrm{L}$ & 1.91 & 0.95 & 89.1 & 1.91 & 1.43 & 3.2 \\
bar & 0.20 & 0.10 & 74.5 & 0.21 & 0.10 & 37.0 & $\mathrm{rs}$ & 0.65 & 0.48 & 85.2 & 0.73 & 0.65 & 96.8 \\
& & & & & & & bar & 0.54 & 0.33 & 17.9 & 0.80 & 0.34 & 100.1 \\
\hline
\end{tabular}
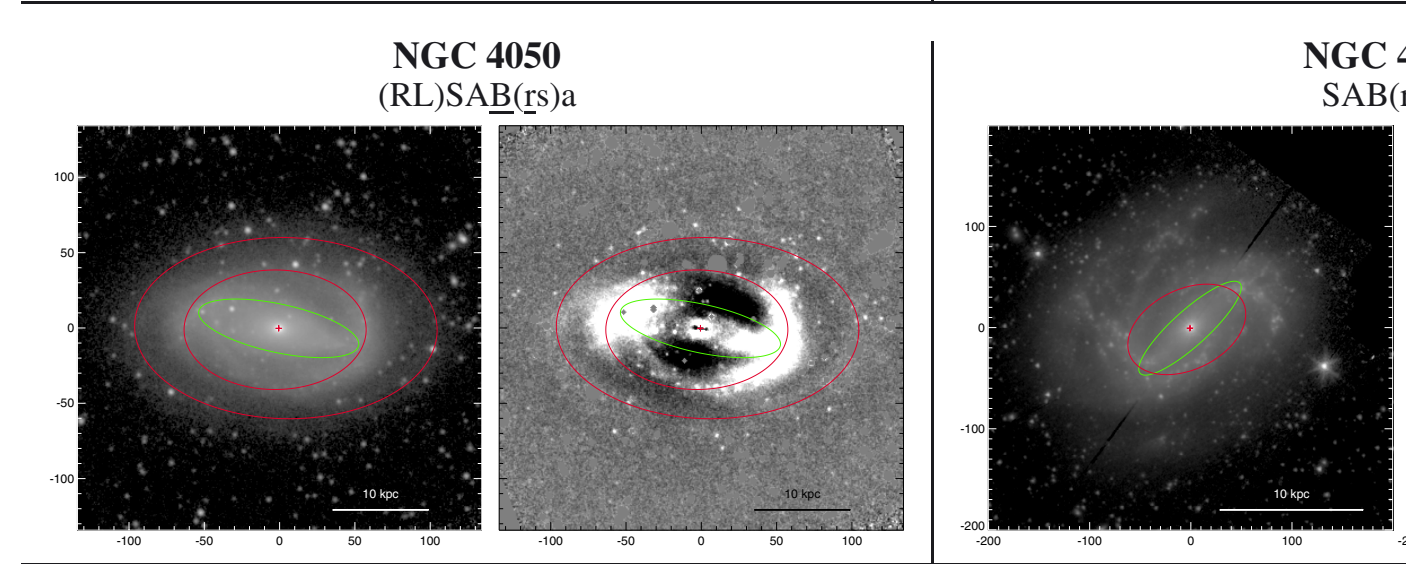

NGC 4051

$\mathrm{SAB}(\mathrm{rs}) \mathrm{b}$

\begin{tabular}{ccccccc|ccccccc}
\hline \hline Feature & $\begin{array}{c}D_{\mathrm{r}, \mathrm{b}} \\
\left({ }^{\prime}\right)\end{array}$ & $\begin{array}{c}d_{\mathrm{r}, \mathrm{b}} \\
\left({ }^{\prime}\right)\end{array}$ & $\begin{array}{c}\mathrm{PA}_{\mathrm{r}, \mathrm{b}} \\
\left({ }^{\circ}\right)\end{array}$ & $\begin{array}{c}D_{\mathrm{r}, \mathrm{b}, 0} \\
\left({ }^{\prime}\right)\end{array}$ & $\begin{array}{c}d_{\mathrm{r}, \mathrm{b}, 0} \\
\left({ }^{\prime}\right)\end{array}$ & $\begin{array}{c}\theta_{\mathrm{r}, \mathrm{b}} \\
\left({ }^{\circ}\right)\end{array}$ & Feature & $\begin{array}{c}D_{\mathrm{r}, \mathrm{b}} \\
\left({ }^{\prime}\right)\end{array}$ & $\begin{array}{c}d_{\mathrm{r}, \mathrm{b}} \\
\left({ }^{\prime}\right)\end{array}$ & $\begin{array}{c}\mathrm{PA}_{\mathrm{r}, \mathrm{b}} \\
\left({ }^{\circ}\right)\end{array}$ & $\begin{array}{c}D_{\mathrm{r}, \mathrm{b}, 0} \\
\left({ }^{\prime}\right)\end{array}$ & $\begin{array}{c}d_{\mathrm{r}, \mathrm{b}, 0} \\
\left({ }^{\prime}\right)\end{array}$ & $\begin{array}{c}\theta_{\mathrm{r}, \mathrm{b}} \\
\left({ }^{\circ}\right)\end{array}$ \\
\hline $\mathrm{RL}$ & 3.36 & 2.01 & 88.8 & 3.38 & 3.03 & 165.9 & bar & 2.22 & 0.59 & 132.1 & 2.23 & 0.68 & 11.6 \\
$\underline{\mathrm{rs}}$ & 2.02 & 1.33 & 90.1 & 2.04 & 1.99 & 142.6 & $\mathrm{rs}$ & 2.07 & 1.32 & 115.9 & 2.07 & 1.53 & 170.3 \\
$\mathrm{bar}$ & 1.81 & 0.54 & 77.8 & 1.89 & 0.78 & 157.3 & & & & & & & \\
\hline
\end{tabular}

NGC 4062

$\mathrm{SA}(\mathrm{rs}) \mathrm{b}$

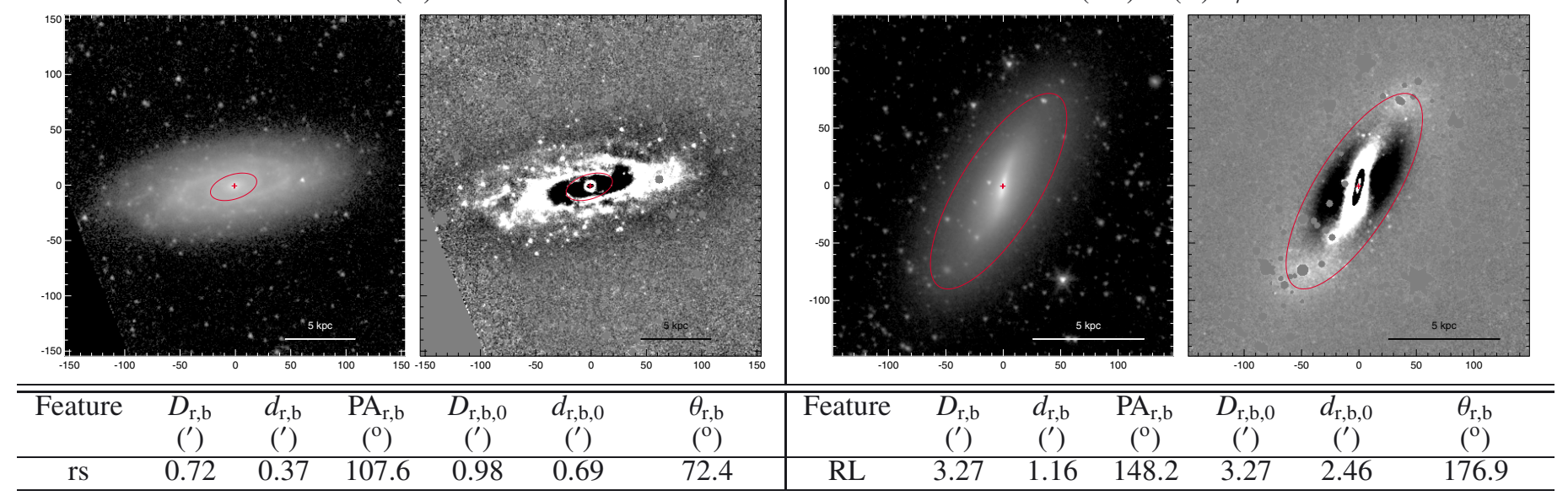

NGC 4045

$\left(\mathrm{R}_{1}^{\prime} \mathrm{L}\right) \mathrm{SAB}(\underline{\mathrm{rs}}, \mathrm{nl}) \mathrm{ab}$

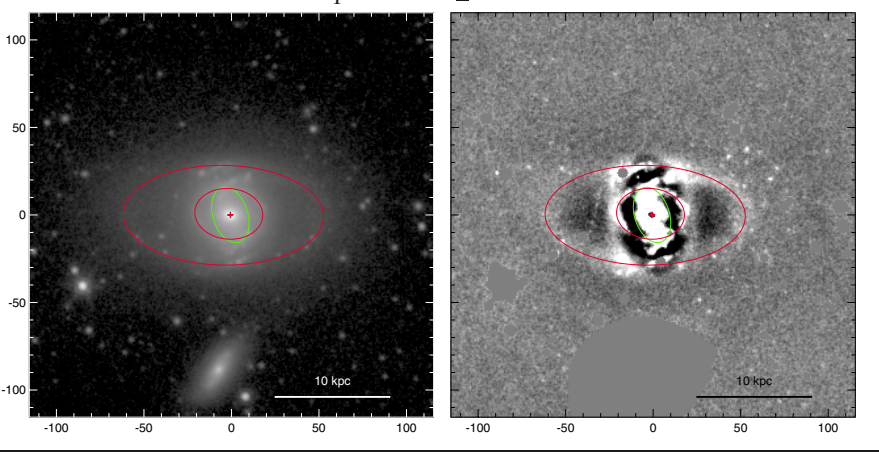

.




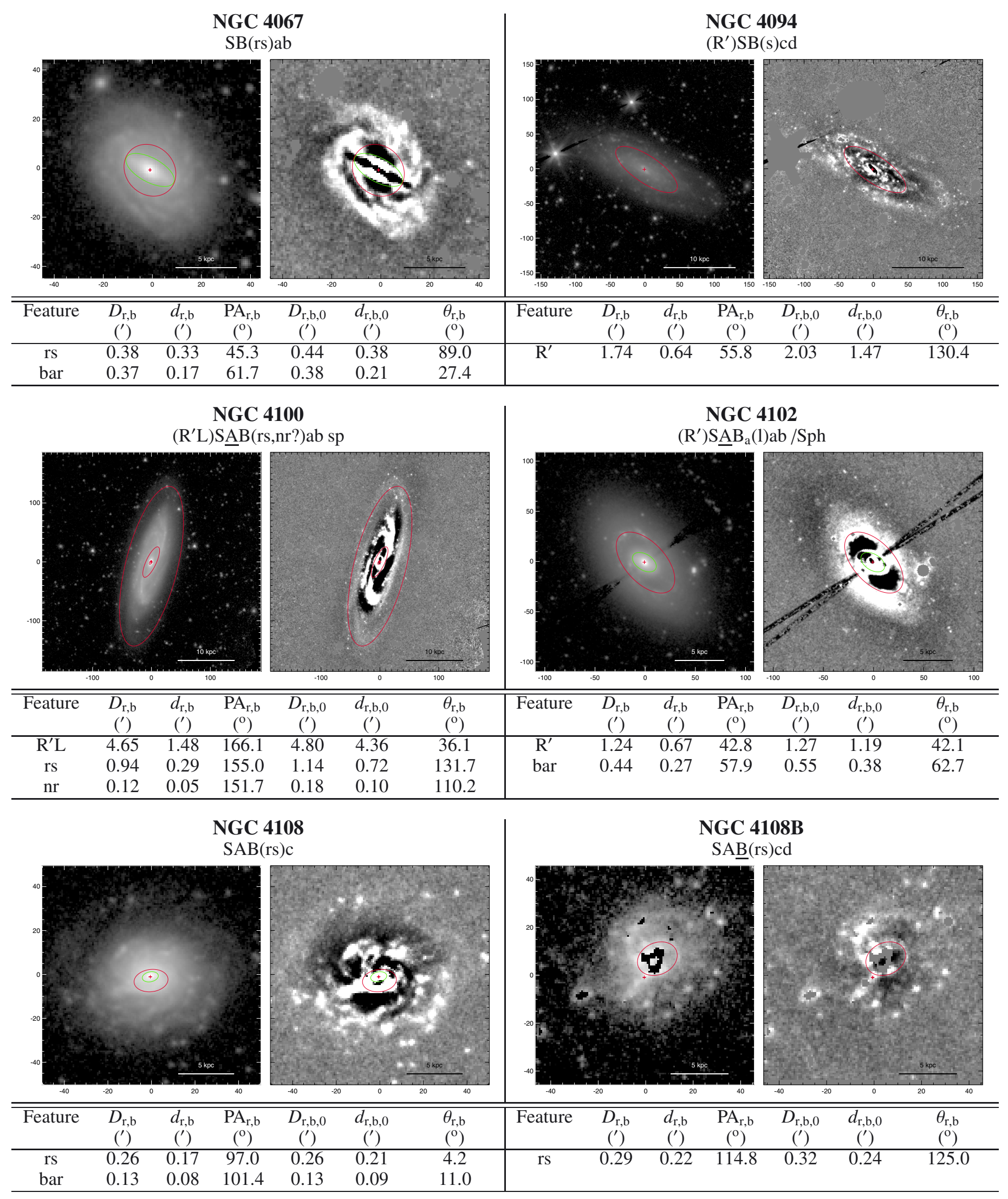


S. Comerón et al.: ARRAKIS

NGC 4116

$\mathrm{SB}(\mathrm{rs}) \mathrm{d}$

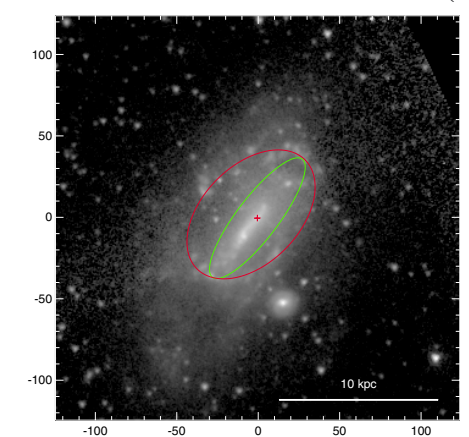

NGC 4120

$\mathrm{SB}(\mathrm{rs}:) \mathrm{d}$

\begin{tabular}{|c|c|c|c|c|c|c|c|c|c|c|c|c|c|}
\hline Feature & $\begin{array}{c}D_{\mathrm{r}, \mathrm{b}} \\
\left({ }^{\prime}\right)\end{array}$ & $\begin{array}{c}d_{\mathrm{r}, \mathrm{b}} \\
\left(^{\prime}\right)\end{array}$ & $\begin{array}{c}\mathrm{PA}_{\mathrm{r}, \mathrm{b}} \\
\left({ }^{\mathrm{o}}\right)\end{array}$ & $\begin{array}{c}D_{\mathrm{r}, \mathrm{b}, 0} \\
\left({ }^{\prime}\right)\end{array}$ & $\begin{array}{c}d_{\mathrm{r}, \mathrm{b}, 0} \\
\left({ }^{\prime}\right)\end{array}$ & $\begin{array}{l}\theta_{\mathrm{r}, \mathrm{b}} \\
\left({ }^{\circ}\right)\end{array}$ & Feature & $\begin{array}{c}D_{\mathrm{r}, \mathrm{b}} \\
\left({ }^{\prime}\right)\end{array}$ & $\begin{array}{c}d_{\mathrm{r}, \mathrm{b}} \\
\left({ }^{\prime}\right)\end{array}$ & $\begin{array}{c}\mathrm{PA}_{\mathrm{r}, \mathrm{b}} \\
\left({ }^{\circ}\right)\end{array}$ & $\begin{array}{c}D_{\mathrm{r}, \mathrm{b}, 0} \\
\left(^{\prime}\right)\end{array}$ & $\begin{array}{c}d_{\mathrm{r}, \mathrm{b}, 0} \\
\left(^{\prime}\right)\end{array}$ & $\begin{array}{l}\theta_{\mathrm{r}, \mathrm{b}} \\
\left({ }^{\circ}\right)\end{array}$ \\
\hline rs & 1.58 & 1.00 & 135.4 & 1.88 & 1.51 & 111.8 & rs & 0.42 & 0.20 & 168.9 & 0.68 & 0.42 & 88.6 \\
\hline bar & 1.52 & 0.43 & 142.7 & 1.53 & 0.76 & 175.2 & & & & & & & \\
\hline
\end{tabular}

NGC 4123

$\mathrm{SB}_{\mathrm{x}}(\mathrm{rs}) \mathrm{b}$
NGC 4136

$\mathrm{SAB}(\mathrm{rs}) \mathrm{bc}$
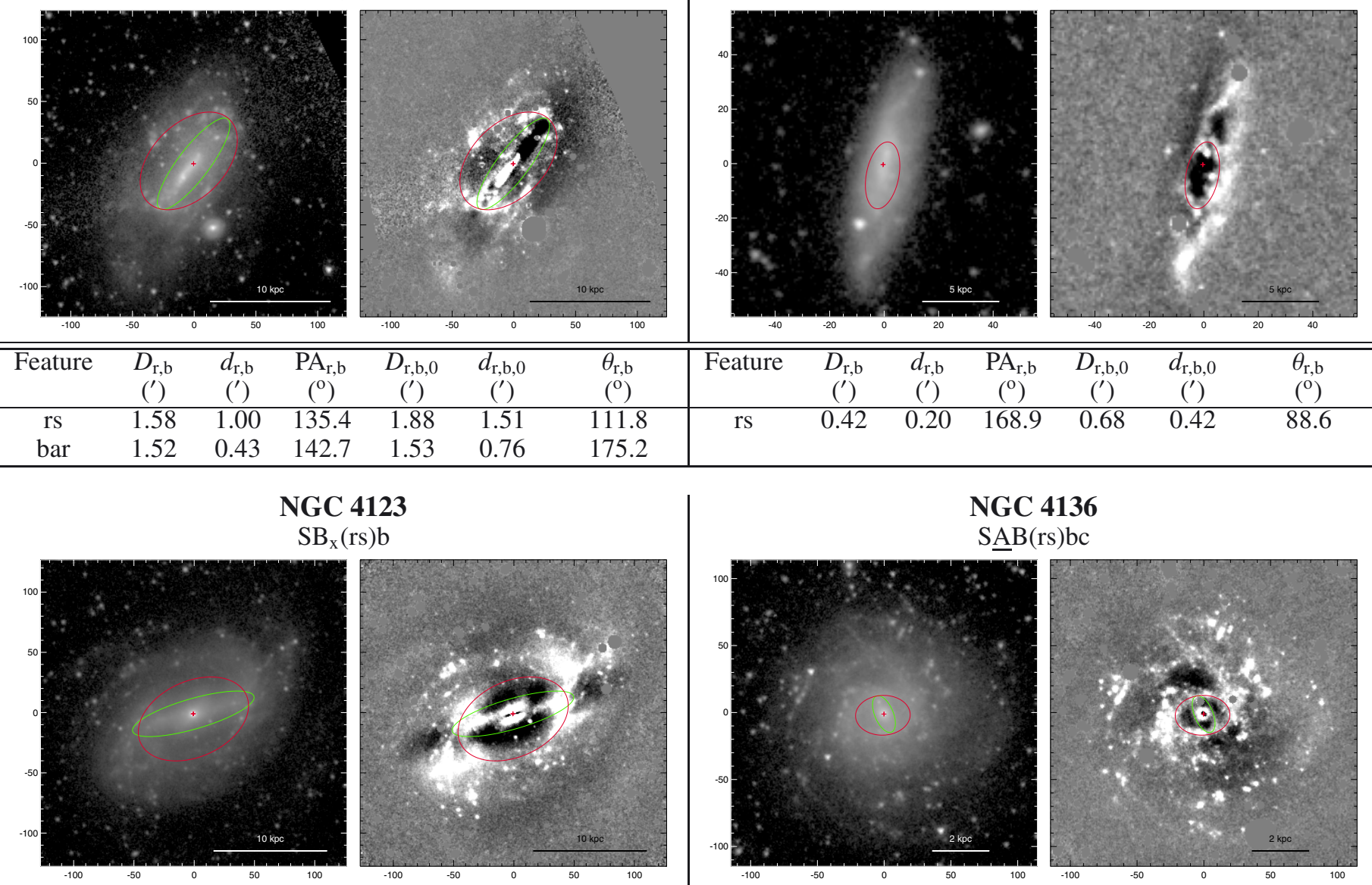

\begin{tabular}{|c|c|c|c|c|c|c|c|c|c|c|c|c|c|}
\hline Feature & $\begin{array}{c}D_{\mathrm{r}, \mathrm{b}} \\
\left({ }^{\prime}\right)\end{array}$ & $\begin{array}{c}d_{\mathrm{r}, \mathrm{b}} \\
(')\end{array}$ & $\begin{array}{c}\mathrm{PA}_{\mathrm{r}, \mathrm{b}} \\
\left({ }^{\circ}\right)\end{array}$ & $\begin{array}{c}D_{\mathrm{r}, \mathrm{b}, 0} \\
\left(^{\prime}\right)\end{array}$ & $\begin{array}{c}d_{\mathrm{r}, \mathrm{b}, 0} \\
\left(^{\prime}\right)\end{array}$ & $\begin{array}{c}\theta_{\mathrm{r}, \mathrm{b}} \\
\left({ }^{\circ}\right)\end{array}$ & Feature & $\begin{array}{c}D_{\mathrm{r}, \mathrm{b}} \\
\left({ }^{\prime}\right)\end{array}$ & $\begin{array}{c}d_{\mathrm{r}, \mathrm{b}} \\
\left({ }^{\prime}\right)\end{array}$ & $\begin{array}{c}\mathrm{PA}_{\mathrm{r}, \mathrm{b}} \\
\left({ }^{\circ}\right)\end{array}$ & $\begin{array}{c}D_{\mathrm{r}, \mathrm{b}, 0} \\
\left(^{\prime}\right)\end{array}$ & $\begin{array}{c}d_{\mathrm{r}, \mathrm{b}, 0} \\
\left(^{\prime}\right)\end{array}$ & $\begin{array}{c}\theta_{\mathrm{r}, \mathrm{b}} \\
\left({ }^{\circ}\right)\end{array}$ \\
\hline bar & 1.75 & 0.42 & 106.1 & 1.84 & 0.59 & 155.0 & rs & 0.68 & 0.50 & 92.4 & 0.74 & 0.52 & 57.9 \\
\hline rs & 1.61 & 1.05 & 114.4 & 1.68 & 1.47 & 141.1 & bar & 0.49 & 0.23 & 22.9 & 0.50 & 0.25 & 154.3 \\
\hline
\end{tabular}

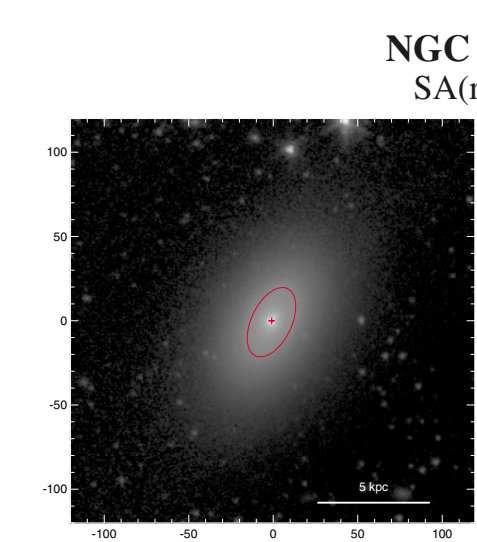

GC 4138

$\mathrm{SA}(\mathrm{r}) 0^{+}$

\begin{tabular}{ccccccc|ccccccc}
\hline \hline Feature & $\begin{array}{c}D_{\mathrm{r}, \mathrm{b}} \\
\left({ }^{\prime}\right)\end{array}$ & $\begin{array}{c}d_{\mathrm{r}, \mathrm{b}} \\
\left({ }^{\prime}\right)\end{array}$ & $\begin{array}{c}\mathrm{PA}_{\mathrm{r}, \mathrm{b}} \\
\left({ }^{\circ}\right)\end{array}$ & $\begin{array}{c}D_{\mathrm{r}, \mathrm{b}, 0} \\
\left({ }^{\prime}\right)\end{array}$ & $\begin{array}{c}d_{\mathrm{r}, \mathrm{b}, 0} \\
\left({ }^{\prime}\right)\end{array}$ & $\begin{array}{c}\theta_{\mathrm{r}, \mathrm{b}} \\
\left({ }^{(}\right)\end{array}$ & Feature & $\begin{array}{c}D_{\mathrm{r}, \mathrm{b}} \\
\left({ }^{\prime}\right)\end{array}$ & $\begin{array}{c}d_{\mathrm{r}, \mathrm{b}} \\
\left({ }^{\prime}\right)\end{array}$ & $\begin{array}{c}\mathrm{PA}_{\mathrm{r}, \mathrm{b}} \\
\left({ }^{\circ}\right)\end{array}$ & $\begin{array}{c}D_{\mathrm{r}, \mathrm{b}, 0} \\
\left({ }^{\prime}\right)\end{array}$ & $\begin{array}{c}d_{\mathrm{r}, \mathrm{b}, 0} \\
\left({ }^{\prime}\right)\end{array}$ & $\begin{array}{c}\theta_{\mathrm{r}, \mathrm{b}} \\
\left({ }^{\circ}\right)\end{array}$ \\
\hline $\mathrm{r}$ & 0.74 & 0.40 & 154.3 & 0.76 & 0.68 & 35.8 & $\mathrm{rs}$ & 0.38 & 0.28 & 57.2 & 0.41 & 0.34 & 125.9 \\
& & & & & & & $\mathrm{bar}$ & 0.24 & 0.11 & 86.2 & 0.25 & 0.15 & 17.9 \\
\hline
\end{tabular}


NGC 4145

$\mathrm{SAB}(\mathrm{rs}) \mathrm{d}$
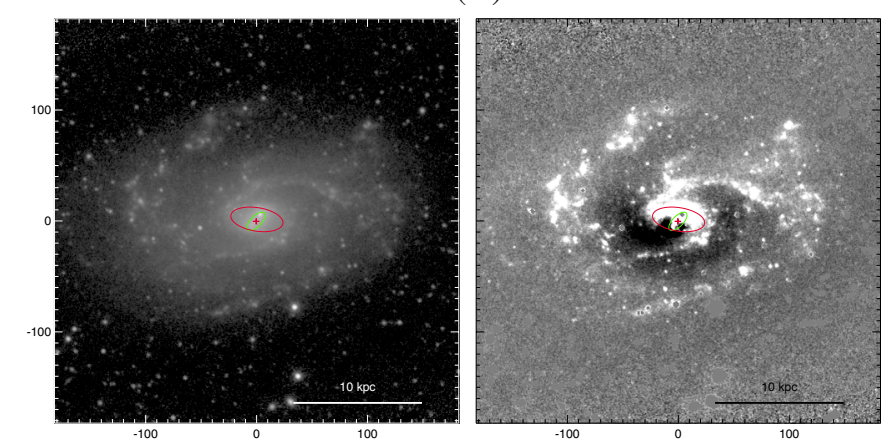

\begin{tabular}{ccccccc|ccccccc}
\hline \hline Feature & $\begin{array}{c}D_{\mathrm{r}, \mathrm{b}} \\
\left({ }^{\prime}\right)\end{array}$ & $\begin{array}{c}d_{\mathrm{r}, \mathrm{b}} \\
\left({ }^{\prime}\right)\end{array}$ & $\begin{array}{c}\mathrm{PA}_{\mathrm{r}, \mathrm{b}} \\
\left({ }^{\prime}\right)\end{array}$ & $\begin{array}{c}D_{\mathrm{r}, \mathrm{b}, 0} \\
\left({ }^{\prime}\right)\end{array}$ & $\begin{array}{c}d_{\mathrm{r}, \mathrm{b}, 0} \\
\left({ }^{\prime}\right)\end{array}$ & $\begin{array}{c}\theta_{\mathrm{r}, \mathrm{b}} \\
\left({ }^{\circ}\right)\end{array}$ & Feature & $\begin{array}{c}D_{\mathrm{r}, \mathrm{b}} \\
\left({ }^{\prime}\right)\end{array}$ & $\begin{array}{c}d_{\mathrm{r}, \mathrm{b}} \\
\left({ }^{\prime}\right)\end{array}$ & $\begin{array}{c}\mathrm{PA}_{\mathrm{r}, \mathrm{b}} \\
\left({ }^{\circ}\right)\end{array}$ & $\begin{array}{c}D_{\mathrm{r}, \mathrm{b}, 0} \\
()^{2}\end{array}$ & $\begin{array}{c}d_{\mathrm{r}, \mathrm{b}, 0} \\
\left({ }^{\prime}\right)\end{array}$ & $\begin{array}{c}\theta_{\mathrm{r}, \mathrm{b}} \\
\left({ }^{\circ}\right)\end{array}$ \\
\hline $\mathrm{rs}$ & 0.79 & 0.35 & 81.1 & 0.88 & 0.55 & 141.6 & $\mathrm{rs}$ & 0.32 & 0.27 & 93.5 & 0.38 & 0.30 & 107.1 \\
$\mathrm{bar}$ & 0.36 & 0.16 & 135.6 & 0.49 & 0.20 & 60.9 & $\mathrm{bar}$ & 0.17 & 0.11 & 40.9 & 0.22 & 0.11 & 94.3 \\
\hline
\end{tabular}

NGC 4158

$(\mathrm{RL}) \mathrm{SAB}_{\mathrm{a}}(\mathrm{r}) \mathrm{a}$
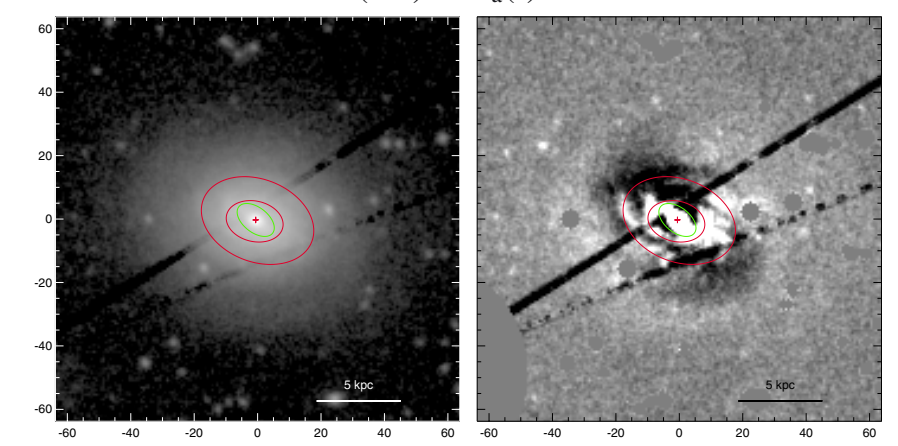

\begin{tabular}{|c|c|c|c|c|c|c|c|c|c|c|c|c|c|}
\hline Feature & $\begin{array}{c}D_{\mathrm{r}, \mathrm{b}} \\
\left({ }^{\prime}\right)\end{array}$ & $\begin{array}{c}d_{\mathrm{r}, \mathrm{b}} \\
(')\end{array}$ & $\begin{array}{c}\mathrm{PA}_{\mathrm{r}, \mathrm{b}} \\
\left({ }^{\mathrm{O}}\right)\end{array}$ & $\begin{array}{c}D_{\mathrm{r}, \mathrm{b}, 0} \\
\left(^{\prime}\right)\end{array}$ & $\begin{array}{c}d_{\mathrm{r}, \mathrm{b}, 0} \\
\left({ }^{\prime}\right)\end{array}$ & $\begin{array}{l}\theta_{\mathrm{r}, \mathrm{b}} \\
\left({ }^{\circ}\right)\end{array}$ & Feature & $\begin{array}{c}D_{\mathrm{r}, \mathrm{b}} \\
\left({ }^{\prime}\right)\end{array}$ & $\begin{array}{c}d_{\mathrm{r}, \mathrm{b}} \\
\left({ }^{\prime}\right)\end{array}$ & $\begin{array}{c}\mathrm{PA}_{\mathrm{r}, \mathrm{b}} \\
\left({ }^{\circ}\right)\end{array}$ & $\begin{array}{c}D_{\mathrm{r}, \mathrm{b}, 0} \\
\left({ }^{\prime}\right)\end{array}$ & $\begin{array}{c}d_{\mathrm{r}, \mathrm{b}, 0} \\
\left(^{\prime}\right)\end{array}$ & $\begin{array}{c}\theta_{\mathrm{r}, \mathrm{b}} \\
\left({ }^{\circ}\right)\end{array}$ \\
\hline$\overline{R L}$ & 0.62 & 0.44 & 68.5 & 0.62 & 0.57 & 5.0 & $\mathrm{r}$ & 0.37 & 0.22 & 166.8 & 0.37 & 0.34 & 161.7 \\
\hline $\mathrm{r}$ & 0.30 & 0.21 & 77.4 & 0.31 & 0.27 & 32.4 & & & & & & & \\
\hline bar & 0.23 & 0.13 & 52.0 & 0.24 & 0.17 & 151.3 & & & & & & & \\
\hline
\end{tabular}
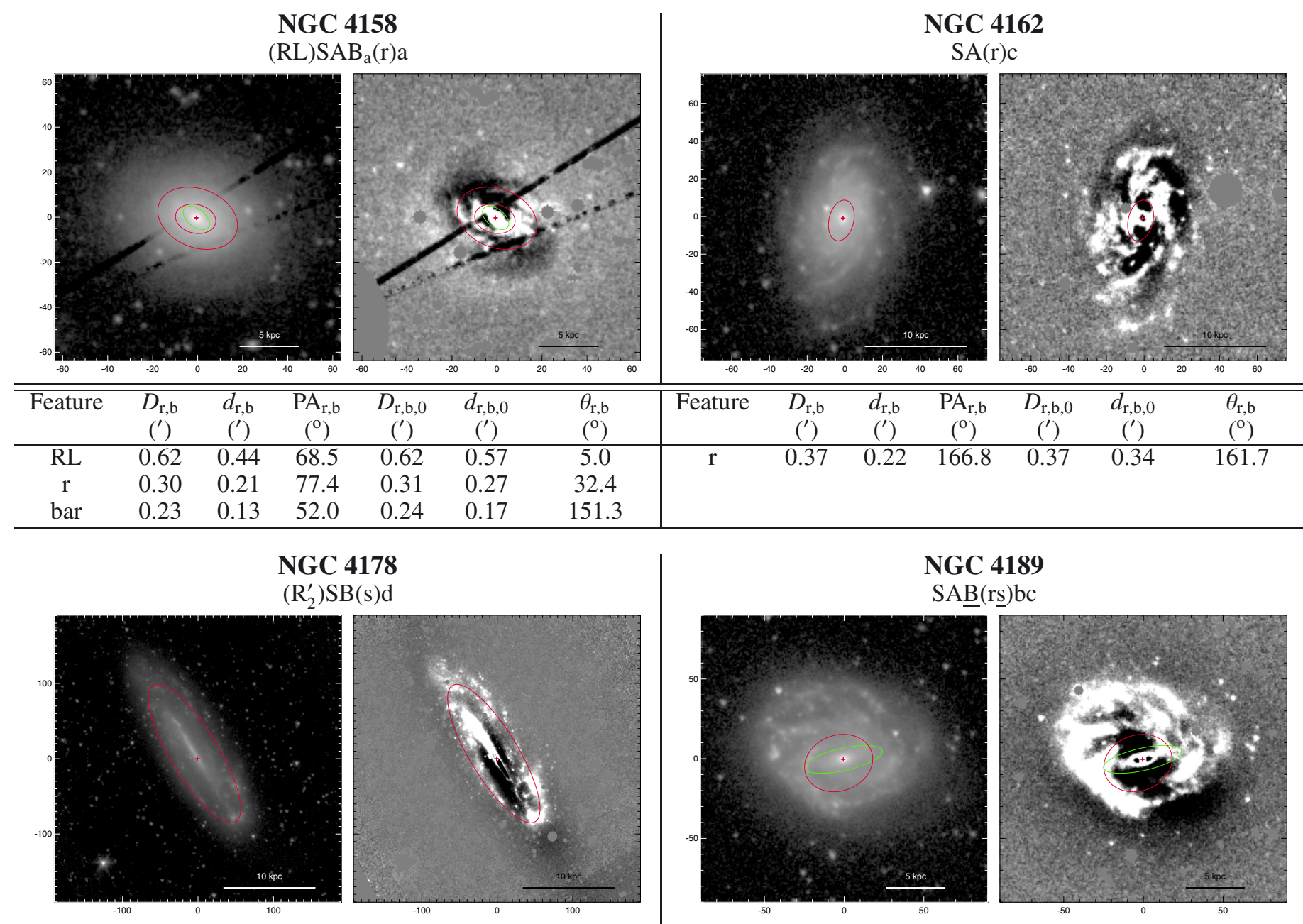

SAB 4152

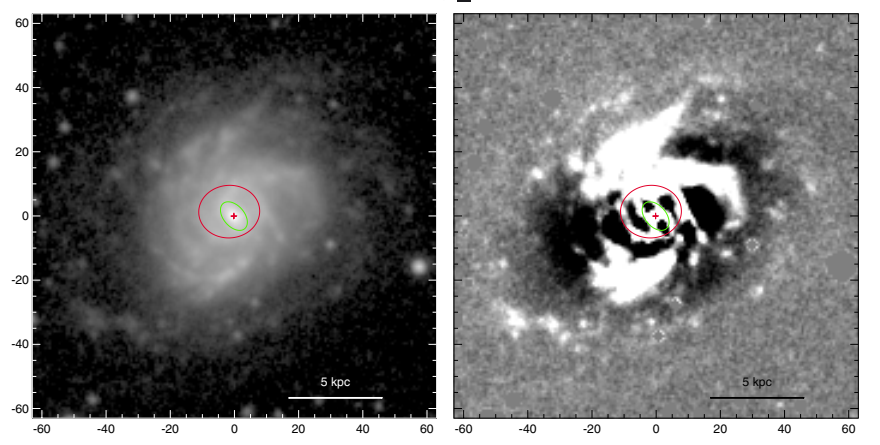

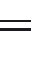


NGC 4192

$\left(\mathrm{R}^{\prime}\right) \mathrm{SAB}_{\mathrm{x}}(\mathrm{rs}, \mathrm{nd}) \mathrm{a}$
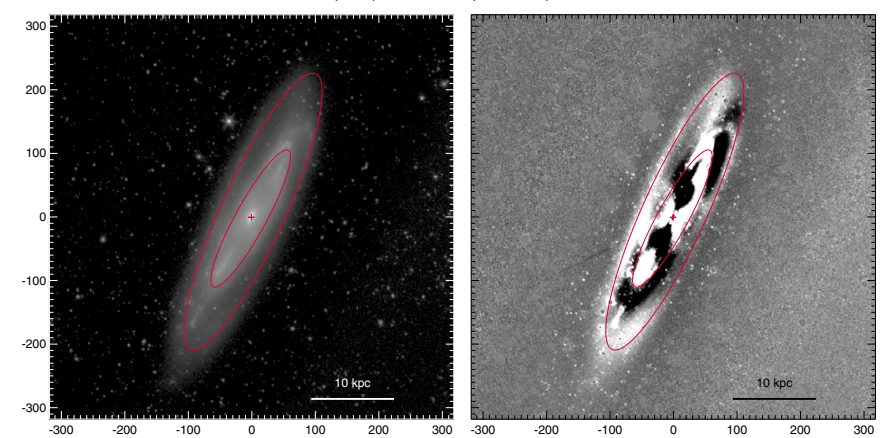

NGC 4193

(RL)SAB(rs)a

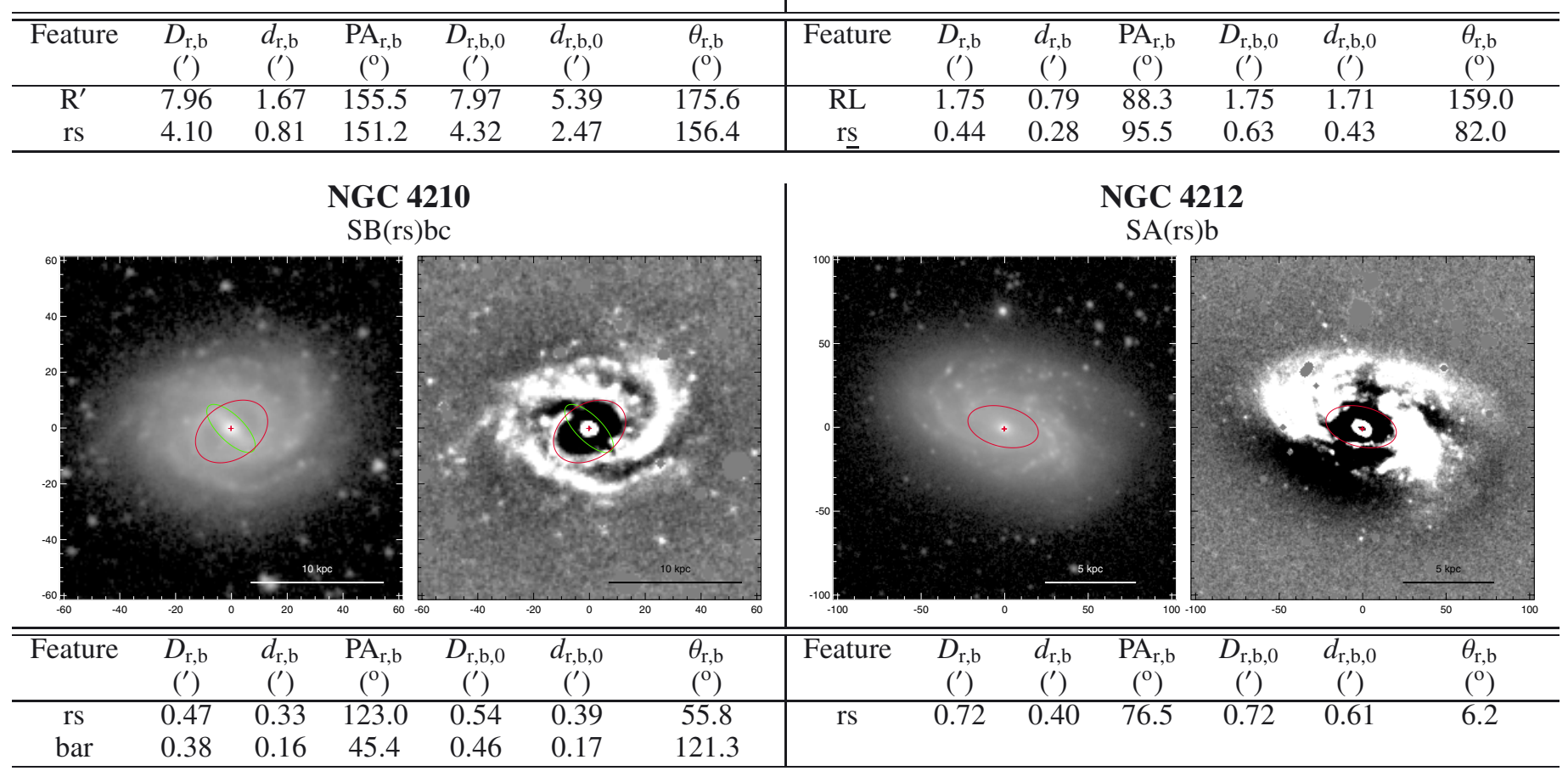

NGC 4216

$\left(\mathrm{R}^{\prime}\right) \mathrm{SAB}_{\text {box }, \mathrm{a}}(\mathrm{r}, \mathrm{nd}) \mathrm{a}$

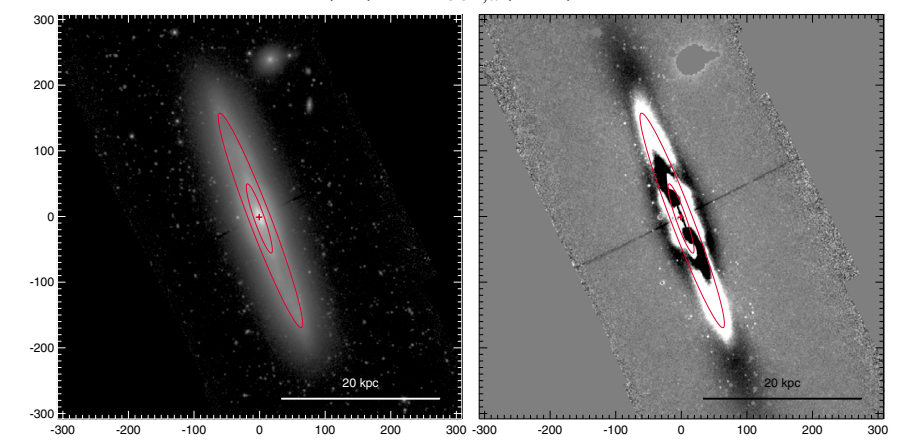

NGC 4220

(L) $\mathrm{SAB}(\mathrm{r}) 0^{+}$

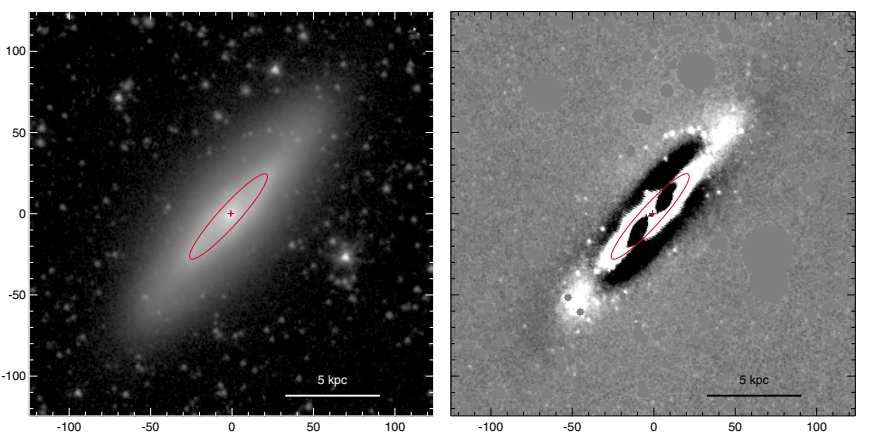

\begin{tabular}{|c|c|c|c|c|c|c|c|c|c|c|c|c|c|}
\hline Feature & $\begin{array}{c}D_{\mathrm{r}, \mathrm{b}} \\
\left(^{\prime}\right)\end{array}$ & $\begin{array}{c}d_{\mathrm{r}, \mathrm{b}} \\
\left(^{\prime}\right)\end{array}$ & $\begin{array}{c}\mathrm{PA}_{\mathrm{r}, \mathrm{b}} \\
\left({ }^{\circ}\right)\end{array}$ & $\begin{array}{c}D_{\mathrm{r}, \mathrm{b}, 0} \\
\left(^{\prime}\right)\end{array}$ & $\begin{array}{c}d_{\mathrm{r}, \mathrm{b}, 0} \\
\left(^{\prime}\right)\end{array}$ & $\begin{array}{l}\theta_{\mathrm{r}, \mathrm{b}} \\
\left({ }^{\circ}\right)\end{array}$ & Feature & $\begin{array}{c}D_{\mathrm{r}, \mathrm{b}} \\
\left({ }^{\prime}\right)\end{array}$ & $\begin{array}{c}d_{\mathrm{r}, \mathrm{b}} \\
\left({ }^{\prime}\right)\end{array}$ & $\begin{array}{c}\mathrm{PA}_{\mathrm{r}, \mathrm{b}} \\
\left({ }^{\circ}\right)\end{array}$ & $\begin{array}{c}D_{\mathrm{r}, \mathrm{b}, 0} \\
\left(^{\prime}\right)\end{array}$ & $\begin{array}{c}d_{\mathrm{r}, \mathrm{b}, 0} \\
\left(^{\prime}\right)\end{array}$ & $\begin{array}{c}\theta_{\mathrm{r}, \mathrm{b}} \\
\left({ }^{\circ}\right)\end{array}$ \\
\hline$\overline{\mathrm{R}^{\prime}}$ & 5.84 & 0.55 & 20.9 & 5.85 & 3.02 & 3.9 & $\bar{r}$ & 1.17 & 0.21 & 137.8 & 1.17 & 0.70 & 172.7 \\
\hline$r$ & 1.86 & 0.26 & 19.2 & 1.89 & 1.42 & 165.5 & & & & & & & \\
\hline
\end{tabular}




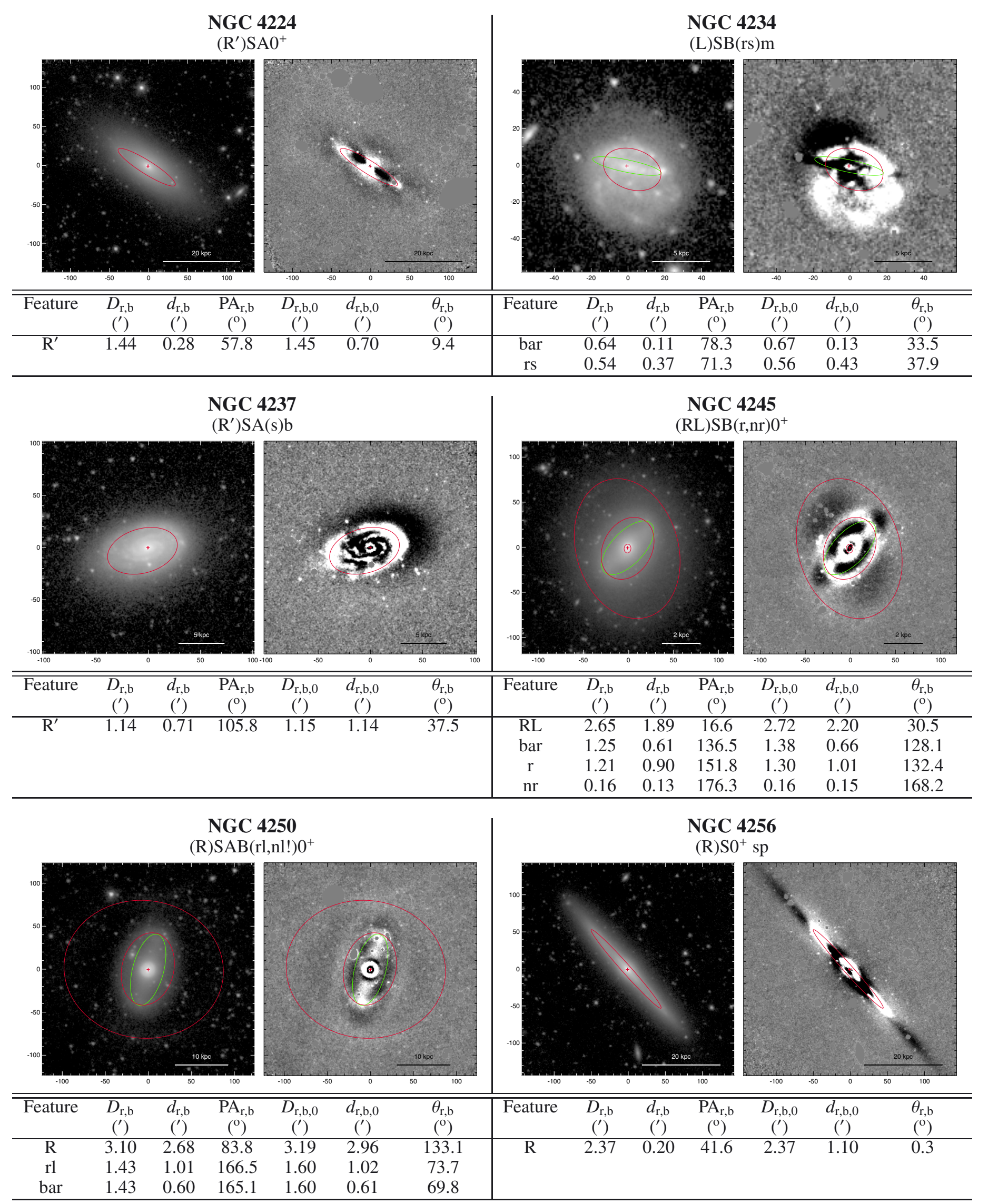


NGC 4258

(R)SAB(rs,nb or $\left.n d_{\mathrm{sp}}\right) \mathrm{a}$
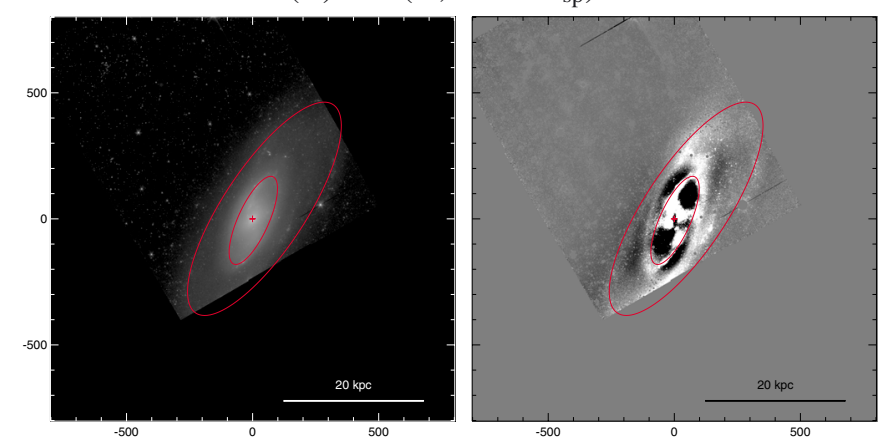

\begin{tabular}{|c|c|c|c|c|c|c|c|c|c|c|c|c|c|}
\hline Feature & $\begin{array}{c}D_{\mathrm{r}, \mathrm{b}} \\
\left({ }^{\prime}\right)\end{array}$ & $\begin{array}{c}d_{\mathrm{r}, \mathrm{b}} \\
\left(^{\prime}\right)\end{array}$ & $\begin{array}{c}\mathrm{PA}_{\mathrm{r}, \mathrm{b}} \\
\left({ }^{\mathrm{O}}\right)\end{array}$ & $\begin{array}{c}D_{\mathrm{r}, \mathrm{b}, 0} \\
\left(^{\prime}\right)\end{array}$ & $\begin{array}{c}d_{\mathrm{r}, \mathrm{b}, 0} \\
\left({ }^{\prime}\right)\end{array}$ & $\begin{array}{l}\theta_{\mathrm{r}, \mathrm{b}} \\
\left({ }^{\circ}\right)\end{array}$ & Feature & $\begin{array}{c}D_{\mathrm{r}, \mathrm{b}} \\
\left({ }^{\prime}\right)\end{array}$ & $\begin{array}{c}d_{\mathrm{r}, \mathrm{b}} \\
\left({ }^{\prime}\right)\end{array}$ & $\begin{array}{c}\mathrm{PA}_{\mathrm{r}, \mathrm{b}} \\
\left({ }^{\circ}\right)\end{array}$ & $\begin{array}{c}D_{\mathrm{r}, \mathrm{b}, 0} \\
\left(^{\prime}\right)\end{array}$ & $\begin{array}{c}d_{\mathrm{r}, \mathrm{b}, 0} \\
{ }^{\prime}\left({ }^{\prime}\right)\end{array}$ & $\begin{array}{c}\theta_{\mathrm{r}, \mathrm{b}} \\
\left({ }^{\circ}\right)\end{array}$ \\
\hline $\mathrm{R}$ & 16.51 & 5.47 & 146.6 & 23.39 & 15.26 & 69.9 & r $\underline{s}$ & 1.12 & 0.62 & 57.9 & 1.61 & 1.12 & 89.4 \\
\hline rs & 6.33 & 2.03 & 155.7 & 10.90 & 4.66 & 66.2 & & & & & & & \\
\hline
\end{tabular}

\section{NGC 4268}

$\mathrm{SA}:(\underline{\mathrm{rs}}) 0^{+} \mathrm{sp}$

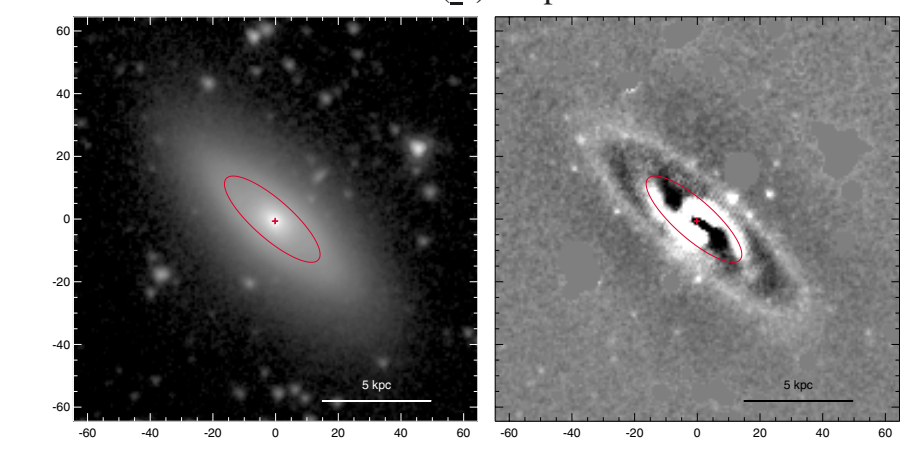

\begin{tabular}{|c|c|c|c|c|c|c|c|c|c|c|c|c|c|}
\hline Feature & $\begin{array}{c}D_{\mathrm{r}, \mathrm{b}} \\
\left(^{\prime}\right)\end{array}$ & $\begin{array}{c}d_{\mathrm{r}, \mathrm{b}} \\
\left(^{\prime}\right)\end{array}$ & $\begin{array}{c}\mathrm{PA}_{\mathrm{r}, \mathrm{b}} \\
\left({ }^{\mathrm{O}}\right)\end{array}$ & $\begin{array}{c}D_{\mathrm{r}, \mathrm{b}, 0} \\
\left({ }^{\prime}\right)\end{array}$ & $\begin{array}{c}d_{\mathrm{r}, \mathrm{b}, 0} \\
\left({ }^{\prime}\right)\end{array}$ & $\begin{array}{l}\theta_{\mathrm{r}, \mathrm{b}} \\
\left({ }^{\circ}\right)\end{array}$ & Feature & $\begin{array}{c}D_{\mathrm{r}, \mathrm{b}} \\
\left(^{\prime}\right)\end{array}$ & $\begin{array}{c}d_{\mathrm{r}, \mathrm{b}} \\
\left(^{\prime}\right)\end{array}$ & $\begin{array}{c}\mathrm{PA}_{\mathrm{r}, \mathrm{b}} \\
\left({ }^{\mathrm{o}}\right)\end{array}$ & $\begin{array}{c}D_{\mathrm{r}, \mathrm{b}, 0} \\
\left(^{\prime}\right)\end{array}$ & $\begin{array}{c}d_{\mathrm{r}, \mathrm{b}, 0} \\
\left(^{\prime}\right)\end{array}$ & $\begin{array}{l}\theta_{\mathrm{r}, \mathrm{b}} \\
\left({ }^{\circ}\right)\end{array}$ \\
\hline \multirow[t]{3}{*}{ rs } & 0.66 & 0.21 & 48.6 & 0.67 & 0.42 & 13.6 & $\overline{\mathrm{R}}$ & 5.92 & 1.50 & 100.8 & 5.93 & 4.41 & 177.3 \\
\hline & & & & & & & $r$ & 2.79 & 0.91 & 100.3 & 2.81 & 2.66 & 155.6 \\
\hline & & & & & & & $\mathrm{nr}$ & 0.26 & 0.08 & 102.7 & 0.26 & 0.24 & 29.5 \\
\hline
\end{tabular}

NGC 4286

$\mathrm{d}(\mathrm{RL}) \mathrm{SA}(1) 0^{\circ}, \mathrm{N} / \mathrm{Sph}$

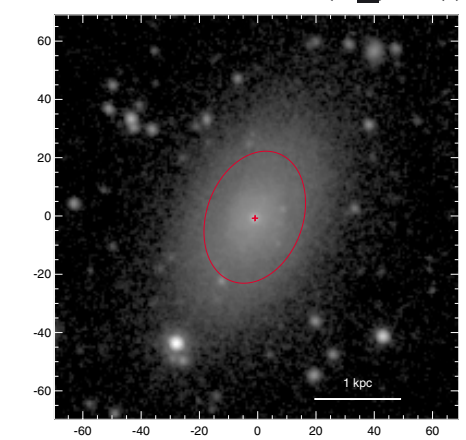

NGC 4260

$\mathrm{SA} \underline{\mathrm{B}}(\mathrm{rs}) \mathrm{a}$

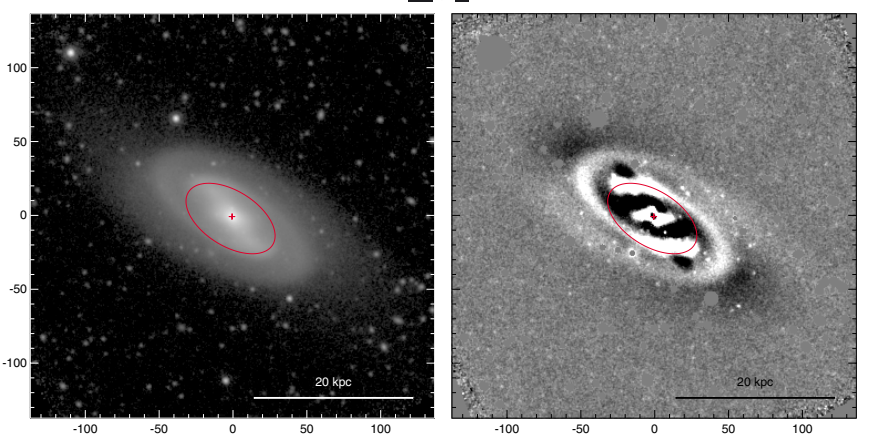

NGC 4274

(R)SB(r,nr)0/a

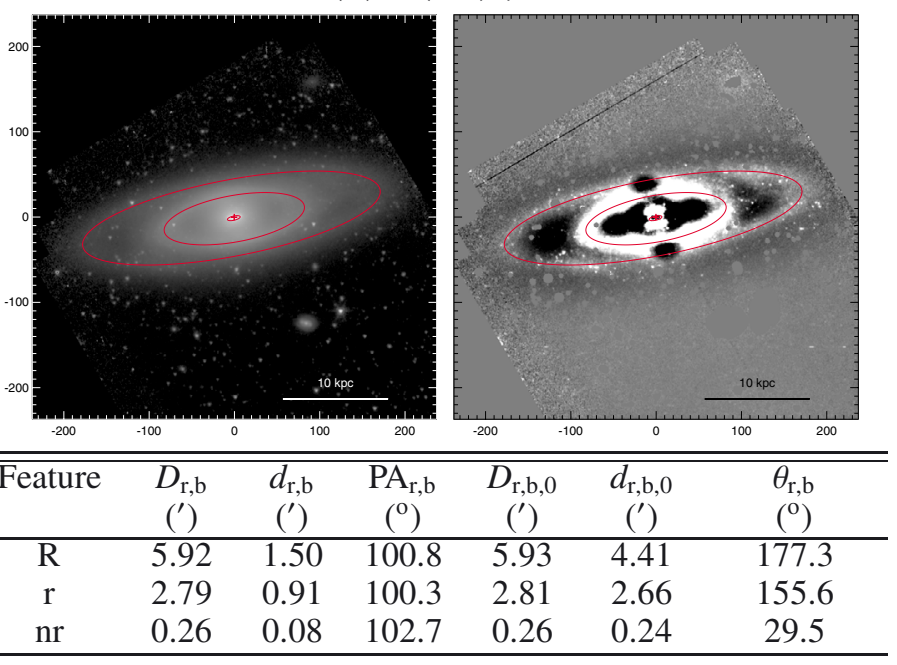

NGC 4293

(R) $\mathrm{SB}_{\mathrm{x}}(\mathrm{rs}) 0 / \mathrm{a}$
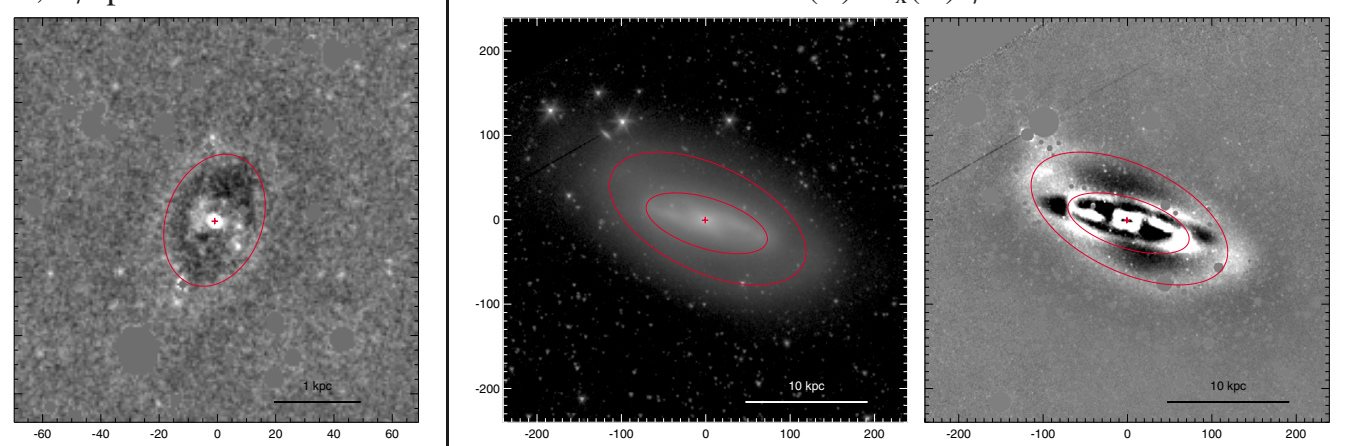

\begin{tabular}{|c|c|c|c|c|c|c|c|c|c|c|c|c|c|}
\hline Feature & $\begin{array}{c}D_{\mathrm{r}, \mathrm{b}} \\
\left({ }^{\prime}\right)\end{array}$ & $\begin{array}{c}d_{\mathrm{r}, \mathrm{b}} \\
\left(^{\prime}\right)\end{array}$ & $\begin{array}{c}\mathrm{PA}_{\mathrm{r}, \mathrm{b}} \\
\left(^{\circ}\right)\end{array}$ & $\begin{array}{c}D_{\mathrm{r}, \mathrm{b}, 0} \\
\left(^{\prime}\right)\end{array}$ & $\begin{array}{c}d_{\mathrm{r}, \mathrm{b}, 0} \\
\left({ }^{\prime}\right)\end{array}$ & $\begin{array}{c}\theta_{\mathrm{r}, \mathrm{b}} \\
\left(^{\circ}\right)\end{array}$ & Feature & $\begin{array}{c}D_{\mathrm{r}, \mathrm{b}} \\
\left(^{\prime}\right)\end{array}$ & $\begin{array}{c}d_{\mathrm{r}, \mathrm{b}} \\
\left(^{\prime}\right)\end{array}$ & $\begin{array}{c}\mathrm{PA}_{\mathrm{r}, \mathrm{b}} \\
\left({ }^{\circ}\right)\end{array}$ & $\begin{array}{c}D_{\mathrm{r}, \mathrm{b}, 0} \\
\left(^{\prime}\right)\end{array}$ & $\begin{array}{c}d_{\mathrm{r}, \mathrm{b}, 0} \\
\left.\text { (') }^{\prime}\right)\end{array}$ & $\begin{array}{l}\theta_{\mathrm{r}, \mathrm{b}} \\
\left(^{\circ}\right)\end{array}$ \\
\hline $\mathrm{RL}$ & 0.78 & 0.55 & 160.2 & 0.91 & 0.77 & 74.3 & $\begin{array}{l}\mathrm{R} \\
\mathrm{rs}\end{array}$ & $\begin{array}{l}4.20 \\
2.49\end{array}$ & $\begin{array}{l}2.11 \\
0.99\end{array}$ & $\begin{array}{l}64.4 \\
72.8\end{array}$ & $\begin{array}{l}4.51 \\
2.71\end{array}$ & $\begin{array}{l}4.19 \\
1.96\end{array}$ & $\begin{array}{l}86.2 \\
36.5\end{array}$ \\
\hline
\end{tabular}


NGC 4298

$\left(\mathrm{R}^{\prime}\right) \mathrm{SA}(\mathrm{rs}) \mathrm{bc}$
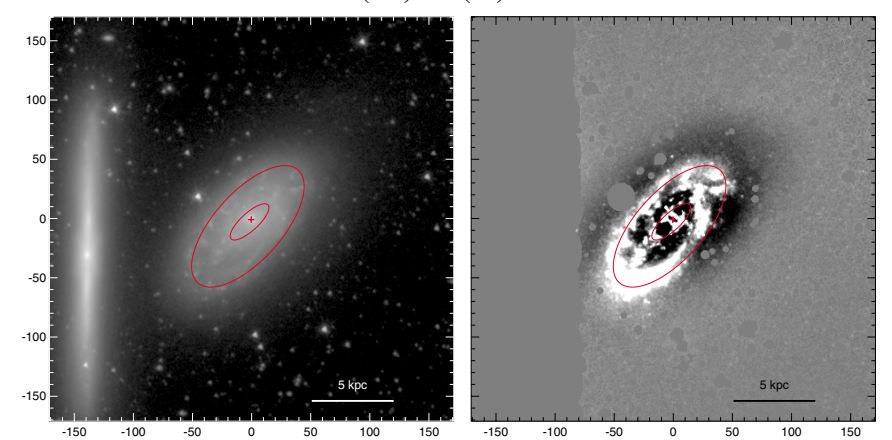

\begin{tabular}{|c|c|c|c|c|c|c|c|c|c|c|c|c|c|}
\hline Feature & $\begin{array}{c}D_{\mathrm{r}, \mathrm{b}} \\
\left({ }^{\prime}\right)\end{array}$ & $\begin{array}{c}d_{\mathrm{r}, \mathrm{b}} \\
\left(^{\prime}\right)\end{array}$ & $\begin{array}{c}\mathrm{PA}_{\mathrm{r}, \mathrm{b}} \\
\left({ }^{\mathrm{O}}\right)\end{array}$ & $\begin{array}{c}D_{\mathrm{r}, \mathrm{b}, 0} \\
\left(^{\prime}\right)\end{array}$ & $\begin{array}{c}d_{\mathrm{r}, \mathrm{b}, 0} \\
\left({ }^{\prime}\right)\end{array}$ & $\begin{array}{l}\theta_{\mathrm{r}, \mathrm{b}} \\
\left({ }^{\circ}\right)\end{array}$ & Feature & $\begin{array}{c}D_{\mathrm{r}, \mathrm{b}} \\
\left({ }^{\prime}\right)\end{array}$ & $\begin{array}{c}d_{\mathrm{r}, \mathrm{b}} \\
\left({ }^{\prime}\right)\end{array}$ & $\begin{array}{c}\mathrm{PA}_{\mathrm{r}, \mathrm{b}} \\
\left({ }^{\circ}\right)\end{array}$ & $\begin{array}{c}D_{\mathrm{r}, \mathrm{b}, 0} \\
\left(^{\prime}\right)\end{array}$ & $\begin{array}{c}d_{\mathrm{r}, \mathrm{b}, 0} \\
{ }^{\prime}\left({ }^{\prime}\right)\end{array}$ & $\begin{array}{l}\theta_{\mathrm{r}, \mathrm{b}} \\
\left({ }^{\circ}\right)\end{array}$ \\
\hline$\overline{\mathrm{R}^{\prime}}$ & 2.13 & 0.96 & 138.3 & 2.20 & 1.62 & 22.8 & bar & 1.86 & 0.56 & 2.1 & 1.95 & 0.60 & 42.4 \\
\hline rs & 0.71 & 0.25 & 132.5 & 0.71 & 0.43 & 3.4 & rs & 1.59 & 1.33 & 81.0 & 1.76 & 1.35 & 106.8 \\
\hline
\end{tabular}
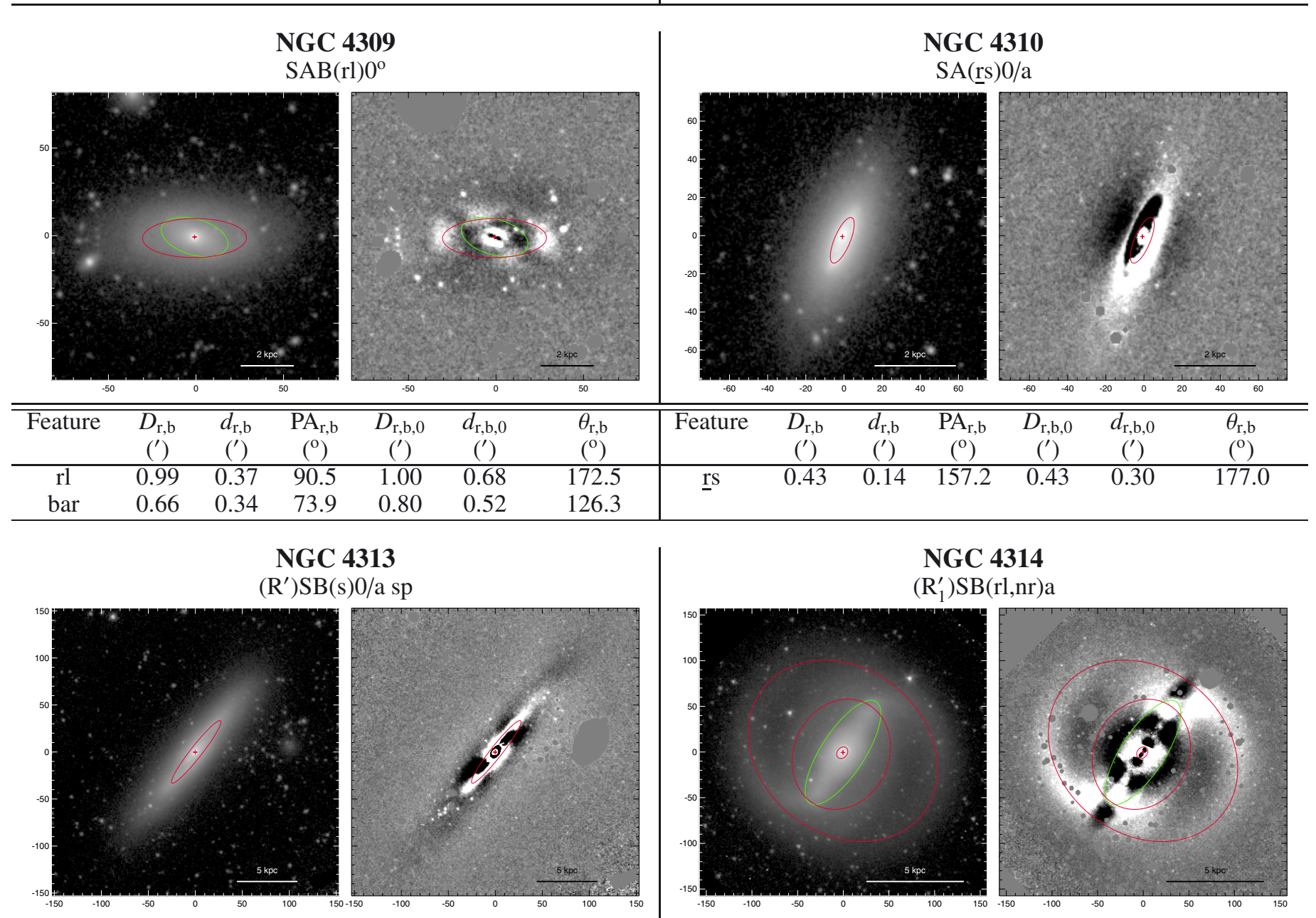

NGC 4314
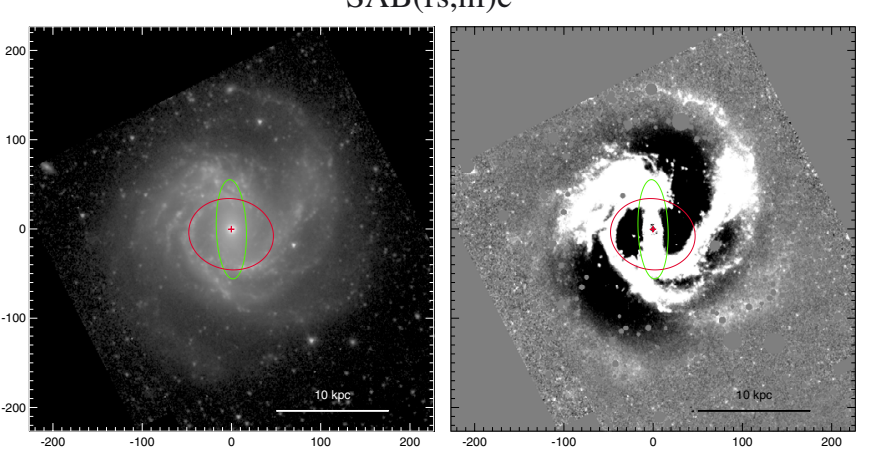

NGC 4303

$\mathrm{SAB}(\mathrm{rs}, \mathrm{nl}) \mathrm{C}$ 
NGC 4316

$\mathrm{SB}(\mathrm{rs}$ ?) c sp
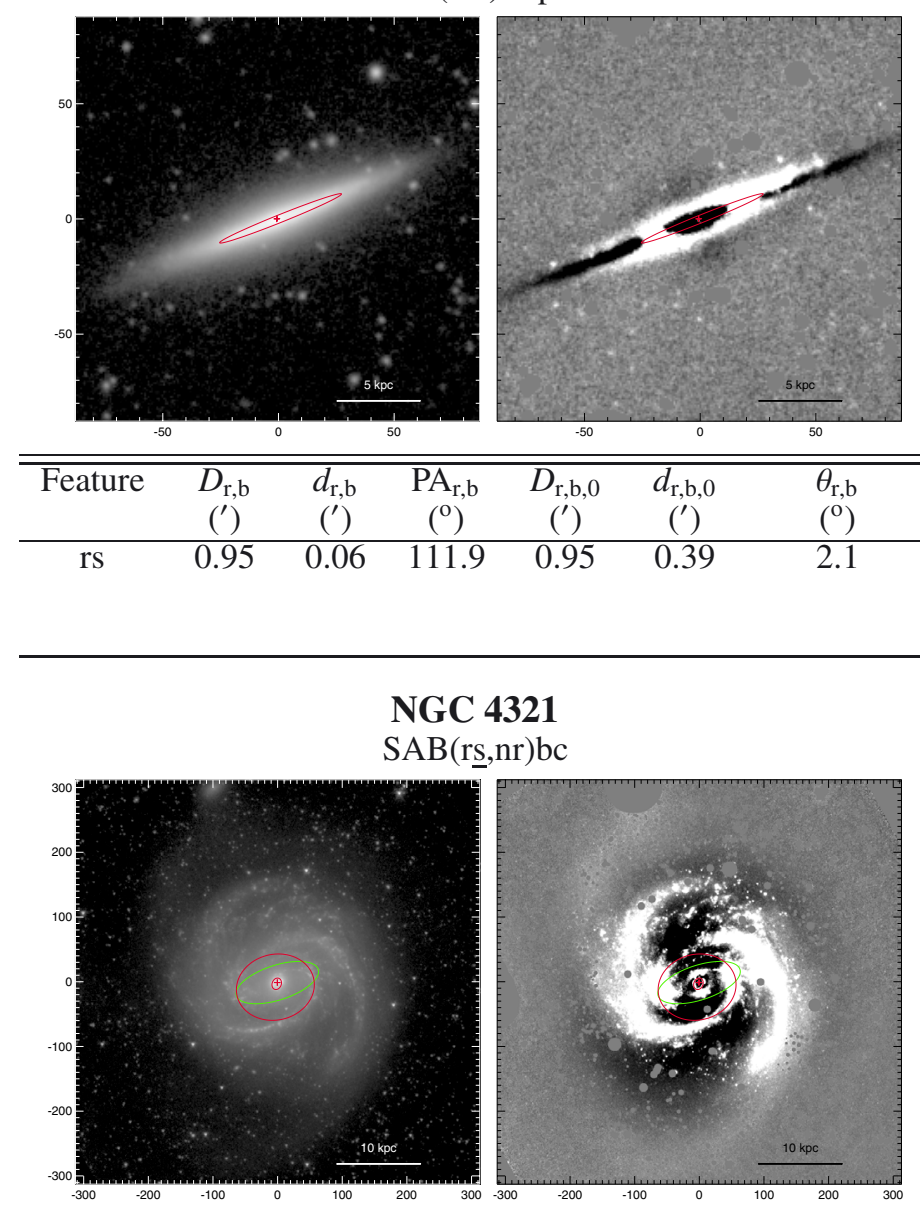

\begin{tabular}{|c|c|c|c|c|c|c|c|c|c|c|c|c|c|}
\hline Feature & $\begin{array}{c}D_{\mathrm{r}, \mathrm{b}} \\
\left(^{\prime}\right)\end{array}$ & $\begin{array}{c}d_{\mathrm{r}, \mathrm{b}} \\
\left(^{\prime}\right)\end{array}$ & $\begin{array}{c}\mathrm{PA}_{\mathrm{r}, \mathrm{b}} \\
\left({ }^{\circ}\right)\end{array}$ & $\begin{array}{c}D_{\mathrm{r}, \mathrm{b}, 0} \\
\left(^{\prime}\right)\end{array}$ & $\begin{array}{c}d_{\mathrm{r}, \mathrm{b}, 0} \\
\left(^{\prime}\right)\end{array}$ & $\begin{array}{l}\theta_{\mathrm{r}, \mathrm{b}} \\
\left({ }^{\circ}\right)\end{array}$ & Feature & $\begin{array}{c}D_{\mathrm{r}, \mathrm{b}} \\
\left({ }^{\prime}\right)\end{array}$ & $\begin{array}{c}d_{\mathrm{r}, \mathrm{b}} \\
\left(^{\prime}\right)\end{array}$ & $\begin{array}{c}\overline{\mathrm{PA}_{\mathrm{r}, \mathrm{b}}} \\
\left({ }^{\circ}\right)\end{array}$ & $\begin{array}{c}D_{\mathrm{r}, \mathrm{b}, 0} \\
\left(^{\prime}\right)\end{array}$ & $\begin{array}{c}d_{\mathrm{r}, \mathrm{b}, 0} \\
\left({ }^{\prime}\right)\end{array}$ & $\begin{array}{c}\theta_{\mathrm{r}, \mathrm{b}} \\
\left({ }^{\mathrm{o}}\right)\end{array}$ \\
\hline bar & 2.22 & 0.90 & 107.9 & 2.52 & 0.96 & 122.5 & $\mathrm{r}$ & 0.72 & 0.32 & 54.0 & 0.75 & 0.71 & 119.1 \\
\hline rs & 2.03 & 1.70 & 103.0 & 2.38 & 1.75 & 106.3 & & & & & & & \\
\hline$\overline{n r}$ & 0.30 & 0.24 & 158.6 & 0.30 & 0.29 & 1.6 & & & & & & & \\
\hline
\end{tabular}

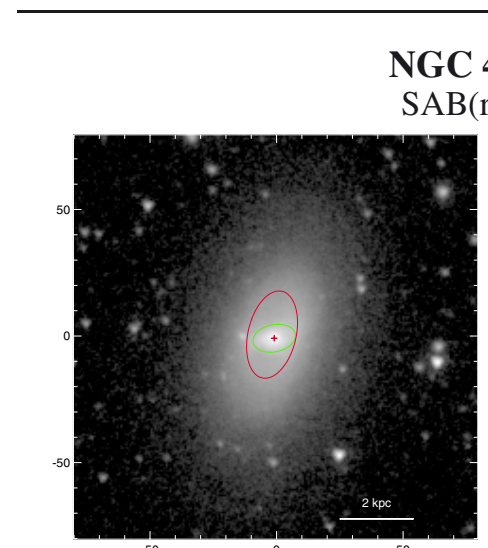

NGC 4336

$\mathrm{SAB}(\mathrm{r}) 0 / \mathrm{a}$
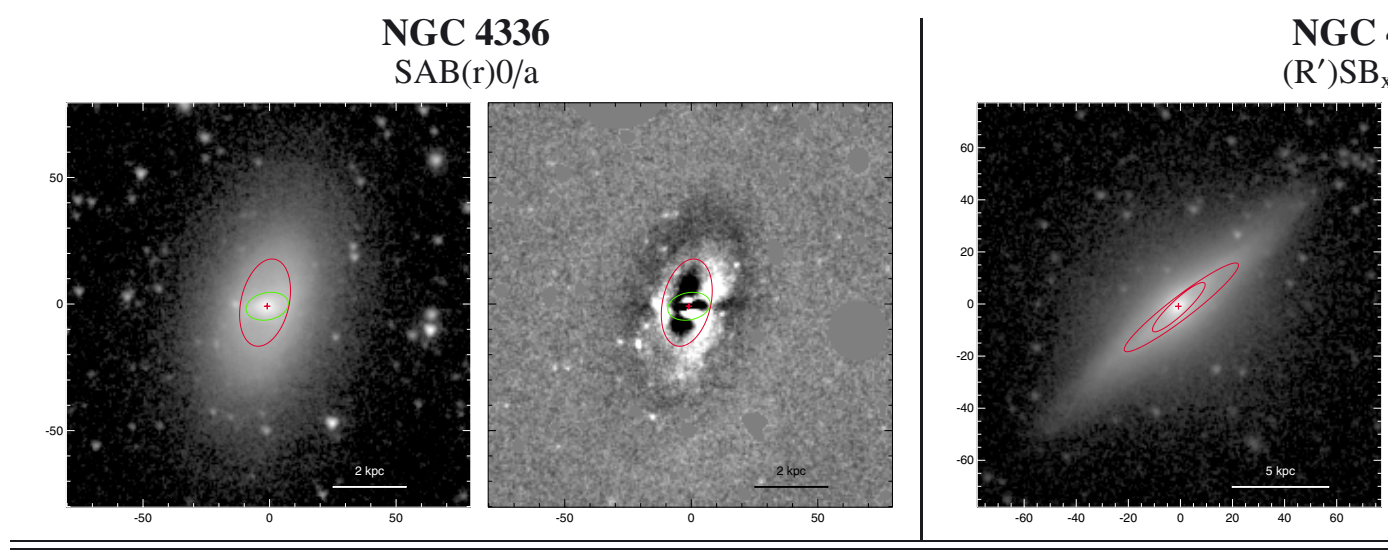

$\left(\mathrm{R}^{\prime}\right) \mathrm{SA} \underline{\mathrm{B}}(\underline{\mathrm{rs}})$ a pec

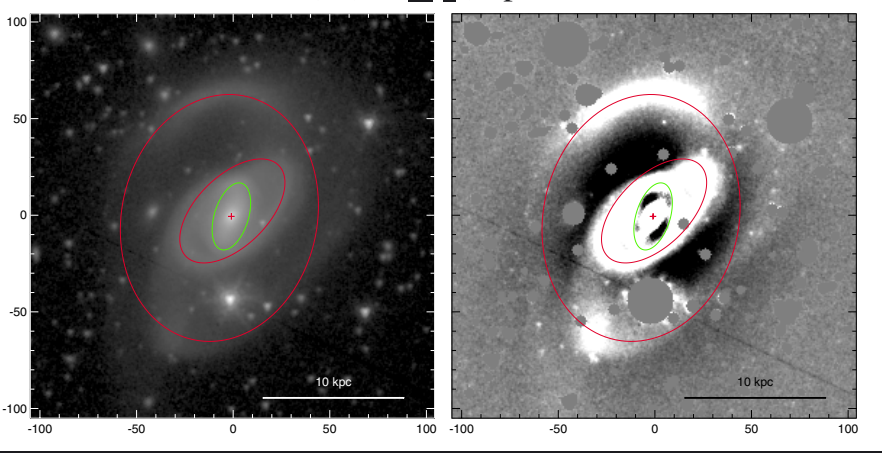

\begin{tabular}{ccccccc} 
Feature & $\begin{array}{c}D_{\mathrm{r}, \mathrm{b}} \\
\left({ }^{\prime}\right)\end{array}$ & $\begin{array}{c}d_{\mathrm{r}, \mathrm{b}} \\
\left({ }^{\prime}\right)\end{array}$ & $\begin{array}{c}\mathrm{PA}_{\mathrm{r}, \mathrm{b}} \\
\left({ }^{\circ}\right)\end{array}$ & $\begin{array}{c}D_{\mathrm{r}, \mathrm{b}, 0} \\
\left({ }^{\prime}\right)\end{array}$ & $\begin{array}{c}d_{\mathrm{r}, \mathrm{b}, 0} \\
\left({ }^{\prime}\right)\end{array}$ & $\begin{array}{c}\theta_{\mathrm{r}, \mathrm{b}} \\
\left({ }^{\circ}\right)\end{array}$ \\
\hline $\mathrm{R}^{\prime}$ & 2.16 & 1.69 & 168.0 & 2.32 & 2.00 & 53.9 \\
$\underline{\mathrm{rs}}$ & 1.12 & 0.61 & 134.4 & 1.16 & 0.76 & 154.5 \\
$\mathrm{~b}$ & 0.60 & 0.30 & 162.8 & 0.61 & 0.37 & 19.2 \\
\hline
\end{tabular}

NGC 4324
(L)SA(r) $0^{+}$

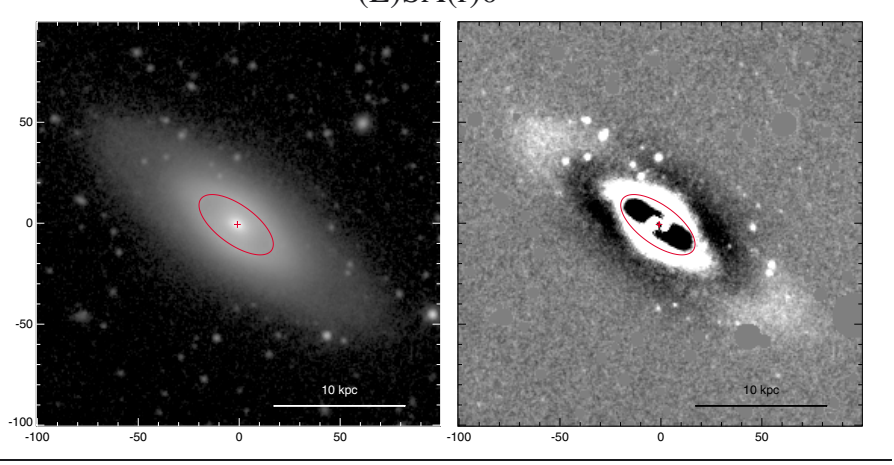

\begin{tabular}{|c|c|c|c|c|c|c|c|c|c|c|c|c|c|}
\hline Feature & $\begin{array}{c}D_{\mathrm{r}, \mathrm{b}} \\
\left(^{\prime}\right)\end{array}$ & $\begin{array}{c}d_{\mathrm{r}, \mathrm{b}} \\
\left(^{\prime}\right)\end{array}$ & $\begin{array}{c}\mathrm{PA}_{\mathrm{r}, \mathrm{b}} \\
\left({ }^{\mathrm{O}}\right)\end{array}$ & $\begin{array}{c}D_{\mathrm{r}, \mathrm{b}, 0} \\
\left(^{\prime}\right)\end{array}$ & $\begin{array}{c}d_{\mathrm{r}, \mathrm{b}, 0} \\
\left(^{\prime}\right)\end{array}$ & $\begin{array}{l}\theta_{\mathrm{r}, \mathrm{b}} \\
\left({ }^{\circ}\right)\end{array}$ & Feature & $\begin{array}{c}D_{\mathrm{r}, \mathrm{b}} \\
\left({ }^{\prime}\right)\end{array}$ & $\begin{array}{c}d_{\mathrm{r}, \mathrm{b}} \\
\left({ }^{\prime}\right)\end{array}$ & $\begin{array}{c}\mathrm{PA}_{\mathrm{r}, \mathrm{b}} \\
\left({ }^{\mathrm{o}}\right)\end{array}$ & $\begin{array}{c}D_{\mathrm{r}, \mathrm{b}, 0} \\
\left(^{\prime}\right)\end{array}$ & $\begin{array}{c}d_{\mathrm{r}, \mathrm{b}, 0} \\
\left({ }^{\prime}\right)\end{array}$ & $\begin{array}{l}\theta_{\mathrm{r}, \mathrm{b}} \\
\left({ }^{\mathrm{o}}\right)\end{array}$ \\
\hline $\mathrm{r}$ & 0.59 & 0.32 & 167.0 & 0.60 & 0.53 & 26.8 & $\overline{\mathrm{R}^{\prime}}$ & 0.92 & 0.14 & 127.5 & 0.93 & 0.41 & 171.3 \\
\hline bar & 0.28 & 0.18 & 101.7 & 0.45 & 0.19 & 102.2 & $\mathrm{r}$ & 0.45 & 0.10 & 132.9 & 0.46 & 0.28 & 12.6 \\
\hline
\end{tabular}


NGC 4344

$\mathrm{dSA}(\mathrm{r}) 0^{+}, \mathrm{N}$
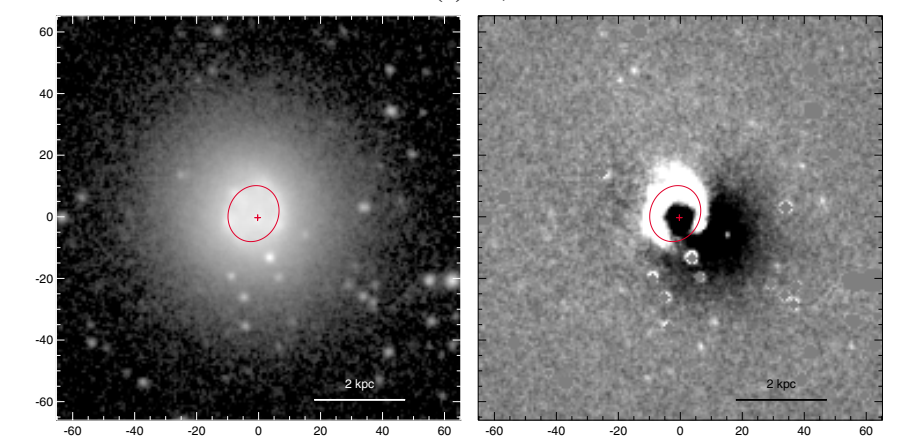

NGC 4348

$\mathrm{SB}(\mathrm{rs}) \mathrm{bc} \mathrm{sp}$

\begin{tabular}{|c|c|c|c|c|c|c|c|c|c|c|c|c|c|}
\hline Feature & $\begin{array}{c}D_{\mathrm{r}, \mathrm{b}} \\
\left({ }^{\prime}\right)\end{array}$ & $\begin{array}{c}d_{\mathrm{r}, \mathrm{b}} \\
\left({ }^{\prime}\right)\end{array}$ & $\begin{array}{c}\mathrm{PA}_{\mathrm{r}, \mathrm{b}} \\
\left({ }^{\circ}\right)\end{array}$ & $\begin{array}{c}D_{\mathrm{r}, \mathrm{b}, 0} \\
\left(^{\prime}\right)\end{array}$ & $\begin{array}{c}d_{\mathrm{r}, \mathrm{b}, 0} \\
\left({ }^{\prime}\right)\end{array}$ & $\begin{array}{c}\theta_{\mathrm{r}, \mathrm{b}} \\
\left({ }^{\circ}\right)\end{array}$ & Feature & $\begin{array}{c}D_{\mathrm{r}, \mathrm{b}} \\
\left({ }^{\prime}\right)\end{array}$ & $\begin{array}{c}d_{\mathrm{r}, \mathrm{b}} \\
\left(^{\prime}\right)\end{array}$ & $\begin{array}{c}\mathrm{PA}_{\mathrm{r}, \mathrm{b}} \\
\left({ }^{\circ}\right)\end{array}$ & $\begin{array}{c}D_{\mathrm{r}, \mathrm{b}, 0} \\
\left({ }^{\prime}\right)\end{array}$ & $\begin{array}{c}d_{\mathrm{r}, \mathrm{b}, 0} \\
\left(^{\prime}\right)\end{array}$ & $\begin{array}{l}\theta_{\mathrm{r}, \mathrm{b}} \\
\left({ }^{\circ}\right)\end{array}$ \\
\hline $\mathrm{r}$ & 0.31 & 0.27 & 155.8 & 0.35 & 0.27 & 94.8 & rs & 0.68 & 0.14 & 33.8 & 0.71 & 0.58 & 151.3 \\
\hline
\end{tabular}

NGC 4351

$\left(\mathrm{R}^{\prime}\right) \mathrm{SAB}(\mathrm{s}) \mathrm{dm}$ imbedded
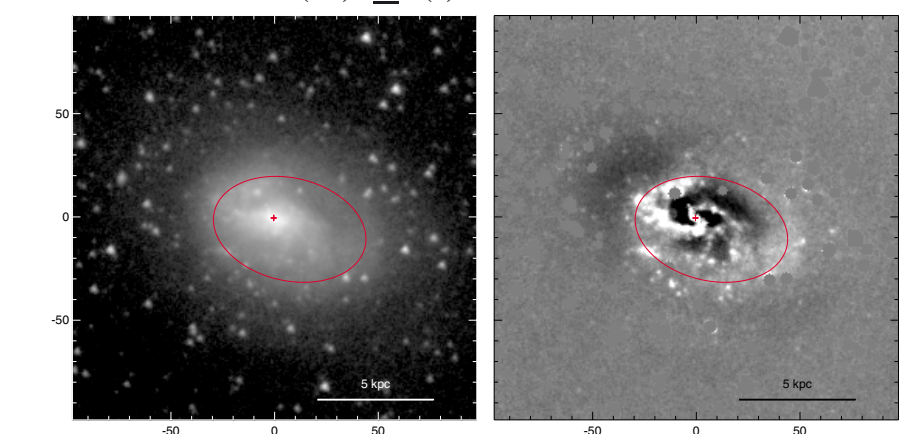

NGC 4355

( $\left.\mathrm{R}^{\prime} \mathrm{L}\right) \mathrm{SAB}(\mathrm{l}) 0 / \mathrm{a}$

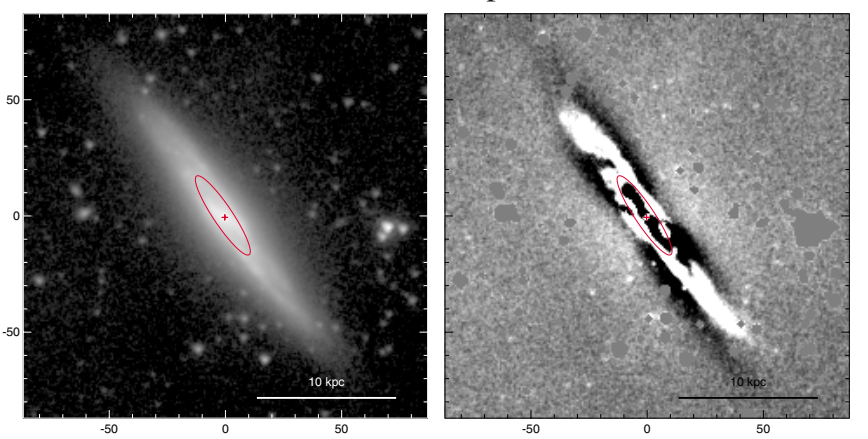

\section{(R'L) -}

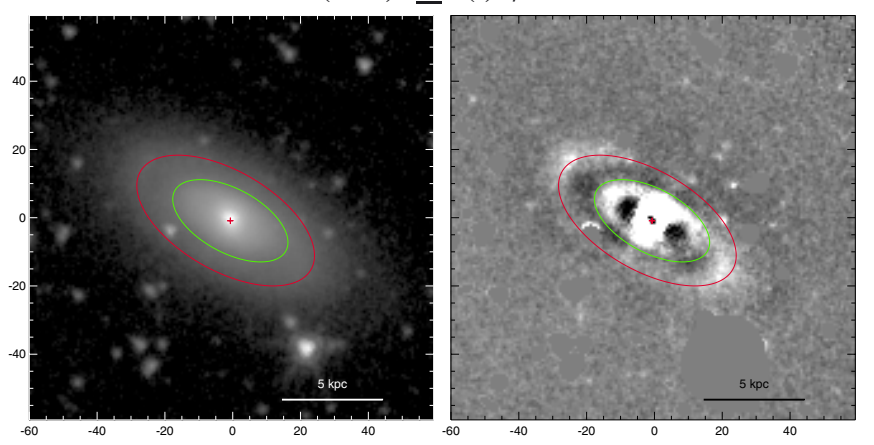

\begin{tabular}{|c|c|c|c|c|c|c|c|c|c|c|c|c|c|}
\hline Feature & $\begin{array}{c}D_{\mathrm{r}, \mathrm{b}} \\
\left(^{\prime}\right)\end{array}$ & $\begin{array}{c}d_{\mathrm{r}, \mathrm{b}} \\
\left(^{\prime}\right)\end{array}$ & $\begin{array}{c}\mathrm{PA}_{\mathrm{r}, \mathrm{b}} \\
\left({ }^{\circ}\right)\end{array}$ & $\begin{array}{c}D_{\mathrm{r}, \mathrm{b}, 0} \\
\left(^{\prime}\right)\end{array}$ & $\begin{array}{c}d_{\mathrm{r}, \mathrm{b}, 0} \\
\left({ }^{\prime}\right)\end{array}$ & $\begin{array}{c}\theta_{\mathrm{r}, \mathrm{b}} \\
\left({ }^{\circ}\right)\end{array}$ & Feature & $\begin{array}{c}D_{\mathrm{r}, \mathrm{b}} \\
\left(^{\prime}\right)\end{array}$ & $\begin{array}{c}d_{\mathrm{r}, \mathrm{b}} \\
\left({ }^{\prime}\right)\end{array}$ & $\begin{array}{c}\mathrm{PA}_{\mathrm{r}, \mathrm{b}} \\
\left({ }^{\circ}\right)\end{array}$ & $\begin{array}{c}D_{\mathrm{r}, \mathrm{b}, 0} \\
\text { (') }^{\prime}\end{array}$ & $\begin{array}{c}d_{\mathrm{r}, \mathrm{b}, 0} \\
\left(^{\prime}\right)\end{array}$ & $\begin{array}{l}\theta_{\mathrm{r}, \mathrm{b}} \\
\left(^{\circ}\right)\end{array}$ \\
\hline$\overline{\mathrm{R}^{\prime}}$ & 1.25 & 0.83 & 76.9 & 1.38 & 1.11 & 51.4 & $\begin{array}{l}\mathrm{R}^{\prime} \mathrm{L} \\
\text { bar }\end{array}$ & $\begin{array}{l}0.97 \\
0.63\end{array}$ & $\begin{array}{l}0.49 \\
0.32\end{array}$ & $\begin{array}{l}60.1 \\
61.6\end{array}$ & $\begin{array}{l}1.01 \\
0.63\end{array}$ & $\begin{array}{l}0.95 \\
0.62\end{array}$ & $\begin{array}{l}129.5 \\
127.3\end{array}$ \\
\hline
\end{tabular}

NGC 4369

(R)SB(rs)0/a: pec

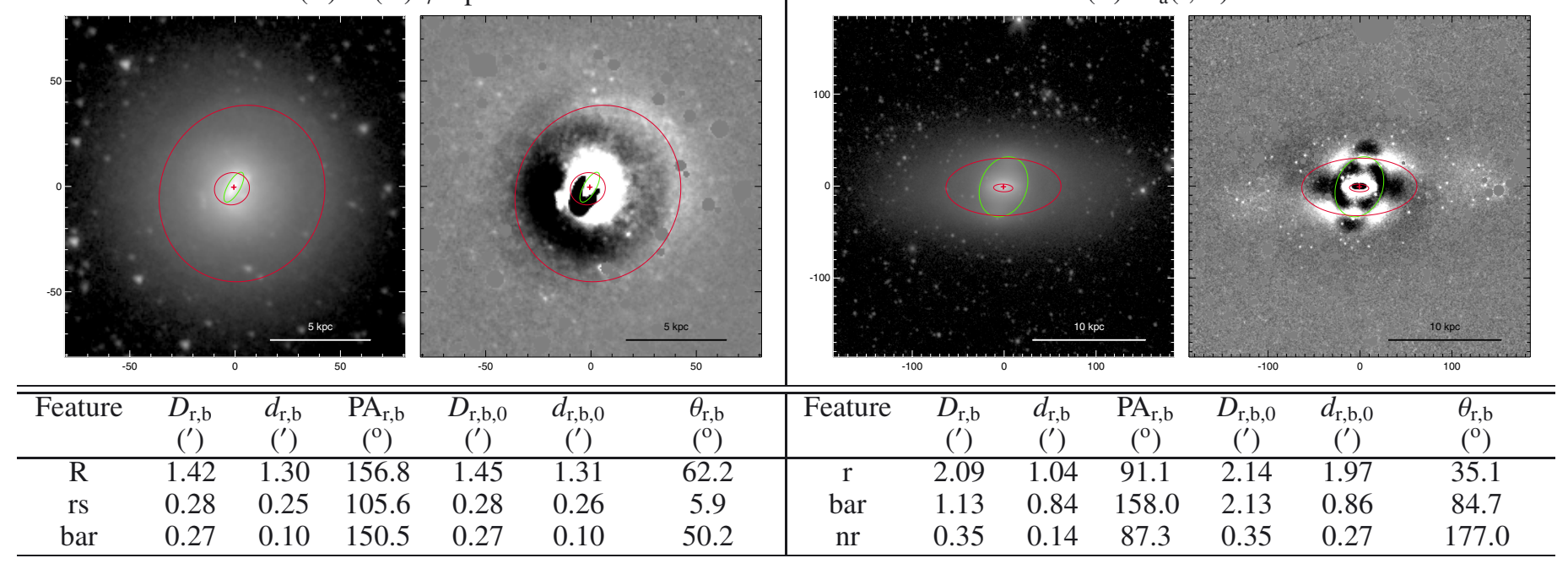

NGC 4371

(L) $\mathrm{SB}_{\mathrm{a}}(\mathrm{r}, \mathrm{nr}) 0^{\circ}$ 
NGC 4378

$\left(\mathrm{R}^{\prime}\right) \mathrm{SA}(1) \mathrm{a}$

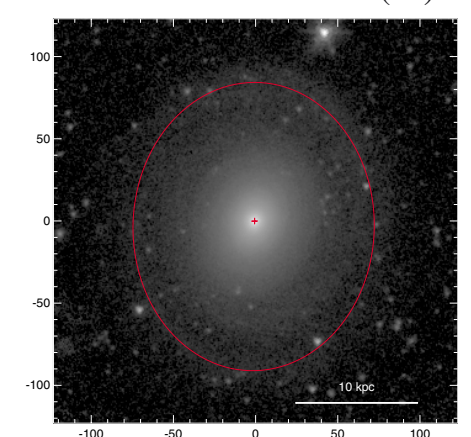

NGC 4380

(R)SA(r,l)ab

\begin{tabular}{|c|c|c|c|c|c|c|c|c|c|c|c|c|c|}
\hline 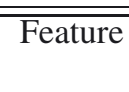 & $\begin{array}{c}D_{\mathrm{r}, \mathrm{b}} \\
\left({ }^{\prime}\right)\end{array}$ & $\begin{array}{c}d_{\mathrm{r}, \mathrm{b}} \\
\left({ }^{\prime}\right)\end{array}$ & $\begin{array}{c}\mathrm{PA}_{\mathrm{r}, \mathrm{b}} \\
\left({ }^{\circ}\right)\end{array}$ & $\begin{array}{c}D_{\mathrm{r}, \mathrm{b}, 0} \\
\left({ }^{\prime}\right)\end{array}$ & $\begin{array}{c}d_{\mathrm{r}, \mathrm{b}, 0} \\
\left({ }^{\prime}\right)\end{array}$ & $\begin{array}{c}\theta_{\mathrm{r}, \mathrm{b}} \\
\left({ }^{\circ}\right)\end{array}$ & Feature & $\begin{array}{c}D_{\mathrm{r}, \mathrm{b}} \\
\left({ }^{\prime}\right)\end{array}$ & $\begin{array}{c}d_{\mathrm{r}, \mathrm{b}} \\
\left({ }^{\prime}\right)\end{array}$ & $\begin{array}{c}\mathrm{PA}_{\mathrm{r}, \mathrm{b}} \\
\left({ }^{\circ}\right)\end{array}$ & $\begin{array}{c}D_{\mathrm{r}, \mathrm{b}, 0} \\
\left({ }^{\prime}\right)\end{array}$ & $\begin{array}{c}d_{\mathrm{r}, \mathrm{b}, 0} \\
\left({ }^{\prime}\right)\end{array}$ & $\begin{array}{l}\theta_{\mathrm{r}, \mathrm{b}} \\
\left({ }^{\circ}\right)\end{array}$ \\
\hline$\overline{\mathrm{R}^{\prime}}$ & 2.93 & 2.45 & 178.5 & 2.99 & 2.85 & 43.2 & $\overline{\mathrm{R}}$ & $\begin{array}{l}2.02 \\
0.08\end{array}$ & $\begin{array}{l}1.07 \\
0.54\end{array}$ & 156.5 & 2.03 & $\begin{array}{l}1.93 \\
092\end{array}$ & $\begin{array}{l}169.8 \\
1325\end{array}$ \\
\hline
\end{tabular}

NGC 4384

(L) $\mathrm{SB}(\mathrm{rs}) \mathrm{dm}$
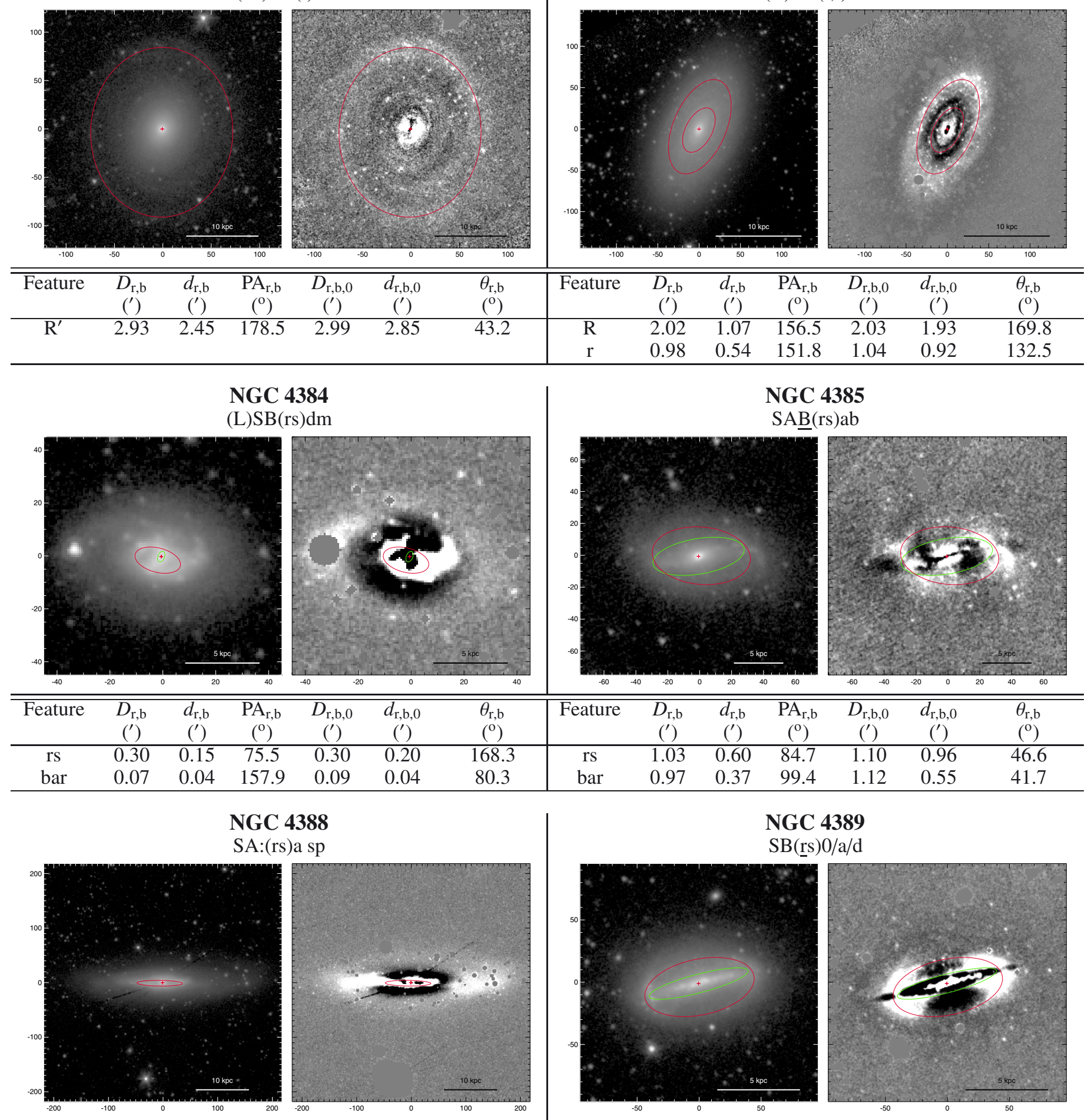

NGC 4389

$\mathrm{SB}(\underline{\mathrm{rs}}) 0 / \mathrm{a} / \mathrm{d}$

\begin{tabular}{ccccccc|ccccccc}
\hline \hline Feature & $\begin{array}{c}D_{\mathrm{r}, \mathrm{b}} \\
\left({ }^{\prime}\right)\end{array}$ & $\begin{array}{c}d_{\mathrm{r}, \mathrm{b}} \\
\left({ }^{\prime}\right)\end{array}$ & $\begin{array}{c}\mathrm{PA}_{\mathrm{r}, \mathrm{b}} \\
\left({ }^{\circ}\right)\end{array}$ & $\begin{array}{c}D_{\mathrm{r}, \mathrm{b}, 0} \\
\left({ }^{\prime}\right)\end{array}$ & $\begin{array}{c}d_{\mathrm{r}, \mathrm{b}, 0} \\
\left({ }^{\prime}\right)\end{array}$ & $\begin{array}{c}\theta_{\mathrm{r}, \mathrm{b}} \\
\left({ }^{\circ}\right)\end{array}$ & Feature & $\begin{array}{c}D_{\mathrm{r}, \mathrm{b}} \\
\left({ }^{\prime}\right)\end{array}$ & $\begin{array}{c}d_{\mathrm{r}, \mathrm{b}} \\
\left({ }^{\prime}\right)\end{array}$ & $\begin{array}{c}\mathrm{PA}_{\mathrm{r}, \mathrm{b}} \\
\left({ }^{\circ}\right)\end{array}$ & $\begin{array}{c}D_{\mathrm{r}, \mathrm{b}, 0} \\
\left({ }^{\prime}\right)\end{array}$ & $\begin{array}{c}d_{\mathrm{r}, \mathrm{b}, 0} \\
\left({ }^{\prime}\right)\end{array}$ & $\begin{array}{c}\theta_{\mathrm{r}, \mathrm{b}} \\
\left({ }^{\circ}\right)\end{array}$ \\
\hline rs & 1.39 & 0.17 & 89.3 & 1.40 & 0.62 & 172.4 & $\underline{\mathrm{rs}}$ & 1.50 & 0.75 & 101.9 & 1.52 & 1.16 & 15.7 \\
& & & & & & & $\underline{b a r}$ & 1.38 & 0.25 & 104.6 & 1.40 & 0.38 & 13.4 \\
\hline
\end{tabular}


NGC 4394

(R)SB(rs,nl)0/a
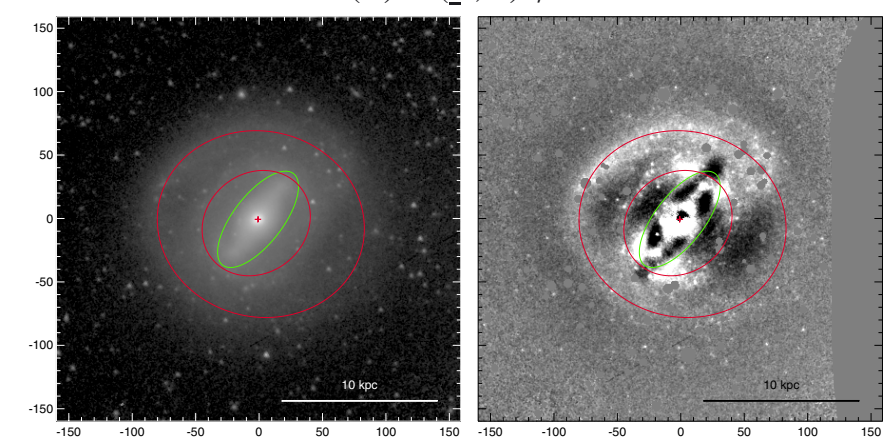

\begin{tabular}{|c|c|c|c|c|c|c|c|c|c|c|c|c|c|}
\hline Feature & $\begin{array}{c}D_{\mathrm{r}, \mathrm{b}} \\
\left(^{\prime}\right)\end{array}$ & $\begin{array}{c}d_{\mathrm{r}, \mathrm{b}} \\
\left({ }^{\prime}\right)\end{array}$ & $\begin{array}{c}\mathrm{PA}_{\mathrm{r}, \mathrm{b}} \\
\left({ }^{\circ}\right)\end{array}$ & $\begin{array}{c}D_{\mathrm{r}, \mathrm{b}, 0} \\
\left({ }^{\prime}\right)\end{array}$ & $\begin{array}{c}d_{\mathrm{r}, \mathrm{b}, 0} \\
\left(^{\prime}\right)\end{array}$ & $\begin{array}{l}\theta_{\mathrm{r}, \mathrm{b}} \\
\left({ }^{\circ}\right)\end{array}$ & Feature & $\begin{array}{c}D_{\mathrm{r}, \mathrm{b}} \\
\left({ }^{\prime}\right)\end{array}$ & $\begin{array}{c}d_{\mathrm{r}, \mathrm{b}} \\
\left(^{\prime}\right)\end{array}$ & $\begin{array}{c}\mathrm{PA}_{\mathrm{r}, \mathrm{b}} \\
\left({ }^{\circ}\right)\end{array}$ & $\begin{array}{c}D_{\mathrm{r}, \mathrm{b}, 0} \\
\left(^{\prime}\right)\end{array}$ & $\begin{array}{c}d_{\mathrm{r}, \mathrm{b}, 0} \\
\left(^{\prime}\right)\end{array}$ & $\begin{array}{l}\theta_{\mathrm{r}, \mathrm{b}} \\
\left({ }^{\circ}\right)\end{array}$ \\
\hline$\overline{\mathrm{R}}$ & 2.74 & 2.45 & 77.5 & 2.99 & 2.60 & 113.0 & $\overline{\mathrm{R}^{\prime}}$ & 1.86 & 0.20 & 89.8 & 1.95 & 1.28 & 23.2 \\
\hline bar & 1.52 & 0.65 & 142.2 & 1.60 & 0.72 & 38.3 & & & & & & & \\
\hline$\underline{r} s$ & 1.50 & 1.31 & 129.2 & 1.58 & 1.44 & 58.5 & & & & & & & \\
\hline
\end{tabular}

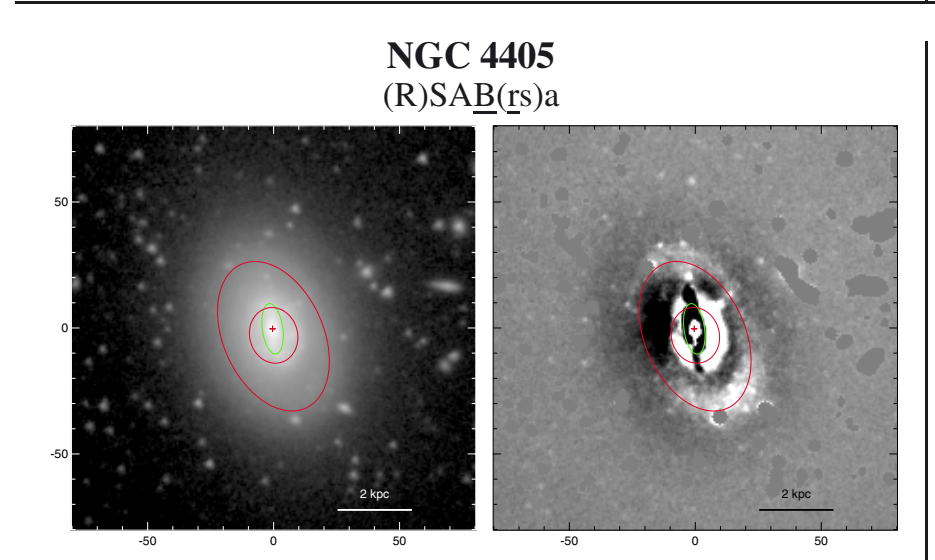

\begin{tabular}{|c|c|c|c|c|c|c|c|c|c|c|c|c|c|}
\hline$\overline{\text { Feature }}$ & $\begin{array}{c}D_{\mathrm{r}, \mathrm{b}} \\
\left({ }^{\prime}\right)\end{array}$ & $\begin{array}{c}d_{\mathrm{r}, \mathrm{b}} \\
\left({ }^{\prime}\right)\end{array}$ & $\begin{array}{c}\mathrm{PA}_{\mathrm{r}, \mathrm{b}} \\
\left({ }^{\circ}\right)\end{array}$ & $\begin{array}{c}D_{\mathrm{r}, \mathrm{b}, 0} \\
\left(^{\prime}\right)\end{array}$ & $\begin{array}{c}d_{\mathrm{r}, \mathrm{b}, 0} \\
\left(^{\prime}\right)\end{array}$ & $\begin{array}{l}\theta_{\mathrm{r}, \mathrm{b}} \\
\left({ }^{\circ}\right)\end{array}$ & Feature & $\begin{array}{c}D_{\mathrm{r}, \mathrm{b}} \\
\left({ }^{\prime}\right)\end{array}$ & $\begin{array}{c}d_{\mathrm{r}, \mathrm{b}} \\
\left(^{\prime}\right)\end{array}$ & $\begin{array}{c}\mathrm{PA}_{\mathrm{r}, \mathrm{b}} \\
\left({ }^{\circ}\right)\end{array}$ & $\begin{array}{c}D_{\mathrm{r}, \mathrm{b}, 0} \\
\left(^{\prime}\right)\end{array}$ & $\begin{array}{c}d_{\mathrm{r}, \mathrm{b}, 0} \\
\left(^{\prime}\right)\end{array}$ & $\begin{array}{l}\theta_{\mathrm{r}, \mathrm{b}} \\
\left({ }^{\circ}\right)\end{array}$ \\
\hline$\overline{\mathrm{R}}$ & 1.05 & 0.66 & 24.2 & 1.05 & 0.87 & 13.3 & rs & 0.57 & 0.43 & 32.6 & 0.68 & 0.43 & 94.5 \\
\hline$\underline{\mathrm{r} s}$ & 0.37 & 0.32 & 13.8 & 0.42 & 0.37 & 96.4 & bar & 0.16 & 0.03 & 109.7 & 0.16 & 0.03 & 173.4 \\
\hline$\overline{\mathrm{bar}}$ & 0.34 & 0.14 & 7.9 & 0.34 & 0.18 & 163.6 & & & & & & & \\
\hline
\end{tabular}

NGC 4412

$\mathrm{SAB}(r \underline{\mathrm{B}}) \mathrm{bc}$
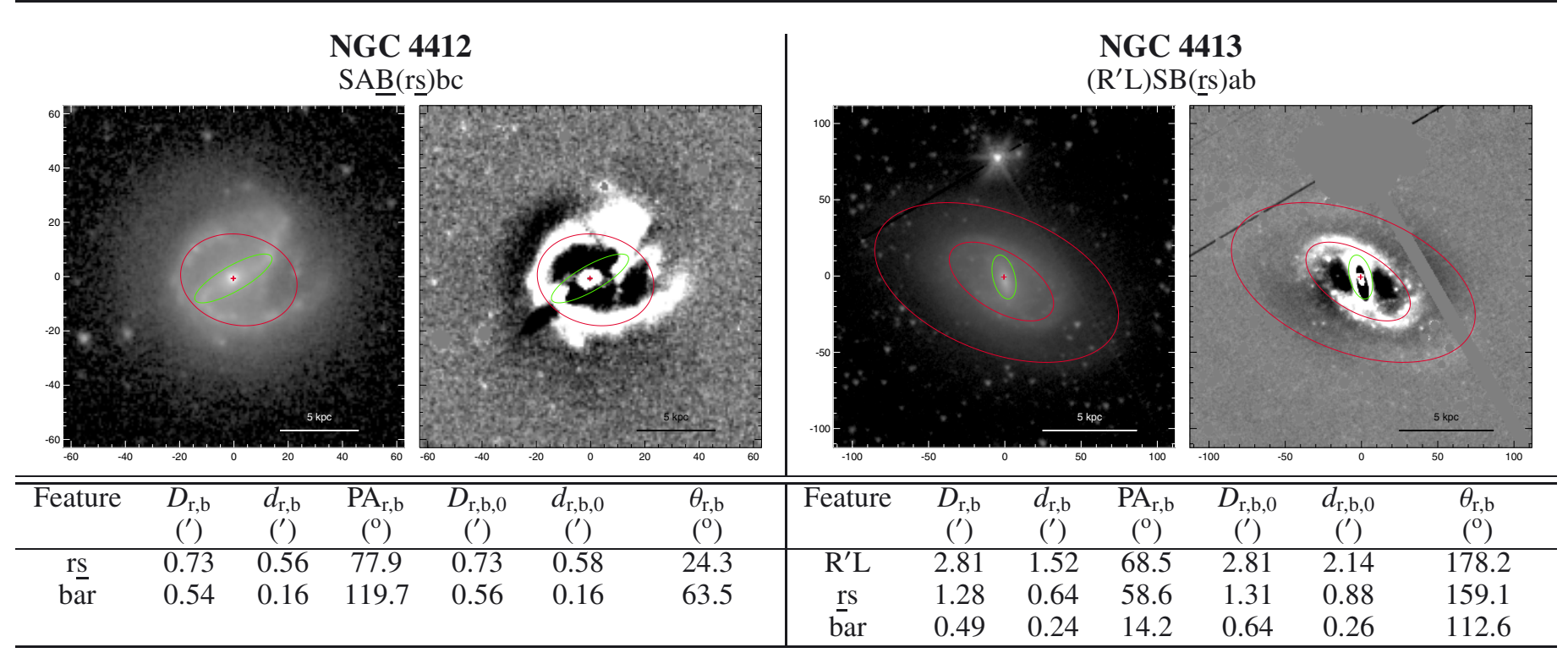

\begin{tabular}{|c|c|c|c|c|c|c|c|c|c|c|c|c|c|}
\hline Feature & $\begin{array}{c}D_{\mathrm{r}, \mathrm{b}} \\
\left({ }^{\prime}\right)\end{array}$ & $\begin{array}{c}d_{\mathrm{r}, \mathrm{b}} \\
\left(^{\prime}\right)\end{array}$ & $\begin{array}{c}\mathrm{PA}_{\mathrm{r}, \mathrm{b}} \\
\left({ }^{\circ}\right)\end{array}$ & $\begin{array}{c}D_{\mathrm{r}, \mathrm{b}, 0} \\
\left(^{\prime}\right)\end{array}$ & $\begin{array}{c}d_{\mathrm{r}, \mathrm{b}, 0} \\
\left(^{\prime}\right)\end{array}$ & $\begin{array}{c}\theta_{\mathrm{r}, \mathrm{b}} \\
\left({ }^{\circ}\right)\end{array}$ & Feature & $\begin{array}{c}D_{\mathrm{r}, \mathrm{b}} \\
\left({ }^{\prime}\right)\end{array}$ & $\begin{array}{c}d_{\mathrm{r}, \mathrm{b}} \\
\left({ }^{\prime}\right)\end{array}$ & $\begin{array}{c}\mathrm{PA}_{\mathrm{r}, \mathrm{b}} \\
\left({ }^{\circ}\right)\end{array}$ & $\begin{array}{c}D_{\mathrm{r}, \mathrm{b}, 0} \\
\left({ }^{\prime}\right)\end{array}$ & $\begin{array}{c}d_{\mathrm{r}, \mathrm{b}, 0} \\
\left(^{\prime}\right)\end{array}$ & $\begin{array}{l}\theta_{\mathrm{r}, \mathrm{b}} \\
\left({ }^{\circ}\right)\end{array}$ \\
\hline $\mathrm{rs}$ & 0.73 & 0.56 & 77.9 & 0.73 & 0.58 & 24.3 & $\mathrm{R}^{\prime} \mathrm{L}$ & 2.81 & 1.52 & 68.5 & 2.81 & 2.14 & 178.2 \\
\hline bār & 0.54 & 0.16 & 119.7 & 0.56 & 0.16 & 63.5 & $\underline{\mathrm{rs}}$ & $\begin{array}{l}1.28 \\
0.49\end{array}$ & $\begin{array}{l}0.64 \\
024\end{array}$ & $\begin{array}{l}58.6 \\
142\end{array}$ & $\begin{array}{l}1.31 \\
0.64\end{array}$ & $\begin{array}{l}0.88 \\
026\end{array}$ & $\begin{array}{l}159.1 \\
1126\end{array}$ \\
\hline
\end{tabular}

NGC 4402

$\left(\mathrm{R}^{\prime}\right) \mathrm{SAB}(\mathrm{s}) \mathrm{c}$

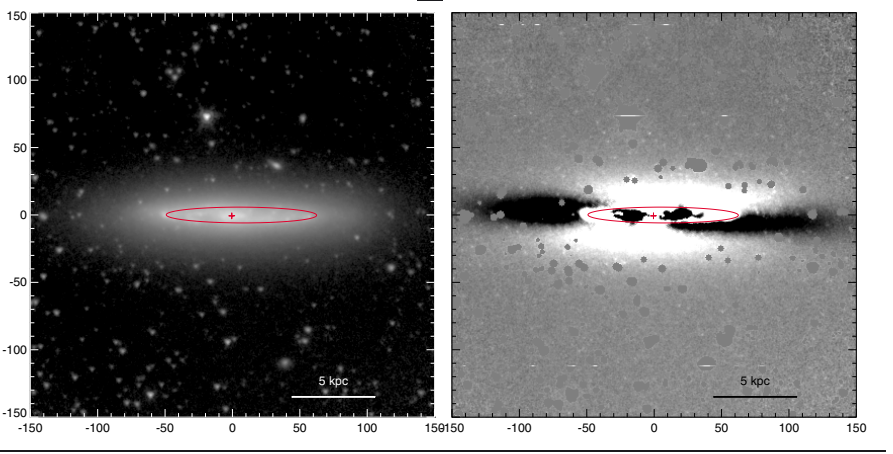

NGC 4411A

$\mathrm{SB}(\mathrm{rs}) \mathrm{cd}$

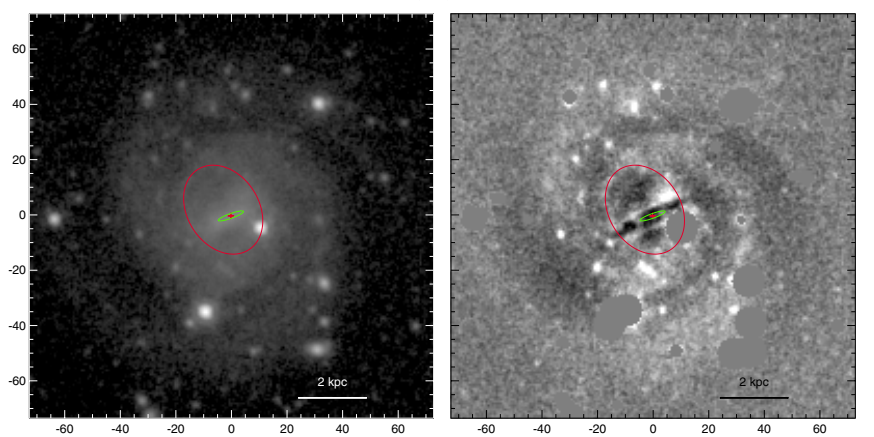


NGC 4414

$\mathrm{SA}(\mathrm{rl}) \mathrm{bc}$
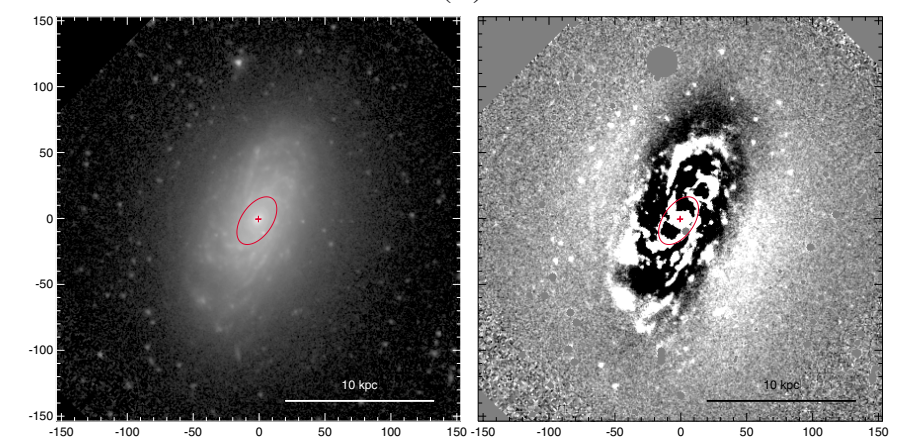

\begin{tabular}{|c|c|c|c|c|c|c|c|c|c|c|c|c|c|}
\hline Feature & $\begin{array}{c}D_{\mathrm{r}, \mathrm{b}} \\
\left(^{\prime}\right) \\
\end{array}$ & $\begin{array}{c}d_{\mathrm{r}, \mathrm{b}} \\
\left({ }^{\prime}\right)\end{array}$ & $\begin{array}{c}\mathrm{PA}_{\mathrm{r}, \mathrm{b}} \\
\left({ }^{\circ}\right)\end{array}$ & $\begin{array}{c}D_{\mathrm{r}, \mathrm{b}, 0} \\
\left({ }^{\prime}\right)\end{array}$ & $\begin{array}{c}d_{\mathrm{r}, \mathrm{b}, 0} \\
\left(^{\prime}\right)\end{array}$ & $\begin{array}{l}\theta_{\mathrm{r}, \mathrm{b}} \\
\left({ }^{\circ}\right)\end{array}$ & "Feature & $\begin{array}{c}D_{\mathrm{r}, \mathrm{b}} \\
\left({ }^{\prime}\right)\end{array}$ & $\begin{array}{c}d_{\mathrm{r}, \mathrm{b}} \\
\left({ }^{\prime}\right)\end{array}$ & $\begin{array}{c}\mathrm{PA}_{\mathrm{r}, \mathrm{b}} \\
\left({ }^{\circ}\right)\end{array}$ & $\begin{array}{c}D_{\mathrm{r}, \mathrm{b}, 0} \\
\left({ }^{\prime}\right)\end{array}$ & $\begin{array}{c}d_{\mathrm{r}, \mathrm{b}, 0} \\
\left(^{\prime}\right)\end{array}$ & $\begin{array}{l}\theta_{\mathrm{r}, \mathrm{b}} \\
\left({ }^{\circ}\right)\end{array}$ \\
\hline $\mathrm{rl}$ & 0.68 & 0.39 & 145.2 & 0.82 & 0.60 & 122.0 & & $\begin{array}{l}.08 \\
0.41\end{array}$ & $\begin{array}{l}1.01 \\
0.32\end{array}$ & $\begin{array}{l}98.4 \\
66.6\end{array}$ & $\begin{array}{l}1.17 \\
0.43\end{array}$ & $\begin{array}{l}1.07 \\
0.36\end{array}$ & $\begin{array}{c}82.4 \\
139.6\end{array}$ \\
\hline
\end{tabular}
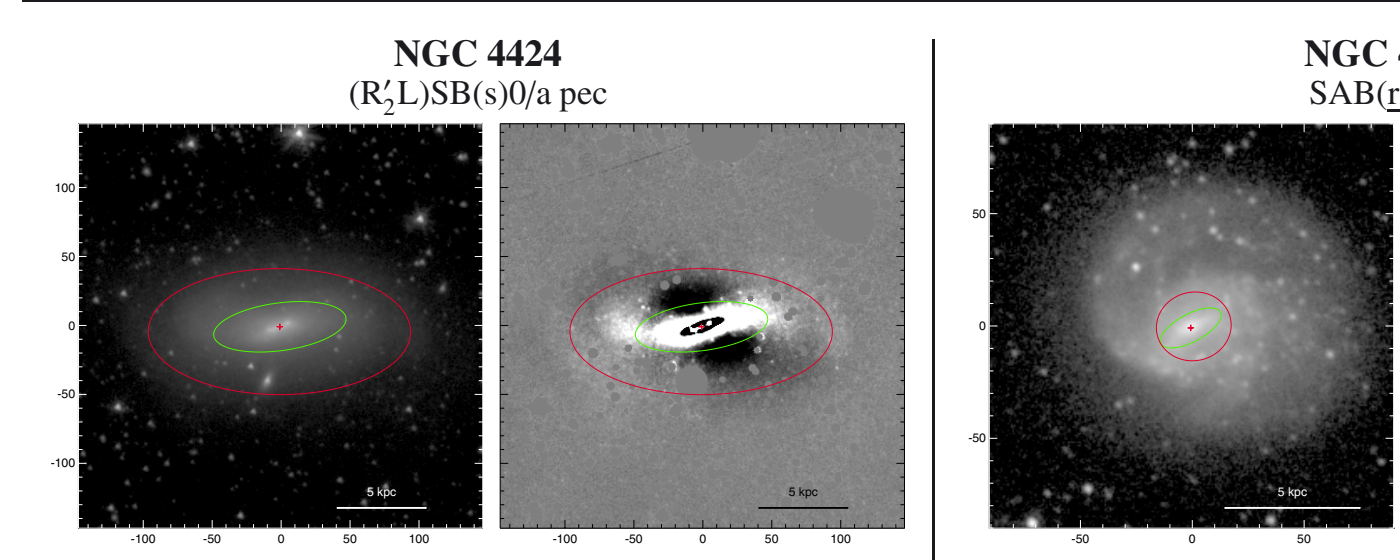

NGC 4430

$\mathrm{SAB}(\underline{\mathrm{r}} \mathrm{s}) \mathrm{dm}$

\begin{tabular}{ccccccc|ccccccc}
\hline \hline Feature & $\begin{array}{c}D_{\mathrm{r}, \mathrm{b}} \\
\left({ }^{\prime}\right)\end{array}$ & $\begin{array}{c}d_{\mathrm{r}, \mathrm{b}} \\
\left({ }^{\prime}\right)\end{array}$ & $\begin{array}{c}\mathrm{PA}_{\mathrm{r}, \mathrm{b}} \\
\left({ }^{\circ}\right)\end{array}$ & $\begin{array}{c}D_{\mathrm{r}, \mathrm{b}, 0} \\
\left({ }^{\prime}\right)\end{array}$ & $\begin{array}{c}d_{\mathrm{r}, \mathrm{b}, 0} \\
\left({ }^{\prime}\right)\end{array}$ & $\begin{array}{c}\theta_{\mathrm{r}, \mathrm{b}} \\
\left({ }^{\circ}\right)\end{array}$ & Feature & $\begin{array}{c}D_{\mathrm{r}, \mathrm{b}} \\
\left({ }^{\prime}\right)\end{array}$ & $\begin{array}{c}d_{\mathrm{r}, \mathrm{b}} \\
\left({ }^{\prime}\right)\end{array}$ & $\begin{array}{c}\mathrm{PA}_{\mathrm{r}, \mathrm{b}} \\
\left({ }^{\circ}\right)\end{array}$ & $\begin{array}{c}D_{\mathrm{r}, \mathrm{b}, 0} \\
\left({ }^{\prime}\right)\end{array}$ & $\begin{array}{c}d_{\mathrm{r}, \mathrm{b}, 0} \\
\left({ }^{\prime}\right)\end{array}$ & $\begin{array}{c}\theta_{\mathrm{r}, \mathrm{b}} \\
\left({ }^{\circ}\right)\end{array}$ \\
\hline $\mathrm{R}_{2}^{\prime} \mathrm{L}$ & 3.18 & 1.53 & 89.9 & 3.25 & 2.70 & 22.5 & $\underline{\mathrm{rs}}$ & 0.56 & 0.51 & 102.8 & 0.62 & 0.54 & 72.6 \\
$\mathrm{bar}$ & 1.62 & 0.58 & 97.6 & 1.73 & 0.98 & 29.1 & $\mathrm{bar}$ & 0.50 & 0.21 & 118.1 & 0.55 & 0.22 & 50.5 \\
\hline
\end{tabular}

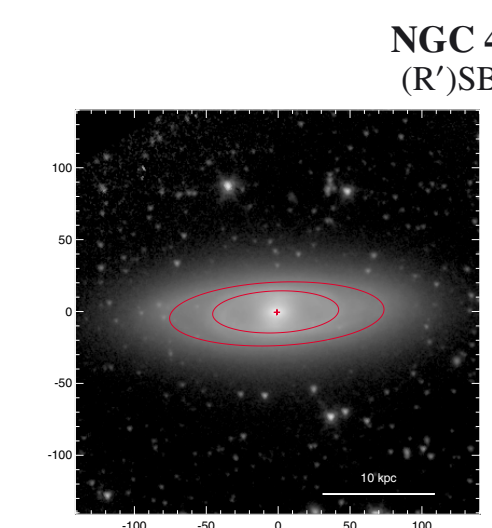

NGC 4448

$\left(\mathrm{R}^{\prime}\right) \mathrm{SB}(\mathrm{r}) \mathrm{a}$

\begin{tabular}{|c|c|c|c|c|c|c|c|c|c|c|c|c|c|}
\hline Feature & $\begin{array}{c}D_{\mathrm{r}, \mathrm{b}} \\
\left({ }^{\prime}\right)\end{array}$ & $\begin{array}{c}d_{\mathrm{r}, \mathrm{b}} \\
\left({ }^{\prime}\right)\end{array}$ & $\begin{array}{c}\mathrm{PA}_{\mathrm{r}, \mathrm{b}} \\
\left({ }^{\circ}\right)\end{array}$ & $\begin{array}{c}D_{\mathrm{r}, \mathrm{b}, 0} \\
\left({ }^{\prime}\right)\end{array}$ & $\begin{array}{c}d_{\mathrm{r}, \mathrm{b}, 0} \\
\left(^{\prime}\right)\end{array}$ & $\begin{array}{c}\theta_{\mathrm{r}, \mathrm{b}} \\
\left({ }^{\circ}\right)\end{array}$ & Feature & $\begin{array}{c}D_{\mathrm{r}, \mathrm{b}} \\
\left({ }^{\prime}\right)\end{array}$ & $\begin{array}{c}d_{\mathrm{r}, \mathrm{b}} \\
\left({ }^{\prime}\right)\end{array}$ & $\begin{array}{c}\mathrm{PA}_{\mathrm{r}, \mathrm{b}} \\
\left({ }^{\circ}\right)\end{array}$ & $\begin{array}{c}D_{\mathrm{r}, \mathrm{b}, 0} \\
\left(^{\prime}\right)\end{array}$ & $\begin{array}{c}d_{\mathrm{r}, \mathrm{b}, 0} \\
\left({ }^{\prime}\right)\end{array}$ & $\begin{array}{l}\theta_{\mathrm{r}, \mathrm{b}} \\
\left({ }^{\circ}\right)\end{array}$ \\
\hline$\overline{\mathrm{R}^{\prime}}$ & 2.50 & 0.74 & 92.6 & 2.50 & 2.28 & 10.9 & $\overline{\mathrm{R}^{\prime}}$ & 3.43 & 2.20 & 164.1 & 3.56 & 3.04 & 145.7 \\
\hline$r$ & 1.47 & 0.49 & 92.2 & 1.51 & 1.47 & 82.6 & $\begin{array}{l}\mathrm{rs} \\
\mathrm{bar}\end{array}$ & $\begin{array}{l}1.51 \\
1.46\end{array}$ & $\begin{array}{l}0.96 \\
0.68\end{array}$ & $\begin{array}{l}10.0 \\
7.0\end{array}$ & $\begin{array}{l}1.64 \\
1.52\end{array}$ & $\begin{array}{l}1.28 \\
0.94\end{array}$ & $\begin{array}{l}44.2 \\
25.0\end{array}$ \\
\hline
\end{tabular}

NGC 4416

(RL)SB(rs)dm

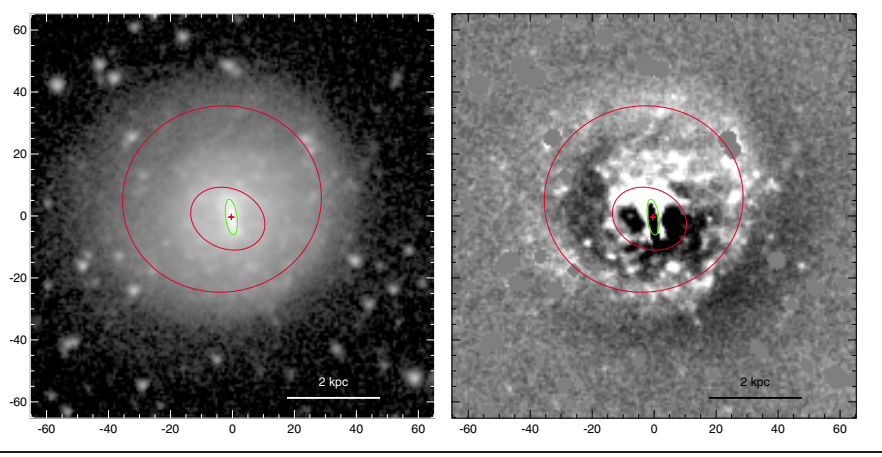

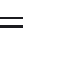




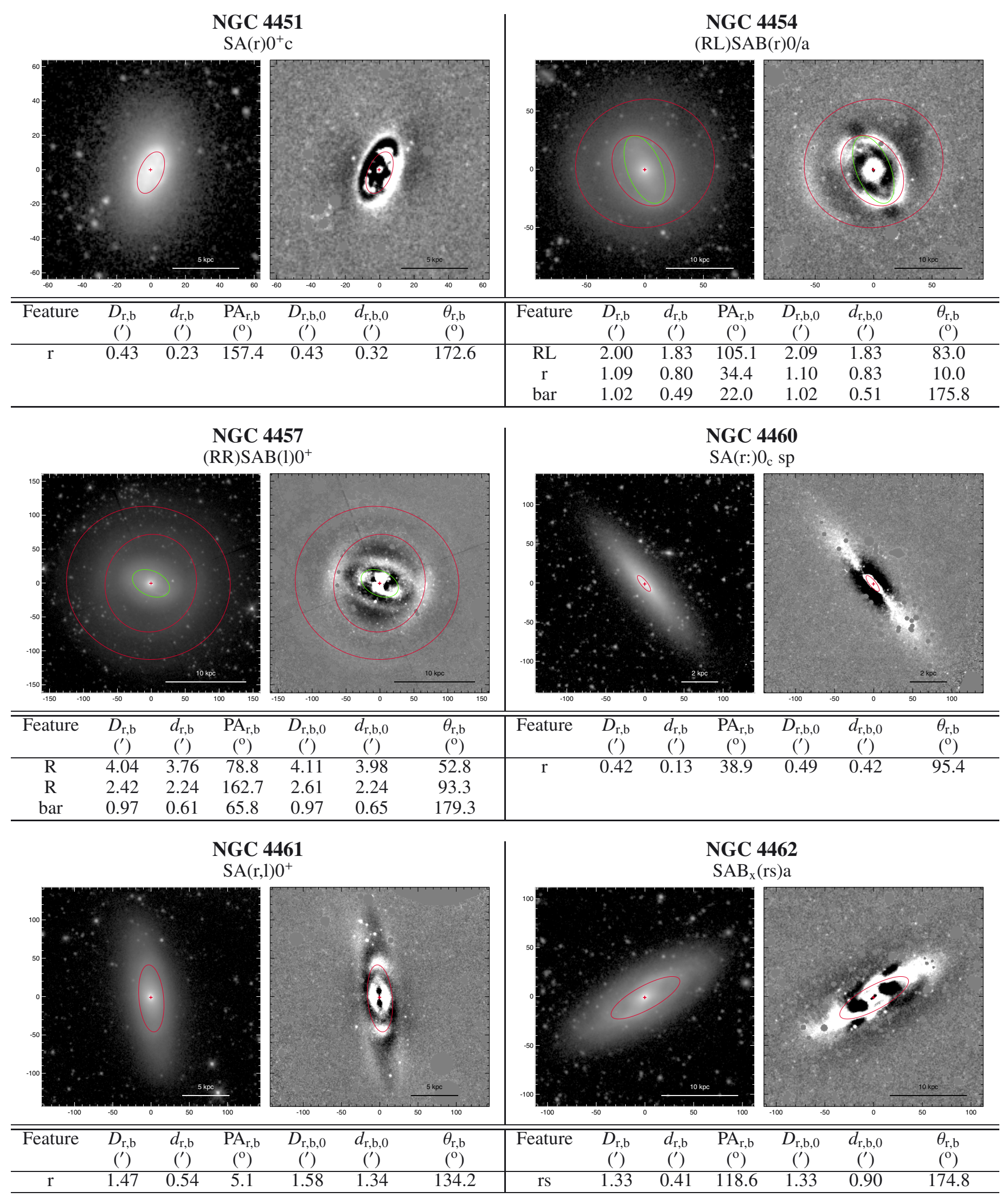




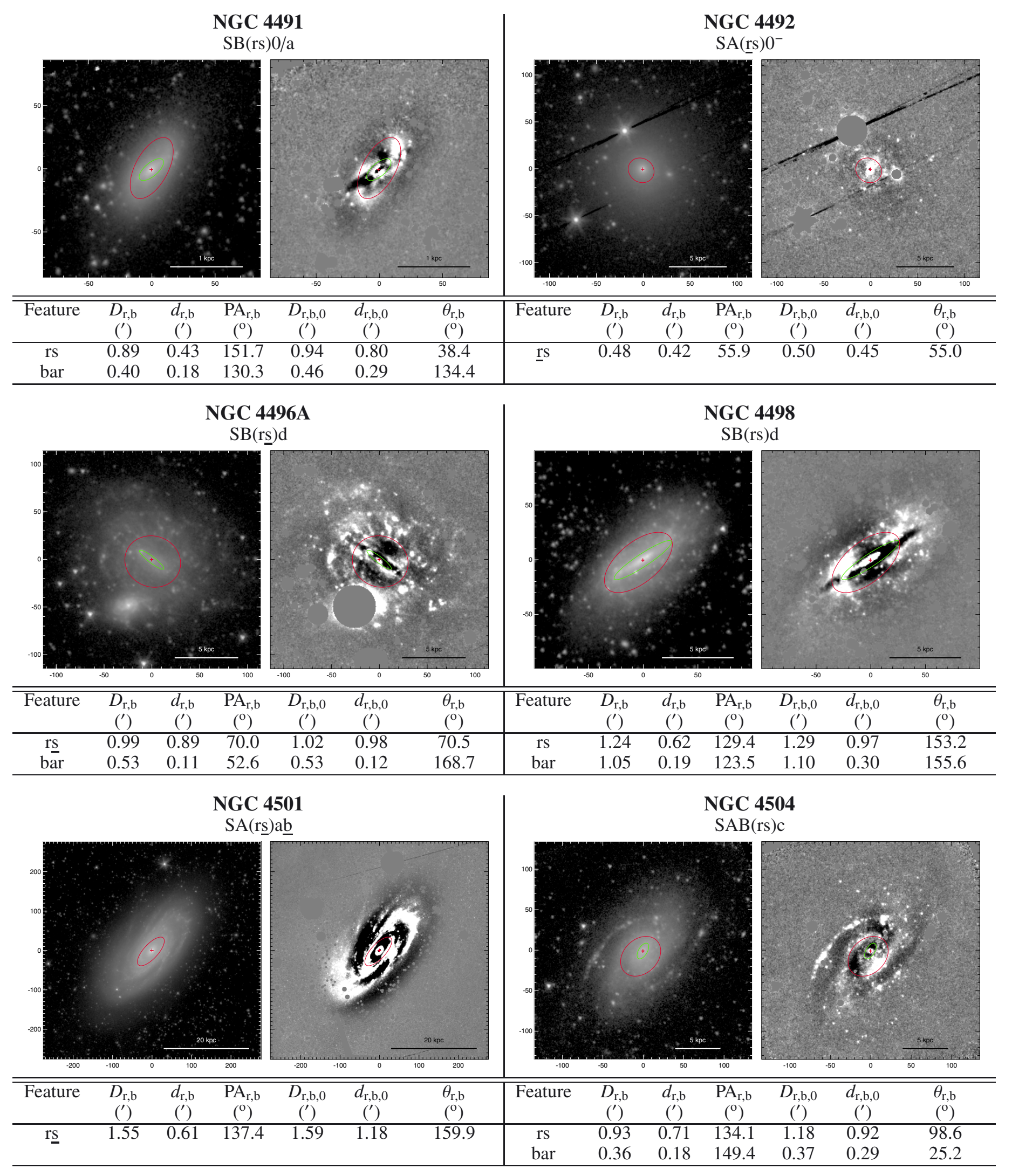




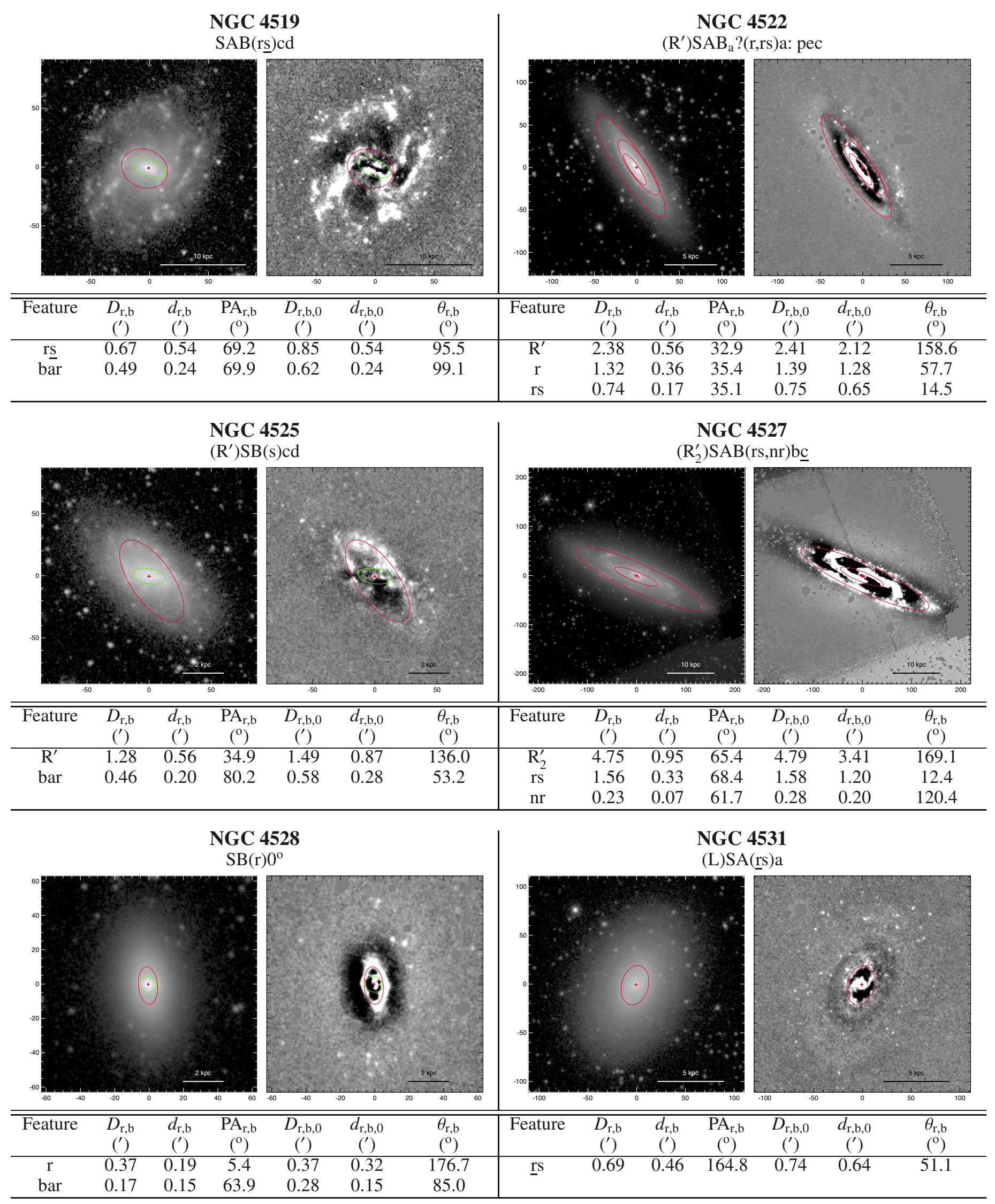


NGC 4536

$\mathrm{SAB}(\mathrm{rs}) \underline{\mathrm{b} c}$
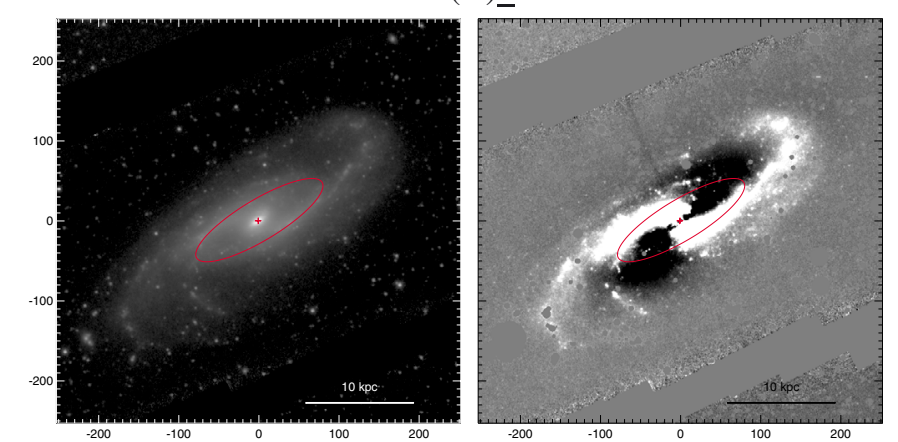

\begin{tabular}{ccccccc|ccccccc}
\hline \hline Feature & $\begin{array}{c}D_{\mathrm{r}, \mathrm{b}} \\
\left({ }^{\prime}\right)\end{array}$ & $\begin{array}{c}d_{\mathrm{r}, \mathrm{b}}\left({ }^{\prime}\right) \\
\left(\mathrm{PA}_{\mathrm{r}, \mathrm{b}}\right. \\
(\mathrm{o})\end{array}$ & $\begin{array}{c}D_{\mathrm{r}, \mathrm{b}, 0} \\
\left({ }^{\prime}\right)\end{array}$ & $\begin{array}{c}d_{\mathrm{r}, \mathrm{b}, 0} \\
\left({ }^{\prime}\right)\end{array}$ & $\begin{array}{c}\theta_{\mathrm{r}, \mathrm{b}} \\
\left({ }^{\circ}\right)\end{array}$ & Feature & $\begin{array}{c}D_{\mathrm{r}, \mathrm{b}} \\
\left({ }^{\prime}\right)\end{array}$ & $\begin{array}{c}d_{\mathrm{r}, \mathrm{b}} \\
\left({ }^{\prime}\right)\end{array}$ & $\begin{array}{c}\mathrm{PA}_{\mathrm{r}, \mathrm{b}} \\
\left({ }^{\circ}\right)\end{array}$ & $\begin{array}{c}D_{\mathrm{r}, \mathrm{b}, 0} \\
\left({ }^{\prime}\right)\end{array}$ & $\begin{array}{c}d_{\mathrm{r}, \mathrm{b}, 0} \\
\left({ }^{\prime}\right)\end{array}$ & $\begin{array}{c}\theta_{\mathrm{r}, \mathrm{b}} \\
\left({ }^{\circ}\right)\end{array}$ \\
\hline $\mathrm{rs}$ & 3.05 & 0.85 & 121.1 & 3.11 & 1.92 & 164.8 & $\mathrm{rs}$ & 0.69 & 0.66 & 111.6 & 0.72 & 0.66 & 72.5 \\
& & & & & & & $\mathrm{bar}$ & 0.30 & 0.12 & 53.6 & 0.30 & 0.13 & 177.4 \\
\hline
\end{tabular}

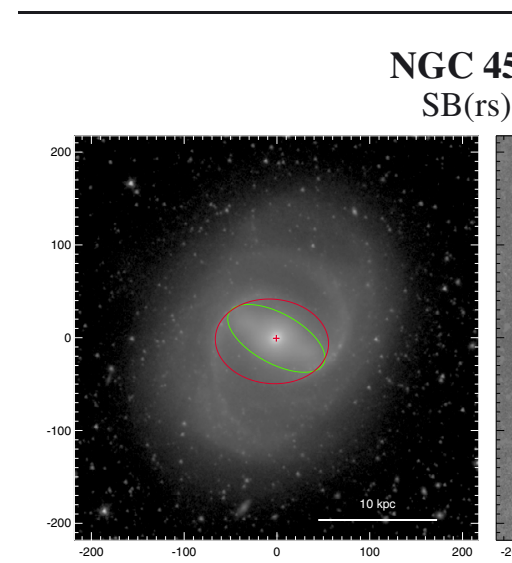

NGC 4548

$\mathrm{SB}(\mathrm{rs}) \mathrm{a}$

\begin{tabular}{|c|c|c|c|c|c|c|c|c|c|c|c|c|c|}
\hline Feature & $\begin{array}{c}D_{\mathrm{r}, \mathrm{b}} \\
\left(^{\prime}\right)\end{array}$ & $\begin{array}{c}d_{\mathrm{r}, \mathrm{b}} \\
\left(^{\prime}\right)\end{array}$ & $\begin{array}{c}\mathrm{PA}_{\mathrm{r}, \mathrm{b}} \\
\left({ }^{\circ}\right)\end{array}$ & $\begin{array}{c}D_{\mathrm{r}, \mathrm{b}, 0} \\
\left(^{\prime}\right)\end{array}$ & $\begin{array}{c}d_{\mathrm{r}, \mathrm{b}, 0} \\
\left({ }^{\prime}\right)\end{array}$ & $\begin{array}{c}\theta_{\mathrm{r}, \mathrm{b}} \\
\left({ }^{\circ}\right)\end{array}$ & Feature & $\begin{array}{c}D_{\mathrm{r}, \mathrm{b}} \\
\left(^{\prime}\right)\end{array}$ & $\begin{array}{c}d_{\mathrm{r}, \mathrm{b}} \\
\left({ }^{\prime}\right)\end{array}$ & $\begin{array}{c}\mathrm{PA}_{\mathrm{r}, \mathrm{b}} \\
\left(^{\mathrm{o}}\right)\end{array}$ & $\begin{array}{c}D_{\mathrm{r}, \mathrm{b}, 0} \\
\left(^{\prime}\right)\end{array}$ & $\begin{array}{c}d_{\mathrm{r}, \mathrm{b}, 0} \\
\left(^{\prime}\right)\end{array}$ & $\begin{array}{c}\theta_{\mathrm{r}, \mathrm{b}} \\
\left.\mathrm{o}^{\mathrm{o}}\right)\end{array}$ \\
\hline rs & 2.04 & 1.52 & 85.2 & 2.56 & 1.56 & 103.7 & $\mathrm{r}$ & 3.12 & 0.22 & 135.6 & 3.13 & 1.33 & 3.3 \\
\hline bar & 1.93 & 0.90 & 61.3 & 2.49 & 0.90 & 91.5 & & & & & & & \\
\hline
\end{tabular}
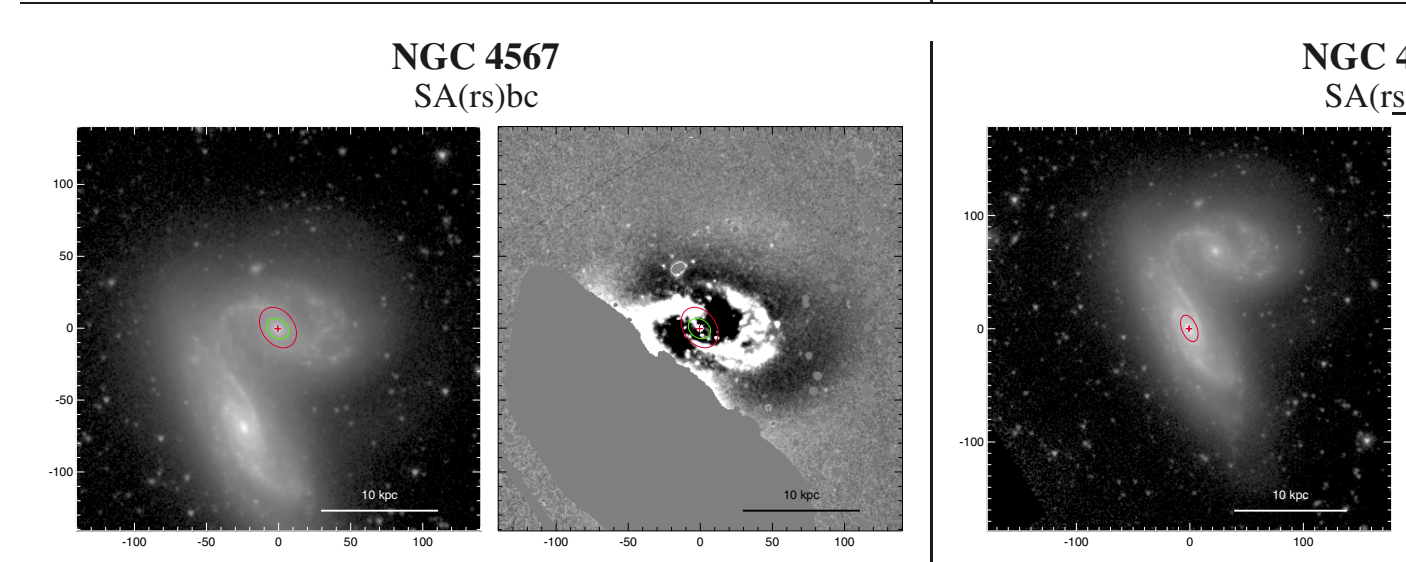

NGC 4568

$\mathrm{SA}(\mathrm{rs}) \mathrm{bc}$

\begin{tabular}{ccccccc|ccccccc}
\hline \hline Feature & $\begin{array}{c}D_{\mathrm{r}, \mathrm{b}} \\
\left({ }^{\prime}\right)\end{array}$ & $\begin{array}{c}d_{\mathrm{r}, \mathrm{b}} \\
\left({ }^{\prime}\right)\end{array}$ & $\begin{array}{c}\mathrm{PA}_{\mathrm{r}, \mathrm{b}} \\
\left({ }^{\circ}\right)\end{array}$ & $\begin{array}{c}D_{\mathrm{r}, \mathrm{b}, 0} \\
\left({ }^{\prime}\right)\end{array}$ & $\begin{array}{c}d_{\mathrm{r}, \mathrm{b}, 0} \\
\left({ }^{\prime}\right)\end{array}$ & $\begin{array}{c}\theta_{\mathrm{r}, \mathrm{b}} \\
\left({ }^{\circ}\right)\end{array}$ & Feature & $\begin{array}{c}D_{\mathrm{r}, \mathrm{b}} \\
\left({ }^{\prime}\right)\end{array}$ & $\begin{array}{c}d_{\mathrm{r}, \mathrm{b}} \\
\left({ }^{\prime}\right)\end{array}$ & $\begin{array}{c}\mathrm{PA}_{\mathrm{r}, \mathrm{b}} \\
\left({ }^{\circ}\right)\end{array}$ & $\begin{array}{c}D_{\mathrm{r}, \mathrm{b}, 0} \\
\left({ }^{\prime}\right)\end{array}$ & $\begin{array}{c}d_{\mathrm{r}, \mathrm{b}, 0} \\
\left({ }^{\prime}\right)\end{array}$ & $\begin{array}{c}\theta_{\mathrm{r}, \mathrm{b}} \\
\left({ }^{\circ}\right)\end{array}$ \\
\hline $\mathrm{rs}$ & 0.51 & 0.37 & 36.6 & 0.71 & 0.41 & 106.7 & rs & 0.41 & 0.22 & 22.4 & 0.50 & 0.40 & 108.9 \\
$\mathrm{bar}$ & 0.28 & 0.21 & 56.0 & 0.36 & 0.25 & 109.9 & & & & & & & \\
\hline
\end{tabular}


NGC 4569

(L) $\mathrm{SB}_{\mathrm{x}}\left(\mathrm{rs}, \mathrm{x}_{1} \mathrm{r}\right) \mathrm{a}$
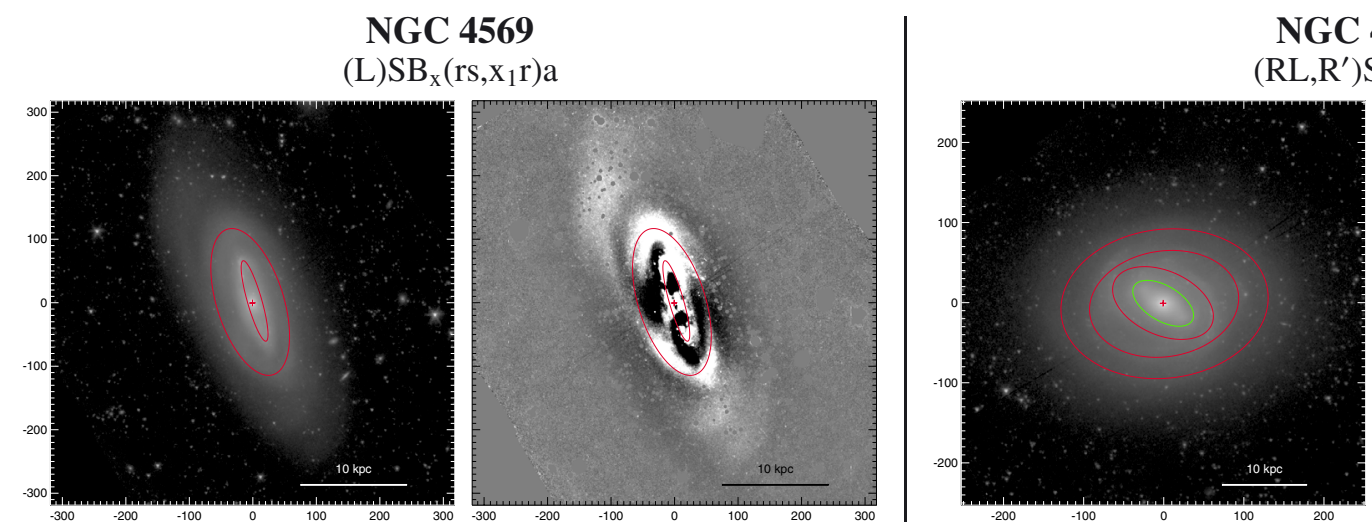

NGC 4579

$\left(\mathrm{RL}, \mathrm{R}^{\prime}\right) \mathrm{SB}(\mathrm{rs}) \mathrm{a}$

\begin{tabular}{ccccccc|ccccccc}
\hline \hline Feature & $\begin{array}{c}D_{\mathrm{r}, \mathrm{b}} \\
\left({ }^{\prime}\right)\end{array}$ & $\begin{array}{c}d_{\mathrm{r}, \mathrm{b}} \\
\left({ }^{\prime}\right)\end{array}$ & $\begin{array}{c}\mathrm{PA}_{\mathrm{r}, \mathrm{b}} \\
\left({ }^{\circ}\right)\end{array}$ & $\begin{array}{c}D_{\mathrm{r}, \mathrm{b}, 0} \\
\left({ }^{\prime}\right)\end{array}$ & $\begin{array}{c}d_{\mathrm{r}, \mathrm{b}, 0} \\
\left({ }^{\prime}\right)\end{array}$ & $\begin{array}{c}\theta_{\mathrm{r}, \mathrm{b}} \\
\left({ }^{(}\right)\end{array}$ & Feature & $\begin{array}{c}D_{\mathrm{r}, \mathrm{b}} \\
\left({ }^{\prime}\right)\end{array}$ & $\begin{array}{c}d_{\mathrm{r}, \mathrm{b}} \\
\left({ }^{\prime}\right)\end{array}$ & $\begin{array}{c}\mathrm{PA}_{\mathrm{r}, \mathrm{b}} \\
\left({ }^{\circ}\right)\end{array}$ & $\begin{array}{c}D_{\mathrm{r}, \mathrm{b}, 0} \\
\left({ }^{\prime}\right)\end{array}$ & $\begin{array}{c}d_{\mathrm{r}, \mathrm{b}, 0} \\
\left({ }^{\prime}\right)\end{array}$ & $\begin{array}{c}\theta_{\mathrm{r}, \mathrm{b}} \\
\left({ }^{\circ}\right)\end{array}$ \\
\hline $\mathrm{rs}$ & 4.01 & 1.76 & 17.5 & 4.73 & 3.66 & 121.4 & $\mathrm{RL}$ & 4.33 & 3.10 & 96.7 & 4.39 & 4.10 & 27.5 \\
$\mathrm{x}_{1} \mathrm{r}$ & 2.20 & 0.38 & 16.0 & 2.34 & 0.87 & 156.3 & $\mathrm{R}$ & 3.13 & 2.20 & 98.7 & 3.18 & 2.90 & 29.6 \\
& & & & & & & $\mathrm{rs}$ & 2.22 & 1.34 & 66.1 & 2.44 & 1.62 & 135.4 \\
& & & & & & & $\mathrm{bar}$ & 1.41 & 0.73 & 59.2 & 1.59 & 0.87 & 132.3 \\
\hline
\end{tabular}

NGC 4580

$\left(\mathrm{R}^{\prime}\right) \mathrm{SA}\left(\mathrm{rs}_{1}, \mathrm{rs}_{2}\right) \mathrm{a}$
NGC 4591

$\mathrm{SAB}(\mathrm{rs}) \mathrm{a}$
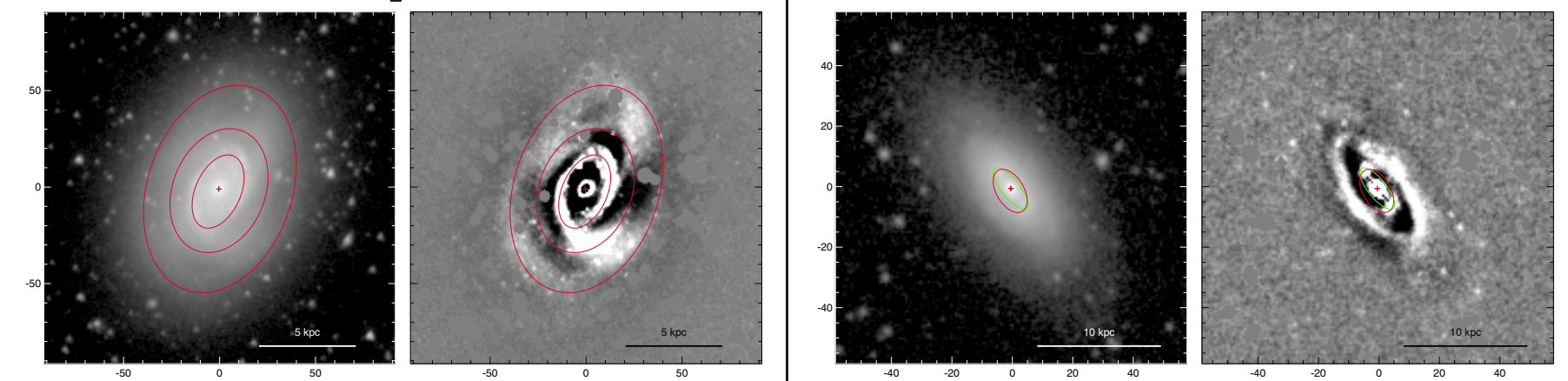

\begin{tabular}{ccccccc|ccccccc}
\hline \hline Feature & $\begin{array}{c}D_{\mathrm{r}, \mathrm{b}} \\
\left({ }^{\prime}\right)\end{array}$ & $\begin{array}{c}d_{\mathrm{r}, \mathrm{b}} \\
\left({ }^{\prime}\right)\end{array}$ & $\begin{array}{c}\mathrm{PA}_{\mathrm{r}, \mathrm{b}} \\
\left({ }^{\circ}\right)\end{array}$ & $\begin{array}{c}D_{\mathrm{r}, \mathrm{b}, 0} \\
\left({ }^{\prime}\right)\end{array}$ & $\begin{array}{c}d_{\mathrm{r}, \mathrm{b}, 0} \\
\left({ }^{\prime}\right)\end{array}$ & $\begin{array}{c}\theta_{\mathrm{r}, \mathrm{b}} \\
\left({ }^{(}\right)\end{array}$ & Feature & $\begin{array}{c}D_{\mathrm{r}, \mathrm{b}} \\
\left({ }^{\prime}\right)\end{array}$ & $\begin{array}{c}d_{\mathrm{r}, \mathrm{b}} \\
\left({ }^{\prime}\right)\end{array}$ & $\begin{array}{c}\mathrm{PA}_{\mathrm{r}, \mathrm{b}} \\
\left({ }^{\circ}\right)\end{array}$ & $\begin{array}{c}D_{\mathrm{r}, \mathrm{b}, 0} \\
\left({ }^{\prime}\right)\end{array}$ & $\begin{array}{c}d_{\mathrm{r}, \mathrm{b}, 0} \\
\left({ }^{\prime}\right)\end{array}$ & $\begin{array}{c}\theta_{\mathrm{r}, \mathrm{b}} \\
\left({ }^{\circ}\right)\end{array}$ \\
\hline $\mathrm{R}^{\prime}$ & 1.86 & 1.26 & 161.6 & 1.88 & 1.64 & 20.8 & bar & 0.28 & 0.10 & 38.7 & 0.28 & 0.19 & 6.2 \\
$\mathrm{rs}$ & 1.12 & 0.81 & 158.3 & 1.12 & 1.06 & 19.4 & $\mathrm{rs}$ & 0.26 & 0.15 & 31.1 & 0.30 & 0.26 & 115.2 \\
$\mathrm{rs}$ & 0.67 & 0.40 & 156.2 & 0.68 & 0.52 & 2.4 & & & & & & & \\
\hline
\end{tabular}

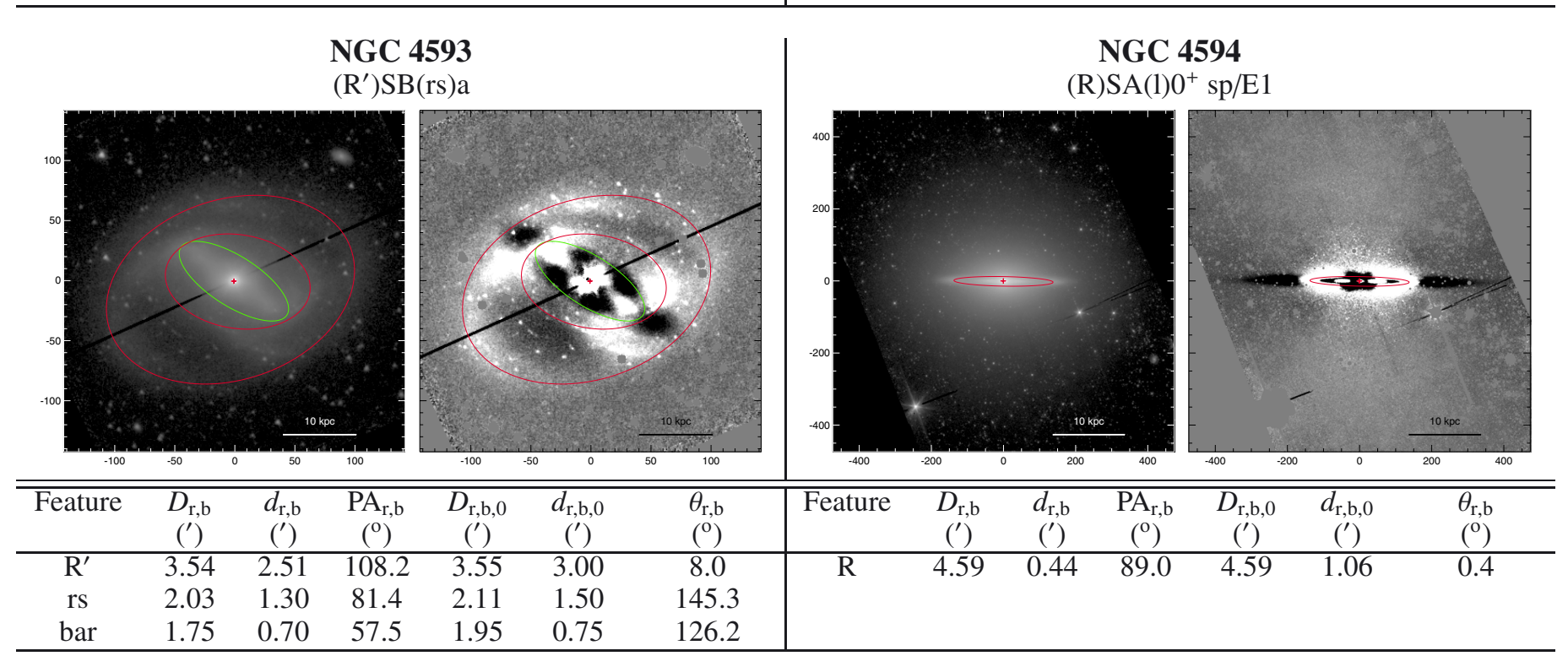




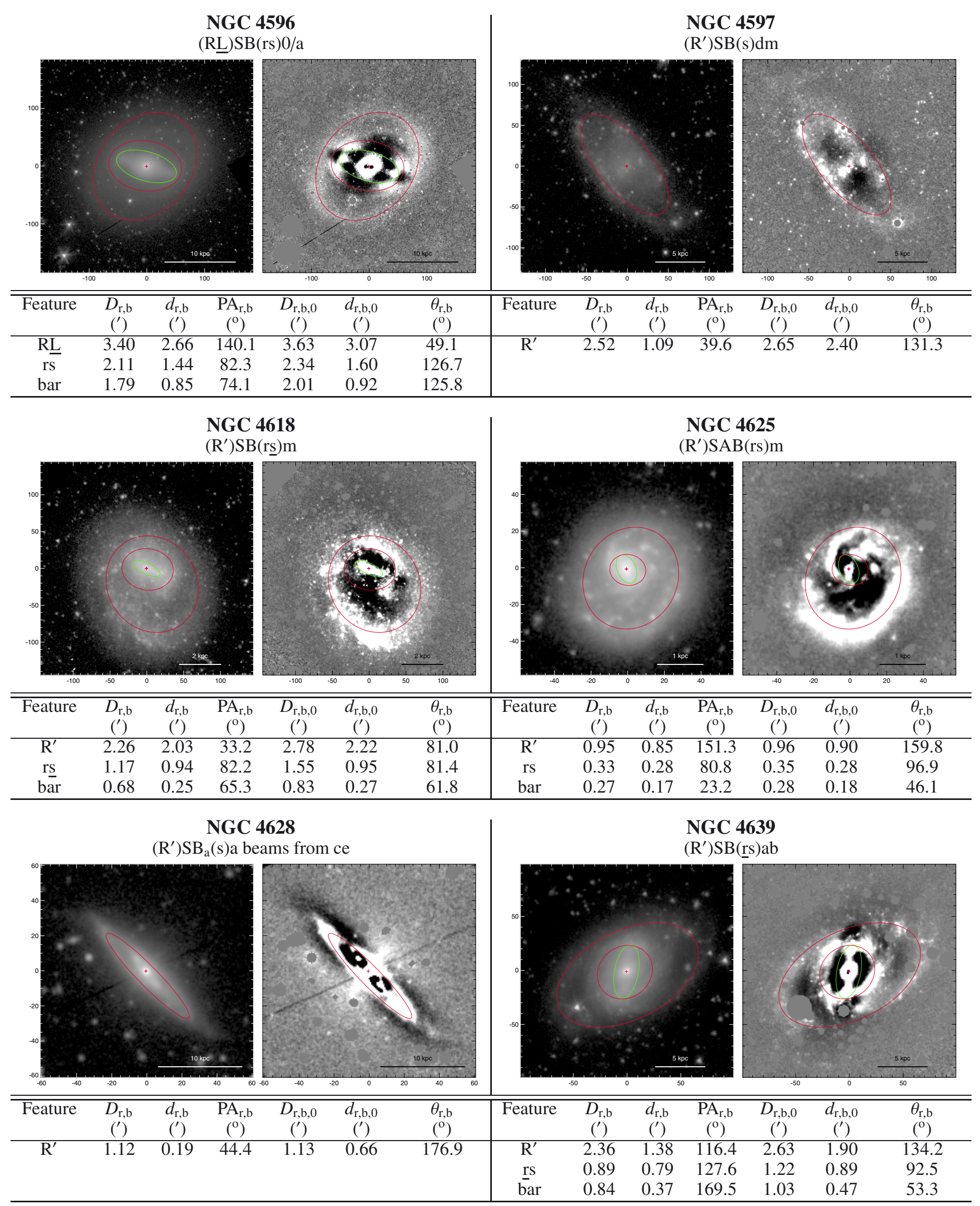




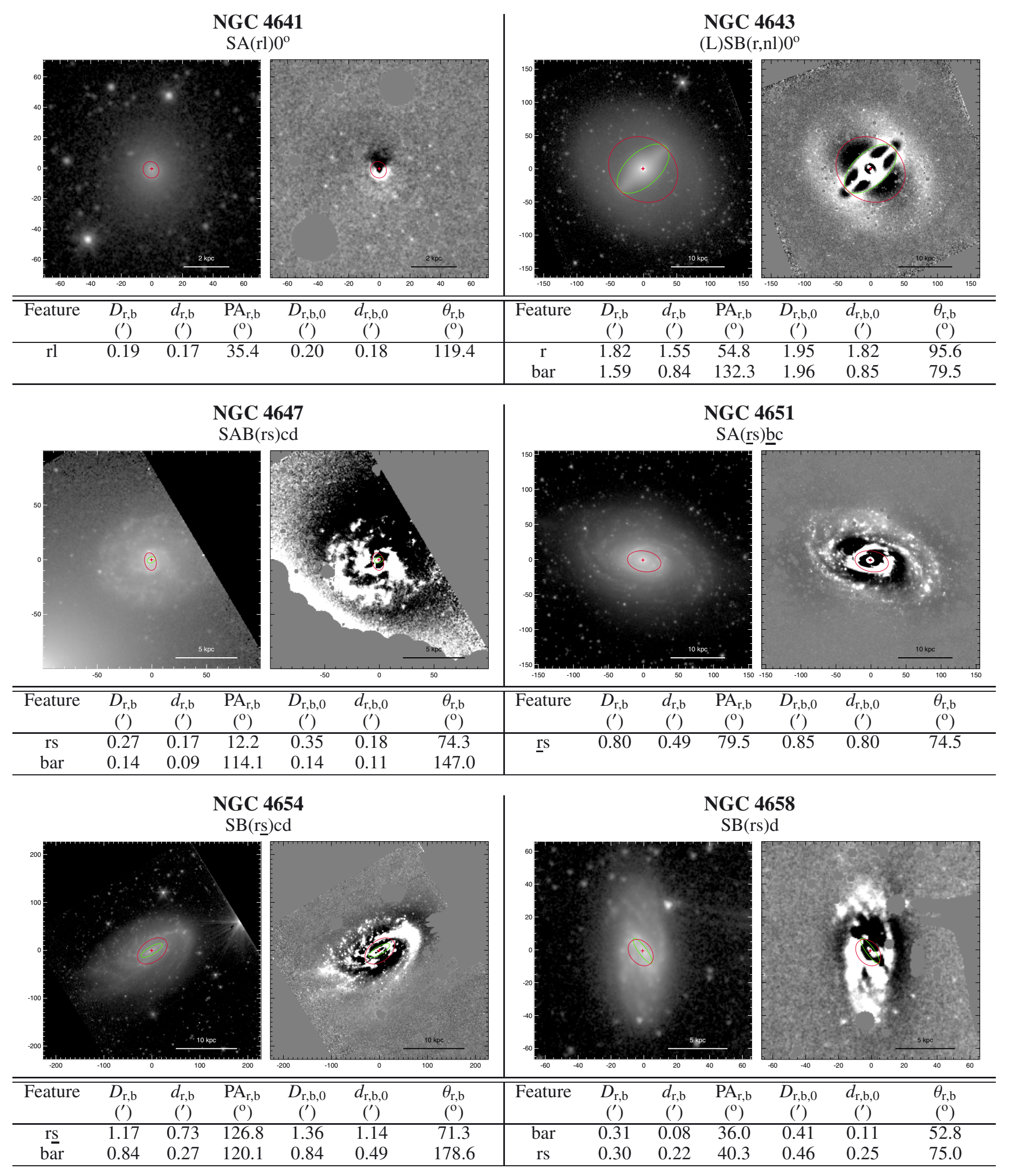


NGC 4659

(R) $\operatorname{SAB}(1) 0^{\circ}$
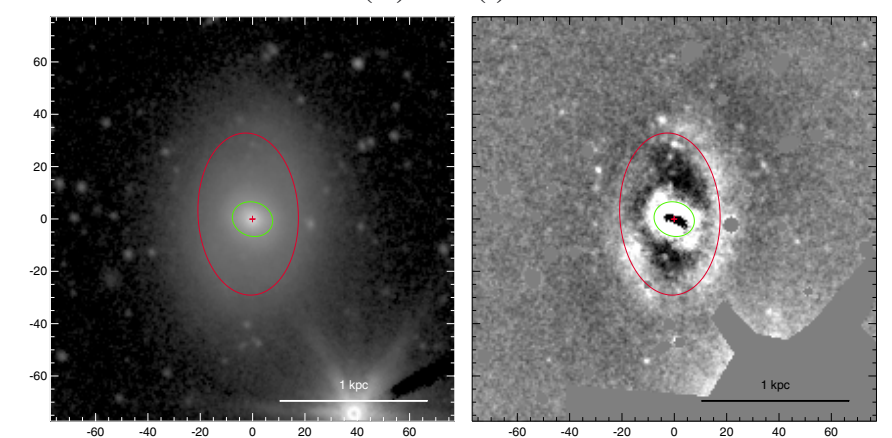

NGC 4680

$\mathrm{SAB}(\mathrm{rs}) \mathrm{b} / \mathrm{Sph}$

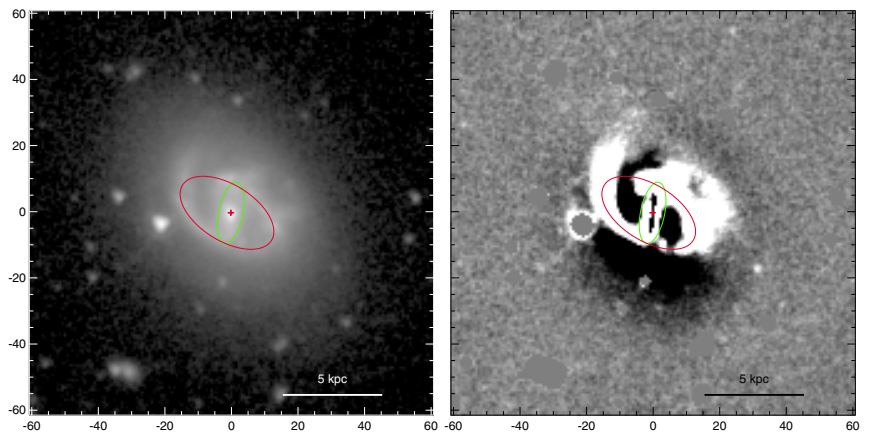

\begin{tabular}{|c|c|c|c|c|c|c|c|c|c|c|c|c|c|}
\hline Feature & $\begin{array}{c}D_{\mathrm{r}, \mathrm{b}} \\
\left({ }^{\prime}\right)\end{array}$ & $\begin{array}{c}d_{\mathrm{r}, \mathrm{b}} \\
\left({ }^{\prime}\right)\end{array}$ & $\begin{array}{c}\mathrm{PA}_{\mathrm{r}, \mathrm{b}} \\
\left({ }^{\circ}\right)\end{array}$ & $\begin{array}{c}D_{\mathrm{r}, \mathrm{b}, 0} \\
\left({ }^{\prime}\right)\end{array}$ & $\begin{array}{c}d_{\mathrm{r}, \mathrm{b}, 0} \\
\left({ }^{\prime}\right)\end{array}$ & $\begin{array}{l}\theta_{\mathrm{r}, \mathrm{b}} \\
\left({ }^{\circ}\right)\end{array}$ & Feature & $\begin{array}{c}D_{\mathrm{r}, \mathrm{b}} \\
\left({ }^{\prime}\right)\end{array}$ & $\begin{array}{c}d_{\mathrm{r}, \mathrm{b}} \\
\left({ }^{\prime}\right)\end{array}$ & $\begin{array}{c}\mathrm{PA}_{\mathrm{r}, \mathrm{b}} \\
\left({ }^{\circ}\right)\end{array}$ & $\begin{array}{c}D_{\mathrm{r}, \mathrm{b}, 0} \\
\left({ }^{\prime}\right)\end{array}$ & $\begin{array}{c}d_{\mathrm{r}, \mathrm{b}, 0} \\
\left(^{\prime}\right)\end{array}$ & $\begin{array}{l}\theta_{\mathrm{r}, \mathrm{b}} \\
\left({ }^{\circ}\right)\end{array}$ \\
\hline $\mathrm{R}$ & 1.04 & 0.64 & 2.8 & 1.06 & 0.86 & 25.0 & rs & 0.53 & 0.28 & 58.1 & 0.55 & 0.35 & 31.3 \\
\hline bar & 0.26 & 0.22 & 72.4 & 0.36 & 0.22 & 86.1 & bar & 0.31 & 0.12 & 168.5 & 0.37 & 0.13 & 121.0 \\
\hline
\end{tabular}

NGC 4682

$\mathrm{SA}(\mathrm{r}) \mathrm{bc}$

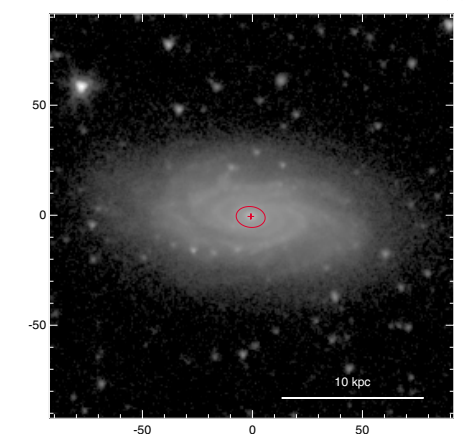

NGC 4689

(R)SA(rs)bc
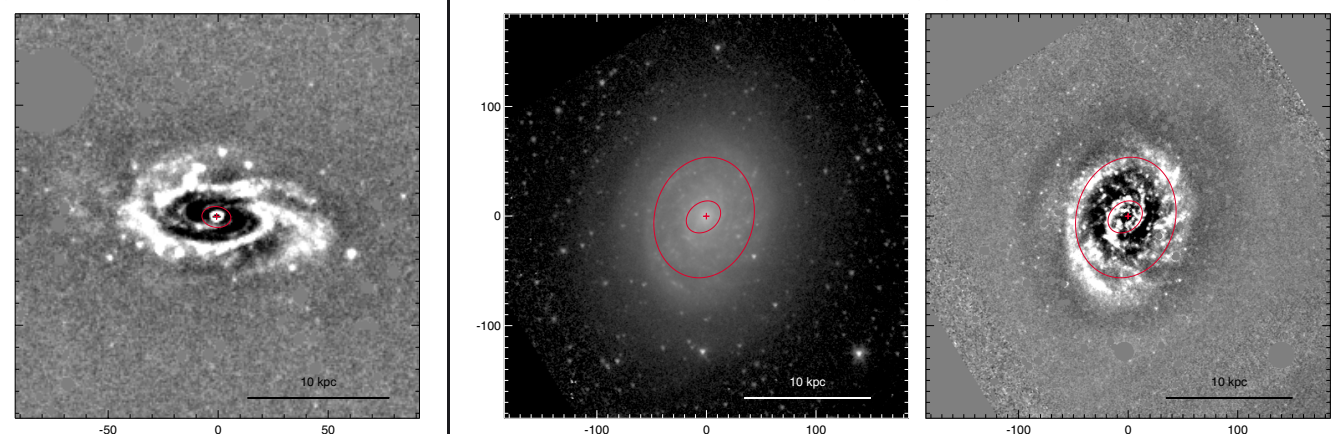

\begin{tabular}{|c|c|c|c|c|c|c|c|c|c|c|c|c|c|}
\hline Feature & $\begin{array}{c}D_{\mathrm{r}, \mathrm{b}} \\
\left({ }^{\prime}\right)\end{array}$ & $\begin{array}{c}d_{\mathrm{r}, \mathrm{b}} \\
(')\end{array}$ & $\begin{array}{c}\mathrm{PA}_{\mathrm{r}, \mathrm{b}} \\
\left({ }^{\circ}\right)\end{array}$ & $\begin{array}{c}D_{\mathrm{r}, \mathrm{b}, 0} \\
\left(^{\prime}\right)\end{array}$ & $\begin{array}{c}d_{\mathrm{r}, \mathrm{b}, 0} \\
\left(^{\prime}\right)\end{array}$ & $\begin{array}{l}\theta_{\mathrm{r}, \mathrm{b}} \\
\left(^{\circ}\right)\end{array}$ & Feature & $\begin{array}{c}D_{\mathrm{r}, \mathrm{b}} \\
\left({ }^{\prime}\right)\end{array}$ & $\begin{array}{c}d_{\mathrm{r}, \mathrm{b}} \\
\left(^{\prime}\right)\end{array}$ & $\begin{array}{c}\mathrm{PA}_{\mathrm{r}, \mathrm{b}} \\
\left(^{\circ}\right)\end{array}$ & $\begin{array}{c}D_{\mathrm{r}, \mathrm{b}, 0} \\
\left(^{\prime}\right)\end{array}$ & $\begin{array}{c}d_{\mathrm{r}, \mathrm{b}, 0} \\
\left(^{\prime}\right)\end{array}$ & $\begin{array}{l}\theta_{\mathrm{r}, \mathrm{b}} \\
\left(^{\circ}\right)\end{array}$ \\
\hline $\mathrm{r}$ & 0.22 & 0.16 & 82.6 & 0.32 & 0.22 & 90.9 & $\bar{R}$ & 1.86 & 1.51 & 165.3 & 1.88 & 1.85 & 62.0 \\
\hline & & & & & & & rs & 0.57 & 0.44 & 127.2 & 0.64 & 0.48 & 120.1 \\
\hline
\end{tabular}

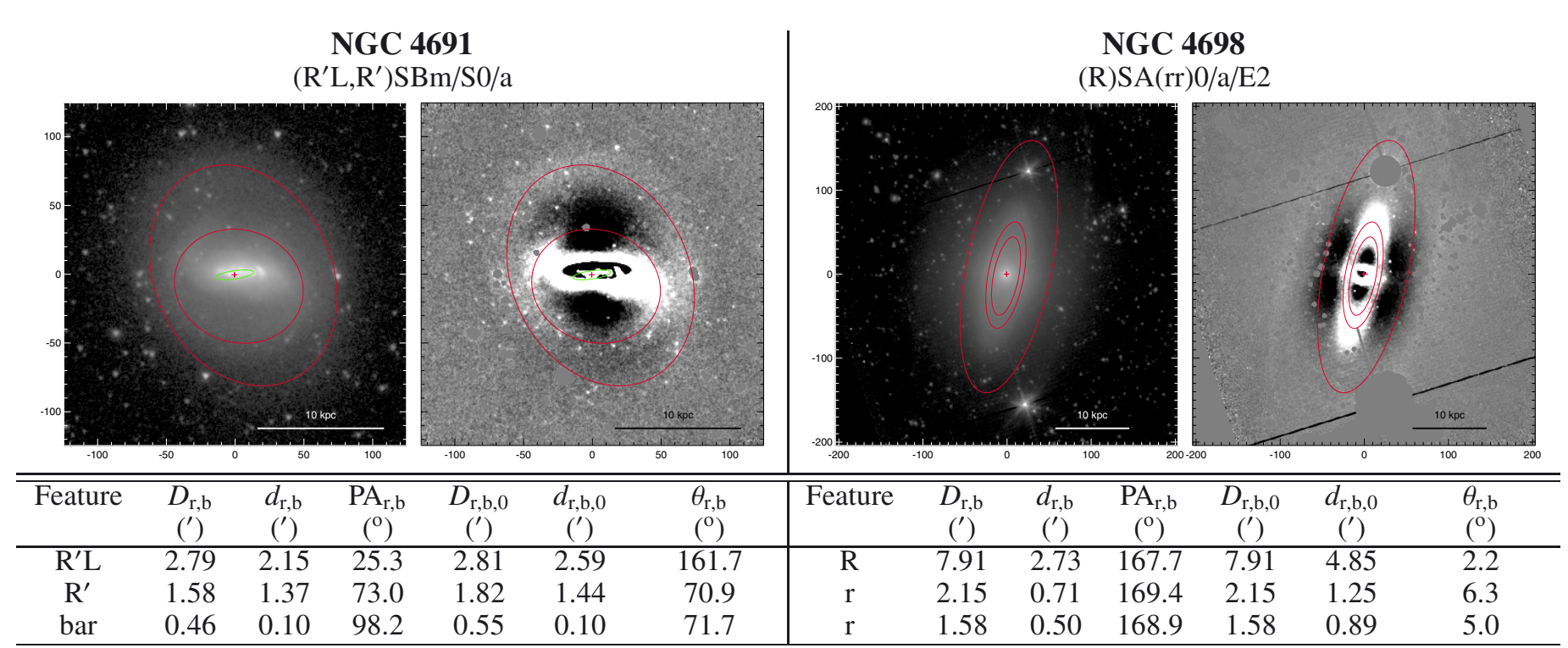


NGC 4699

$\left(\mathrm{R}^{\prime}\right) \mathrm{SB}(1) 0 / \mathrm{a}$
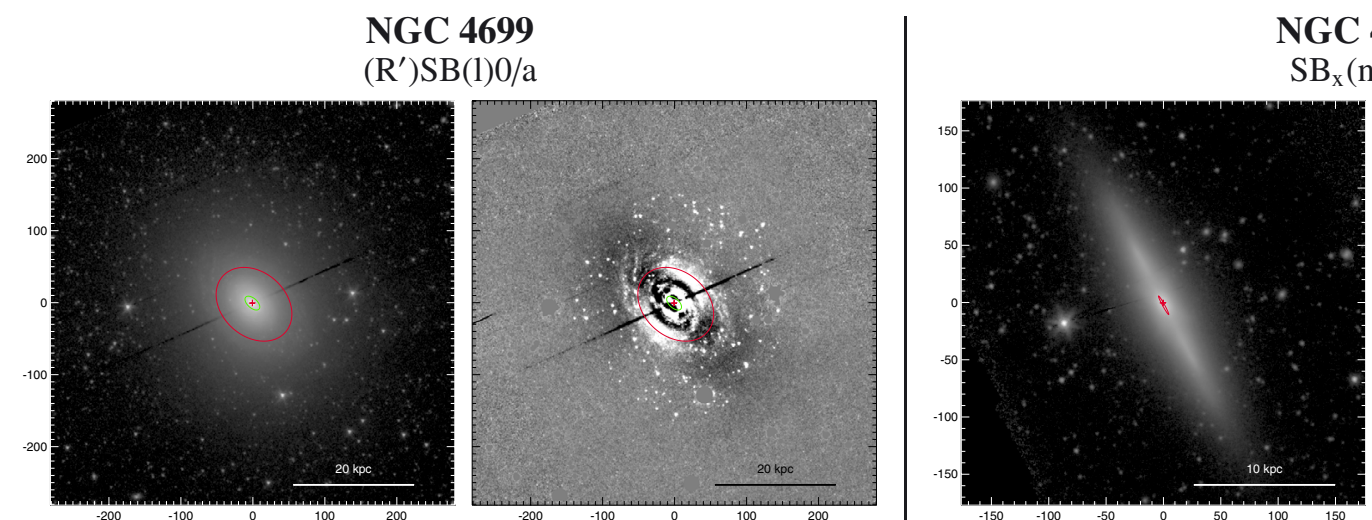

GC 4710

$\mathrm{SB}_{\mathrm{x}}(\mathrm{nr}) 0^{+}$

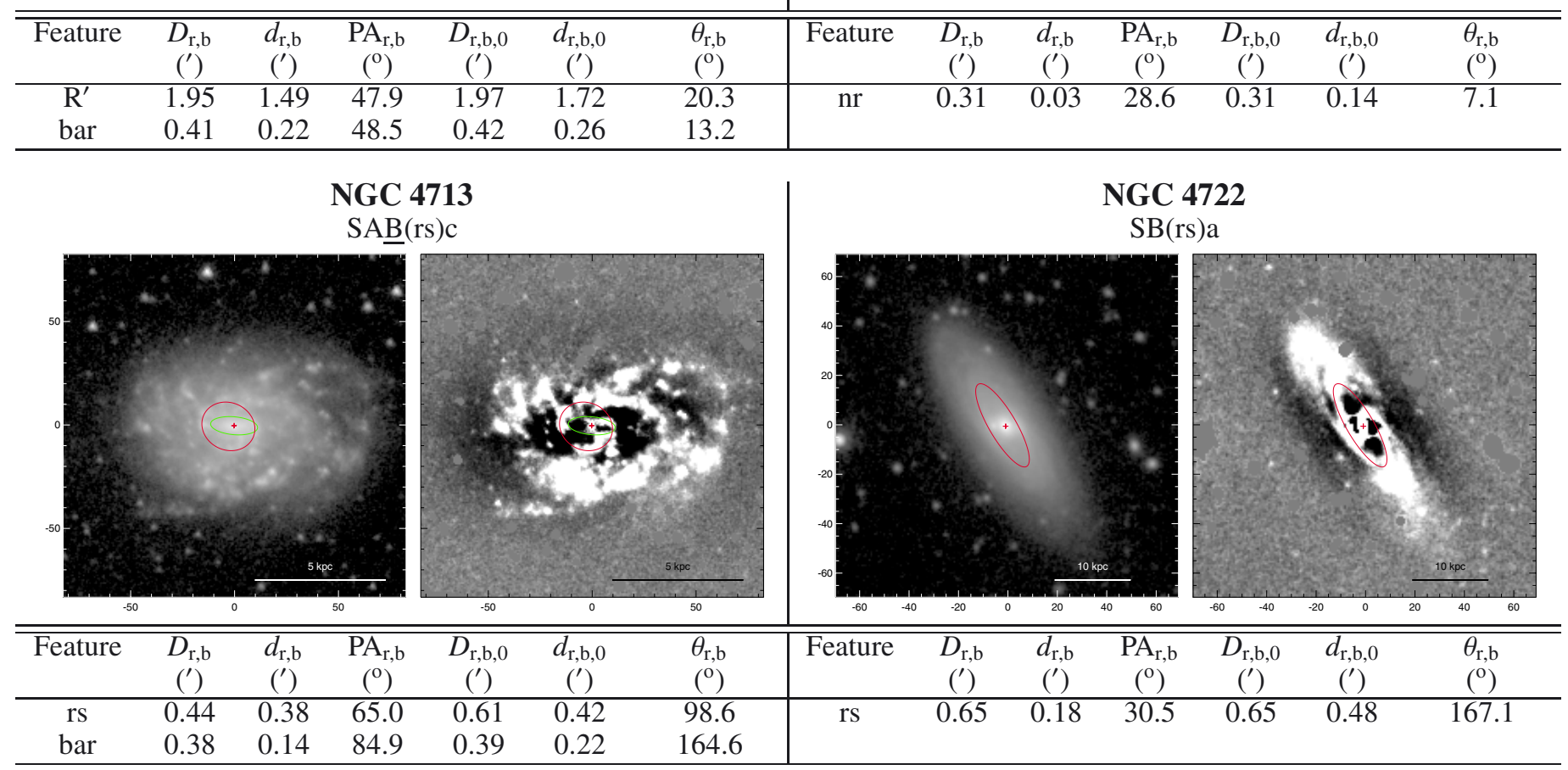

NGC 4725

$\mathrm{SAB}(\mathrm{r}, \mathrm{nb}) \mathrm{a}$
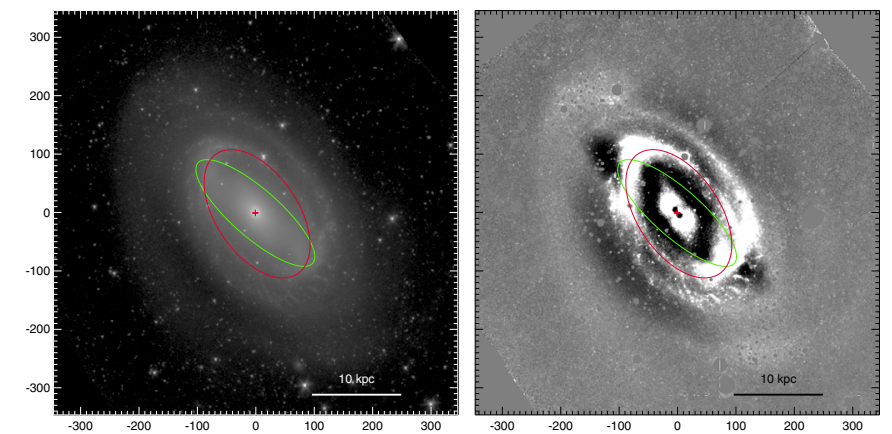

NGC 4736

(R)SAB(rl,nr',nl,nb)a

\begin{tabular}{|c|c|c|c|c|c|c|c|c|c|c|c|c|c|}
\hline Feature & $\begin{array}{c}D_{\mathrm{r}, \mathrm{b}} \\
\left({ }^{\prime}\right)\end{array}$ & $\begin{array}{c}d_{\mathrm{r}, \mathrm{b}} \\
\left(^{\prime}\right)\end{array}$ & $\begin{array}{c}\mathrm{PA}_{\mathrm{r}, \mathrm{b}} \\
\left(^{\circ}\right)\end{array}$ & $\begin{array}{c}D_{\mathrm{r}, \mathrm{b}, 0} \\
\left(^{\prime}\right)\end{array}$ & $\begin{array}{c}d_{\mathrm{r}, \mathrm{b}, 0} \\
\left(^{\prime}\right)\end{array}$ & $\begin{array}{c}\theta_{\mathrm{r}, \mathrm{b}} \\
\left({ }^{\circ}\right)\end{array}$ & Feature & $\begin{array}{c}D_{\mathrm{r}, \mathrm{b}} \\
\left(^{\prime}\right)\end{array}$ & $\begin{array}{c}d_{\mathrm{r}, \mathrm{b}} \\
\left({ }^{\prime}\right)\end{array}$ & $\begin{array}{c}\mathrm{PA}_{\mathrm{r}, \mathrm{b}} \\
\left(^{\circ}\right)\end{array}$ & $\begin{array}{c}D_{\mathrm{r}, \mathrm{b}, 0} \\
\left.\text { (') }^{\prime}\right)\end{array}$ & $\begin{array}{c}d_{\mathrm{r}, \mathrm{b}, 0} \\
\left(^{\prime}\right)\end{array}$ & $\begin{array}{c}\theta_{\mathrm{r}, \mathrm{b}} \\
\left({ }^{\circ}\right)\end{array}$ \\
\hline bar & 4.35 & 1.37 & 48.8 & 4.53 & 1.93 & 23.5 & $\overline{\mathrm{R}}$ & 10.58 & 8.68 & 134.8 & 10.94 & 10.13 & 47.1 \\
\hline \multirow[t]{3}{*}{$r$} & 4.14 & 2.34 & 34.2 & 4.14 & 3.42 & 0.7 & bar & 4.71 & 3.48 & 94.4 & 5.07 & 3.91 & 131.4 \\
\hline & & & & & & & $\mathrm{rl}$ & 4.48 & 3.47 & 103.9 & 4.70 & 3.99 & 136.6 \\
\hline & & & & & & & $\mathrm{nr}^{\prime}$ & 1.48 & 1.13 & 114.0 & 1.50 & 1.34 & 152.9 \\
\hline
\end{tabular}


NGC 4746

$\left(\mathrm{R}^{\prime}\right) \mathrm{Sc} \mathrm{sp}$
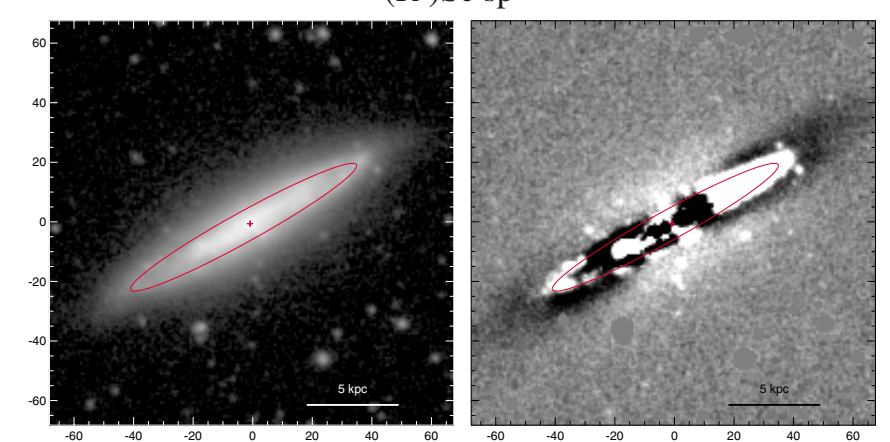

\begin{tabular}{|c|c|c|c|c|c|c|c|c|c|c|c|c|c|}
\hline Feature & $\begin{array}{c}D_{\mathrm{r}, \mathrm{b}} \\
\left({ }^{\prime}\right)\end{array}$ & $\begin{array}{c}d_{\mathrm{r}, \mathrm{b}} \\
\left({ }^{\prime}\right)\end{array}$ & $\begin{array}{c}\mathrm{PA}_{\mathrm{r}, \mathrm{b}} \\
\left({ }^{\circ}\right)\end{array}$ & $\begin{array}{c}D_{\mathrm{r}, \mathrm{b}, 0} \\
\left(^{\prime}\right)\end{array}$ & $\begin{array}{c}d_{\mathrm{r}, \mathrm{b}, 0} \\
\left({ }^{\prime}\right)\end{array}$ & $\begin{array}{c}\theta_{\mathrm{r}, \mathrm{b}} \\
\left({ }^{\circ}\right)\end{array}$ & Feature & $\begin{array}{c}D_{\mathrm{r}, \mathrm{b}} \\
\left({ }^{\prime}\right)\end{array}$ & $\begin{array}{c}d_{\mathrm{r}, \mathrm{b}} \\
\left({ }^{\prime}\right)\end{array}$ & $\begin{array}{c}\mathrm{PA}_{\mathrm{r}, \mathrm{b}} \\
(\mathrm{o})\end{array}$ & $\begin{array}{c}D_{\mathrm{r}, \mathrm{b}, 0} \\
\left({ }^{\prime}\right)\end{array}$ & $\begin{array}{c}d_{\mathrm{r}, \mathrm{b}, 0} \\
\left({ }^{\prime}\right)\end{array}$ & $\begin{array}{l}\theta_{\mathrm{r}, \mathrm{b}} \\
\left({ }^{\circ}\right)\end{array}$ \\
\hline$\overline{\mathrm{R}^{\prime}}$ & 1.45 & 0.17 & 119.0 & 1.45 & 0.77 & 176.2 & $\begin{array}{l}\overline{\mathrm{R}^{\prime}} \\
\mathrm{rs}\end{array}$ & $\begin{array}{l}1.52 \\
0.55\end{array}$ & $\begin{array}{l}1.33 \\
0.49\end{array}$ & $\begin{array}{l}156.7 \\
146.0\end{array}$ & $\begin{array}{l}1.69 \\
0.63\end{array}$ & $\begin{array}{l}1.51 \\
0.53\end{array}$ & $\begin{array}{l}101.5 \\
103.6\end{array}$ \\
\hline
\end{tabular}

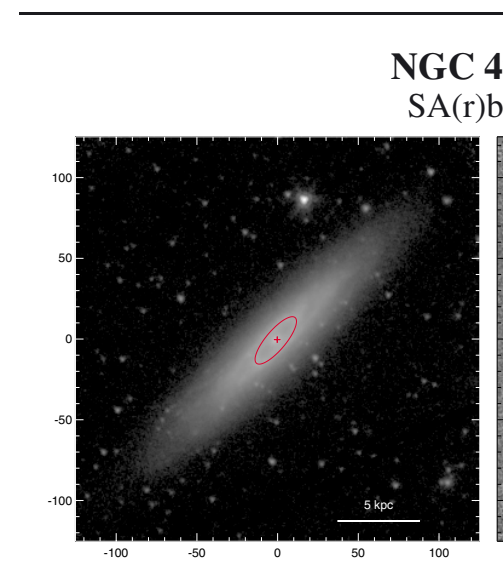

NGC 4771

$\mathrm{SA}(\mathrm{r}) \mathrm{b}$ sp

\begin{tabular}{|c|c|c|c|c|c|c|c|c|c|c|c|c|c|}
\hline Feature & $\begin{array}{c}D_{\mathrm{r}, \mathrm{b}} \\
\left({ }^{\prime}\right)\end{array}$ & $\begin{array}{c}d_{\mathrm{r}, \mathrm{b}} \\
(')\end{array}$ & $\begin{array}{c}\mathrm{PA}_{\mathrm{r}, \mathrm{b}} \\
\left({ }^{\circ}\right)\end{array}$ & $\begin{array}{c}D_{\mathrm{r}, \mathrm{b}, 0} \\
\left(^{\prime}\right)\end{array}$ & $\begin{array}{c}d_{\mathrm{r}, \mathrm{b}, 0} \\
\left({ }^{\prime}\right)\end{array}$ & $\begin{array}{c}\theta_{\mathrm{r}, \mathrm{b}} \\
\left({ }^{\circ}\right)\end{array}$ & Feature & $\begin{array}{c}D_{\mathrm{r}, \mathrm{b}} \\
\left({ }^{\prime}\right)\end{array}$ & $\begin{array}{c}d_{\mathrm{r}, \mathrm{b}} \\
\left({ }^{\prime}\right)\end{array}$ & $\begin{array}{c}\mathrm{PA}_{\mathrm{r}, \mathrm{b}} \\
\left({ }^{\circ}\right)\end{array}$ & $\begin{array}{c}D_{\mathrm{r}, \mathrm{b}, 0} \\
\left(^{\prime}\right)\end{array}$ & $\begin{array}{c}d_{\mathrm{r}, \mathrm{b}, 0} \\
\left(^{\prime}\right)\end{array}$ & $\begin{array}{c}\theta_{\mathrm{r}, \mathrm{b}} \\
\left({ }^{\circ}\right)\end{array}$ \\
\hline $\mathrm{r}$ & 0.62 & 0.20 & 139.8 & 0.93 & 0.58 & 72.3 & $\begin{array}{l}\mathrm{R}^{\prime} \\
\mathrm{r}\end{array}$ & $\begin{array}{l}3.85 \\
2.36\end{array}$ & $\begin{array}{l}1.93 \\
0.61\end{array}$ & $\begin{array}{l}147.4 \\
1453\end{array}$ & $\begin{array}{l}3.86 \\
2.36\end{array}$ & $\begin{array}{l}3.68 \\
116\end{array}$ & $\begin{array}{c}19.5 \\
1780\end{array}$ \\
\hline
\end{tabular}
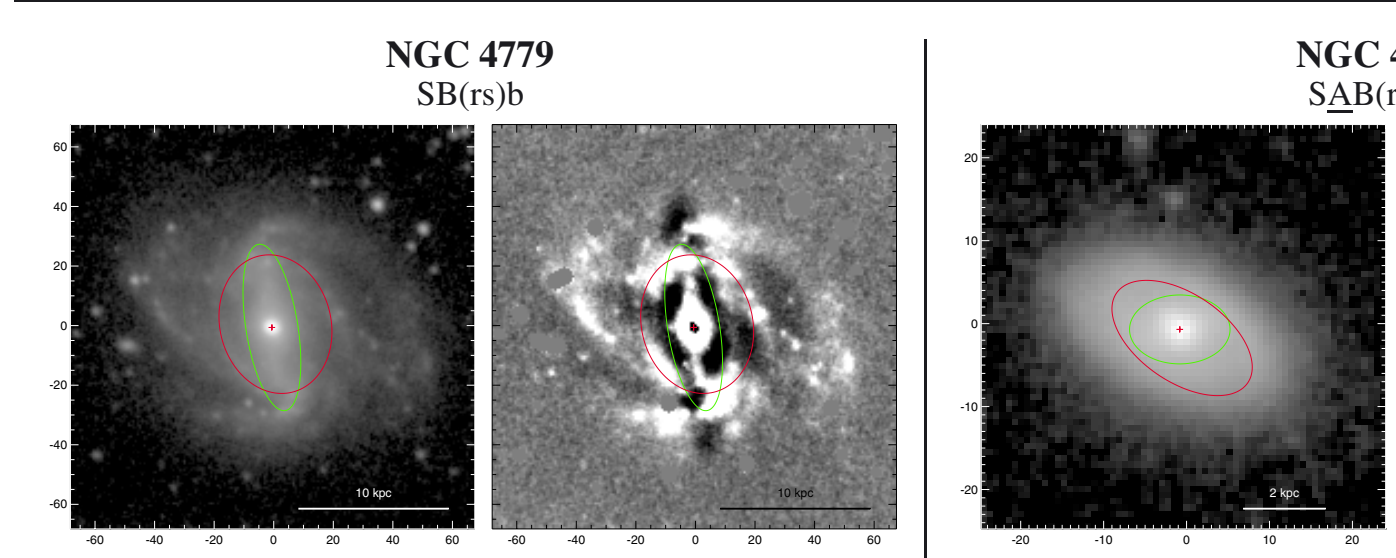

NGC 4791

$\mathrm{SABB}(\mathrm{rl}) 0^{+}$

\begin{tabular}{ccccccc|ccccccc}
\hline \hline Feature & $\begin{array}{c}D_{\mathrm{r}, \mathrm{b}} \\
\left({ }^{\prime}\right)\end{array}$ & $\begin{array}{c}d_{\mathrm{r}, \mathrm{b}} \\
\left({ }^{\prime}\right)\end{array}$ & $\begin{array}{c}\mathrm{PA}_{\mathrm{r}, \mathrm{b}} \\
\left({ }^{\circ}\right)\end{array}$ & $\begin{array}{c}D_{\mathrm{r}, \mathrm{b}, 0} \\
\left({ }^{\prime}\right)\end{array}$ & $\begin{array}{c}d_{\mathrm{r}, \mathrm{b}, 0} \\
\left({ }^{\prime}\right)\end{array}$ & $\begin{array}{c}\theta_{\mathrm{r}, \mathrm{b}} \\
\left({ }^{\circ}\right)\end{array}$ & Feature & $\begin{array}{c}D_{\mathrm{r}, \mathrm{b}} \\
\left({ }^{\prime}\right)\end{array}$ & $\begin{array}{c}d_{\mathrm{r}, \mathrm{b}} \\
\left({ }^{\prime}\right)\end{array}$ & $\begin{array}{c}\mathrm{PA}_{\mathrm{r}, \mathrm{b}} \\
\left({ }^{\circ}\right)\end{array}$ & $\begin{array}{c}D_{\mathrm{r}, \mathrm{b}, 0} \\
\left({ }^{\prime}\right)\end{array}$ & $\begin{array}{c}d_{\mathrm{r}, \mathrm{b}, 0} \\
\left({ }^{\prime}\right)\end{array}$ & $\begin{array}{c}\theta_{\mathrm{r}, \mathrm{b}} \\
\left({ }^{\circ}\right)\end{array}$ \\
\hline bar & 0.95 & 0.29 & 9.2 & 1.13 & 0.31 & 114.2 & $\mathrm{rl}$ & 0.32 & 0.18 & 55.7 & 0.33 & 0.25 & 155.8 \\
rs & 0.79 & 0.62 & 14.0 & 0.94 & 0.65 & 107.7 & bar & 0.21 & 0.14 & 90.2 & 0.23 & 0.17 & 55.1 \\
\hline
\end{tabular}




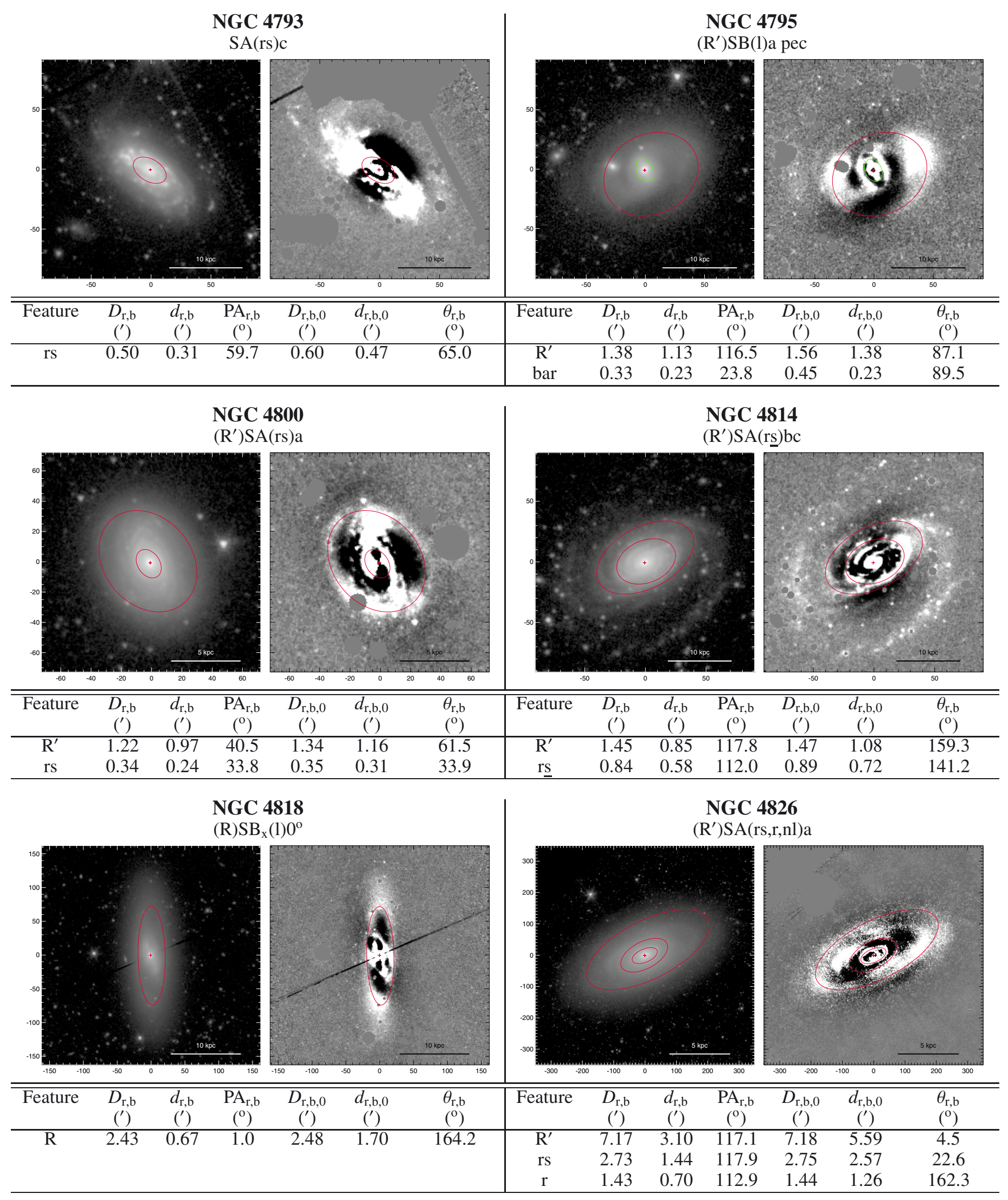


NGC 4845

$\left(\mathrm{R}^{\prime}\right) \mathrm{SAB}_{\mathrm{x}}(\mathrm{rs}, \mathrm{nd}) 0 / \mathrm{a}$
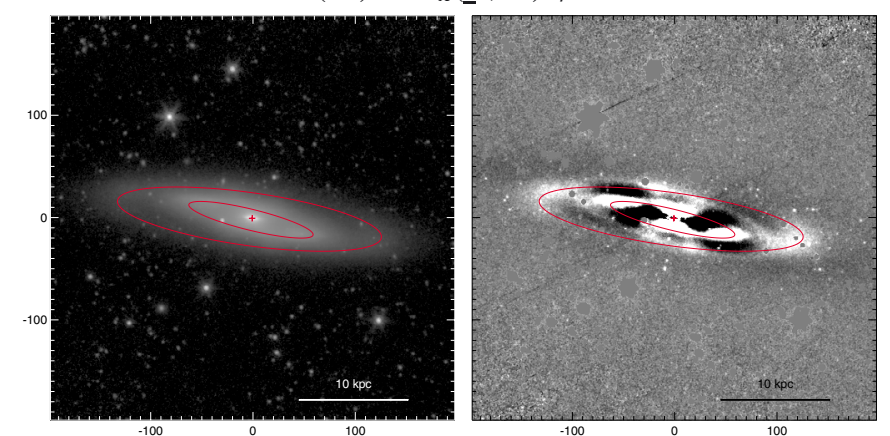

\begin{tabular}{|c|c|c|c|c|c|c|c|c|c|c|c|c|c|}
\hline Feature & $\begin{array}{c}D_{\mathrm{r}, \mathrm{b}} \\
\left({ }^{\prime}\right)\end{array}$ & $\begin{array}{c}d_{\mathrm{r}, \mathrm{b}} \\
\left({ }^{\prime}\right)\end{array}$ & $\begin{array}{c}\mathrm{PA}_{\mathrm{r}, \mathrm{b}} \\
\left({ }^{\circ}\right)\end{array}$ & $\begin{array}{c}D_{\mathrm{r}, \mathrm{b}, 0} \\
\left(^{\prime}\right)\end{array}$ & $\begin{array}{c}d_{\mathrm{r}, \mathrm{b}, 0} \\
\left(^{\prime}\right)\end{array}$ & $\begin{array}{l}\theta_{\mathrm{r}, \mathrm{b}} \\
\left({ }^{\circ}\right)\end{array}$ & Feature & $\begin{array}{c}D_{\mathrm{r}, \mathrm{b}} \\
\left({ }^{\prime}\right)\end{array}$ & $\begin{array}{c}d_{\mathrm{r}, \mathrm{b}} \\
\left({ }^{\prime}\right)\end{array}$ & $\begin{array}{c}\mathrm{PA}_{\mathrm{r}, \mathrm{b}} \\
\left(^{\circ}\right)\end{array}$ & $\begin{array}{c}D_{\mathrm{r}, \mathrm{b}, 0} \\
\left(^{\prime}\right)\end{array}$ & $\begin{array}{c}d_{\mathrm{r}, \mathrm{b}, 0} \\
\left({ }^{\prime}\right)\end{array}$ & $\begin{array}{l}\theta_{\mathrm{r}, \mathrm{b}} \\
\left({ }^{\circ}\right)\end{array}$ \\
\hline$\overline{\mathrm{R}^{\prime}}$ & 4.35 & 0.87 & 82.0 & 4.47 & 3.30 & 19.9 & $\overline{\mathrm{RL}}$ & 2.44 & 0.65 & 36.2 & 2.46 & 1.79 & 169.3 \\
\hline rs & 2.09 & 0.34 & 76.2 & 2.17 & 1.29 & 159.5 & & & & & & & \\
\hline
\end{tabular}

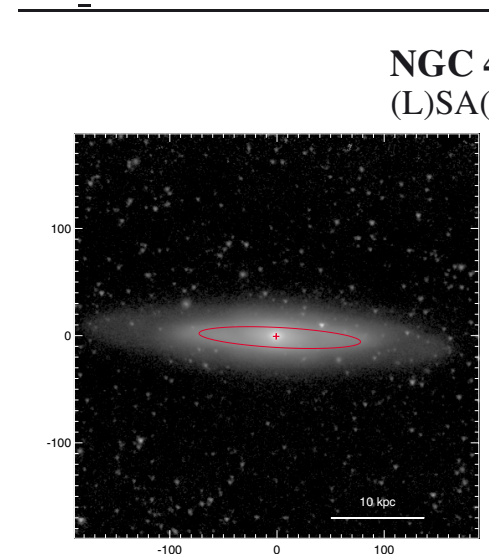

NGC 4866

(L) $\mathrm{SA}(\mathrm{rl}) 0^{+}$

\begin{tabular}{ccccccc} 
& \multicolumn{2}{c}{${ }^{0}$} & \multicolumn{2}{c}{${ }^{-100}$} & ${ }^{0}$ & ${ }^{100}$ \\
\hline \hline Feature & $\begin{array}{c}D_{\mathrm{r}, \mathrm{b}} \\
\left(^{\prime}\right)\end{array}$ & $\begin{array}{c}d_{\mathrm{r}, \mathrm{b}} \\
\left(^{\prime}\right)\end{array}$ & $\begin{array}{c}\mathrm{PA}_{\mathrm{r}, \mathrm{b}} \\
\left(^{\mathrm{o}}\right)\end{array}$ & $\begin{array}{c}D_{\mathrm{r}, \mathrm{b}, 0} \\
\left(^{\prime}\right)\end{array}$ & $\begin{array}{c}d_{\mathrm{r}, \mathrm{b}, 0} \\
\left(^{\prime}\right)\end{array}$ & $\begin{array}{c}\theta_{\mathrm{r}, \mathrm{b}} \\
\left({ }^{\circ}\right)\end{array}$ \\
\hline $\mathrm{rl}$ & 2.52 & 0.31 & 87.0 & 2.53 & 1.29 & 175.4
\end{tabular}

$\mathrm{rl}$

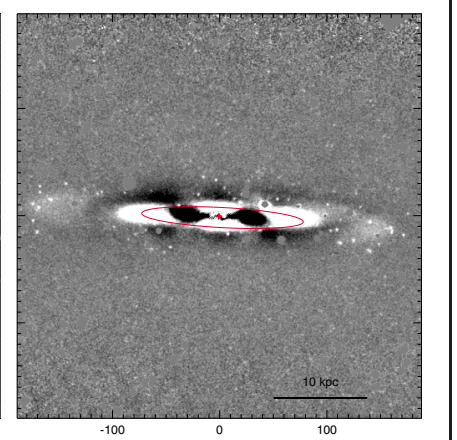

NGC 4880

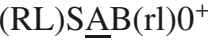

NGC 4856

(RL)SB0-

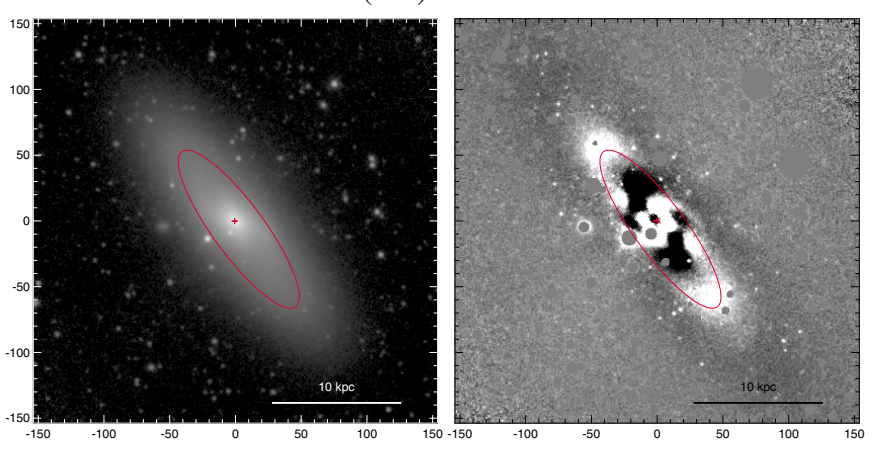

eron et al.: ARRAKIS 


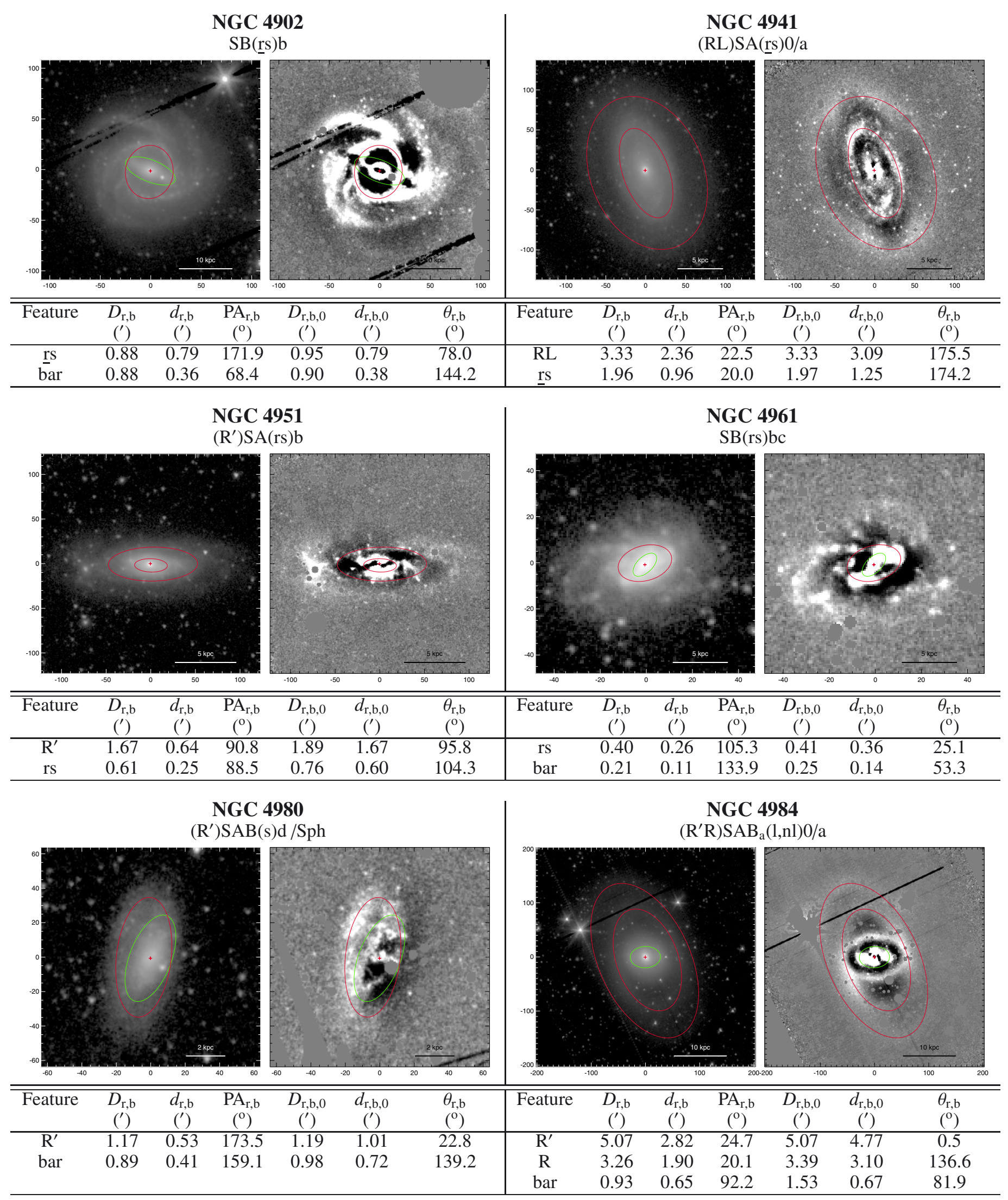


NGC 4995

$\mathrm{SAB}_{\mathrm{a}}(\mathrm{rs}) \mathrm{ab}$
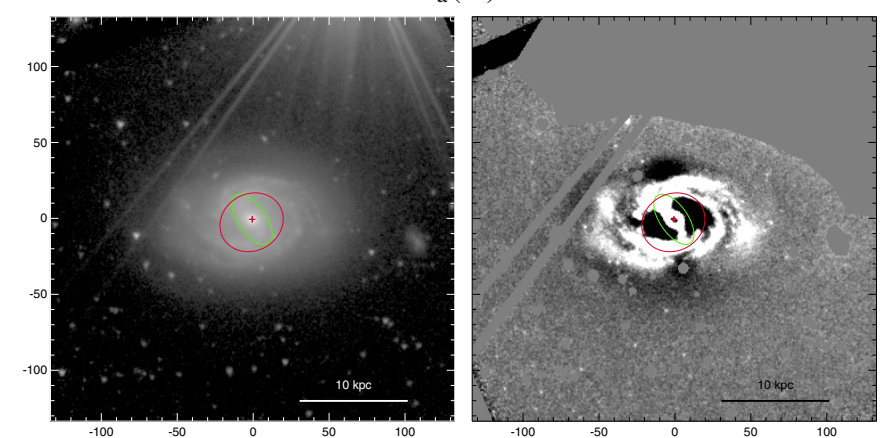

NGC 5005

$\left(\mathrm{R}_{2}^{\prime}\right) \mathrm{SAB}_{\mathrm{x}}(\underline{\mathrm{rs}}) \mathrm{ab}$

\begin{tabular}{|c|c|c|c|c|c|c|c|c|c|c|c|c|c|}
\hline Feature & $\begin{array}{c}D_{\mathrm{r}, \mathrm{b}} \\
\left({ }^{\prime}\right)\end{array}$ & $\begin{array}{c}d_{\mathrm{r}, \mathrm{b}} \\
(')\end{array}$ & $\begin{array}{c}\mathrm{PA}_{\mathrm{r}, \mathrm{b}} \\
\left({ }^{\circ}\right)\end{array}$ & $\begin{array}{c}D_{\mathrm{r}, \mathrm{b}, 0} \\
\left(^{\prime}\right)\end{array}$ & $\begin{array}{c}d_{\mathrm{r}, \mathrm{b}, 0} \\
\left({ }^{\prime}\right)\end{array}$ & $\begin{array}{l}\theta_{\mathrm{r}, \mathrm{b}} \\
\left(^{\circ}\right)\end{array}$ & Feature & $\begin{array}{c}D_{\mathrm{r}, \mathrm{b}} \\
\left(^{\prime}\right)\end{array}$ & $\begin{array}{c}d_{\mathrm{r}, \mathrm{b}} \\
\left({ }^{\prime}\right)\end{array}$ & $\begin{array}{c}\mathrm{PA}_{\mathrm{r}, \mathrm{b}} \\
\left(^{\circ}\right)\end{array}$ & $\begin{array}{c}D_{\mathrm{r}, \mathrm{b}, 0} \\
\left(^{\prime}\right)\end{array}$ & $\begin{array}{c}d_{\mathrm{r}, \mathrm{b}, 0} \\
\left(^{\prime}\right)\end{array}$ & $\begin{array}{l}\theta_{\mathrm{r}, \mathrm{b}} \\
\left(^{\circ}\right)\end{array}$ \\
\hline rs & 0.72 & 0.63 & 118.6 & 1.01 & 0.69 & 82.4 & $\overline{\mathrm{R}_{2}^{\prime}}$ & 3.53 & 1.23 & 63.2 & 3.66 & 3.01 & 151.8 \\
\hline bar & 0.64 & 0.31 & 34.5 & 0.93 & 0.33 & 107.1 & rs & 1.24 & 0.42 & 71.5 & 1.29 & 1.02 & 28.4 \\
\hline
\end{tabular}

NGC 5016

$\mathrm{SAB}(\underline{\mathrm{rs}}) \mathrm{c}$
NGC 5033

$\left(\mathrm{R}^{\prime}\right) \mathrm{SA}(\mathrm{rs}) \mathrm{c}$
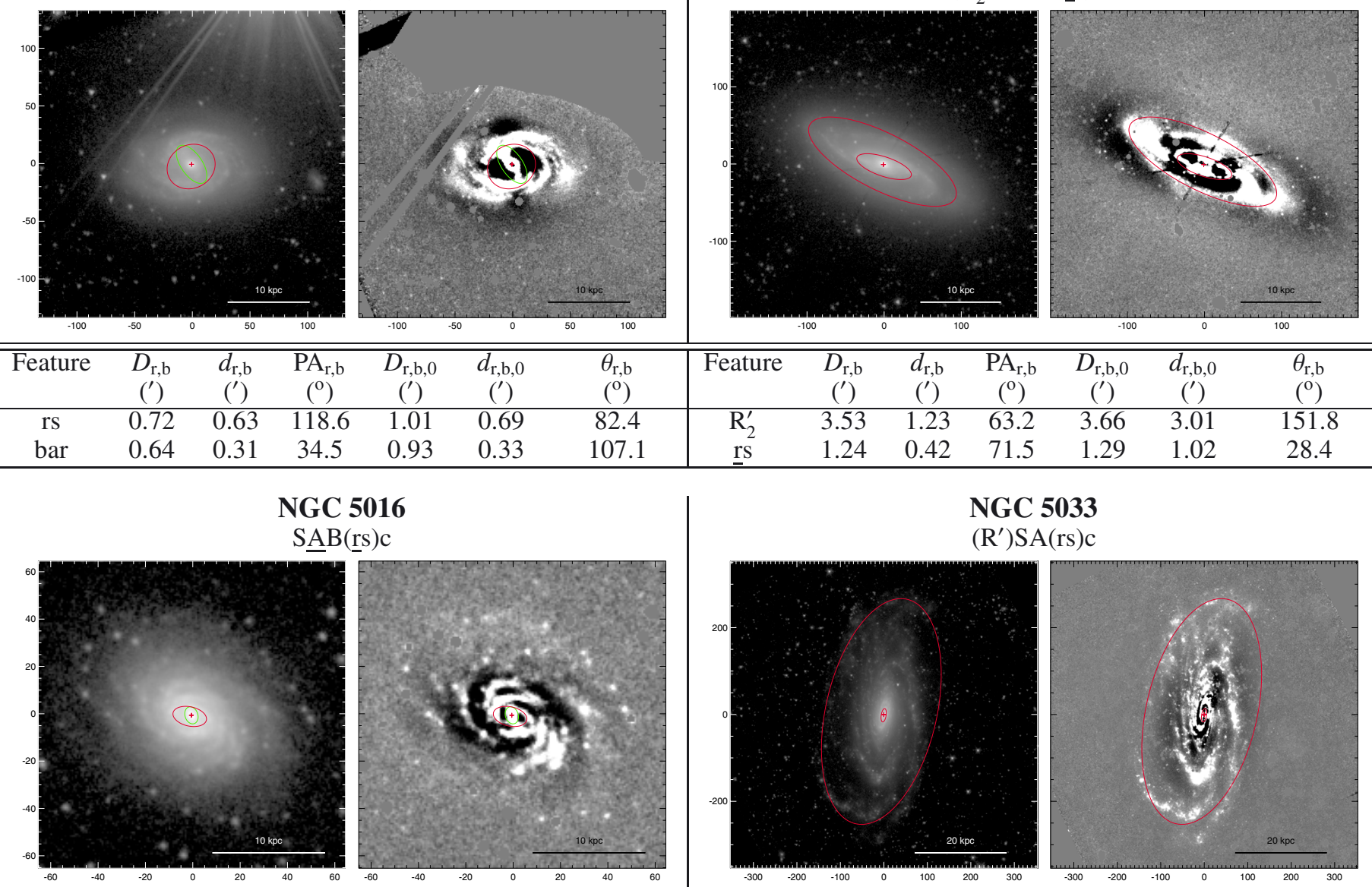

\section{.}


NGC 5068

$\mathrm{SB}(\mathrm{rs}) \mathrm{d}$
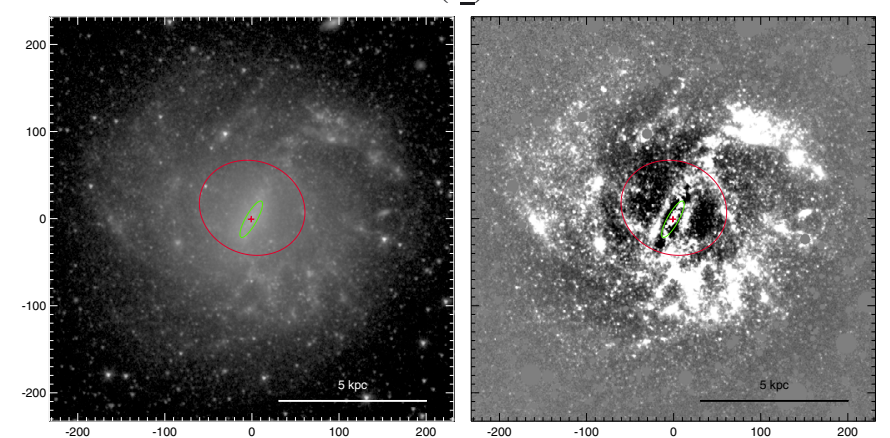

NGC 5078

$\mathrm{E}(\mathrm{d}) 5 / \mathrm{S}(\mathrm{r}) \mathrm{b}$ sp

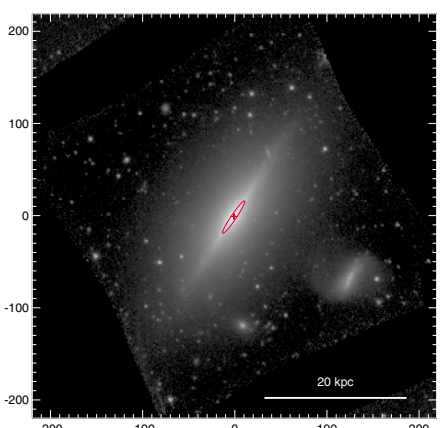

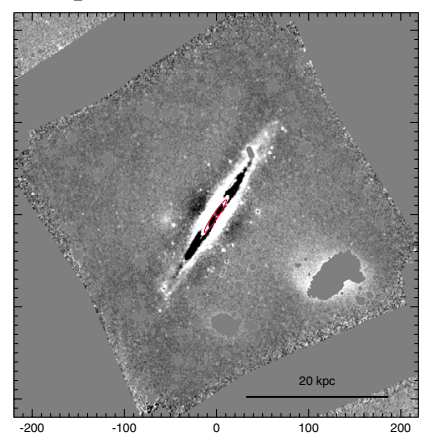

\begin{tabular}{ccccccc|ccccccc}
\hline \hline Feature & $\begin{array}{c}D_{\mathrm{r}, \mathrm{b}} \\
\left({ }^{\prime}\right)\end{array}$ & $\begin{array}{c}d_{\mathrm{r}, \mathrm{b}}\left({ }^{\prime}\right) \\
\left(\mathrm{PA}_{\mathrm{r}, \mathrm{b}}\right. \\
(\mathrm{o})\end{array}$ & $\begin{array}{c}D_{\mathrm{r}, \mathrm{b}, 0} \\
\left({ }^{\prime}\right)\end{array}$ & $\begin{array}{c}d_{\mathrm{r}, \mathrm{b}, 0} \\
\left({ }^{\prime}\right)\end{array}$ & $\begin{array}{c}\theta_{\mathrm{r}, \mathrm{b}} \\
\left({ }^{\circ}\right)\end{array}$ & Feature & $\begin{array}{c}D_{\mathrm{r}, \mathrm{b}} \\
\left({ }^{\prime}\right)\end{array}$ & $\begin{array}{c}d_{\mathrm{r}, \mathrm{b}} \\
\left({ }^{\prime}\right)\end{array}$ & $\begin{array}{c}\mathrm{PA}_{\mathrm{r}, \mathrm{b}} \\
\left({ }^{\circ}\right)\end{array}$ & $\begin{array}{c}D_{\mathrm{r}, \mathrm{b}, 0} \\
\left({ }^{\prime}\right)\end{array}$ & $\begin{array}{c}d_{\mathrm{r}, \mathrm{b}, 0} \\
\left({ }^{\prime}\right)\end{array}$ & $\begin{array}{c}\theta_{\mathrm{r}, \mathrm{b}} \\
\left({ }^{\circ}\right)\end{array}$ \\
\hline $\mathrm{rs}$ & 2.06 & 1.79 & 69.2 & 2.07 & 1.82 & 145.0 & $\mathrm{r}$ & 0.71 & 0.09 & 146.0 & 0.71 & 0.27 & 177.5 \\
$\mathrm{bar}$ & 0.79 & 0.21 & 150.5 & 0.81 & 0.22 & 52.2 & & & & & & & \\
\hline
\end{tabular}

NGC 5079

$\left(\mathrm{R}^{\prime}\right) \mathrm{SAB}(\mathrm{s}) \mathrm{b}$
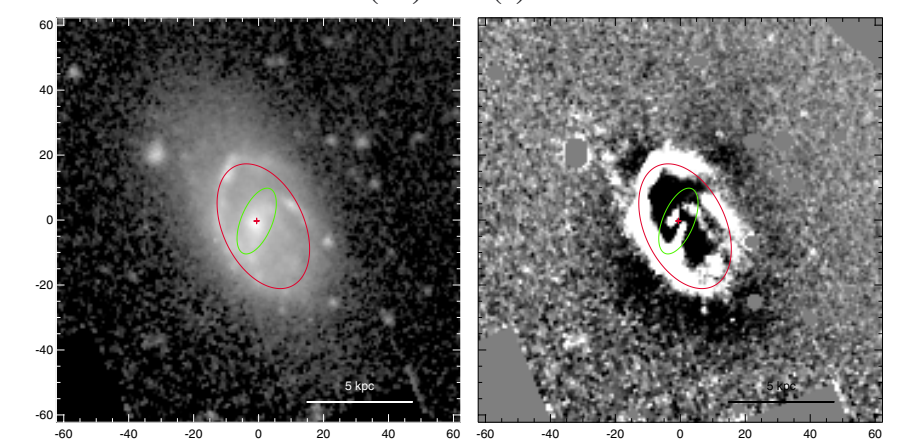

NGC 5085

$\mathrm{SA}(\mathrm{rs}) \mathrm{bc}$

\begin{tabular}{|c|c|c|c|c|c|c|c|c|c|c|c|c|c|}
\hline Feature & $\begin{array}{c}D_{\mathrm{r}, \mathrm{b}} \\
\left({ }^{\prime}\right)\end{array}$ & $\begin{array}{c}d_{\mathrm{r}, \mathrm{b}} \\
\left({ }^{\prime}\right)\end{array}$ & $\begin{array}{c}\mathrm{PA}_{\mathrm{r}, \mathrm{b}} \\
\left({ }^{\circ}\right)\end{array}$ & $\begin{array}{c}D_{\mathrm{r}, \mathrm{b}, 0} \\
\left(^{\prime}\right)\end{array}$ & $\begin{array}{c}d_{\mathrm{r}, \mathrm{b}, 0} \\
\left({ }^{\prime}\right)\end{array}$ & $\begin{array}{c}\theta_{\mathrm{r}, \mathrm{b}} \\
\left({ }^{\circ}\right)\end{array}$ & Feature & $\begin{array}{c}D_{\mathrm{r}, \mathrm{b}} \\
\left(^{\prime}\right)\end{array}$ & $\begin{array}{c}d_{\mathrm{r}, \mathrm{b}} \\
\left({ }^{\prime}\right)\end{array}$ & $\begin{array}{c}\mathrm{PA}_{\mathrm{r}, \mathrm{b}} \\
\left({ }^{\circ}\right)\end{array}$ & $\begin{array}{c}D_{\mathrm{r}, \mathrm{b}, 0} \\
\left(^{\prime}\right)\end{array}$ & $\begin{array}{c}d_{\mathrm{r}, \mathrm{b}, 0} \\
\left(^{\prime}\right)\end{array}$ & $\begin{array}{l}\theta_{\mathrm{r}, \mathrm{b}} \\
\left({ }^{\circ}\right)\end{array}$ \\
\hline$\overline{\mathrm{R}^{\prime}}$ & 0.68 & 0.42 & 25.2 & 0.82 & 0.68 & 100.4 & $\mathrm{rs}$ & 0.28 & 0.19 & 45.8 & 0.28 & 0.21 & 171.6 \\
\hline bar & 0.36 & 0.16 & 157.6 & 0.61 & 0.19 & 108.2 & & & & & & & \\
\hline
\end{tabular}

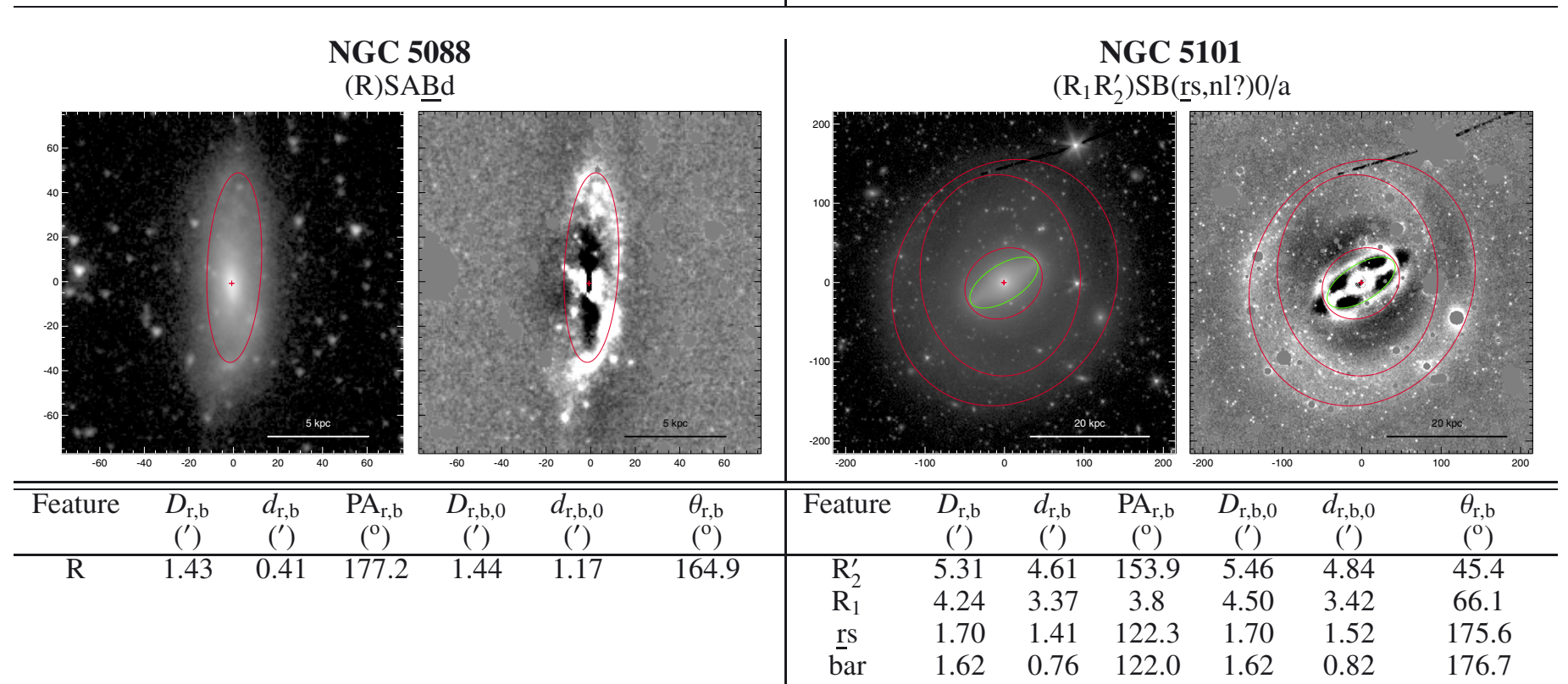




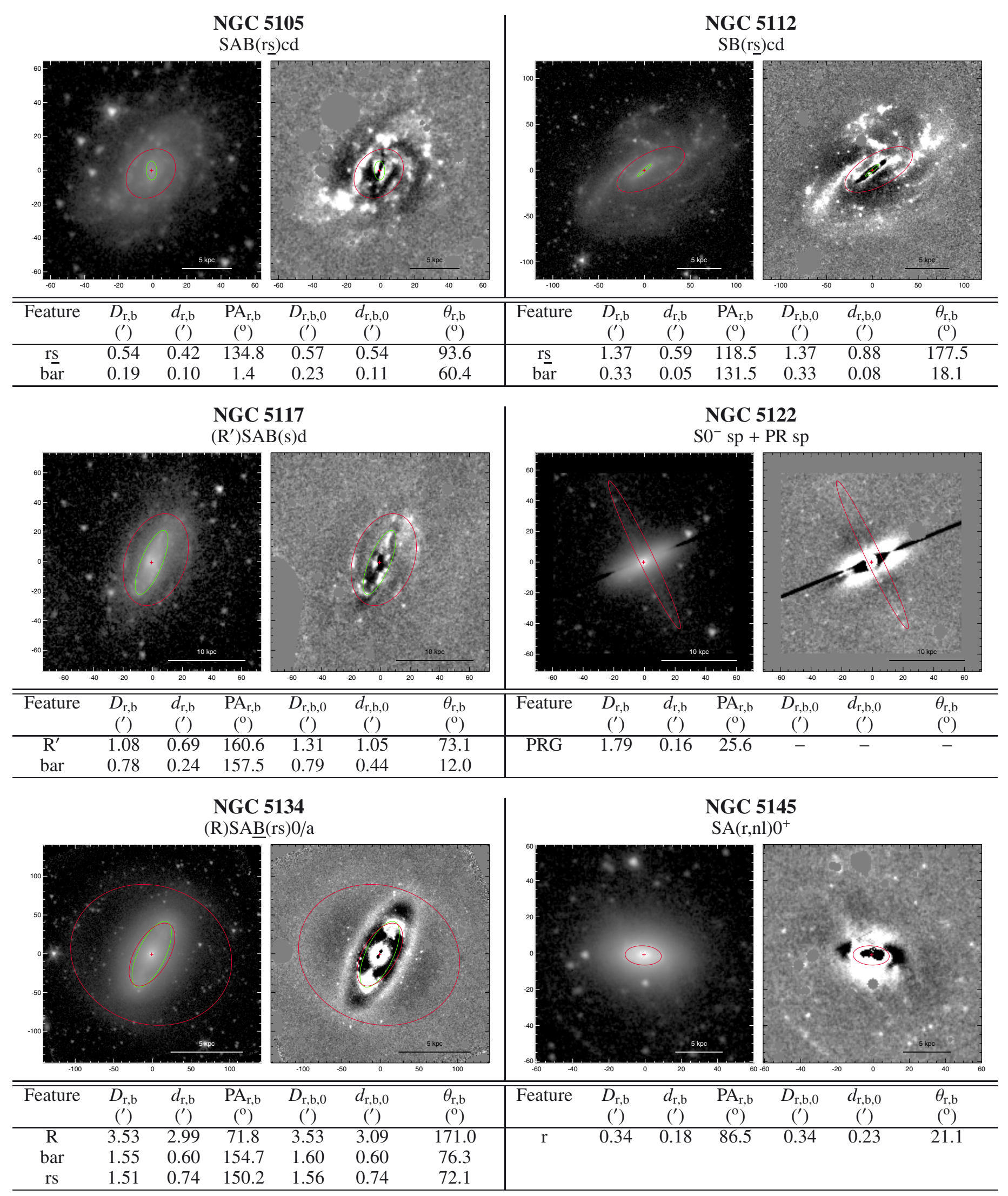


NGC 5147

$\left(\mathrm{R}^{\prime}\right) \mathrm{SB}(\mathrm{s}) \mathrm{c}$
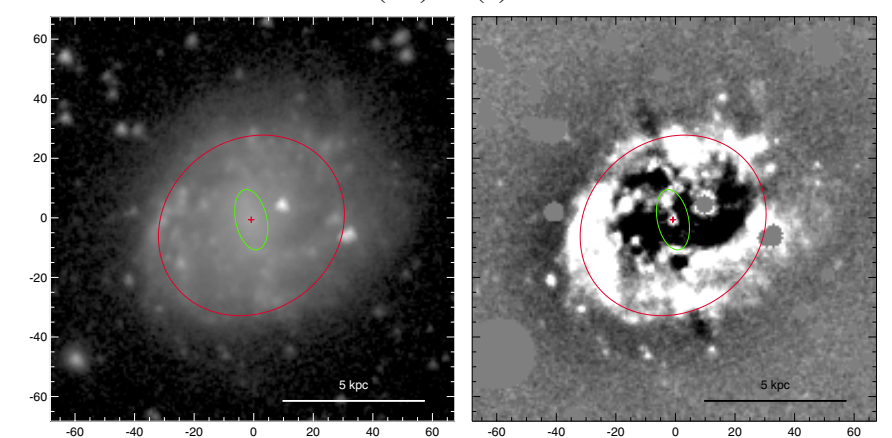

\begin{tabular}{|c|c|c|c|c|c|c|c|c|c|c|c|c|c|}
\hline Feature & $\begin{array}{c}D_{\mathrm{r}, \mathrm{b}} \\
\left({ }^{\prime}\right)\end{array}$ & $\begin{array}{c}d_{\mathrm{r}, \mathrm{b}} \\
\left({ }^{\prime}\right)\end{array}$ & $\begin{array}{c}\mathrm{PA}_{\mathrm{r}, \mathrm{b}} \\
\left({ }^{\mathrm{O}}\right)\end{array}$ & $\begin{array}{c}D_{\mathrm{r}, \mathrm{b}, 0} \\
\left(^{\prime}\right)\end{array}$ & $\begin{array}{c}d_{\mathrm{r}, \mathrm{b}, 0} \\
\left({ }^{\prime}\right)\end{array}$ & $\begin{array}{l}\theta_{\mathrm{r}, \mathrm{b}} \\
\left({ }^{\circ}\right)\end{array}$ & Feature & $\begin{array}{c}D_{\mathrm{r}, \mathrm{b}} \\
\left({ }^{\prime}\right)\end{array}$ & $\begin{array}{c}d_{\mathrm{r}, \mathrm{b}} \\
\left({ }^{\prime}\right)\end{array}$ & $\begin{array}{c}\mathrm{PA}_{\mathrm{r}, \mathrm{b}} \\
\left({ }^{\circ}\right)\end{array}$ & $\begin{array}{c}D_{\mathrm{r}, \mathrm{b}, 0} \\
\left({ }^{\prime}\right)\end{array}$ & $\begin{array}{c}d_{\mathrm{r}, \mathrm{b}, 0} \\
\left(^{\prime}\right)\end{array}$ & $\begin{array}{l}\theta_{\mathrm{r}, \mathrm{b}} \\
\left({ }^{\circ}\right)\end{array}$ \\
\hline $\mathrm{R}^{\prime}$ & 1.10 & 0.96 & 128.3 & 1.19 & 1.09 & 77.2 & $\overline{\mathrm{R}^{\prime}}$ & 4.85 & 0.29 & 126.0 & 4.85 & 1.97 & 177.9 \\
\hline bar & 0.35 & 0.18 & 12.0 & 0.42 & 0.18 & 76.8 & & & & & & & \\
\hline
\end{tabular}

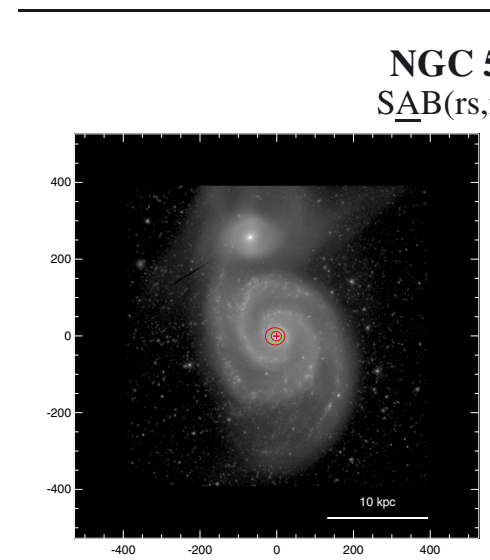

NGC 5194

$\mathrm{AB}\left(\mathrm{rs}, \mathrm{nr}^{\prime}\right) \mathrm{bc}$

\begin{tabular}{|c|c|c|c|c|c|c|c|c|c|c|c|c|c|}
\hline$\overline{\text { Feature }}$ & $\begin{array}{c}D_{\mathrm{r}, \mathrm{b}} \\
\left({ }^{\prime}\right)\end{array}$ & $\begin{array}{c}d_{\mathrm{r}, \mathrm{b}} \\
\left({ }^{\prime}\right)\end{array}$ & $\begin{array}{c}\mathrm{PA}_{\mathrm{r}, \mathrm{b}} \\
\left({ }^{\circ}\right)\end{array}$ & $\begin{array}{c}D_{\mathrm{r}, \mathrm{b}, 0} \\
\left(^{\prime}\right)\end{array}$ & $\begin{array}{c}d_{\mathrm{r}, \mathrm{b}, 0} \\
\left({ }^{\prime}\right)\end{array}$ & $\begin{array}{l}\overline{\theta_{\mathrm{r}, \mathrm{b}}} \\
\left({ }^{\circ}\right)\end{array}$ & $\overline{\text { Feature }}$ & $\begin{array}{c}D_{\mathrm{r}, \mathrm{b}} \\
\left({ }^{\prime}\right)\end{array}$ & $\begin{array}{c}d_{\mathrm{r}, \mathrm{b}} \\
\left(^{\prime}\right)\end{array}$ & $\begin{array}{c}\mathrm{PA}_{\mathrm{r}, \mathrm{b}} \\
\left({ }^{\circ}\right)\end{array}$ & $\begin{array}{c}D_{\mathrm{r}, \mathrm{b}, 0} \\
\text { (') }^{\prime}\end{array}$ & $\begin{array}{c}d_{\mathrm{r}, \mathrm{b}, 0} \\
\left({ }^{\prime}\right)\end{array}$ & $\begin{array}{l}\overline{\theta_{\mathrm{r}, \mathrm{b}}} \\
\left({ }^{\circ}\right)\end{array}$ \\
\hline rs & 0.84 & 0.75 & 87.0 & 0.97 & 0.77 & 73.4 & $\mathrm{r}$ & 1.54 & 1.41 & 130.5 & 1.91 & 1.51 & 81.7 \\
\hline bar & 0.74 & 0.50 & 138.4 & 0.88 & 0.50 & 96.3 & bar & 1.34 & 0.84 & 177.8 & 1.74 & 0.85 & 80.1 \\
\hline $\mathrm{nr}^{\prime}$ & 0.44 & 0.41 & 79.8 & 0.50 & 0.42 & 77.3 & & & & & & & \\
\hline
\end{tabular}
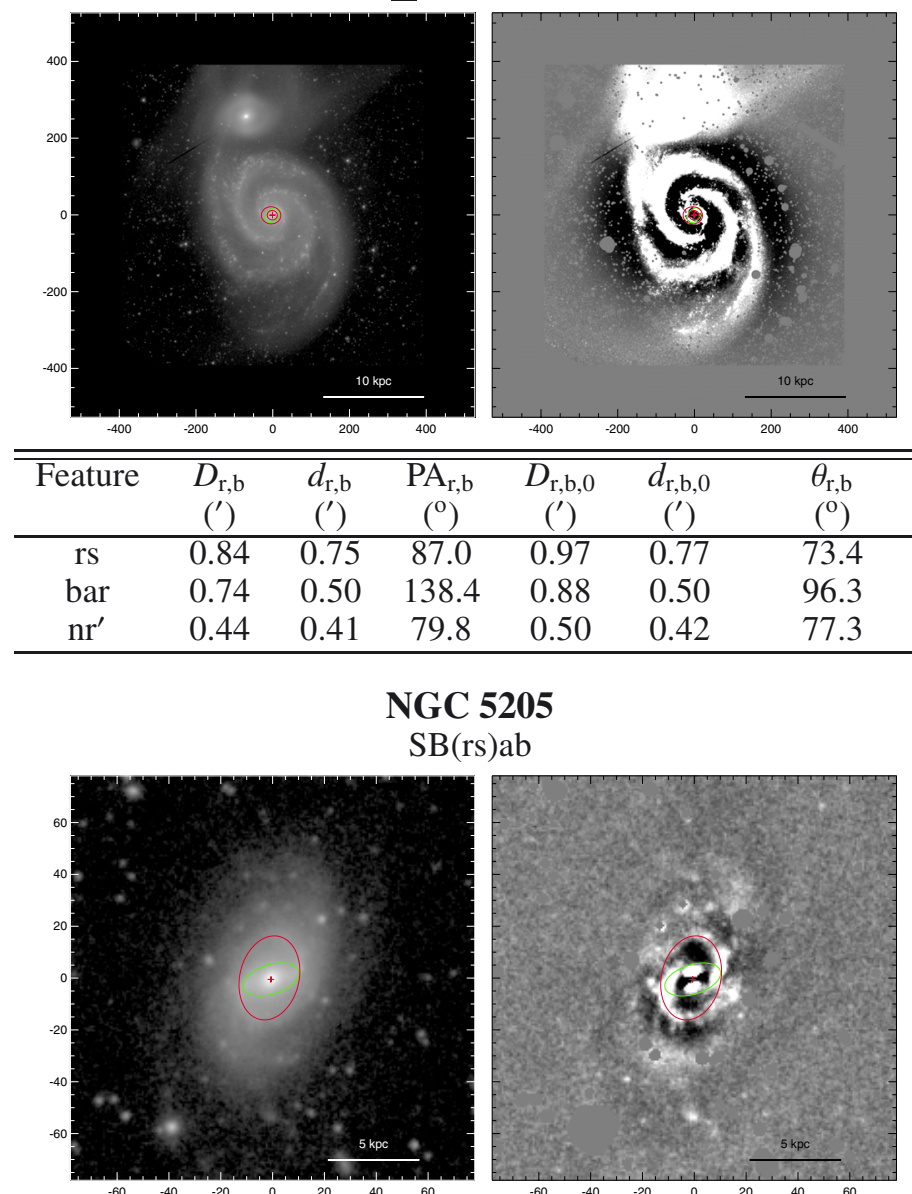

NGC 5195

$\mathrm{SAB}(\mathrm{r}) 0 / \mathrm{a}$

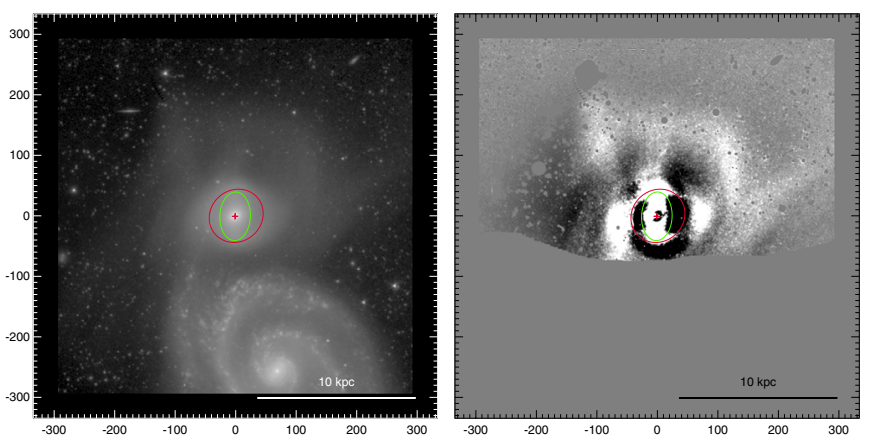

\section{NGC 5170}

$\left(\mathrm{R}^{\prime}\right) \mathrm{SAB}_{\mathrm{x}, \text { box }}(\mathrm{l}) 0 / \mathrm{a} \mathrm{sp}$

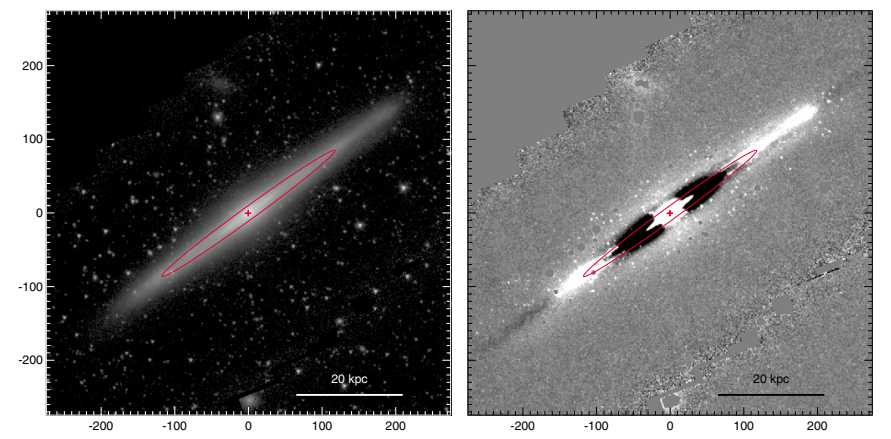

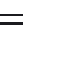


NGC 5236

$\mathrm{SAB}(\mathrm{s}, \mathrm{nr}) \mathrm{c}$
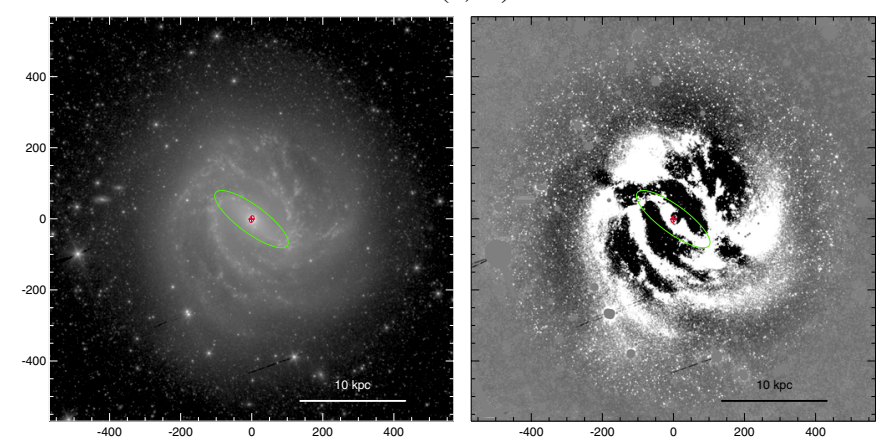

\begin{tabular}{|c|c|c|c|c|c|c|c|c|c|c|c|c|c|}
\hline Feature & $\begin{array}{c}D_{\mathrm{r}, \mathrm{b}} \\
\left({ }^{\prime}\right)\end{array}$ & $\begin{array}{c}d_{\mathrm{r}, \mathrm{b}} \\
\left({ }^{\prime}\right)\end{array}$ & $\begin{array}{c}\mathrm{PA}_{\mathrm{r}, \mathrm{b}} \\
\left({ }^{\circ}\right)\end{array}$ & $\begin{array}{c}D_{\mathrm{r}, \mathrm{b}, 0} \\
\left(^{\prime}\right)\end{array}$ & $\begin{array}{c}d_{\mathrm{r}, \mathrm{b}, 0} \\
\left(^{\prime}\right)\end{array}$ & $\begin{array}{l}\theta_{\mathrm{r}, \mathrm{b}} \\
\left({ }^{\circ}\right)\end{array}$ & Feature & $\begin{array}{c}D_{\mathrm{r}, \mathrm{b}} \\
\left({ }^{\prime}\right)\end{array}$ & $\begin{array}{c}d_{\mathrm{r}, \mathrm{b}} \\
\left({ }^{\prime}\right)\end{array}$ & $\begin{array}{c}\mathrm{PA}_{\mathrm{r}, \mathrm{b}} \\
\left({ }^{\circ}\right)\end{array}$ & $\begin{array}{c}D_{\mathrm{r}, \mathrm{b}, 0} \\
\left(^{\prime}\right)\end{array}$ & $\begin{array}{c}d_{\mathrm{r}, \mathrm{b}, 0} \\
\left(^{\prime}\right)\end{array}$ & $\begin{array}{l}\theta_{\mathrm{r}, \mathrm{b}} \\
\left({ }^{\circ}\right)\end{array}$ \\
\hline bar & 4.18 & 1.29 & 53.7 & 4.33 & 1.32 & 51.5 & $\mathrm{r}$ & 0.30 & 0.25 & 5.3 & 0.34 & 0.26 & 108.8 \\
\hline $\mathrm{nr}$ & 0.33 & 0.20 & 158.5 & 0.33 & 0.21 & 151.5 & & & & & & & \\
\hline
\end{tabular}

NGC 5248

$\left(\mathrm{R}^{\prime}\right) \mathrm{SAB}(\mathrm{s}, \mathrm{nr}) \mathrm{bc}$
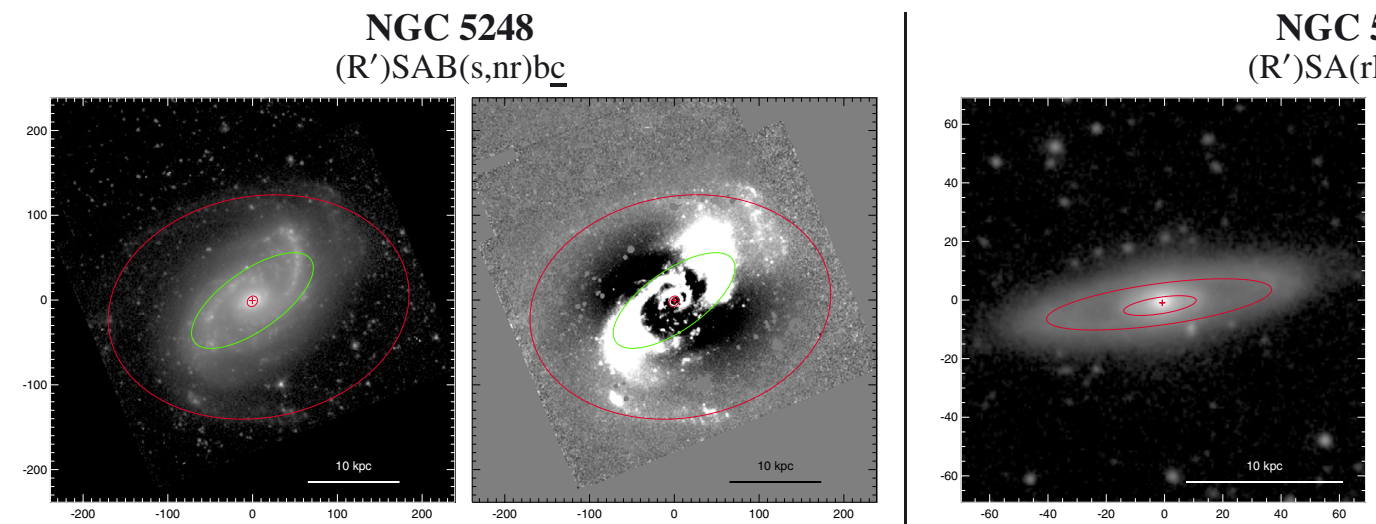

NGC 5289

$\left.\mathrm{R}^{\prime}\right) \mathrm{SA}(\mathrm{rl}) 0 / \mathrm{a} \mathrm{sp}$

\begin{tabular}{|c|c|c|c|c|c|c|c|c|c|c|c|c|c|}
\hline Feature & $\begin{array}{c}D_{\mathrm{r}, \mathrm{b}} \\
\left({ }^{\prime}\right)\end{array}$ & $\begin{array}{c}d_{\mathrm{r}, \mathrm{b}} \\
\left(^{\prime}\right)\end{array}$ & $\begin{array}{c}\mathrm{PA}_{\mathrm{r}, \mathrm{b}} \\
\left({ }^{\circ}\right)\end{array}$ & $\begin{array}{c}D_{\mathrm{r}, \mathrm{b}, 0} \\
\left(^{\prime}\right)\end{array}$ & $\begin{array}{c}d_{\mathrm{r}, \mathrm{b}, 0} \\
\left({ }^{\prime}\right)\end{array}$ & $\begin{array}{l}\theta_{\mathrm{r}, \mathrm{b}} \\
\left({ }^{\circ}\right)\end{array}$ & $\overline{\text { Feature }}$ & $\begin{array}{c}D_{\mathrm{r}, \mathrm{b}} \\
\left(^{\prime}\right)\end{array}$ & $\begin{array}{c}d_{\mathrm{r}, \mathrm{b}} \\
\left(^{\prime}\right)\end{array}$ & $\begin{array}{c}\mathrm{PA}_{\mathrm{r}, \mathrm{b}} \\
\left({ }^{\circ}\right)\end{array}$ & $\begin{array}{c}D_{\mathrm{r}, \mathrm{b}, 0} \\
\text { (') }^{\prime}\end{array}$ & $\begin{array}{c}d_{\mathrm{r}, \mathrm{b}, 0} \\
\left(^{\prime}\right)\end{array}$ & $\begin{array}{l}\theta_{\mathrm{r}, \mathrm{b}} \\
\left({ }^{\circ}\right)\end{array}$ \\
\hline$\overline{\mathrm{R}^{\prime}}$ & 5.97 & 4.36 & 100.2 & 5.99 & 5.55 & 163.7 & $\overline{\mathrm{R}^{\prime}}$ & 1.31 & 0.24 & 97.7 & 1.32 & 0.90 & 168.2 \\
\hline bar & 2.81 & 1.17 & 125.2 & 2.94 & 1.44 & 29.5 & $\mathrm{rl}$ & 0.42 & 0.09 & 98.8 & 0.42 & 0.36 & 170.8 \\
\hline $\mathrm{nr}$ & 0.22 & 0.19 & 139.5 & 0.26 & 0.20 & 68.9 & & & & & & & \\
\hline
\end{tabular}

NGC 5300

$\mathrm{SAB}(\mathrm{rs}) \mathrm{c}$
NGC 5313

$\mathrm{SA}(\mathrm{r}) \mathrm{b}$
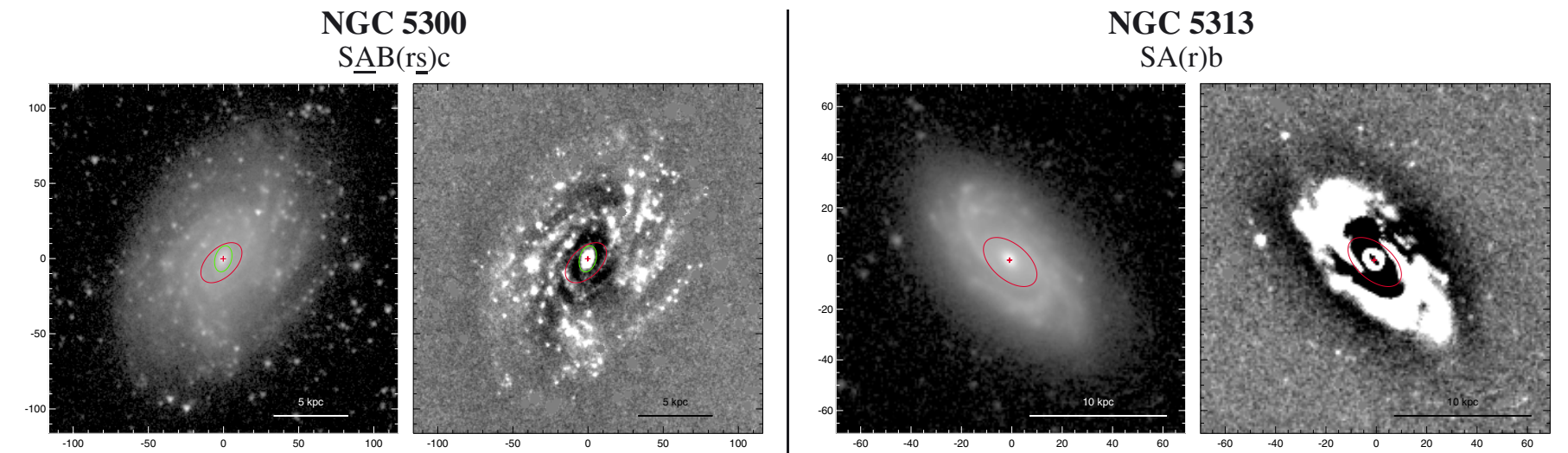

\begin{tabular}{|c|c|c|c|c|c|c|c|c|c|c|c|c|c|}
\hline Feature & $\begin{array}{c}D_{\mathrm{r}, \mathrm{b}} \\
\left({ }^{\prime}\right)\end{array}$ & $\begin{array}{c}d_{\mathrm{r}, \mathrm{b}} \\
(')\end{array}$ & $\begin{array}{c}\mathrm{PA}_{\mathrm{r}, \mathrm{b}} \\
\left({ }^{\circ}\right)\end{array}$ & $\begin{array}{c}D_{\mathrm{r}, \mathrm{b}, 0} \\
\left({ }^{\prime}\right)\end{array}$ & $\begin{array}{c}d_{\mathrm{r}, \mathrm{b}, 0} \\
\left(^{\prime}\right)\end{array}$ & $\begin{array}{l}\theta_{\mathrm{r}, \mathrm{b}} \\
\left(^{\circ}\right)\end{array}$ & Feature & $\begin{array}{c}D_{\mathrm{r}, \mathrm{b}} \\
\left({ }^{\prime}\right)\end{array}$ & $\begin{array}{c}d_{\mathrm{r}, \mathrm{b}} \\
\left(^{\prime}\right)\end{array}$ & $\begin{array}{c}\mathrm{PA}_{\mathrm{r}, \mathrm{b}} \\
\left({ }^{\circ}\right)\end{array}$ & $\begin{array}{c}D_{\mathrm{r}, \mathrm{b}, 0} \\
\left(^{\prime}\right)\end{array}$ & $\begin{array}{c}d_{\mathrm{r}, \mathrm{b}, 0} \\
\left(^{\prime}\right)\end{array}$ & $\begin{array}{l}\theta_{\mathrm{r}, \mathrm{b}} \\
\left({ }^{\circ}\right)\end{array}$ \\
\hline rs & 0.55 & 0.32 & 133.6 & 0.61 & 0.45 & 135.7 & $\mathrm{r}$ & 0.42 & 0.24 & 49.8 & 0.43 & 0.40 & 38.1 \\
\hline bar & 0.30 & 0.18 & 163.9 & 0.33 & 0.26 & 47.7 & & & & & & & \\
\hline
\end{tabular}




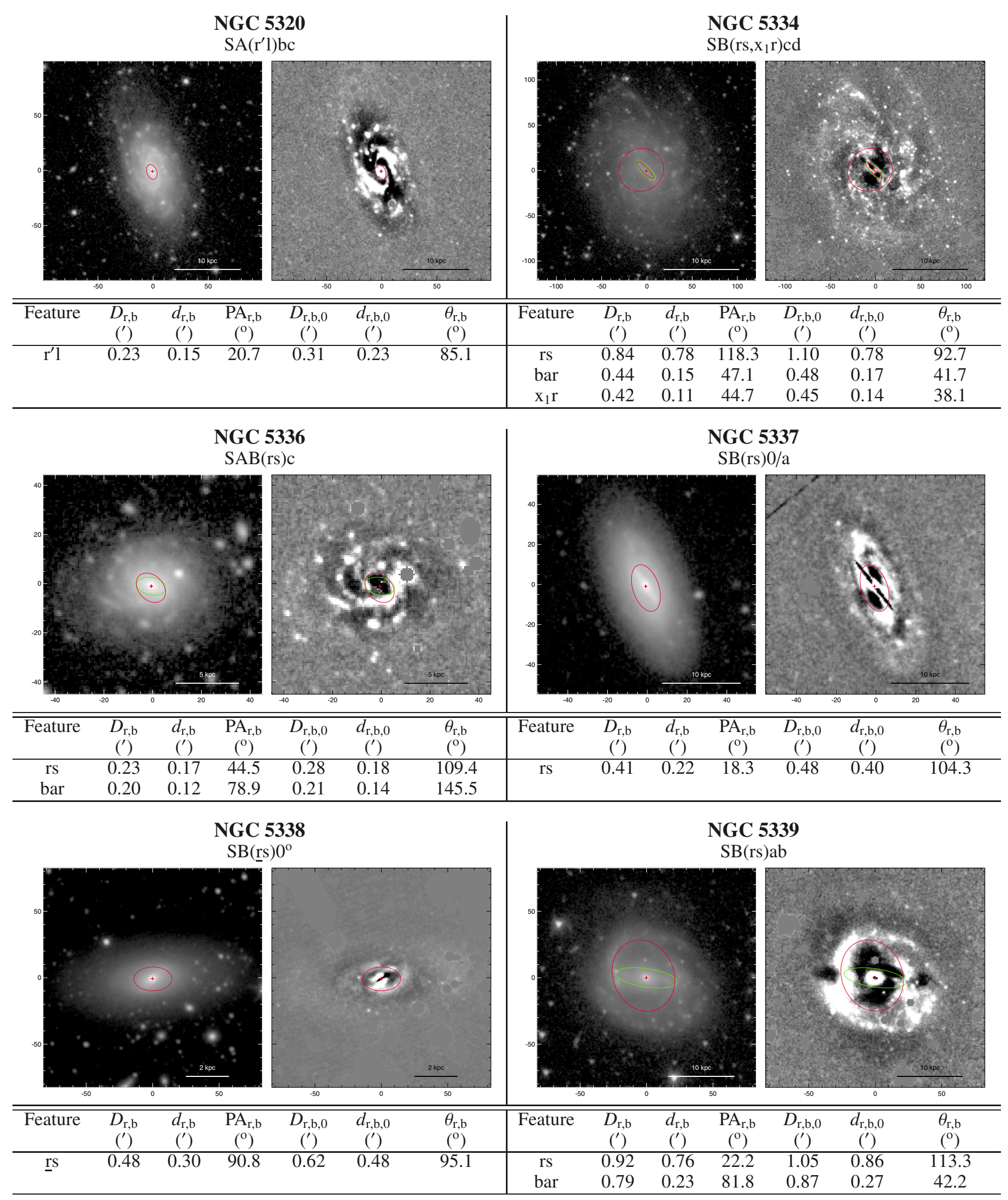




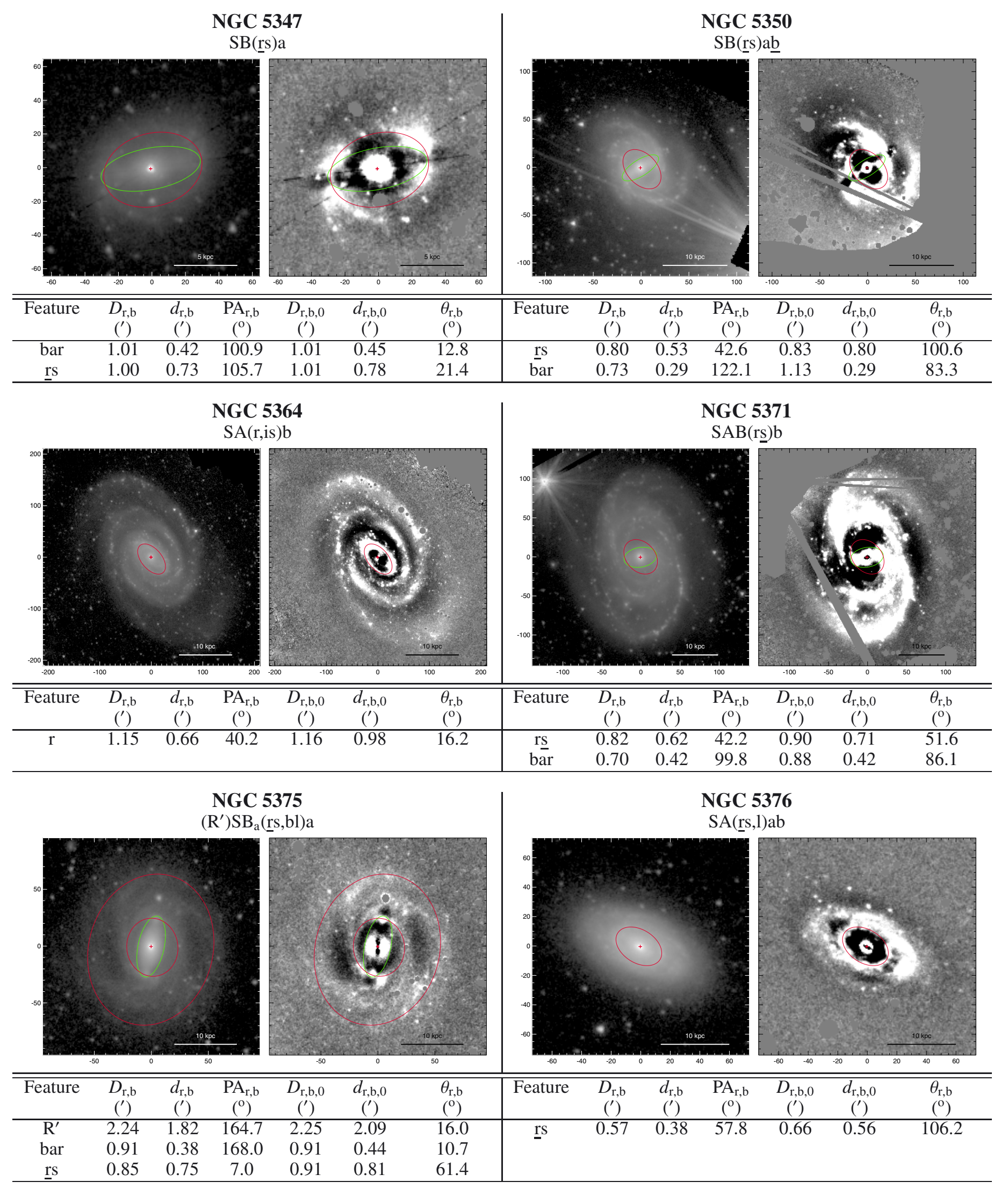


NGC 5377

$\left(\mathrm{R}_{1}\right) \mathrm{SAB}(\mathrm{s}, \mathrm{nl}) 0 / \mathrm{a}$
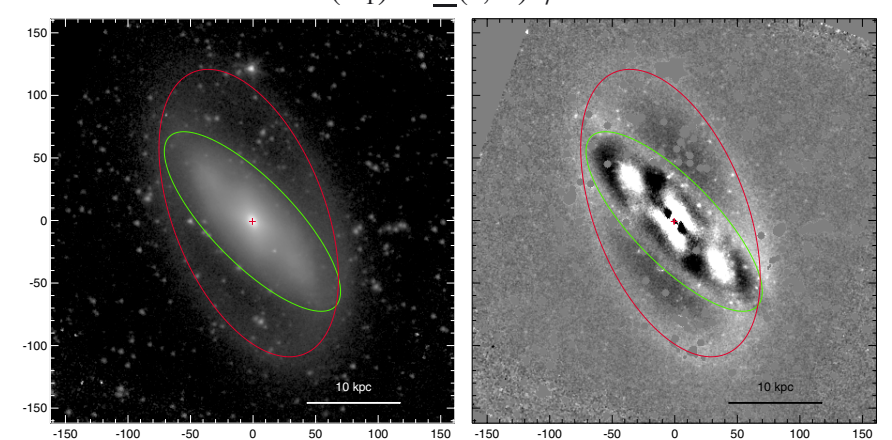

NGC 5383

SB(rs,nr)a

\begin{tabular}{ccccccc|ccccccc}
\hline \hline Feature & $\begin{array}{c}D_{\mathrm{r}, \mathrm{b}} \\
\left({ }^{\prime}\right)\end{array}$ & $\begin{array}{c}d_{\mathrm{r}, \mathrm{b}} \\
\left({ }^{\prime}\right)\end{array}$ & $\begin{array}{c}\mathrm{PA}_{\mathrm{r}, \mathrm{b}} \\
\left({ }^{\circ}\right)\end{array}$ & $\begin{array}{c}D_{\mathrm{r}, \mathrm{b}, 0} \\
\left({ }^{\prime}\right)\end{array}$ & $\begin{array}{c}d_{\mathrm{r}, \mathrm{b}, 0} \\
\left({ }^{\prime}\right)\end{array}$ & $\begin{array}{c}\theta_{\mathrm{r}, \mathrm{b}} \\
\left({ }^{\circ}\right)\end{array}$ & Feature & $\begin{array}{c}D_{\mathrm{r}, \mathrm{b}} \\
\left({ }^{\prime}\right)\end{array}$ & $\begin{array}{c}d_{\mathrm{r}, \mathrm{b}} \\
\left({ }^{\prime}\right)\end{array}$ & $\begin{array}{c}\mathrm{PA}_{\mathrm{r}, \mathrm{b}} \\
\left({ }^{\circ}\right)\end{array}$ & $\begin{array}{c}D_{\mathrm{r}, \mathrm{b}, 0} \\
\left({ }^{\prime}\right)\end{array}$ & $\begin{array}{c}d_{\mathrm{r}, \mathrm{b}, 0} \\
\left({ }^{\prime}\right)\end{array}$ & $\begin{array}{c}\theta_{\mathrm{r}, \mathrm{b}} \\
\left({ }^{\circ}\right)\end{array}$ \\
\hline $\mathrm{R}_{1}$ & 4.05 & 2.03 & 21.4 & 4.19 & 3.64 & 146.7 & $\mathrm{rs}$ & 1.98 & 1.72 & 102.4 & 2.11 & 1.96 & 106.2 \\
bar & 3.17 & 1.11 & 44.3 & 3.62 & 1.81 & 39.0 & $\mathrm{bar}$ & 1.67 & 0.61 & 123.5 & 1.69 & 0.73 & 16.6 \\
& & & & & & & $\mathrm{nr}$ & 0.29 & 0.14 & 79.1 & 0.31 & 0.16 & 139.6 \\
\hline
\end{tabular}
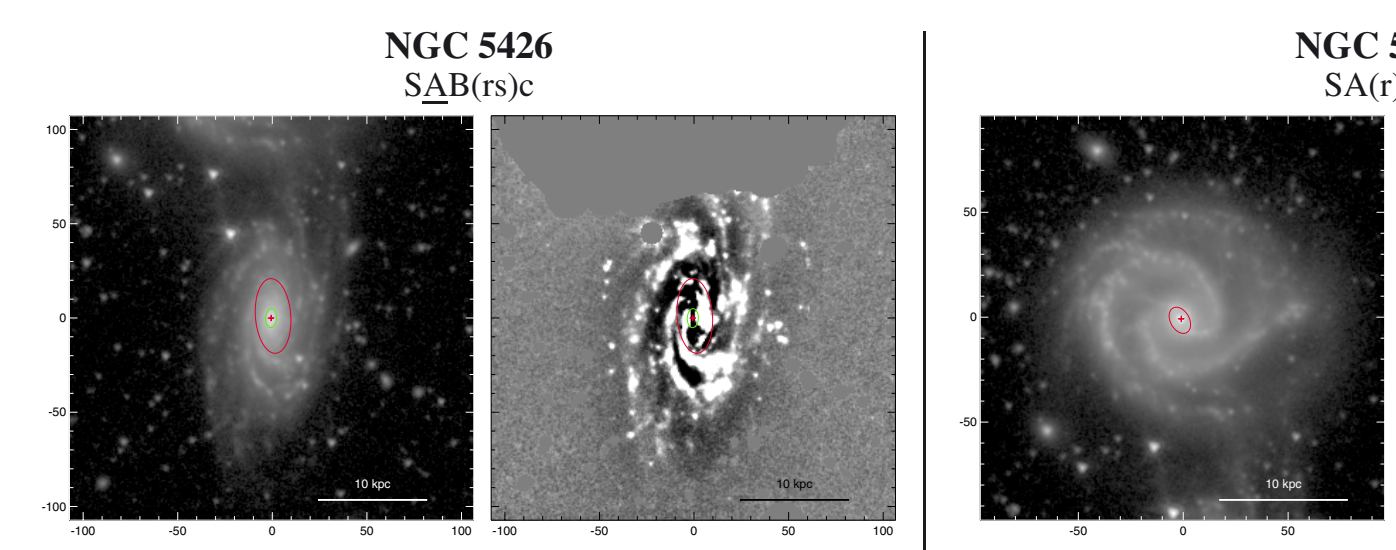

NGC 5427

$\mathrm{SA}(\mathrm{r}) \mathrm{bc}$

\begin{tabular}{|c|c|c|c|c|c|c|c|c|c|c|c|c|c|}
\hline Feature & $\begin{array}{c}D_{\mathrm{r}, \mathrm{b}} \\
\left({ }^{\prime}\right)\end{array}$ & $\begin{array}{c}d_{\mathrm{r}, \mathrm{b}} \\
\left(^{\prime}\right)\end{array}$ & $\begin{array}{c}\mathrm{PA}_{\mathrm{r}, \mathrm{b}} \\
\left({ }^{\circ}\right)\end{array}$ & $\begin{array}{c}D_{\mathrm{r}, \mathrm{b}, 0} \\
\left(^{\prime}\right)\end{array}$ & $\begin{array}{c}d_{\mathrm{r}, \mathrm{b}, 0} \\
\left({ }^{\prime}\right)\end{array}$ & $\begin{array}{c}\theta_{\mathrm{r}, \mathrm{b}} \\
\left({ }^{\circ}\right)\end{array}$ & Feature & $\begin{array}{c}D_{\mathrm{r}, \mathrm{b}} \\
\left({ }^{\prime}\right)\end{array}$ & $\begin{array}{c}d_{\mathrm{r}, \mathrm{b}} \\
\left({ }^{\prime}\right)\end{array}$ & $\begin{array}{c}\mathrm{PA}_{\mathrm{r}, \mathrm{b}} \\
\left({ }^{\circ}\right)\end{array}$ & $\begin{array}{c}\overline{D_{\mathrm{r}, \mathrm{b}, 0}} \\
\left(^{\prime}\right)\end{array}$ & $\begin{array}{c}d_{\mathrm{r}, \mathrm{b}, 0} \\
\left({ }^{\prime}\right)\end{array}$ & $\begin{array}{l}\theta_{\mathrm{r}, \mathrm{b}} \\
\left({ }^{\circ}\right)\end{array}$ \\
\hline rs & 0.66 & 0.31 & 4.2 & 0.78 & 0.53 & 49.8 & $r$ & 0.22 & 0.15 & 29.3 & 0.24 & 0.16 & 130.2 \\
\hline bar & 0.17 & 0.10 & 177.2 & 0.20 & 0.16 & 69.8 & & & & & & & \\
\hline
\end{tabular}

NGC 5430

$\left(\mathrm{R}^{\prime}\right) \mathrm{SB}(\mathrm{s}, \mathrm{tb}) \mathrm{b}$
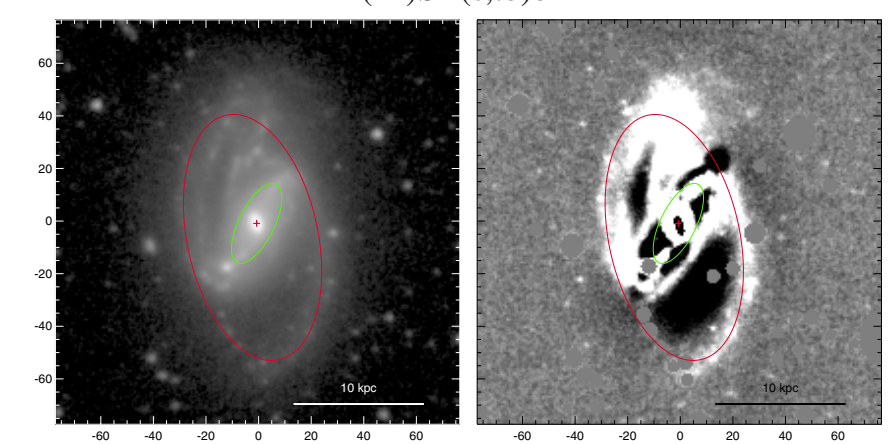

\section{NGC 5443}

$\left(\mathrm{R}^{\prime} \mathrm{L}\right) \mathrm{SAB}_{\mathrm{x}}(\underline{\mathrm{rs}}) \mathrm{a} \mathrm{sp}$

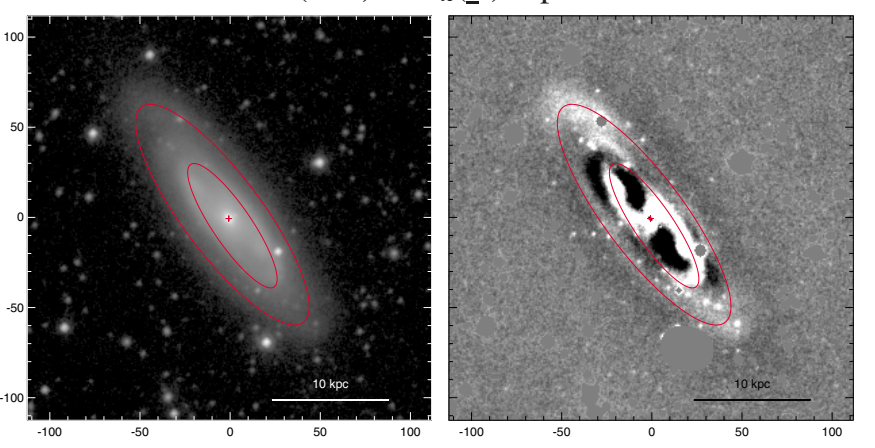

\begin{tabular}{|c|c|c|c|c|c|c|c|c|c|c|c|c|c|}
\hline Feature & $\begin{array}{c}D_{\mathrm{r}, \mathrm{b}} \\
\left({ }^{\prime}\right)\end{array}$ & $\begin{array}{c}d_{\mathrm{r}, \mathrm{b}} \\
\left({ }^{\prime}\right)\end{array}$ & $\begin{array}{c}\mathrm{PA}_{\mathrm{r}, \mathrm{b}} \\
\left({ }^{\circ}\right)\end{array}$ & $\begin{array}{c}D_{\mathrm{r}, \mathrm{b}, 0} \\
\left(^{\prime}\right)\end{array}$ & $\begin{array}{c}d_{\mathrm{r}, \mathrm{b}, 0} \\
\left(^{\prime}\right)\end{array}$ & $\begin{array}{l}\theta_{\mathrm{r}, \mathrm{b}} \\
\left({ }^{\circ}\right)\end{array}$ & Feature & $\begin{array}{c}D_{\mathrm{r}, \mathrm{b}} \\
\left({ }^{\prime}\right)\end{array}$ & $\begin{array}{c}d_{\mathrm{r}, \mathrm{b}} \\
\left({ }^{\prime}\right)\end{array}$ & $\begin{array}{c}\mathrm{PA}_{\mathrm{r}, \mathrm{b}} \\
\left({ }^{\circ}\right)\end{array}$ & $\begin{array}{c}D_{\mathrm{r}, \mathrm{b}, 0} \\
\left(^{\prime}\right)\end{array}$ & $\begin{array}{c}d_{\mathrm{r}, \mathrm{b}, 0} \\
\left(^{\prime}\right)\end{array}$ & $\begin{array}{l}\theta_{\mathrm{r}, \mathrm{b}} \\
\left({ }^{\circ}\right)\end{array}$ \\
\hline$\overline{\mathrm{R}^{\prime}}$ & 1.59 & 0.83 & 12.1 & 1.63 & 1.23 & 22.0 & $\overline{\mathrm{R}^{\prime} \mathrm{L}}$ & 2.50 & 0.73 & 36.9 & 2.52 & 1.94 & 10.8 \\
\hline bar & 0.56 & 0.23 & 153.6 & 0.65 & 0.29 & 133.2 & $\underline{\mathrm{r} s}$ & 1.38 & 0.36 & 34.3 & 1.38 & 0.96 & 176.2 \\
\hline
\end{tabular}


S. Comerón et al.: ARRAKIS

NGC 5448

$\left(\mathrm{R}_{1}\right) \mathrm{SAB}(\underline{\mathrm{rs}}) \mathrm{a}$
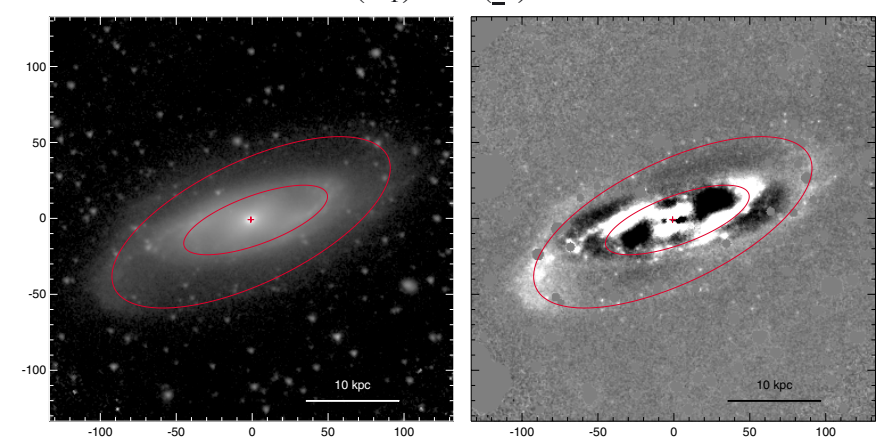

\begin{tabular}{|c|c|c|c|c|c|c|c|c|c|c|c|c|c|}
\hline Feature & $\begin{array}{c}D_{\mathrm{r}, \mathrm{b}} \\
\left({ }^{\prime}\right)\end{array}$ & $\begin{array}{c}d_{\mathrm{r}, \mathrm{b}} \\
\left({ }^{\prime}\right)\end{array}$ & $\begin{array}{c}\mathrm{PA}_{\mathrm{r}, \mathrm{b}} \\
\left({ }^{\mathrm{O}}\right)\end{array}$ & $\begin{array}{c}D_{\mathrm{r}, \mathrm{b}, 0} \\
\left(^{\prime}\right)\end{array}$ & $\begin{array}{c}d_{\mathrm{r}, \mathrm{b}, 0} \\
\left({ }^{\prime}\right)\end{array}$ & $\begin{array}{l}\theta_{\mathrm{r}, \mathrm{b}} \\
\left({ }^{\circ}\right)\end{array}$ & Feature & $\begin{array}{c}D_{\mathrm{r}, \mathrm{b}} \\
\left({ }^{\prime}\right)\end{array}$ & $\begin{array}{c}d_{\mathrm{r}, \mathrm{b}} \\
\left({ }^{\prime}\right)\end{array}$ & $\begin{array}{c}\mathrm{PA}_{\mathrm{r}, \mathrm{b}} \\
\left({ }^{\circ}\right)\end{array}$ & $\begin{array}{c}D_{\mathrm{r}, \mathrm{b}, 0} \\
\left(^{\prime}\right)\end{array}$ & $\begin{array}{c}d_{\mathrm{r}, \mathrm{b}, 0} \\
{ }^{\prime}\left({ }^{\prime}\right)\end{array}$ & $\begin{array}{l}\theta_{\mathrm{r}, \mathrm{b}} \\
\left({ }^{\circ}\right)\end{array}$ \\
\hline$\overline{\mathrm{R}_{1}}$ & 3.34 & 1.34 & 115.7 & 3.62 & 2.96 & 43.3 & $\mathrm{rs}$ & 1.81 & 1.60 & 178.7 & $\begin{array}{l}1.88 \\
\end{array}$ & 1.65 & 124.1 \\
\hline rs & 1.67 & 0.54 & 110.2 & 1.67 & 1.28 & 179.9 & bār & 0.84 & 0.58 & 80.4 & 0.87 & 0.60 & 48.7 \\
\hline
\end{tabular}

NGC 5472

$\left(\mathrm{R}^{\prime}\right) \mathrm{SAB}_{\mathrm{a}} 0 / \mathrm{a}$
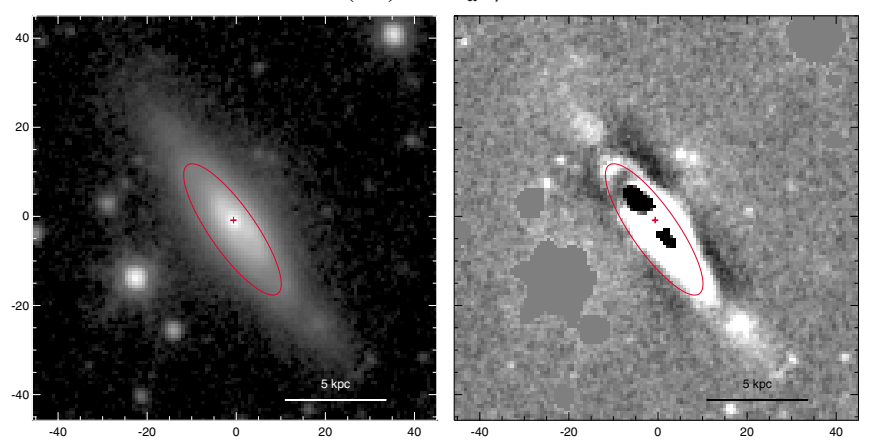

\begin{tabular}{|c|c|c|c|c|c|c|c|c|c|c|c|c|c|}
\hline Feature & $\begin{array}{c}D_{\mathrm{r}, \mathrm{b}} \\
\left({ }^{\prime}\right)\end{array}$ & $\begin{array}{c}d_{\mathrm{r}, \mathrm{b}} \\
\left({ }^{\prime}\right)\end{array}$ & $\begin{array}{c}\mathrm{PA}_{\mathrm{r}, \mathrm{b}} \\
\left({ }^{\circ}\right)\end{array}$ & $\begin{array}{c}D_{\mathrm{r}, \mathrm{b}, 0} \\
\left(^{\prime}\right)\end{array}$ & $\begin{array}{c}d_{\mathrm{r}, \mathrm{b}, 0} \\
\left({ }^{\prime}\right)\end{array}$ & $\begin{array}{c}\theta_{\mathrm{r}, \mathrm{b}} \\
\left({ }^{\circ}\right)\end{array}$ & Feature & $\begin{array}{c}D_{\mathrm{r}, \mathrm{b}} \\
\left({ }^{\prime}\right)\end{array}$ & $\begin{array}{c}d_{\mathrm{r}, \mathrm{b}} \\
\left({ }^{\prime}\right)\end{array}$ & $\begin{array}{c}\mathrm{PA}_{\mathrm{r}, \mathrm{b}} \\
\left({ }^{\circ}\right)\end{array}$ & $\begin{array}{c}D_{\mathrm{r}, \mathrm{b}, 0} \\
\left(^{\prime}\right)\end{array}$ & $\begin{array}{c}d_{\mathrm{r}, \mathrm{b}, 0} \\
\left({ }^{\prime}\right)\end{array}$ & $\begin{array}{l}\theta_{\mathrm{r}, \mathrm{b}} \\
\left({ }^{\circ}\right)\end{array}$ \\
\hline$\overline{\mathrm{R}^{\prime}}$ & 0.59 & 0.17 & 35.0 & 0.59 & 0.45 & 172. & $\mathrm{rs}$ & 0.40 & 0.09 & 500 & 040 & 033 & 188 \\
\hline
\end{tabular}

\section{NGC 5523}

$\mathrm{SA}(\mathrm{rs}) \mathrm{cd}$
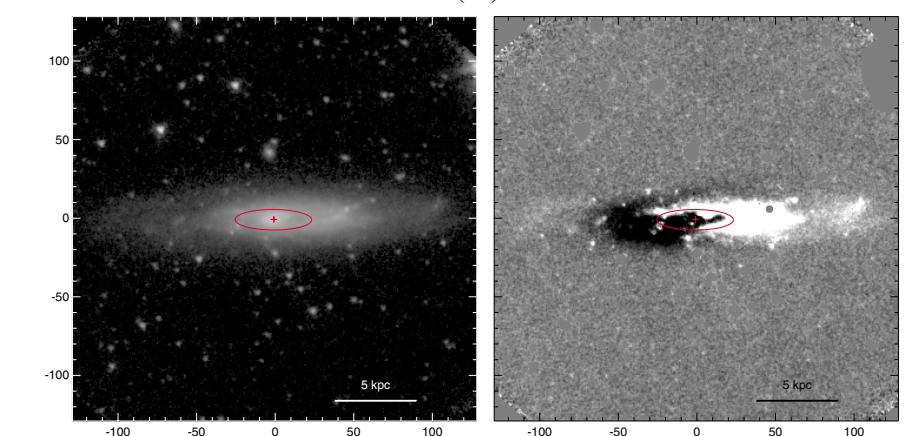

\begin{tabular}{ccccccc|ccccccc}
\hline \hline Feature & $\begin{array}{c}D_{\mathrm{r}, \mathrm{b}} \\
\left({ }^{\prime}\right)\end{array}$ & $\begin{array}{c}d_{\mathrm{r}, \mathrm{b}} \\
\left({ }^{\prime}\right)\end{array}$ & $\begin{array}{c}\mathrm{PA}_{\mathrm{r}, \mathrm{b}} \\
\left({ }^{\circ}\right)\end{array}$ & $\begin{array}{c}D_{\mathrm{r}, \mathrm{b}, 0} \\
\left({ }^{\prime}\right)\end{array}$ & $\begin{array}{c}d_{\mathrm{r}, \mathrm{b}, 0} \\
\left({ }^{\prime}\right)\end{array}$ & $\begin{array}{c}\theta_{\mathrm{r}, \mathrm{b}} \\
\left({ }^{\circ}\right)\end{array}$ & Feature & $\begin{array}{c}D_{\mathrm{r}, \mathrm{b}} \\
\left({ }^{\prime}\right)\end{array}$ & $\begin{array}{c}d_{\mathrm{r}, \mathrm{b}} \\
\left({ }^{\prime}\right)\end{array}$ & $\begin{array}{c}\mathrm{PA}_{\mathrm{r}, \mathrm{b}} \\
\left({ }^{\circ}\right)\end{array}$ & $\begin{array}{c}D_{\mathrm{r}, \mathrm{b}, 0} \\
\left({ }^{\prime}\right)\end{array}$ & $\begin{array}{c}d_{\mathrm{r}, \mathrm{b}, 0} \\
\left({ }^{\prime}\right)\end{array}$ & $\begin{array}{c}\theta_{\mathrm{r}, \mathrm{b}} \\
\left({ }^{\circ}\right)\end{array}$ \\
\hline rs & 0.81 & 0.22 & 89.8 & 0.89 & 0.72 & 135.6 & $\mathrm{R}{ }^{\prime} \mathrm{L}$ & 1.21 & 0.83 & 170.3 & 1.30 & 0.86 & 58.7 \\
& & & & & & & & $\mathrm{rs}$ & 0.50 & 0.37 & 77.6 & 0.53 & 0.39 \\
& & & & & & & $\mathrm{bar}$ & 0.50 & 0.22 & 74.9 & 0.53 & 0.23 & 130.2 \\
\hline
\end{tabular}

NGC 5457

$\mathrm{SAB}(\mathrm{rs}) \mathrm{c}$

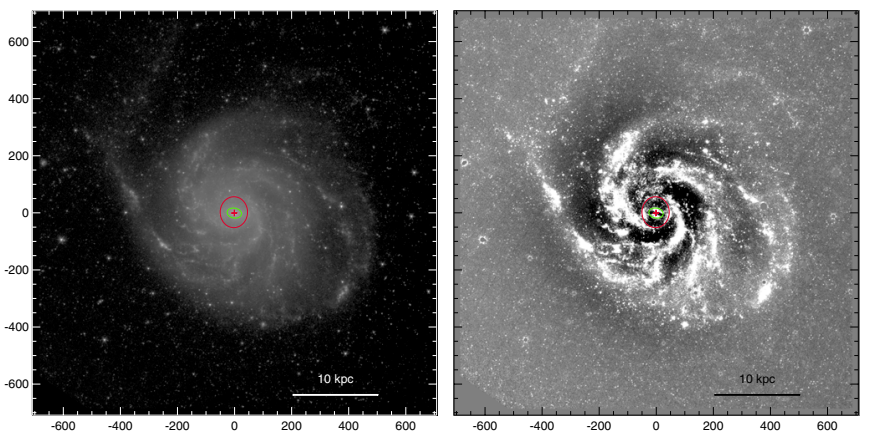

\section{NGC 5492}

$\mathrm{SA}(\mathrm{rs}) \mathrm{b}: \mathrm{sp}$

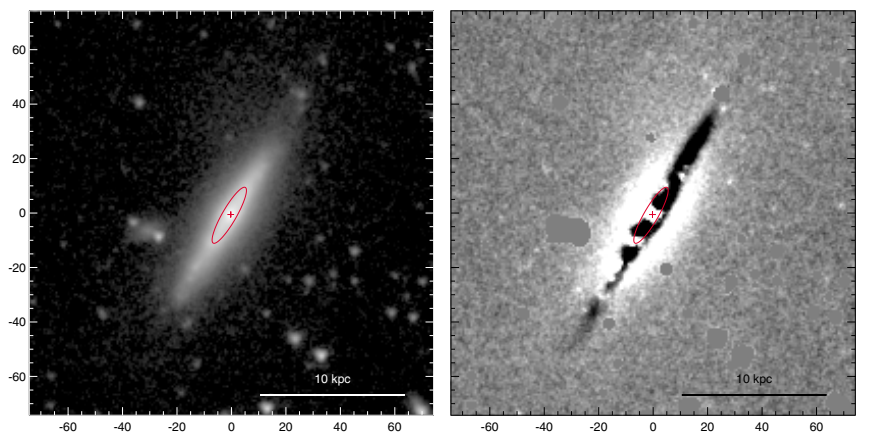

\section{NGC 5534}

( $\left.\mathrm{R}^{\prime} \mathrm{L}\right) \mathrm{SB}(\mathrm{rs})$ a pec

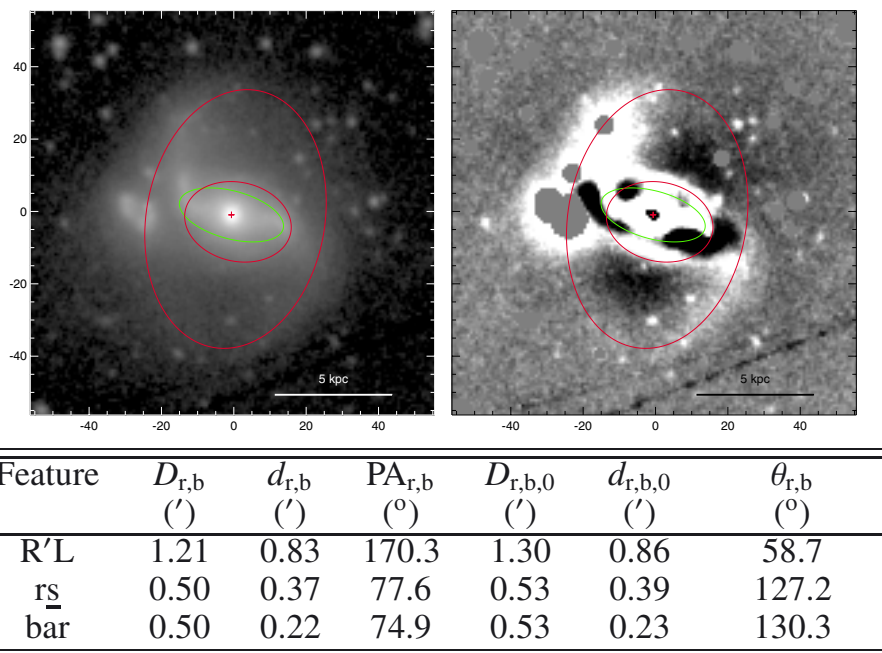


NGC 5566

$\left(\mathrm{R}^{\prime}\right) \mathrm{SAB}\left(\mathrm{r}, \mathrm{nr}^{\prime}\right) \mathrm{a}$

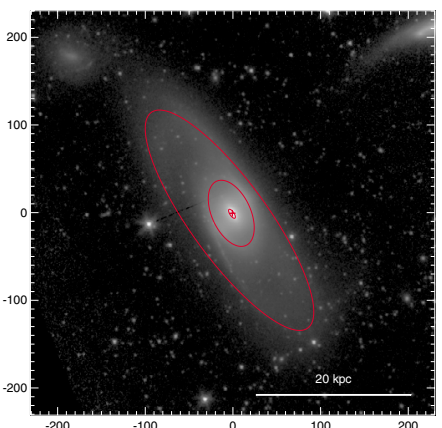

NGC 5577

$\mathrm{SA}(\mathrm{rs}) \mathrm{cd}$
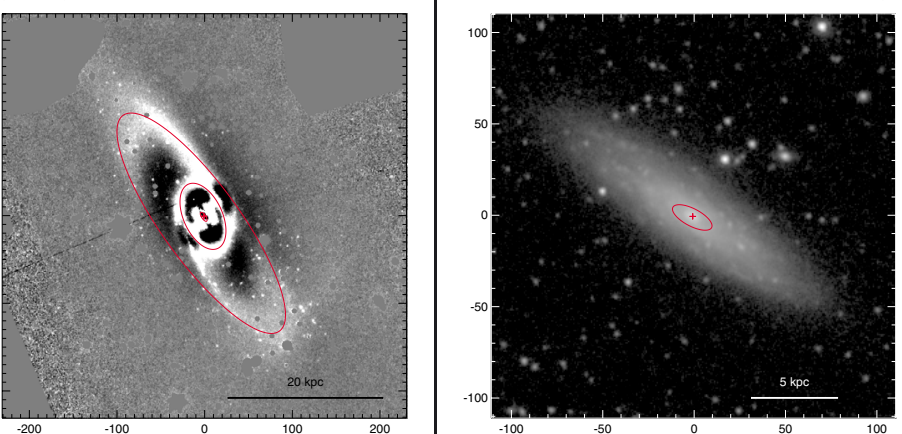

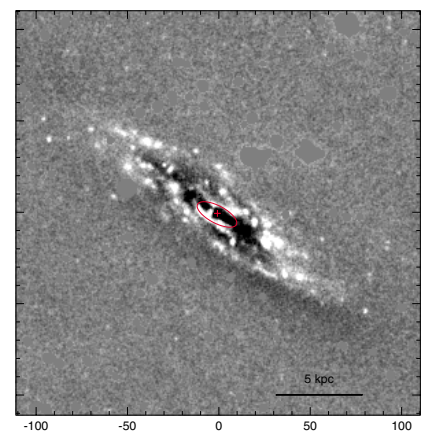

\begin{tabular}{|c|c|c|c|c|c|c|c|c|c|c|c|c|c|}
\hline Feature & $\begin{array}{c}D_{\mathrm{r}, \mathrm{b}} \\
\left({ }^{\prime}\right)\end{array}$ & $\begin{array}{c}d_{\mathrm{r}, \mathrm{b}} \\
\left({ }^{\prime}\right)\end{array}$ & $\begin{array}{c}\mathrm{PA}_{\mathrm{r}, \mathrm{b}} \\
\left({ }^{\circ}\right)\end{array}$ & $\begin{array}{c}D_{\mathrm{r}, \mathrm{b}, 0} \\
\left(^{\prime}\right)\end{array}$ & $\begin{array}{c}d_{\mathrm{r}, \mathrm{b}, 0} \\
\left({ }^{\prime}\right)\end{array}$ & $\begin{array}{l}\theta_{\mathrm{r}, \mathrm{b}} \\
\left({ }^{\circ}\right)\end{array}$ & Feature & $\begin{array}{c}D_{\mathrm{r}, \mathrm{b}} \\
\left({ }^{\prime}\right)\end{array}$ & $\begin{array}{c}d_{\mathrm{r}, \mathrm{b}} \\
\left({ }^{\prime}\right)\end{array}$ & $\begin{array}{c}\mathrm{PA}_{\mathrm{r}, \mathrm{b}} \\
\left(^{\circ}\right)\end{array}$ & $\begin{array}{c}D_{\mathrm{r}, \mathrm{b}, 0} \\
\left(^{\prime}\right)\end{array}$ & $\begin{array}{c}d_{\mathrm{r}, \mathrm{b}, 0} \\
{ }^{\prime}\left({ }^{\prime}\right)\end{array}$ & $\begin{array}{c}\theta_{\mathrm{r}, \mathrm{b}} \\
\left({ }^{\circ}\right)\end{array}$ \\
\hline$\overline{\mathrm{R}^{\prime}}$ & 5.07 & 1.47 & 36.0 & 5.09 & 3.99 & 7.4 & rs & 0.40 & 0.16 & 62.3 & 0.60 & 0.37 & 72.2 \\
\hline $\mathrm{r}$ & 1.35 & 0.74 & 24.8 & 2.10 & 1.28 & 102.6 & & & & & & & \\
\hline $\mathrm{nr}^{\prime}$ & 0.20 & 0.08 & 34.1 & 0.22 & 0.20 & 100.3 & & & & & & & \\
\hline
\end{tabular}
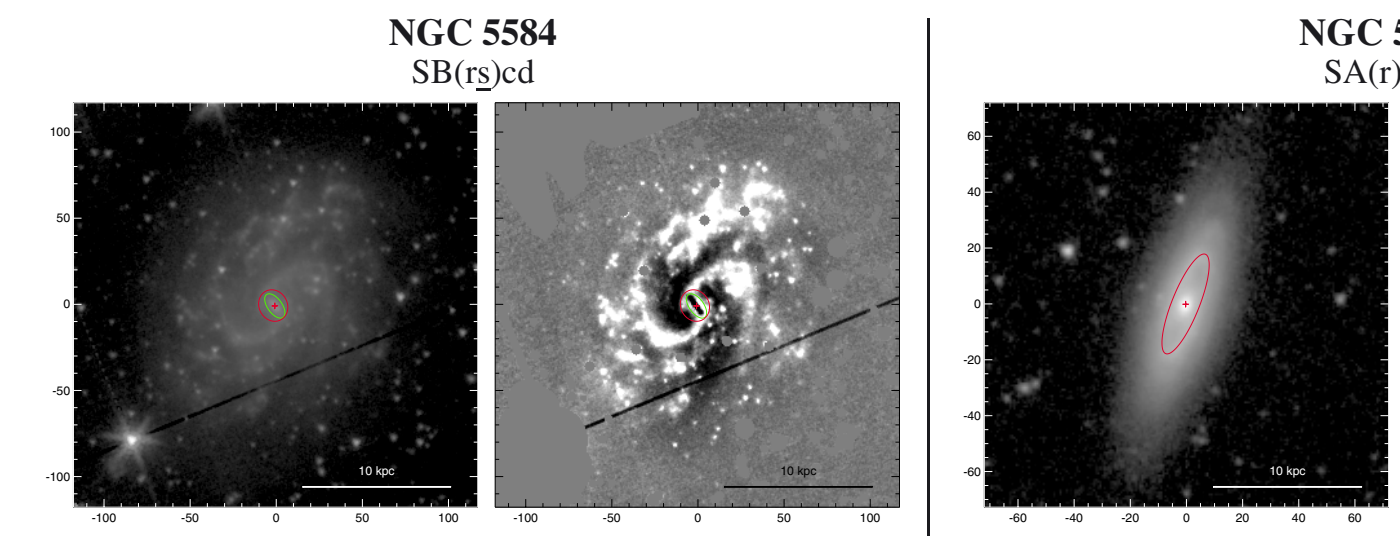

NGC 5587

SA(r)0/a

\begin{tabular}{|c|c|c|c|c|c|c|c|c|c|c|c|c|c|}
\hline Feature & $\begin{array}{c}D_{\mathrm{r}, \mathrm{b}} \\
\left(^{\prime}\right)\end{array}$ & $\begin{array}{c}d_{\mathrm{r}, \mathrm{b}} \\
\left(^{\prime}\right)\end{array}$ & $\begin{array}{c}\mathrm{PA}_{\mathrm{r}, \mathrm{b}} \\
\left({ }^{\mathrm{o}}\right)\end{array}$ & $\begin{array}{c}D_{\mathrm{r}, \mathrm{b}, 0} \\
\left(^{\prime}\right)\end{array}$ & $\begin{array}{c}d_{\mathrm{r}, \mathrm{b}, 0} \\
\left({ }^{\prime}\right)\end{array}$ & $\begin{array}{c}\theta_{\mathrm{r}, \mathrm{b}} \\
\left({ }^{\circ}\right)\end{array}$ & Feature & $\begin{array}{c}D_{\mathrm{r}, \mathrm{b}} \\
\left({ }^{\prime}\right)\end{array}$ & $\begin{array}{c}d_{\mathrm{r}, \mathrm{b}} \\
\left({ }^{\prime}\right)\end{array}$ & $\begin{array}{c}\mathrm{PA}_{\mathrm{r}, \mathrm{b}} \\
\left({ }^{\circ}\right)\end{array}$ & $\begin{array}{c}D_{\mathrm{r}, \mathrm{b}, 0} \\
\left(^{\prime}\right)\end{array}$ & $\begin{array}{c}d_{\mathrm{r}, \mathrm{b}, 0} \\
\left({ }^{\prime}\right)\end{array}$ & $\begin{array}{l}\theta_{\mathrm{r}, \mathrm{b}} \\
\left(^{\circ}\right)\end{array}$ \\
\hline rs & 0.31 & 0.27 & 27.4 & 0.42 & 0.29 & 80.0 & $r$ & 0.64 & 0.16 & 158.2 & 0.67 & 0.45 & 157.0 \\
\hline bār & 0.27 & 0.14 & 35.9 & 0.36 & 0.15 & 70.1 & & & & & & & \\
\hline
\end{tabular}

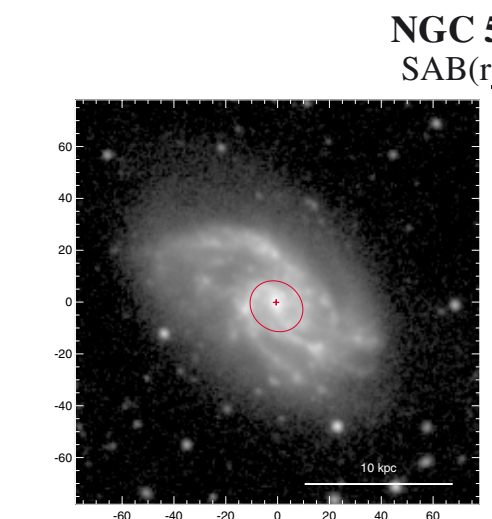

GC 5595

$\mathrm{AB}(\mathrm{rs}) \mathrm{cd}$

NGC 5600

$\mathrm{SB}(\mathrm{rs}) \mathrm{dm}$

\begin{tabular}{|c|c|c|c|c|c|c|c|c|c|c|c|c|c|}
\hline Feature & $\begin{array}{c}D_{\mathrm{r}, \mathrm{b}} \\
\left(^{\prime}\right)\end{array}$ & $\begin{array}{c}d_{\mathrm{r}, \mathrm{b}} \\
\left(^{\prime}\right)\end{array}$ & $\begin{array}{c}\mathrm{PA}_{\mathrm{r}, \mathrm{b}} \\
\left({ }^{\circ}\right)\end{array}$ & $\begin{array}{c}D_{\mathrm{r}, \mathrm{b}, 0} \\
\left(^{\prime}\right)\end{array}$ & $\begin{array}{c}d_{\mathrm{r}, \mathrm{b}, 0} \\
\left.\text { (') }^{\prime}\right)\end{array}$ & $\begin{array}{c}\theta_{\mathrm{r}, \mathrm{b}} \\
\left({ }^{\circ}\right)\end{array}$ & Feature & $\begin{array}{c}D_{\mathrm{r}, \mathrm{b}} \\
\left(^{\prime}\right)\end{array}$ & $\begin{array}{c}d_{\mathrm{r}, \mathrm{b}} \\
\left(^{\prime}\right)\end{array}$ & $\begin{array}{c}\mathrm{PA}_{\mathrm{r}, \mathrm{b}} \\
\left({ }^{\circ}\right)\end{array}$ & $\begin{array}{c}D_{\mathrm{r}, \mathrm{b}, 0} \\
\left(^{\prime}\right)\end{array}$ & $\begin{array}{c}d_{\mathrm{r}, \mathrm{b}, 0} \\
\left(^{\prime}\right)\end{array}$ & $\begin{array}{l}\theta_{\mathrm{r}, \mathrm{b}} \\
\left({ }^{\circ}\right)\end{array}$ \\
\hline $\mathrm{rs}$ & 0.36 & 0.31 & 53.0 & 0.51 & 0.36 & 87.9 & $\begin{array}{c}\text { bar } \\
\text { rs }\end{array}$ & $\begin{array}{l}0.44 \\
0.44\end{array}$ & $\begin{array}{l}0.20 \\
0.35\end{array}$ & $\begin{array}{l}155.6 \\
144.0\end{array}$ & $\begin{array}{l}0.45 \\
0.45\end{array}$ & $\begin{array}{l}0.21 \\
0.37\end{array}$ & $\begin{array}{l}166.1 \\
147.0\end{array}$ \\
\hline
\end{tabular}


NGC 5602

(RL)SA(l) $0^{+}$
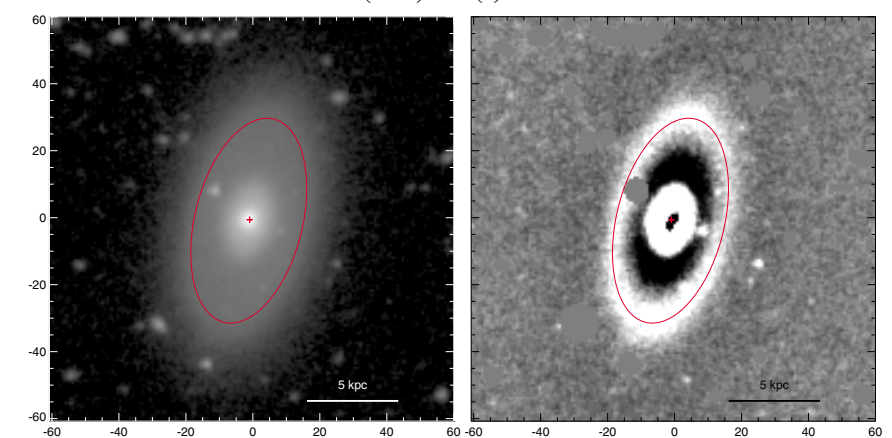

\begin{tabular}{|c|c|c|c|c|c|c|c|c|c|c|c|c|c|}
\hline Feature & $\begin{array}{c}D_{\mathrm{r}, \mathrm{b}} \\
\left({ }^{\prime}\right)\end{array}$ & $\begin{array}{c}d_{\mathrm{r}, \mathrm{b}} \\
\left({ }^{\prime}\right)\end{array}$ & $\begin{array}{c}\mathrm{PA}_{\mathrm{r}, \mathrm{b}} \\
\left({ }^{\circ}\right)\end{array}$ & $\begin{array}{c}D_{\mathrm{r}, \mathrm{b}, 0} \\
\left(^{\prime}\right)\end{array}$ & $\begin{array}{c}d_{\mathrm{r}, \mathrm{b}, 0} \\
\left({ }^{\prime}\right)\end{array}$ & $\begin{array}{l}\theta_{\mathrm{r}, \mathrm{b}} \\
\left({ }^{\circ}\right)\end{array}$ & Feature & $\begin{array}{c}D_{\mathrm{r}, \mathrm{b}} \\
\left({ }^{\prime}\right)\end{array}$ & $\begin{array}{c}d_{\mathrm{r}, \mathrm{b}} \\
\left({ }^{\prime}\right)\end{array}$ & $\begin{array}{c}\mathrm{PA}_{\mathrm{r}, \mathrm{b}} \\
\left(^{\circ}\right)\end{array}$ & $\begin{array}{c}D_{\mathrm{r}, \mathrm{b}, 0} \\
\left(^{\prime}\right)\end{array}$ & $\begin{array}{c}d_{\mathrm{r}, \mathrm{b}, 0} \\
\left(^{\prime}\right)\end{array}$ & $\begin{array}{l}\theta_{\mathrm{r}, \mathrm{b}} \\
\left({ }^{\circ}\right)\end{array}$ \\
\hline$\overline{\mathrm{RL}}$ & 1.05 & 0.54 & 166.2 & 1.05 & 0.97 & 165.1 & $r$ & 0.46 & 0.30 & 10.3 & 0.46 & 0.39 & 157.7 \\
\hline
\end{tabular}

NGC 5636

$\mathrm{SAB}(\mathrm{r}) 0 / \mathrm{a}$
NGC 5633

$\mathrm{SAB}(\mathrm{r}) \mathrm{b}$

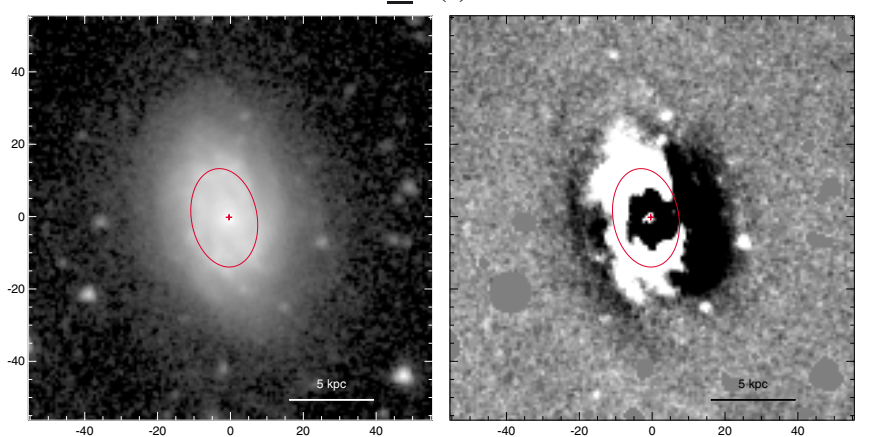

\section{NGC 5665}

$\mathrm{SAB}(\mathrm{rs}) \mathrm{c}$ pec $/ \mathrm{Sph}$
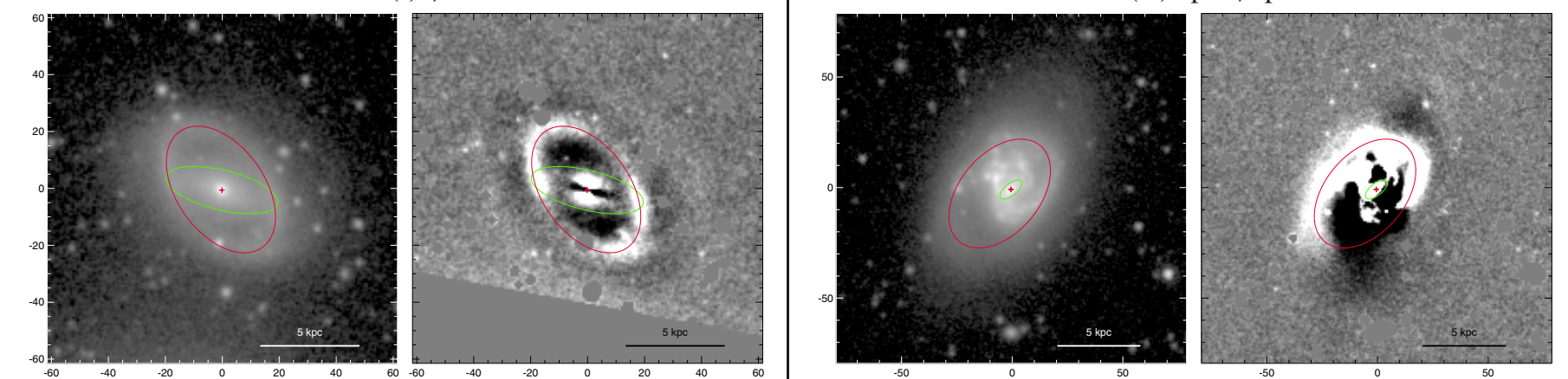

\begin{tabular}{ccccccc|ccccccc}
\hline \hline Feature & $\begin{array}{c}D_{\mathrm{r}, \mathrm{b}} \\
\left({ }^{\prime}\right)\end{array}$ & $\begin{array}{c}d_{\mathrm{r}, \mathrm{b}} \\
\left({ }^{\prime}\right)\end{array}$ & $\begin{array}{c}\mathrm{PA}_{\mathrm{r}, \mathrm{b}} \\
\left({ }^{\mathrm{O}}\right)\end{array}$ & $\begin{array}{c}D_{\mathrm{r}, \mathrm{b}, 0} \\
\left({ }^{\prime}\right)\end{array}$ & $\begin{array}{c}d_{\mathrm{r}, \mathrm{b}, 0} \\
\left({ }^{\prime}\right)\end{array}$ & $\begin{array}{c}\theta_{\mathrm{r}, \mathrm{b}} \\
\left({ }^{\circ}\right)\end{array}$ & Feature & $\begin{array}{c}D_{\mathrm{r}, \mathrm{b}} \\
\left({ }^{\prime}\right)\end{array}$ & $\begin{array}{c}d_{\mathrm{r}, \mathrm{b}} \\
\left({ }^{\prime}\right)\end{array}$ & $\begin{array}{c}\mathrm{PA}_{\mathrm{r}, \mathrm{b}} \\
\left({ }^{\circ}\right)\end{array}$ & $\begin{array}{c}D_{\mathrm{r}, \mathrm{b}, 0} \\
\left({ }^{\prime}\right)\end{array}$ & $\begin{array}{c}d_{\mathrm{r}, \mathrm{b}, 0} \\
\left({ }^{\prime}\right)\end{array}$ & $\begin{array}{c}\theta_{\mathrm{r}, \mathrm{b}} \\
\left({ }^{\circ}\right)\end{array}$ \\
\hline r & 0.84 & 0.52 & 35.3 & 0.85 & 0.56 & 152.1 & $\mathrm{rs}$ & 0.95 & 0.60 & 139.8 & 1.09 & 0.85 & 123.9 \\
bar & 0.68 & 0.23 & 76.3 & 0.68 & 0.26 & 20.6 & $\mathrm{bar}$ & 0.20 & 0.09 & 128.9 & 0.24 & 0.12 & 133.3 \\
\hline
\end{tabular}

NGC 5668

$\mathrm{SAB}(\mathrm{rs}) \mathrm{cd}$
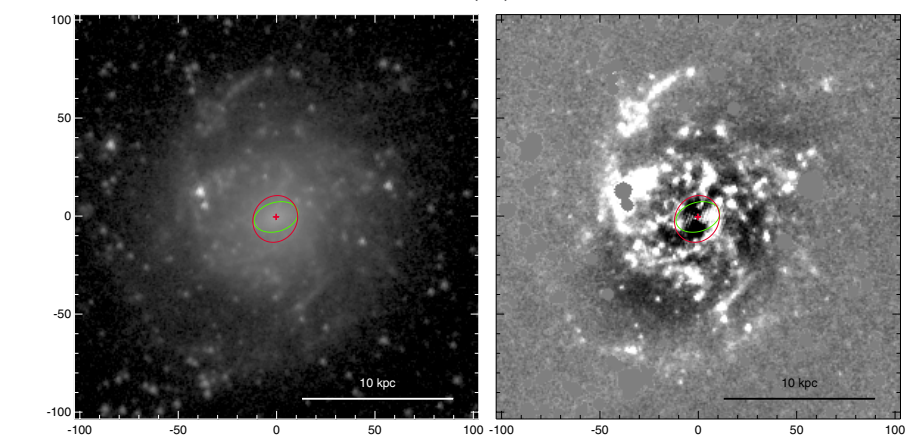

\begin{tabular}{|c|c|c|c|c|c|c|c|c|c|c|c|c|c|}
\hline Feature & $\begin{array}{c}D_{\mathrm{r}, \mathrm{b}} \\
\left({ }^{\prime}\right)\end{array}$ & $\begin{array}{c}d_{\mathrm{r}, \mathrm{b}} \\
\left({ }^{\prime}\right)\end{array}$ & $\begin{array}{c}\mathrm{PA}_{\mathrm{r}, \mathrm{b}} \\
\left({ }^{\circ}\right)\end{array}$ & $\begin{array}{c}D_{\mathrm{r}, \mathrm{b}, 0} \\
\left({ }^{\prime}\right)\end{array}$ & $\begin{array}{c}d_{\mathrm{r}, \mathrm{b}, 0} \\
\left.\text { (') }^{\prime}\right)\end{array}$ & $\begin{array}{l}\theta_{\mathrm{r}, \mathrm{b}} \\
\left({ }^{\circ}\right)\end{array}$ & Feature & $\begin{array}{c}D_{\mathrm{r}, \mathrm{b}} \\
\left({ }^{\prime}\right)\end{array}$ & $\begin{array}{c}d_{\mathrm{r}, \mathrm{b}} \\
\left(^{\prime}\right)\end{array}$ & $\begin{array}{c}\mathrm{PA}_{\mathrm{r}, \mathrm{b}} \\
\left({ }^{\circ}\right)\end{array}$ & $\begin{array}{c}D_{\mathrm{r}, \mathrm{b}, 0} \\
\left({ }^{\prime}\right)\end{array}$ & $\begin{array}{c}d_{\mathrm{r}, \mathrm{b}, 0} \\
\left(^{\prime}\right)\end{array}$ & $\begin{array}{l}\theta_{\mathrm{r}, \mathrm{b}} \\
\left({ }^{\circ}\right)\end{array}$ \\
\hline rs & 0.41 & 0.37 & 146.7 & 0.43 & 0.41 & 79.8 & $\mathrm{rs}$ & 0.38 & 0.31 & 89.1 & 0.45 & 0.35 & 70.5 \\
\hline bar & 0.38 & 0.25 & 106.5 & 0.41 & 0.27 & 134.3 & bar & 0.17 & 0.04 & 40.5 & 0.17 & 0.05 & 152.4 \\
\hline
\end{tabular}

NGC 5669

$\mathrm{SB}(\mathrm{rs}) \mathrm{d}$

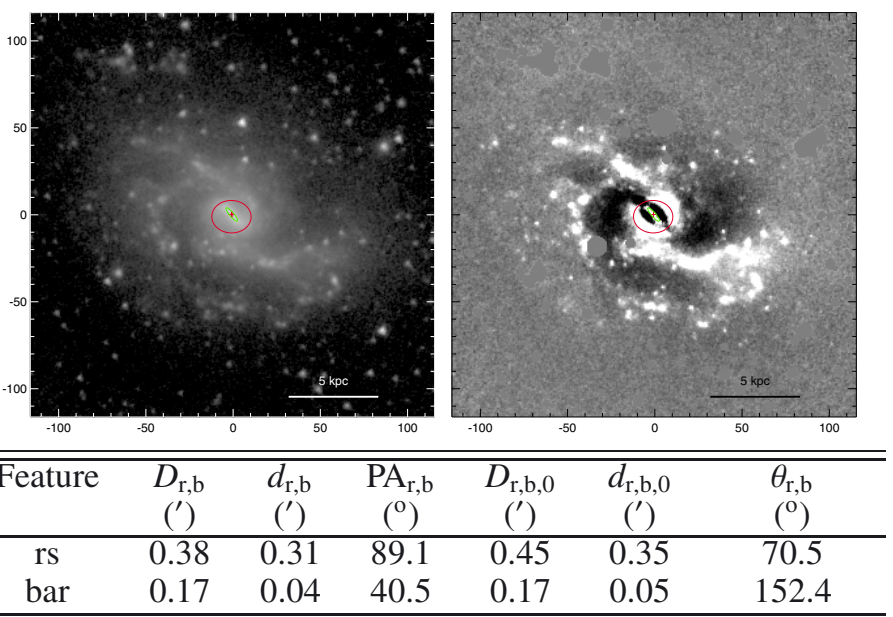


NGC 5678

$\left(\mathrm{R}^{\prime}:\right) \mathrm{SA}(\mathrm{rs}) \mathrm{b} / \mathrm{RG}$
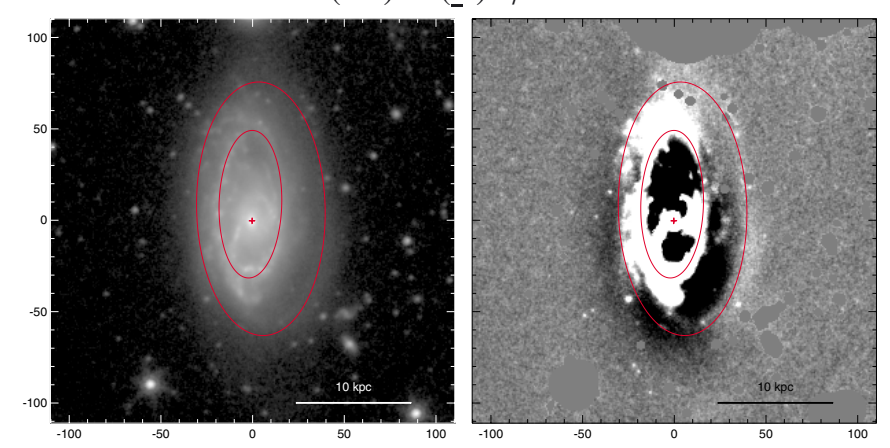

\begin{tabular}{|c|c|c|c|c|c|c|c|c|c|c|c|c|c|}
\hline Feature & $\begin{array}{c}D_{\mathrm{r}, \mathrm{b}} \\
\left({ }^{\prime}\right)\end{array}$ & $\begin{array}{c}d_{\mathrm{r}, \mathrm{b}} \\
\left({ }^{\prime}\right)\end{array}$ & $\begin{array}{c}\mathrm{PA}_{\mathrm{r}, \mathrm{b}} \\
\left({ }^{\circ}\right)\end{array}$ & $\begin{array}{c}\overline{D_{\mathrm{r}, \mathrm{b}, 0}} \\
\left(^{\prime}\right)\end{array}$ & $\begin{array}{c}d_{\mathrm{r}, \mathrm{b}, 0} \\
\left(^{\prime}\right)\end{array}$ & $\begin{array}{l}\theta_{\mathrm{r}, \mathrm{b}} \\
\left({ }^{\circ}\right)\end{array}$ & Feature & $\begin{array}{c}D_{\mathrm{r}, \mathrm{b}} \\
\left({ }^{\prime}\right)\end{array}$ & $\begin{array}{c}d_{\mathrm{r}, \mathrm{b}} \\
\left({ }^{\prime}\right)\end{array}$ & $\begin{array}{c}\mathrm{PA}_{\mathrm{r}, \mathrm{b}} \\
\left({ }^{\circ}\right)\end{array}$ & $\begin{array}{c}D_{\mathrm{r}, \mathrm{b}, 0} \\
\left(^{\prime}\right)\end{array}$ & $\begin{array}{c}d_{\mathrm{r}, \mathrm{b}, 0} \\
\left(^{\prime}\right)\end{array}$ & $\begin{array}{l}\theta_{\mathrm{r}, \mathrm{b}} \\
\left({ }^{\circ}\right)\end{array}$ \\
\hline$\overline{\mathrm{R}^{\prime}}$ & 2.31 & 1.17 & 1.3 & 2.32 & 2.14 & 169.1 & $\overline{\mathrm{R}^{\prime}}$ & 2.50 & 0.40 & 83.9 & 2.50 & 1.49 & 179.1 \\
\hline$\underline{\mathrm{rs}}$ & 1.35 & 0.57 & 178.3 & 1.36 & 1.03 & 164.9 & $\mathrm{rl}$ & 1.05 & 0.25 & 87.0 & 1.10 & 0.88 & 31.5 \\
\hline
\end{tabular}

NGC 5690

$\left(\mathrm{R}^{\prime}\right) \mathrm{SB}(\mathrm{s}) \mathrm{d} \mathrm{sp}$
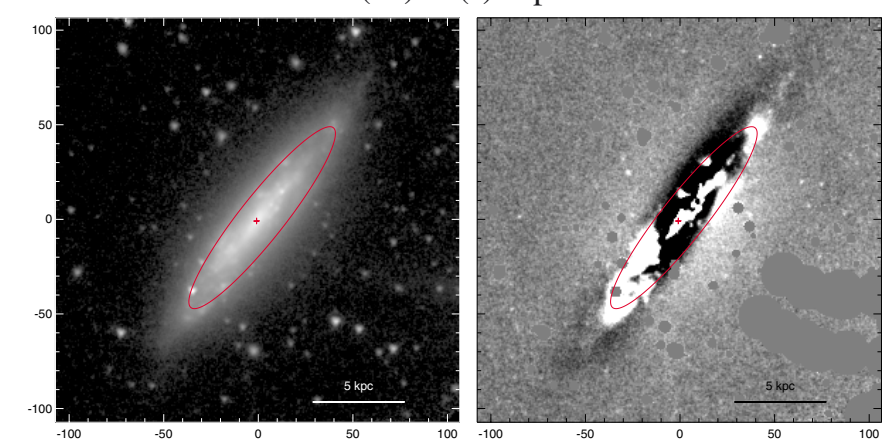

\begin{tabular}{|c|c|c|c|c|c|c|c|c|c|c|c|c|c|}
\hline Feature & $\begin{array}{c}D_{\mathrm{r}, \mathrm{b}} \\
\left({ }^{\prime}\right)\end{array}$ & $\begin{array}{c}d_{\mathrm{r}, \mathrm{b}} \\
\left({ }^{\prime}\right)\end{array}$ & $\begin{array}{c}\mathrm{PA}_{\mathrm{r}, \mathrm{b}} \\
\left({ }^{\circ}\right)\end{array}$ & $\begin{array}{c}D_{\mathrm{r}, \mathrm{b}, 0} \\
\left({ }^{\prime}\right)\end{array}$ & $\begin{array}{c}d_{\mathrm{r}, \mathrm{b}, 0} \\
\left(^{\prime}\right)\end{array}$ & $\begin{array}{l}\theta_{\mathrm{r}, \mathrm{b}} \\
\left({ }^{\circ}\right)\end{array}$ & Feature & $\begin{array}{c}D_{\mathrm{r}, \mathrm{b}} \\
\left({ }^{\prime}\right)\end{array}$ & $\begin{array}{c}d_{\mathrm{r}, \mathrm{b}} \\
\left(^{\prime}\right)\end{array}$ & $\begin{array}{c}\mathrm{PA}_{\mathrm{r}, \mathrm{b}} \\
\left({ }^{\circ}\right)\end{array}$ & $\begin{array}{c}D_{\mathrm{r}, \mathrm{b}, 0} \\
\left({ }^{\prime}\right)\end{array}$ & $\begin{array}{c}d_{\mathrm{r}, \mathrm{b}, 0} \\
\left.{ }^{\prime}()^{\prime}\right)\end{array}$ & $\begin{array}{l}\theta_{\mathrm{r}, \mathrm{b}} \\
\left({ }^{\circ}\right)\end{array}$ \\
\hline $\mathrm{R}^{\prime}$ & 2.03 & 0.39 & 141.6 & 2.03 & 1.60 & 177.2 & $\begin{array}{c}\mathrm{R}_{1}^{\prime} \\
\mathrm{rl}\end{array}$ & $\begin{array}{l}3.21 \\
1.34\end{array}$ & $\begin{array}{l}2.72 \\
1.08\end{array}$ & $\begin{array}{c}77.2 \\
178.3\end{array}$ & $\begin{array}{l}3.24 \\
1.37\end{array}$ & $\begin{array}{l}2.80 \\
1.10\end{array}$ & $\begin{array}{c}32.7 \\
123.6\end{array}$ \\
\hline & & & & & & & bar & 1.28 & 0.74 & 177.2 & 1.31 & 0.75 & 125.1 \\
\hline
\end{tabular}

NGC 5713

( $\left.\mathrm{R}^{\prime}\right) \mathrm{SB}(\mathrm{rs}) \mathrm{ab}$ : pec
NGC 5689

$\left(\mathrm{R}^{\prime}\right) \mathrm{SAB}_{\text {boxy }}(\mathrm{rl}, \mathrm{nd}) 0 / \mathrm{a}$

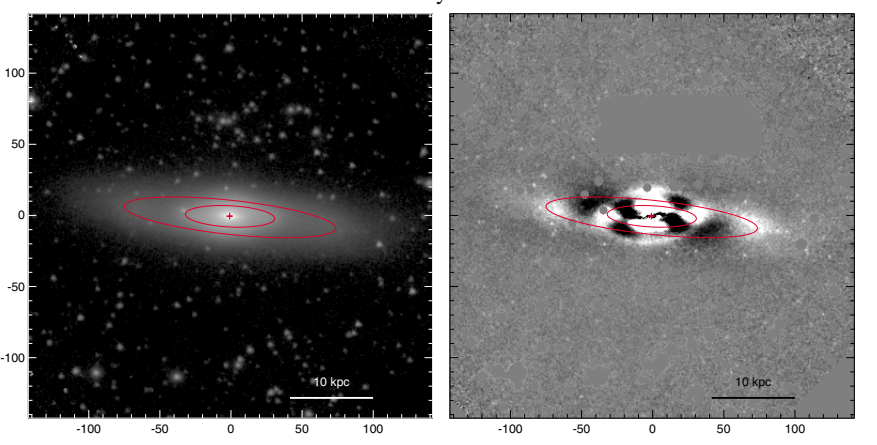

NGC 5701

$\left(\mathrm{R}_{1}^{\prime}\right) \mathrm{SB}(\mathrm{rl}) 0 / \mathrm{a}$

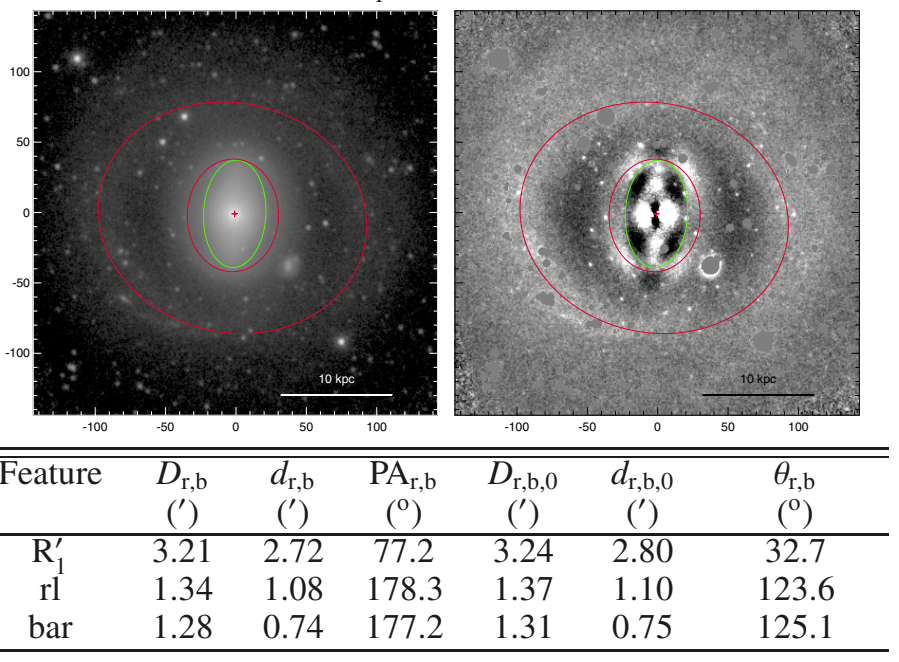

NGC 5719

( $\left.\mathrm{R}^{\prime}\right) \mathrm{SB}(\mathrm{nr}) 0 / \mathrm{a} \mathrm{sp}$

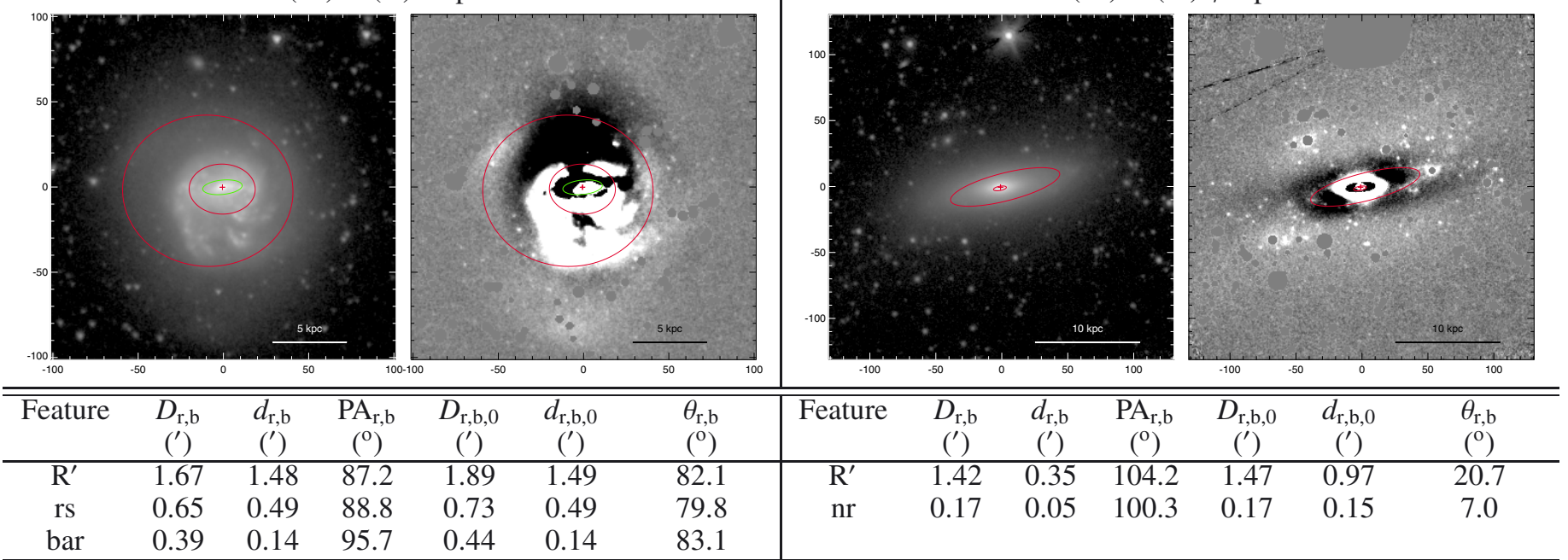


NGC 5728

$\left(\mathrm{R}_{1}\right) \mathrm{SB}(\mathrm{rs}, \mathrm{nr}, \mathrm{nb}) 0 / \mathrm{a}$

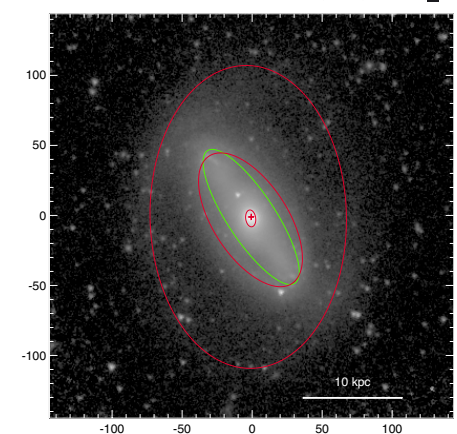

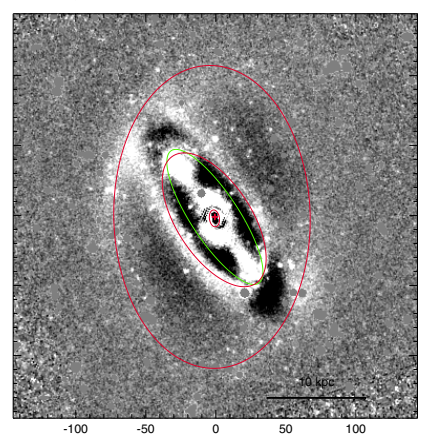

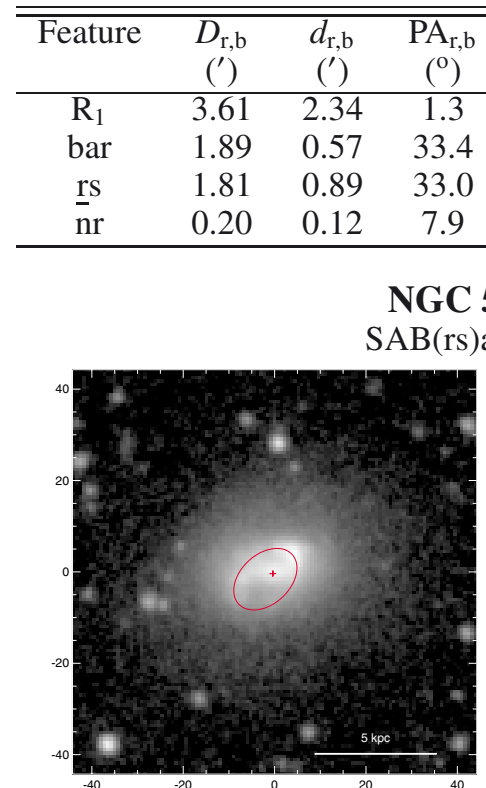

GC 5744

ab: pec

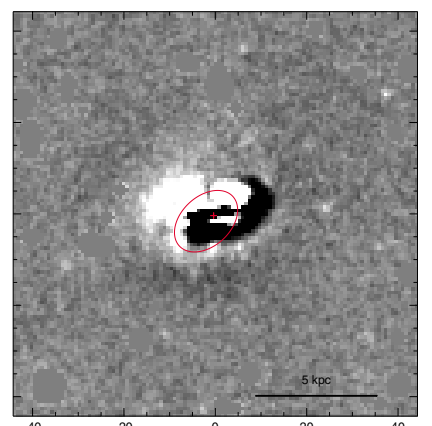

NGC 5740

(R)SAB(r)ab

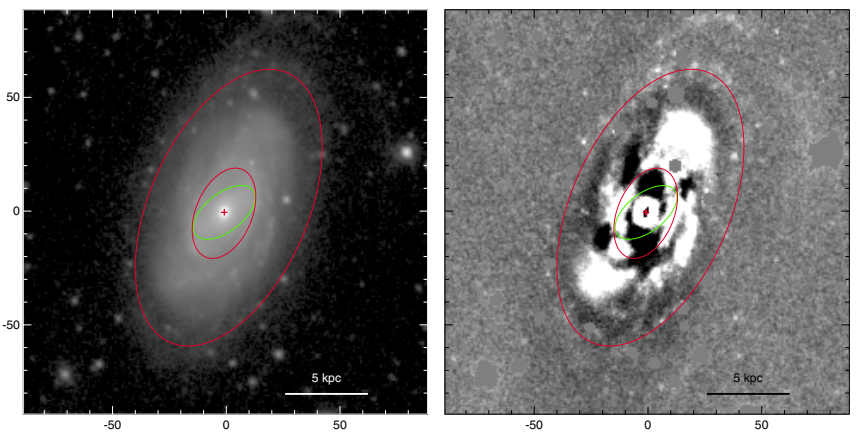

\begin{tabular}{ccc|ccccccc}
$\begin{array}{c}D_{\mathrm{r}, \mathrm{b}, 0} \\
\left({ }^{\prime}\right)\end{array}$ & $\begin{array}{c}d_{\mathrm{r}, \mathrm{b}, 0} \\
\left({ }^{\prime}\right)\end{array}$ & $\begin{array}{c}\theta_{\mathrm{r}, \mathrm{b}} \\
\left({ }^{\circ}\right)\end{array}$ & Feature & $\begin{array}{c}D_{\mathrm{r}, \mathrm{b}} \\
\left({ }^{\prime}\right)\end{array}$ & $\begin{array}{c}d_{\mathrm{r}, \mathrm{b}} \\
\left({ }^{\prime}\right)\end{array}$ & $\begin{array}{c}\mathrm{PA}_{\mathrm{r}, \mathrm{b}} \\
\left({ }^{\circ}\right)\end{array}$ & $\begin{array}{c}D_{\mathrm{r}, \mathrm{b}, 0} \\
\left({ }^{\prime}\right)\end{array}$ & $\begin{array}{c}d_{\mathrm{r}, \mathrm{b}, 0} \\
\left({ }^{\prime}\right)\end{array}$ & $\begin{array}{c}\theta_{\mathrm{r}, \mathrm{b}} \\
\left({ }^{\circ}\right)\end{array}$ \\
\hline 3.65 & 3.17 & 161.9 & $\mathrm{R}$ & 2.16 & 1.17 & 156.3 & 2.34 & 2.04 & 127.3 \\
2.06 & 0.72 & 36.5 & $\mathrm{r}$ & 0.70 & 0.41 & 157.3 & 0.80 & 0.68 & 108.0 \\
1.99 & 1.11 & 40.8 & bar & 0.53 & 0.28 & 127.8 & 0.78 & 0.36 & 115.3 \\
0.20 & 0.16 & 3.1 & & & & & & & \\
\hline
\end{tabular}

NGC 5746

$\left(\mathrm{R}^{\prime}\right) \mathrm{SB}_{\mathrm{x}}(\mathrm{r}, \mathrm{nd}) 0 / \mathrm{a} \mathrm{sp}$

\begin{tabular}{|c|c|c|c|c|c|c|c|c|c|c|c|c|c|}
\hline Feature & $\begin{array}{c}D_{\mathrm{r}, \mathrm{b}} \\
\left({ }^{\prime}\right)\end{array}$ & $\begin{array}{c}d_{\mathrm{r}, \mathrm{b}} \\
\left(^{\prime}\right)\end{array}$ & $\begin{array}{c}\mathrm{PA}_{\mathrm{r}, \mathrm{b}} \\
\left({ }^{\circ}\right)\end{array}$ & $\begin{array}{c}D_{\mathrm{r}, \mathrm{b}, 0} \\
\left(^{\prime}\right)\end{array}$ & $\begin{array}{c}d_{\mathrm{r}, \mathrm{b}, 0} \\
\left({ }^{\prime}\right)\end{array}$ & $\begin{array}{c}\theta_{\mathrm{r}, \mathrm{b}} \\
\left({ }^{\circ}\right)\end{array}$ & Feature & $\begin{array}{c}D_{\mathrm{r}, \mathrm{b}} \\
\left({ }^{\prime}\right)\end{array}$ & $\begin{array}{c}d_{\mathrm{r}, \mathrm{b}} \\
\left({ }^{\prime}\right)\end{array}$ & $\begin{array}{c}\mathrm{PA}_{\mathrm{r}, \mathrm{b}} \\
\left(^{\mathrm{o}}\right)\end{array}$ & $\begin{array}{c}D_{\mathrm{r}, \mathrm{b}, 0} \\
\left(^{\prime}\right)\end{array}$ & $\begin{array}{c}d_{\mathrm{r}, \mathrm{b}, 0} \\
\left(^{\prime}\right)\end{array}$ & $\begin{array}{l}\theta_{\mathrm{r}, \mathrm{b}} \\
\left({ }^{\circ}\right)\end{array}$ \\
\hline rs & 0.27 & 0.18 & 132.9 & 0.29 & 0.23 & 43.8 & $\begin{array}{r}\mathrm{R}^{\prime} \\
\mathrm{r}\end{array}$ & $\begin{array}{l}5.06 \\
2.43\end{array}$ & $\begin{array}{l}0.39 \\
0.25\end{array}$ & $\begin{array}{l}170.7 \\
171.4\end{array}$ & $\begin{array}{l}5.09 \\
2.47\end{array}$ & $\begin{array}{l}2.02 \\
1.28\end{array}$ & $\begin{array}{c}6.8 \\
12.4\end{array}$ \\
\hline
\end{tabular}

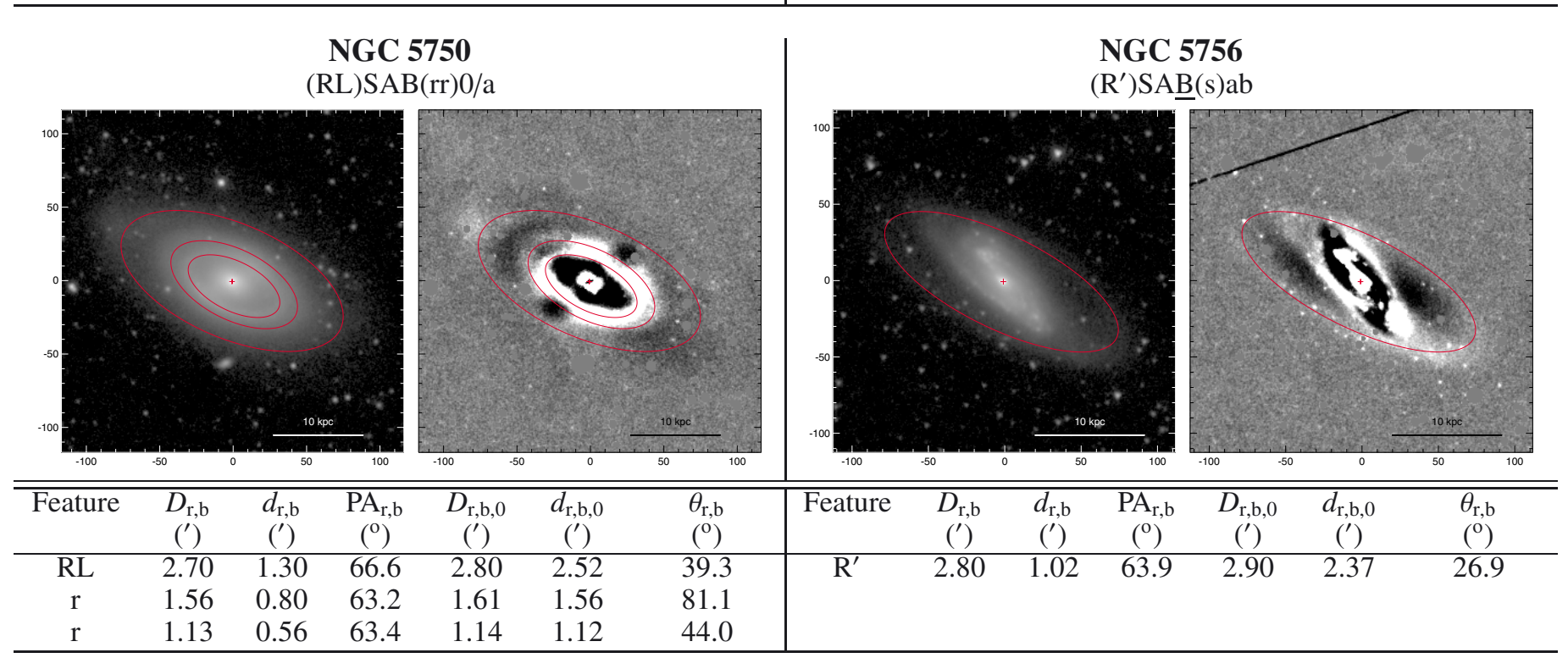


NGC 5757

$\left(\mathrm{R}_{2}^{\prime}\right) \mathrm{SB}(\mathrm{rs}) \underline{\mathrm{ab}}$
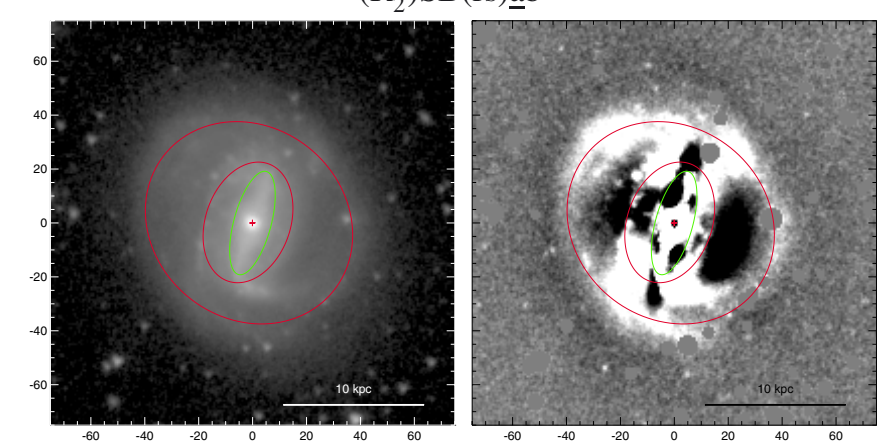

NGC 5768

$\mathrm{SAB}(\mathrm{rs}) \mathrm{bc}$

\begin{tabular}{|c|c|c|c|c|c|c|c|c|c|c|c|c|c|}
\hline Feature & $\begin{array}{c}D_{\mathrm{r}, \mathrm{b}} \\
\left({ }^{\prime}\right)\end{array}$ & $\begin{array}{c}d_{\mathrm{r}, \mathrm{b}} \\
\left({ }^{\prime}\right)\end{array}$ & $\begin{array}{c}\mathrm{PA}_{\mathrm{r}, \mathrm{b}} \\
\left({ }^{\circ}\right)\end{array}$ & $\begin{array}{c}D_{\mathrm{r}, \mathrm{b}, 0} \\
\left({ }^{\prime}\right)\end{array}$ & $\begin{array}{c}d_{\mathrm{r}, \mathrm{b}, 0} \\
\left(^{\prime}\right)\end{array}$ & $\begin{array}{l}\theta_{\mathrm{r}, \mathrm{b}} \\
\left(^{\circ}\right)\end{array}$ & Feature & $\begin{array}{c}D_{\mathrm{r}, \mathrm{b}} \\
\left({ }^{\prime}\right)\end{array}$ & $\begin{array}{c}d_{\mathrm{r}, \mathrm{b}} \\
\left({ }^{\prime}\right)\end{array}$ & $\begin{array}{c}\mathrm{PA}_{\mathrm{r}, \mathrm{b}} \\
\left({ }^{\circ}\right)\end{array}$ & $\begin{array}{c}D_{\mathrm{r}, \mathrm{b}, 0} \\
\left({ }^{\prime}\right)\end{array}$ & $\begin{array}{c}d_{\mathrm{r}, \mathrm{b}, 0} \\
\left(^{\prime}\right)\end{array}$ & $\begin{array}{c}\theta_{\mathrm{r}, \mathrm{b}} \\
\left({ }^{\circ}\right)\end{array}$ \\
\hline$\overline{\mathrm{R}_{2}^{\prime}}$ & 1.35 & 1.19 & 50.7 & 1.46 & 1.31 & 67.2 & rs & 0.55 & 0.50 & 113.8 & 0.55 & 0.54 & 152.8 \\
\hline rs & 0.77 & 0.53 & 161.2 & 0.87 & 0.56 & 118.4 & bar & 0.36 & 0.16 & 149.2 & 0.37 & 0.17 & 31.4 \\
\hline bar & 0.66 & 0.23 & 164.8 & 0.73 & 0.25 & 127.8 & & & & & & & \\
\hline
\end{tabular}

NGC 5770

$\mathrm{SAB}(\mathrm{r}, \mathrm{bl}) 0^{+}$
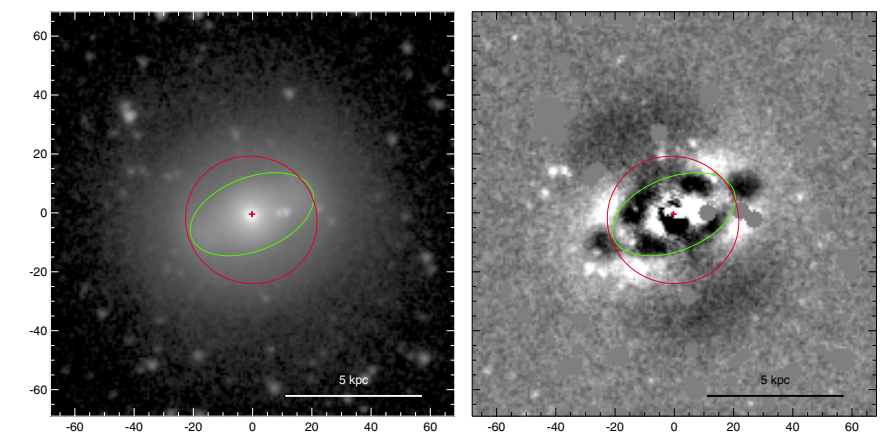

NGC 5777

(R) $\mathrm{SB}_{\text {box }}(1, \mathrm{nl}) 0^{+} \mathrm{sp}$

\begin{tabular}{|c|c|c|c|c|c|c|c|c|c|c|c|c|c|}
\hline Feature & $\begin{array}{c}D_{\mathrm{r}, \mathrm{b}} \\
\left(^{\prime}\right)\end{array}$ & $\begin{array}{c}d_{\mathrm{r}, \mathrm{b}} \\
(')\end{array}$ & $\begin{array}{c}\mathrm{PA}_{\mathrm{r}, \mathrm{b}} \\
\left(^{\mathrm{O}}\right)\end{array}$ & $\begin{array}{c}D_{\mathrm{r}, \mathrm{b}, 0} \\
\left(^{\prime}\right)\end{array}$ & $\begin{array}{c}d_{\mathrm{r}, \mathrm{b}, 0} \\
\left(^{\prime}\right)\end{array}$ & $\begin{array}{l}\theta_{\mathrm{r}, \mathrm{b}} \\
\left({ }^{\circ}\right)\end{array}$ & Feature & $\begin{array}{c}D_{\mathrm{r}, \mathrm{b}} \\
\left({ }^{\prime}\right)\end{array}$ & $\begin{array}{c}d_{\mathrm{r}, \mathrm{b}} \\
\left({ }^{\prime}\right)\end{array}$ & $\begin{array}{c}\mathrm{PA}_{\mathrm{r}, \mathrm{b}} \\
\left(^{\mathrm{O}}\right)\end{array}$ & $\begin{array}{c}D_{\mathrm{r}, \mathrm{b}, 0} \\
\left(^{\prime}\right)\end{array}$ & $\begin{array}{c}d_{\mathrm{r}, \mathrm{b}, 0} \\
\left(^{\prime}\right)\end{array}$ & $\begin{array}{l}\theta_{\mathrm{r}, \mathrm{b}} \\
\left({ }^{\circ}\right)\end{array}$ \\
\hline$r$ & 0.75 & 0.72 & 71.4 & 0.79 & 0.73 & 74.8 & $\mathrm{R}$ & 2.08 & 0.17 & 142.8 & 2.08 & 0.79 & 177.5 \\
\hline bar & 0.73 & 0.41 & 111.5 & 0.79 & 0.42 & 75.3 & & & & & & & \\
\hline
\end{tabular}

NGC 5781

(R)SB(rs)a/d
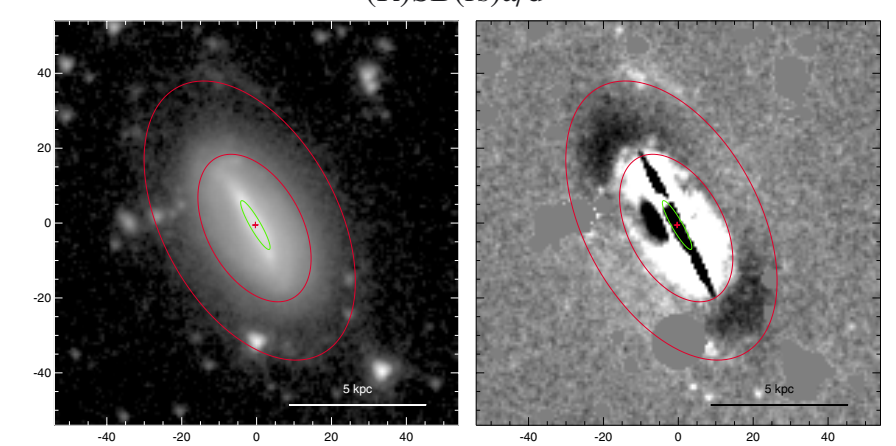

\section{NGC 5792}

$\left(\mathrm{R}_{1}^{\prime}\right) \mathrm{SAB}(\mathrm{r}, \mathrm{nd}, \mathrm{nr}) \mathrm{ab}$

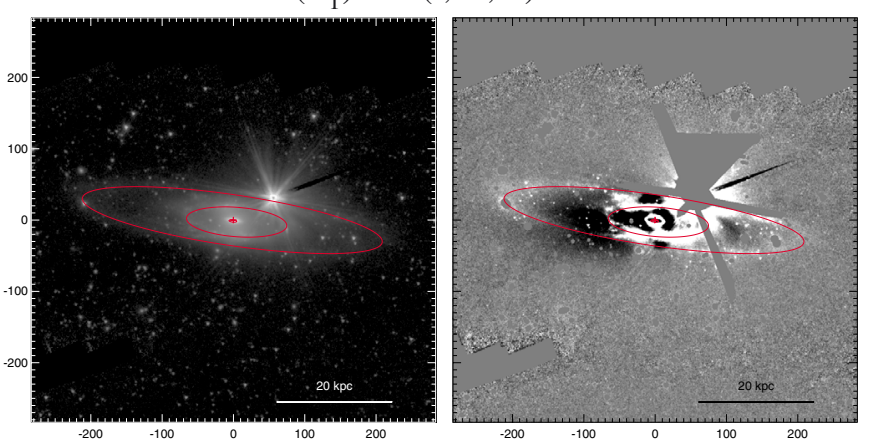

\begin{tabular}{ccccccc|ccccccc}
\hline \hline Feature & $\begin{array}{c}D_{\mathrm{r}, \mathrm{b}} \\
\left({ }^{\prime}\right)\end{array}$ & $\begin{array}{c}d_{\mathrm{r}, \mathrm{b}} \\
\left({ }^{\prime}\right)\end{array}$ & $\begin{array}{c}\mathrm{PA}_{\mathrm{r}, \mathrm{b}} \\
\left({ }^{\circ}\right)\end{array}$ & $\begin{array}{c}D_{\mathrm{r}, \mathrm{b}, 0} \\
\left({ }^{\prime}\right)\end{array}$ & $\begin{array}{c}d_{\mathrm{r}, \mathrm{b}, 0} \\
\left({ }^{\prime}\right)\end{array}$ & $\begin{array}{c}\theta_{\mathrm{r}, \mathrm{b}} \\
\left({ }^{\circ}\right)\end{array}$ & Feature & $\begin{array}{c}D_{\mathrm{r}, \mathrm{b}} \\
\left({ }^{\prime}\right)\end{array}$ & $\begin{array}{c}d_{\mathrm{r}, \mathrm{b}} \\
\left({ }^{\prime}\right)\end{array}$ & $\begin{array}{c}\mathrm{PA}_{\mathrm{r}, \mathrm{b}} \\
\left({ }^{\circ}\right)\end{array}$ & $\begin{array}{c}D_{\mathrm{r}, \mathrm{b}, 0} \\
\left({ }^{\prime}\right)\end{array}$ & $\begin{array}{c}d_{\mathrm{r}, \mathrm{b}, 0} \\
\left({ }^{\prime}\right)\end{array}$ & $\begin{array}{c}\theta_{\mathrm{r}, \mathrm{b}} \\
\left({ }^{\circ}\right)\end{array}$ \\
\hline $\mathrm{R}$ & 1.36 & 0.78 & 28.5 & 1.59 & 1.31 & 111.4 & $\mathrm{R}_{1}^{\prime}$ & 7.09 & 1.19 & 81.6 & 7.55 & 3.38 & 156.3 \\
$\mathrm{rs}$ & 0.72 & 0.43 & 28.6 & 0.86 & 0.70 & 107.8 & $\mathrm{r}$ & 2.35 & 0.69 & 85.6 & 2.42 & 2.02 \\
$\mathrm{bar}$ & 0.25 & 0.05 & 30.0 & 0.25 & 0.09 & 169.2 & $\mathrm{nr}$ & 0.18 & 0.06 & 84.1 & 0.20 & 0.16 & 154.2 \\
\hline
\end{tabular}


S. Comerón et al.: ARRAKIS

NGC 5806

( $\left.\mathrm{R}^{\prime}\right) \mathrm{SAB}(\mathrm{rs}, \mathrm{nr}) \mathrm{ab}$

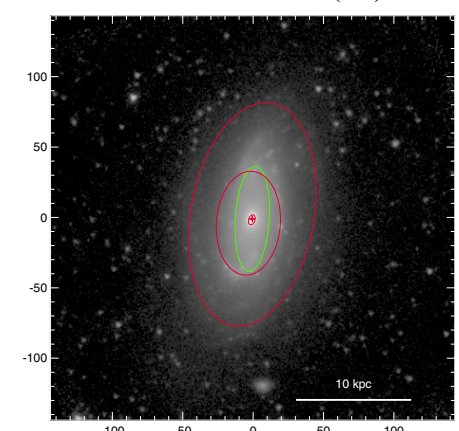

NGC 5809

$\mathrm{SA}(\mathrm{rs}) \mathrm{a} / \mathrm{Sph}$

\begin{tabular}{|c|c|c|c|c|c|c|c|c|c|c|c|c|c|}
\hline Feature & $\begin{array}{c}D_{\mathrm{r}, \mathrm{b}} \\
\left({ }^{\prime}\right)\end{array}$ & $\begin{array}{c}d_{\mathrm{r}, \mathrm{b}} \\
\left(^{\prime}\right)\end{array}$ & $\begin{array}{c}\mathrm{PA}_{\mathrm{r}, \mathrm{b}} \\
\left(^{\mathrm{O}}\right)\end{array}$ & $\begin{array}{c}\overline{D_{\mathrm{r}, \mathrm{b}, 0}} \\
\left(^{\prime}\right)\end{array}$ & $\begin{array}{c}d_{\mathrm{r}, \mathrm{b}, 0} \\
\left(^{\prime}\right)\end{array}$ & $\begin{array}{l}\theta_{\mathrm{r}, \mathrm{b}} \\
\left({ }^{\circ}\right)\end{array}$ & Feature & $\begin{array}{c}D_{\mathrm{r}, \mathrm{b}} \\
\left({ }^{\prime}\right)\end{array}$ & $\begin{array}{c}d_{\mathrm{r}, \mathrm{b}} \\
\left(^{\prime}\right)\end{array}$ & $\begin{array}{c}\mathrm{PA}_{\mathrm{r}, \mathrm{b}} \\
\left(^{\circ}\right)\end{array}$ & $\begin{array}{c}D_{\mathrm{r}, \mathrm{b}, 0} \\
\left(^{\prime}\right)\end{array}$ & $\begin{array}{c}d_{\mathrm{r}, \mathrm{b}, 0} \\
\left(^{\prime}\right)\end{array}$ & $\begin{array}{c}\theta_{\mathrm{r}, \mathrm{b}} \\
\left({ }^{\circ}\right)\end{array}$ \\
\hline$\overline{\mathrm{R}^{\prime}}$ & 2.68 & 1.46 & 170.1 & 2.77 & 2.56 & 42.5 & rs & 0.37 & 0.12 & 147.2 & 0.37 & 0.28 & 177.6 \\
\hline bar & 1.25 & 0.40 & 176.3 & 1.30 & 0.70 & 22.2 & & & & & & & \\
\hline rs & 1.23 & 0.76 & 175.7 & 1.44 & 1.17 & 65.5 & & & & & & & \\
\hline $\mathrm{nr}$ & 0.12 & 0.07 & 162.5 & 0.14 & 0.12 & 107.7 & & & & & & & \\
\hline
\end{tabular}
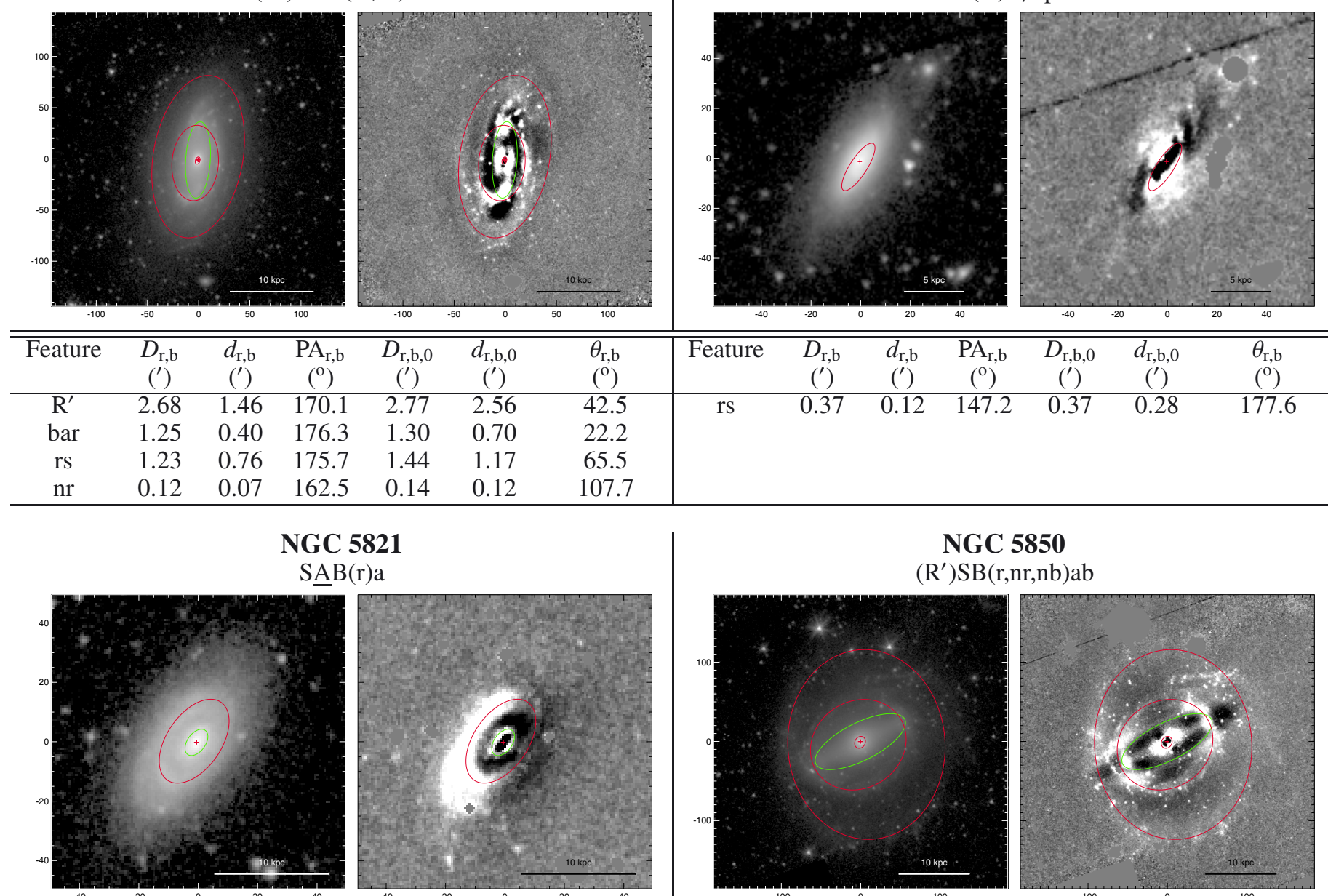

NGC 5850

( $\left.\mathrm{R}^{\prime}\right) \mathrm{SB}(\mathrm{r}, \mathrm{nr}, \mathrm{nb}) \mathrm{ab}$

\begin{tabular}{|c|c|c|c|c|c|c|c|c|c|c|c|c|c|}
\hline Feature & $\begin{array}{c}D_{\mathrm{r}, \mathrm{b}} \\
\left(^{\prime}\right)\end{array}$ & $\begin{array}{c}d_{\mathrm{r}, \mathrm{b}} \\
(')\end{array}$ & $\begin{array}{c}\mathrm{PA}_{\mathrm{r}, \mathrm{b}} \\
\left({ }^{\circ}\right)\end{array}$ & $\begin{array}{c}D_{\mathrm{r}, \mathrm{b}, 0} \\
\left(^{\prime}\right)\end{array}$ & $\begin{array}{c}d_{\mathrm{r}, \mathrm{b}, 0} \\
\left(^{\prime}\right)\end{array}$ & $\begin{array}{l}\theta_{\mathrm{r}, \mathrm{b}} \\
\left({ }^{\circ}\right)\end{array}$ & Feature & $\begin{array}{c}D_{\mathrm{r}, \mathrm{b}} \\
\left(^{\prime}\right)\end{array}$ & $\begin{array}{c}d_{\mathrm{r}, \mathrm{b}} \\
\left(^{\prime}\right)\end{array}$ & $\begin{array}{c}\mathrm{PA}_{\mathrm{r}, \mathrm{b}} \\
\left({ }^{\circ}\right)\end{array}$ & $\begin{array}{c}D_{\mathrm{r}, \mathrm{b}, 0} \\
\left(^{\prime}\right)\end{array}$ & $\begin{array}{c}d_{\mathrm{r}, \mathrm{b}, 0} \\
\left(^{\prime}\right)\end{array}$ & $\begin{array}{l}\theta_{\mathrm{r}, \mathrm{b}} \\
\left({ }^{\circ}\right)\end{array}$ \\
\hline $\mathrm{r}$ & 0.53 & 0.31 & 145.6 & 0.54 & 0.52 & 166.7 & $\overline{\mathrm{R}^{\prime}}$ & 4.00 & 3.31 & 3.8 & 4.26 & 3.84 & 58.6 \\
\hline \multirow[t]{3}{*}{ bar } & 0.17 & 0.10 & 144.0 & 0.18 & 0.17 & 114.9 & bar & 2.09 & 0.79 & 116.7 & 2.42 & 0.85 & 120.0 \\
\hline & & & & & & & $\mathrm{r}$ & 2.09 & 1.83 & 122.8 & 2.47 & 1.91 & 105.5 \\
\hline & & & & & & & $\mathrm{nr}$ & 0.26 & 0.22 & 150.6 & 0.28 & 0.25 & 122.3 \\
\hline
\end{tabular}

NGC 5854

(R) $\mathrm{SB}_{\mathrm{x}}(\mathrm{r}) 0^{+}$
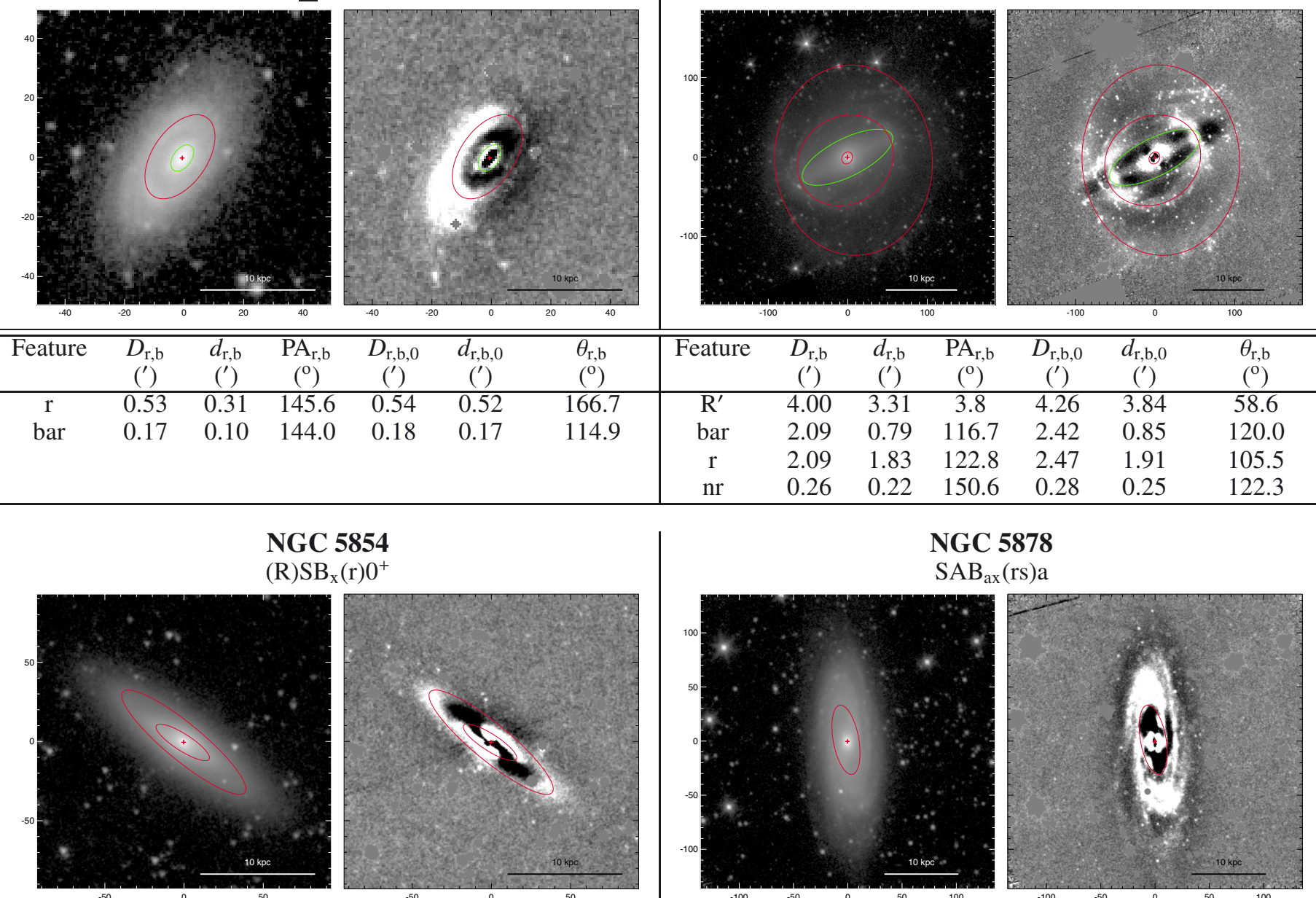

NGC 5878

$\mathrm{SAB}_{\mathrm{ax}}(\mathrm{rs}) \mathrm{a}$

\begin{tabular}{|c|c|c|c|c|c|c|c|c|c|c|c|c|c|}
\hline Feature & $\begin{array}{c}D_{\mathrm{r}, \mathrm{b}} \\
\left({ }^{\prime}\right)\end{array}$ & $\begin{array}{c}d_{\mathrm{r}, \mathrm{b}} \\
\left(^{\prime}\right)\end{array}$ & $\begin{array}{c}\mathrm{PA}_{\mathrm{r}, \mathrm{b}} \\
\left({ }^{\circ}\right)\end{array}$ & $\begin{array}{c}D_{\mathrm{r}, \mathrm{b}, 0} \\
\left(^{\prime}\right)\end{array}$ & $\begin{array}{c}d_{\mathrm{r}, \mathrm{b}, 0} \\
\left(^{\prime}\right)\end{array}$ & $\begin{array}{l}\theta_{\mathrm{r}, \mathrm{b}} \\
\left({ }^{\circ}\right)\end{array}$ & Feature & $\begin{array}{c}D_{\mathrm{r}, \mathrm{b}} \\
\left({ }^{\prime}\right)\end{array}$ & $\begin{array}{c}d_{\mathrm{r}, \mathrm{b}} \\
\left(^{\prime}\right)\end{array}$ & $\begin{array}{c}\mathrm{PA}_{\mathrm{r}, \mathrm{b}} \\
\left({ }^{\circ}\right)\end{array}$ & $\begin{array}{c}D_{\mathrm{r}, \mathrm{b}, 0} \\
\left(^{\prime}\right)\end{array}$ & $\begin{array}{c}d_{\mathrm{r}, \mathrm{b}, 0} \\
\left(^{\prime}\right)\end{array}$ & $\begin{array}{l}\theta_{\mathrm{r}, \mathrm{b}} \\
\left({ }^{\circ}\right)\end{array}$ \\
\hline$\overline{\mathrm{R}}$ & 1.67 & 0.39 & 50.5 & 1.73 & 1.15 & 158.9 & rs & 1.08 & 0.39 & 10.3 & 1.23 & 0.93 & 48.0 \\
\hline $\mathrm{r}$ & 0.66 & 0.18 & 57.4 & 0.68 & 0.53 & 22.4 & & & & & & & \\
\hline
\end{tabular}


NGC 5879

$\mathrm{SAB}_{\mathrm{a}}(\mathrm{rs}) \mathrm{bc}$
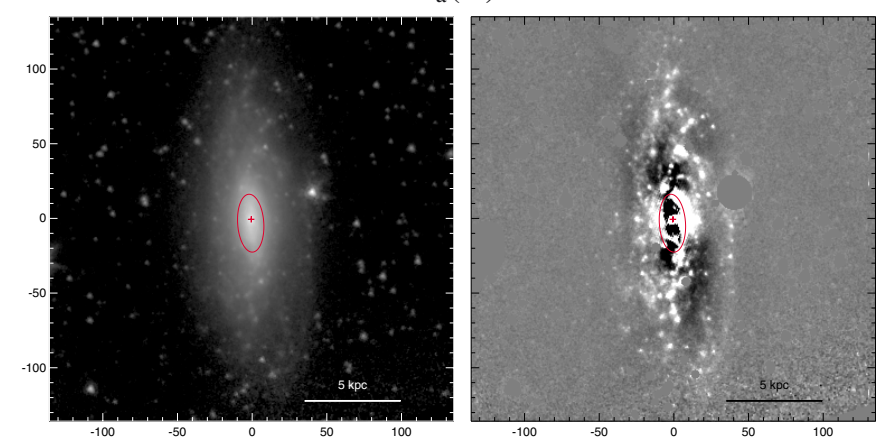

\begin{tabular}{ccccccc|ccccccc}
\hline \hline Feature & $\begin{array}{c}D_{\mathrm{r}, \mathrm{b}} \\
\left({ }^{\prime}\right)\end{array}$ & $\begin{array}{c}d_{\mathrm{r}, \mathrm{b}} \\
\left({ }^{\prime}\right)\end{array}$ & $\begin{array}{c}\mathrm{PA}_{\mathrm{r}, \mathrm{b}} \\
\left({ }^{\circ}\right)\end{array}$ & $\begin{array}{c}D_{\mathrm{r}, \mathrm{b}, 0} \\
\left({ }^{\prime}\right)\end{array}$ & $\begin{array}{c}d_{\mathrm{r}, \mathrm{b}, 0} \\
\left({ }^{\prime}\right)\end{array}$ & $\begin{array}{c}\theta_{\mathrm{r}, \mathrm{b}} \\
\left({ }^{\circ}\right)\end{array}$ & Feature & $\begin{array}{c}D_{\mathrm{r}, \mathrm{b}} \\
\left({ }^{\prime}\right)\end{array}$ & $\begin{array}{c}d_{\mathrm{r}, \mathrm{b}} \\
\left({ }^{\prime}\right)\end{array}$ & $\begin{array}{c}\mathrm{PA}_{\mathrm{r}, \mathrm{b}} \\
\left({ }^{\circ}\right)\end{array}$ & $\begin{array}{c}D_{\mathrm{r}, \mathrm{b}, 0} \\
\left({ }^{\prime}\right)\end{array}$ & $\begin{array}{c}d_{\mathrm{r}, \mathrm{b}, 0} \\
\left({ }^{\prime}\right)\end{array}$ & $\begin{array}{c}\theta_{\mathrm{r}, \mathrm{b}} \\
\left({ }^{\circ}\right)\end{array}$ \\
\hline rs & 0.58 & 0.27 & 5.0 & 0.71 & 0.58 & 85.6 & $\mathrm{rs}$ & 0.45 & 0.39 & 144.1 & 0.48 & 0.41 & 62.0 \\
& & & & & & & $\mathrm{bar}$ & 0.16 & 0.09 & 157.1 & 0.17 & 0.09 & 56.2 \\
\hline
\end{tabular}

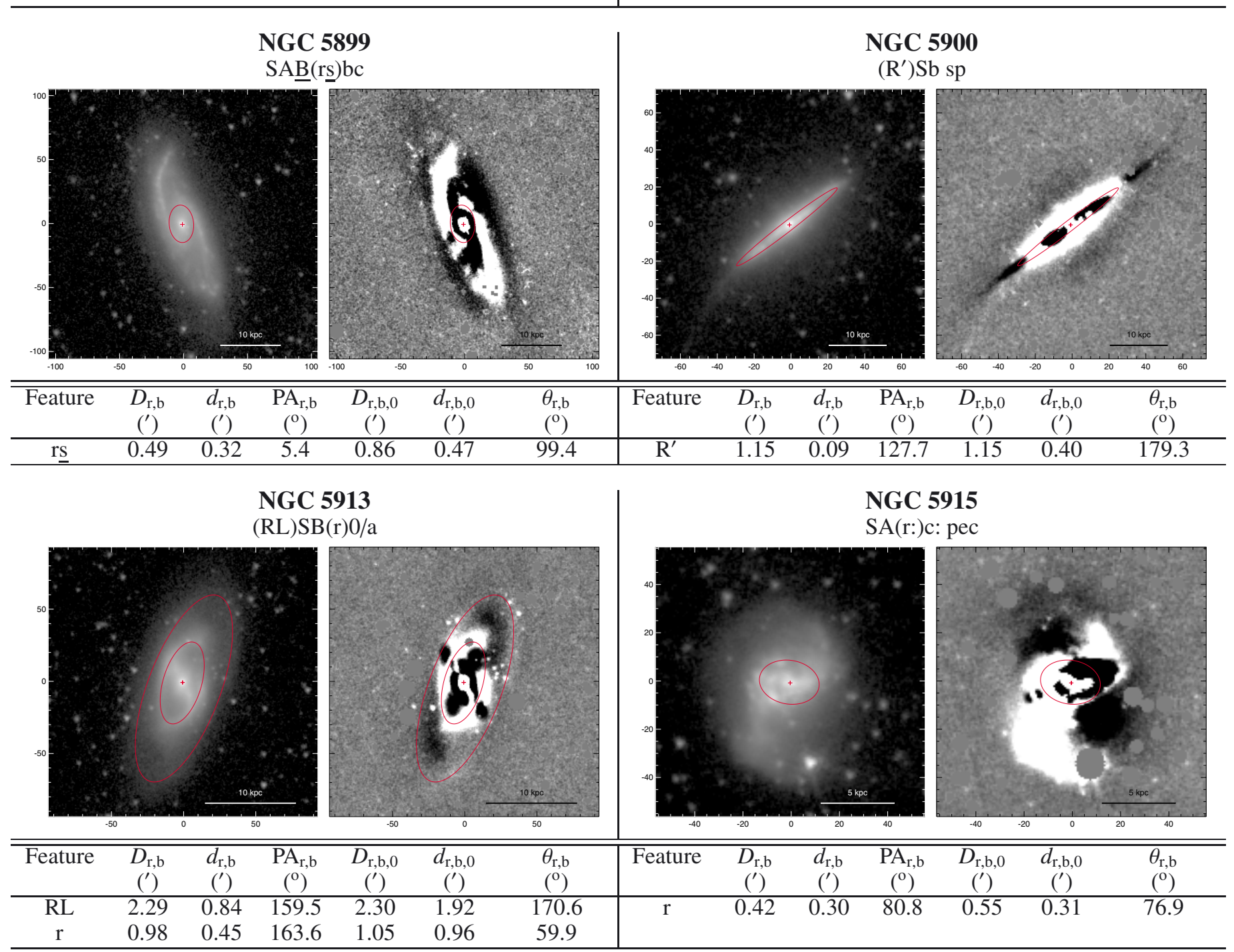

NGC 5892

$\mathrm{SB}(\mathrm{rs}) \mathrm{cd}$

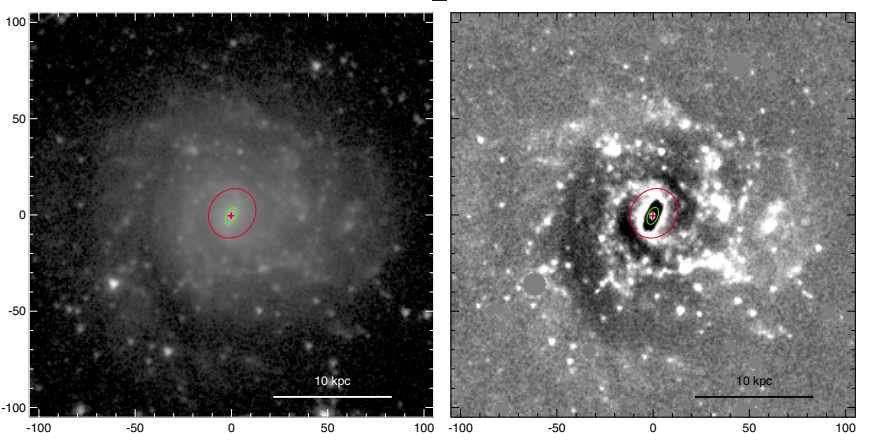

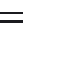


NGC 5921

$\mathrm{SB}(\mathrm{rs}) \mathrm{b}$
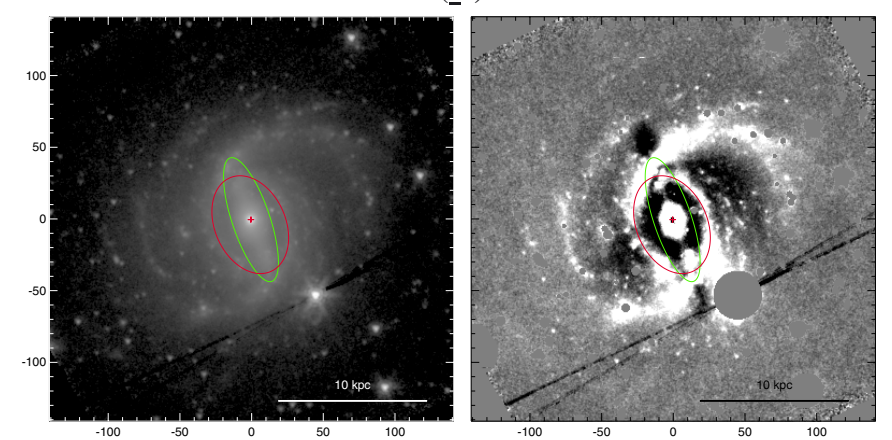

\begin{tabular}{|c|c|c|c|c|c|c|c|c|c|c|c|c|c|}
\hline Feature & $\begin{array}{c}D_{\mathrm{r}, \mathrm{b}} \\
\left({ }^{\prime}\right)\end{array}$ & $\begin{array}{c}d_{\mathrm{r}, \mathrm{b}} \\
\left({ }^{\prime}\right)\end{array}$ & $\begin{array}{c}\mathrm{PA}_{\mathrm{r}, \mathrm{b}} \\
\left({ }^{\circ}\right)\end{array}$ & $\begin{array}{c}D_{\mathrm{r}, \mathrm{b}, 0} \\
\left({ }^{\prime}\right)\end{array}$ & $\begin{array}{c}d_{\mathrm{r}, \mathrm{b}, 0} \\
\left({ }^{\prime}\right)\end{array}$ & $\begin{array}{c}\theta_{\mathrm{r}, \mathrm{b}} \\
\left({ }^{\circ}\right)\end{array}$ & Feature & $\begin{array}{c}D_{\mathrm{r}, \mathrm{b}} \\
\left({ }^{\prime}\right)\end{array}$ & $\begin{array}{c}d_{\mathrm{r}, \mathrm{b}} \\
\left({ }^{\prime}\right)\end{array}$ & $\begin{array}{c}\mathrm{PA}_{\mathrm{r}, \mathrm{b}} \\
\left({ }^{\circ}\right)\end{array}$ & $\begin{array}{c}D_{\mathrm{r}, \mathrm{b}, 0} \\
\left({ }^{\prime}\right)\end{array}$ & $\begin{array}{c}d_{\mathrm{r}, \mathrm{b}, 0} \\
\left(^{\prime}\right)\end{array}$ & $\begin{array}{c}\theta_{\mathrm{r}, \mathrm{b}} \\
\left({ }^{\circ}\right)\end{array}$ \\
\hline bar & 1.52 & 0.44 & 18.6 & 1.72 & 0.47 & 59.6 & rs & 0.42 & 0.26 & 159.4 & 0.50 & 0.41 & 97.1 \\
\hline$\underline{\mathrm{rs}}$ & 1.19 & 0.82 & 23.7 & 1.38 & 0.85 & 69.3 & bar & 0.25 & 0.18 & 73.2 & 0.46 & 0.18 & 90.3 \\
\hline
\end{tabular}

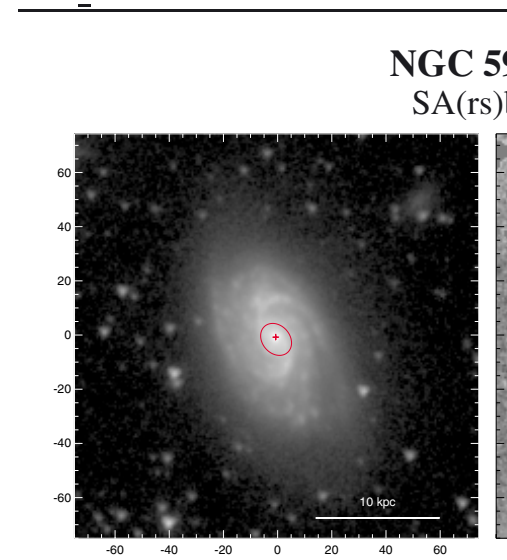

\begin{tabular}{|c|c|c|c|c|c|c|c|c|c|c|c|c|c|}
\hline Feature & $\begin{array}{c}D_{\mathrm{r}, \mathrm{b}} \\
\left(^{\prime}\right)\end{array}$ & $\begin{array}{c}d_{\mathrm{r}, \mathrm{b}} \\
\left(^{\prime}\right)\end{array}$ & $\begin{array}{c}\mathrm{PA}_{\mathrm{r}, \mathrm{b}} \\
\left({ }^{\circ}\right)\end{array}$ & $\begin{array}{c}D_{\mathrm{r}, \mathrm{b}, 0} \\
\left(^{\prime}\right)\end{array}$ & $\begin{array}{c}d_{\mathrm{r}, \mathrm{b}, 0} \\
\left(^{\prime}\right)\end{array}$ & $\begin{array}{c}\theta_{\mathrm{r}, \mathrm{b}} \\
\left.\mathrm{(}^{\mathrm{o}}\right)\end{array}$ & Feature & $\begin{array}{c}D_{\mathrm{r}, \mathrm{b}} \\
\left(^{\prime}\right)\end{array}$ & $\begin{array}{c}d_{\mathrm{r}, \mathrm{b}} \\
\left({ }^{\prime}\right)\end{array}$ & $\begin{array}{c}\mathrm{PA}_{\mathrm{r}, \mathrm{b}} \\
\left({ }^{\mathrm{o}}\right)\end{array}$ & $\begin{array}{c}D_{\mathrm{r}, \mathrm{b}, 0} \\
\left(^{\prime}\right)\end{array}$ & $\begin{array}{c}d_{\mathrm{r}, \mathrm{b}, 0} \\
\left({ }^{\prime}\right)\end{array}$ & $\begin{array}{c}\theta_{\mathrm{r}, \mathrm{b}} \\
\left({ }^{\circ}\right)\end{array}$ \\
\hline rs & 0.22 & 0.17 & 40.8 & 0.30 & 0.20 & 77.9 & $r$ & 0.21 & 0.17 & 72.9 & 0.22 & 0.18 & 47.3 \\
\hline
\end{tabular}

NGC 5954

$\left(\mathrm{R}^{\prime}\right) \mathrm{SA}(\mathrm{rs}) \mathrm{b}$
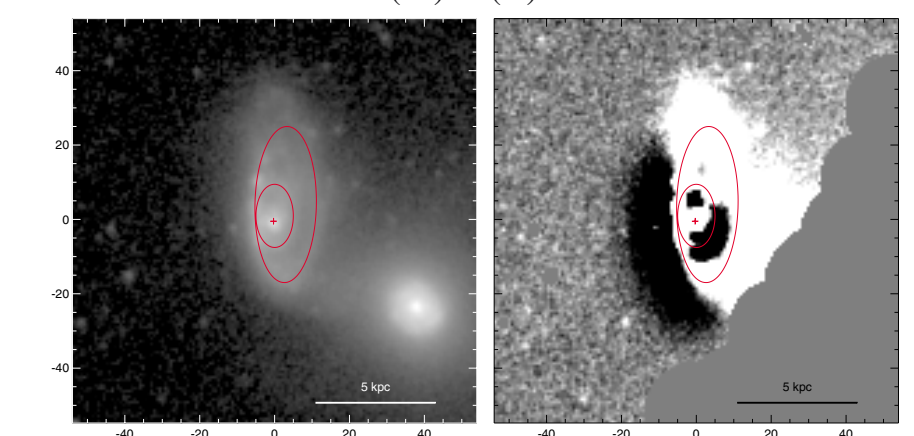

\begin{tabular}{|c|c|c|c|c|c|c|c|c|c|c|c|c|c|}
\hline Feature & $\begin{array}{c}D_{\mathrm{r}, \mathrm{b}} \\
\left(^{\prime}\right)\end{array}$ & $\begin{array}{c}d_{\mathrm{r}, \mathrm{b}} \\
\left(^{\prime}\right)\end{array}$ & $\begin{array}{c}\mathrm{PA}_{\mathrm{r}, \mathrm{b}} \\
\left(^{\mathrm{O}}\right)\end{array}$ & $\begin{array}{c}D_{\mathrm{r}, \mathrm{b}, 0} \\
\left(^{\prime}\right)\end{array}$ & $\begin{array}{c}d_{\mathrm{r}, \mathrm{b}, 0} \\
\left({ }^{\prime}\right)\end{array}$ & $\begin{array}{l}\theta_{\mathrm{r}, \mathrm{b}} \\
\left(^{\circ}\right)\end{array}$ & Feature & $\begin{array}{c}D_{\mathrm{r}, \mathrm{b}} \\
\left(^{\prime}\right)\end{array}$ & $\begin{array}{c}d_{\mathrm{r}, \mathrm{b}} \\
\left(^{\prime}\right)\end{array}$ & $\begin{array}{c}\mathrm{PA}_{\mathrm{r}, \mathrm{b}} \\
\left(^{\mathrm{o}}\right)\end{array}$ & $\begin{array}{c}D_{\mathrm{r}, \mathrm{b}, 0} \\
\left(^{\prime}\right)\end{array}$ & $\begin{array}{c}d_{\mathrm{r}, \mathrm{b}, 0} \\
\left({ }^{\prime}\right)\end{array}$ & $\begin{array}{l}\theta_{\mathrm{r}, \mathrm{b}} \\
\left(^{\mathrm{O}}\right)\end{array}$ \\
\hline$\overline{\mathrm{R}^{\prime}}$ & 0.70 & 0.28 & 178.8 & 0.71 & 0.44 & 167.2 & rs & 0.27 & 0.23 & 15.4 & 0.28 & 0.23 & 103.9 \\
\hline rs & 0.28 & 0.17 & 0.2 & 0.29 & 0.27 & 146.5 & $\overline{\mathrm{bar}}$ & 0.23 & 0.13 & 30.7 & 0.23 & 0.13 & 120.4 \\
\hline
\end{tabular}

NGC 5930

(L)SAB $($ rs) $0 / a$

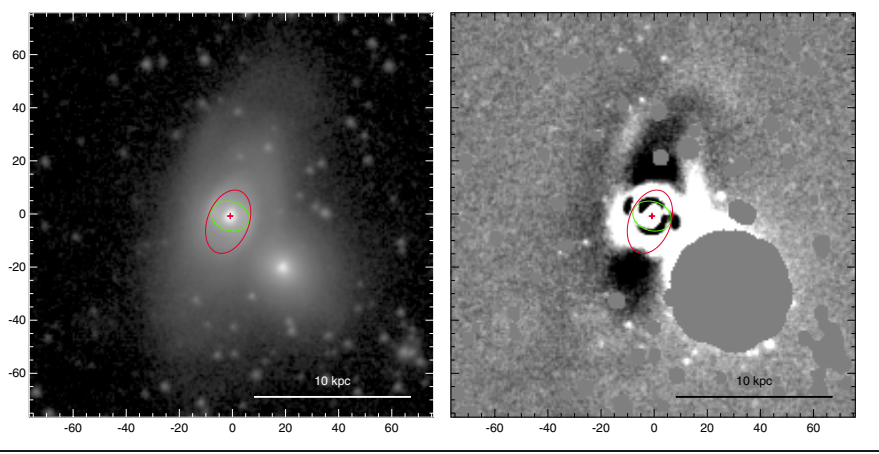

NGC 5953

$\mathrm{SA}(\mathrm{r}) 0^{+}$

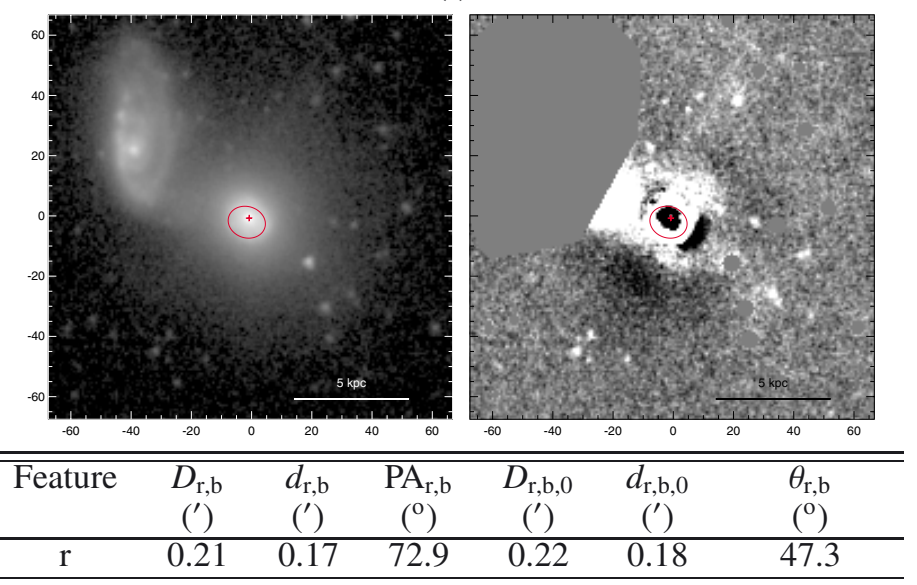

NGC 5956

$\mathrm{SB}(\mathrm{rs}) \mathrm{ab}$

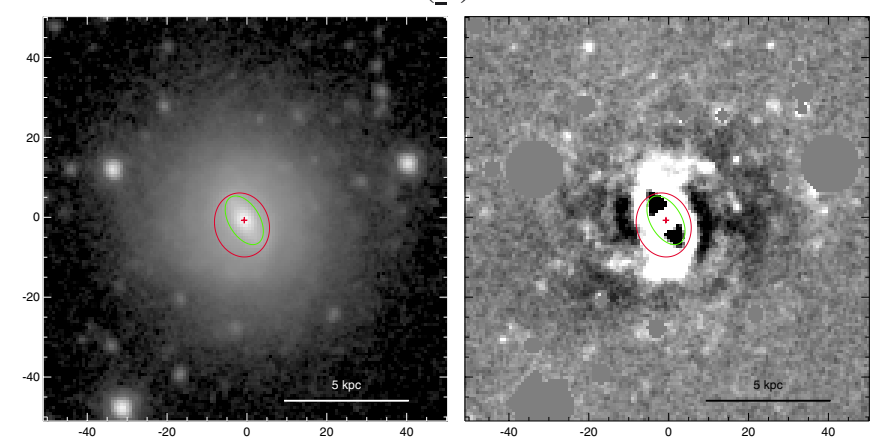

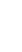




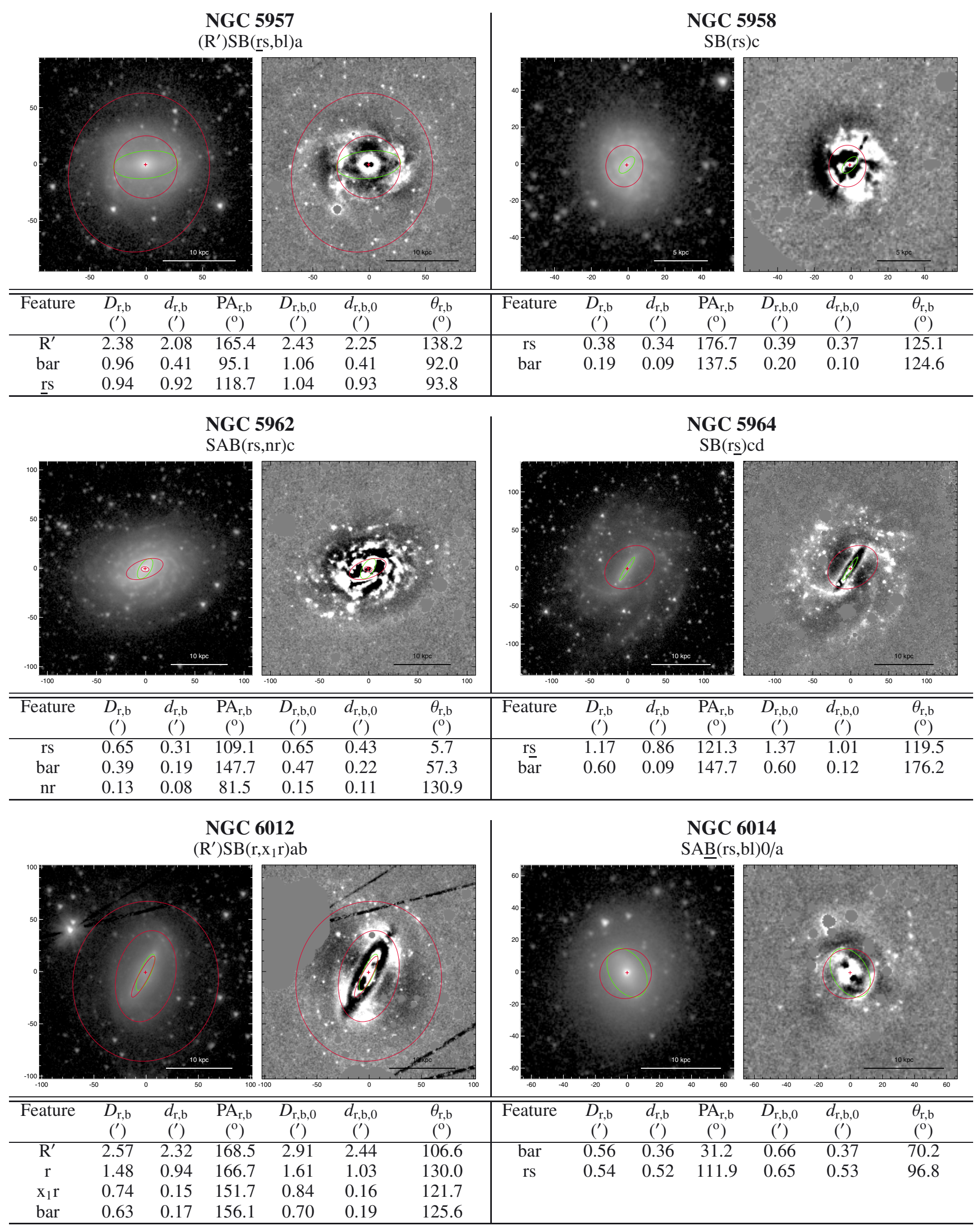


S. Comerón et al.: ARRAKIS

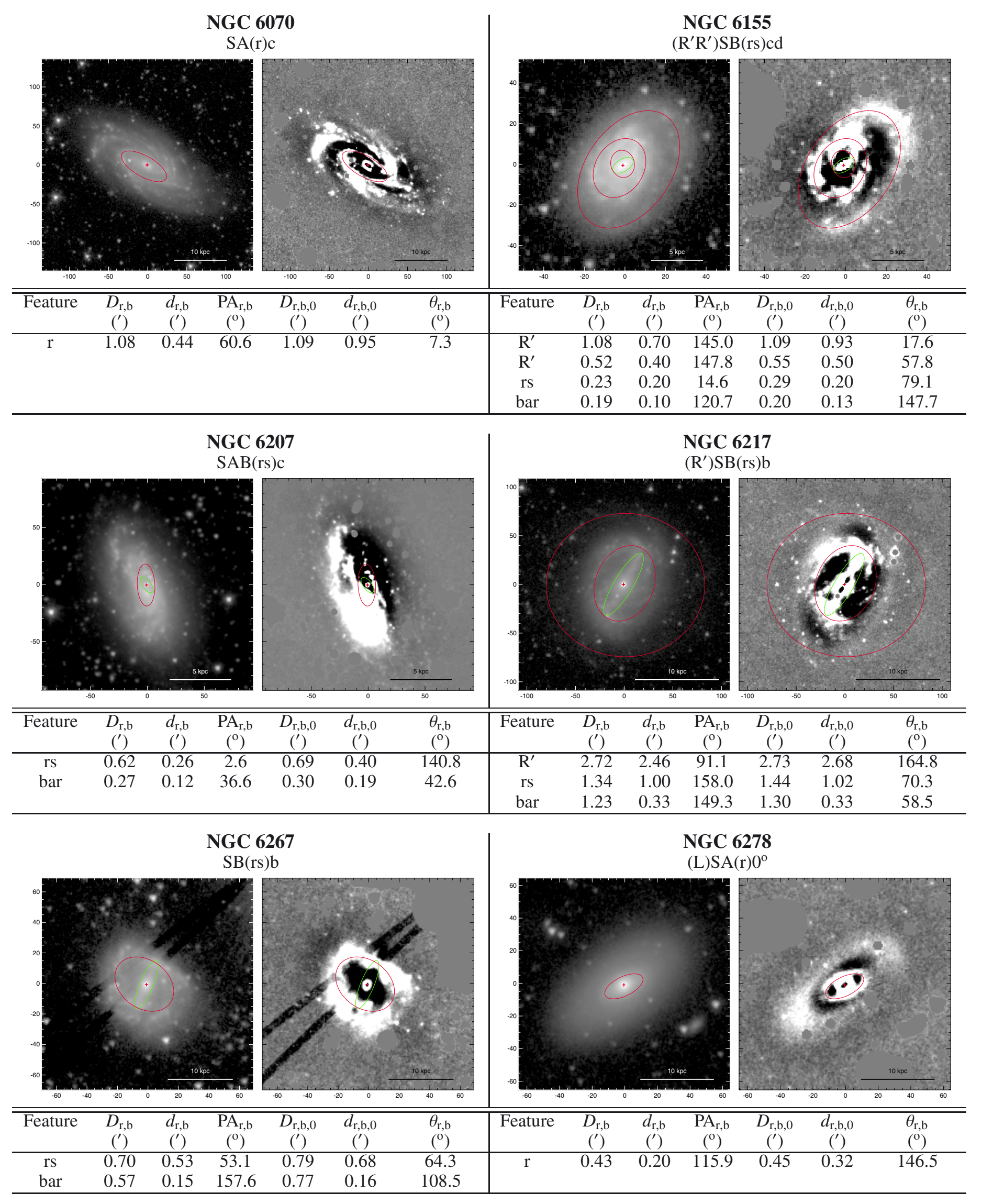




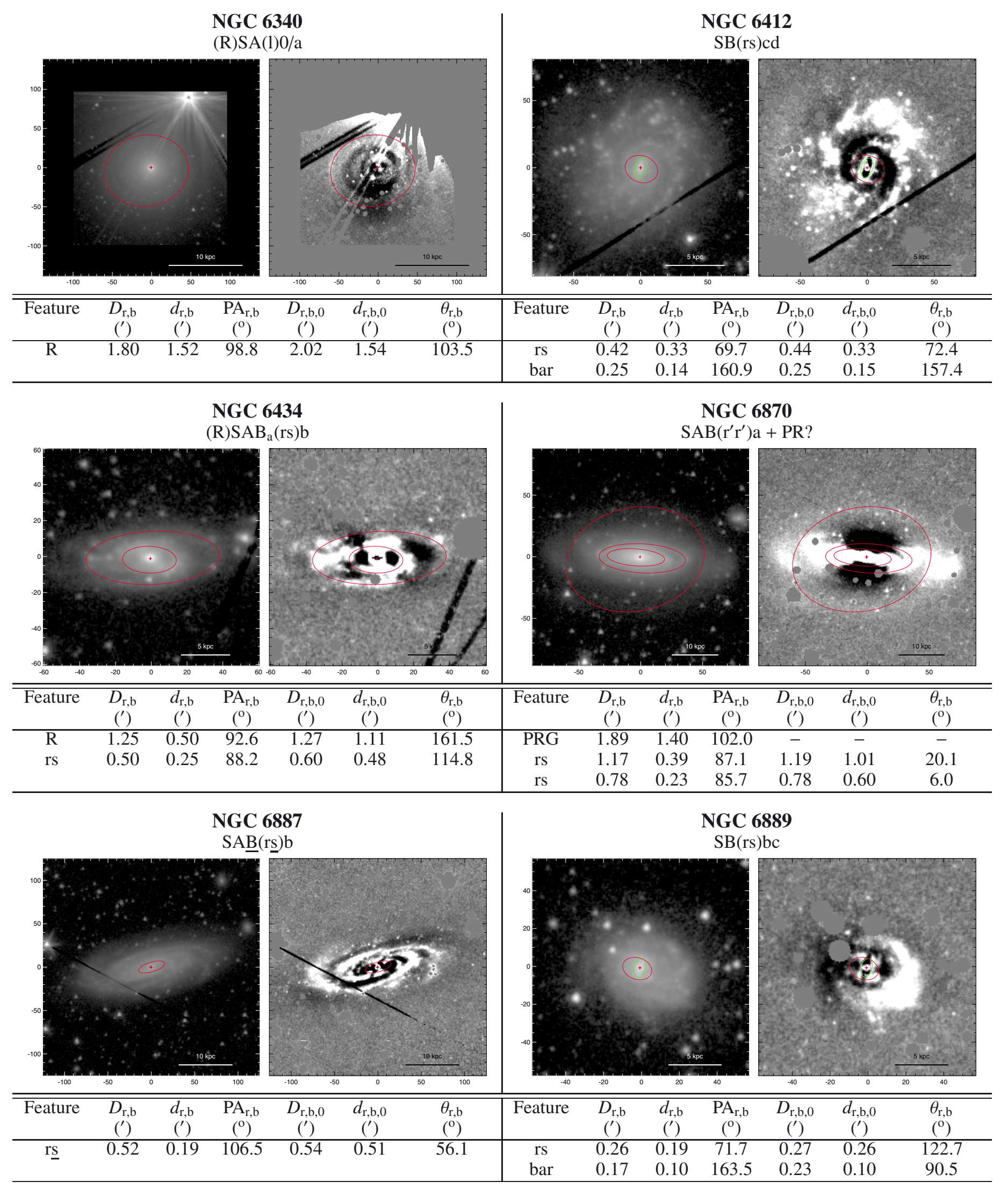


S. Comerón et al.: ARRAKIS

NGC 6902

$\mathrm{SAB}(\mathrm{rs}, \mathrm{nl}) \mathrm{a}$
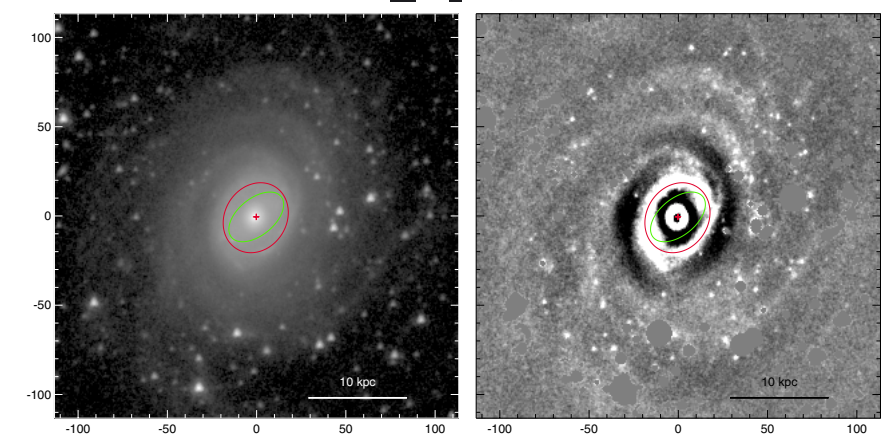

\begin{tabular}{|c|c|c|c|c|c|c|c|c|c|c|c|c|c|}
\hline Feature & $\begin{array}{c}D_{\mathrm{r}, \mathrm{b}} \\
\left({ }^{\prime}\right)\end{array}$ & $\begin{array}{c}d_{\mathrm{r}, \mathrm{b}} \\
\left({ }^{\prime}\right)\end{array}$ & $\begin{array}{c}\mathrm{PA}_{\mathrm{r}, \mathrm{b}} \\
\left({ }^{\mathrm{O}}\right)\end{array}$ & $\begin{array}{c}D_{\mathrm{r}, \mathrm{b}, 0} \\
\left(^{\prime}\right)\end{array}$ & $\begin{array}{c}d_{\mathrm{r}, \mathrm{b}, 0} \\
\left(^{\prime}\right)\end{array}$ & $\begin{array}{l}\theta_{\mathrm{r}, \mathrm{b}} \\
\left({ }^{\circ}\right)\end{array}$ & Feature & $\begin{array}{c}D_{\mathrm{r}, \mathrm{b}} \\
\left({ }^{\prime}\right)\end{array}$ & $\begin{array}{c}d_{\mathrm{r}, \mathrm{b}} \\
\left(^{\prime}\right)\end{array}$ & $\begin{array}{c}\mathrm{PA}_{\mathrm{r}, \mathrm{b}} \\
\left(^{\circ}\right)\end{array}$ & $\begin{array}{c}D_{\mathrm{r}, \mathrm{b}, 0} \\
\left(^{\prime}\right)\end{array}$ & $\begin{array}{c}d_{\mathrm{r}, \mathrm{b}, 0} \\
\left(^{\prime}\right)\end{array}$ & $\begin{array}{c}\theta_{\mathrm{r}, \mathrm{b}} \\
\left({ }^{\circ}\right)\end{array}$ \\
\hline $\begin{array}{l}\underline{\mathrm{r} s} \\
\mathrm{~b}\end{array}$ & $\begin{array}{l}0.68 \\
0.60\end{array}$ & $\begin{array}{l}0.58 \\
0.34\end{array}$ & $\begin{array}{l}147.8 \\
129.4\end{array}$ & $\begin{array}{l}0.71 \\
0.62\end{array}$ & $\begin{array}{l}0.68 \\
0.41\end{array}$ & $\begin{array}{c}91.6 \\
151.3\end{array}$ & $\mathrm{rl}$ & 0.36 & 0.17 & 73.4 & 0.36 & 0.25 & 178.9 \\
\hline
\end{tabular}

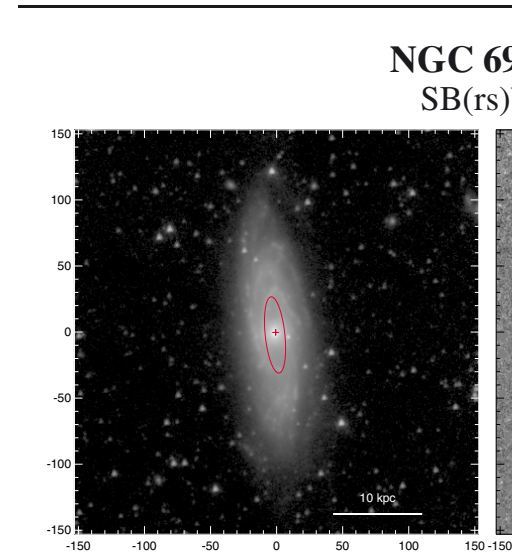

$\mathrm{SB}(\mathrm{rs}) \mathrm{b}$

\begin{tabular}{|c|c|c|c|c|c|c|c|c|c|c|c|c|c|}
\hline Feature & $\begin{array}{c}D_{\mathrm{r}, \mathrm{b}} \\
\left({ }^{\prime}\right)\end{array}$ & $\begin{array}{c}d_{\mathrm{r}, \mathrm{b}} \\
\left(^{\prime}\right)\end{array}$ & $\begin{array}{c}\mathrm{PA}_{\mathrm{r}, \mathrm{b}} \\
\left({ }^{\circ}\right)\end{array}$ & $\begin{array}{c}D_{\mathrm{r}, \mathrm{b}, 0} \\
\left(^{\prime}\right)\end{array}$ & $\begin{array}{c}d_{\mathrm{r}, \mathrm{b}, 0} \\
\left({ }^{\prime}\right)\end{array}$ & $\begin{array}{l}\theta_{\mathrm{r}, \mathrm{b}} \\
\left({ }^{\circ}\right)\end{array}$ & Feature & $\begin{array}{c}D_{\mathrm{r}, \mathrm{b}} \\
\left(^{\prime}\right)\end{array}$ & $\begin{array}{c}d_{\mathrm{r}, \mathrm{b}} \\
\left({ }^{\prime}\right)\end{array}$ & $\begin{array}{c}\mathrm{PA}_{\mathrm{r}, \mathrm{b}} \\
\left({ }^{\circ}\right)\end{array}$ & $\begin{array}{c}D_{\mathrm{r}, \mathrm{b}, 0} \\
\left(^{\prime}\right)\end{array}$ & $\begin{array}{c}d_{\mathrm{r}, \mathrm{b}, 0} \\
\left(^{\prime}\right)\end{array}$ & $\begin{array}{l}\overline{\theta_{\mathrm{r}, \mathrm{b}}} \\
\left(^{\circ}\right)\end{array}$ \\
\hline \multirow[t]{4}{*}{ rs } & 0.97 & 0.25 & 6.0 & 0.97 & 0.76 & 172.9 & $\mathrm{R}_{2}^{\prime}$ & 1.12 & 0.97 & 57.4 & 1.13 & 1.04 & 163.5 \\
\hline & & & & & & & $\mathrm{R}_{1}^{\prime}$ & 0.76 & 0.58 & 179.4 & 0.81 & 0.59 & 108.4 \\
\hline & & & & & & & rs & 0.29 & 0.22 & 78.4 & 0.30 & 0.23 & 15.7 \\
\hline & & & & & & & bar & 0.28 & 0.14 & 79.4 & 0.28 & 0.15 & 14.7 \\
\hline
\end{tabular}

NGC 7070

$\mathrm{SB}(\mathrm{rs}) \mathrm{c}$

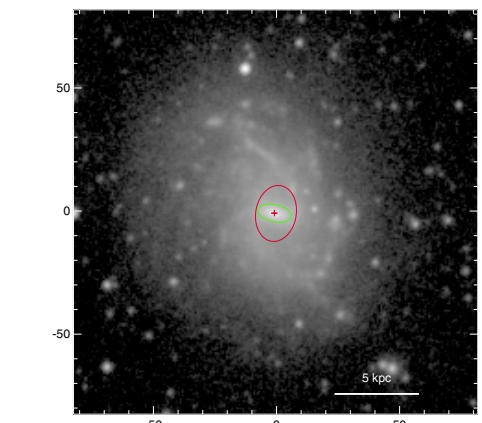

\section{NGC 7098}

(R)SAB ${ }_{\mathrm{a}}\left(\mathrm{r}^{\prime} 1, \mathrm{nl}, \mathrm{nb}\right) 0 / \mathrm{a}$

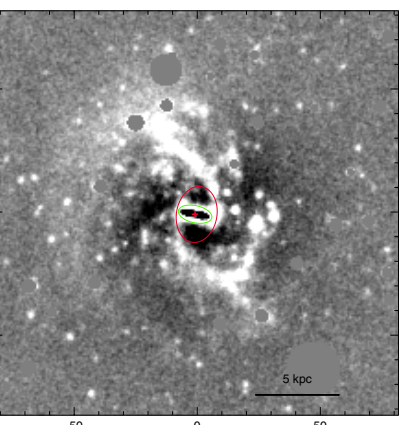

NGC 6923

$\mathrm{SAB}_{a}(\mathrm{rl}) \mathrm{b}$

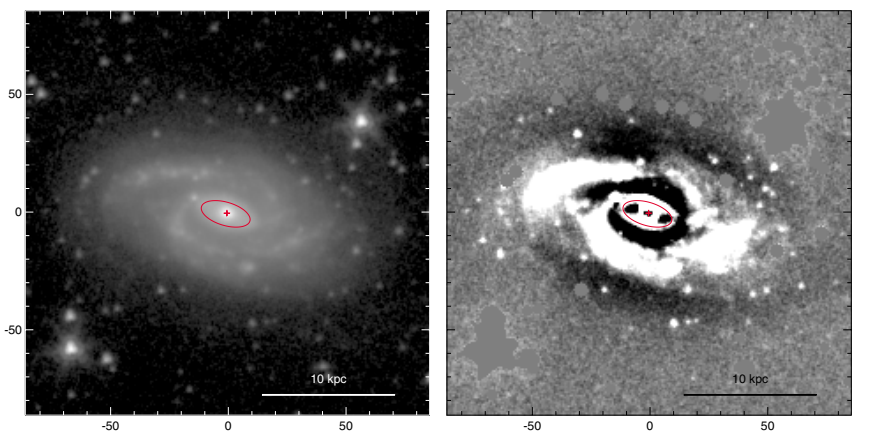

NGC 7051

$\left(\mathrm{R}_{1}^{\prime} \mathrm{R}_{2}^{\prime}\right) \mathrm{SAB}_{\mathrm{a}}(\mathrm{rs}) \mathrm{b}$

\begin{tabular}{|c|c|c|c|c|c|c|c|c|c|c|c|c|c|}
\hline Feature & $\begin{array}{c}D_{\mathrm{r}, \mathrm{b}} \\
\left(^{\prime}\right)\end{array}$ & $\begin{array}{c}d_{\mathrm{r}, \mathrm{b}} \\
\left({ }^{\prime}\right)\end{array}$ & $\begin{array}{c}\mathrm{PA}_{\mathrm{r}, \mathrm{b}} \\
\left({ }^{\circ}\right)\end{array}$ & 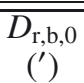 & $\begin{array}{c}d_{\mathrm{r}, \mathrm{b}, 0} \\
\left(^{\prime}\right)\end{array}$ & $\begin{array}{l}\theta_{\mathrm{r}, \mathrm{b}} \\
\left({ }^{\circ}\right)\end{array}$ & Feature & $\begin{array}{c}D_{\mathrm{r}, \mathrm{b}} \\
\left({ }^{\prime}\right)\end{array}$ & $\begin{array}{c}d_{\mathrm{r}, \mathrm{b}} \\
\left(^{\prime}\right)\end{array}$ & $\begin{array}{c}\mathrm{PA}_{\mathrm{r}, \mathrm{b}} \\
\left({ }^{\circ}\right)\end{array}$ & $\begin{array}{c}D_{\mathrm{r}, \mathrm{b}, 0} \\
\text { (') }^{\prime}\end{array}$ & $\begin{array}{c}d_{\mathrm{r}, \mathrm{b}, 0} \\
\left(^{\prime}\right)\end{array}$ & $\begin{array}{l}\theta_{\mathrm{r}, \mathrm{b}} \\
\left({ }^{\circ}\right)\end{array}$ \\
\hline rs & 0.38 & 0.28 & 170.4 & 0.40 & 0.30 & 140.8 & $\bar{R}$ & 3.63 & 2.26 & 80.7 & 4.13 & 3.46 & 62.9 \\
\hline bār & 0.23 & 0.12 & 77.6 & 0.26 & 0.12 & 64.7 & $r^{\prime} 1$ & 1.87 & 1.07 & 67.8 & 1.95 & 1.78 & 134.9 \\
\hline & & & & & & & bar & 1.38 & 0.63 & 50.6 & 1.63 & 0.93 & 133.1 \\
\hline
\end{tabular}


NGC 7107

$\left(\mathrm{R}^{\prime}\right) \mathrm{SB}(\mathrm{rs}) \mathrm{dm}$
NGC 7140

$\left(\mathrm{R}^{\prime}\right) \mathrm{SAB}_{\mathrm{x}}(\mathrm{rs}, \mathrm{nl}) \mathrm{ab}$
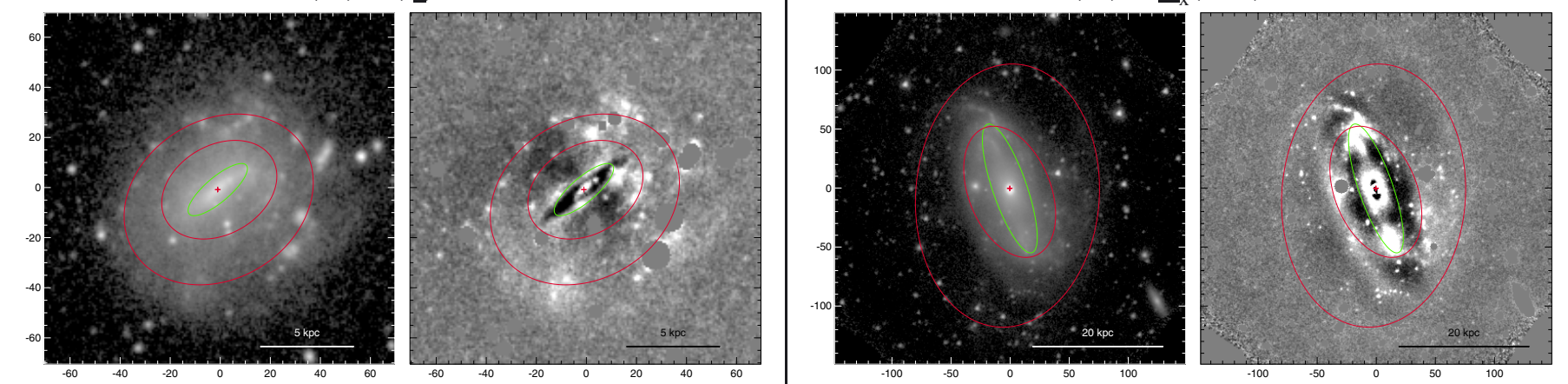

\begin{tabular}{ccccccc|ccccccc}
\hline \hline Feature & $\begin{array}{c}D_{\mathrm{r}, \mathrm{b}} \\
\left({ }^{\prime}\right)\end{array}$ & $\begin{array}{c}d_{\mathrm{r}, \mathrm{b}} \\
\left({ }^{\prime}\right)\end{array}$ & $\begin{array}{c}\mathrm{PA}_{\mathrm{r}, \mathrm{b}} \\
\left({ }^{\circ}\right)\end{array}$ & $\begin{array}{c}D_{\mathrm{r}, \mathrm{b}, 0} \\
\left({ }^{\prime}\right)\end{array}$ & $\begin{array}{c}d_{\mathrm{r}, \mathrm{b}, 0} \\
\left({ }^{\prime}\right)\end{array}$ & $\begin{array}{c}\theta_{\mathrm{r}, \mathrm{b}} \\
\left({ }^{\circ}\right)\end{array}$ & Feature & $\begin{array}{c}D_{\mathrm{r}, \mathrm{b}} \\
\left({ }^{\prime}\right)\end{array}$ & $\begin{array}{c}d_{\mathrm{r}, \mathrm{b}} \\
\left({ }^{\prime}\right)\end{array}$ & $\begin{array}{c}\mathrm{PA}_{\mathrm{r}, \mathrm{b}} \\
\left({ }^{\circ}\right)\end{array}$ & $\begin{array}{c}D_{\mathrm{r}, \mathrm{b}, 0} \\
\left({ }^{\prime}\right)\end{array}$ & $\begin{array}{c}d_{\mathrm{r}, \mathrm{b}, 0} \\
\left({ }^{\prime}\right)\end{array}$ & $\begin{array}{c}\theta_{\mathrm{r}, \mathrm{b}} \\
\left({ }^{\circ}\right)\end{array}$ \\
\hline $\mathrm{R}^{\prime}$ & 1.33 & 1.06 & 121.4 & 1.38 & 1.18 & 38.2 & $\mathrm{R}$ & 3.72 & 2.60 & 176.0 & 4.13 & 3.62 & 113.2 \\
$\mathrm{r} \underline{\mathrm{s}}$ & 0.82 & 0.60 & 120.2 & 0.84 & 0.66 & 30.3 & $\mathrm{rs}$ & 1.94 & 1.16 & 22.0 & 2.19 & 1.59 & 48.6 \\
$\mathrm{~b} \mathrm{a}$ & 0.50 & 0.16 & 130.4 & 0.52 & 0.18 & 34.1 & $\mathrm{bar}$ & 1.92 & 0.48 & 19.1 & 2.02 & 0.71 & 25.3 \\
\hline
\end{tabular}
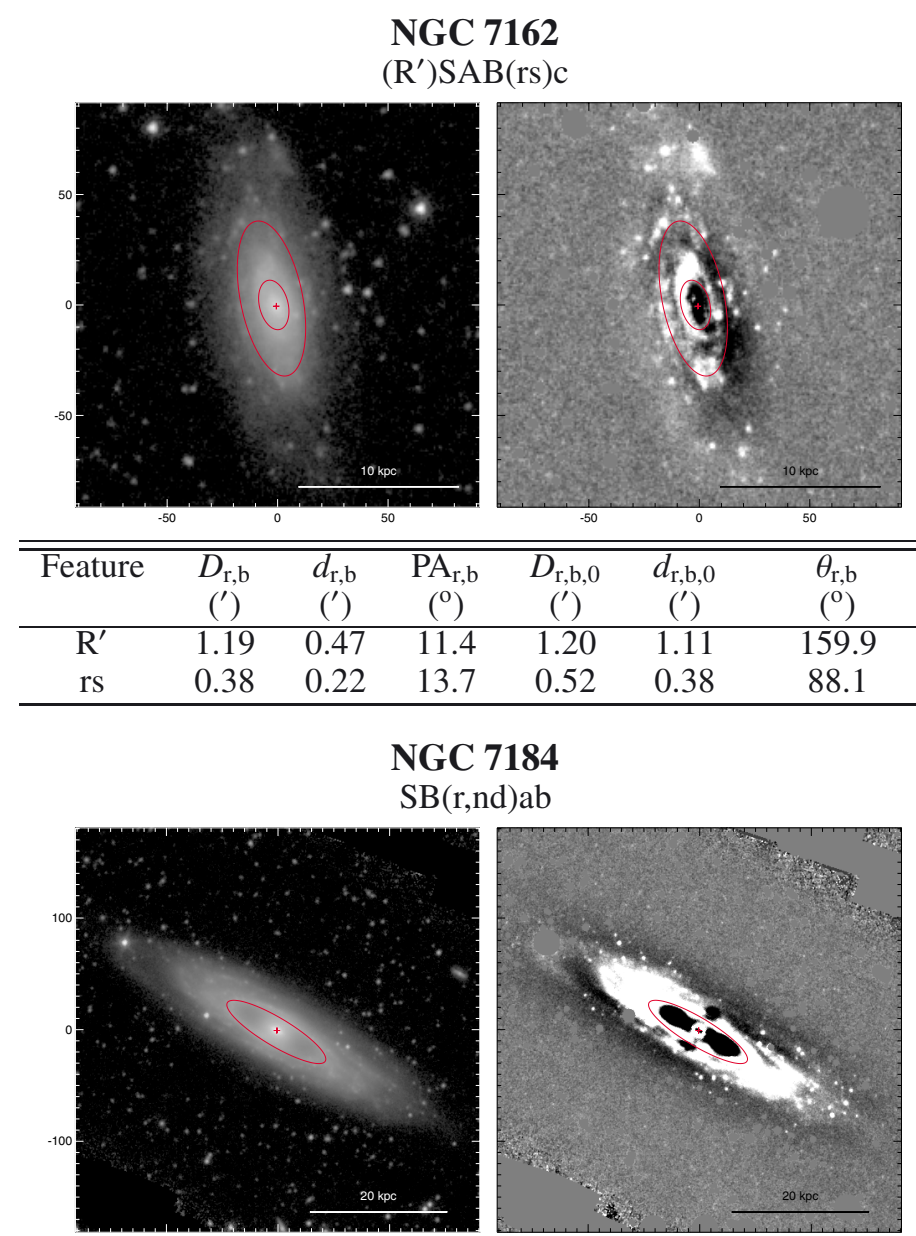

NGC 7179

$\mathrm{SB}_{\mathrm{xa}}(\mathrm{r}) 0 / \mathrm{a}$
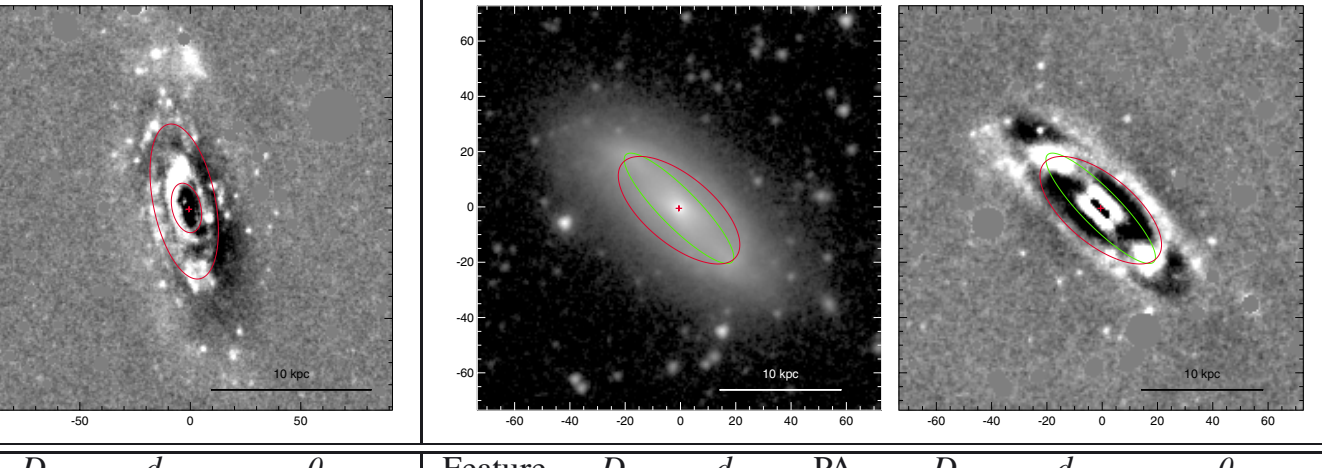

Feature

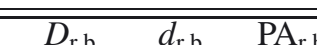

\begin{tabular}{c|c}
$\left({ }^{\circ}\right)$ & \\
\hline 159.9 & bar 0
\end{tabular}

$\left({ }^{\prime}\right)$
0.91
0.89

$\frac{\left({ }^{\prime}\right)}{0.23}$

( $\left.{ }^{\mathrm{O}}\right)$

$D_{\mathrm{r}, \mathrm{b}, 0} \quad d_{\mathrm{r}, \mathrm{b}, 0}$

$\begin{array}{llll} & 0.23 & 44.7 & 0.93\end{array}$

$0.93 \quad 0.44$

0.44
0.80 $\left(^{\mathrm{o}}\right)$

$\begin{array}{ll}0.52 & 0.38\end{array}$

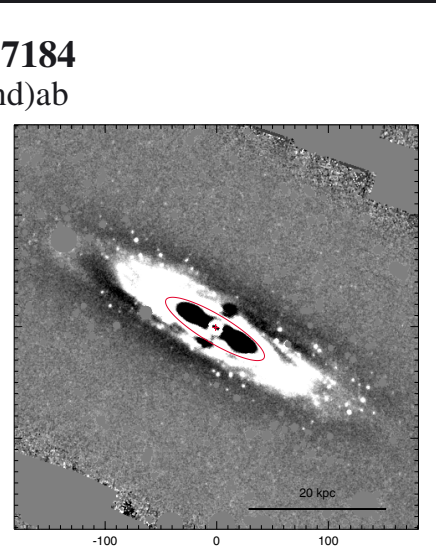

NGC 7188

$\left(\mathrm{R}^{\prime}\right) \mathrm{SAB}(\mathrm{s}) \mathrm{b}$ :

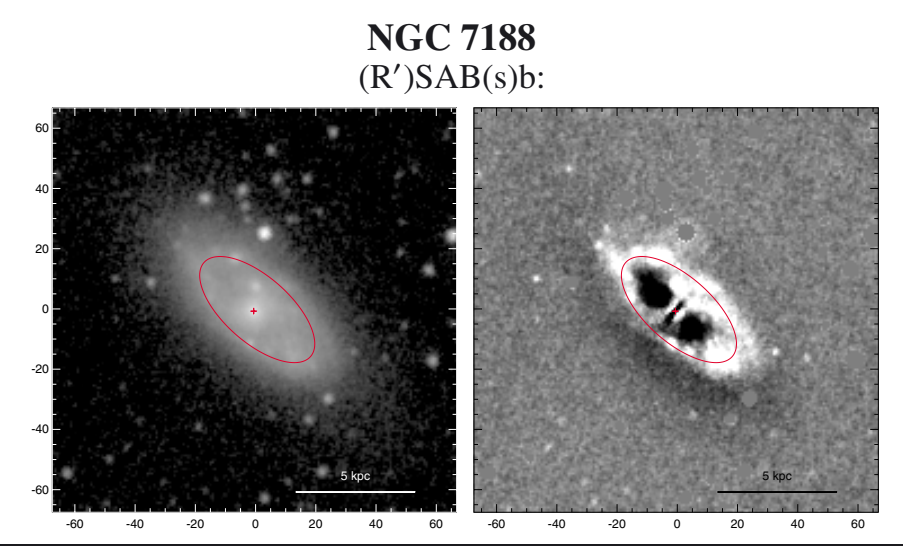

\begin{tabular}{ccccccc|ccccccc}
\hline \hline Feature & $\begin{array}{c}D_{\mathrm{r}, \mathrm{b}} \\
\left({ }^{\prime}\right)\end{array}$ & $\begin{array}{c}d_{\mathrm{r}, \mathrm{b}} \\
\left({ }^{\prime}\right)\end{array}$ & $\begin{array}{c}\mathrm{PA}_{\mathrm{r}, \mathrm{b}} \\
\left({ }^{\circ}\right)\end{array}$ & $\begin{array}{c}D_{\mathrm{r}, \mathrm{b}, 0} \\
\left({ }^{\prime}\right)\end{array}$ & $\begin{array}{c}d_{\mathrm{r}, \mathrm{b}, 0} \\
\left({ }^{\prime}\right)\end{array}$ & $\begin{array}{c}\theta_{\mathrm{r}, \mathrm{b}} \\
\left({ }^{(}\right)\end{array}$ & Feature & $\begin{array}{c}D_{\mathrm{r}, \mathrm{b}} \\
\left({ }^{\prime}\right)\end{array}$ & $\begin{array}{c}d_{\mathrm{r}, \mathrm{b}} \\
\left({ }^{\prime}\right)\end{array}$ & $\begin{array}{c}\mathrm{PA}_{\mathrm{r}, \mathrm{b}} \\
\left({ }^{\circ}\right)\end{array}$ & $\begin{array}{c}D_{\mathrm{r}, \mathrm{b}, 0} \\
\left({ }^{\prime}\right)\end{array}$ & $\begin{array}{c}d_{\mathrm{r}, \mathrm{b}, 0} \\
\left({ }^{\prime}\right)\end{array}$ & $\begin{array}{c}\theta_{\mathrm{r}, \mathrm{b}} \\
\left({ }^{\circ}\right)\end{array}$ \\
\hline $\mathrm{r}$ & 1.71 & 0.42 & 59.1 & 1.71 & 1.64 & 164.6 & $\mathrm{R}^{\prime}$ & 0.79 & 0.37 & 48.6 & 0.80 & 0.74 & 28.3 \\
\hline
\end{tabular}


NGC 7191

$\left(\mathrm{R}^{\prime}\right) \mathrm{SAB}(\mathrm{s}) \mathrm{b}$ pec
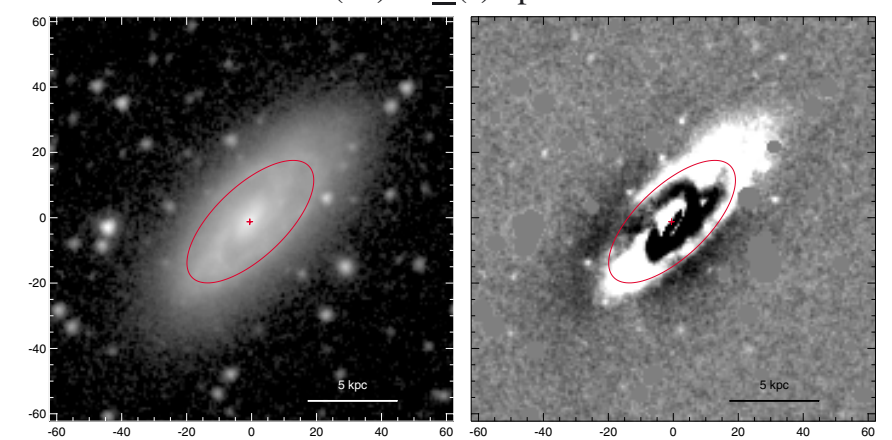

NGC 7205

$\mathrm{SA}(\mathrm{rs}) \mathrm{bc}$

\begin{tabular}{|c|c|c|c|c|c|c|c|c|c|c|c|c|c|}
\hline Feature & $\begin{array}{c}D_{\mathrm{r}, \mathrm{b}} \\
\left({ }^{\prime}\right)\end{array}$ & $\begin{array}{c}d_{\mathrm{r}, \mathrm{b}} \\
\left({ }^{\prime}\right)\end{array}$ & $\begin{array}{c}\mathrm{PA}_{\mathrm{r}, \mathrm{b}} \\
\left({ }^{\circ}\right)\end{array}$ & $\begin{array}{c}D_{\mathrm{r}, \mathrm{b}, 0} \\
\left(^{\prime}\right)\end{array}$ & $\begin{array}{c}d_{\mathrm{r}, \mathrm{b}, 0} \\
\left.\text { (') }^{\prime}\right)\end{array}$ & $\begin{array}{l}\theta_{\mathrm{r}, \mathrm{b}} \\
\left({ }^{\circ}\right)\end{array}$ & Feature & $\begin{array}{c}D_{\mathrm{r}, \mathrm{b}} \\
\left({ }^{\prime}\right)\end{array}$ & $\begin{array}{c}d_{\mathrm{r}, \mathrm{b}} \\
\left({ }^{\prime}\right)\end{array}$ & $\begin{array}{c}\mathrm{PA}_{\mathrm{r}, \mathrm{b}} \\
\left(^{\circ}\right)\end{array}$ & $\begin{array}{c}D_{\mathrm{r}, \mathrm{b}, 0} \\
\left(^{\prime}\right)\end{array}$ & $\begin{array}{c}d_{\mathrm{r}, \mathrm{b}, 0} \\
\left(^{\prime}\right)\end{array}$ & $\begin{array}{l}\theta_{\mathrm{r}, \mathrm{b}} \\
\left({ }^{\circ}\right)\end{array}$ \\
\hline$\overline{\mathrm{R}^{\prime}}$ & 0.83 & 0.37 & 133.5 & 0.90 & 0.80 & 122.4 & rs & 0.47 & 0.25 & 63.1 & 0.52 & 0.46 & 115.7 \\
\hline
\end{tabular}

NGC 7213

(L) $\mathrm{SA}(\mathrm{r}, 1) 0^{\circ}$
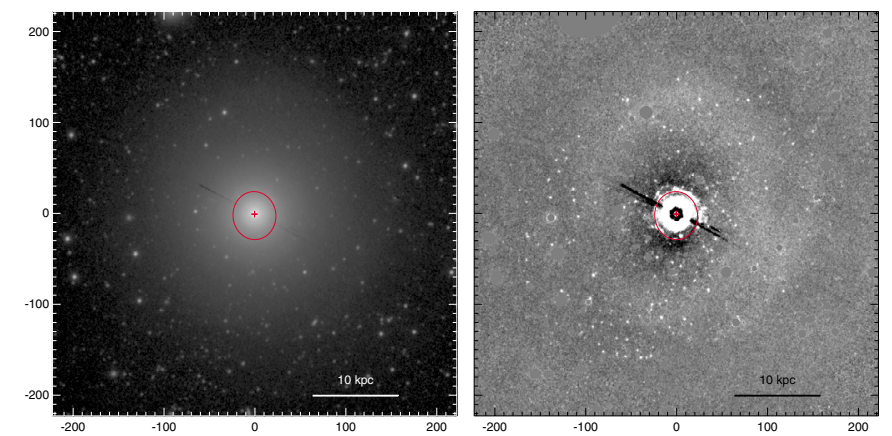

NGC 7218

$\mathrm{SB}(\mathrm{rs}) \mathrm{cd}$

\begin{tabular}{|c|c|c|c|c|c|c|c|c|c|c|c|c|c|}
\hline Feature & $\begin{array}{c}D_{\mathrm{r}, \mathrm{b}} \\
\left({ }^{\prime}\right)\end{array}$ & $\begin{array}{c}d_{\mathrm{r}, \mathrm{b}} \\
\left(^{\prime}\right)\end{array}$ & $\begin{array}{c}\mathrm{PA}_{\mathrm{r}, \mathrm{b}} \\
\left({ }^{\circ}\right)\end{array}$ & $\begin{array}{c}D_{\mathrm{r}, \mathrm{b}, 0} \\
\left(^{\prime}\right)\end{array}$ & $\begin{array}{c}d_{\mathrm{r}, \mathrm{b}, 0} \\
\left({ }^{\prime}\right)\end{array}$ & $\begin{array}{l}\theta_{\mathrm{r}, \mathrm{b}} \\
\left({ }^{\circ}\right)\end{array}$ & Feature & $\begin{array}{c}D_{\mathrm{r}, \mathrm{b}} \\
\left({ }^{\prime}\right)\end{array}$ & $\begin{array}{c}d_{\mathrm{r}, \mathrm{b}} \\
(')\end{array}$ & $\begin{array}{c}\mathrm{PA}_{\mathrm{r}, \mathrm{b}} \\
\left({ }^{\circ}\right)\end{array}$ & $\begin{array}{c}D_{\mathrm{r}, \mathrm{b}, 0} \\
\left(^{\prime}\right)\end{array}$ & $\begin{array}{c}d_{\mathrm{r}, \mathrm{b}, 0} \\
\left({ }^{\prime}\right)\end{array}$ & $\begin{array}{l}\theta_{\mathrm{r}, \mathrm{b}} \\
\left(^{\circ}\right)\end{array}$ \\
\hline $\mathrm{r}$ & 0.88 & 0.79 & 5.2 & 0.90 & 0.82 & 42.9 & rs & 0.54 & 0.37 & 28.2 & 0.82 & 0.53 & 83.3 \\
\hline
\end{tabular}

NGC 7219

$\left(\mathrm{R}_{2}^{\prime}\right) \mathrm{SAB}(\mathrm{r}) \mathrm{a}$
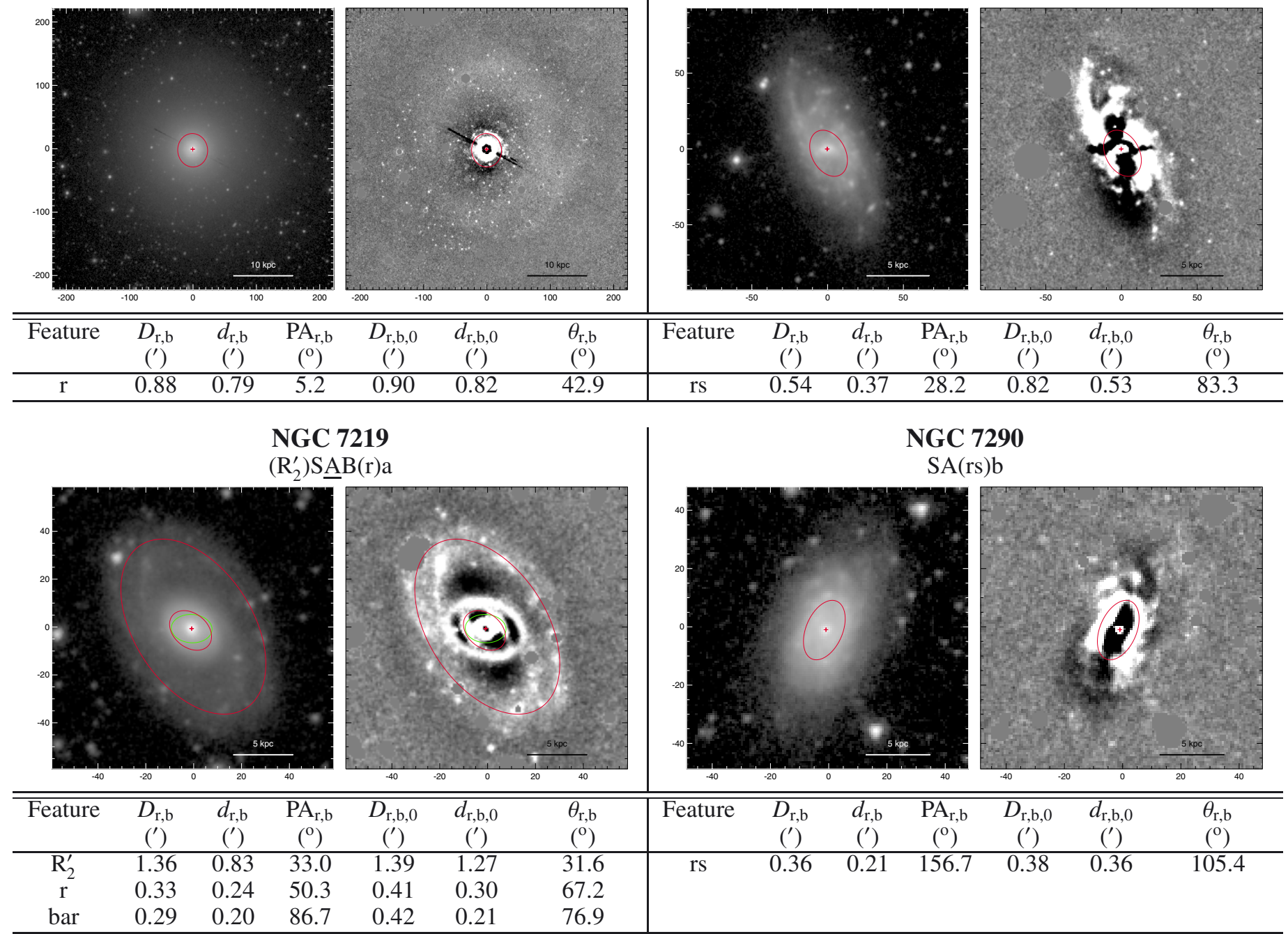


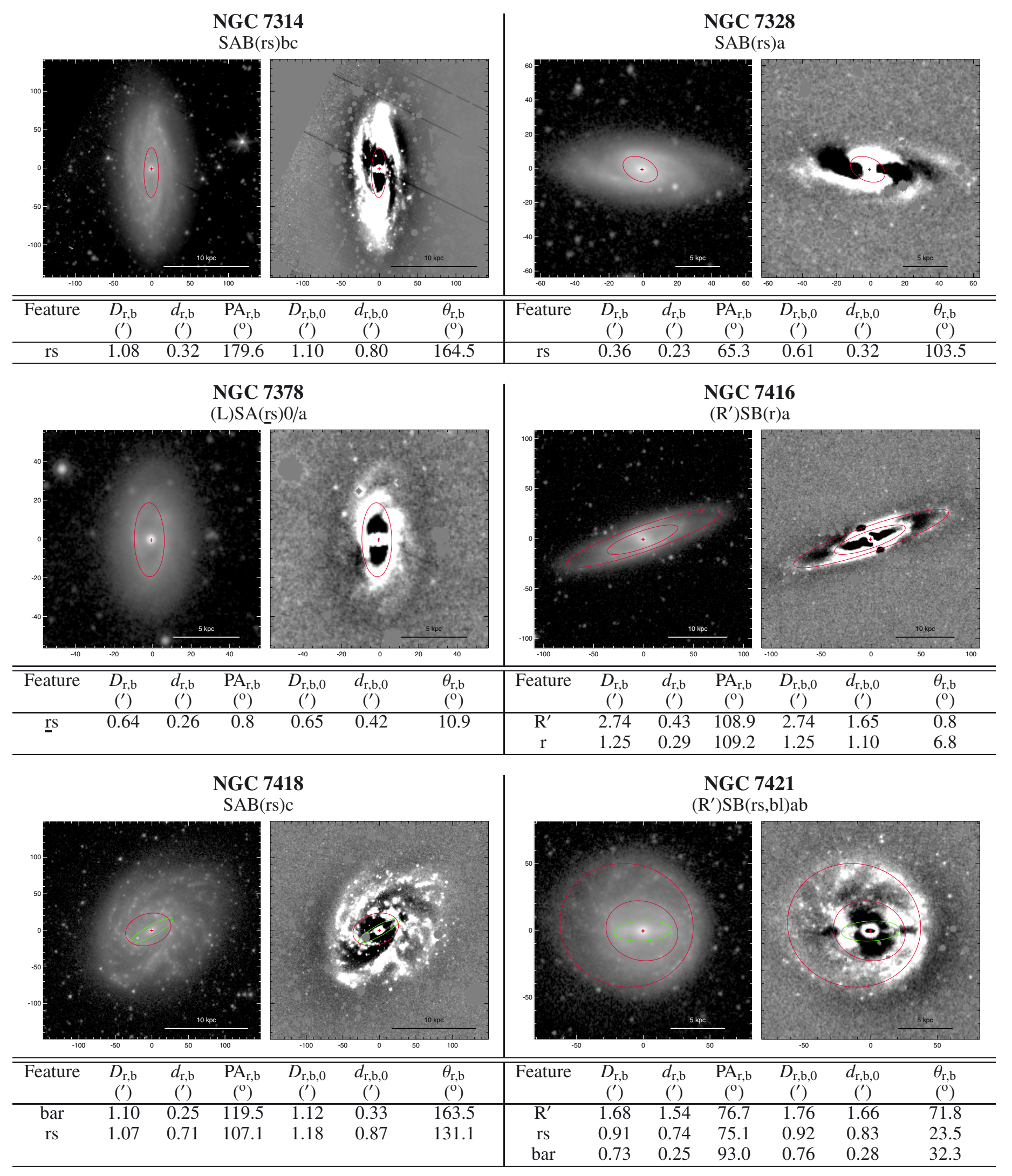




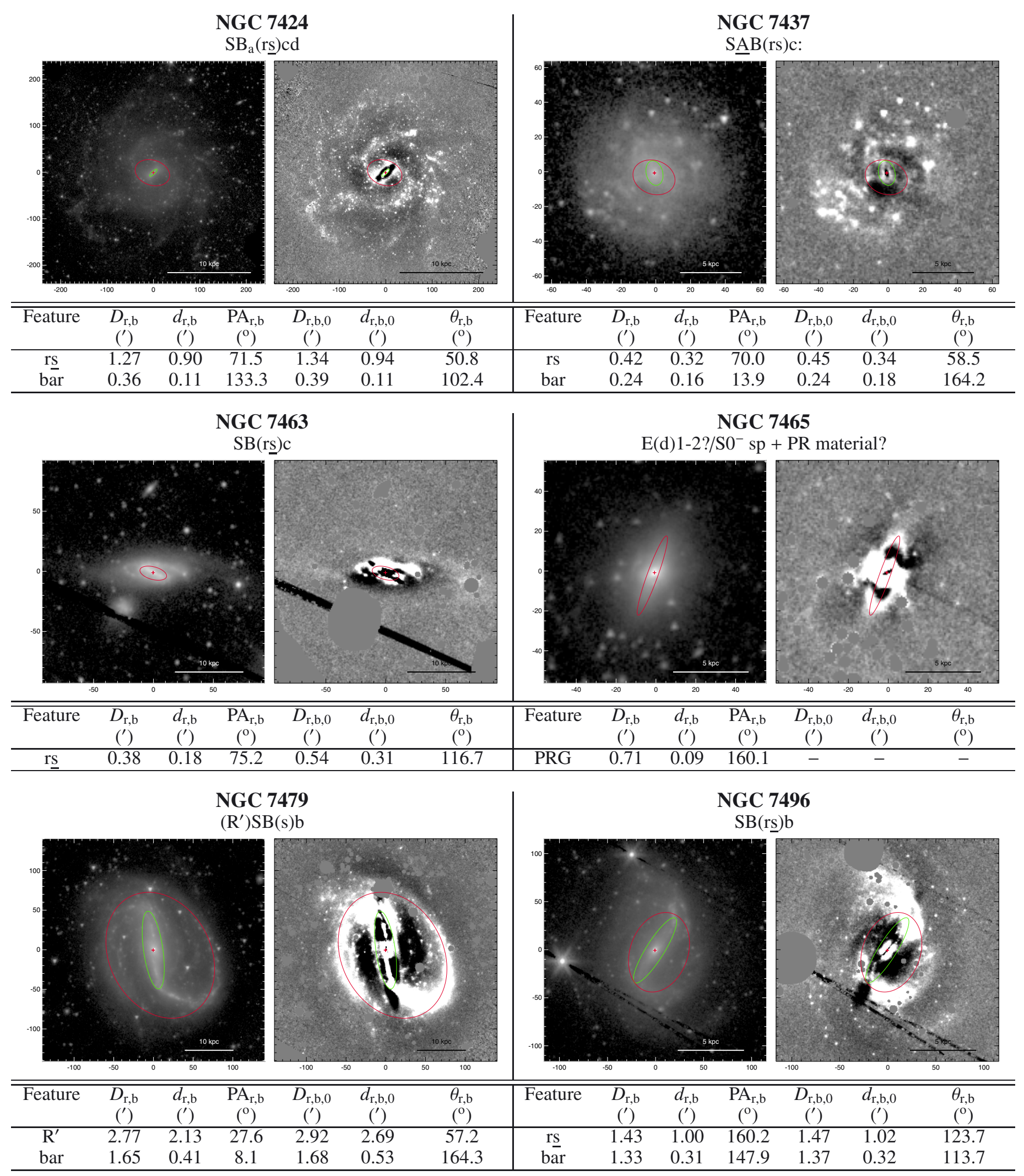


NGC 7513

$\mathrm{SB}(\mathrm{rs}) \mathrm{a}$
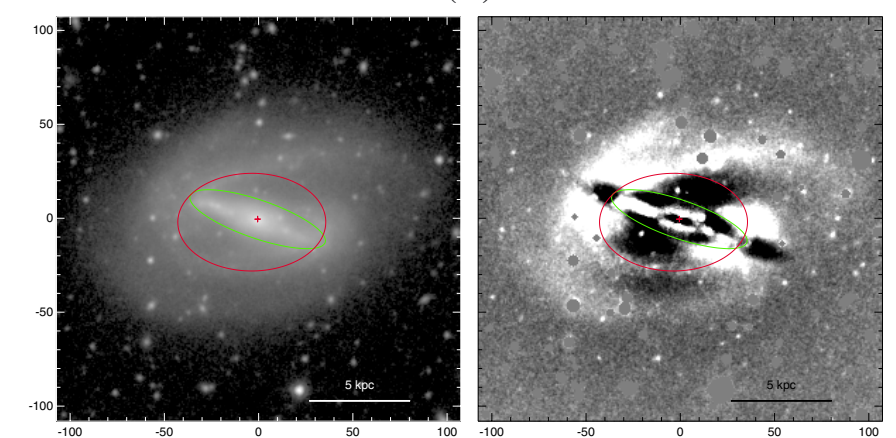

\begin{tabular}{ccccccc|ccccccc}
\hline \hline Feature & $\begin{array}{c}D_{\mathrm{r}, \mathrm{b}} \\
\left({ }^{\prime}\right)\end{array}$ & $\begin{array}{c}d_{\mathrm{r}, \mathrm{b}} \\
\left({ }^{\prime}\right)\end{array}$ & $\begin{array}{c}\mathrm{PA}_{\mathrm{r}, \mathrm{b}} \\
\left({ }^{\prime}\right)\end{array}$ & $\begin{array}{c}D_{\mathrm{r}, \mathrm{b}, 0} \\
\left({ }^{\prime}\right)\end{array}$ & $\begin{array}{c}d_{\mathrm{r}, \mathrm{b}, 0} \\
\left({ }^{\prime}\right)\end{array}$ & $\begin{array}{c}\theta_{\mathrm{r}, \mathrm{b}} \\
\left({ }^{\circ}\right)\end{array}$ & Feature & $\begin{array}{c}D_{\mathrm{r}, \mathrm{b}} \\
\left({ }^{\prime}\right)\end{array}$ & $\begin{array}{c}d_{\mathrm{r}, \mathrm{b}} \\
\left({ }^{\prime}\right)\end{array}$ & $\begin{array}{c}\mathrm{PA}_{\mathrm{r}, \mathrm{b}} \\
\left({ }^{\circ}\right)\end{array}$ & $\begin{array}{c}D_{\mathrm{r}, \mathrm{b}, 0} \\
()^{\prime}\end{array}$ & $\begin{array}{c}d_{\mathrm{r}, \mathrm{b}, 0} \\
\left({ }^{\prime}\right)\end{array}$ & $\begin{array}{c}\theta_{\mathrm{r}, \mathrm{b}} \\
\left({ }^{\circ}\right)\end{array}$ \\
\hline $\mathrm{rs}$ & 1.31 & 0.87 & 90.2 & 1.46 & 1.17 & 127.5 & $\mathrm{bar}$ & 0.99 & 0.33 & 11.7 & 1.03 & 0.59 & 156.3 \\
$\mathrm{bar}$ & 1.27 & 0.34 & 71.0 & 1.51 & 0.43 & 131.9 & $\mathrm{r}$ & 0.95 & 0.39 & 17.1 & 0.96 & 0.72 & 164.7 \\
\hline
\end{tabular}

NGC 7537

$\mathrm{SA}(\mathrm{rs}) \mathrm{c}$
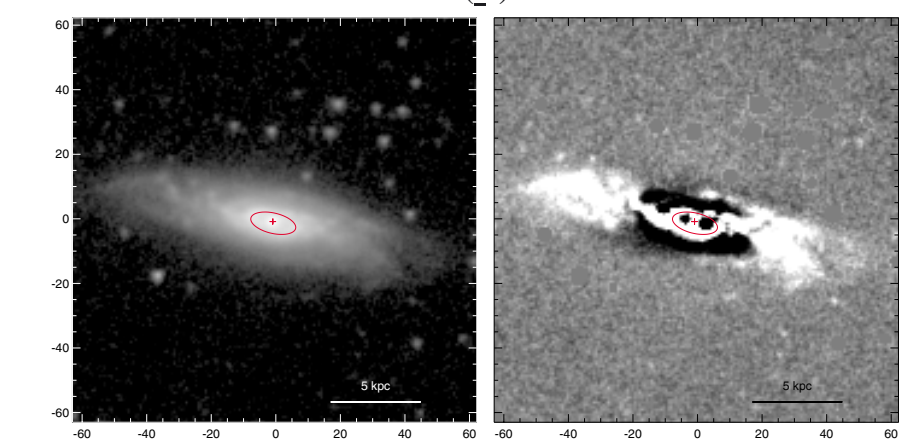

\begin{tabular}{|c|c|c|c|c|c|c|c|c|c|c|c|c|c|}
\hline "Feature & $\begin{array}{c}D_{\mathrm{r}, \mathrm{b}} \\
\left({ }^{\prime}\right)\end{array}$ & $\begin{array}{c}d_{\mathrm{r}, \mathrm{b}} \\
\left(^{\prime}\right)\end{array}$ & $\begin{array}{c}\mathrm{PA}_{\mathrm{r}, \mathrm{b}} \\
\left({ }^{\circ}\right)\end{array}$ & $\begin{array}{c}D_{\mathrm{r}, \mathrm{b}, 0} \\
\left(^{\prime}\right)\end{array}$ & $\begin{array}{c}d_{\mathrm{r}, \mathrm{b}, 0} \\
\left(^{\prime}\right)\end{array}$ & $\begin{array}{c}\theta_{\mathrm{r}, \mathrm{b}} \\
\left({ }^{\circ}\right)\end{array}$ & Feature & $\begin{array}{c}D_{\mathrm{r}, \mathrm{b}} \\
\left({ }^{\prime}\right)\end{array}$ & $\begin{array}{c}d_{\mathrm{r}, \mathrm{b}} \\
\left(^{\prime}\right)\end{array}$ & $\begin{array}{c}\mathrm{PA}_{\mathrm{r}, \mathrm{b}} \\
\left({ }^{\circ}\right)\end{array}$ & $\begin{array}{c}D_{\mathrm{r}, \mathrm{b}, 0} \\
\left(^{\prime}\right)\end{array}$ & $\begin{array}{c}d_{\mathrm{r}, \mathrm{b}, 0} \\
\left(^{\prime}\right)\end{array}$ & $\begin{array}{c}\theta_{\mathrm{r}, \mathrm{b}} \\
\left({ }^{\circ}\right)\end{array}$ \\
\hline \multirow[t]{3}{*}{$\underline{r} s$} & 0.24 & 0.11 & 76.9 & 0.34 & 0.24 & 90.5 & $\mathrm{R}_{1}^{\prime}$ & $\begin{array}{l}3.08 \\
1.96\end{array}$ & $\begin{array}{l}2.52 \\
123\end{array}$ & $\begin{array}{l}179.1 \\
0997\end{array}$ & 3.14 & 2.57 & 135.0 \\
\hline & & & & & & & $1 \underline{s}$ & 1.96 & 1.23 & 99.7 & 2.02 & 1.24 & 63.3 \\
\hline & & & & & & & bar & 1.20 & $\begin{array}{l}0.43 \\
0.10\end{array}$ & $\begin{array}{l}91.2 \\
1047\end{array}$ & 1.23 & 0.44 & 54.1 \\
\hline
\end{tabular}

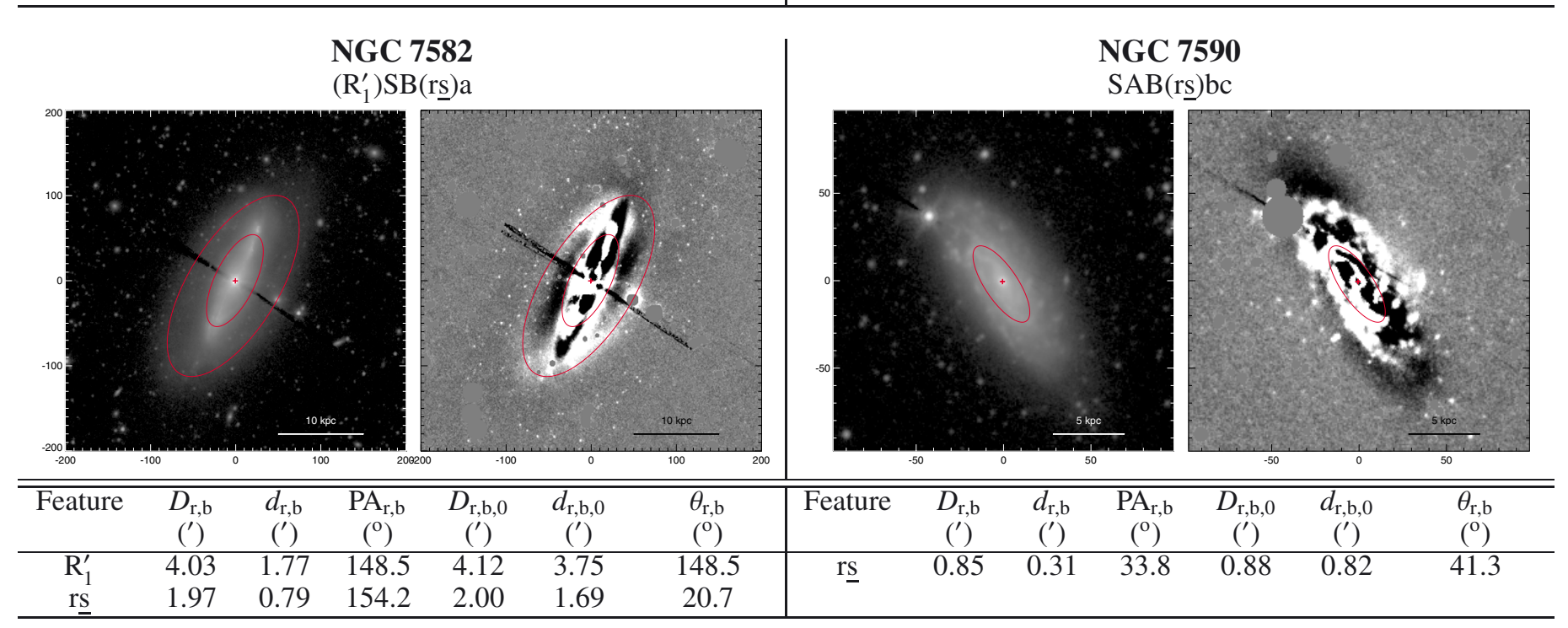

NGC 7531

$\mathrm{SAB}_{\mathrm{x}}(\mathrm{r}) \mathrm{a}$

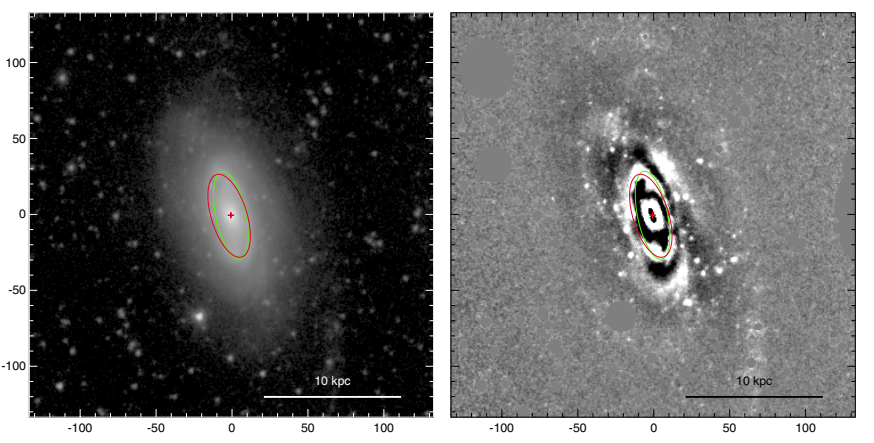

NGC 7552

$\left(\mathrm{R}_{1}^{\prime}\right) \mathrm{SB}(\mathrm{rs}, \mathrm{nr}) \mathrm{a}$

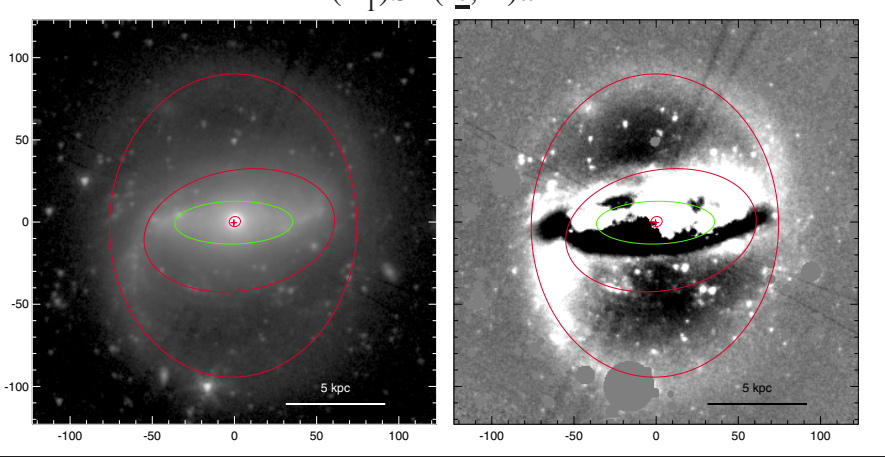




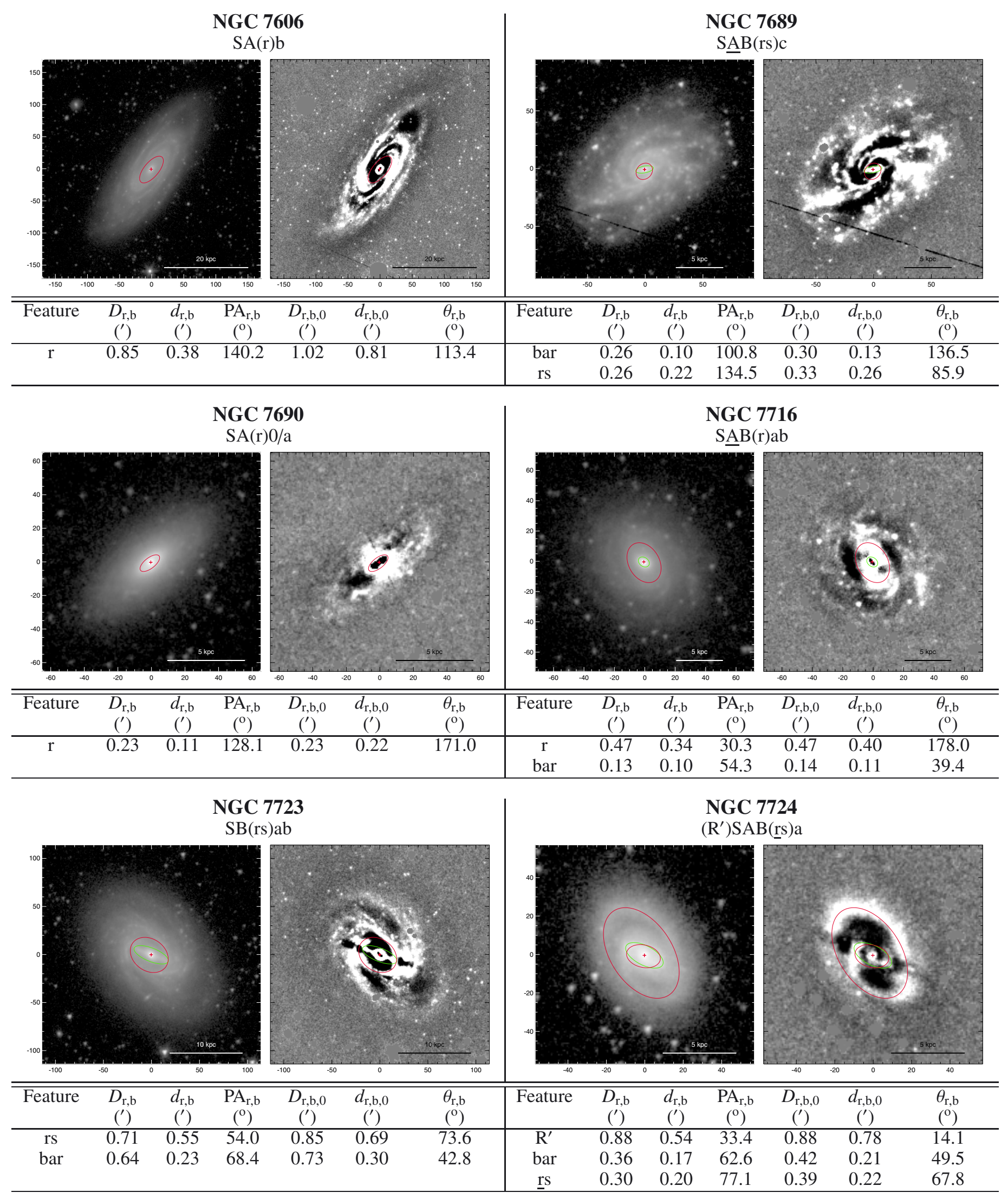




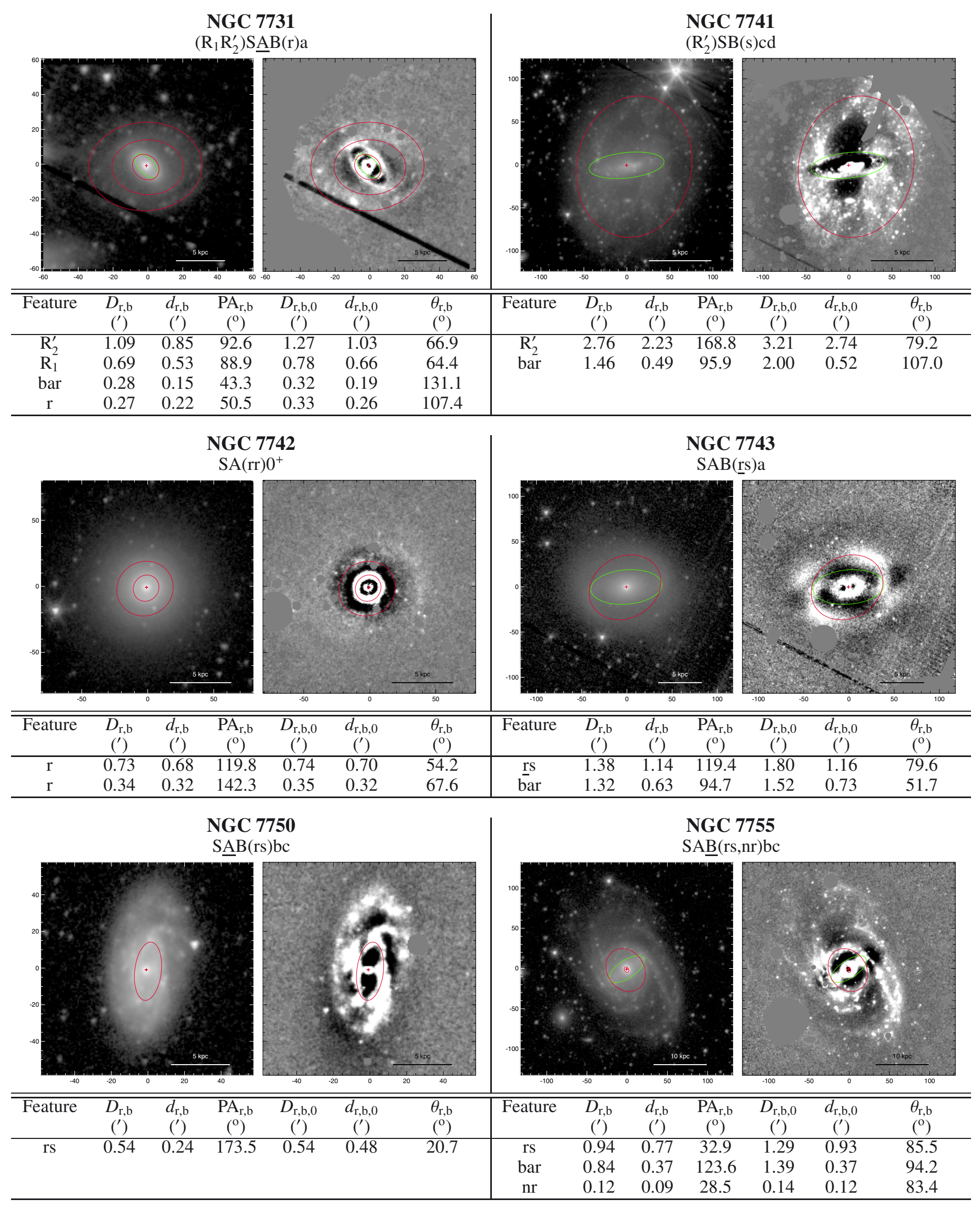


NGC 7817

$\mathrm{SAB}(\mathrm{rs}, \mathrm{nd}) \mathrm{bc}$
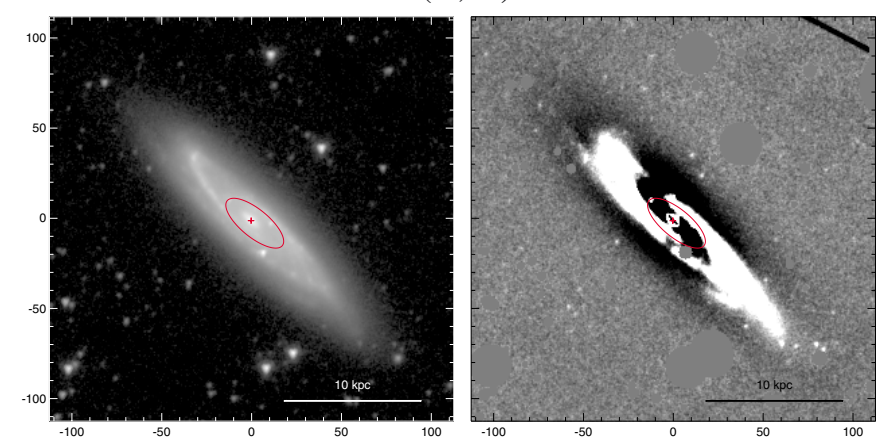

PGC 2492

$\mathrm{SAB}(\underline{\mathrm{rs}}) \mathrm{a} / \mathrm{d}$

\begin{tabular}{ccccccc|ccccccc}
\hline \hline Feature & $\begin{array}{c}D_{\mathrm{r}, \mathrm{b}} \\
\left({ }^{\prime}\right)\end{array}$ & $\begin{array}{c}d_{\mathrm{r}, \mathrm{b}} \\
\left({ }^{\prime}\right)\end{array}$ & $\begin{array}{c}\mathrm{PA}_{\mathrm{r}, \mathrm{b}} \\
\left({ }^{\circ}\right)\end{array}$ & $\begin{array}{c}D_{\mathrm{r}, \mathrm{b}, 0} \\
\left({ }^{\prime}\right)\end{array}$ & $\begin{array}{c}d_{\mathrm{r}, \mathrm{b}, 0} \\
\left({ }^{\prime}\right)\end{array}$ & $\begin{array}{c}\theta_{\mathrm{r}, \mathrm{b}} \\
\left({ }^{\circ}\right)\end{array}$ & Feature & $\begin{array}{c}D_{\mathrm{r}, \mathrm{b}} \\
\left({ }^{\prime}\right)\end{array}$ & $\begin{array}{c}d_{\mathrm{r}, \mathrm{b}} \\
\left({ }^{\prime}\right)\end{array}$ & $\begin{array}{c}\mathrm{PA}_{\mathrm{r}, \mathrm{b}} \\
\left({ }^{\circ}\right)\end{array}$ & $\begin{array}{c}D_{\mathrm{r}, \mathrm{b}, 0} \\
\left({ }^{\prime}\right)\end{array}$ & $\begin{array}{c}d_{\mathrm{r}, \mathrm{b}, 0} \\
\left({ }^{\prime}\right)\end{array}$ & $\begin{array}{c}\theta_{\mathrm{r}, \mathrm{b}} \\
\left({ }^{\circ}\right)\end{array}$ \\
\hline $\mathrm{rs}$ & 0.66 & 0.26 & 50.8 & 0.86 & 0.63 & 71.7 & $\underline{\mathrm{rs}}$ & 0.75 & 0.67 & 168.7 & 0.97 & 0.75 & 88.4 \\
& & & & & & & bar & 0.37 & 0.17 & 171.5 & 0.38 & 0.24 & 13.4 \\
\hline
\end{tabular}

PGC 3853

$\left(\mathrm{R}^{\prime}\right) \mathrm{SB}(\mathrm{rs}) \mathrm{cd}$
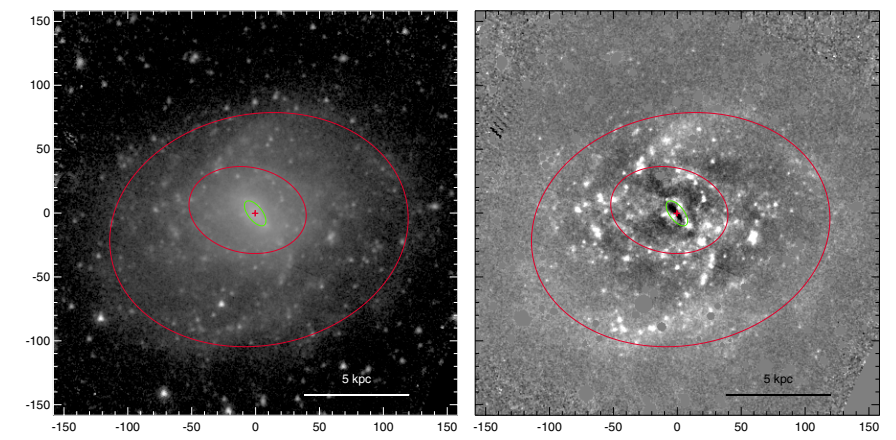

\section{PGC 6228 \\ $\mathrm{SB}(\mathrm{rs}) \mathrm{dm}$}
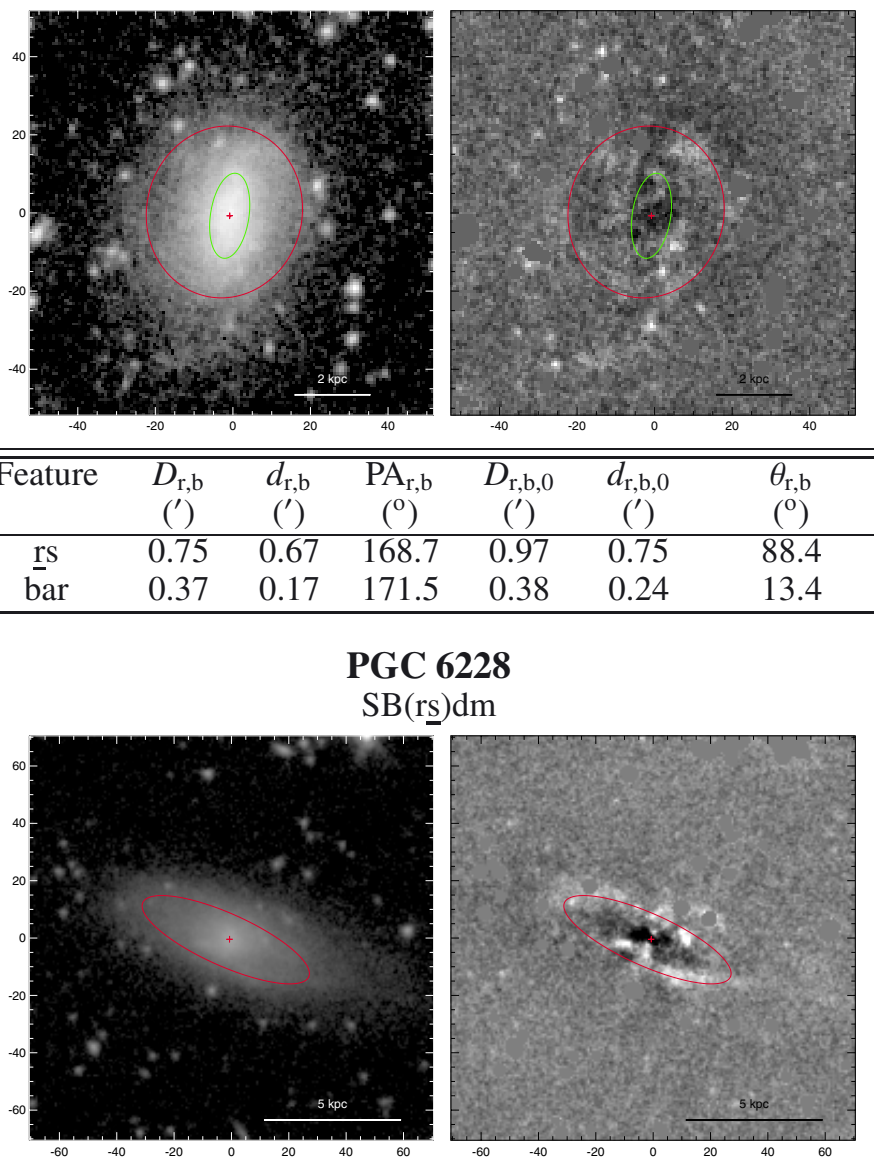

\begin{tabular}{ccccccc|ccccccc}
\hline \hline Feature & $\begin{array}{c}D_{\mathrm{r}, \mathrm{b}} \\
\left({ }^{\prime}\right)\end{array}$ & $\begin{array}{c}d_{\mathrm{r}, \mathrm{b}} \\
\left({ }^{\prime}\right)\end{array}$ & $\begin{array}{c}\mathrm{PA}_{\mathrm{r}, \mathrm{b}} \\
\left({ }^{\circ}\right)\end{array}$ & $\begin{array}{c}D_{\mathrm{r}, \mathrm{b}, 0} \\
\left({ }^{\prime}\right)\end{array}$ & $\begin{array}{c}d_{\mathrm{r}, \mathrm{b}, 0} \\
\left({ }^{\prime}\right)\end{array}$ & $\begin{array}{c}\theta_{\mathrm{r}, \mathrm{b}} \\
\left({ }^{\circ}\right)\end{array}$ & Feature & $\begin{array}{c}D_{\mathrm{r}, \mathrm{b}} \\
\left({ }^{\prime}\right)\end{array}$ & $\begin{array}{c}d_{\mathrm{r}, \mathrm{b}} \\
\left({ }^{\prime}\right)\end{array}$ & $\begin{array}{c}\mathrm{PA}_{\mathrm{r}, \mathrm{b}} \\
\left({ }^{\circ}\right)\end{array}$ & $\begin{array}{c}D_{\mathrm{r}, \mathrm{b}, 0} \\
\left({ }^{\prime}\right)\end{array}$ & $\begin{array}{c}d_{\mathrm{r}, \mathrm{b}, 0} \\
\left({ }^{\prime}\right)\end{array}$ & $\begin{array}{c}\theta_{\mathrm{r}, \mathrm{b}} \\
\left({ }^{\circ}\right)\end{array}$ \\
\hline $\mathrm{R}^{\prime}$ & 3.93 & 3.03 & 100.8 & 4.12 & 3.87 & 117.8 & $\underline{\mathrm{rs}}$ & 1.06 & 0.31 & 65.9 & 1.14 & 0.75 & 149.4 \\
$\mathrm{rs}$ & 1.55 & 1.12 & 78.9 & 1.76 & 1.32 & 123.5 & & & & & & & \\
$\underline{\mathrm{bar}}$ & 0.39 & 0.19 & 38.1 & 0.51 & 0.19 & 104.6 & & & & & & \\
\hline
\end{tabular}

PGC 6626

$\left(\mathrm{R}^{\prime}\right) \mathrm{SB}(\mathrm{rs}) \mathrm{cd}$

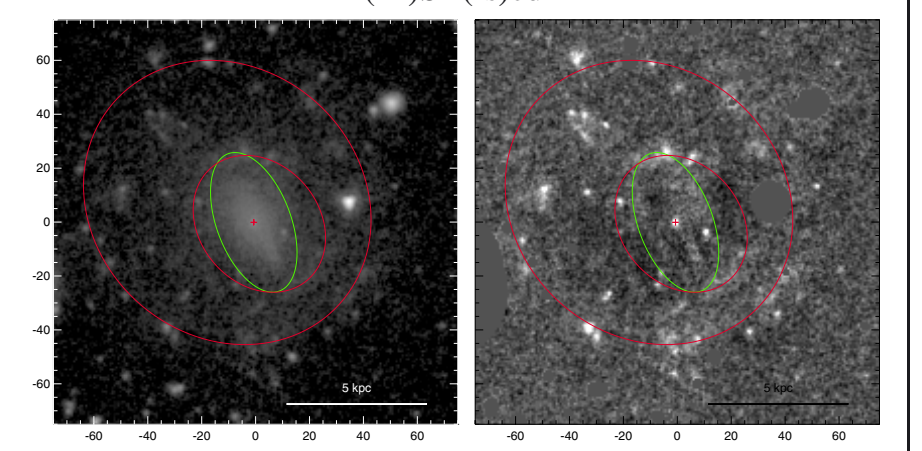

PGC 11248

$\mathrm{SB}(\mathrm{rs}) \mathrm{d}$

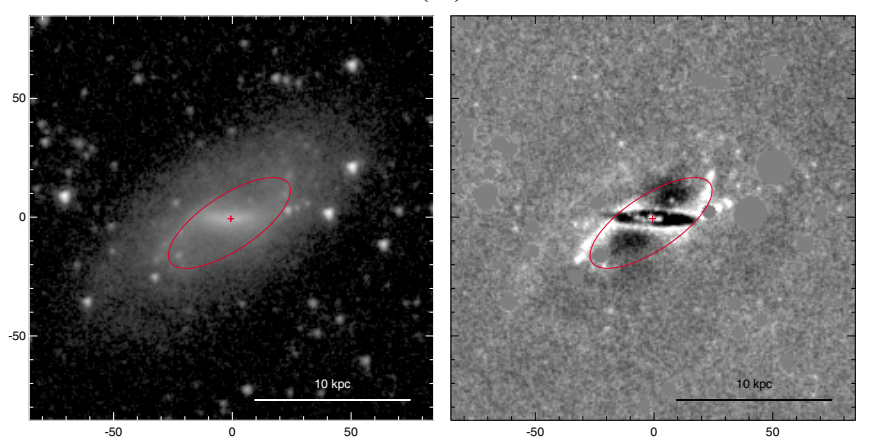

\begin{tabular}{ccccccc|ccccccc}
\hline \hline Feature & $\begin{array}{c}D_{\mathrm{r}, \mathrm{b}} \\
\left({ }^{\prime}\right)\end{array}$ & $\begin{array}{c}d_{\mathrm{r}, \mathrm{b}}\left({ }^{\prime}\right) \\
\mathrm{P}^{\prime}\end{array}$ & $\begin{array}{c}\mathrm{PA}_{\mathrm{r}, \mathrm{b}} \\
\left({ }^{\circ}\right)\end{array}$ & $\begin{array}{c}D_{\mathrm{r}, \mathrm{b}, 0} \\
\left({ }^{\prime}\right)\end{array}$ & $\begin{array}{c}d_{\mathrm{r}, \mathrm{b}, 0} \\
\left({ }^{\prime}\right)\end{array}$ & $\begin{array}{c}\theta_{\mathrm{r}, \mathrm{b}} \\
\left({ }^{\circ}\right)\end{array}$ & Feature & $\begin{array}{c}D_{\mathrm{r}, \mathrm{b}} \\
(,)\end{array}$ & $\begin{array}{c}d_{\mathrm{r}, \mathrm{b}} \\
\left({ }^{\prime}\right)\end{array}$ & $\begin{array}{c}\mathrm{PA}_{\mathrm{r}, \mathrm{b}} \\
\left({ }^{\circ}\right)\end{array}$ & $\begin{array}{c}D_{\mathrm{r}, \mathrm{b}, 0} \\
\left({ }^{\prime}\right)\end{array}$ & $\begin{array}{c}d_{\mathrm{r}, \mathrm{b}, 0} \\
\left({ }^{\prime}\right)\end{array}$ & $\begin{array}{c}\theta_{\mathrm{r}, \mathrm{b}} \\
\left({ }^{\circ}\right)\end{array}$ \\
\hline $\mathrm{R}^{\prime}$ & 1.88 & 1.67 & 47.7 & 2.02 & 1.72 & 115.0 & $\mathrm{rs}$ & 1.00 & 0.39 & 123.6 & 1.00 & 0.87 & 174.5 \\
$\mathrm{rs}$ & 0.92 & 0.75 & 40.4 & 0.99 & 0.77 & 116.4 & & & & & & & \\
$\mathrm{bar}$ & 0.91 & 0.46 & 20.6 & 1.00 & 0.47 & 105.9 & & & & & & & \\
\end{tabular}


PGC 12633

$\mathrm{SAB}(\mathrm{rs}) \mathrm{ab}$
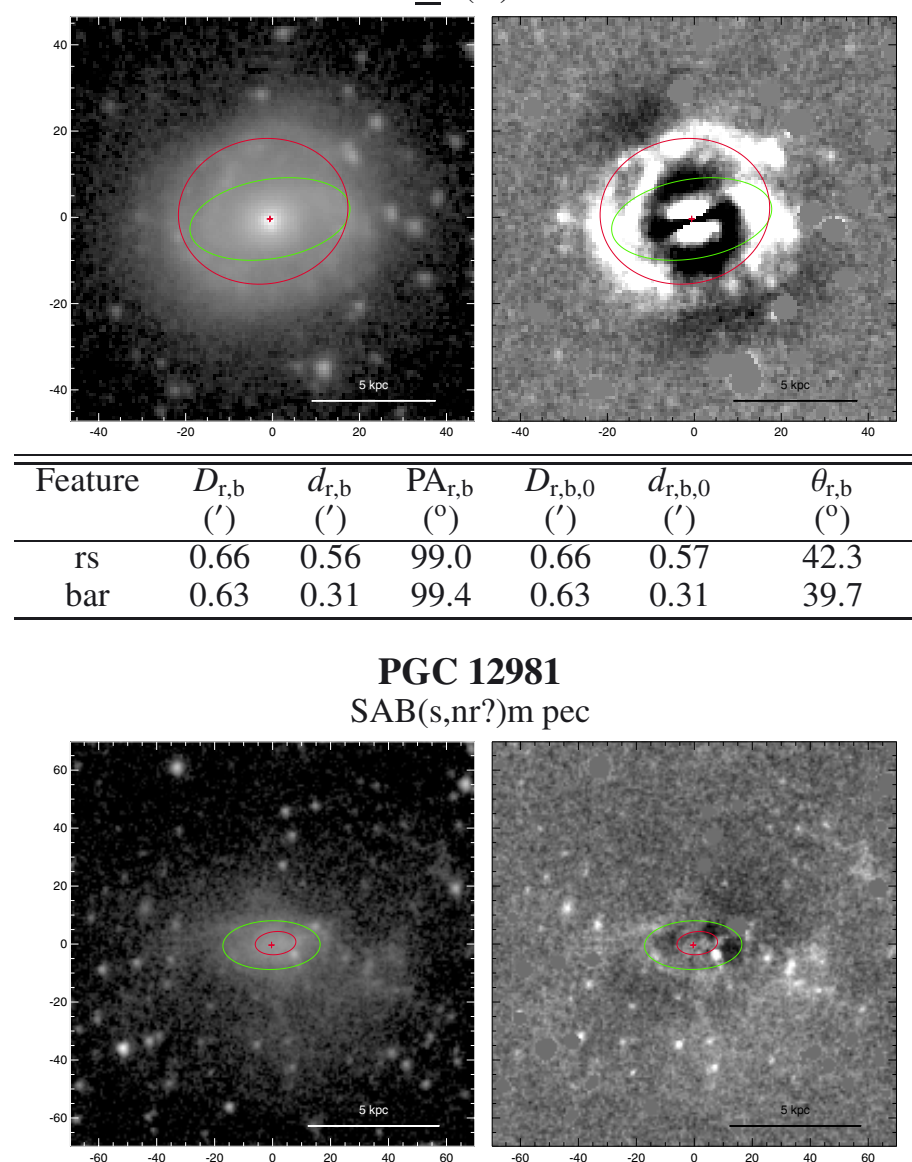

$\begin{array}{ccc}\left({ }^{\prime}\right) & \left({ }^{\prime}\right) & \left(^{\circ}\right) \\ 0.66 & 0.57 & 42.3 \\ 0.63 & 0.31 & 39.7\end{array}$

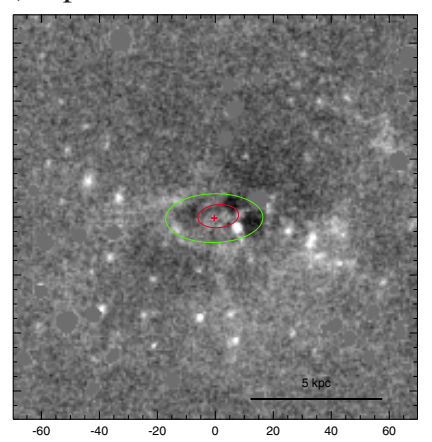

PGC 12664

$\mathrm{SA} \underline{\mathrm{B}}(\mathrm{rs}) \mathrm{cd}$
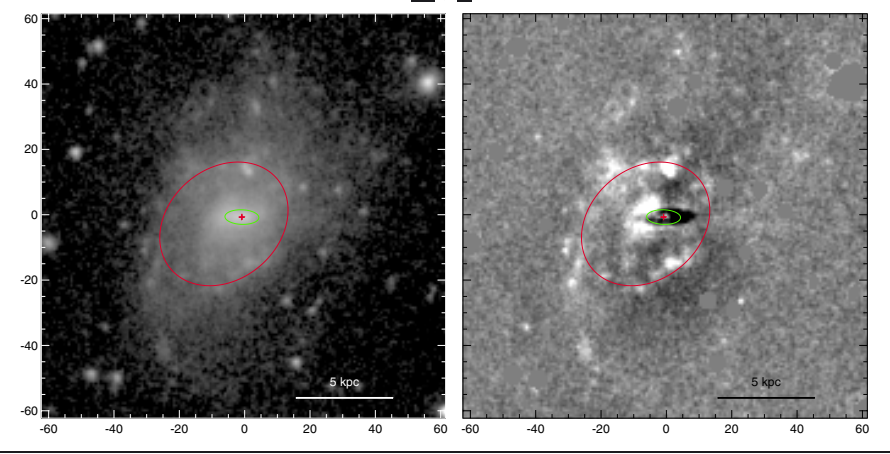

\begin{tabular}{|cc} 
Feature & \\
b $\underline{\mathrm{s}}$ & 0
\end{tabular}

$\begin{array}{lll}\left({ }^{\prime}\right) & \left({ }^{\prime}\right) & \left({ }^{\circ}\right)\end{array}$

$\begin{array}{lll}0.72 & 0.57 & 130.3\end{array}$

bar

0.17

\begin{tabular}{ll}
$0.07 \quad 88.1$ \\
\hline
\end{tabular}

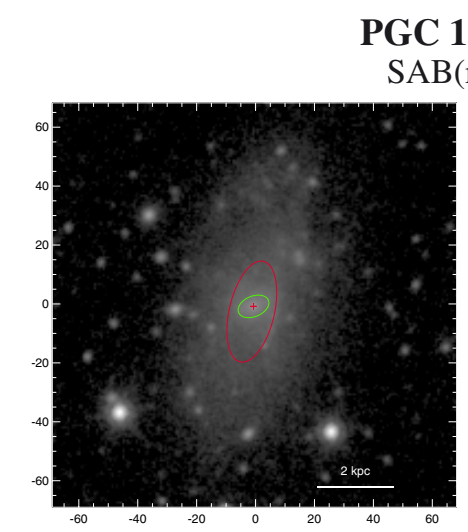

PGC 13684
SAB $(\mathrm{rs}) \mathrm{c}$

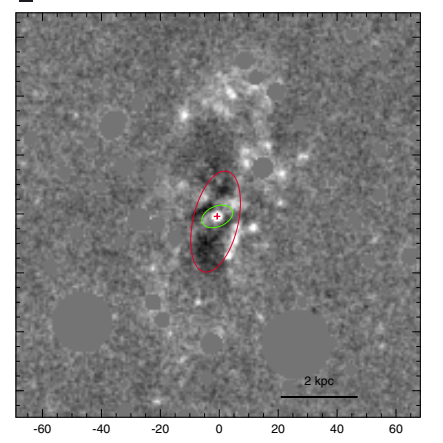

\begin{tabular}{ccccccc|ccccccc}
\hline \hline Feature & $\begin{array}{c}D_{\mathrm{r}, \mathrm{b}} \\
\left({ }^{\prime}\right)\end{array}$ & $\begin{array}{c}d_{\mathrm{r}, \mathrm{b}}\left({ }^{\prime}\right) \\
\left(\mathrm{PA}_{\mathrm{r}, \mathrm{b}}\right. \\
\left({ }^{\circ}\right.\end{array}$ & $\begin{array}{c}D_{\mathrm{r}, \mathrm{b}, 0} \\
\left({ }^{\prime}\right)\end{array}$ & $\begin{array}{c}d_{\mathrm{r}, \mathrm{b}, 0} \\
\left({ }^{\prime}\right)\end{array}$ & $\begin{array}{c}\theta_{\mathrm{r}, \mathrm{b}} \\
\left({ }^{(}\right)\end{array}$ & Feature & $\begin{array}{c}D_{\mathrm{r}, \mathrm{b}} \\
\left({ }^{\prime}\right)\end{array}$ & $\begin{array}{c}d_{\mathrm{r}, \mathrm{b}} \\
\left({ }^{\prime}\right)\end{array}$ & $\begin{array}{c}\mathrm{PA}_{\mathrm{r}, \mathrm{b}} \\
\left({ }^{\circ}\right)\end{array}$ & $\begin{array}{c}D_{\mathrm{r}, \mathrm{b}, 0} \\
\left({ }^{\prime}\right)\end{array}$ & $\begin{array}{c}d_{\mathrm{r}, \mathrm{b}, 0} \\
\left({ }^{\prime}\right)\end{array}$ & $\begin{array}{c}\theta_{\mathrm{r}, \mathrm{b}} \\
\left({ }^{\circ}\right)\end{array}$ \\
\hline $\mathrm{bar}$ & 0.56 & 0.28 & 91.2 & 0.57 & 0.36 & 166.4 & $\mathrm{rs}$ & 0.59 & 0.26 & 167.0 & 0.59 & 0.48 & 2.2 \\
$\mathrm{nr}$ & 0.24 & 0.13 & 95.3 & 0.24 & 0.17 & 172.0 & $\mathrm{bar}$ & 0.18 & 0.12 & 112.7 & 0.31 & 0.13 & 103.0 \\
\hline
\end{tabular}
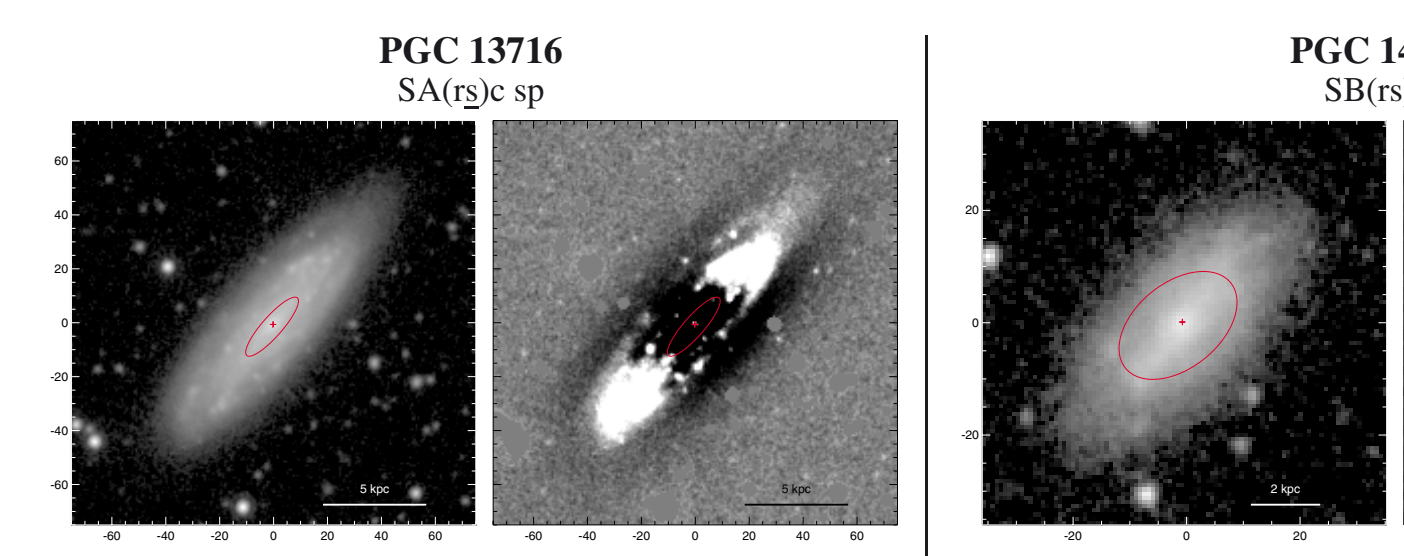

GC 14037

SB(rs)c:

\begin{tabular}{ccccccc|ccccccc}
\hline \hline Feature & $\begin{array}{c}D_{\mathrm{r}, \mathrm{b}} \\
\left({ }^{\prime}\right)\end{array}$ & $\begin{array}{c}d_{\mathrm{r}, \mathrm{b}}\left({ }^{\prime}\right) \\
\left(\mathrm{PA}_{\mathrm{r}, \mathrm{b}}\right.\end{array}$ & $\begin{array}{c}D_{\mathrm{r}, \mathrm{b}, 0} \\
\left({ }^{\prime}\right)\end{array}$ & $\begin{array}{c}d_{\mathrm{r}, \mathrm{b}, 0} \\
\left({ }^{\prime}\right)\end{array}$ & $\begin{array}{c}\theta_{\mathrm{r}, \mathrm{b}} \\
\left({ }^{\circ}\right)\end{array}$ & Feature & $\begin{array}{c}D_{\mathrm{r}, \mathrm{b}} \\
\left({ }^{\prime}\right)\end{array}$ & $\begin{array}{c}d_{\mathrm{r}, \mathrm{b}} \\
\left({ }^{\prime}\right)\end{array}$ & $\begin{array}{c}\mathrm{PA}_{\mathrm{r}, \mathrm{b}} \\
\left({ }^{\circ}\right)\end{array}$ & $\begin{array}{c}D_{\mathrm{r}, \mathrm{b}, 0} \\
\left({ }^{\prime}\right)\end{array}$ & $\begin{array}{c}d_{\mathrm{r}, \mathrm{b}, 0} \\
\left({ }^{\prime}\right)\end{array}$ & $\begin{array}{c}\theta_{\mathrm{r}, \mathrm{b}} \\
\left({ }^{\circ}\right)\end{array}$ \\
\hline $\mathrm{rs}$ & 0.48 & 0.12 & 138.7 & 0.48 & 0.29 & 4.9 & rs & 0.40 & 0.25 & 129.1 & 0.56 & 0.39 & 102.8 \\
\hline
\end{tabular}




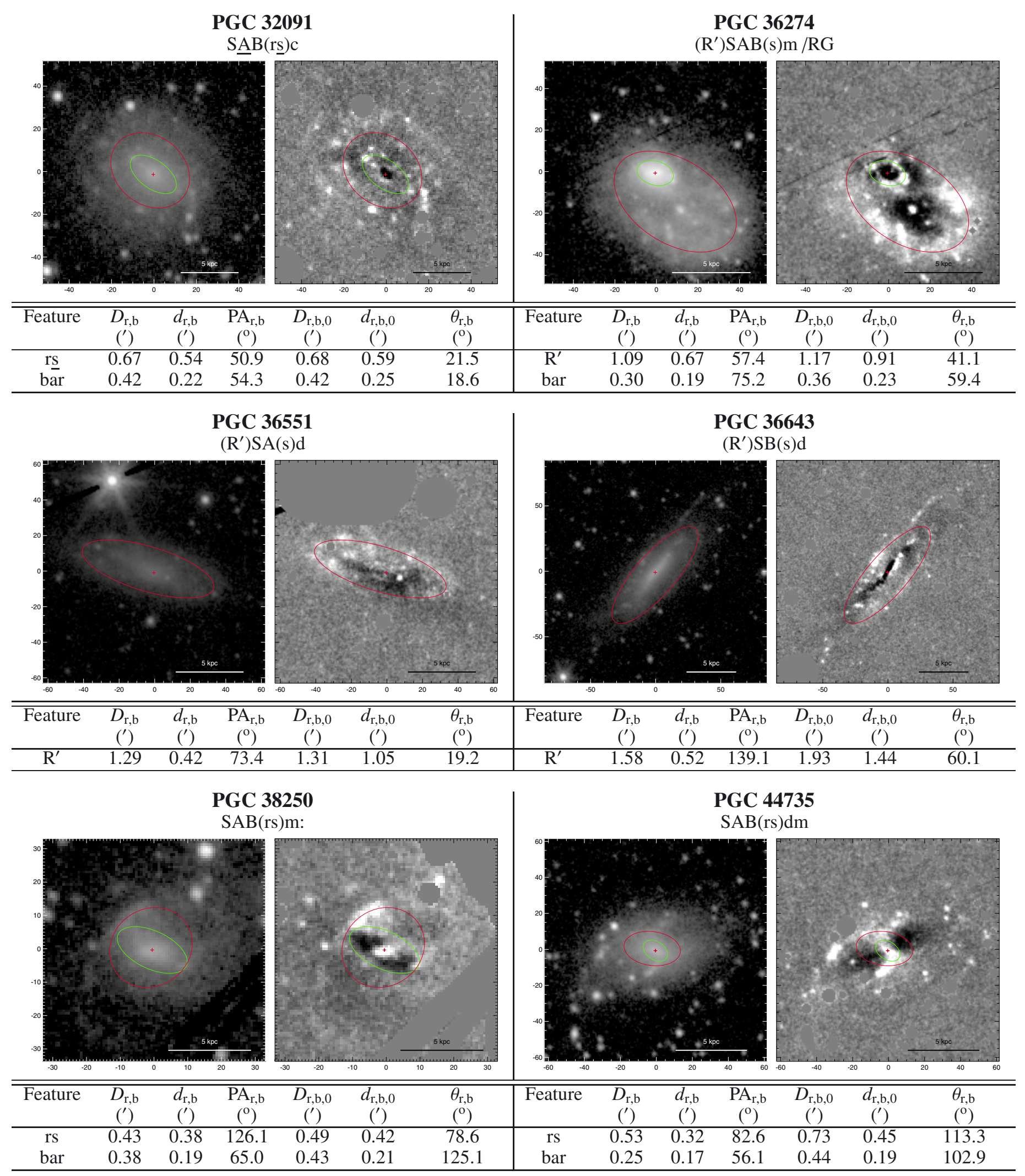




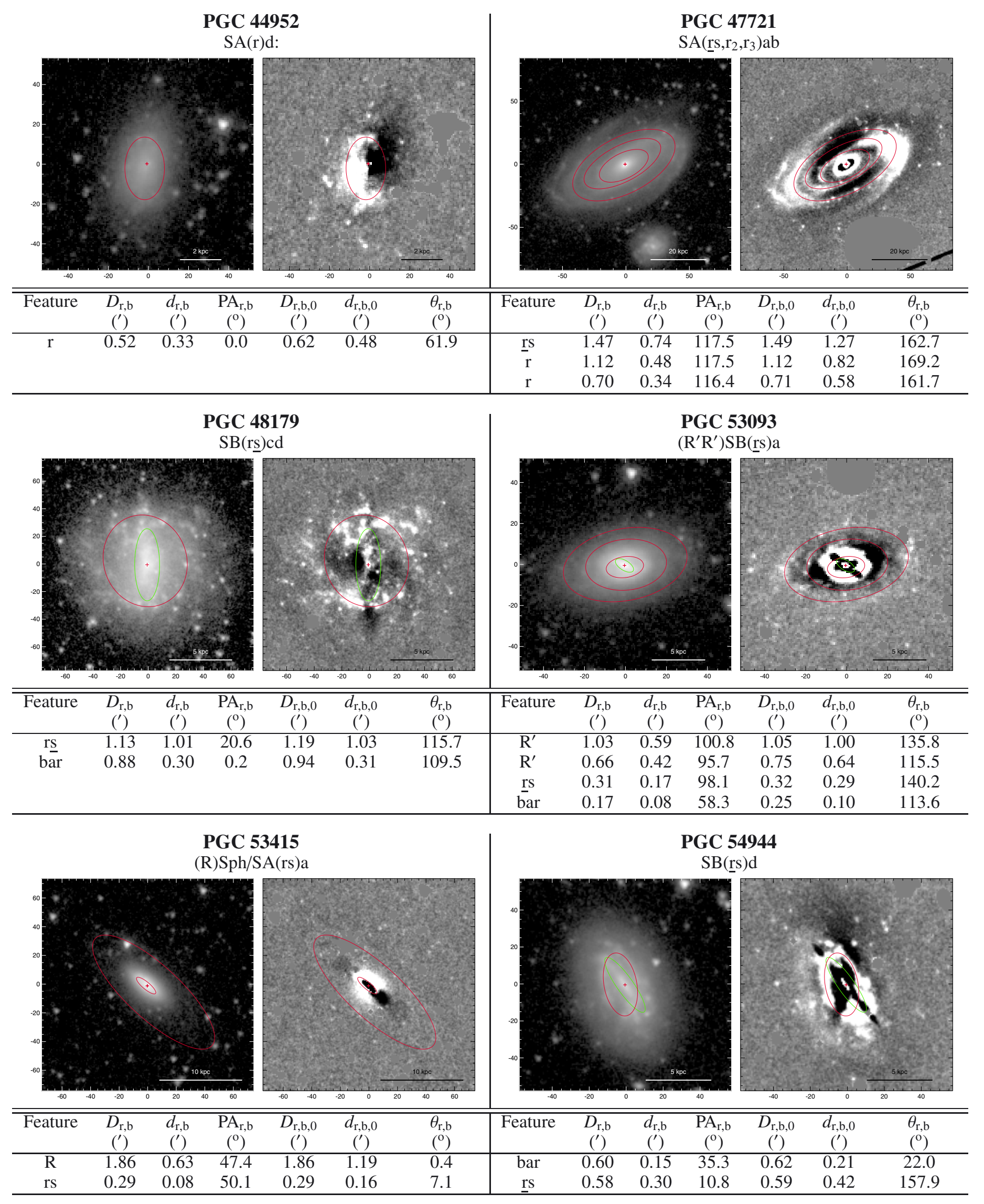




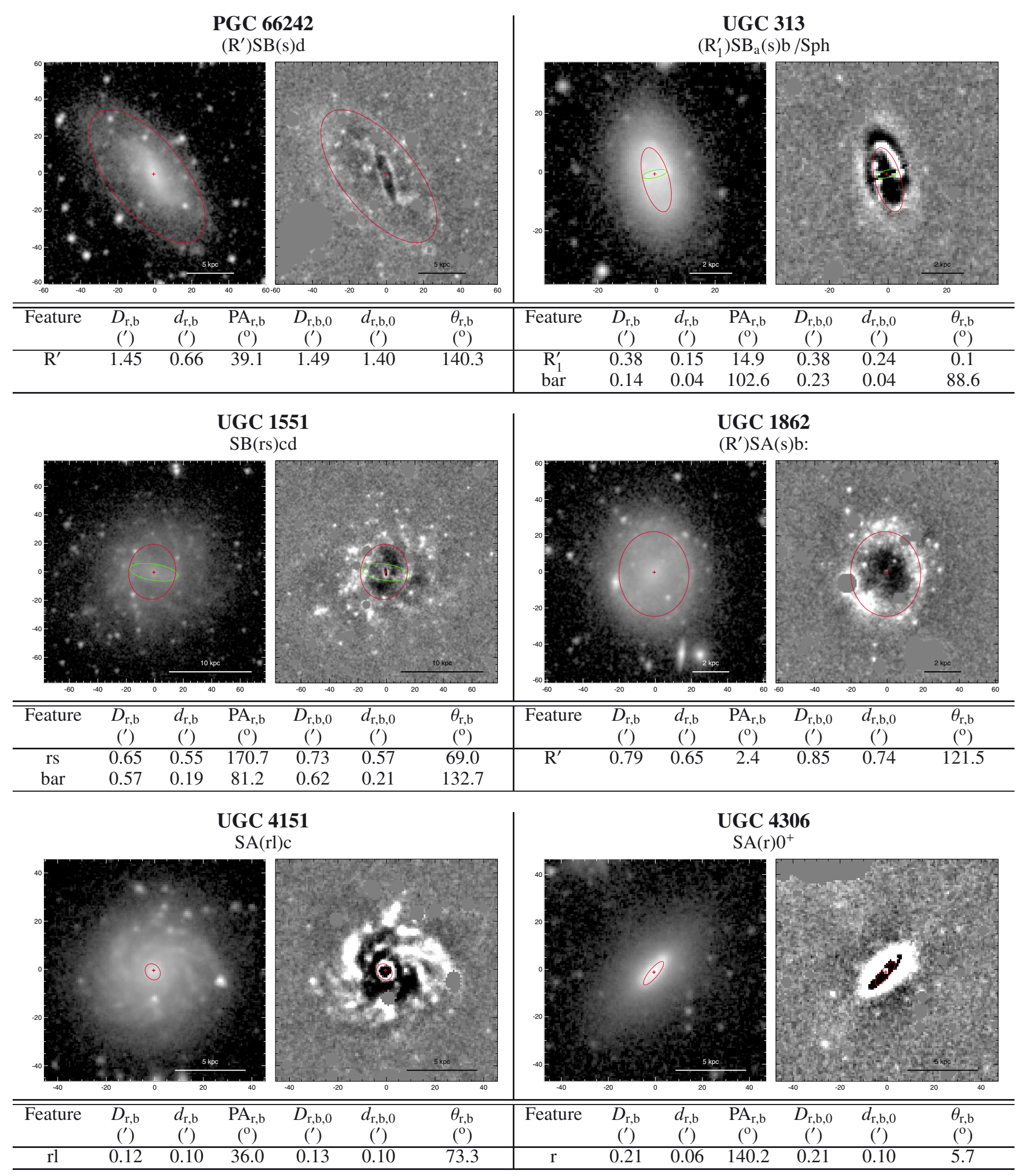




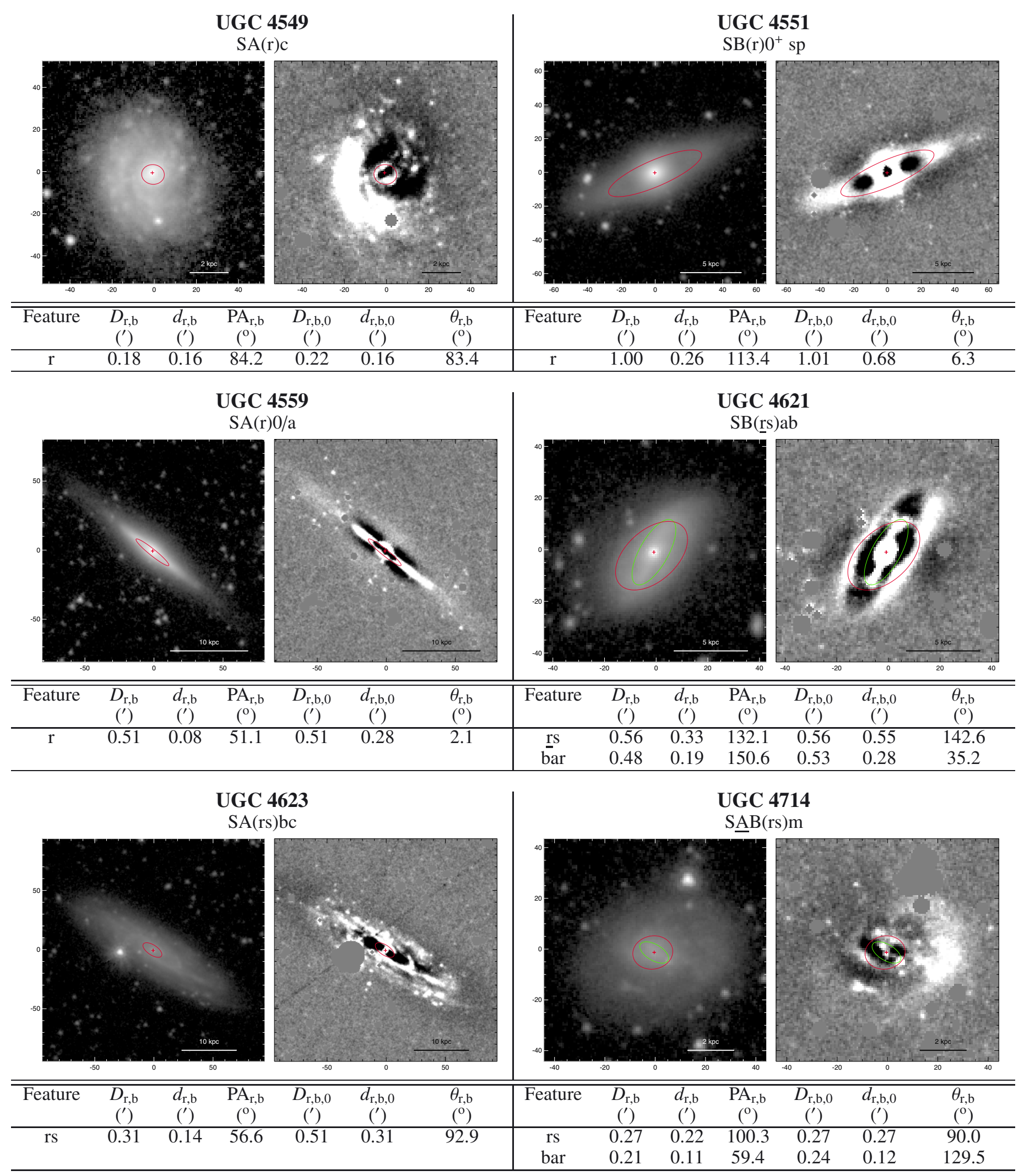




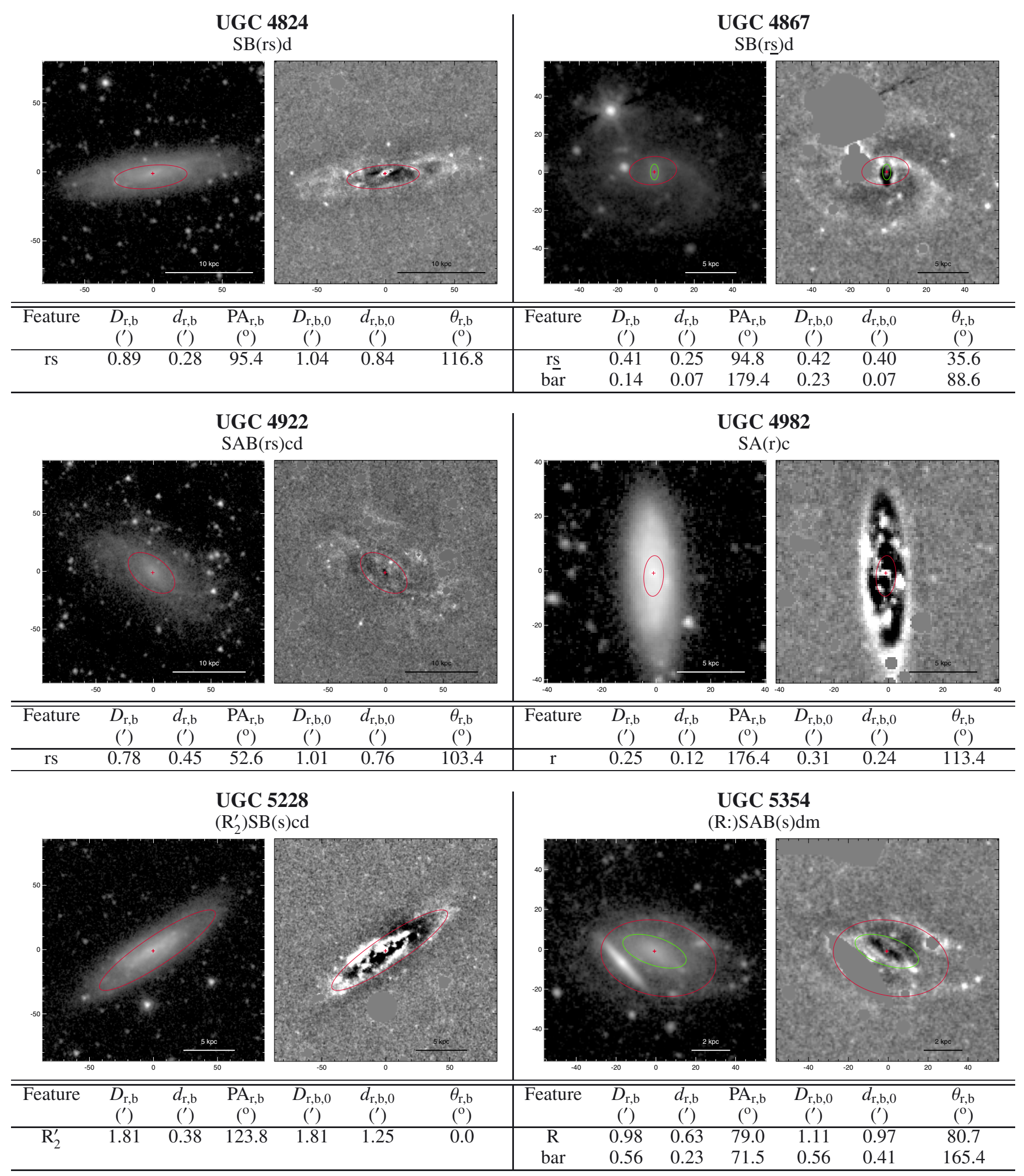




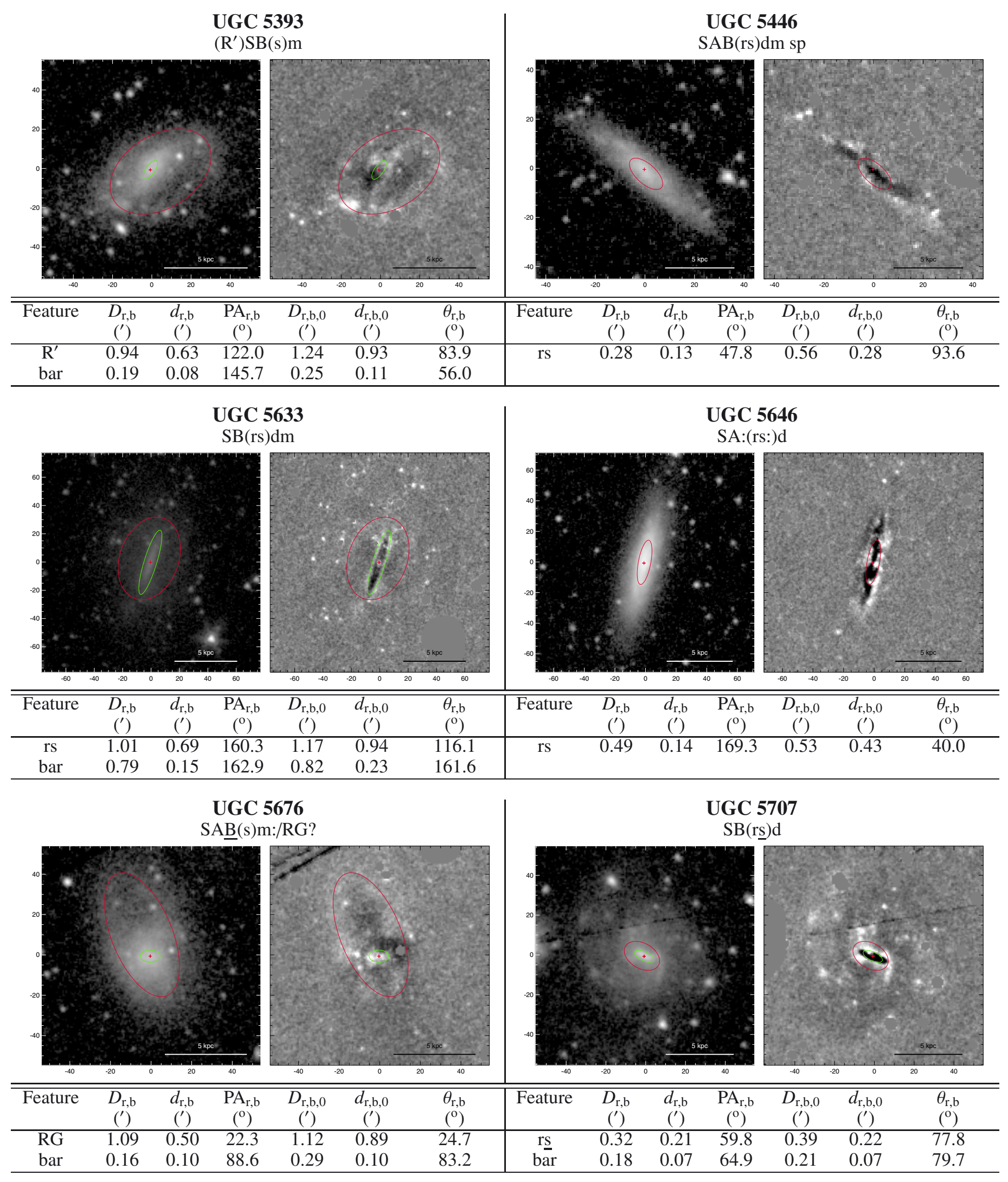


UGC 5814

(RL)SB0/a (shells?) pec
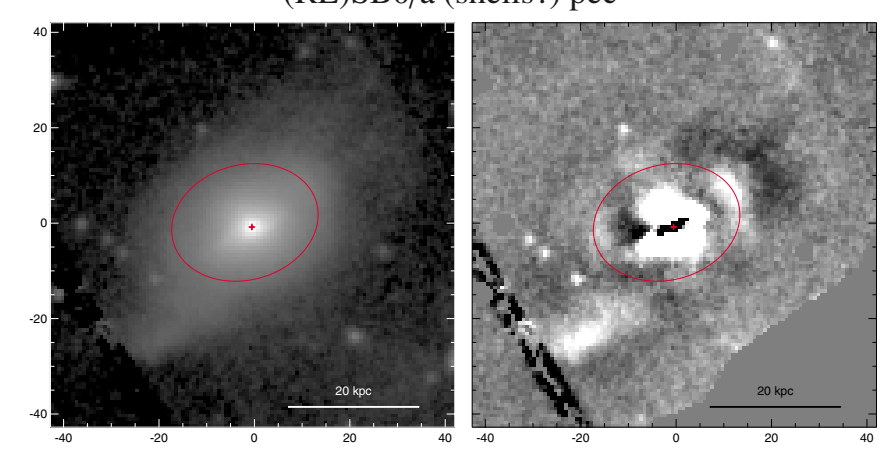

UGC 5841

$\left(\mathrm{R}^{\prime}\right) \mathrm{SB}(\mathrm{rs}:) \mathrm{b}$

\begin{tabular}{ccccccc|ccccccc}
\hline \hline Feature & $\begin{array}{c}D_{\mathrm{r}, \mathrm{b}} \\
\left({ }^{\prime}\right)\end{array}$ & $\begin{array}{c}d_{\mathrm{r}, \mathrm{b}} \\
\left({ }^{\prime}\right)\end{array}$ & $\begin{array}{c}\mathrm{PA}_{\mathrm{r}, \mathrm{b}} \\
(\mathrm{o})\end{array}$ & $\begin{array}{c}D_{\mathrm{r}, \mathrm{b}, 0} \\
\left({ }^{\prime}\right)\end{array}$ & $\begin{array}{c}d_{\mathrm{r}, \mathrm{b}, 0} \\
\left({ }^{\prime}\right)\end{array}$ & $\begin{array}{c}\theta_{\mathrm{r}, \mathrm{b}} \\
\left({ }^{\circ}\right)\end{array}$ & Feature & $\begin{array}{c}D_{\mathrm{r}, \mathrm{b}} \\
\left({ }^{\prime}\right)\end{array}$ & $\begin{array}{c}d_{\mathrm{r}, \mathrm{b}} \\
\left({ }^{\prime}\right)\end{array}$ & $\begin{array}{c}\mathrm{PA}_{\mathrm{r}, \mathrm{b}} \\
\left({ }^{\circ}\right)\end{array}$ & $\begin{array}{c}D_{\mathrm{r}, \mathrm{b}, 0} \\
\left({ }^{\prime}\right)\end{array}$ & $\begin{array}{c}d_{\mathrm{r}, \mathrm{b}, 0} \\
\left({ }^{\prime}\right)\end{array}$ & $\begin{array}{c}\theta_{\mathrm{r}, \mathrm{b}} \\
\left({ }^{\circ}\right)\end{array}$ \\
\hline $\mathrm{RL}$ & 0.52 & 0.41 & 105.2 & 0.74 & 0.48 & 104.1 & $\mathrm{R}^{\prime}$ & 0.76 & 0.45 & 127.0 & 0.82 & 0.74 & 116.8 \\
& & & & & & & $\mathrm{bar}$ & 0.43 & 0.14 & 136.7 & 0.43 & 0.25 & 11.6 \\
& & & & & & $\mathrm{rs}$ & 0.38 & 0.21 & 136.1 & 0.40 & 0.35 & 33.1 \\
\hline
\end{tabular}

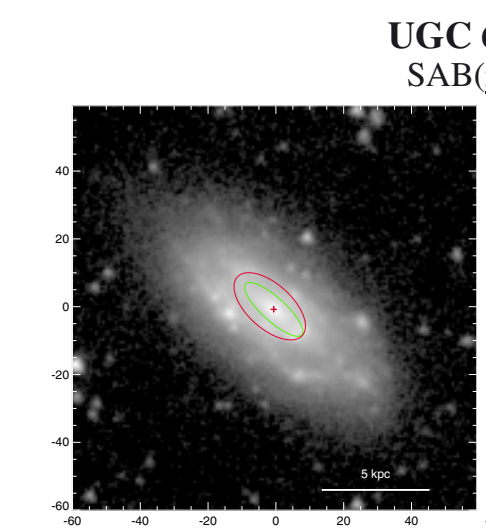

\section{3}

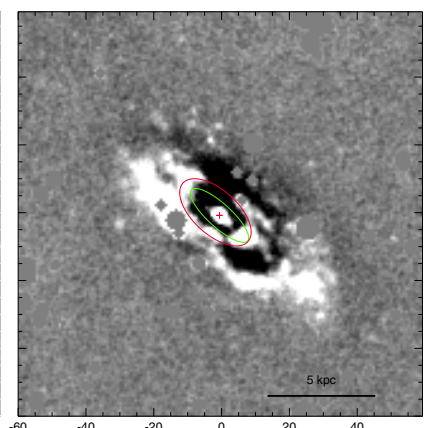

UGC 6162

$\left(\mathrm{R}^{\prime}\right) \mathrm{SA}(\mathrm{s}) \mathrm{d}$

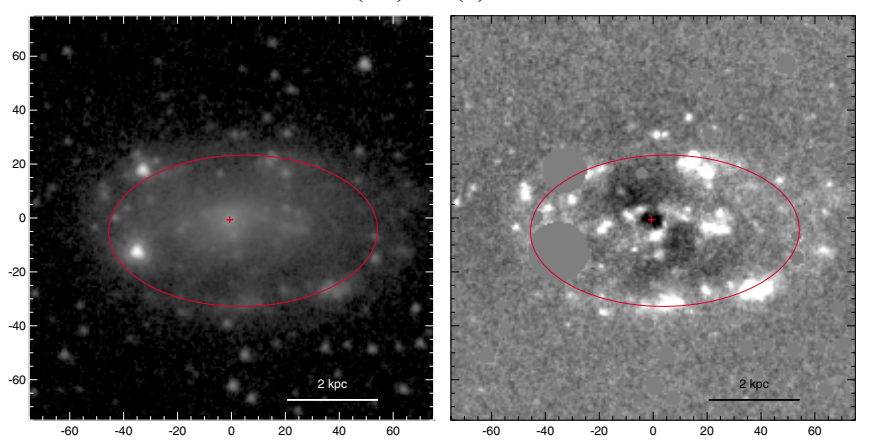

\begin{tabular}{ccccccc|ccccccc}
\hline \hline Feature & $\begin{array}{c}D_{\mathrm{r}, \mathrm{b}} \\
\left({ }^{\prime}\right)\end{array}$ & $\begin{array}{c}d_{\mathrm{r}, \mathrm{b}} \\
\left({ }^{\prime}\right)\end{array}$ & $\begin{array}{c}\mathrm{PA}_{\mathrm{r}, \mathrm{b}} \\
\left({ }^{\circ}\right)\end{array}$ & $\begin{array}{c}D_{\mathrm{r}, \mathrm{b}, 0} \\
\left({ }^{\prime}\right)\end{array}$ & $\begin{array}{c}d_{\mathrm{r}, \mathrm{b}, 0} \\
\left({ }^{\prime}\right)\end{array}$ & $\begin{array}{c}\theta_{\mathrm{r}, \mathrm{b}} \\
\left({ }^{\circ}\right)\end{array}$ & Feature & $\begin{array}{c}D_{\mathrm{r}, \mathrm{b}} \\
\left({ }^{\prime}\right)\end{array}$ & $\begin{array}{c}d_{\mathrm{r}, \mathrm{b}} \\
\left({ }^{\prime}\right)\end{array}$ & $\begin{array}{c}\mathrm{PA}_{\mathrm{r}, \mathrm{b}} \\
\left({ }^{\circ}\right)\end{array}$ & $\begin{array}{c}D_{\mathrm{r}, \mathrm{b}, 0} \\
\left({ }^{\prime}\right)\end{array}$ & $\begin{array}{c}d_{\mathrm{r}, \mathrm{b}, 0} \\
\left({ }^{\prime}\right)\end{array}$ & $\begin{array}{c}\theta_{\mathrm{r}, \mathrm{b}} \\
\left({ }^{\circ}\right)\end{array}$ \\
\hline$\underline{\mathrm{rs}}$ & 0.43 & 0.22 & 48.0 & 0.45 & 0.42 & 129.8 & $\mathrm{R}^{\prime}$ & 1.67 & 0.94 & 90.6 & 1.74 & 1.54 & 38.9 \\
$\underline{\mathrm{bar}}$ & 0.38 & 0.12 & 47.7 & 0.38 & 0.23 & 173.5 & & & & & & & \\
\hline
\end{tabular}

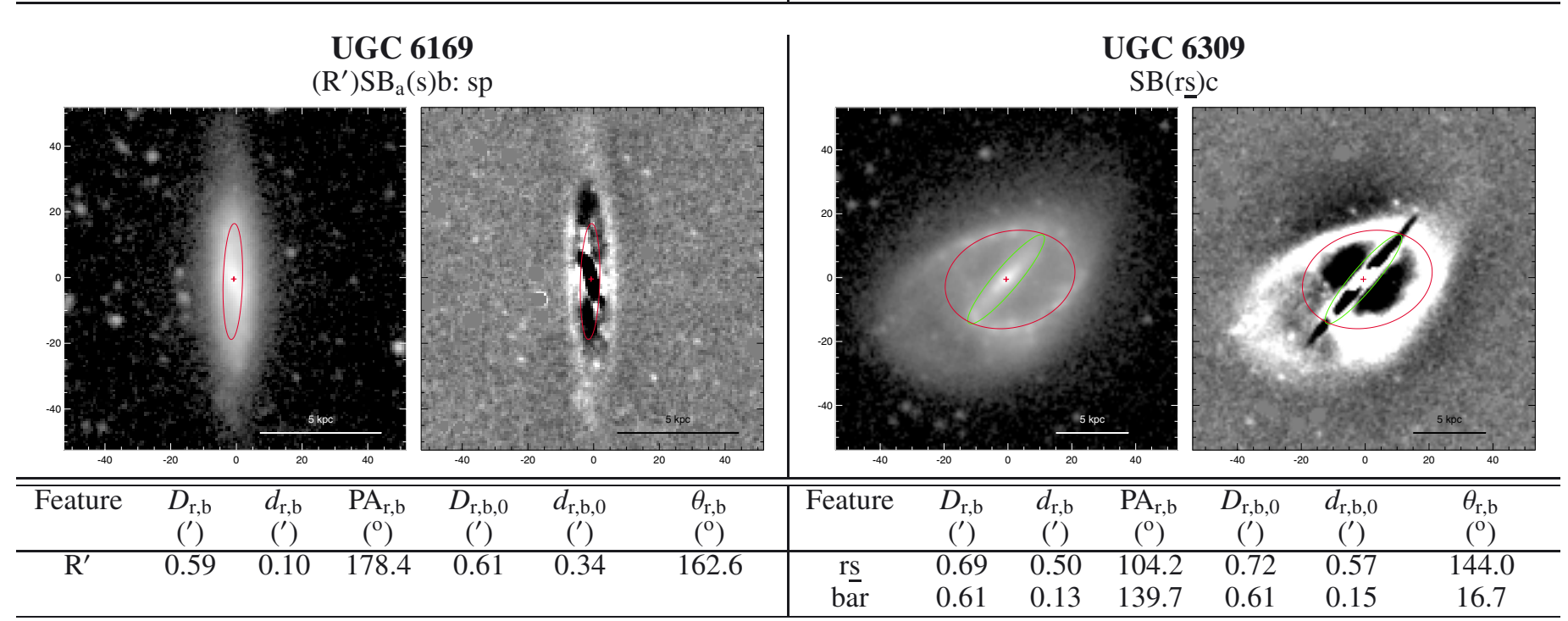


UGC 6335

$\mathrm{SAB}(\mathrm{rs}) \mathrm{c}$
UGC 6517

$\mathrm{SA}(\mathrm{r}) \mathrm{b}$
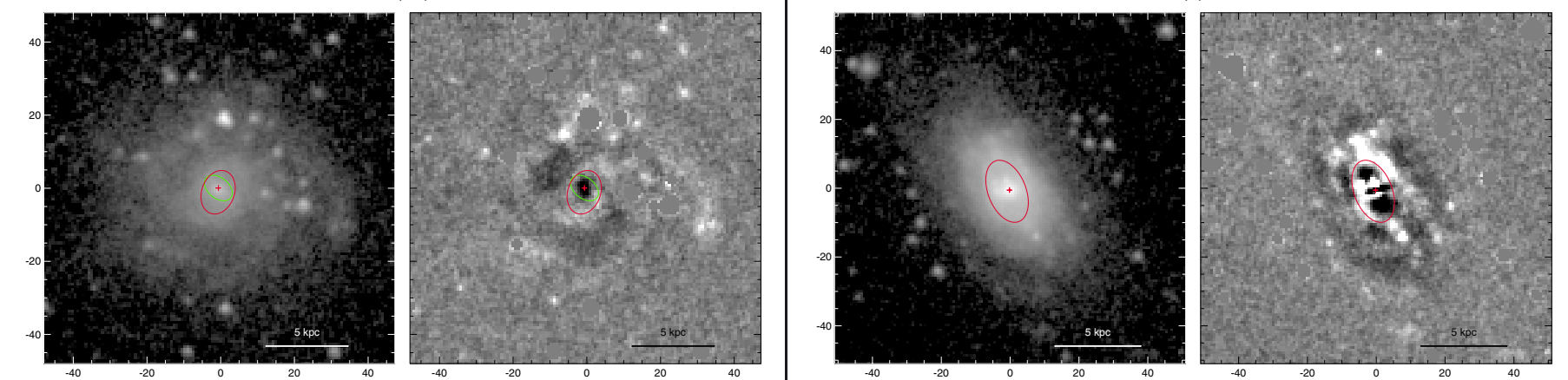

\begin{tabular}{ccccccc|ccccccc}
\hline \hline Feature & $\begin{array}{c}D_{\mathrm{r}, \mathrm{b}} \\
\left({ }^{\prime}\right)\end{array}$ & $\begin{array}{c}d_{\mathrm{r}, \mathrm{b}} \\
\left({ }^{\prime}\right)\end{array}$ & $\begin{array}{c}\mathrm{PA}_{\mathrm{r}, \mathrm{b}} \\
\left({ }^{\circ}\right)\end{array}$ & $\begin{array}{c}D_{\mathrm{r}, \mathrm{b}, 0} \\
\left({ }^{\prime}\right)\end{array}$ & $\begin{array}{c}d_{\mathrm{r}, \mathrm{b}, 0} \\
\left({ }^{\prime}\right)\end{array}$ & $\begin{array}{c}\theta_{\mathrm{r}, \mathrm{b}} \\
\left({ }^{\circ}\right)\end{array}$ & Feature & $\begin{array}{c}D_{\mathrm{r}, \mathrm{b}} \\
\left({ }^{\prime}\right)\end{array}$ & $\begin{array}{c}d_{\mathrm{r}, \mathrm{b}} \\
\left({ }^{\prime}\right)\end{array}$ & $\begin{array}{c}\mathrm{PA}_{\mathrm{r}, \mathrm{b}} \\
\left({ }^{\circ}\right)\end{array}$ & $\begin{array}{c}D_{\mathrm{r}, \mathrm{b}, 0} \\
\left({ }^{\prime}\right)\end{array}$ & $\begin{array}{c}d_{\mathrm{r}, \mathrm{b}, 0} \\
\left({ }^{\prime}\right)\end{array}$ & $\begin{array}{c}\theta_{\mathrm{r}, \mathrm{b}} \\
\left({ }^{\circ}\right)\end{array}$ \\
\hline rs & 0.20 & 0.15 & 160.4 & 0.24 & 0.15 & 91.1 & $\mathrm{r}$ & 0.32 & 0.18 & 21.4 & 0.35 & 0.28 & 131.3 \\
bar & 0.14 & 0.09 & 51.4 & 0.15 & 0.11 & 154.5 & & & & & & & \\
\hline
\end{tabular}

UGC 6983

SB(r)s)dm pec

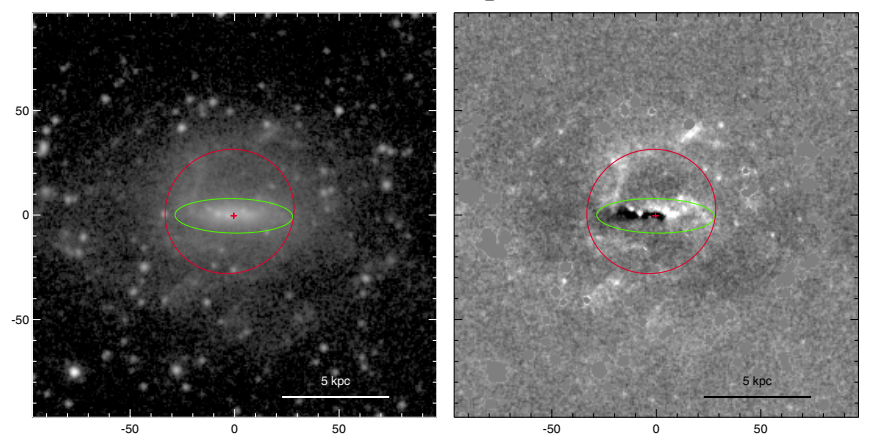

UGC 7184

SB(rs)d

\begin{tabular}{ccccccc|ccccccc}
\hline \hline Feature & $\begin{array}{c}D_{\mathrm{r}, \mathrm{b}} \\
\left({ }^{\prime}\right)\end{array}$ & $\begin{array}{c}d_{\mathrm{r}, \mathrm{b}} \\
\left({ }^{\prime}\right)\end{array}$ & $\begin{array}{c}\mathrm{PA}_{\mathrm{r}, \mathrm{b}} \\
\left({ }^{\circ}\right)\end{array}$ & $\begin{array}{c}D_{\mathrm{r}, \mathrm{b}, 0} \\
\left({ }^{\prime}\right)\end{array}$ & $\begin{array}{c}d_{\mathrm{r}, \mathrm{b}, 0} \\
\left({ }^{\prime}\right)\end{array}$ & $\begin{array}{c}\theta_{\mathrm{r}, \mathrm{b}} \\
\left({ }^{(}\right)\end{array}$ & Feature & $\begin{array}{c}D_{\mathrm{r}, \mathrm{b}} \\
\left({ }^{\prime}\right)\end{array}$ & $\begin{array}{c}d_{\mathrm{r}, \mathrm{b}} \\
\left({ }^{\prime}\right)\end{array}$ & $\begin{array}{c}\mathrm{PA}_{\mathrm{r}, \mathrm{b}} \\
\left({ }^{\circ}\right)\end{array}$ & $\begin{array}{c}D_{\mathrm{r}, \mathrm{b}, 0} \\
\left({ }^{\prime}\right)\end{array}$ & $\begin{array}{c}d_{\mathrm{r}, \mathrm{b}, 0} \\
\left({ }^{\prime}\right)\end{array}$ & $\begin{array}{c}\theta_{\mathrm{r}, \mathrm{b}} \\
\left({ }^{\circ}\right)\end{array}$ \\
\hline $\mathrm{r}$ & 1.04 & 0.99 & 113.3 & 1.51 & 1.03 & 86.9 & $\mathrm{rs}$ & 0.64 & 0.28 & 147.5 & 0.65 & 0.54 & 25.0 \\
$\mathrm{bar}$ & 0.94 & 0.28 & 89.0 & 0.94 & 0.42 & 3.5 & bar & 0.47 & 0.12 & 163.5 & 0.57 & 0.21 & 41.5 \\
\hline
\end{tabular}

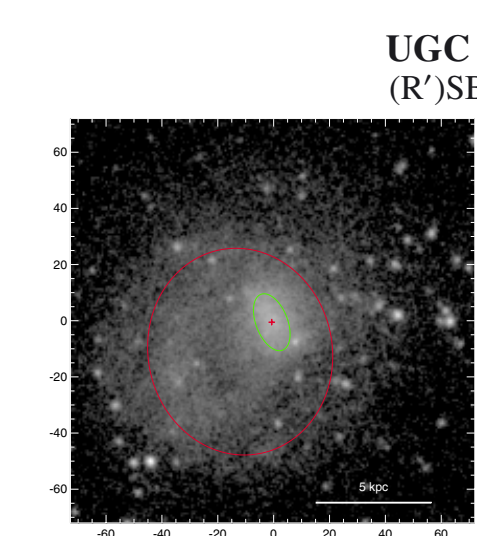

UGC 7239

$\left(\mathrm{R}^{\prime}\right) \mathrm{SB}(\mathrm{s}) \mathrm{m}$

UGC 7848

$\mathrm{SAB}_{\mathrm{a}}\left(\mathrm{s}, \mathrm{x}_{1} \mathrm{r}\right) \mathrm{bc}$
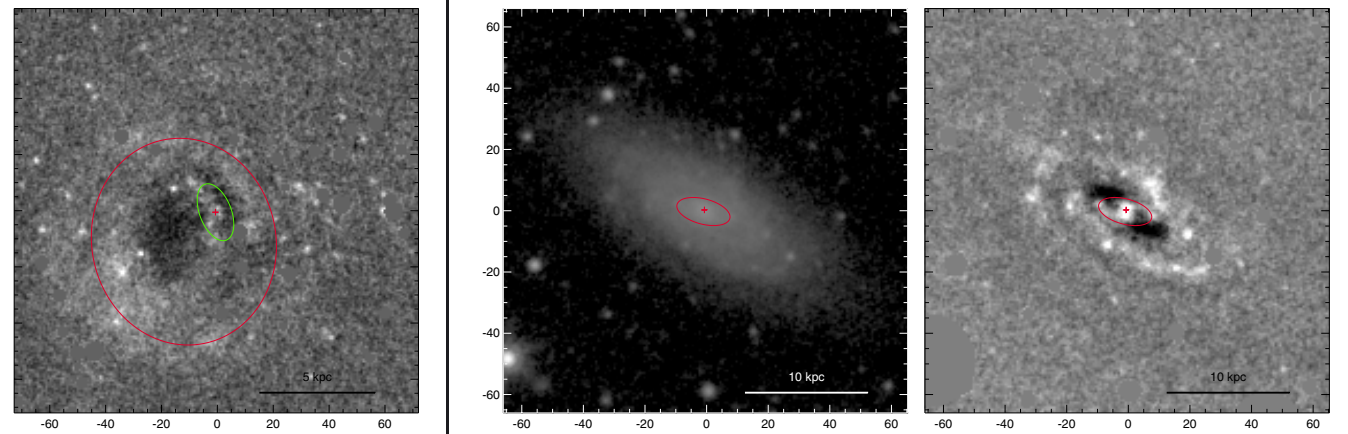

\begin{tabular}{ccccccc|ccccccc}
\hline \hline Feature & $\begin{array}{c}D_{\mathrm{r}, \mathrm{b}} \\
\left({ }^{\prime}\right)\end{array}$ & $\begin{array}{c}d_{\mathrm{r}, \mathrm{b}} \\
\left({ }^{\prime}\right)\end{array}$ & $\begin{array}{c}\mathrm{PA}_{\mathrm{r}, \mathrm{b}} \\
\left({ }^{\circ}\right)\end{array}$ & $\begin{array}{c}D_{\mathrm{r}, \mathrm{b}, 0} \\
\left({ }^{\prime}\right)\end{array}$ & $\begin{array}{c}d_{\mathrm{r}, \mathrm{b}, 0} \\
\left({ }^{\prime}\right)\end{array}$ & $\begin{array}{c}\theta_{\mathrm{r}, \mathrm{b}} \\
\left({ }^{(}\right)\end{array}$ & Feature & $\begin{array}{c}D_{\mathrm{r}, \mathrm{b}} \\
\left({ }^{\prime}\right)\end{array}$ & $\begin{array}{c}d_{\mathrm{r}, \mathrm{b}} \\
\left({ }^{\prime}\right)\end{array}$ & $\begin{array}{c}\mathrm{PA}_{\mathrm{r}, \mathrm{b}} \\
\left({ }^{\circ}\right)\end{array}$ & $\begin{array}{c}D_{\mathrm{r}, \mathrm{b}, 0} \\
\left({ }^{\prime}\right)\end{array}$ & $\begin{array}{c}d_{\mathrm{r}, \mathrm{b}, 0} \\
\left({ }^{\prime}\right)\end{array}$ & $\begin{array}{c}\theta_{\mathrm{r}, \mathrm{b}} \\
\left({ }^{\circ}\right)\end{array}$ \\
\hline $\mathrm{R}^{\prime}$ & 1.24 & 1.10 & 11.1 & 1.54 & 1.11 & 86.1 & $\mathrm{x}_{1} \mathrm{r}$ & 0.30 & 0.14 & 75.3 & 0.40 & 0.24 & 59.9 \\
$\mathrm{bar}$ & 0.36 & 0.19 & 21.3 & 0.45 & 0.19 & 89.2 & & & & & & & \\
\hline
\end{tabular}


UGC 8155

$\mathrm{SA}(\mathrm{rs}, 1) \mathrm{a}$
UGC 8909

$\mathrm{SAB}(\mathrm{rs}) \mathrm{c}$ :
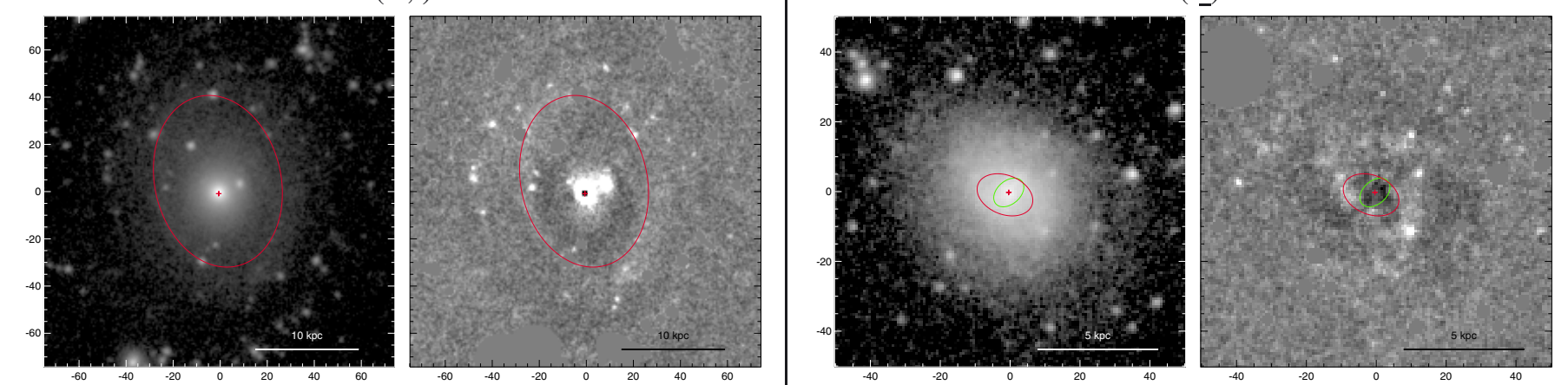

\begin{tabular}{ccccccc|ccccccc}
\hline \hline Feature & $\begin{array}{c}D_{\mathrm{r}, \mathrm{b}} \\
\left({ }^{\prime}\right)\end{array}$ & $\begin{array}{c}d_{\mathrm{r}, \mathrm{b}} \\
\left({ }^{\prime}\right)\end{array}$ & $\begin{array}{c}\mathrm{PA}_{\mathrm{r}, \mathrm{b}} \\
\left({ }^{\circ}\right)\end{array}$ & $\begin{array}{c}D_{\mathrm{r}, \mathrm{b}, 0} \\
\left({ }^{\prime}\right)\end{array}$ & $\begin{array}{c}d_{\mathrm{r}, \mathrm{b}, 0} \\
\left({ }^{\prime}\right)\end{array}$ & $\begin{array}{c}\theta_{\mathrm{r}, \mathrm{b}} \\
\left({ }^{\circ}\right)\end{array}$ & Feature & $\begin{array}{c}D_{\mathrm{r}, \mathrm{b}} \\
\left({ }^{\prime}\right)\end{array}$ & $\begin{array}{c}d_{\mathrm{r}, \mathrm{b}} \\
\left({ }^{\prime}\right)\end{array}$ & $\begin{array}{c}\mathrm{PA}_{\mathrm{r}, \mathrm{b}} \\
\left({ }^{\circ}\right)\end{array}$ & $\begin{array}{c}D_{\mathrm{r}, \mathrm{b}, 0} \\
\left({ }^{\prime}\right)\end{array}$ & $\begin{array}{c}d_{\mathrm{r}, \mathrm{b}, 0} \\
\left({ }^{\prime}\right)\end{array}$ & $\begin{array}{c}\theta_{\mathrm{r}, \mathrm{b}} \\
\left({ }^{\circ}\right)\end{array}$ \\
\hline $\mathrm{rs}$ & 1.24 & 0.90 & 12.8 & 1.24 & 1.13 & 172.4 & $\mathrm{rs}$ & 0.28 & 0.19 & 68.5 & 0.28 & 0.23 & 21.2 \\
& & & & & & & & & & & & &
\end{tabular}

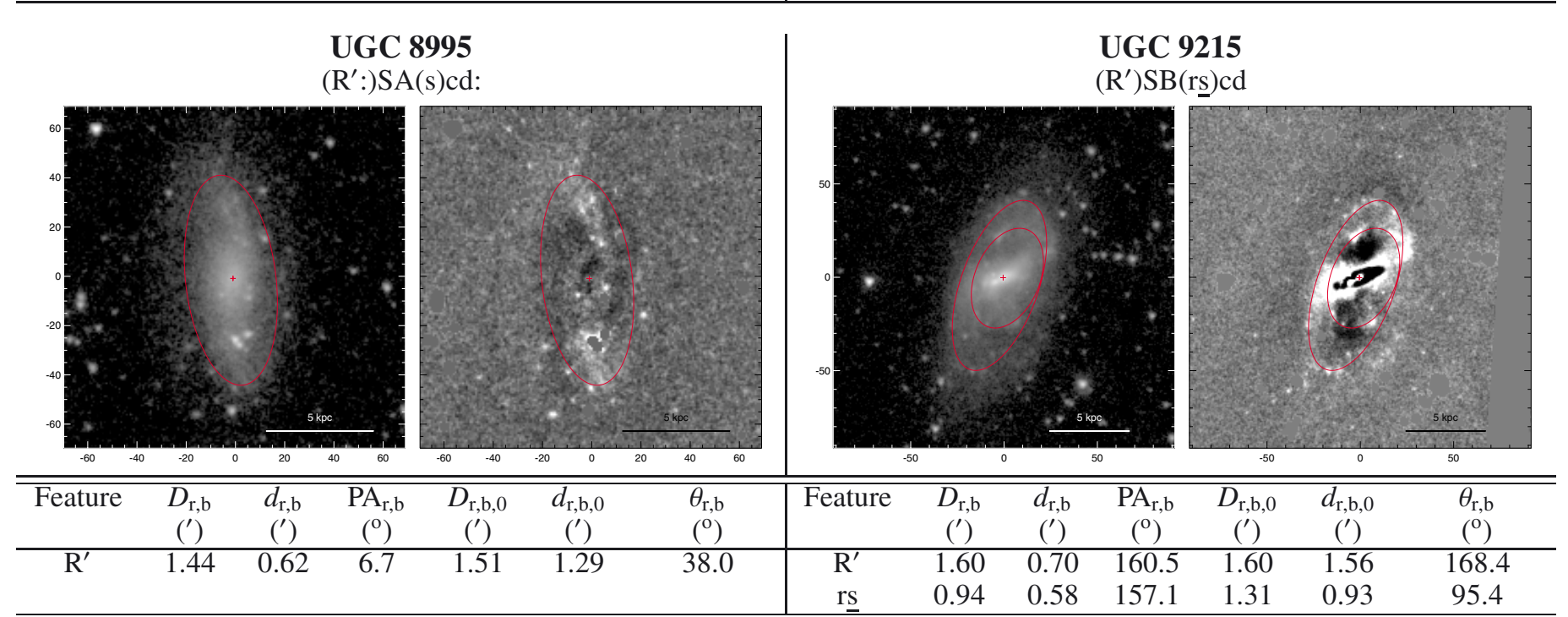

UGC 8995

UGC 9215

$\left(\mathrm{R}^{\prime}\right) \mathrm{SB}(\mathrm{rs}) \mathrm{cd}$

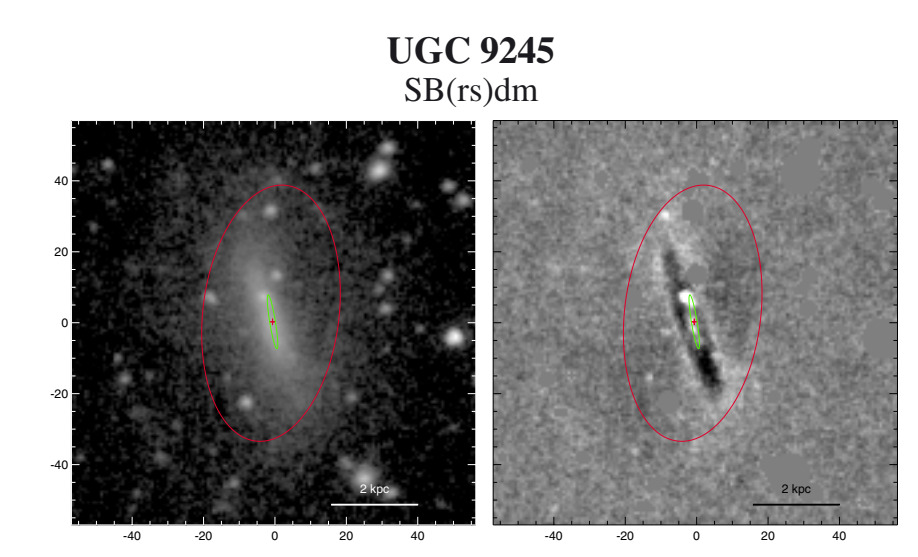

\section{UGC 9291}

$\mathrm{SAB}(\mathrm{rs}) \mathrm{bc}$

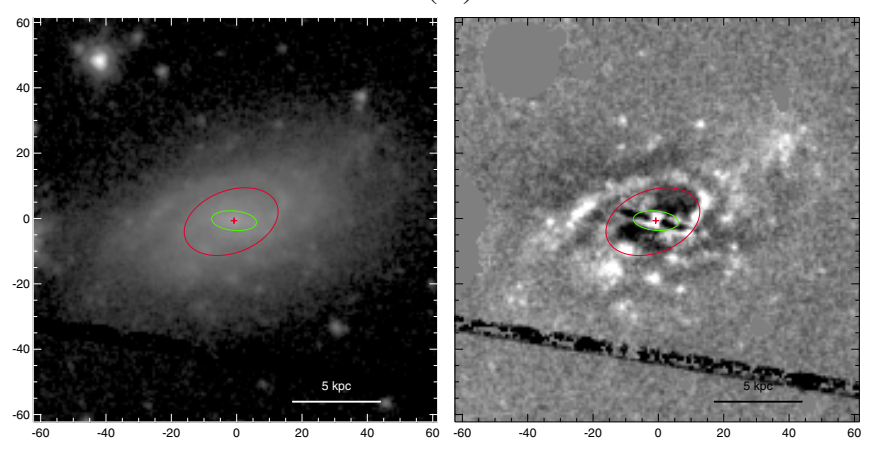

\begin{tabular}{ccccccc|ccccccc}
\hline \hline Feature & $\begin{array}{c}D_{\mathrm{r}, \mathrm{b}} \\
\left({ }^{\prime}\right)\end{array}$ & $\begin{array}{c}d_{\mathrm{r}, \mathrm{b}} \\
\left({ }^{\prime}\right)\end{array}$ & $\begin{array}{c}\mathrm{PA}_{\mathrm{r}, \mathrm{b}} \\
\left({ }^{\circ}\right)\end{array}$ & $\begin{array}{c}D_{\mathrm{r}, \mathrm{b}, 0} \\
\left({ }^{\prime}\right)\end{array}$ & $\begin{array}{c}d_{\mathrm{r}, \mathrm{b}, 0} \\
\left({ }^{\prime}\right)\end{array}$ & $\begin{array}{c}\theta_{\mathrm{r}, \mathrm{b}} \\
\left({ }^{(}\right)\end{array}$ & Feature & $\begin{array}{c}D_{\mathrm{r}, \mathrm{b}} \\
\left({ }^{\prime}\right)\end{array}$ & $\begin{array}{c}d_{\mathrm{r}, \mathrm{b}} \\
\left({ }^{\prime}\right)\end{array}$ & $\begin{array}{c}\mathrm{PA}_{\mathrm{r}, \mathrm{b}} \\
\left({ }^{\circ}\right)\end{array}$ & $\begin{array}{c}D_{\mathrm{r}, \mathrm{b}, 0} \\
\left({ }^{\prime}\right)\end{array}$ & $\begin{array}{c}d_{\mathrm{r}, \mathrm{b}, 0} \\
\left({ }^{\prime}\right)\end{array}$ & $\begin{array}{c}\theta_{\mathrm{r}, \mathrm{b}} \\
\left({ }^{\circ}\right)\end{array}$ \\
\hline rs & 1.21 & 0.64 & 173.2 & 1.21 & 0.88 & 171.7 & $\mathrm{rs}$ & 0.50 & 0.32 & 110.9 & 0.59 & 0.49 & 71.3 \\
bar & 0.26 & 0.03 & 9.3 & 0.26 & 0.04 & 16.6 & bar & 0.23 & 0.10 & 84.7 & 0.27 & 0.16 & 136.4 \\
\hline
\end{tabular}


UGC 9356

( $\left.\mathrm{R}^{\prime} \mathrm{L}\right) \mathrm{SAB}(\mathrm{rs}) \mathrm{bc}$ :

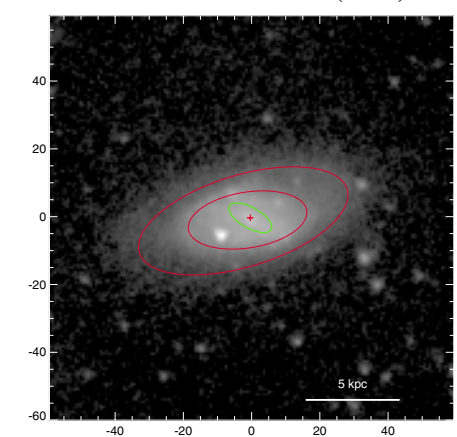

UGC 9364

$\left(\mathrm{R}^{\prime}\right) \mathrm{SA}(\mathrm{s}) \mathrm{cd}$

\begin{tabular}{|c|c|c|c|c|c|c|c|c|c|c|c|c|c|}
\hline Feature & $\begin{array}{c}D_{\mathrm{r}, \mathrm{b}} \\
\left({ }^{\prime}\right)\end{array}$ & $\begin{array}{c}d_{\mathrm{r}, \mathrm{b}} \\
\left({ }^{\prime}\right)\end{array}$ & $\begin{array}{c}\mathrm{PA}_{\mathrm{r}, \mathrm{b}} \\
\left({ }^{\circ}\right)\end{array}$ & $\begin{array}{c}D_{\mathrm{r}, \mathrm{b}, 0} \\
\left({ }^{\prime}\right)\end{array}$ & $\begin{array}{c}d_{\mathrm{r}, \mathrm{b}, 0} \\
\left({ }^{\prime}\right)\end{array}$ & $\begin{array}{l}\theta_{\mathrm{r}, \mathrm{b}} \\
\left({ }^{\circ}\right)\end{array}$ & Feature & $\begin{array}{c}D_{\mathrm{r}, \mathrm{b}} \\
\left({ }^{\prime}\right)\end{array}$ & $\begin{array}{c}d_{\mathrm{r}, \mathrm{b}} \\
\left({ }^{\prime}\right)\end{array}$ & $\begin{array}{c}\mathrm{PA}_{\mathrm{r}, \mathrm{b}} \\
\left({ }^{\circ}\right)\end{array}$ & $\begin{array}{c}D_{\mathrm{r}, \mathrm{b}, 0} \\
\left(^{\prime}\right)\end{array}$ & $\begin{array}{c}d_{\mathrm{r}, \mathrm{b}, 0} \\
\left({ }^{\prime}\right)\end{array}$ & $\begin{array}{l}\theta_{\mathrm{r}, \mathrm{b}} \\
\left({ }^{\circ}\right)\end{array}$ \\
\hline$\overline{\mathrm{R}^{\prime} \mathrm{L}}$ & 1.07 & 0.45 & 107.0 & 1.08 & 0.86 & 13.6 & $\mathrm{R}^{\prime}$ & 0.91 & 0.40 & 17.8 & 0.92 & 0.81 & 164.8 \\
\hline rs & 0.59 & 0.27 & 99.2 & 0.61 & 0.51 & 152.9 & & & & & & & \\
\hline bar & 0.24 & 0.10 & 60.8 & 0.37 & 0.12 & 113.4 & & & & & & & \\
\hline
\end{tabular}
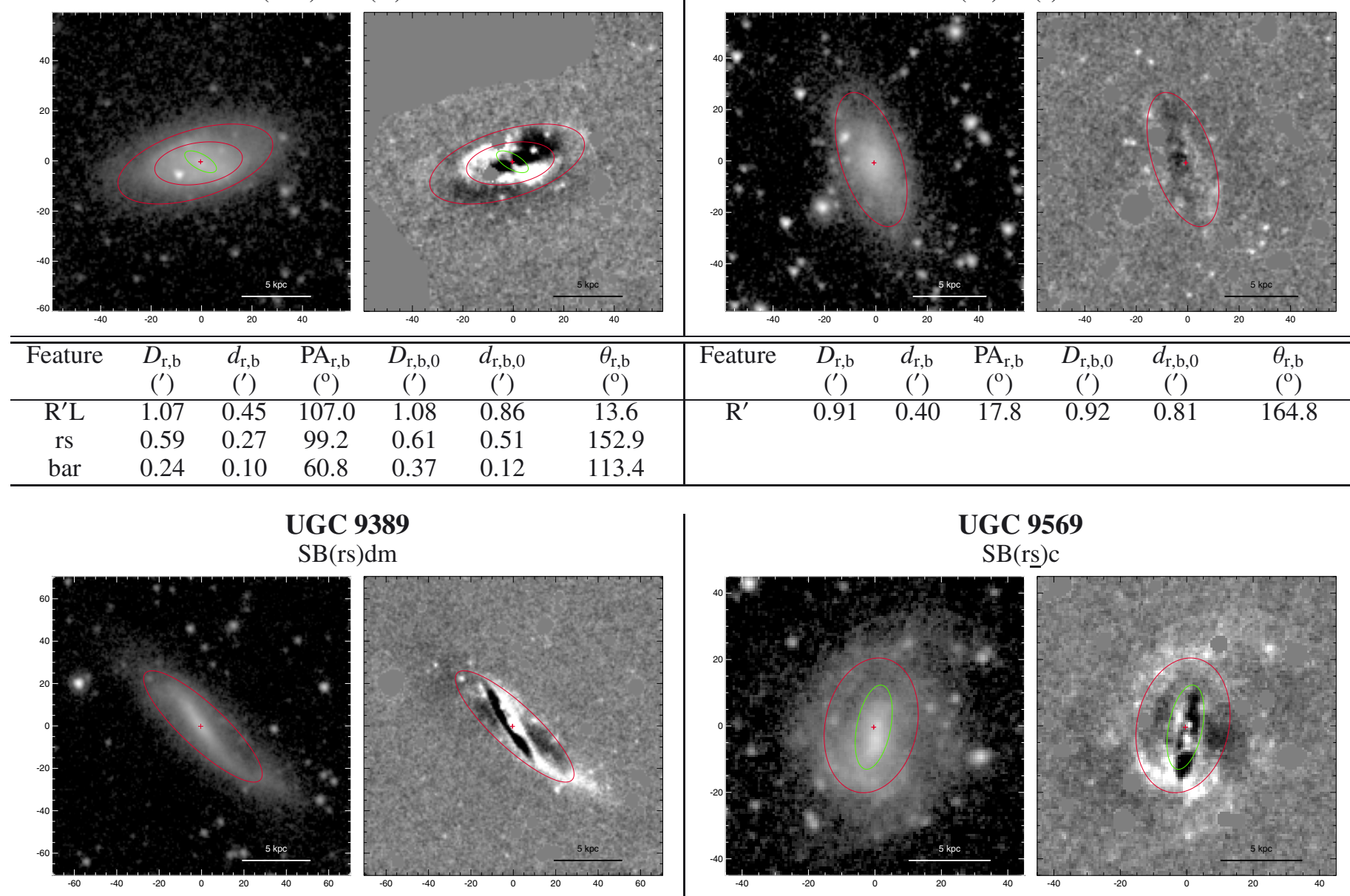

UGC 9569

$\mathrm{SB}(\mathrm{r} \underline{\mathrm{r}}) \mathrm{c}$

\begin{tabular}{ccccccc|ccccccc}
\hline \hline Feature & $\begin{array}{c}D_{\mathrm{r}, \mathrm{b}} \\
\left({ }^{\prime}\right)\end{array}$ & $\begin{array}{c}d_{\mathrm{r}, \mathrm{b}} \\
\left({ }^{\prime}\right)\end{array}$ & $\begin{array}{c}\mathrm{PA}_{\mathrm{r}, \mathrm{b}} \\
\left({ }^{\circ}\right)\end{array}$ & $\begin{array}{c}D_{\mathrm{r}, \mathrm{b}, 0} \\
\left({ }^{\prime}\right)\end{array}$ & $\begin{array}{c}d_{\mathrm{r}, \mathrm{b}, 0} \\
\left({ }^{\prime}\right)\end{array}$ & $\begin{array}{c}\theta_{\mathrm{r}, \mathrm{b}} \\
\left({ }^{\circ}\right)\end{array}$ & Feature & $\begin{array}{c}D_{\mathrm{r}, \mathrm{b}} \\
\left({ }^{\prime}\right)\end{array}$ & $\begin{array}{c}d_{\mathrm{r}, \mathrm{b}} \\
\left({ }^{\prime}\right)\end{array}$ & $\begin{array}{c}\mathrm{PA}_{\mathrm{r}, \mathrm{b}} \\
\left({ }^{\circ}\right)\end{array}$ & $\begin{array}{c}D_{\mathrm{r}, \mathrm{b}, 0} \\
\left({ }^{\prime}\right)\end{array}$ & $\begin{array}{c}d_{\mathrm{r}, \mathrm{b}, 0} \\
\left({ }^{\prime}\right)\end{array}$ & $\begin{array}{c}\theta_{\mathrm{r}, \mathrm{b}} \\
\left({ }^{\circ}\right)\end{array}$ \\
\hline $\mathrm{rs}$ & 1.24 & 0.34 & 47.1 & 1.27 & 1.21 & 136.5 & $\mathrm{rs}$ & 0.69 & 0.46 & 168.1 & 0.80 & 0.51 & 60.3 \\
& & & & & & & $\mathrm{bar}$ & 0.43 & 0.17 & 168.8 & 0.50 & 0.19 & 53.3 \\
\hline
\end{tabular}

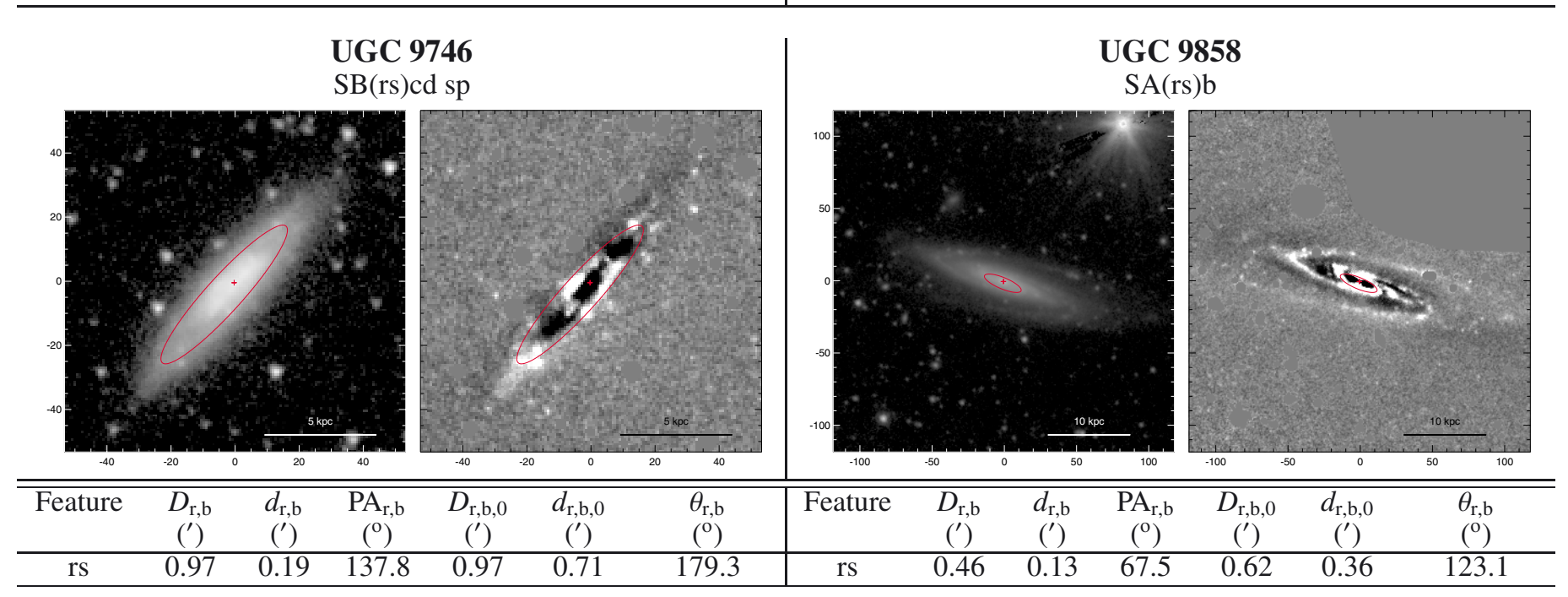


S. Comerón et al.: ARRAKIS

\section{UGC 10054}

$\mathrm{SB}(\mathrm{rs}) \mathrm{d}$
UGC 10791

$\mathrm{SB}(\mathrm{rs}) \mathrm{d}$
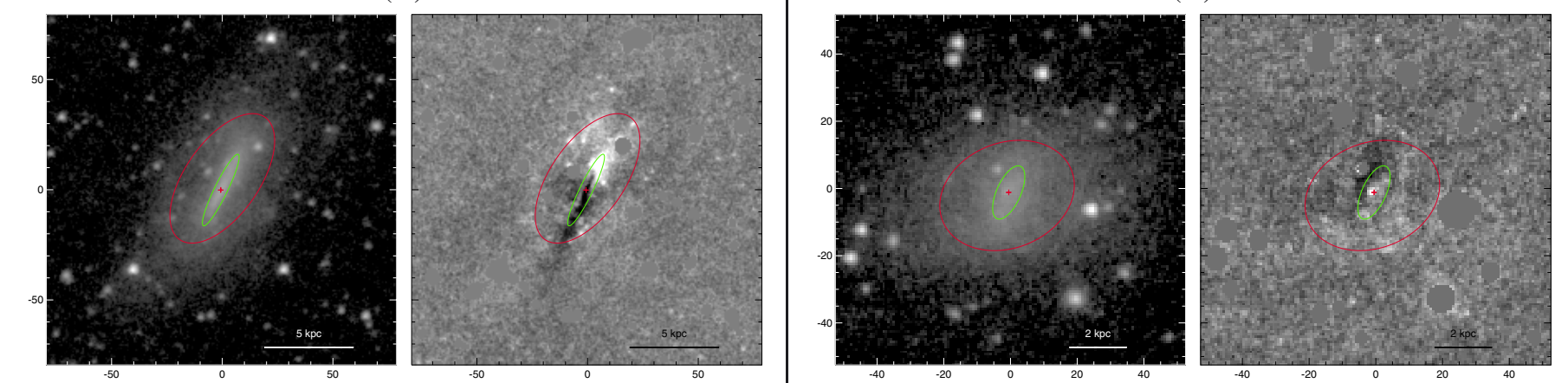

\begin{tabular}{|c|c|c|c|c|c|c|c|c|c|c|c|c|c|}
\hline Feature & $\begin{array}{c}D_{\mathrm{r}, \mathrm{b}} \\
\left({ }^{\prime}\right)\end{array}$ & $\begin{array}{c}d_{\mathrm{r}, \mathrm{b}} \\
\left({ }^{\prime}\right)\end{array}$ & $\begin{array}{c}\mathrm{PA}_{\mathrm{r}, \mathrm{b}} \\
\left({ }^{\circ}\right)\end{array}$ & $\begin{array}{c}D_{\mathrm{r}, \mathrm{b}, 0} \\
\left({ }^{\prime}\right)\end{array}$ & $\begin{array}{c}d_{\mathrm{r}, \mathrm{b}, 0} \\
\left(^{\prime}\right)\end{array}$ & $\begin{array}{l}\theta_{\mathrm{r}, \mathrm{b}} \\
\left({ }^{\circ}\right)\end{array}$ & Feature & $\begin{array}{c}D_{\mathrm{r}, \mathrm{b}} \\
\left({ }^{\prime}\right)\end{array}$ & $\begin{array}{c}d_{\mathrm{r}, \mathrm{b}} \\
\left({ }^{\prime}\right)\end{array}$ & $\begin{array}{c}\mathrm{PA}_{\mathrm{r}, \mathrm{b}} \\
\left({ }^{\circ}\right)\end{array}$ & $\begin{array}{c}D_{\mathrm{r}, \mathrm{b}, 0} \\
\left({ }^{\prime}\right)\end{array}$ & $\begin{array}{c}d_{\mathrm{r}, \mathrm{b}, 0} \\
\left(^{\prime}\right)\end{array}$ & $\begin{array}{c}\theta_{\mathrm{r}, \mathrm{b}} \\
\left({ }^{\circ}\right)\end{array}$ \\
\hline rs & 1.13 & 0.56 & 145.1 & 1.15 & 1.04 & 27.7 & rs & 0.70 & 0.53 & 111.4 & 0.86 & 0.68 & 75.5 \\
\hline bar & 0.60 & 0.10 & 154.0 & 0.64 & 0.18 & 23.4 & bar & 0.29 & 0.12 & 155.9 & 0.42 & 0.13 & 70.0 \\
\hline
\end{tabular}

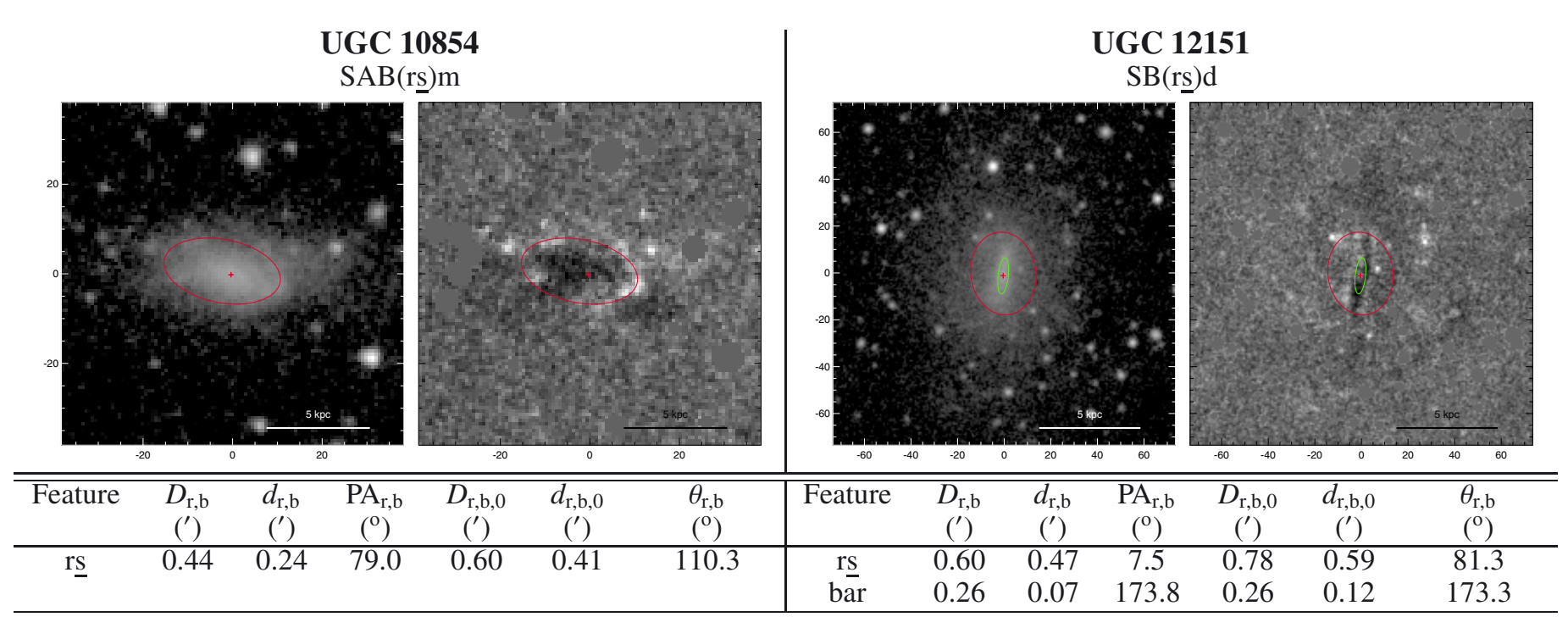




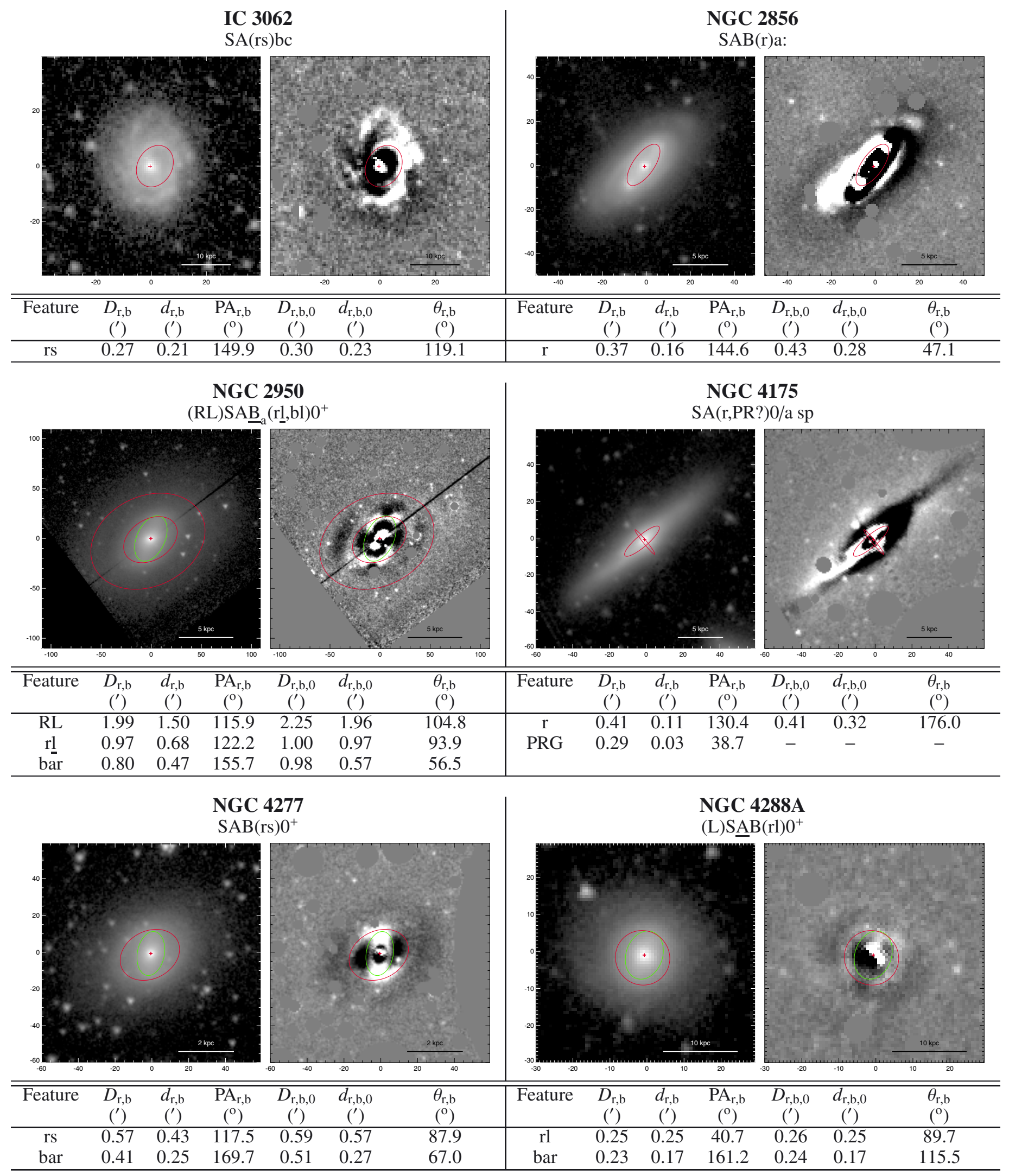


NGC 4342

$\mathrm{S}^{-} \mathrm{sp}+\mathrm{vF}$ PR?
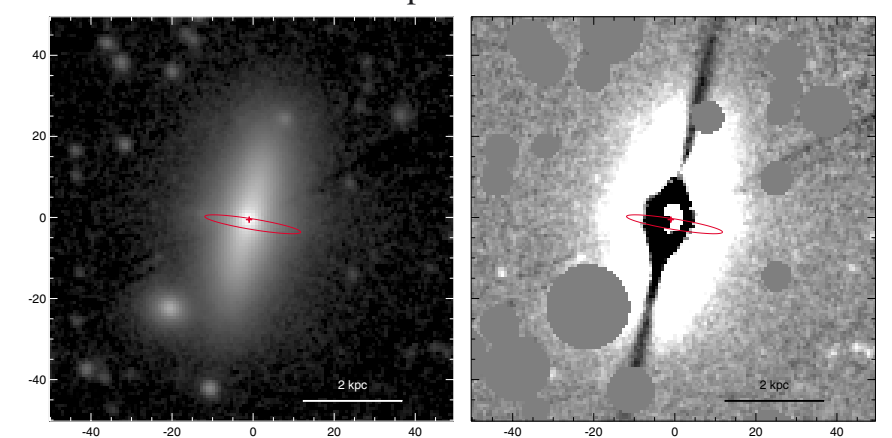

\begin{tabular}{ccccccc|ccccccc}
\hline \hline Feature & $\begin{array}{c}D_{\mathrm{r}, \mathrm{b}} \\
\left({ }^{\prime}\right)\end{array}$ & $\begin{array}{c}d_{\mathrm{r}, \mathrm{b}} \\
\left({ }^{\prime}\right)\end{array}$ & $\begin{array}{c}\mathrm{PA}_{\mathrm{r}, \mathrm{b}} \\
\left({ }^{\circ}\right)\end{array}$ & $\begin{array}{c}D_{\mathrm{r}, \mathrm{b}, 0} \\
\left(^{\prime}\right)\end{array}$ & $\begin{array}{c}d_{\mathrm{r}, \mathrm{b}, 0} \\
\left({ }^{\prime}\right)\end{array}$ & $\begin{array}{c}\theta_{\mathrm{r}, \mathrm{b}} \\
\left({ }^{\circ}\right)\end{array}$ & Feature & $\begin{array}{c}D_{\mathrm{r}, \mathrm{b}} \\
\left({ }^{\circ}\right)\end{array}$ & $\begin{array}{c}d_{\mathrm{r}, \mathrm{b}} \\
\left({ }^{\prime}\right)\end{array}$ & $\begin{array}{c}\mathrm{PA}_{\mathrm{r}, \mathrm{b}} \\
\left({ }^{\circ}\right)\end{array}$ & $\begin{array}{c}D_{\mathrm{r}, \mathrm{b}, 0} \\
\left({ }^{\prime}\right)\end{array}$ & $\begin{array}{c}d_{\mathrm{r}, \mathrm{b}, 0} \\
\left({ }^{\prime}\right)\end{array}$ & $\begin{array}{c}\theta_{\mathrm{r}, \mathrm{b}} \\
\left({ }^{\circ}\right)\end{array}$ \\
\hline PRG & 0.40 & 0.04 & 80.7 & - & - & - & $\mathrm{R}_{1}^{\prime}$ & 0.80 & 0.40 & 108.9 & 0.81 & 0.60 & 169.2 \\
& & & & & & & $\mathrm{bar}$ & 0.16 & 0.13 & 18.8 & 0.25 & 0.13 & 88.7 \\
\hline
\end{tabular}

PGC 43690

$\left(\mathrm{R}_{1}^{\prime}\right) \mathrm{SAB}_{\mathrm{a}}(\mathrm{ls}) \mathrm{a}$

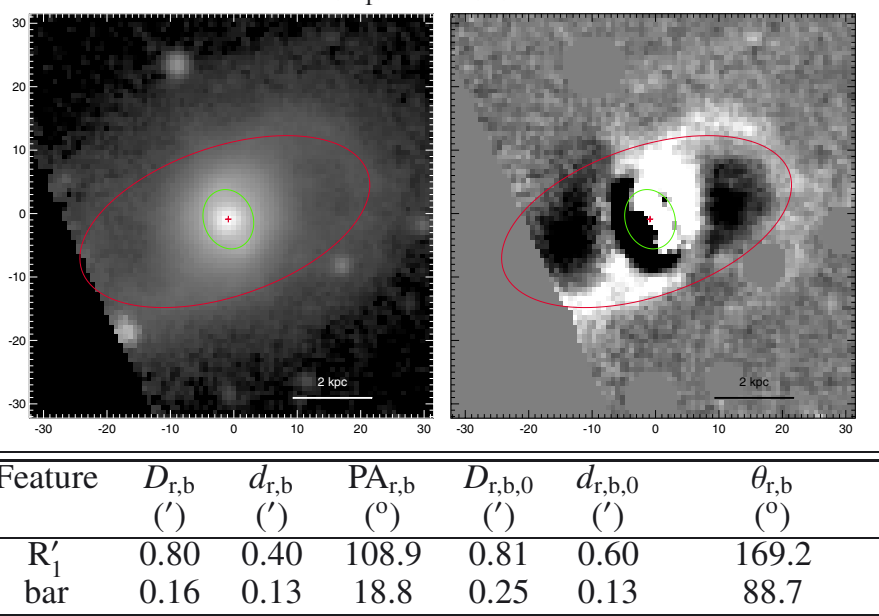

PGC 52269

(R)SB0 ${ }^{+}$

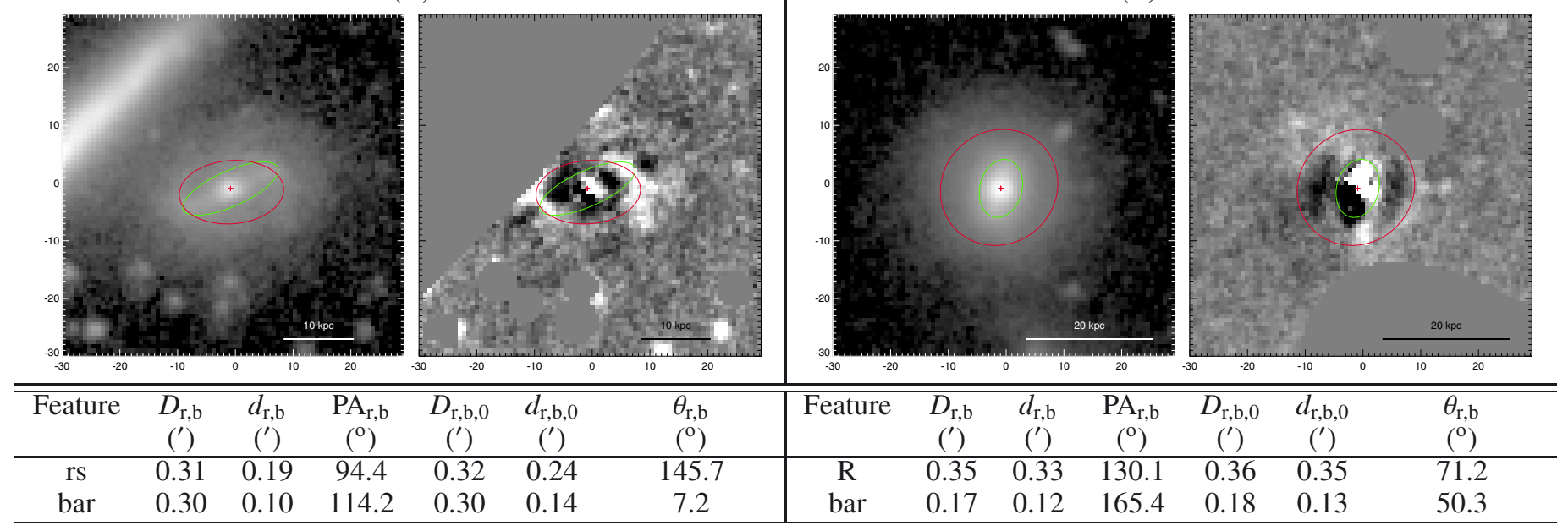

PGC 59663

$\left(\mathrm{R}^{\prime}\right) \mathrm{Sa} \mathrm{sp}$

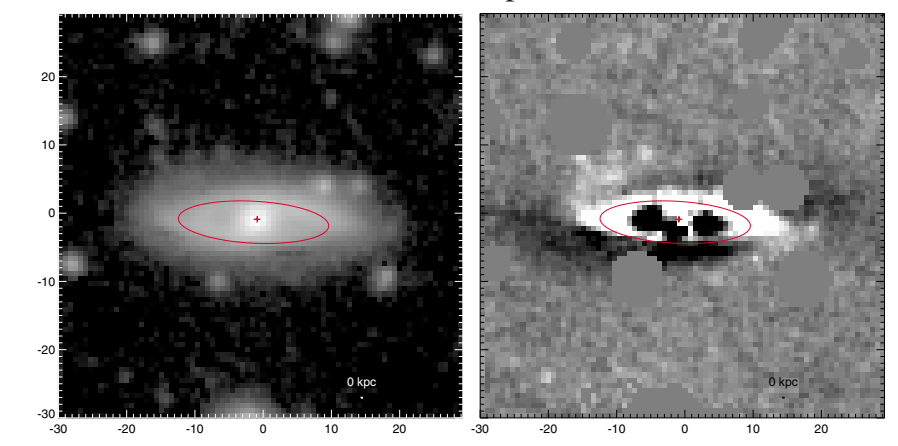

\begin{tabular}{ccccccc|l}
\hline \hline Feature & $\begin{array}{c}D_{\mathrm{r}, \mathrm{b}} \\
\left({ }^{\prime}\right)\end{array}$ & $\begin{array}{c}d_{\mathrm{r}, \mathrm{b}} \\
\left({ }^{\prime}\right)\end{array}$ & $\begin{array}{c}\mathrm{PA}_{\mathrm{r}, \mathrm{b}} \\
\left({ }^{\circ}\right)\end{array}$ & $\begin{array}{c}D_{\mathrm{r}, \mathrm{b}, 0} \\
\left({ }^{\prime}\right)\end{array}$ & $\begin{array}{c}d_{\mathrm{r}, \mathrm{b}, 0} \\
\left({ }^{\prime}\right)\end{array}$ & $\begin{array}{c}\theta_{\mathrm{r}, \mathrm{b}} \\
\left({ }^{\circ}\right)\end{array}$ & \\
\hline $\mathrm{R}^{\prime}$ & 0.37 & 0.10 & 87.0 & 0.41 & 0.29 & 141.9 & \\
\hline
\end{tabular}




\section{Appendix C: Double NGC and IC identifications}

Table C.1. List of ARRAKIS galaxies with a double NGC/IC identification

\begin{tabular}{|c|c|c|c|}
\hline ID used in the $S^{4} G$ & Alternative ID & ID used in the $S^{4} G$ & Alternative ID \\
\hline IC 1826 & IC 1830 & NGC 4336 & IC 3254 \\
\hline IC 3102 & NGC 4223 & NGC 4342 & IC 3256 \\
\hline NGC 723 & NGC 724 & NGC 4351 & NGC 4354 \\
\hline NGC 755 & NGC 763 & NGC 4355 & NGC 4418 \\
\hline NGC 1291 & NGC 1269 & NGC 4405 & IC 788 \\
\hline NGC 1367 & NGC 1371 & NGC 4413 & NGC 4407 \\
\hline NGC 1415 & IC 1983 & NGC 4461 & NGC 4443 \\
\hline NGC 1425 & IC 1988 & NGC 4492 & IC 3438 \\
\hline NGC 1436 & NGC 1437 & NGC 4591 & NGC 4577 \\
\hline NGC 1448 & NGC 1457 & NGC 4618 & IC 3667 \\
\hline NGC 1452 & NGC 1455 & NGC 4625 & IC 3675 \\
\hline NGC 2604 & NGC 2604A & NGC 4722 & IC 3833 \\
\hline NGC 2974 & NGC 2652 & NGC 4845 & NGC 4910 \\
\hline NGC 3301 & NGC 3760 & NGC 4961 & NGC 4960 \\
\hline NGC 3321 & NGC 3322 & NGC 5334 & IC 4338 \\
\hline NGC 3389 & NGC 3373 & NGC 5364 & NGC 5317 \\
\hline NGC 3611 & NGC 3604 & NGC 5371 & NGC 5390 \\
\hline NGC 3626 & NGC 3632 & NGC 5375 & NGC 5396 \\
\hline NGC 3730 & NGC 3732 & NGC 5964 & IC 4551 \\
\hline NGC 4045 & NGC 4046 & NGC 6014 & IC 4586 \\
\hline NGC 4178 & IC 3042 & NGC 6902 & IC 4948 \\
\hline NGC 4189 & IC 3050 & NGC 6923 & IC 5004 \\
\hline NGC 4193 & IC 3051 & NGC 6925 & IC 5015 \\
\hline NGC 4212 & NGC 4208 & NGC 7140 & NGC 7141 \\
\hline NGC 4310 & NGC 4338 & NGC 7552 & IC 5294 \\
\hline NGC 4319 & NGC 4345 & & \\
\hline
\end{tabular}

Notes. The sources used for compiling this table are The Historically Corrected New General Catalogue (HCNGC), NED, and HyperLeda. All double identifications are confirmed by two of those sources except that for NGC 3730 and NGC 3732 (only reported in HyperLeda) and NGC 2974 and NGC 2652 (only reported in the HCNGC). 